\title{
DOE/AL/62350-2IF-Rev.I-Vol, 4
}

UNITED STATES DEPARTMENT OF ENERGY

Albuquerque, New Mexico

\section{Uranium Mill Tailings \\ Remedial Action Project \\ (UMTRAP) \\ Silick Rock, Colorado}

Calculations

Final Design for Construction

Volume IV

$0802 \times 3$

\section{$03 \mathrm{TI}$}

September 1995

\section{(40) \\ MORRISON KNUDSEN CORPORATION \\ ENVIRONMENTAL/GOVERNMENT GROUP}




\section{DISCLAIMER}

This report was prepared as an account of work sponsored by an agency of the United States Government. Neither the United States Government nor any agency thereof, nor any of their employees, makes any warranty, express or implied, or assumes any legal liability or responsibility for the accuracy, completeness, or usefulness of any information, apparatus, product, or process disclosed, or represents that its use would not infringe privately owned rights. Reference herein to any specific commercial product, process, or service by trade name, trademark, manufacturer, or otherwise does not necessarily constitute or imply its endorsement, recommendation, or favoring by the United States Government or any agency thereof. The views and opinions of authors expressed herein do not necessarily state or reflect those of the United States Government or any agency thereof. 


\section{DISCLAIMER}

Portions of this document may be illegible in electronic image products. Images are produced from the best available original document. 


\section{UMTRA PROJECT - SLICK ROCK}

\section{FINAL DESIGN FOR REVIEW}

\section{CALCULATION INDEX}

(Sheet 1 of 3)

Calculation No. Title

\section{VOLUME I}

11-250-04-04 Embankment Design - Embankment MateriaT Properties

11-221-02-01 UC Site - Bedrock Contours

11-270-01-00 Vicinity Properties - Origin of Contamination

11-212-01-03 NC and UC Sites Contaminated Materials Excavation Quantities

11-205-01-02 Demo7ition Debris - Quantity Estimate

\section{VOLUME II}

11-310-01-01 Embankment Design - Slope Stability Analysis

11-310-02-00 Embankment Design - Excavation Stability

11-323-01-02 Embankment Design - Sett7ement and Cover Cracking Analysis

11-275-01-00 Radon Barrier Design - Statistical Analysis of Ra-226 Concentrations for North Continent and Union Carbide Sites

11-340-01-00 Radon Barrier Design - RAECOM Input Data

11-340-02-00 Radon Barrier Design - Design Thickness

11-321-03-01 Cover Design - Frost Penetration Depth 


\section{UMTRA PROJECT - SLICK ROCK \\ FINAL DESIGN FOR REVIEW \\ CALCULATION INDEX}

(Sheet 2 of 3 )

Calculation No.

Title

\section{VOLUME III}

11-209-01-01

Site Hydrology - Rainfall Intensity, Duration, and Frequency Relations

11-315-01-00 Site Hydrology - Probable Maximum Precipitation

11-231-02-02 Erosion Protection - Rock Quality Evaluation [Riprap Types A, B and C]

11-322-01-01 Erosion Protection - Embankment Top and Side STope

11-322-02-02 Erosion Protection - Embankment Toe Apron

11-322-03-00 Erosion Protection - Gradations and Layer Thicknesses

11-314-01-00 UC Site - Temporary Drainage Ditch Design

11-211-01-02 UC Site - Retention Basin Sediment Volume

11-211-02-01 UC Site - Retention Basin Sizing

11-314-02-01 BC Site Temporary Drainage Temporary Drainage Facilities

11-314-03-00 UC Site Temporary Drainage - Water Balance 


\section{UMTRA PROJECT - SLICK ROCK}

FINAL DESIGN FOR REVIEW

CALCULATION INDEX

(Sheet 3 of 3 )

Calculation No. Title

\section{VOLUME IV}

11-324-01-01 Borrow Areas - Site Evaluation

11-330-02-02 Temporary Facilities - Material

Quantities

11-333-01-02 Embankment Quantities - Excavation and Cover Materials

11-333-02-00 BC Site Excavation Quantities - Rippable and Unrippable Materials

11-330-01-03 Site Restoration - Earthwork Quantities and Seeding

11-334-01-02 . Bid ScheduTe Quantities and Material Balance 
CONTRACT NO.

3885-76

DISCIPLINE

Geotech

NO. OF SHEETS (Includes cover sheet) Revol.1

PROJECT

\section{UMTRA - SLICK ROCK}

FEATURE

BORROW AREAS

ITEM

SITE EVALUATION

SOURCES OF DATA

(see below)

\section{SOURCES OF FORMULAE \& REFERENCES}

1. MKES UMTRA-SRK, "Borrow Area Site Plan," Dwg. No. SRK-GE-10-0304.

2. Lambert \& Associates, "Preliminary Boring Logs," MKES Doc. No. 5057-SRK-L-09-00463-00, Letter dated November 10, 1987.

3. U.S. DOE, "Remedial Action Plan \& Site Conceptual Design for Stabilization of the Inactive Uranium Mill Tailings Site @ Slick Rock, Colorado," MKES Doc. No. 5057-SRK-R-03-00041-00, Draft Vol. II, April 1987.

4. MKES UMTRA-SRK, "Dolores River Borrow Site Evaluation," MKES Doc. No. 3885-SRK-R-01-02348-00, January 1993.

PRELIMINARY CALC. $\square \quad$ FINAL CALC. \ SUPERSEDES CALC. NO. $11-231-01-01$

\begin{tabular}{|c|c|c|c|c|c|c|c|}
\hline & & & & & & & \\
\hline 01 & Seesheet IA & Argaymay & $09 / 05 / 95$ & Alims. Ranc- & $9 / 13 / 25$ & A.4 & $9 / 13 / 5$ \\
\hline 00 & & Wolind W.Wmb & $2 / 3 \sqrt[93]{2}$ & Ali M. Banani & $2-8-93$ & $p+4$ Co & $4 / 27 / 9$ \\
\hline $\begin{array}{l}\text { REV. } \\
\text { NO. }\end{array}$ & REVISION & BALCULATION & DATE & $\begin{array}{c}\text { CHECKED } \\
\text { BY }\end{array}$ & DATE & $\begin{array}{c}\text { APPROVED } \\
\text { BY }\end{array}$ & ATE \\
\hline
\end{tabular}


Contract No. $\quad 3885-76$ Designed Checked BYW $A M B$
Sheet File No.

Date Date
$02 / 03 / 93$ $2-8-83$

Table of Contents

Sheet No.

1.0 SUMMARY OF RESULTS $\ldots \ldots \ldots \ldots \ldots \ldots \ldots \ldots \ldots \ldots$

2.0 PURPOSE $\ldots \ldots \ldots \ldots \ldots \ldots \ldots \ldots \ldots \ldots \ldots \ldots \ldots \ldots$

3.0 METHOD $\ldots \ldots \ldots \ldots \ldots \ldots \ldots \ldots \ldots \ldots \ldots \ldots \ldots \ldots \ldots \ldots \ldots$

4.0 DOLORES RIVER BORROW SITE $\ldots \ldots \ldots \ldots \ldots \ldots \ldots$

5.0 DISAPPOINTMENT VALLEY BORROW SITE $\ldots \ldots \ldots \ldots \ldots \ldots$ 
thet paraqumay $L$ frals ipanourgy 925't 5ta245

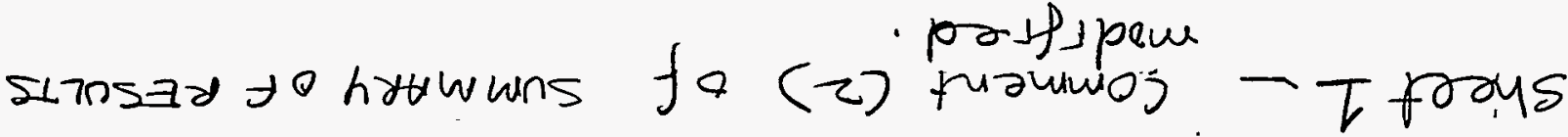
i wasing to $5 / 10+2 C$

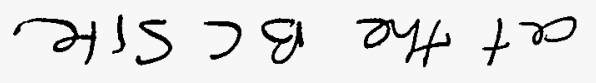

pos losodsip ayt to noltongra zyt moy prunano

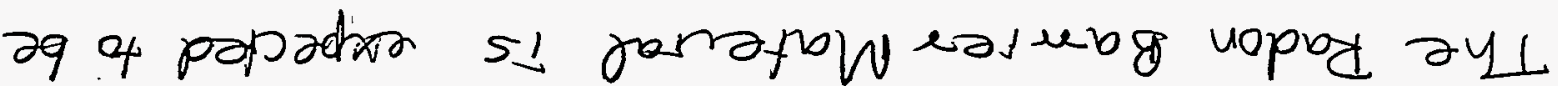
polrafin lameg nopord te asunos

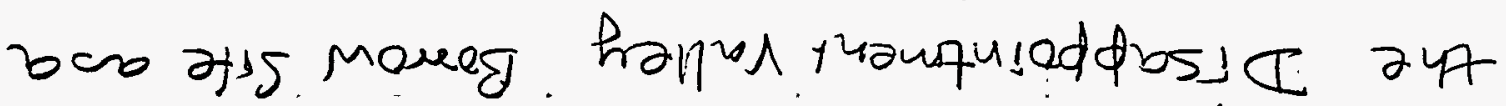

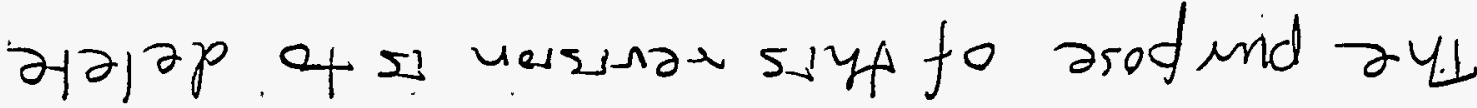

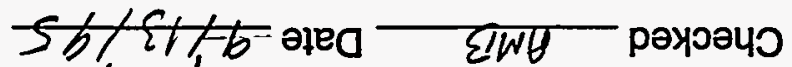

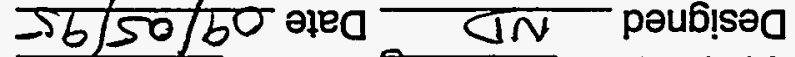

$$
\langle 1 \text { nossinay }
$$

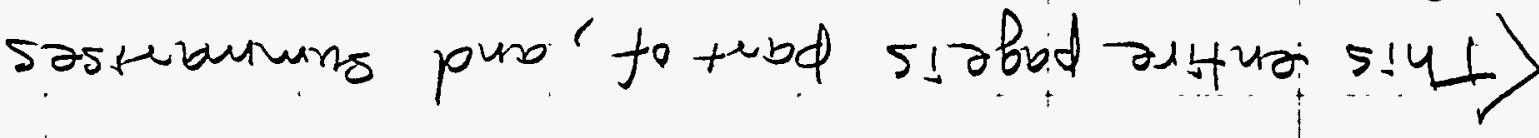




Contract No. $\frac{3885-76}{B Y W}$
Designed
Checked $A M B$

Sheet $\frac{1 B}{\text { File No. } \frac{}{02 / 03 / 93}}$
Date $\frac{2-8-9 ?}{\text { Date }}$

\subsection{SUMMARY OF RESULTS}

(1) For riprap types A, B, \& C, and bedding, the material will come from the Dolores River borrow site. The material available at this site is approximately 300,000 cubic yards.

Reference 4 shows that there will be about $55 \%$ material wastage at the Dolores River borrow site. Even with this $55 \%$ wastage there is twice the required amount of material for riprap types A, B, C, \& bedding (Ref. 4). To reduce the $55 \%$ wastage at the Dolores River borrow site, large size ripraps from the U.C. site can be combined with the Dolores River borrow material (Ref. $1 \& 4$ ).

(2) Raeton barrier material will-come from the +Ejsappointment Valley borrow area. This site-has in excess of 500,000 cubic yards of material within the

C areer bortow area-boundaries and over 300,000 cubic yarts

boundaries.

\subsection{PURPOSE}

The purpose of this calculation is to determine the amount of material available at the following Borrow sites:

(1) Dolores River Borrow Site (Type A, B, \& C riprap, and bedding material)

(2) Disappointment Valley Borrow Site (Radon barrier material)

\subsection{METHOD}

The average depths of available material at each site is established using Boring data from references $2 \& 3$. The amount of material is computed by estimating the plan area and multiplying this value with the average depths of material.

Revol ND 9/5/95

(2) Radon "barrier material will come from the excavation of the disposal cell at BC site. 
Contract No

Designed SITE EVALUATION
Shoot 2

File No.

Date

Date
BYW

$A M B$

\subsection{DOLORES RIVER BORROW SITE}

A plan of the Dolores River borrow site is shown on sheet 3. The estimated plan area of the site, using a planimeter, is:

$A=1,150,700 \mathrm{ft}^{2}$

The following is a summary table of the borehole data:

\section{Dolores River Borrow Site}

\begin{tabular}{|c|c|c|c|}
\hline Borehole & Depth & Soil Profile & $\begin{array}{l}\text { Depth of Gravel/Cobbles } \\
\text { [ft] }\end{array}$ \\
\hline $\begin{array}{l}\text { EP1 } \\
\text { (1) } \\
\end{array}$ & $\begin{array}{l}0^{\prime}-2.5^{\prime} \\
2.5^{\prime}-8.5^{\prime} \\
>8.5^{\prime} \\
\end{array}$ & $\begin{array}{l}\text { Clay } \\
\text { Gravel / Cobbles } \\
\text { Rock }\end{array}$ & 6 \\
\hline EP2 & $\begin{array}{l}0^{\prime}-5^{\prime} \\
5^{\prime}-11.5^{\prime} \\
>11.5^{\prime} \\
\end{array}$ & $\begin{array}{l}\text { Clay } \\
\text { Gravel / Cobbles } \\
\text { Rock } \\
\end{array}$ & 6.5 \\
\hline EP3 & \begin{tabular}{|l|}
$0^{\prime}-3^{\prime}$ \\
$3^{\prime}-10^{\prime}$ \\
$>10^{\prime}$ \\
\end{tabular} & $\begin{array}{l}\text { Clay } \\
\text { Gravel / Cobbles } \\
\text { Rock }\end{array}$ & 7 \\
\hline EP4 & $\begin{array}{l}0^{\prime}-3^{\prime} \\
3^{\prime}-11.5^{\prime} \\
>11.5^{\prime} \\
\end{array}$ & $\begin{array}{l}\text { Clay } \\
\text { Gravel / Cobbles } \\
\text { Rock }\end{array}$ & 8.5 \\
\hline EP5 & $\begin{array}{l}0^{\prime}-5^{\prime} \\
5^{\prime}-9^{\prime} \\
>9^{\prime} \\
\end{array}$ & $\begin{array}{l}\text { Silty clay } \\
\text { Graivel / Cóbbles } \\
\text { Rock }\end{array}$ & $\begin{array}{c}\because \cdots \\
4\end{array}$ \\
\hline $\begin{array}{l}\text { TP503 } \\
\text { (2) }\end{array}$ & $\begin{array}{l}0^{\prime}-2^{\prime} \\
2^{\prime}-11.5^{\prime}\end{array}$ & $\begin{array}{l}\text { Silty sand } \\
\text { Gravel / Cobbles }\end{array}$ & 9.5 \\
\hline TP504 & $\begin{array}{l}0^{\prime}-0.5^{\prime} \\
0.5^{\prime}-10^{\prime} \\
\end{array}$ & $\begin{array}{l}\text { Silty sand } \\
\text { Gravel/Cobbles }\end{array}$ & 9.5 \\
\hline
\end{tabular}

Notes:

(1) From Ref. 2.

(2) From Ref. 3.

Therefore, the amount of material available is:

$\mathrm{Vol}=\underline{1,150,700 \times 7}=\underline{\underline{298,300 \mathrm{cy}}}$ 27

The grain size distribution curves for the Dolores River material are shown on sheet 7 . 
Designed by BYW, 02/03/93

Checked by $A M B, 2-3-95$
UMTRA-SRK, 3885-76, Sheet 3

BORROW AREAS - SITE EVALUATION

This is taken from Ref. 1.

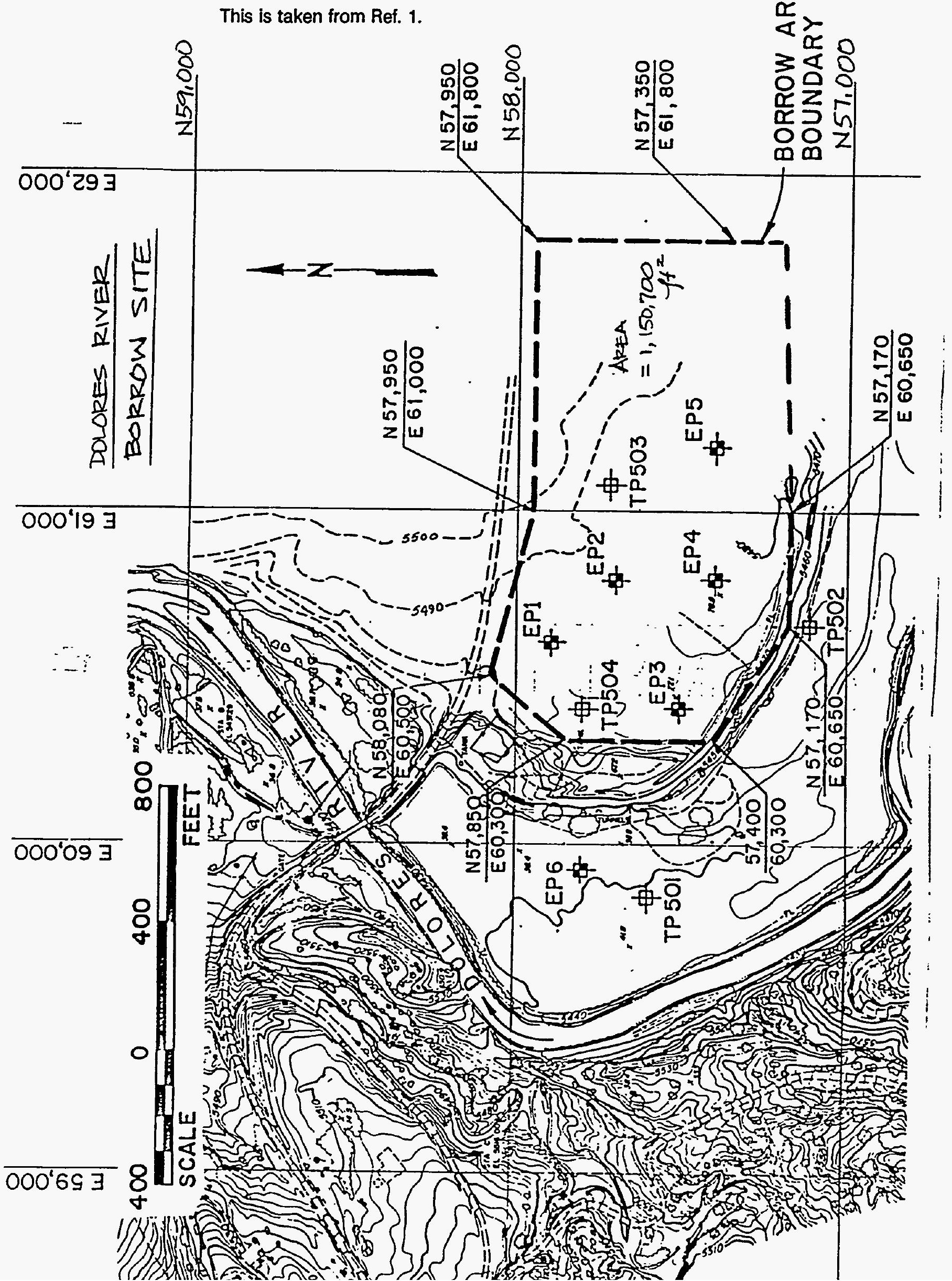


THIS IS TAKEN PROM REF, 4.

PERCENT RETAINED BY WEIGHT Checked by

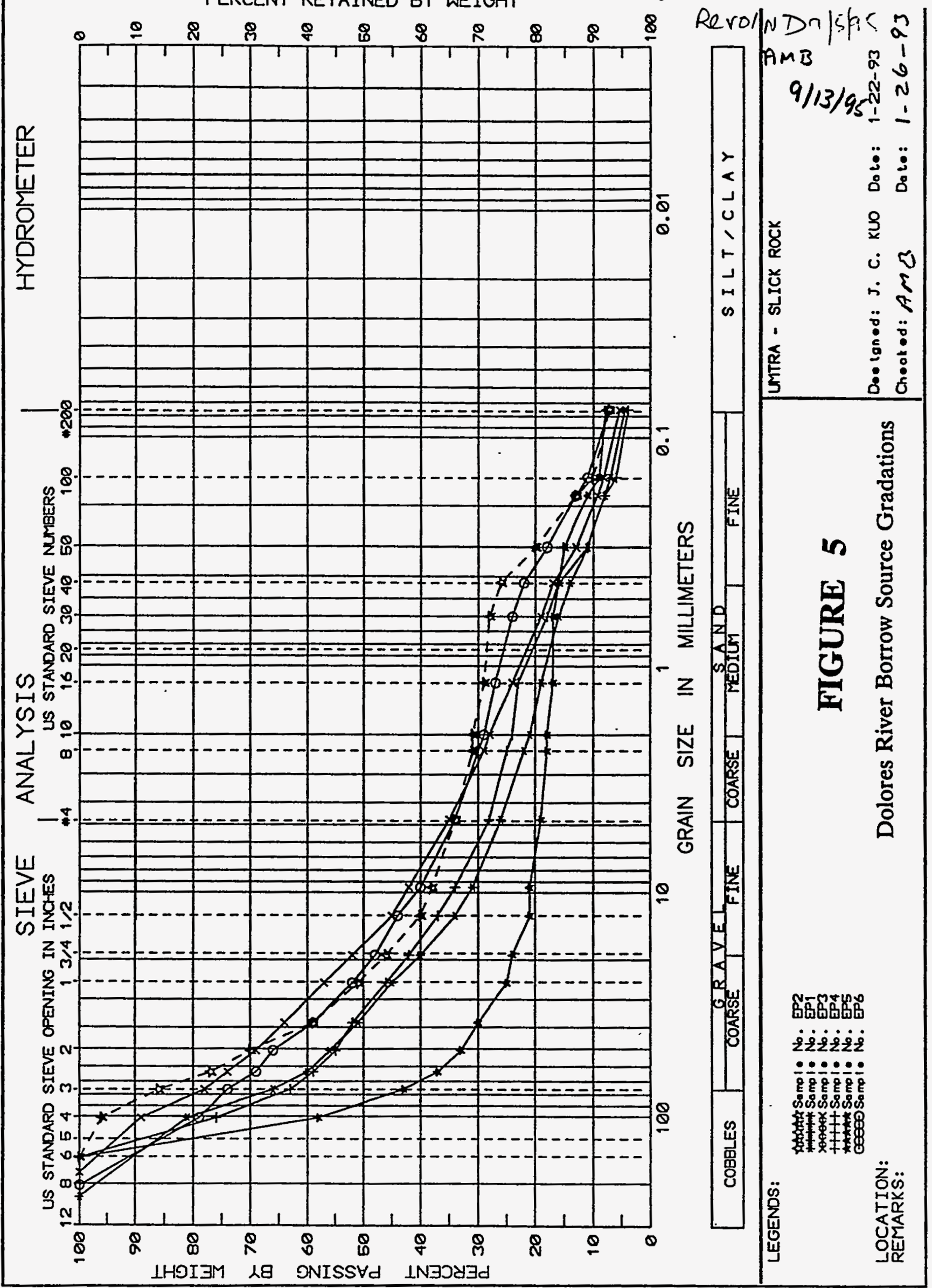


MORRISON KNUDSEN CORPORATION

ENVIRONMENTAL SERVICES DIVISION

CONTRACT NO. 3885-76 DISCIPLINE CIVIL

PROJECT
UMTRA PROJECT

CALCULATION COVER SHEET

CALC. NO. 11-330-02-00 or 02

NO. OF SHEETS 101 (included cover sheet) 14

\section{UMTRA - SLICK ROCK}

FEATURE

TEMPORARY FACILITIES

ITEM

MATERIAL QUANTITIES

SOURCES OF DATA

See sheet 1

SOURCES OF FORMULAE \& REFERENCES

See sheet 1

PRELIMINARY CALC. $\square \quad$ FINAL CALC. $\searrow$ SUPERSEDES CALC. NO.

\begin{tabular}{|c|c|c|c|c|c|c|c|}
\hline & & & & 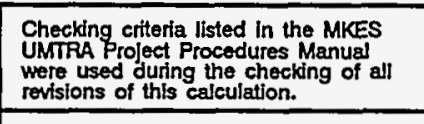 & & & \\
\hline 2 & $\sec \sin 2 B$ & J.S. Randeni: & $1 / 28195$ & fophia Dofhoda & & & $9 / 14 / 56$ \\
\hline 1 & 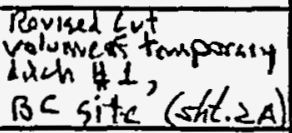 & J.SPandoai & $\begin{array}{l}728699 \\
1125 / 94\end{array}$ & C. Li zardo & $1 / 2+1 / 20$ & PPF.Claie & $1 / 28 / 94$ \\
\hline$\theta \varnothing$ & & Hi Lewrg & $4 \%$ & Belinda y. Worg & $\frac{4-07-9}{4 / 30 / 58}$ & Alsim. Bans: & ifiso \\
\hline $\begin{array}{l}\text { REV. } \\
\text { NO }\end{array}$ & REVISION & $\begin{array}{l}\text { CALCULATION } \\
\text { BY }\end{array}$ & DATE & $\begin{array}{c}\text { CHECKED } \\
\text { BY }\end{array}$ & DATE & $\begin{array}{c}\text { APPROVED } \\
\text { BY }\end{array}$ & DATE \\
\hline
\end{tabular}


Project UMTRA - SRK

Feature TEMPORARY FACILITIES

Item MATERIAL QUANTITIES
Contract No. $3885-76$

Designed HJL

Checked JSR
Sheet

File No.

Date 4-27-93

Date $4 \longdiv { 3 0 / 9 3 }$

\section{SOURCES OF DATA, FORMULAE, AND REFERENCES}

1.) The following MKES Drawings:

SRK-PS-10-0310, Union Carbide Processing Site, Site Plan and Construction Facilities SRK-PS-10-0312, Union Carbide Processing Site, Temporary Site Drainage Plan SRK-PS-10-0313, Union Carbide Processing Site, Temporary Drainage Ditches, Sections, and Details SRK-PS-10-0314, Union Carbide Processing Site, Access Control Area Plan, Sections, and Details SRK-PS-10-0315, Union Carbide Processing Site, Wastewater Retention Basin Sections and Details SRK-PS-10-0316, Union Carbide Processing Site, Contaminated Material Excavation Plan SRK-PS-10-0318, Union Carbide Processing Site, Borings and Test Pits Location Plan SRK-PS-10-0320, North Continent Processing Site, Site Plan and Construction Facilities SRK-PS-10-0321, North Continent Processing Site, Access Roads and Facilities SRK-PS-10-0323, North Continent Processing Site, Contaminated Material Excavation Plan SRK-PS-10-0325, North Contenent Processing Site, Borings and Test Pits Location Plan SRK-DS-10-0330, Burro Canyon Disposal Site, Access Plan SRK-DS-10-0331, Burro Canyon Disposal Site, Site Preparation and Temporary Drainage Facilities SRK-DS-10-0332, Burro Canyon Disposal Site, Access Control Facilities and Wastewater Retention Basin Plan

SRK-DS-10-0333, Burron Canyon Disposal Site, Wastewater Retention Basin and Miscellaneous Section and Details

SRK-DS-10-0338, Burron Canyon Disposal Site, Borings and Test Pits Location Plan

All drawings are rev. $A$ as of the date of this calculation

2.) Telecon with M.B. Leaf of TAC regarding wells to be sealed, $2 / 8 / 93$, MKES Doc. No. 3885-SRK-T-0102359-00

3.) Slick Rock sites well logs, MKES Doc. No. 3885-SRK-X-04-02293-00

4.) MKES-TAC meeting notes, 2/23/93, MKES Doc. No. 3885-SRK-X-02-02367-00

5.) Borehole, Test Pit, and Well Data, Burro Canyon Disposal Site, MKES Doc. No. 3885-SRK-X-04-02294-00

6.) MKES Doc. No. 3885-SRK-L-09-02376-00, 3/15/93, Message from TAC regarding well abandonment at Burro Canyon Disposal Site (attached, see sheet 100) 


\section{CONTENTS}

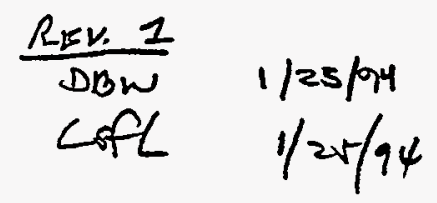

$\frac{R_{F V} 1}{D B W}$

$1 / 25 / 94$

PAGE NO.

ITEM

Revisiom /

I. PURPOSE

II. METHOD

III. SUMMARY OF RESULTS

IV. CALCULATIONS

A.) UC SITE (SUMMARY)

1.) Retention Basin

2.) Ditches 1, 2, and 3

22

3.) Dikes 1 and 2

36

4.) Access Control Facilities

43

5.) Fencing

50

6.) Wells to be sealed

51

B.) NC SITE (SUMMARY)

1.) Access Control Facilities

58

2.) Access Road

60

3.) Fencing

66

4.) Wells to be sealed

67

C.) BC SITE (SUMMARY)

1.) Retention Basin

2.) Ditches

82

3.) Berms 1, 2, and 3

89

4.) Access Control Facilities

89

5.) New Culverts

94

6.) Fencing

98

7.) Wells to be sealed 
사MORRISON KNUDSEN CORPORATION

Project VMTRA-SRK

Contract No? $3885-76$

Sheet $2 \mathrm{~A}$

Feature Temporary facilities \begin{tabular}{ll} 
Designed $D B W$ & File No. \\
Checked $S S A L$ & Date $1 / 21 / 94$ \\
\hline $1 / 24 / 94$
\end{tabular} $\begin{array}{ll}\text { Contract No. } 385-76 & \text { File No, } \\ \text { Designed } \triangle B W & \text { Date } 1 / 21 / 94 \\ \text { Checked } S S A L & \text { Date } 1 / 24 / 94\end{array}$

Item Material Quartics

Tits entire page sport of, and sumpravizes, Revision 1 )

REVISION 1

The purpose of this revision is to update cut volume cali's associated w/ a temporary ditch located SE \&f the proposed tailings pile ombankmentat BC s ute

Sheets $82,83,84,85 \$ 82$ wee replaced in entirety $a D$ she ts $82 A, 83 A, 84 A, 85$ A \& $82 A$

Sheets $74 \$ 4$ ane affected $\$$ show
corrections.

Sheets $86 . \mathrm{B} \& 86 \mathrm{C}$ are added to include the prof le and typical crass section of the ditch.

The excavation avantety of temporary ditch wis calculated using the -3 awe method as described in' Shat 3 .

The length of silt fences and barbed wire? fences in the $B C$ site have changed. (Reval Sits 4,74 and 98. have been revised Sits 4,74 and 98. have been revised
shits 69,70 and 71 have been replaced in th
new sheets.

ENG 375/91 
CA. MORRISON KNUDSEN CORPORATION

Project UMTRA - SRK

Sheet $2 B$

Feature Temporary facilities

Contract No. $3885-76$ File No. item Material Quantities

Designed JSR Date $9 / 13 / 95$ Checked: Sos Date 9 / $13 / 95$

Revision $z$ Repose

This entire sheet is past of $\operatorname{Rer} 2$

(a) The chainilink fence gates have been changed to driveway gates and barbed wire fences have been changed to smooth wire fences. Deer fences have been acted around all the basins at $U C, M C$ and $B C$ sites.

sits 2B,50A,66A, 98A have been added. sits 4,6,13,50,57,66,74, 98 revised

sit $9,54,71$ replaced.

ENG 375/91 


\section{PURPOSE}

The purpose of this calculation is to estimate the material quantities (cut, fill, concrete, aggregate, membrane liner, culvert, fencing, wells to be sealed) that will be required for construction facilities. Results of this calculation will be used to determine the bid schedule.

\section{METHOD}

Excavation quantities were determined by using the Average End Area Method:

$$
\begin{array}{r}
\text { vOLUME }=\frac{1}{2}\left(A_{1}+A_{2}\right) \times(\Delta L) \\
\text { area } 1=A_{1} \\
\text { area } 2=A_{2} \\
\text { distance between } A_{1} \text { and } A_{2}=(\Delta L)
\end{array}
$$

Where the end conditions for the computed volumes were such that the volumes became zero at a point, rather than a line, the Pyramid Volume Formula was used:

$$
\begin{aligned}
& \text { VOLUME }=\frac{1}{3}\left(A_{1}+A_{2}\right) \times(\Delta L) \\
& \text { area } 1=A_{1} \\
& \text { area } 2=A_{2} \\
& \text { distance between } A_{1} \text { and } A_{2}=(\Delta L)
\end{aligned}
$$

All cross sectional areas were measured by planimeter.

Aggregate and concrete volumes were estimated using geometric methods and planimeter, as were the areas for the membrane liner. Sideslope areas for retention basins were estimated using an average perimeter length measured along the sideslope at one half the depth of the basin. This perimeter length was multiplied by the length of the sideslope to obtain the estimated area.

Lengths of fences, culverts, and roads were directly scaled off construction drawings. Cut and fill for roadwork are approximated using scaled dimensions and an assumed depth of one foot. Aggregate for road is approximated using scaled dimensions okroad and assuming a thickness of six inches. 
Project UMTRA - SRK

Feature TEMPORARY FACILITIES

Item MATERIAL QUANTITIES
Contract No. $3885-76$

Designed HJL

Checked
Sheet

File No.

Date 4-27-93

Date $4-30-93$

A.) EARTHWORK

$\underline{\text { UC Site }}$

NC Site

REV. 1

Jse $1 / 28 / 94$

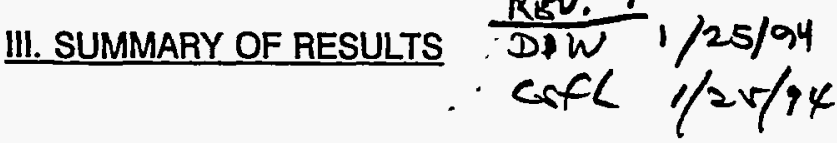

1.) Cut (cy)

22,550

2,220
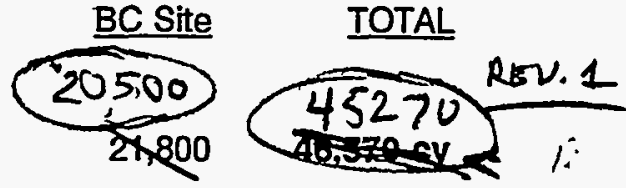

2.) Fill (cy)

12,400

25

4,400

16,825 cy

TOTAL NET CUT

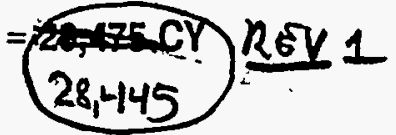

B.) OTHER QUANTITIES

1.) Concrete (cy)

150

50

150

$350 \mathrm{cy}$

2.) Aggregate (cy)

250

0

390

$640 \mathrm{cy}$

3.) Membrane Liner (sf)

112,620

8,130

59,210

179,960 sf

4.) Fencing

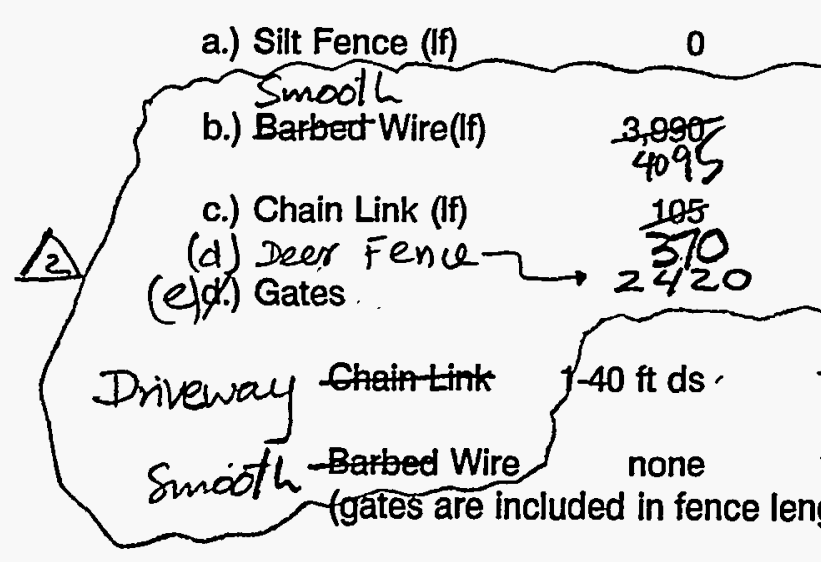

5.) Well Lengths to be sealed (If)

680

0

1-15 ft ss none

2,190

7,160
1220

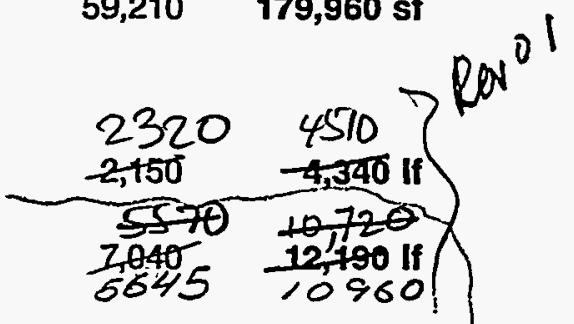

6.) New culvert length (ft)

110

60
0
9

555

930

480

2000

720 If

480

15350

$1-20 \mathrm{ft} d s \quad 1-40 \mathrm{ft} d s$

none

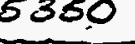

7.) Roadwork

\section{County Road}

a.) Length ( $\mathrm{ft}$ )

915

1,840

3,550

$6,305 \mathrm{ft}$

b.) Excavation qty (cy)

680

1,700

5,260

7,640 cy

c.) Aggregate (cy)

338

850

2,630

3,818 cy

Total for

ReN 2 BY JSR 8/11/94

Che stos 9/13/94 
Contract No. 3885-76

Designed HJL

Checked AFS
Sheet 5

File No.

Date 2-12-93

Date $2 / 16 / 93$

IV. CALCULATIONS - A. UC SITE 

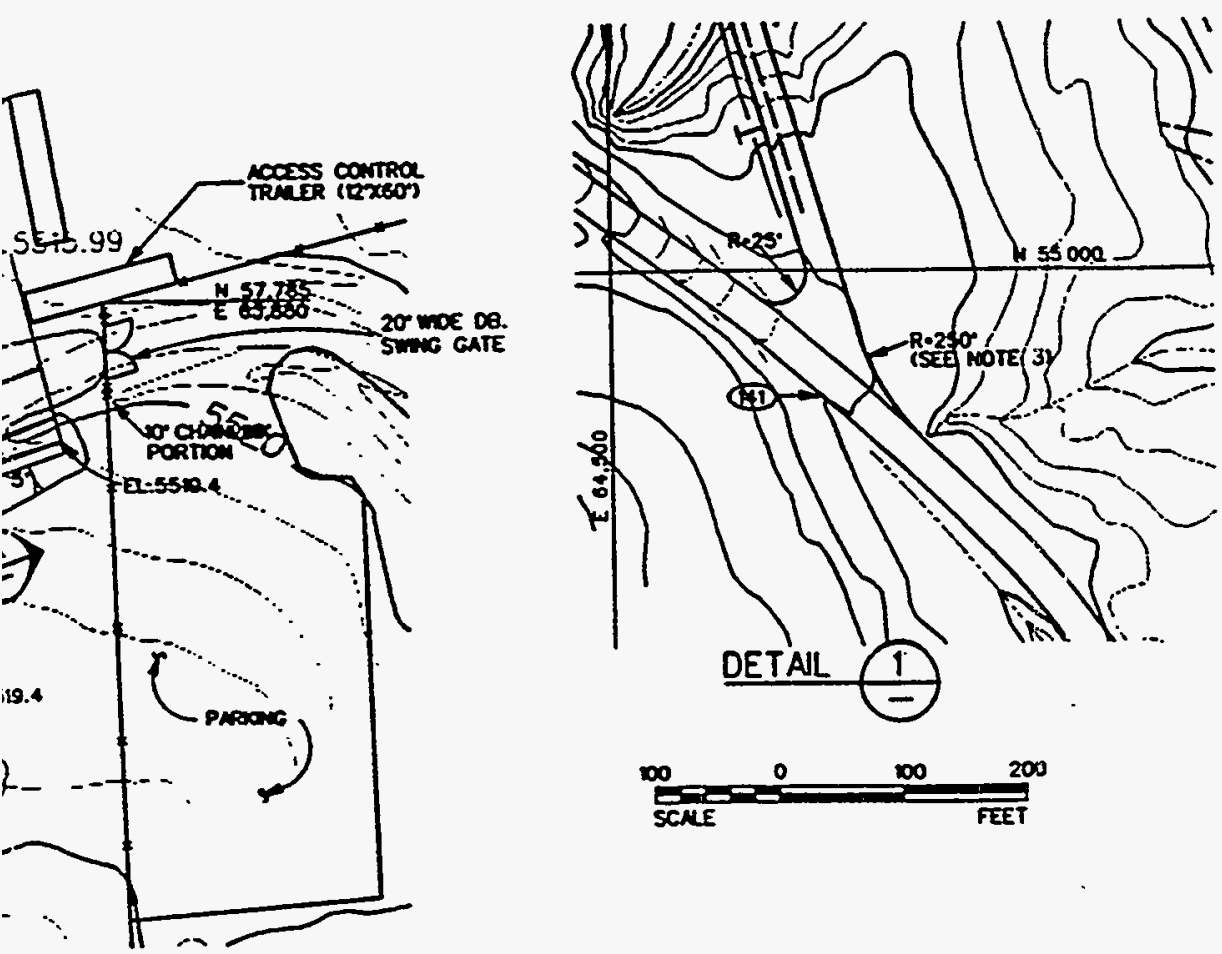

OL FACILITY

PS-10-320)

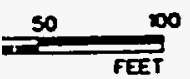

NOTES:

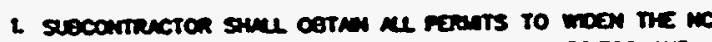

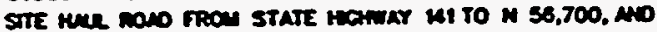
Frou in s7,500 to in stre acoss cate.

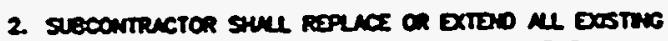

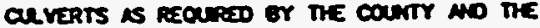
CONTRuctor.

3. SUECONIRACTOR SHUL DETERANE MUGWRTT AO LOCATION of turoenc reous of nocess rowo to the MC stre.

4. TEE SECTIONS MO OETMES OF THE WLSH WATER PETEMTIOH

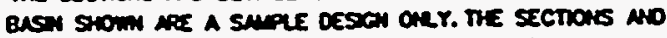

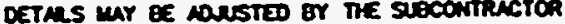

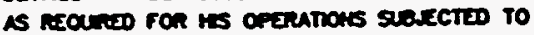
CONTRACTOAS APPROVIK.

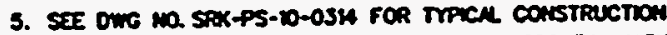

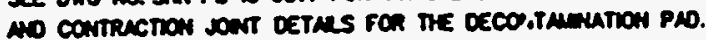
PAO.

6. STORACE TANS MAY BE USED w LEU OF VEVERATE LUND pecarculation baser.

\section{REFERENCE DRAWRGS:}

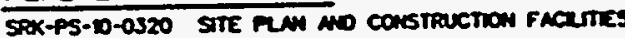
SFOK-PS-D-03H MOCESS CONTROL AREA PLM, SECTIONS MO DETMLS

SRX-PS-D-03E WASTEWATER RETEHTOW BUSN. SECtIONS MO DETALS

\section{LEGEND:}

wraes meprone unc

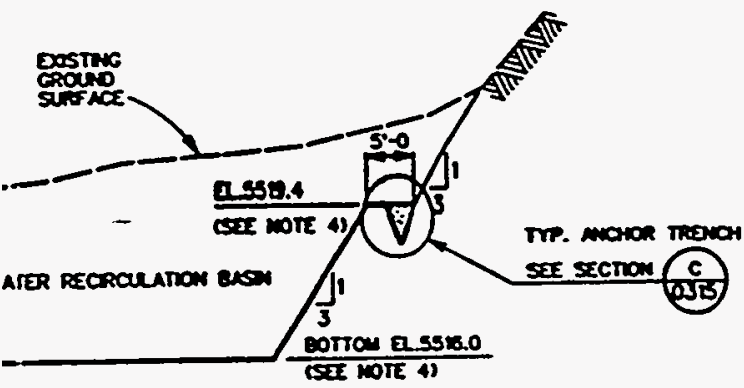

Mr. MONR TREMCH

sex senow C

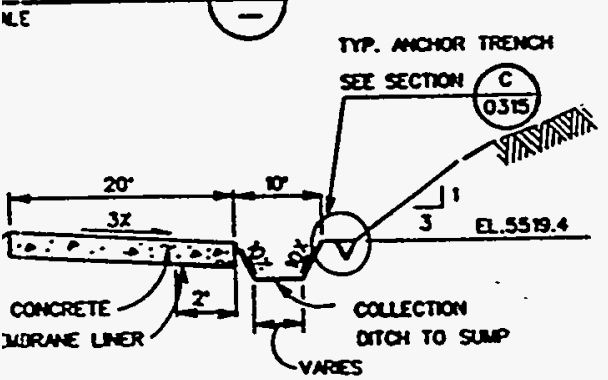

$\frac{\text { TYPICAL SECTION A }}{\text { MOT TO SCNE }}$
U.S. DEPARTMENT OF ENERGY NBUUOUEROUE. NEW MEXICO SLICK ROCK STE NORTH CONTWENT PROCESSHG SITE

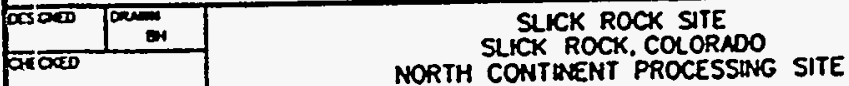

FEXTED ACCESS ROADS AND FACILITIES

\begin{tabular}{|c|c|c|}
\hline 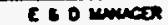 & 0000000 & 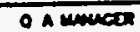 \\
\hline
\end{tabular}




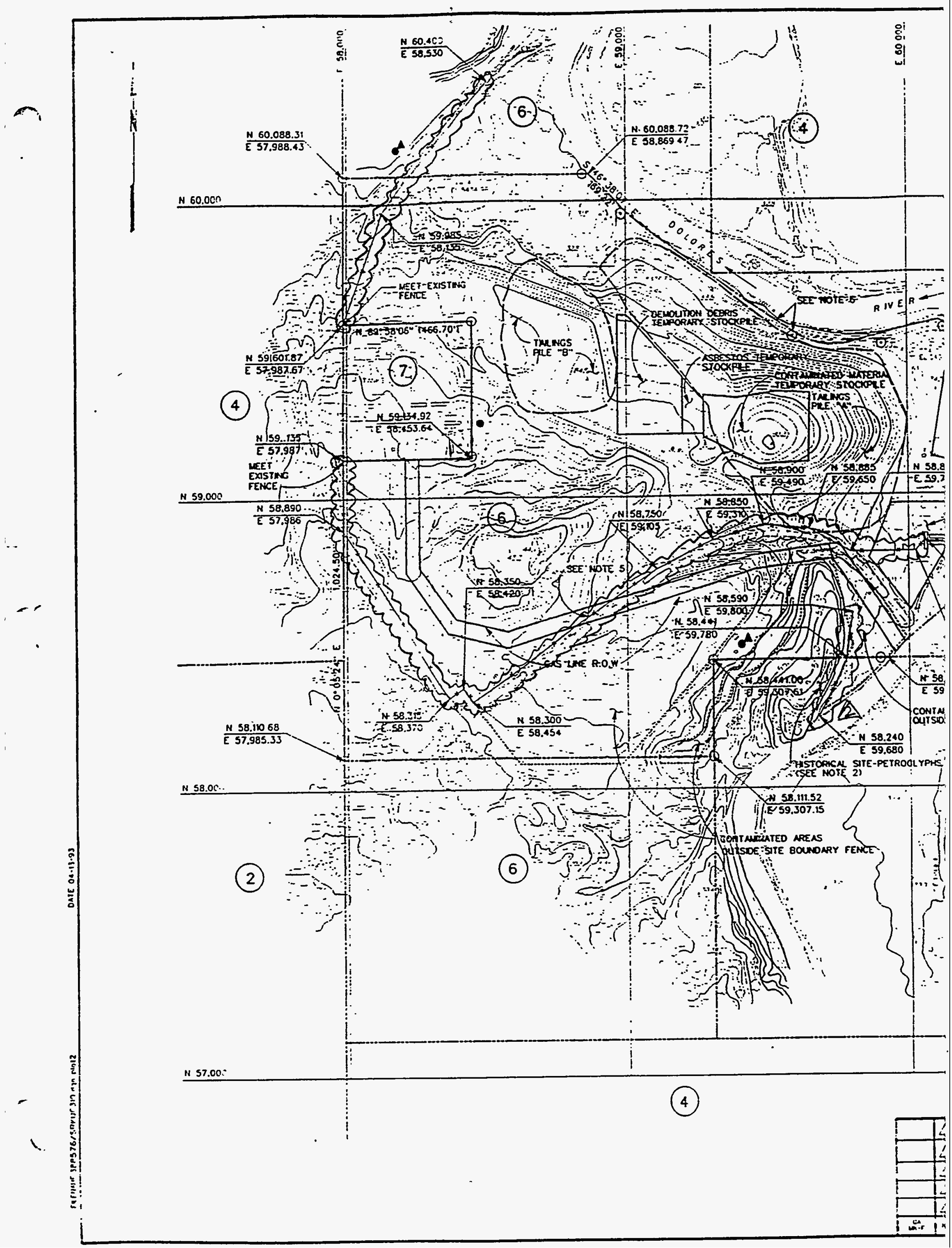




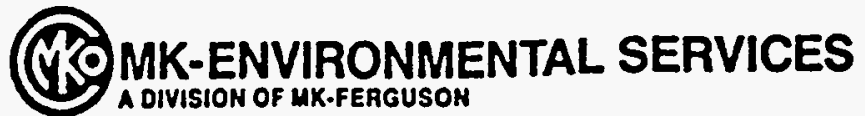

Project \NMTRA-ERK Contract No.3885-76 File No.

Feature TEMTORARY FACILITES

Y idQTERIA- QuA Designed 142 Date $\frac{4.21 .93}{4 / 23 / 92}$
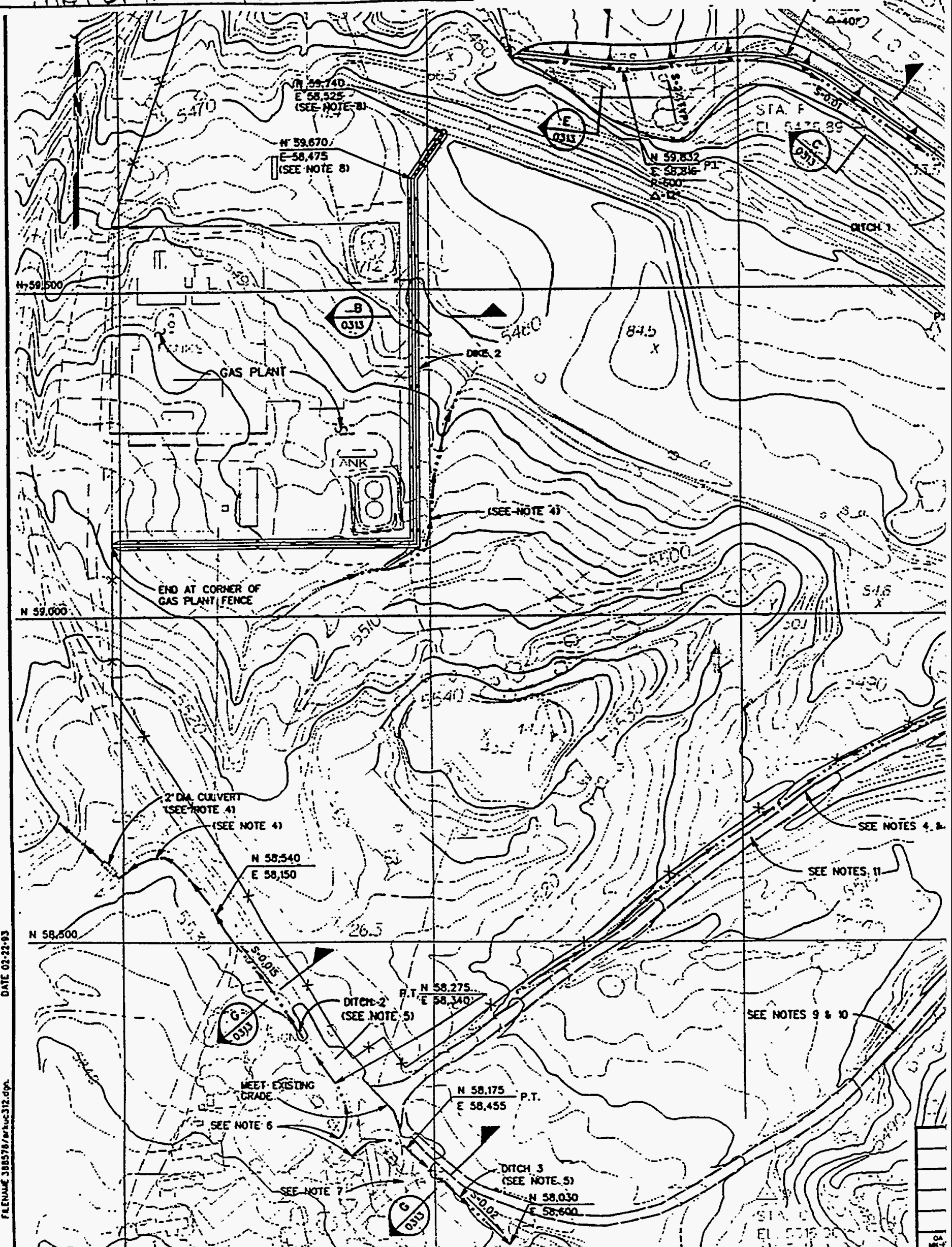


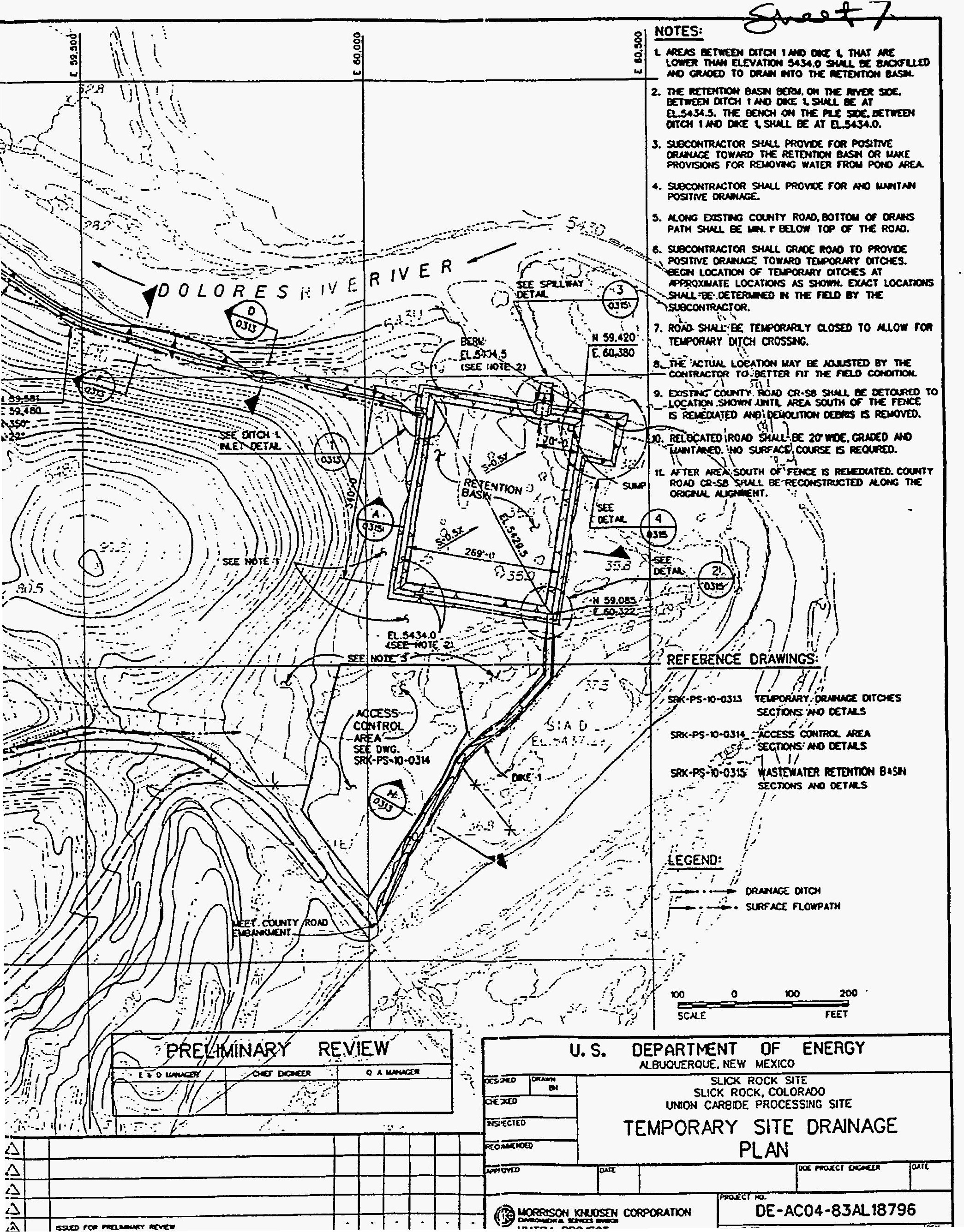



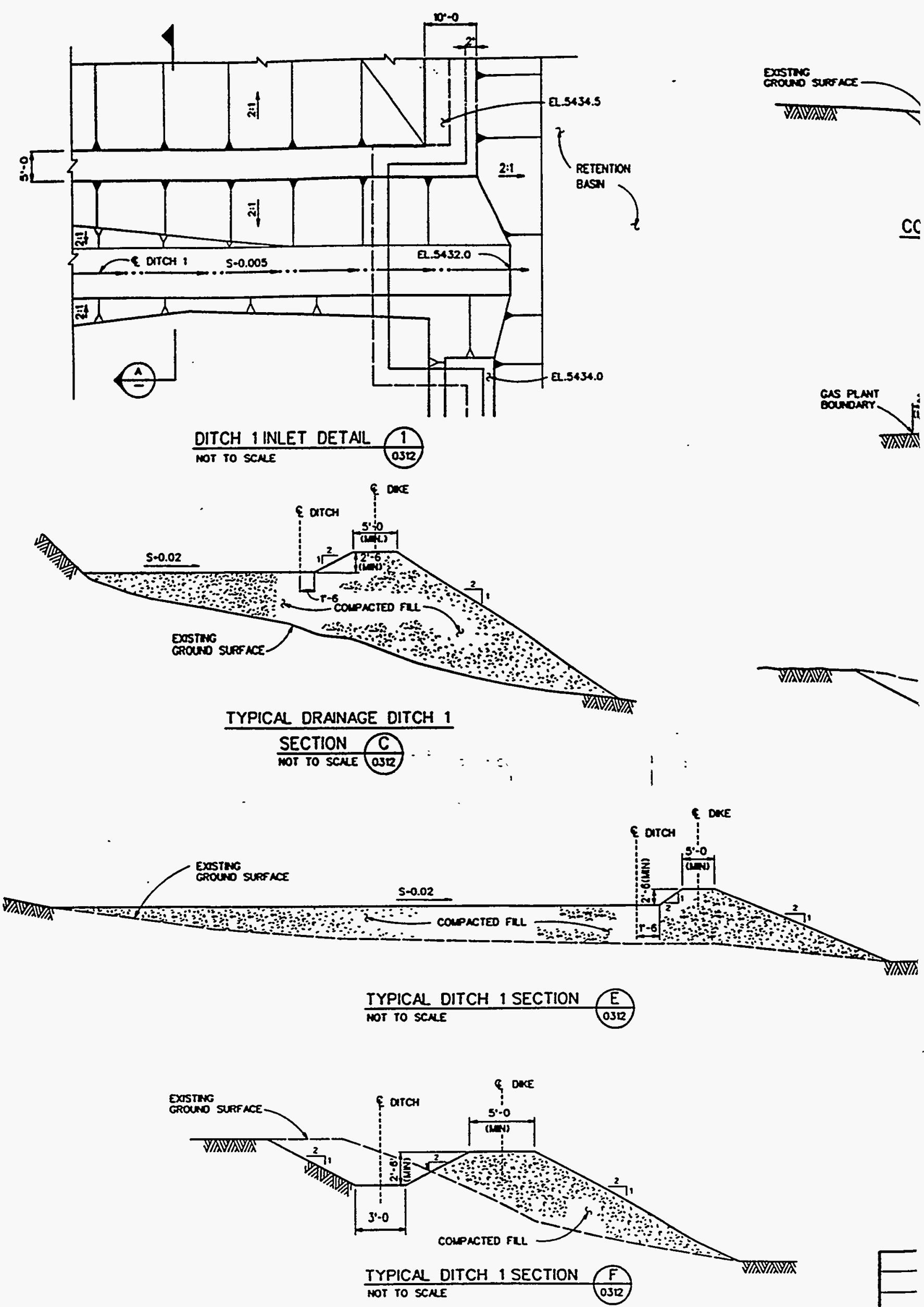


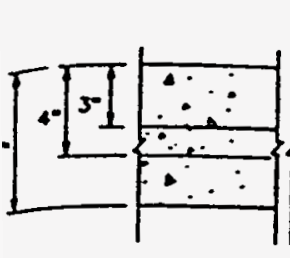

Construction Don (SEE NOTE 6)

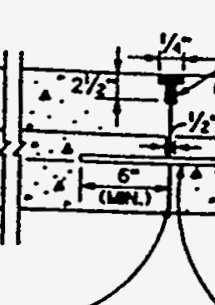

Droge Nowt

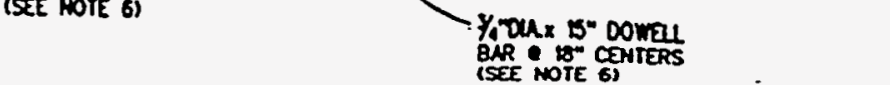

TYP. CONSTRUCTION JOINT DETAL

NOT TO SCME

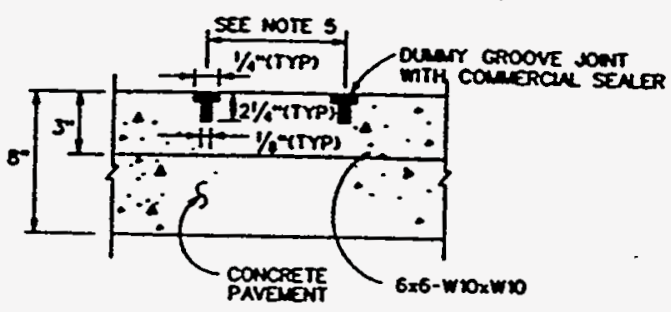

TYP. CONTRACTION JOINT DETALL nOT to SCNE

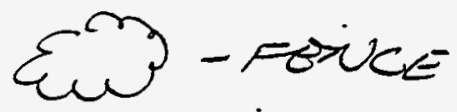

NOTES

- MCESS CONTRO APEA SHU BE COASTRUCTED AFTER RONOVA OF

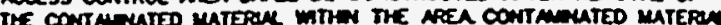

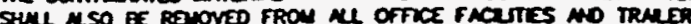
AREA PROR TO Constraction of these Ficumes.

2. Me excavated art sopes swhe ef as recouped ron stabutr

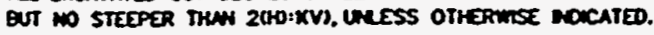

3. AOUCOMT AREAS SWUL EE CRMED TO PRONDE DRUNCE FCR AcoEss control MEEA

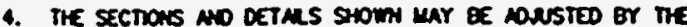
SLCOHIRACTOR AS RECWRED FOR HS OR FER OPERATIONS. SLEXCT to CONIRACTORS APPROVAL.

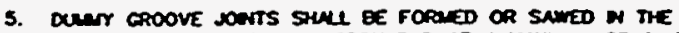
COMCRETE PAVENEAT FOR DECOM PAO AT A Wararen SPACANE of $\mathrm{B}$ FIET.

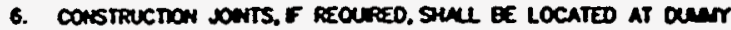
Groove Dats our. DOW' Resurtion of pavit.

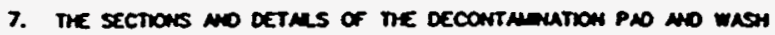
WATER RECRCULATON BASN SHOWM ARE A SHPLE DESTCH OMEY.

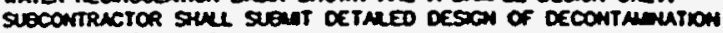
FACUTES FOR APPROVAL.

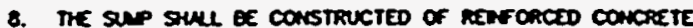
AO EE OESCAED SO A BNOOLE OR FROMT DO LONDER CA EAstr ReVive the sedDerouts.

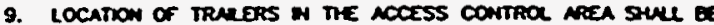
SRECT to THE CONTRACTOR'S APPROVM.

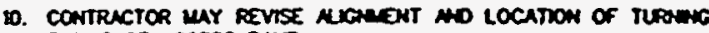
RAOUS OF ACOESS RALP.

I. SLOCOATRACTOR SHUL PROVDE ANO MSTAL DIJR FENCE MO GATES

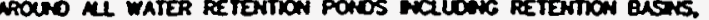
RECROLATION POAOS AO CONSTRUCTION WATER STORUCE PONOS AS APPROYED BY THE CONTRACTOR.

REFERENCE DRAWINGS:

SPax- $\alpha$ - 0 -030S FENE HO GAIE DETALS

SFX-PS-D-OJN SIE PLAN HO CONSTRUCTION FACUTES

SPX-PS-10-03R TEPCRARY STE ORAnucE PLAM

SEX-PS-D-035 WASTEWATER RETEMTION BASH SECTOHS MD DETMLS

\section{LEGEND:}

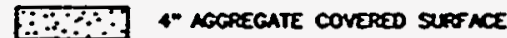
ER:-7.7 concrete pavanant

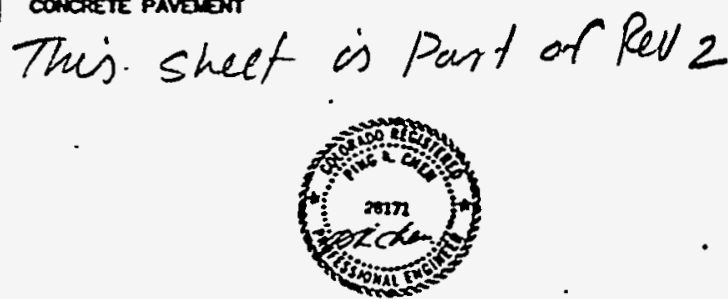

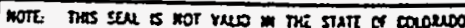

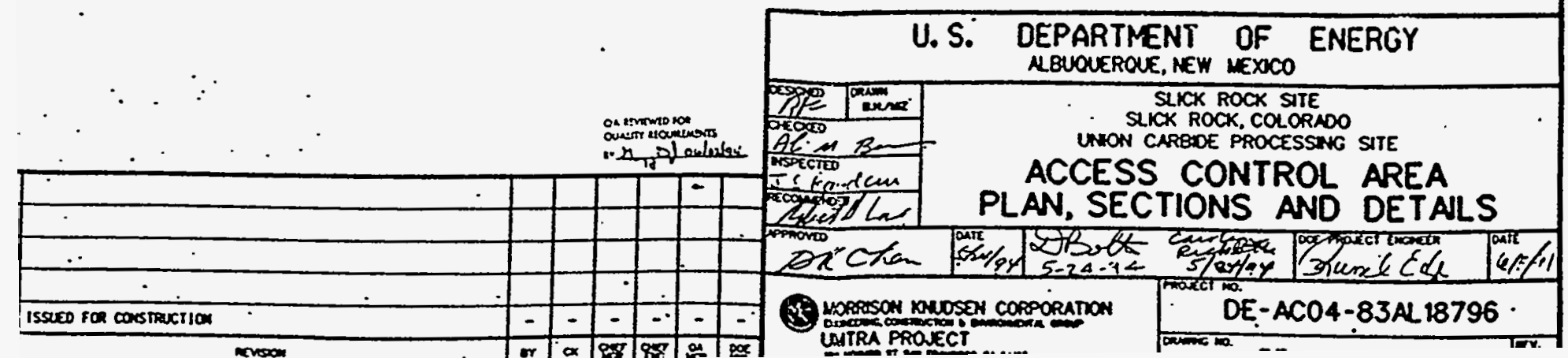




\section{Q. MK-ENVIRONMENTAL SERVICES}

Sheet

10

- Project UMTRA - SRK Feature TEUPORARY FACILITES $1 \mathrm{~mm}$ MATERIAL QUANTTTIES

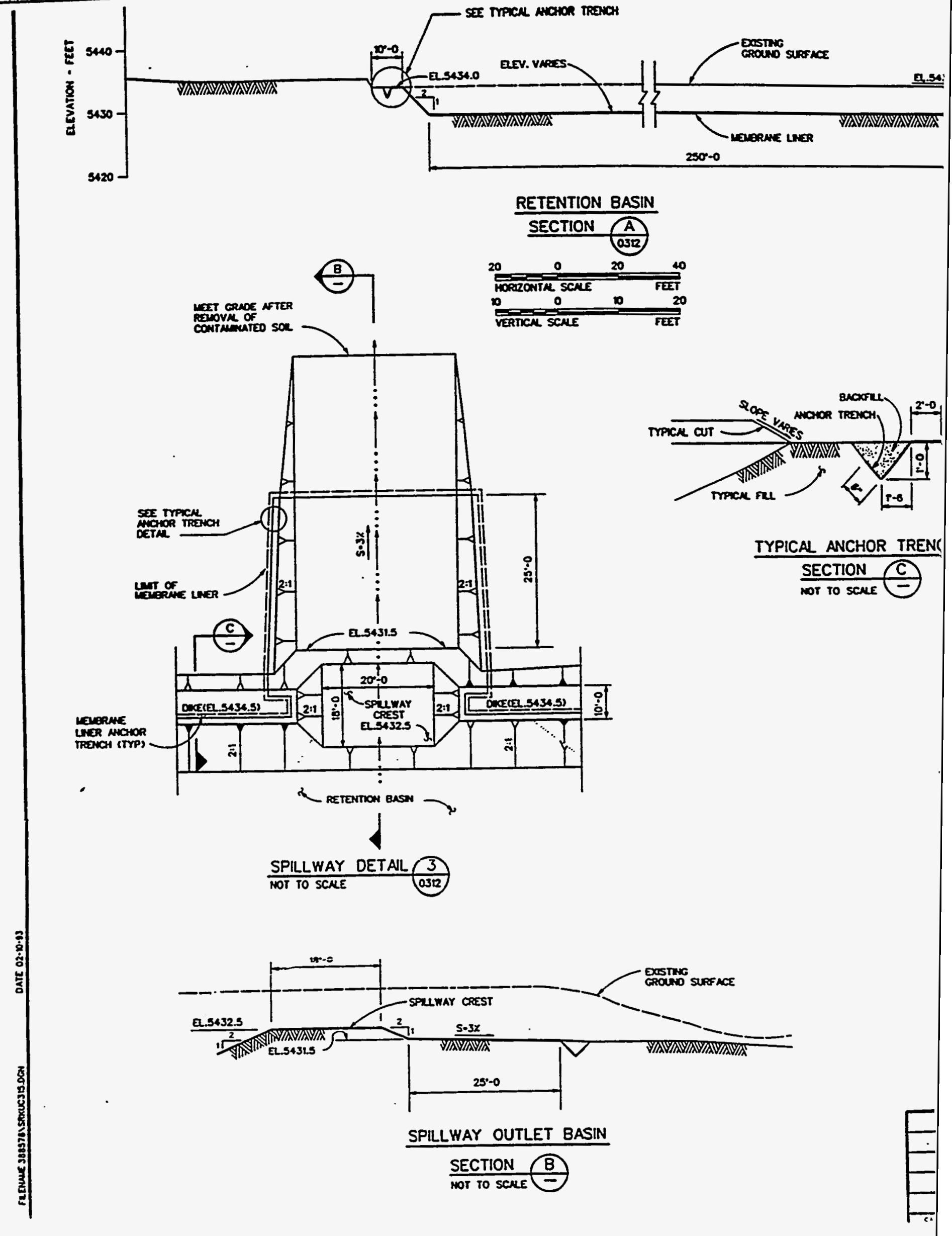

Contract No. $3885-76$ File No. 


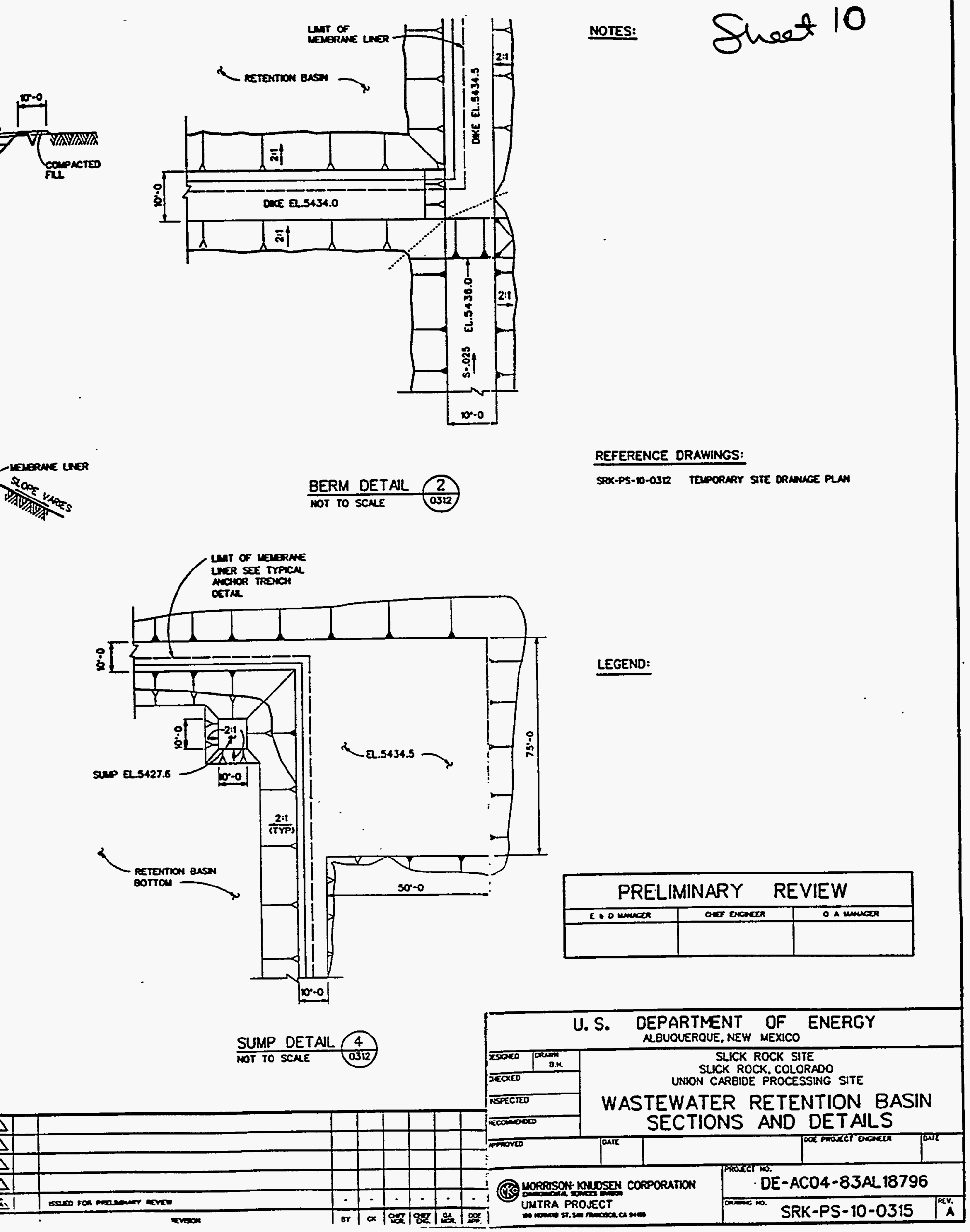




\section{(4.) MK-ENVIRONMENTAL SERVICES}

Project UMTRA - SRIS Contract No.3885-76 File No.

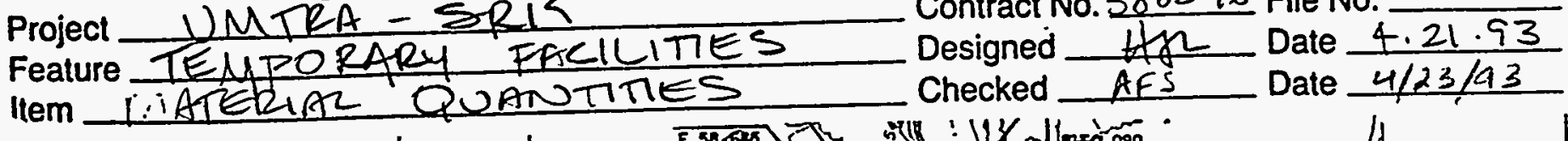

H 80.000

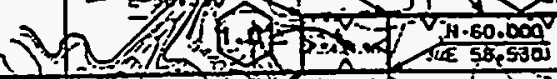

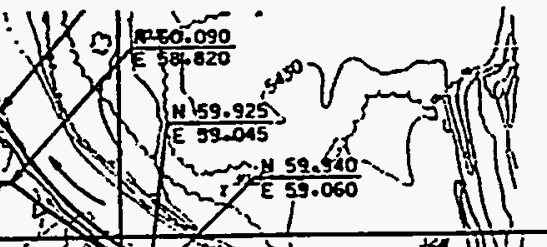
L

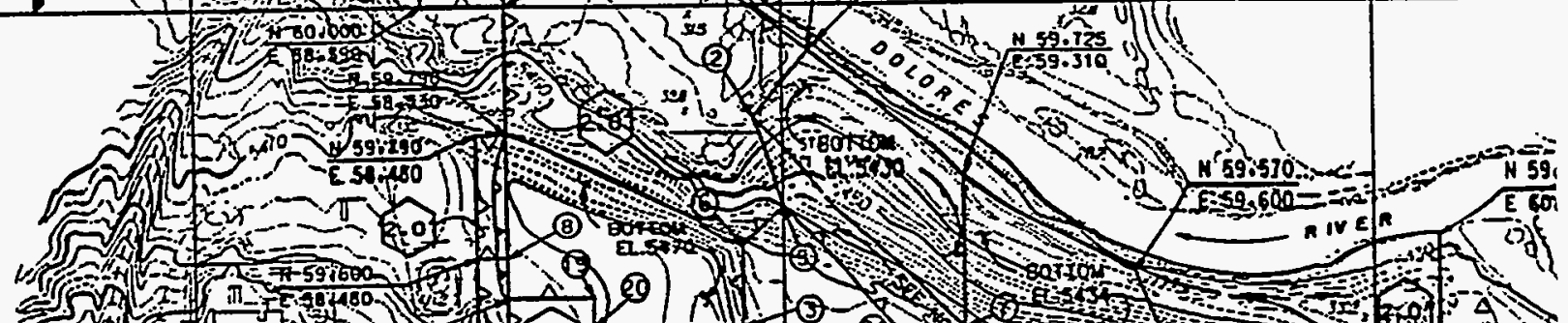

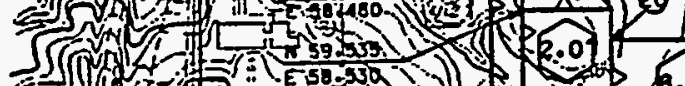

N9.000 s

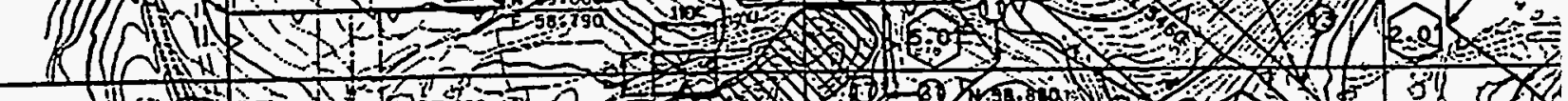

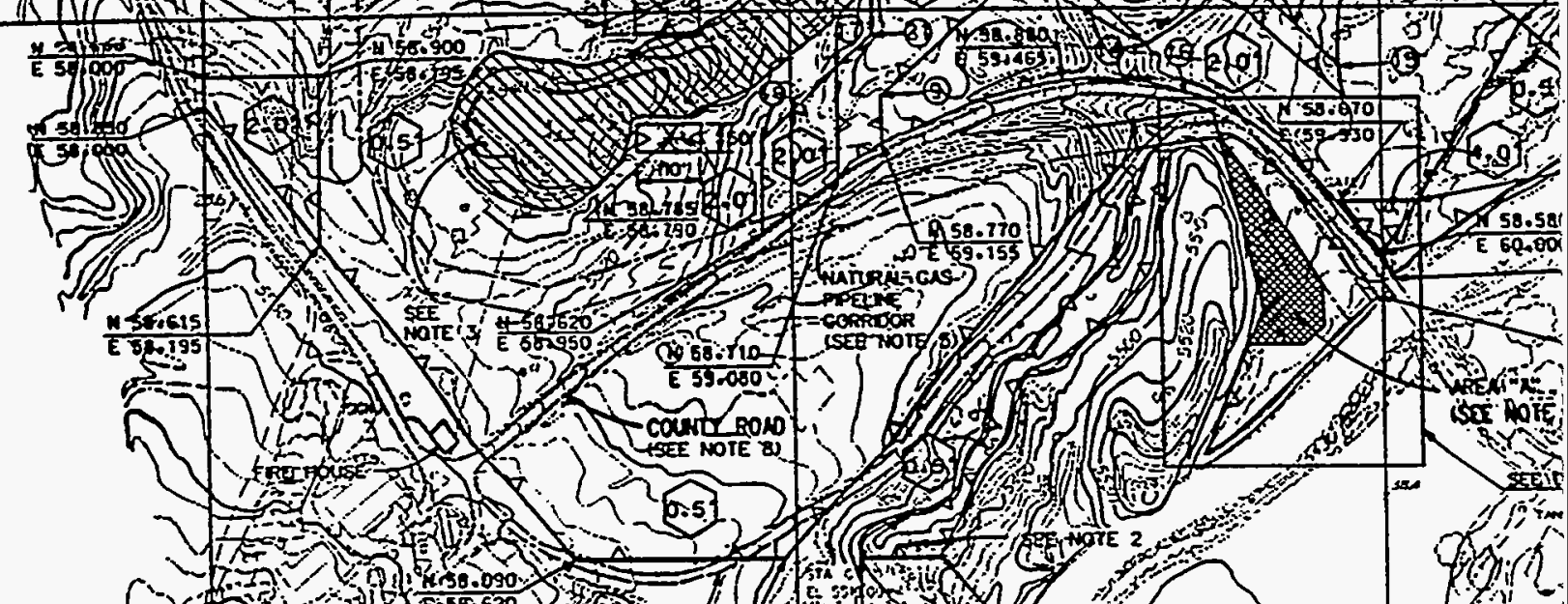
H 58,000

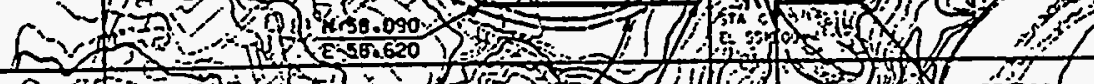
couvry Row

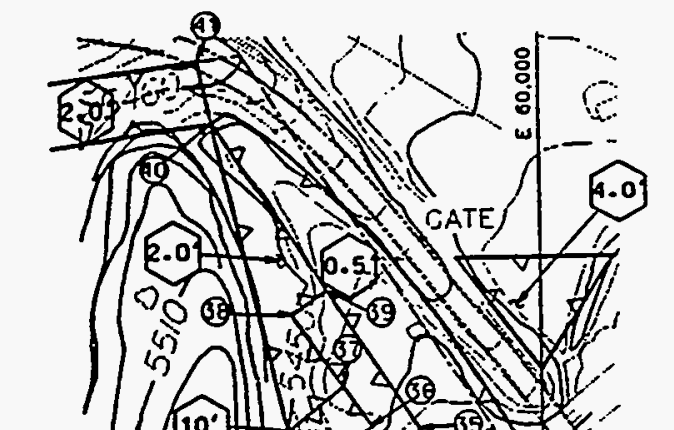

$$
\text { S }
$$
(3),
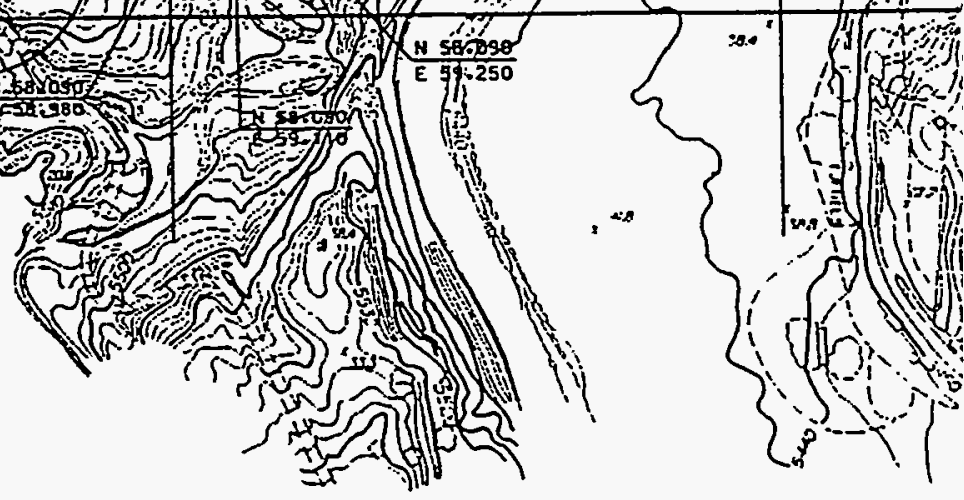
s 58.50 (10.) 10.

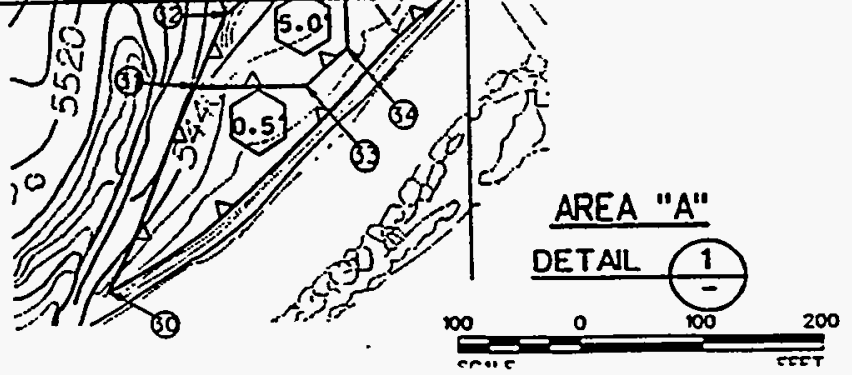




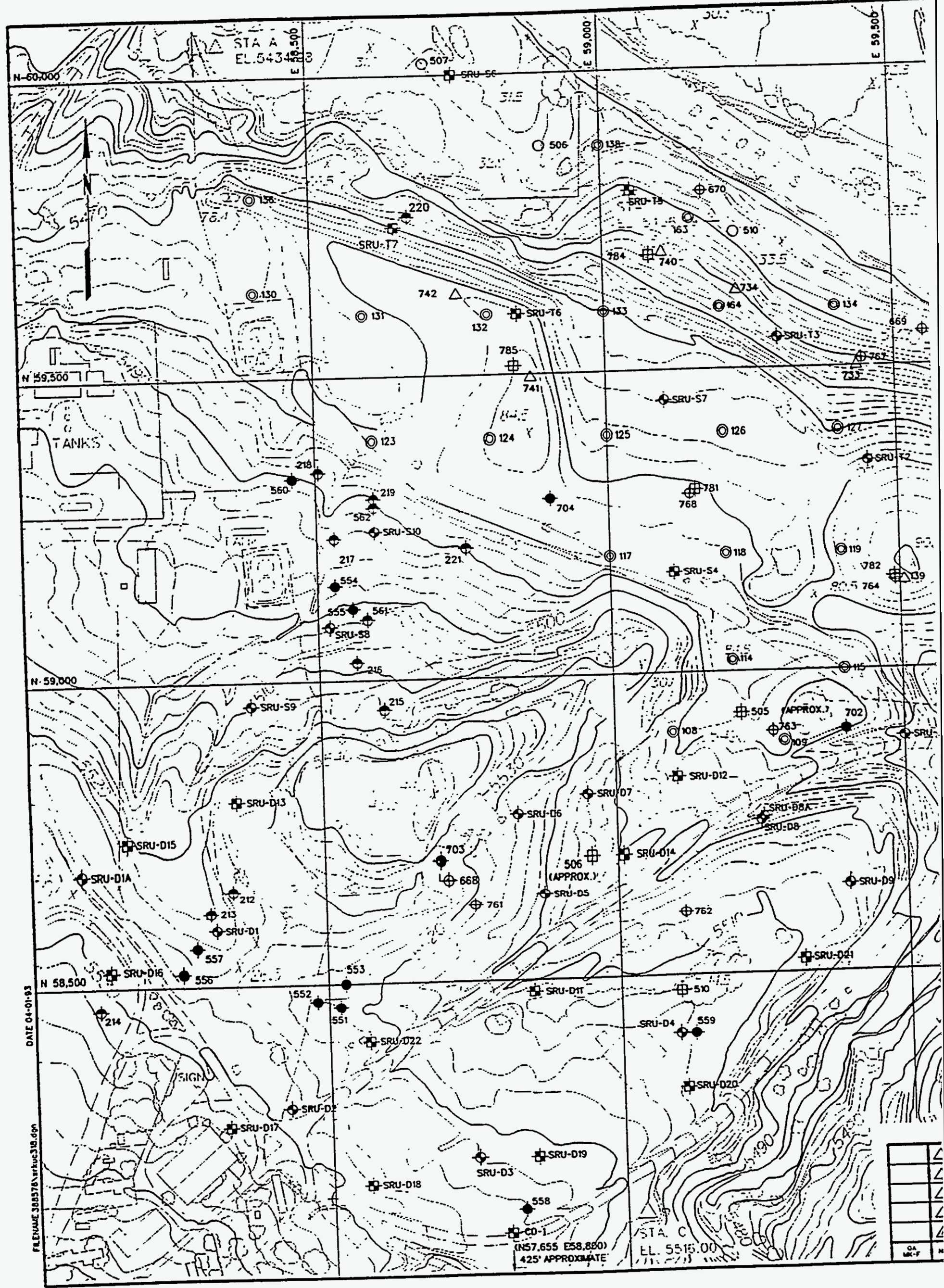




\section{(19.)MK-ENVIRONMENTAL SERVICES}

Project UMTRA - SRK

Feature TEMPORf:RY FACILITES

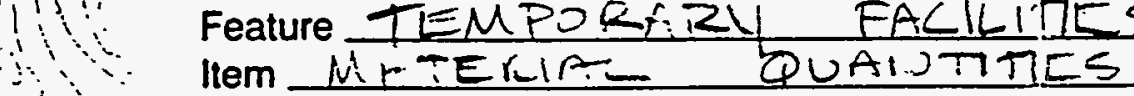
Contract No. $3885 \%$ Sheet $\frac{12}{4 . \overline{27.95}}$ Designed Checked JSR Date $4 / 30 / 93$

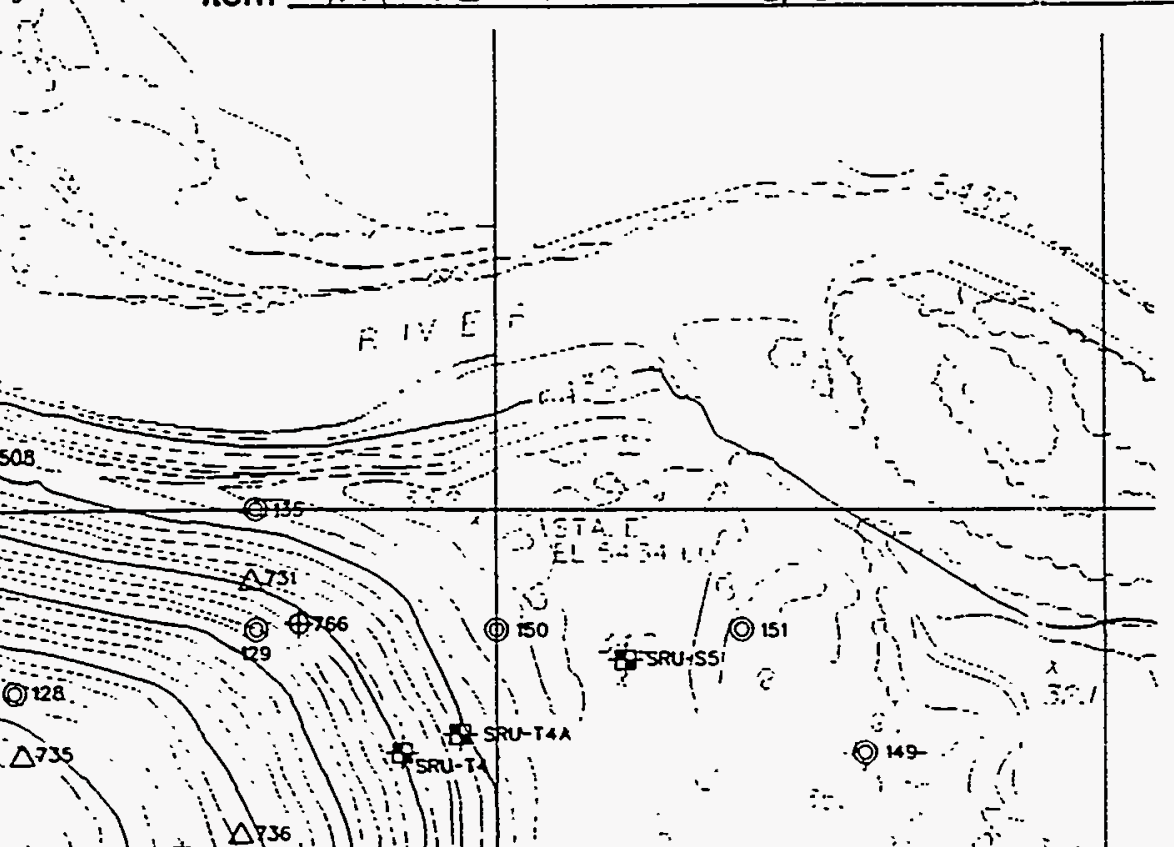

REFERENCE DRAWINGS:

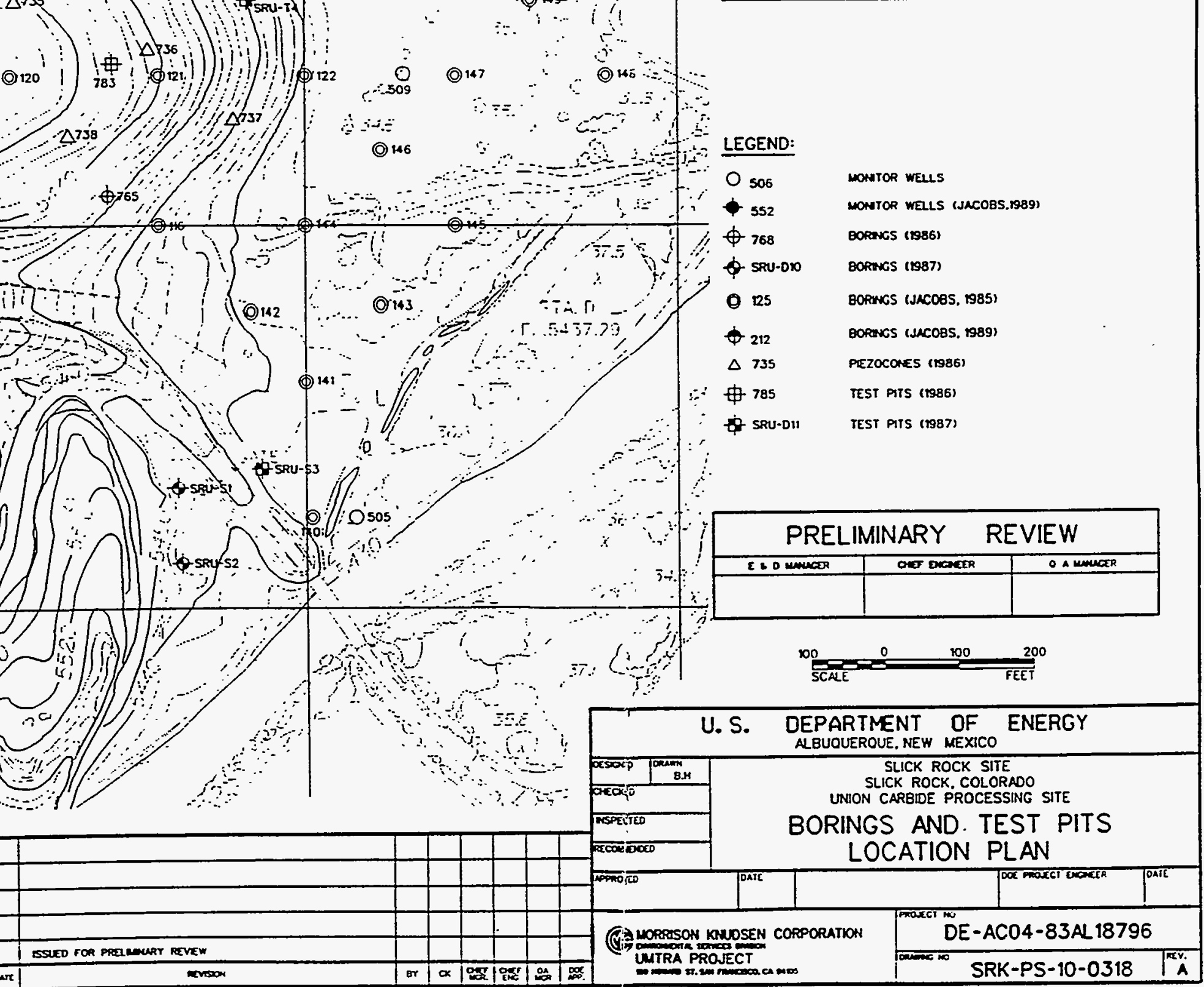


@ROMORISON KNUDSEN CORPORATION

Project UMTRA - SRK

Feature TEMPoraRY FACILTTES

item Materiar quantities
Sheet 13

Contract No.3885-76 File No.

Designed

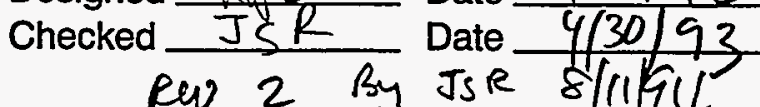

RW 2 By JsR $8 / 1194$

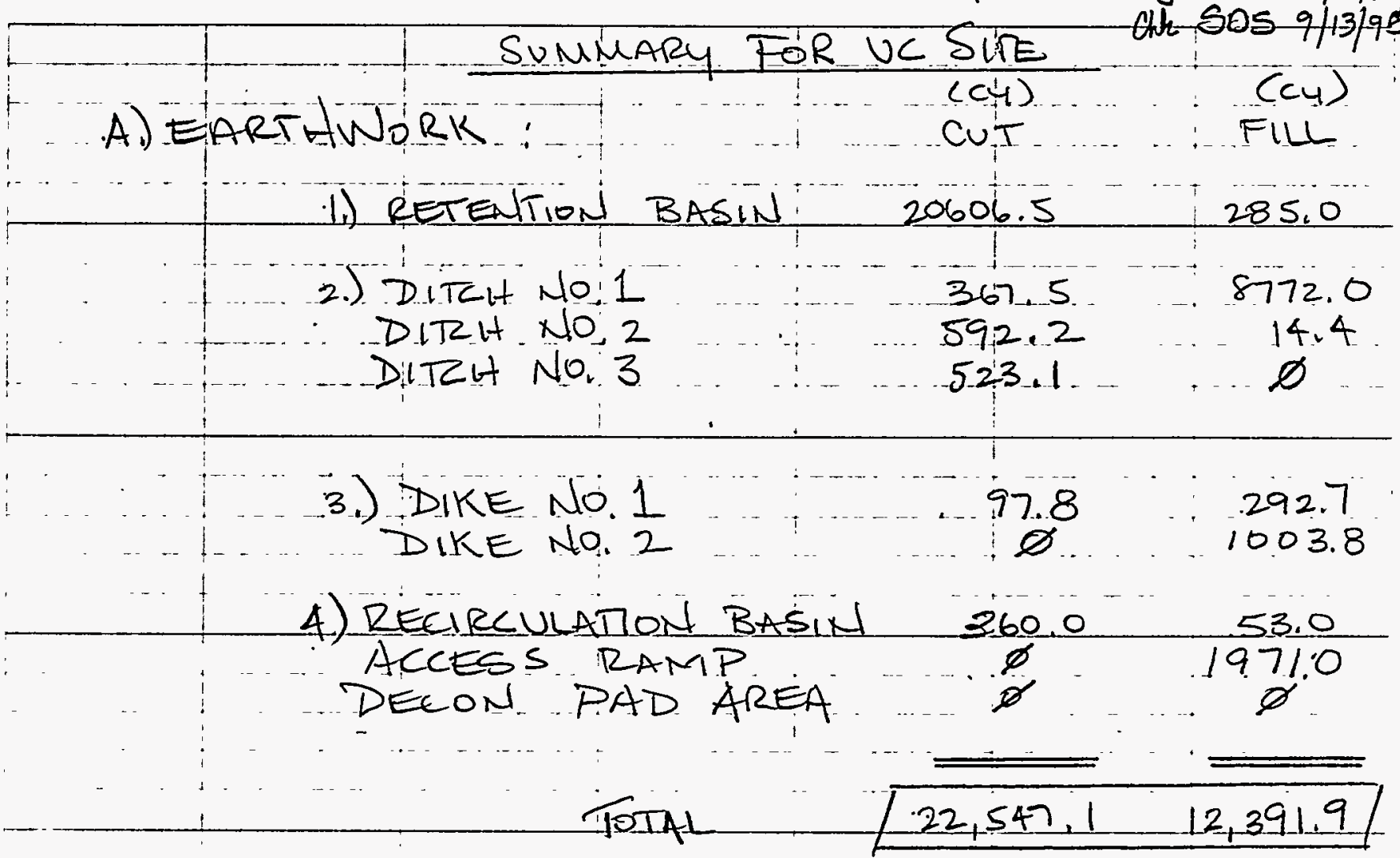

B.) OJHER QUAXTITIES

11) MEMBRANE LINER - BETSIN $109,249.25 .6$. MENIBRANE LINER - RECIRC $\frac{3,372.056}{112,621.2517 .}$

2.) CONCRETE - DECOIP PAD- 152944

3.)

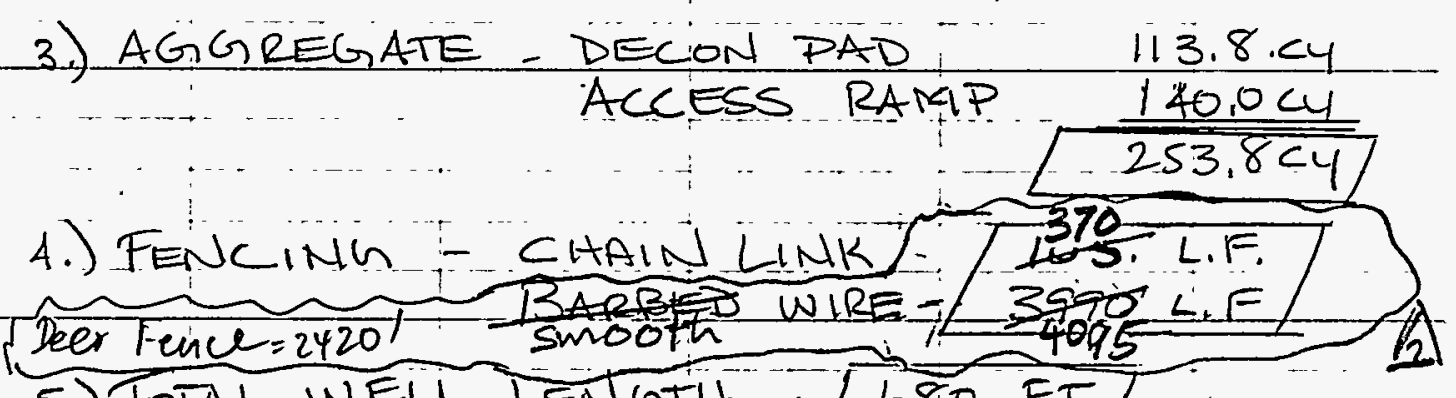

5) TOTAL WELL LENTIH - 680 FT

6.) COUNTY ROAD - LENGTIS $=915$ FT

X XANATION- $677.8 \angle 4$

AGLRECIATE- $33.89 \mathrm{ay}$ 
AT MORRISON KNUDSEN CORPORATION

Project UMTRA - SRK Contract No.3885-76 File No 14

Feature TEMTPORARY FACILITIES

Designed

File No.

Item TMATERIAL QuANTITIES

Checked AFS

Date $\frac{1.29 .93}{7 / 16 / 93}$

1.) Retention Basin

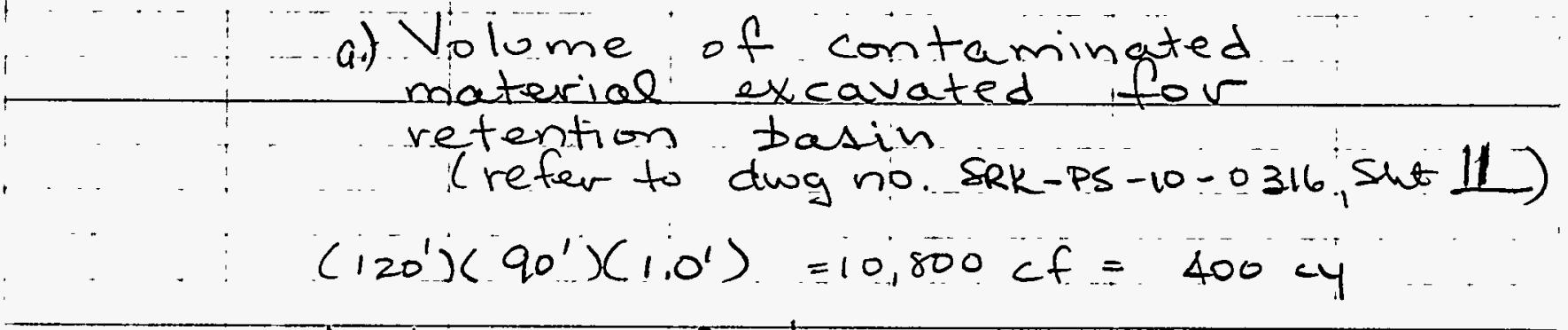

- b) Volume of total material Excavated (See shuts 17 to 21:)

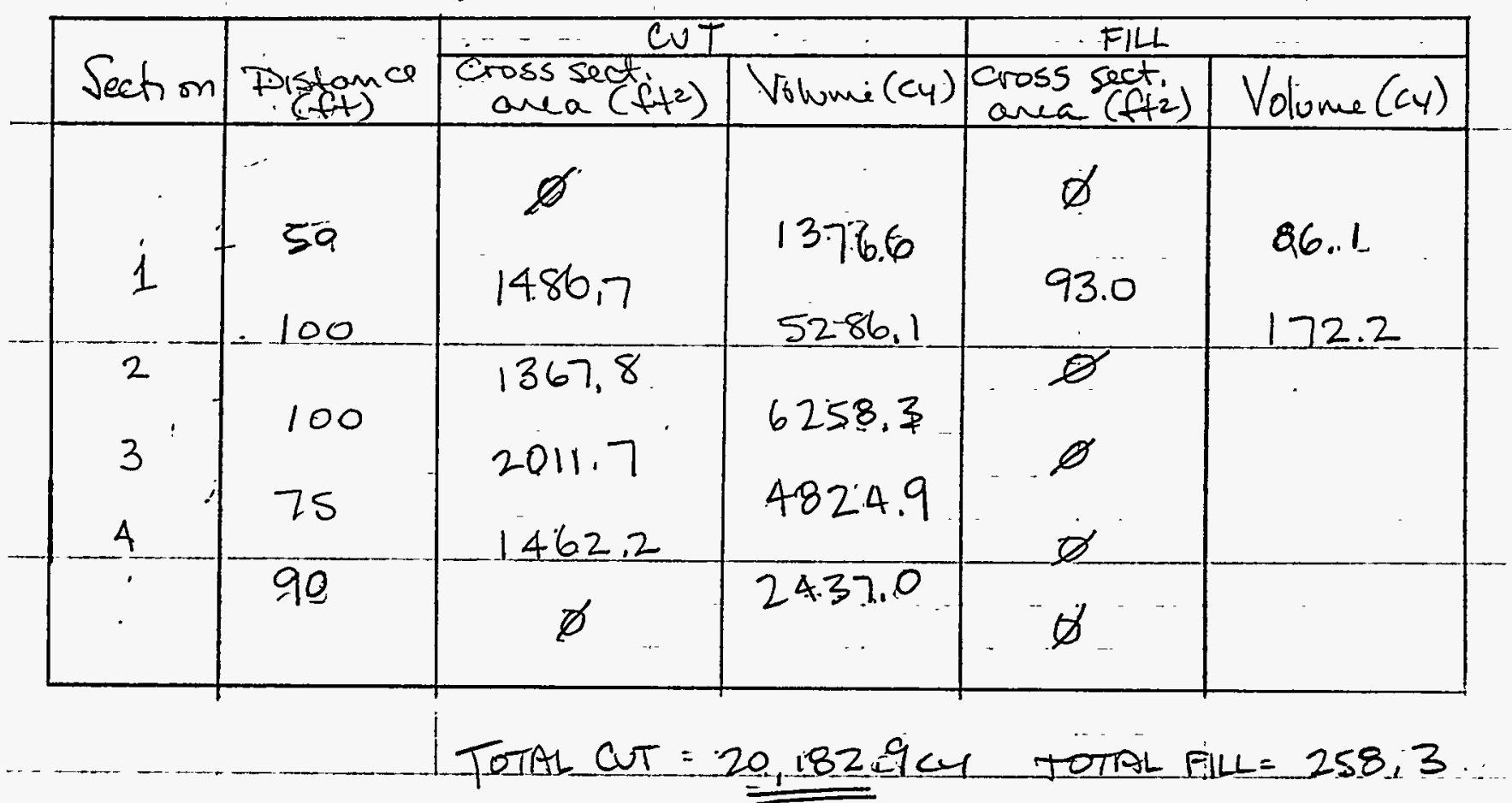

ENG 375/91 
@1 7MORRISON KNUDSEN CORPORATION

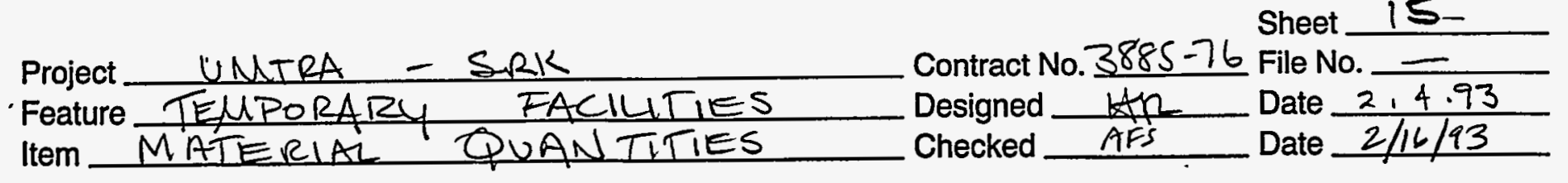

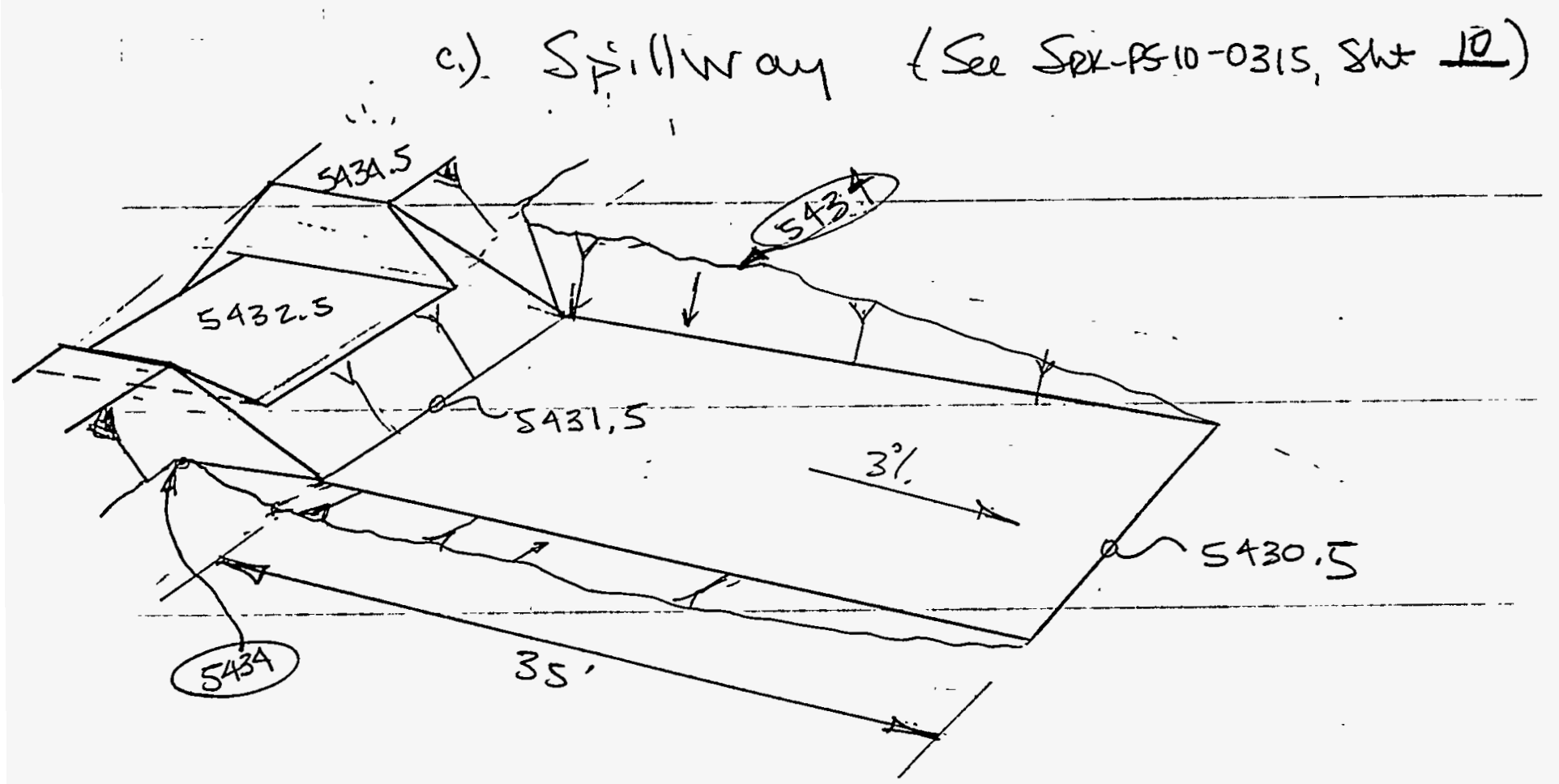

i). Reduce fill by Volume at spillway crest

$$
(18)(20)(2)\left(\frac{1}{27}\right)=26.9 \leq 4
$$

ii) Add to cut spillway ramp

- approximate volume by assuming a wedge

$$
V_{01}=\frac{l \times w \times h}{2}=\frac{(35)(1.0005)(1)(20)\left(\frac{1}{2}\right)}{2}
$$

(i) Sump $=(10 \times 10 \times 2.9)=\frac{13.0 \text { cr }}{=} .50 f=110.6 \mathrm{cy}$ cut

Total cut for

retention basin $=201829.9400+13+10.6=20606.5 \mathrm{cy}$

Total fill for ret, basin $=258.3+26.7=285.0 \mathrm{cy}$

ENQ 375/29

$14242 / 89$ 
(140 )MORRISON KNUDSEN CORPORATION

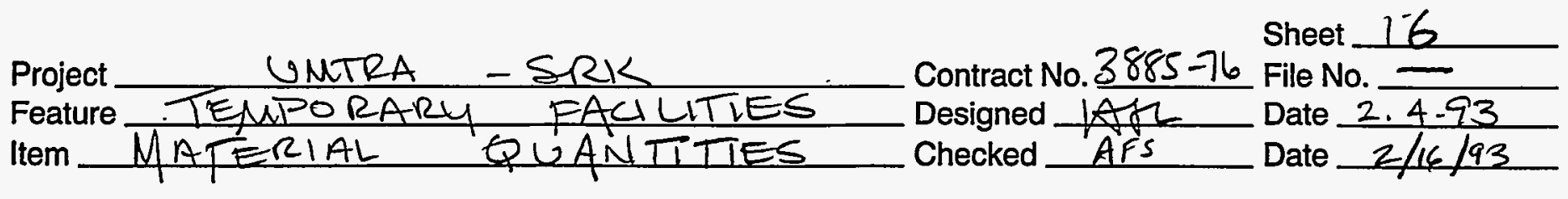

d.) Membrane Liner

i) Bottom Area, neglecting slope

$$
(250)(360)=90,0005 f
$$

ii) Sids Elope area

Aug. $\triangle$ elev.

(from conner elevations)

$$
\begin{aligned}
& =\frac{5.9+4.5+3.6+4.5}{4} \\
& =4.6
\end{aligned}
$$

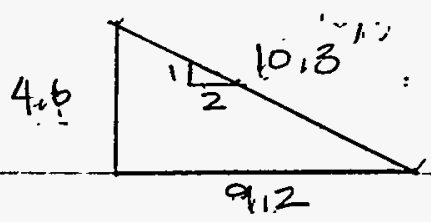

Average 'length of side slope $=10.3$ '

Perimeter, length at approximate midpoint of side slope $=$

$$
2(250+9.2)+2\left(360^{\prime}+9.2\right)=1256.8^{\prime}
$$

Area $=(1256.8)(10.3)=12.945 .0$ sf

(ii) Spill way

Area $=(25+5)(\overline{30})=900 \mathrm{sf}$

iv) Anchor trenches. (see sek-PS-10-0315)

Area $=[2(360+250+18.4)]\left(4,3^{\prime}\right)=5404.2$ sf

Total Liner Area $=10.9,249.2$ sf

ENG 375/89

$\overline{14242 / 89}$ 
Project UNTRA - SRK

Feature TEAMPORARY FACILITES

Item Material QuantiTLES
Sheet 17

File No.

Date 2.493

Date $2 / 16 / 93$
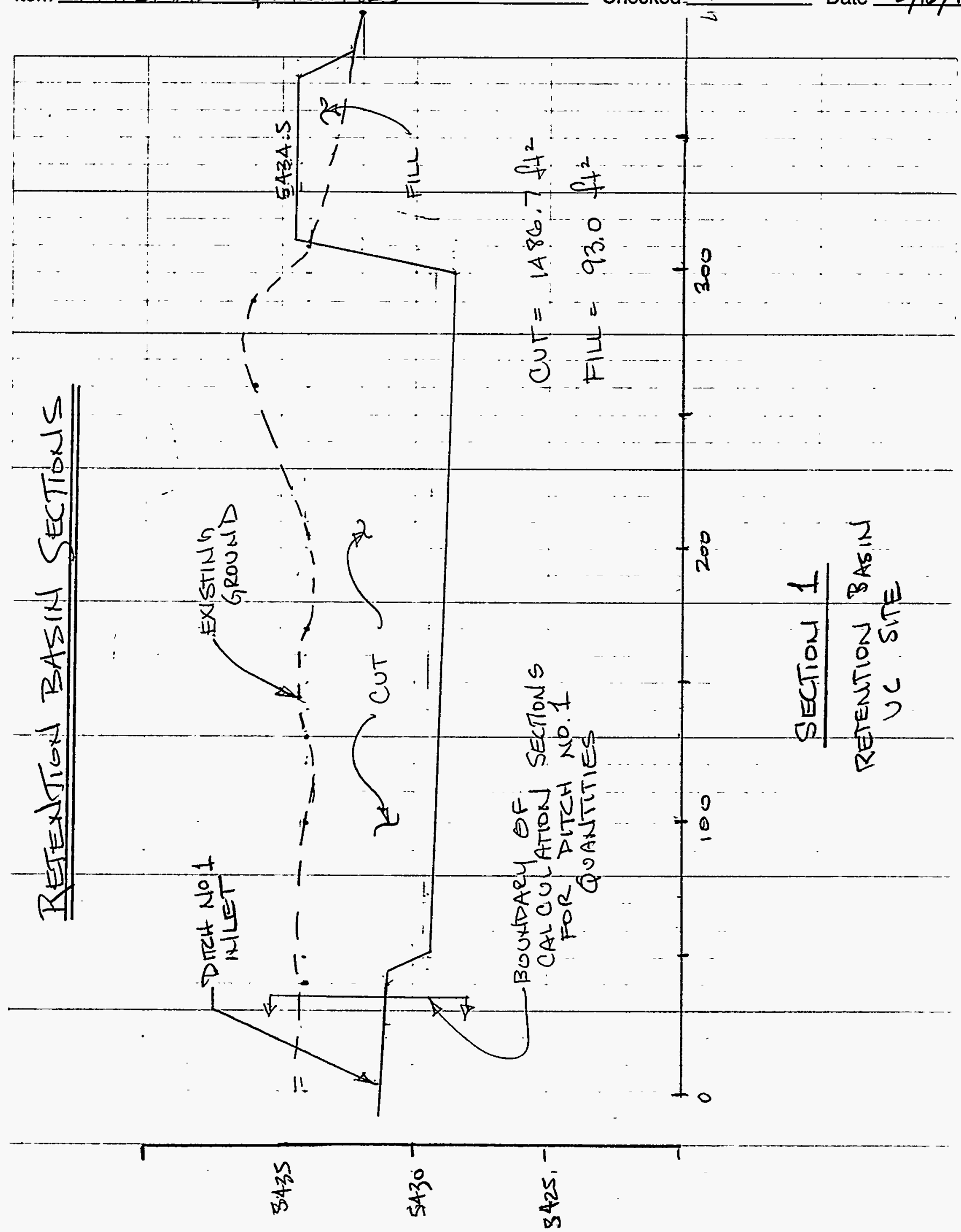
Project UNTRA - SRK

Feature TEAPORARY FACILITHES Item MATERIAL QUANTITIES
Sheet 18

Contract No. $885-76$ File No.

Designed Ahr

Date 2.4 .93

Date $2 / 16 / 93$

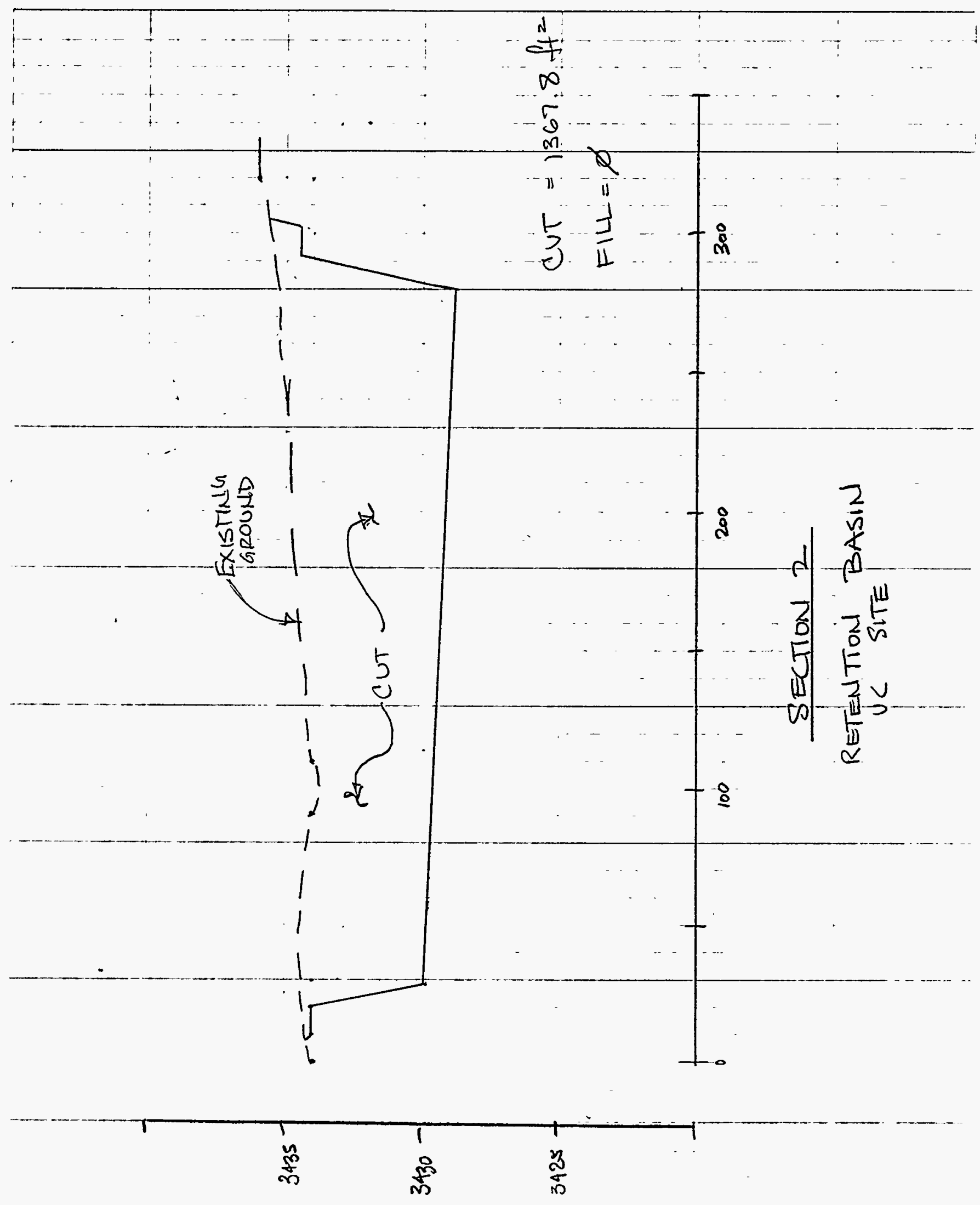


Project VMTRA - SRK

Feature

Item
FACIUTIES MATERIAL QUANTITIES
Contract No. 3885-76

Designed AL Checked AFs
Sheet 19

File No.

Date 2.4 .93

Date $2 / 16 / 93$
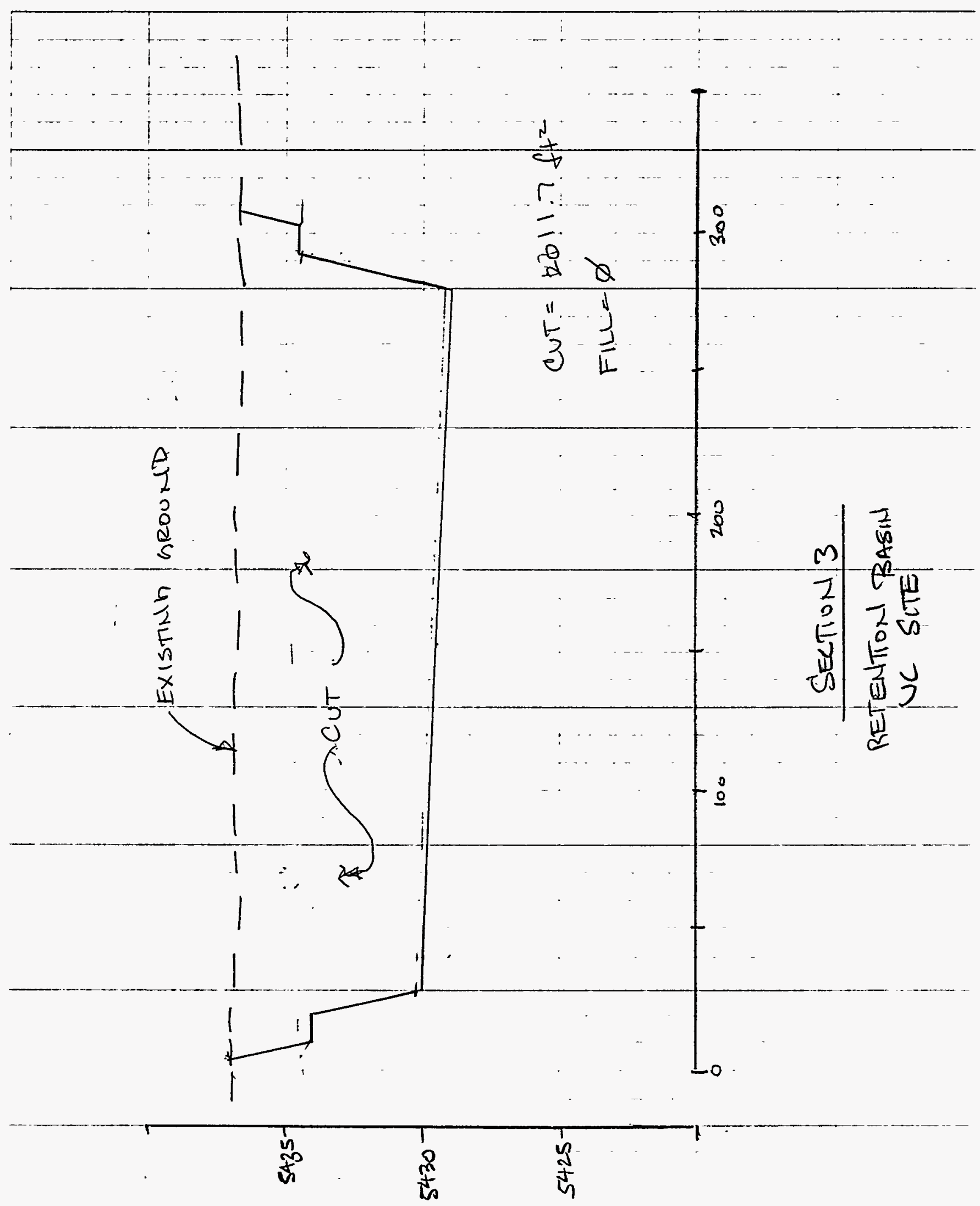
Project UMTRA - SRK

Feature IEMPORARY FACILTTES Item MATERIAL QUANTITIES
Contract No. 3885-76 Designed. Checked
Sheet 20

File No. -

Date 2.4 .93

Date $2 / 16 / 93$

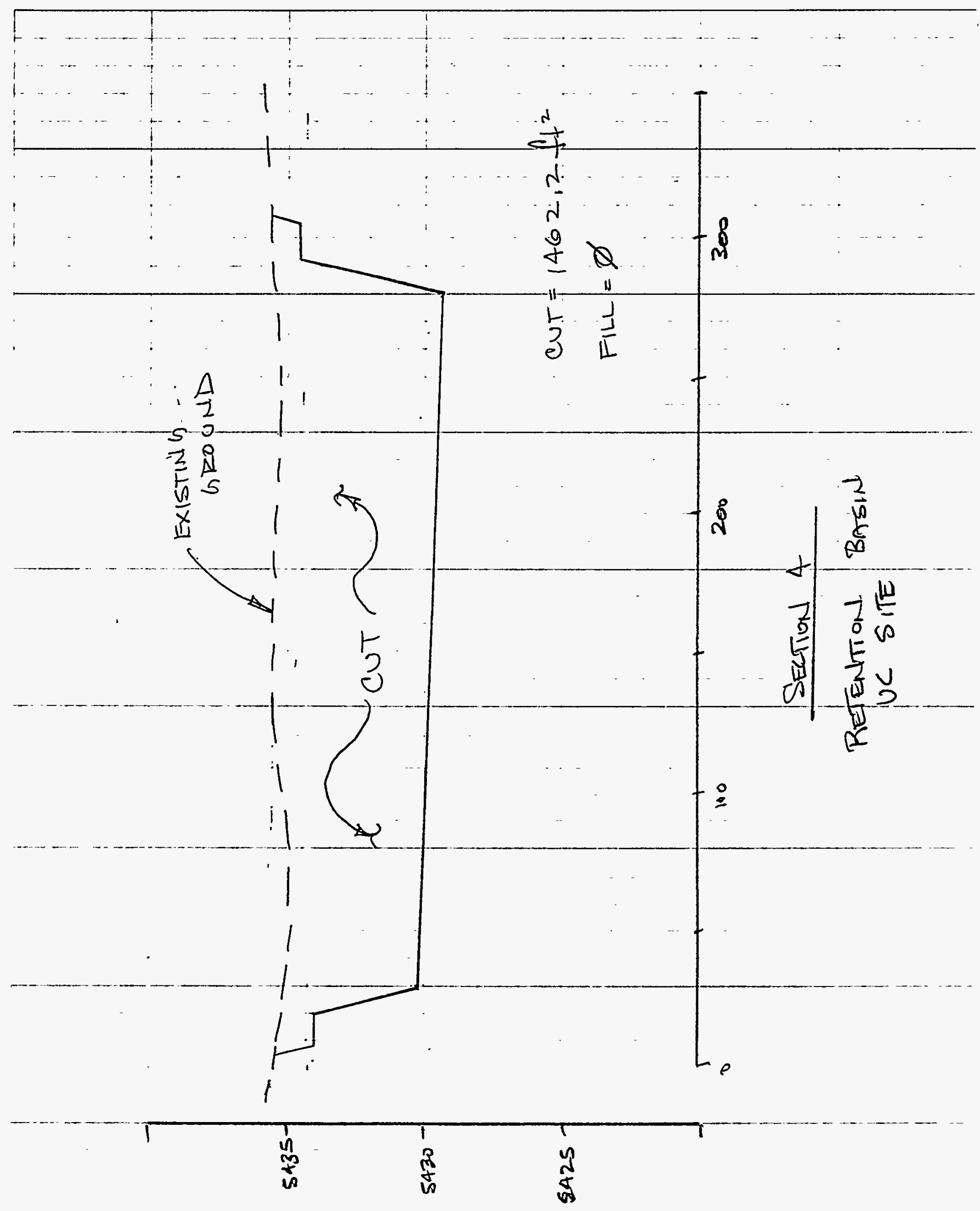




\section{(140)MK-ENVIRONMENTAL SERVICES}

Project UNTRA - SRK Feature TEMPORARY FACILITIES llem MAERITL QUANTTTES : TOW. NS FC Designed Date 4.26 .93 AREA. Checked JSR Date $4 / 30 / 93$

TRACTC rUDIIIVE DRAN

SRK-PS-10-0312
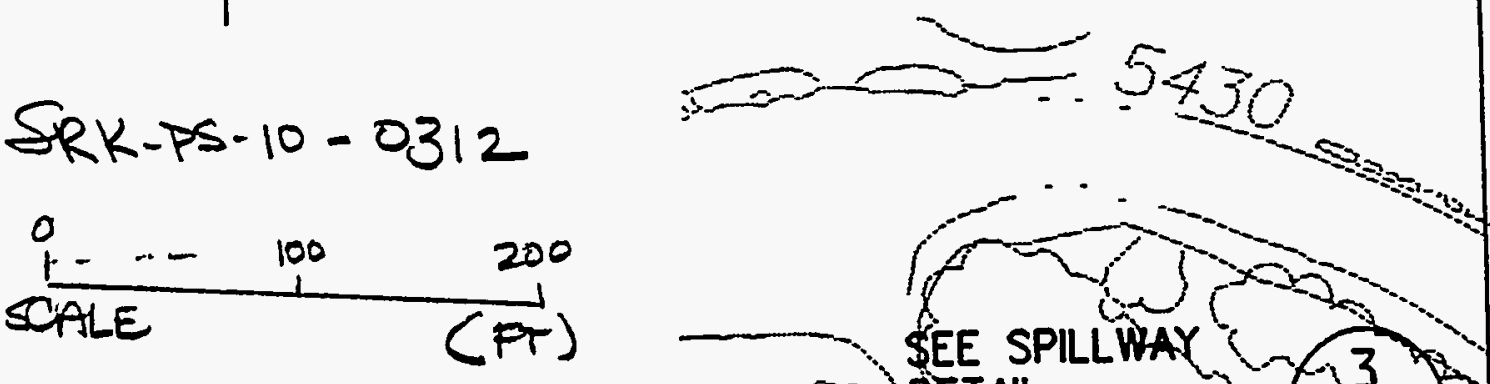

$=$

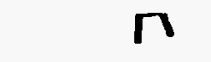
$54+20$

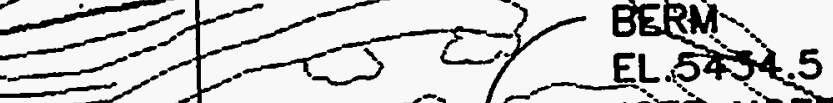
उदु 332 $x \rightarrow 5 T$ (1) $r$ (1) ( $\rightarrow$
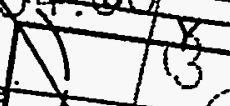
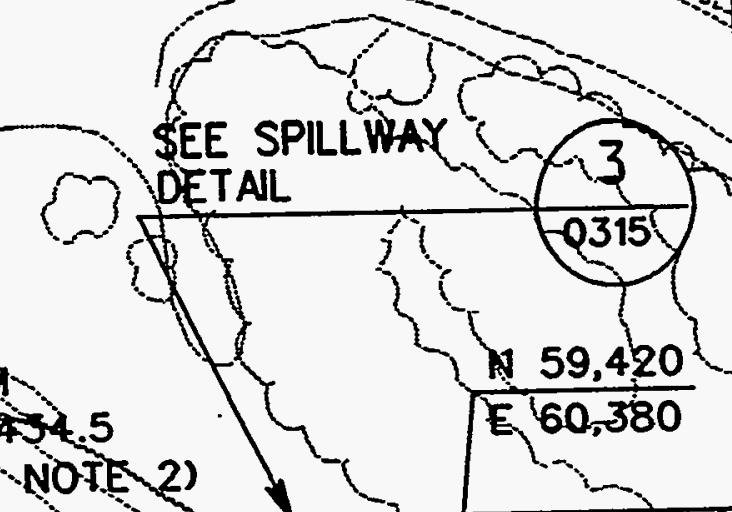

\$EE SPILLWAY

5. ALONG EXISTIN PATH SHALL E

6. SUBCONTRACTI POSITIVE DRAII BEGIN LOCATIC APPROXIMATE SHALL BE DET SUBCONTRACT

7. ROAD SHALL I TEMPORARY [

8. THE ACTUAL CONTRACTOR

9. EXISTING COU LOCATION SHI IS REMEDIATE

10. TEMPORARY : AND MAINTAIN

11. AFTER AREA ROAD CR-S8 ORIGINAL ALIC

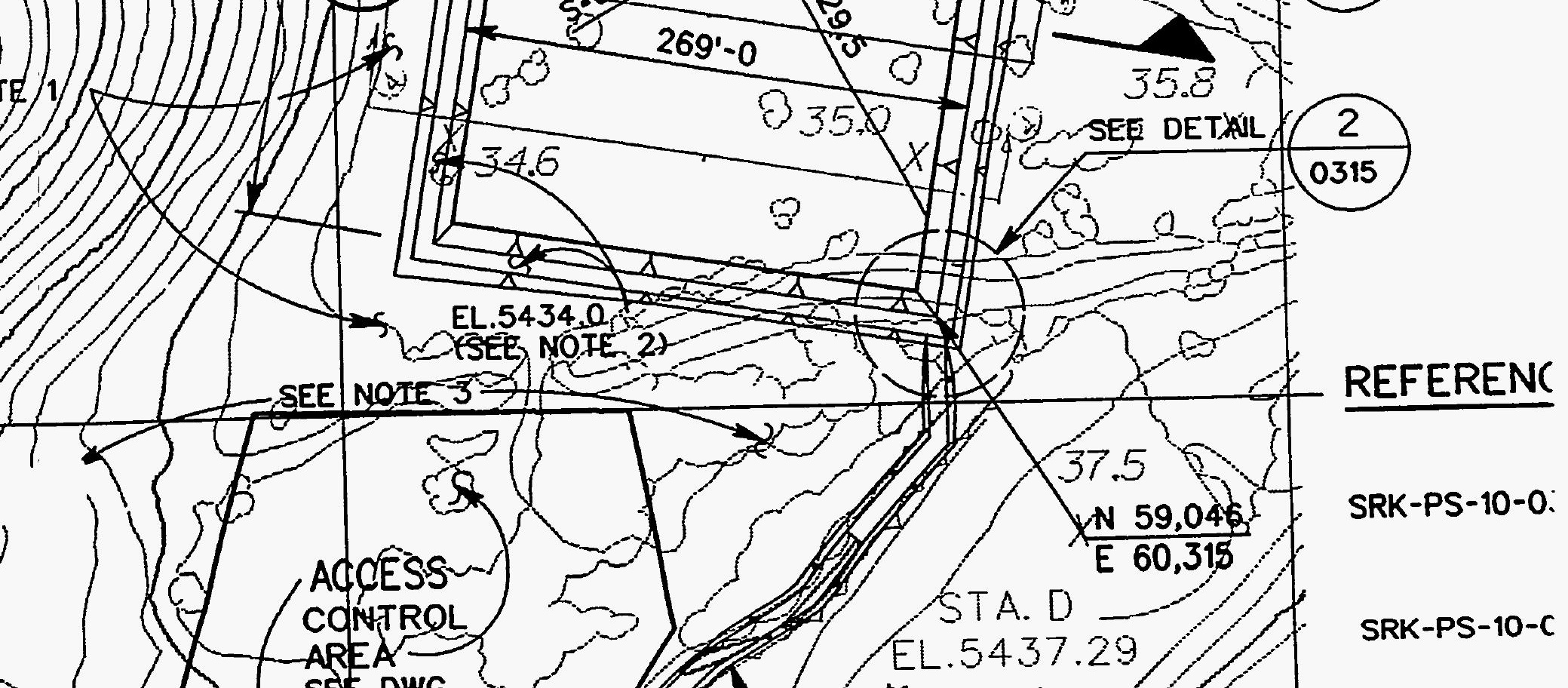


@9. MORRISON KNUDSEN CORPORATION

Project UMTRA - SRK

Sheet 22

Feature TEMPORARY FACILITES

Contract No. $3885-76$ File No.

Item

MATERIAL

QUANTITLES Designed

Checked As

Date $\frac{2.1 \cdot 93}{2 / 16 / 93}$

ii) Ditches

All ditch excavation will be beyond the

boundary of contaminated areas.

DITCH NO 1 (See shuts 24 to 34 .)

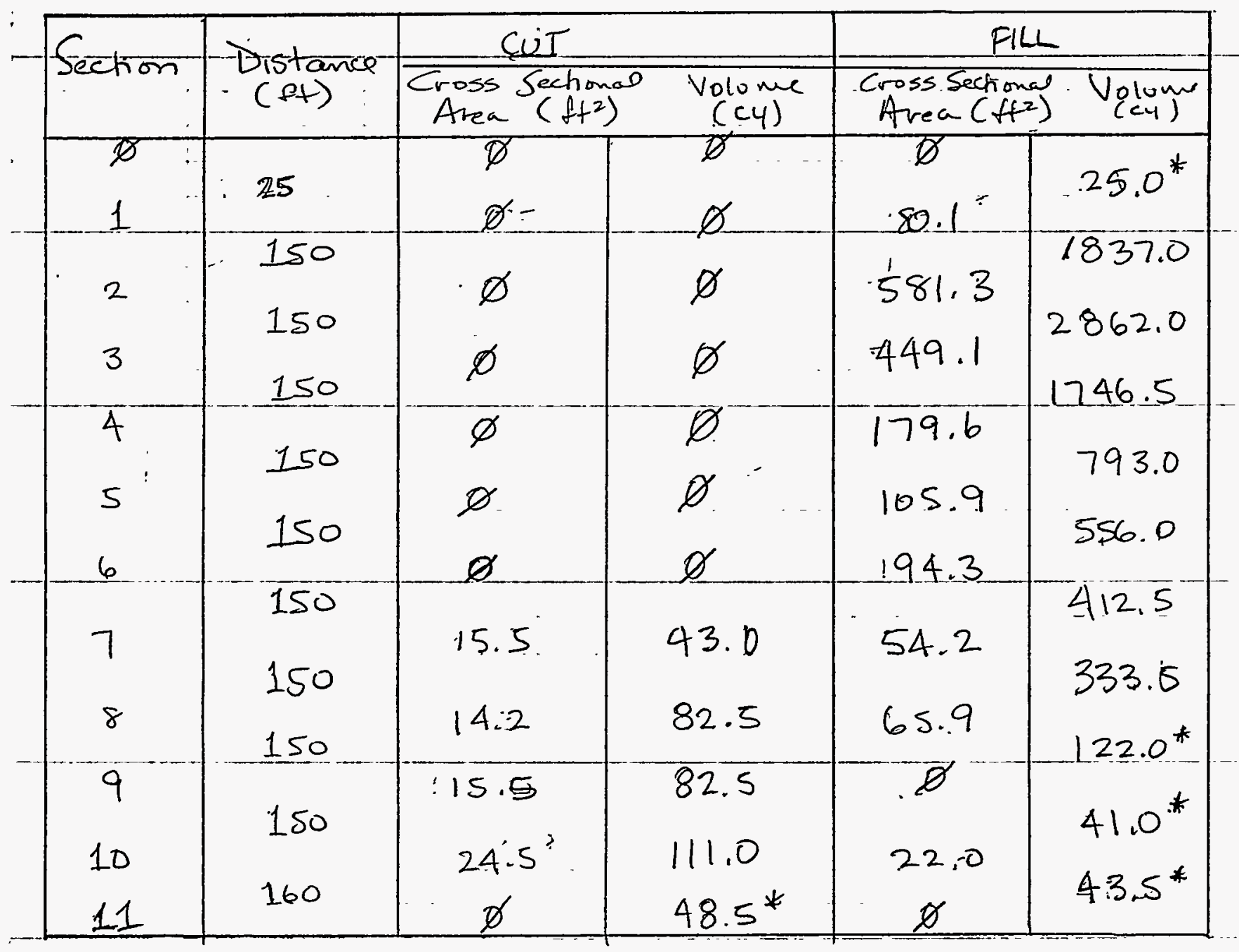

$$
\begin{gathered}
\text { TOTAL } \\
\text { CUT }
\end{gathered}
$$

* iNDICATED WHERE TY AMID VOLUME FORMULA WAS USED

ENG $375 / 91$ 
Project UMTRA - SRK

Feature TEMPORARY FACILITIES Contract $30.385-76$ Sheet 23 Item MATERIM, QuANTITIES

DITH NOH Z - OC SITE (See Shts 25 to 35)

\begin{tabular}{|c|c|c|c|c|c|}
\hline \multirow{2}{*}{ Secrial } & \multirow{2}{*}{$\begin{array}{c}\text { DISTANKCE } \\
\text { (FT) }\end{array}$} & \multicolumn{2}{|l|}{$C U T$} & \multicolumn{2}{|l|}{ FILL } \\
\hline & & $\begin{array}{l}\text { Clooss SETIONAL } \\
\text { ALEA (FTZ) }\end{array}$ & $\begin{array}{l}\text { VOLUME } \\
\text { (C4) }\end{array}$ & $\begin{array}{l}\text { Cooss SECTONAC } \\
A R \in A\left(F T T^{2}\right)\end{array}$ & $\begin{array}{l}\text { VOLUME } \\
(C Y)\end{array}$ \\
\hline (A) & 30 & $\begin{array}{r}\not D \\
38.8 \\
\end{array}$ & $14.4^{*}$ & $\begin{array}{l}\not \\
\varnothing\end{array}$ & $c-1$, \\
\hline (B) & 100 & 10.3 & 90.9 & $\varnothing$ & $\not \varnothing$ \\
\hline (c) & 100 & 5.1 & 28.5 & 3.9 & 7.2 \\
\hline (D) & 100 & 7.8 & 23.9 & $\varnothing$ & 7.2 \\
\hline (E) & 100 & 27.1 & 64,6 & $\not \varnothing$ & $\varnothing$ \\
\hline (B) & 100 & -25.8 & 98,0 & $\varnothing$ & $\varnothing$ \\
\hline (1) & 130 & 72 & 9267 & idx & $\varnothing$ \\
\hline & 40 & $\mathscr{D}$ & $357^{*}$ & & $\varnothing$ \\
\hline & & & & & \\
\hline i & To & TAL CUT & $592.2 \mathrm{cy}$ & TOTAL FILL & $14.4 c y$ \\
\hline & CRH NOO. & $3-v c$ & SITE (See Se & its 25 to & $35)$ \\
\hline & 32 & $\varnothing$ & & $\mathscr{D}$ & $\varnothing$ \\
\hline (A) & & .93 .0 & $40.2^{*}$ & $\varnothing$ & \\
\hline$(\oplus$ & 100 & 15.5 & 200.9 & $\not$ & \\
\hline (I) & 100 & 40.0 & 102.8 & $\varnothing$ & \\
\hline$\Theta$ & .80 & 60.7 & 149.5 & $\varnothing$ & \\
\hline (B) & & $\varnothing$ & $30.0^{*}$ & $\varnothing$ & \\
\hline & & $\because$ & & & \\
\hline
\end{tabular}


《.9. MORRISON KNUDSEN CORPORATION

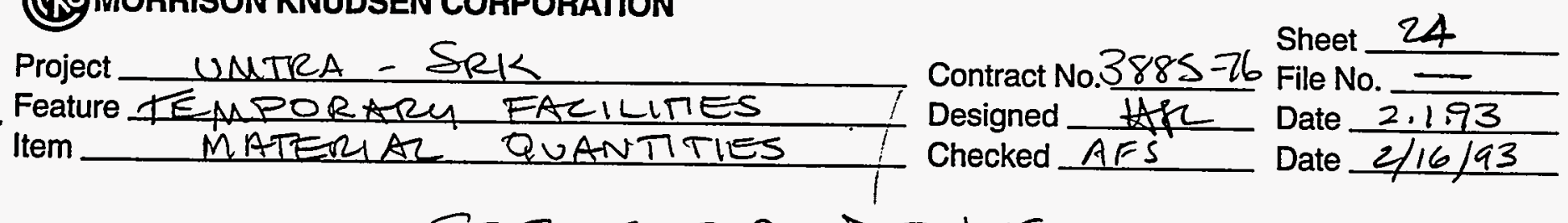

SECTORS FOR DITCHES
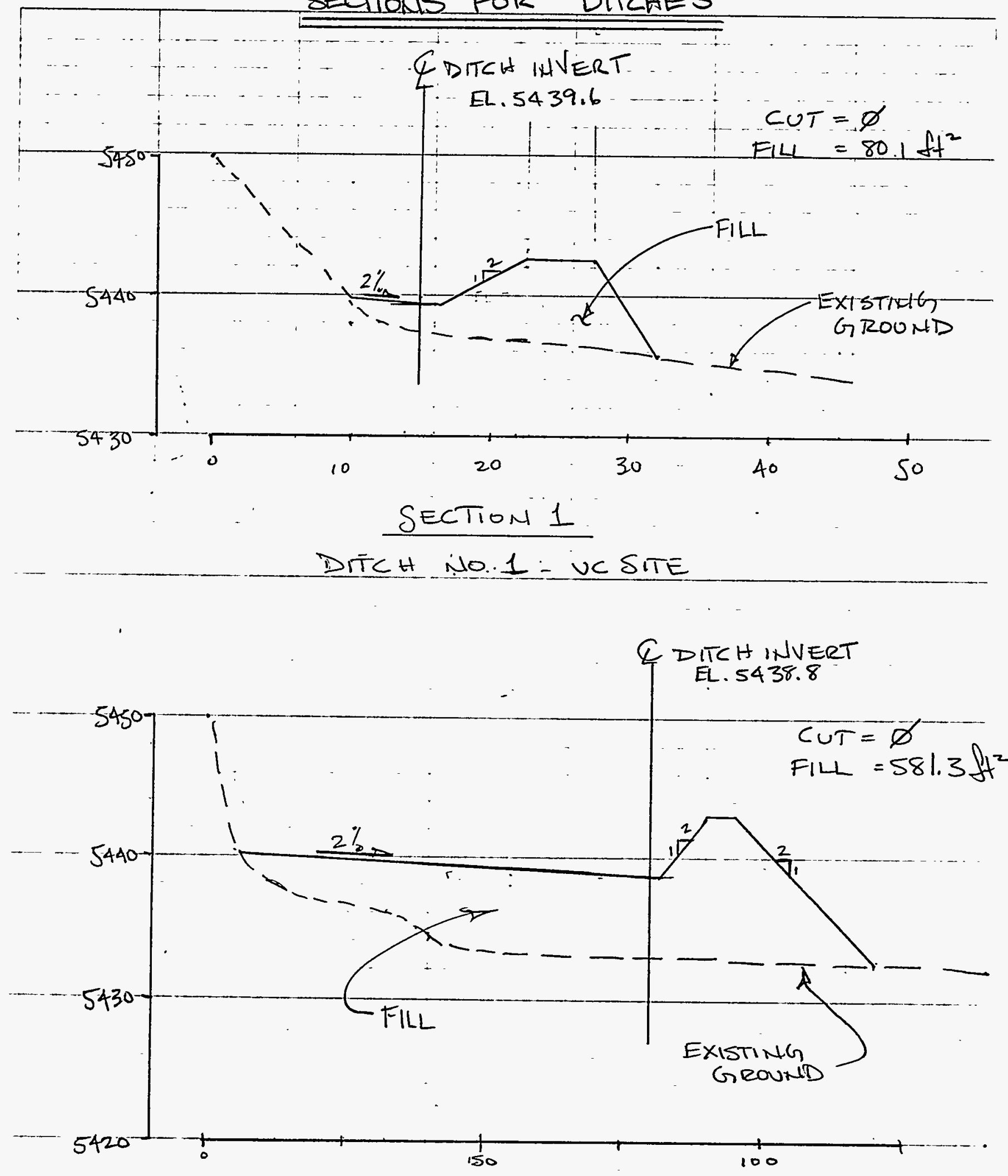

SECTION 2

DITCH NOS - UL SITE

ENG 375/91 
@M MORRISON KNUDSEN CORPORATION

Project UUMTRA - SRK

Feature TEAPPORARY FACILITIES

Item
Contract No $3885-76$

Designed

Checked AFS
Sheet 25

File No.

Date 1.2993

Date $\frac{1.29 .93}{2 / 16 / 93}$

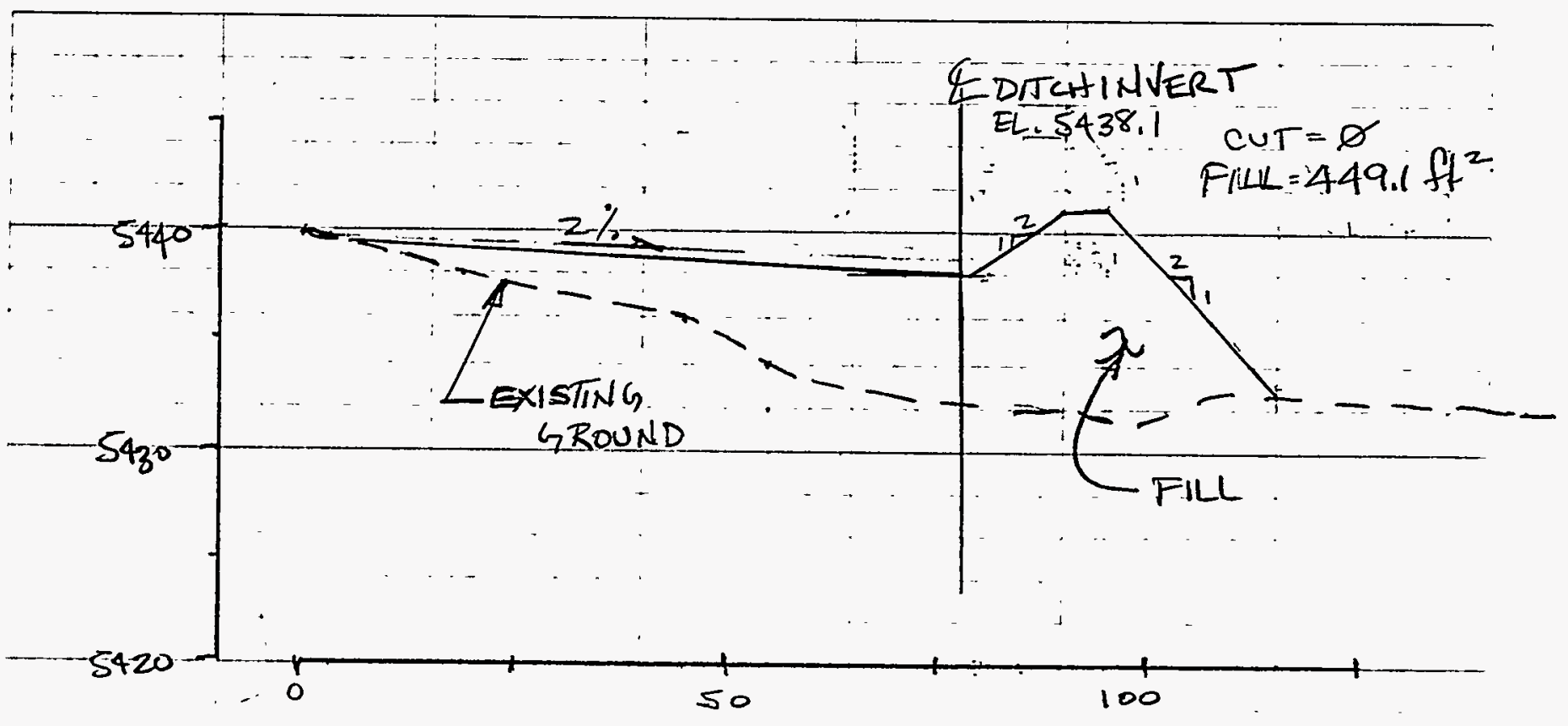

SECTION 3

DITEH No 1 - VC SITE

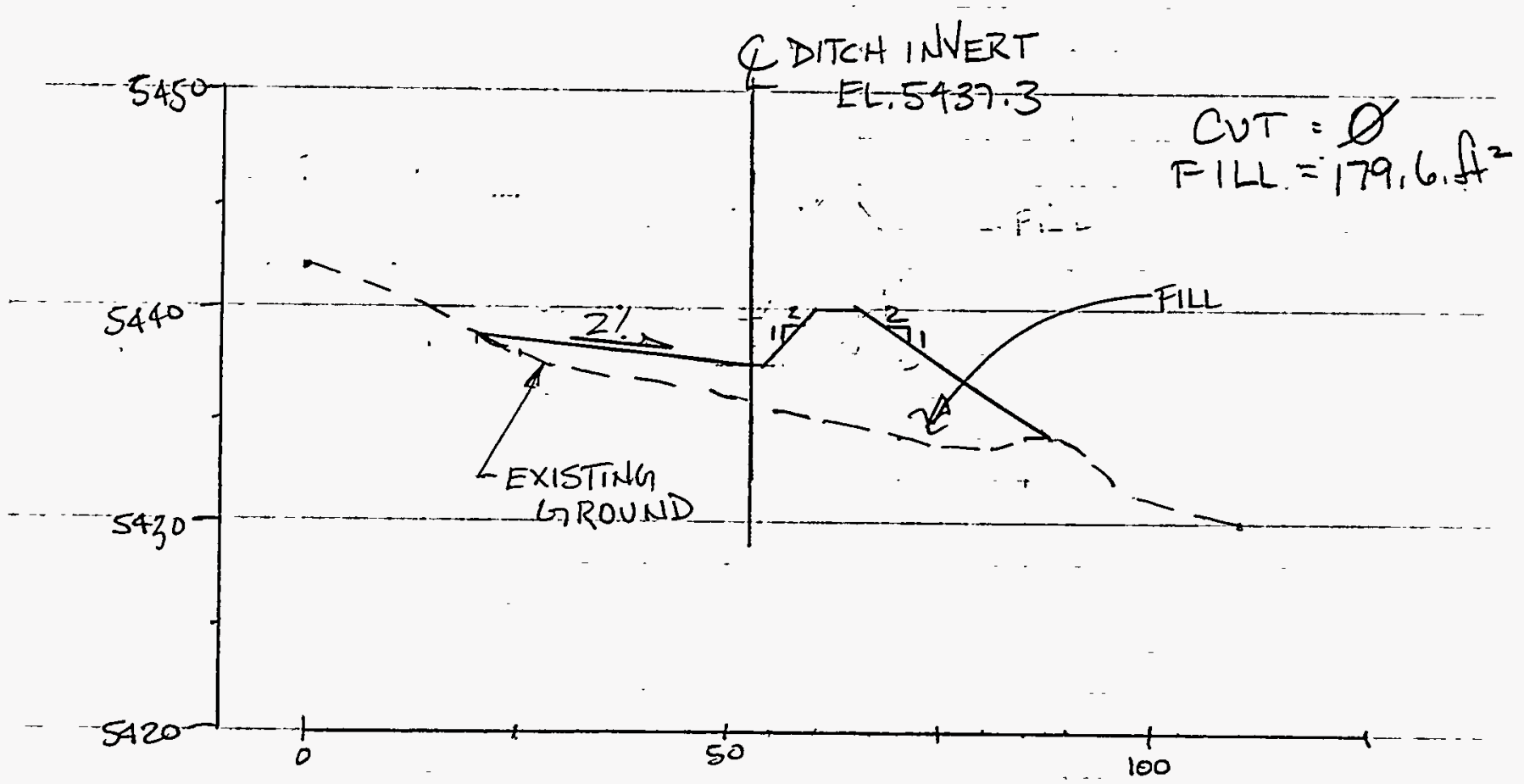

SECTION 4

DITCH NOD - VC SITE

ENG 375/91 
CROMORRISON KNUDSEN CORPORATION
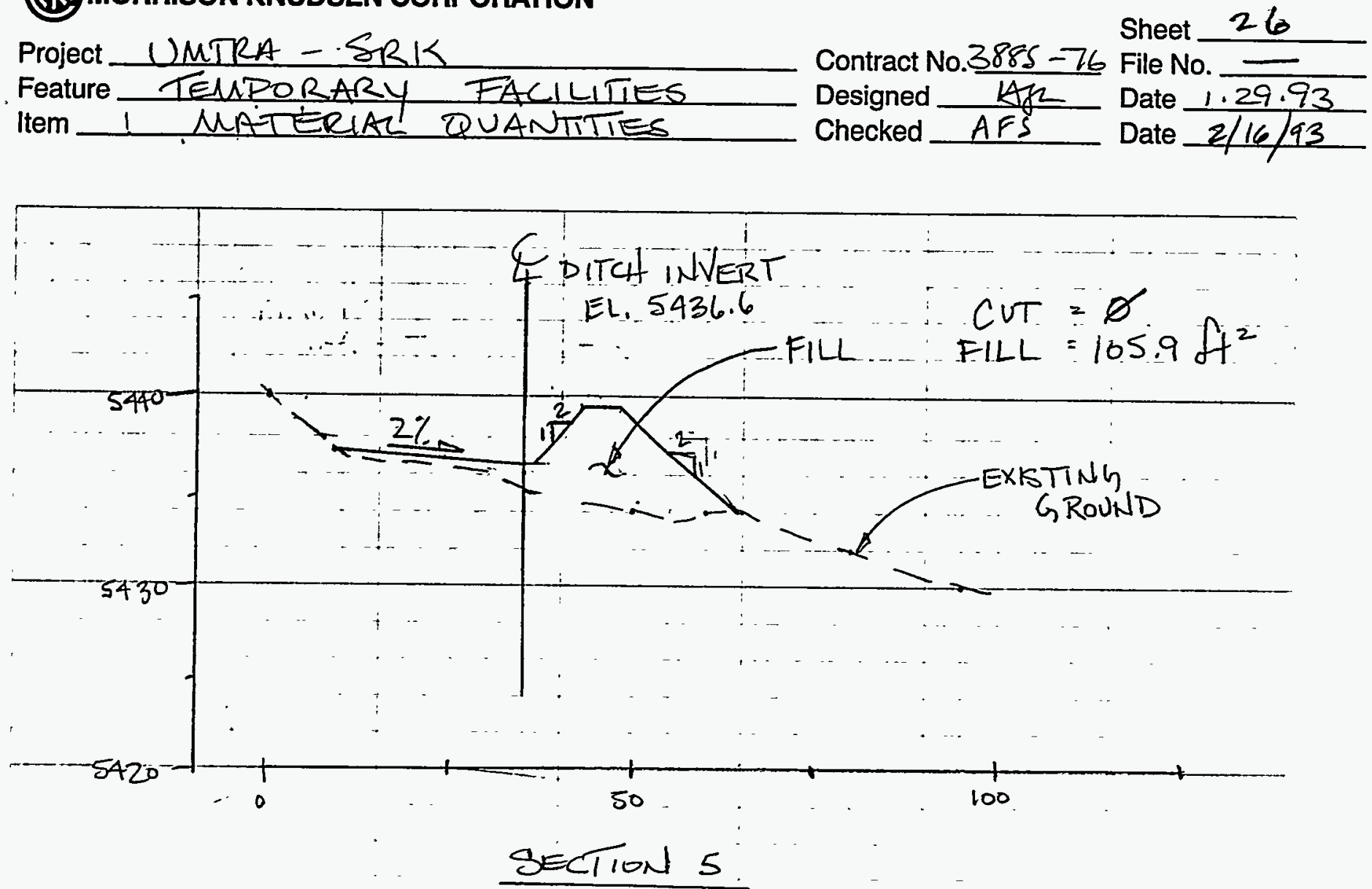

DIKH No 1 - U SITE

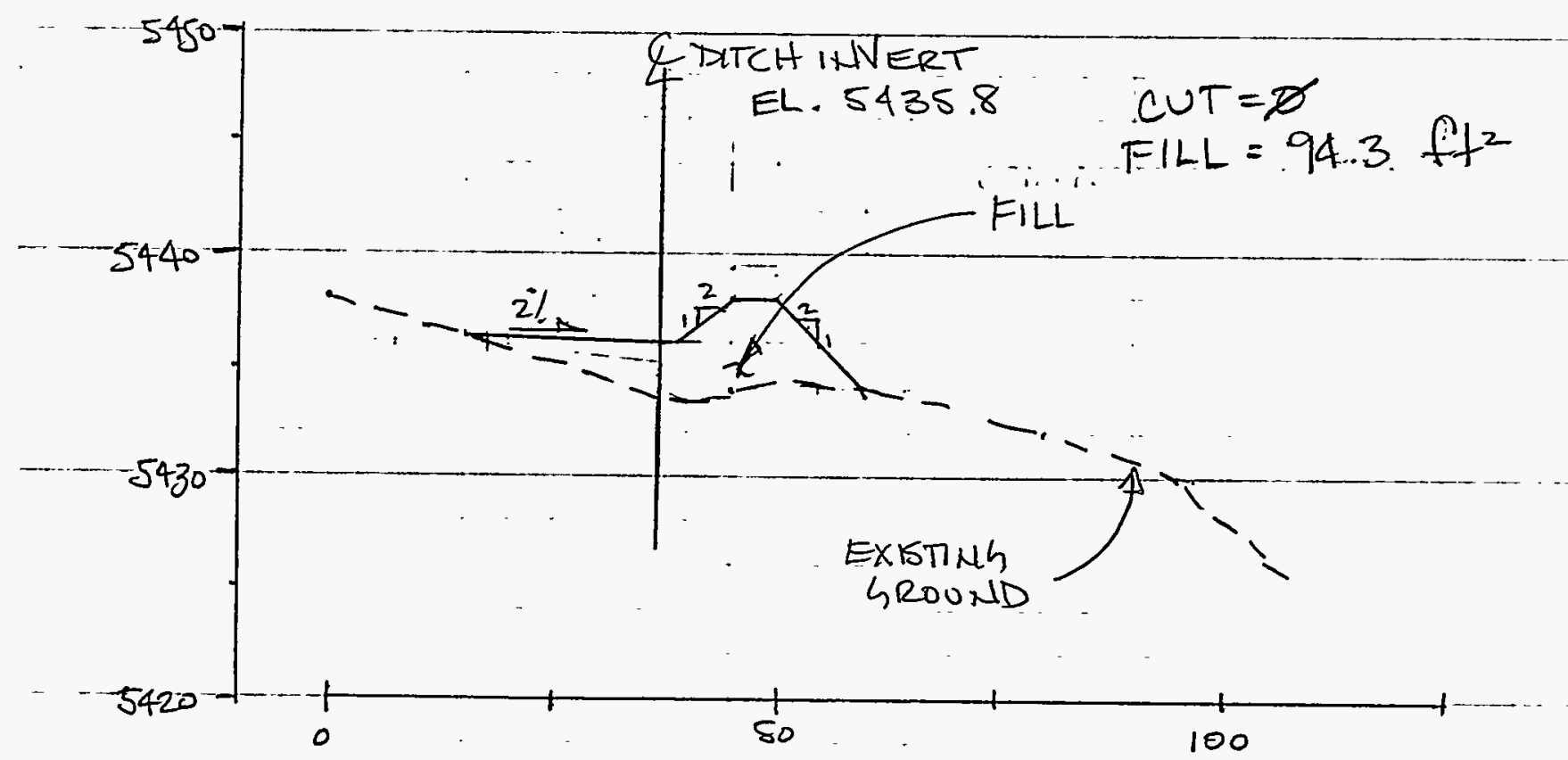

SECTION 6

DITCH NO 1 - UK SITE

ENG 375/91 
49. MORRISON KNUDSEN CORPORATION

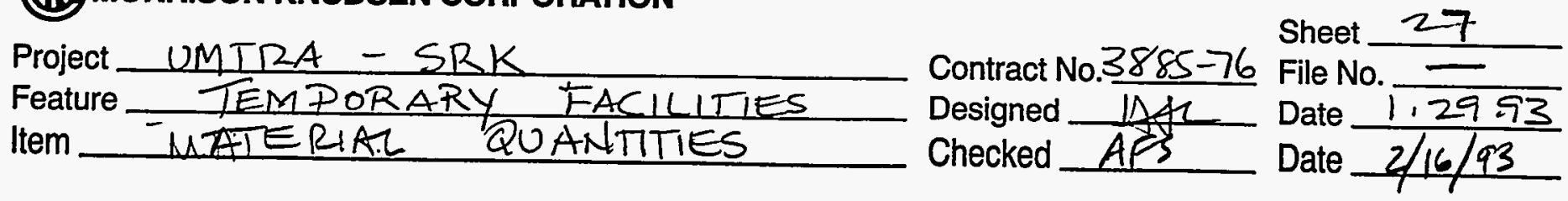

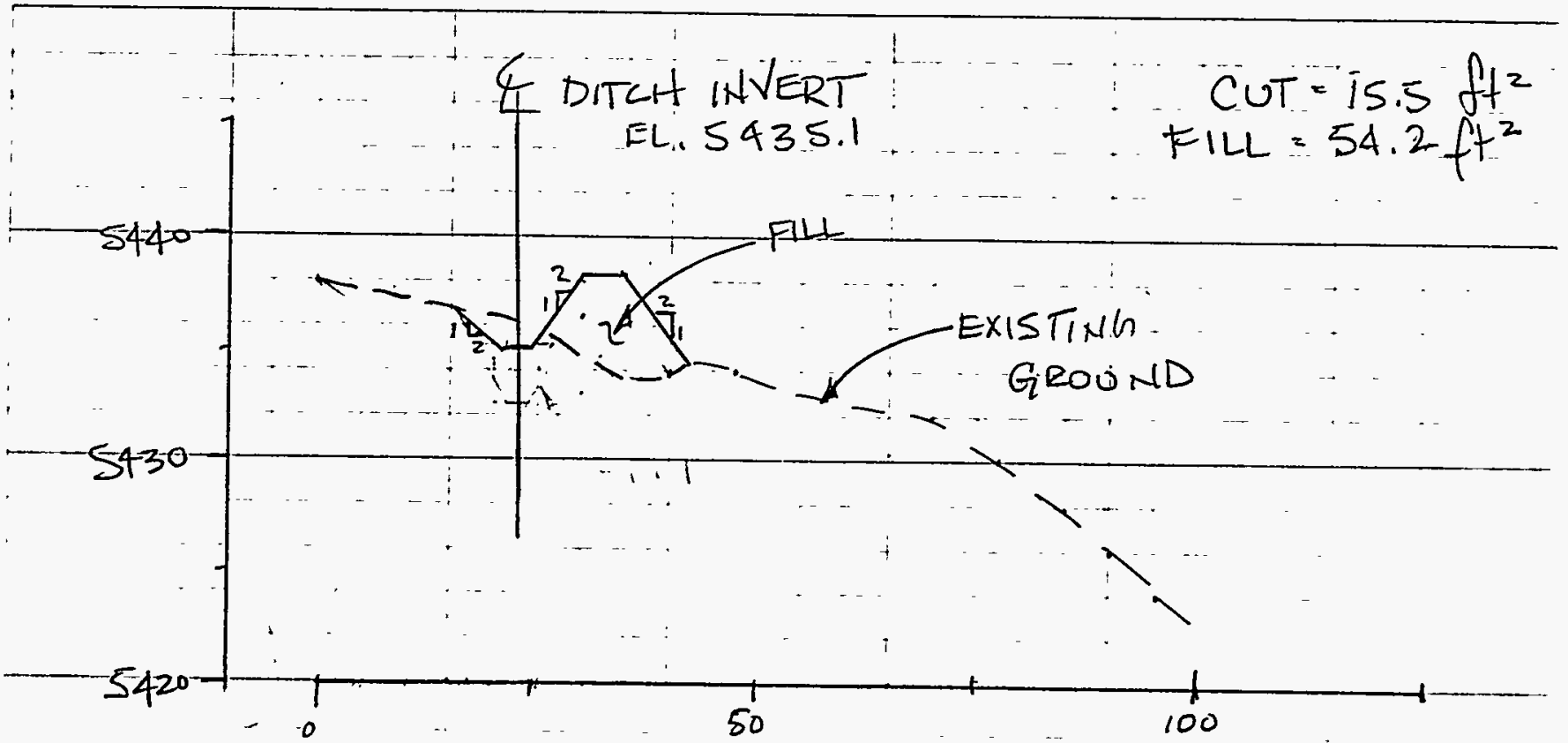

SECTION 7

DITCH NO. 1 - NC SITE

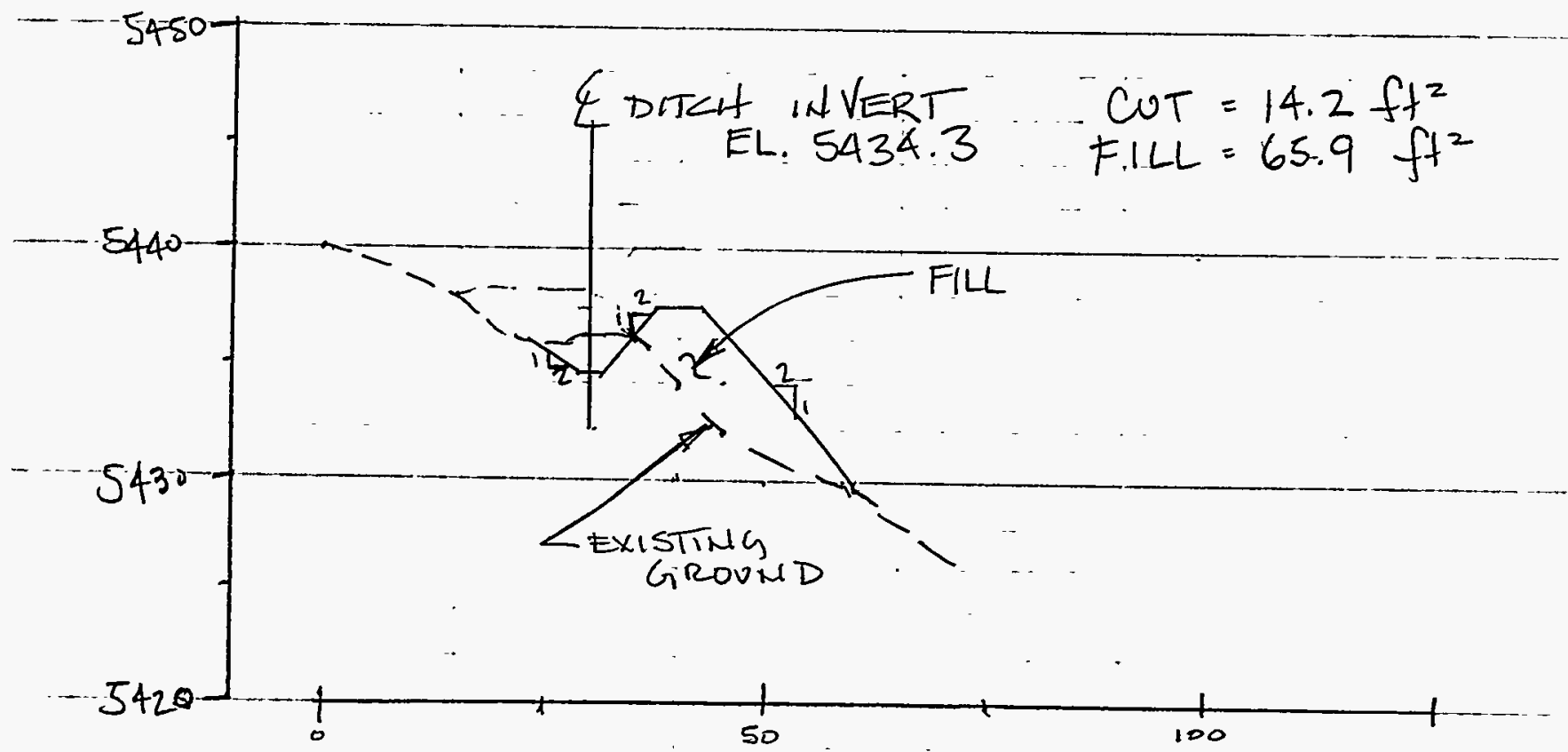

SECTION. 8

DITCH NO 1. VC SITE

ENG 375/91 
숭 MORRISON KNUDSEN CORPORATION

Project UMTRA - SRK

Feature TEMPORAR, FACILITIES

Item
MATERIAL
Contract No.3885-76 Designed

Checked AFS
Sheet 28

File No.

Date $\frac{1.29 .93}{2 / 14 / 93}$

Date $2 / 16 / 93$
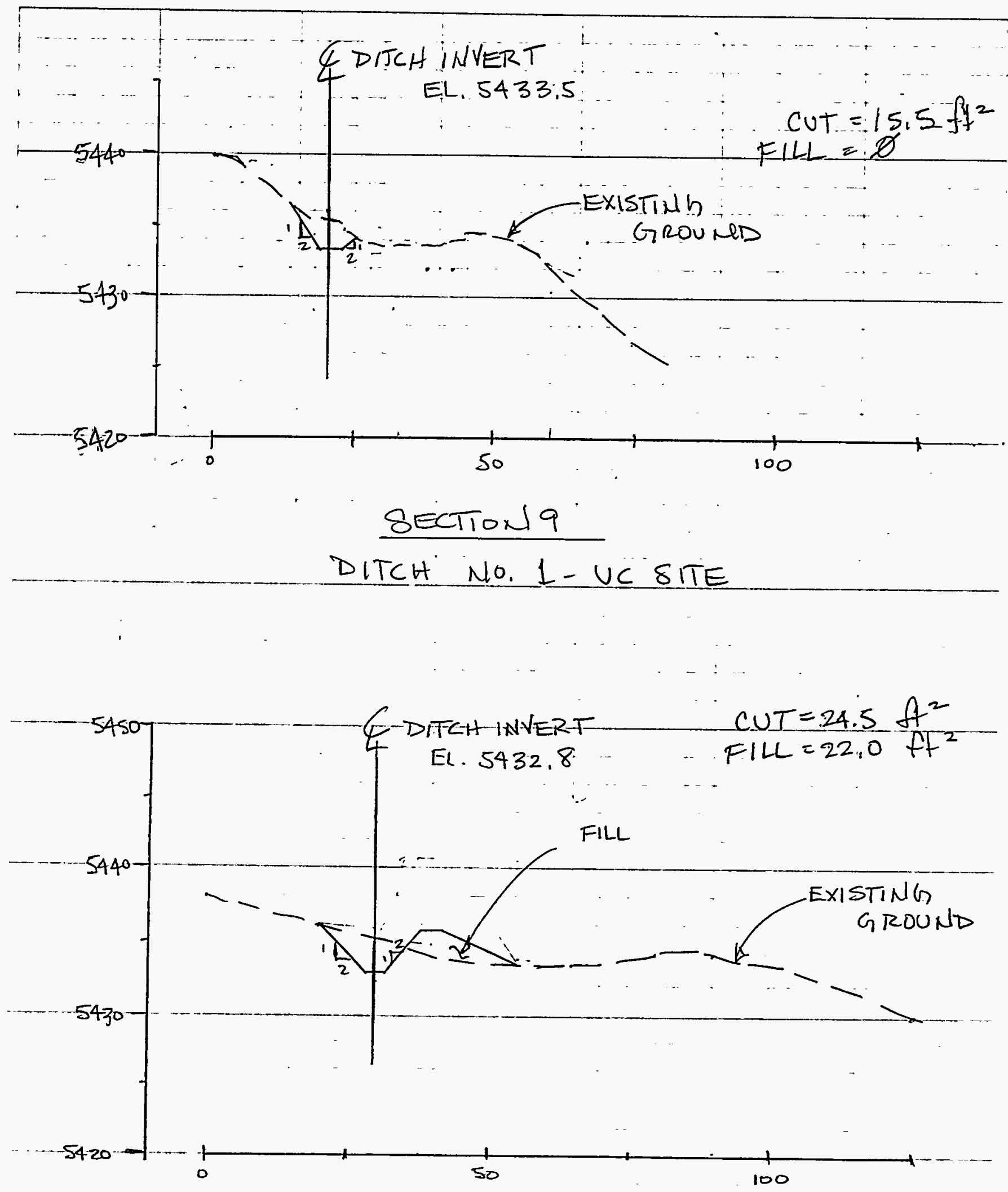

SECTION 10

DITCH NO. 1 - VC SITE

ENG 375/91 
Project UMTRA - SRK

Feature TENPPORARY FACILTIES

Item uaterlál quantities

Contract No. $3885-76$ Designed Ay Checked AYs
Sheet 29

File No. -

Date 2.1 .93

Date $2 / 16 / 93$

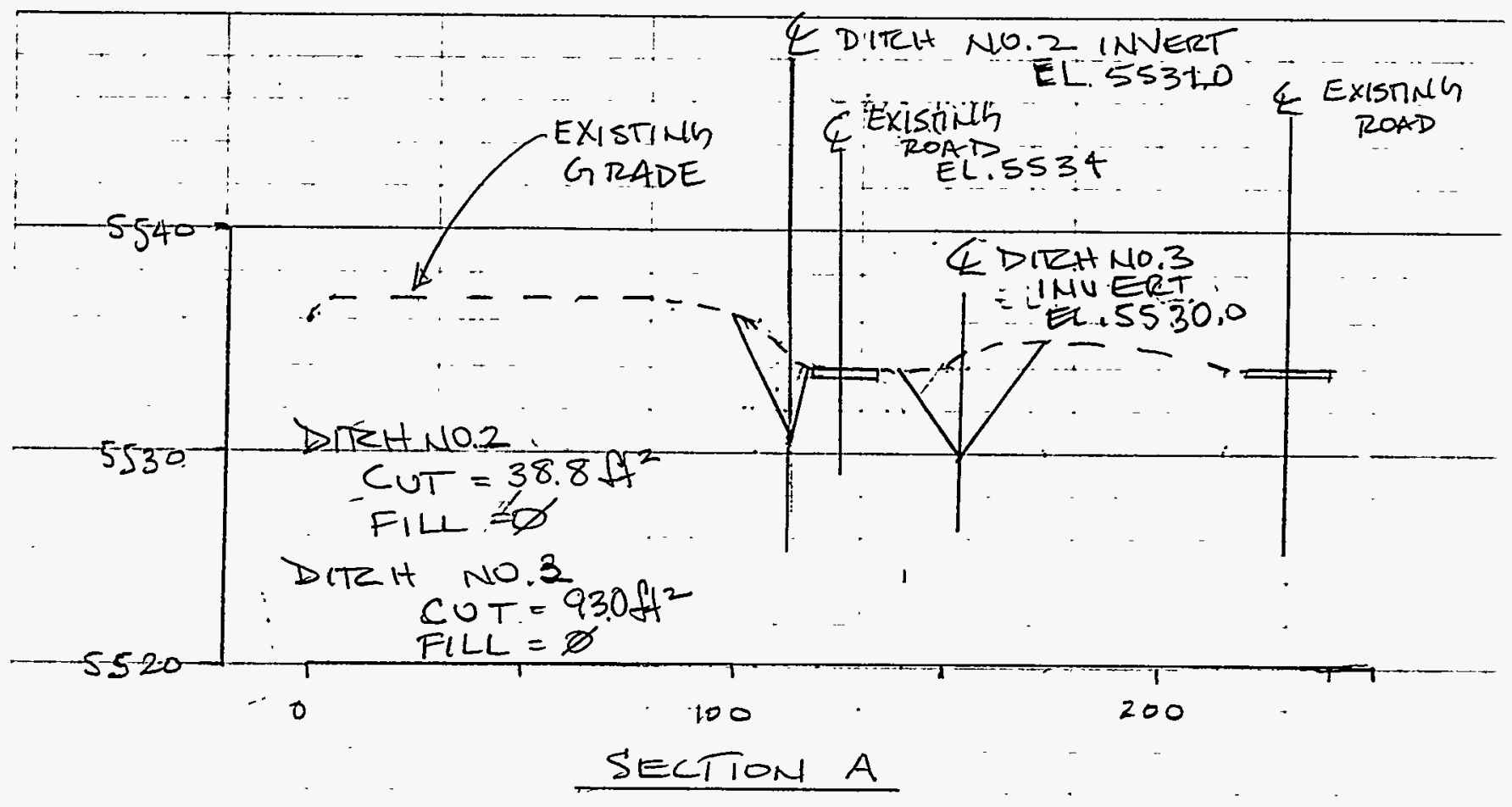

DITCH $2 \sum_{1} 3$ - VC SITE

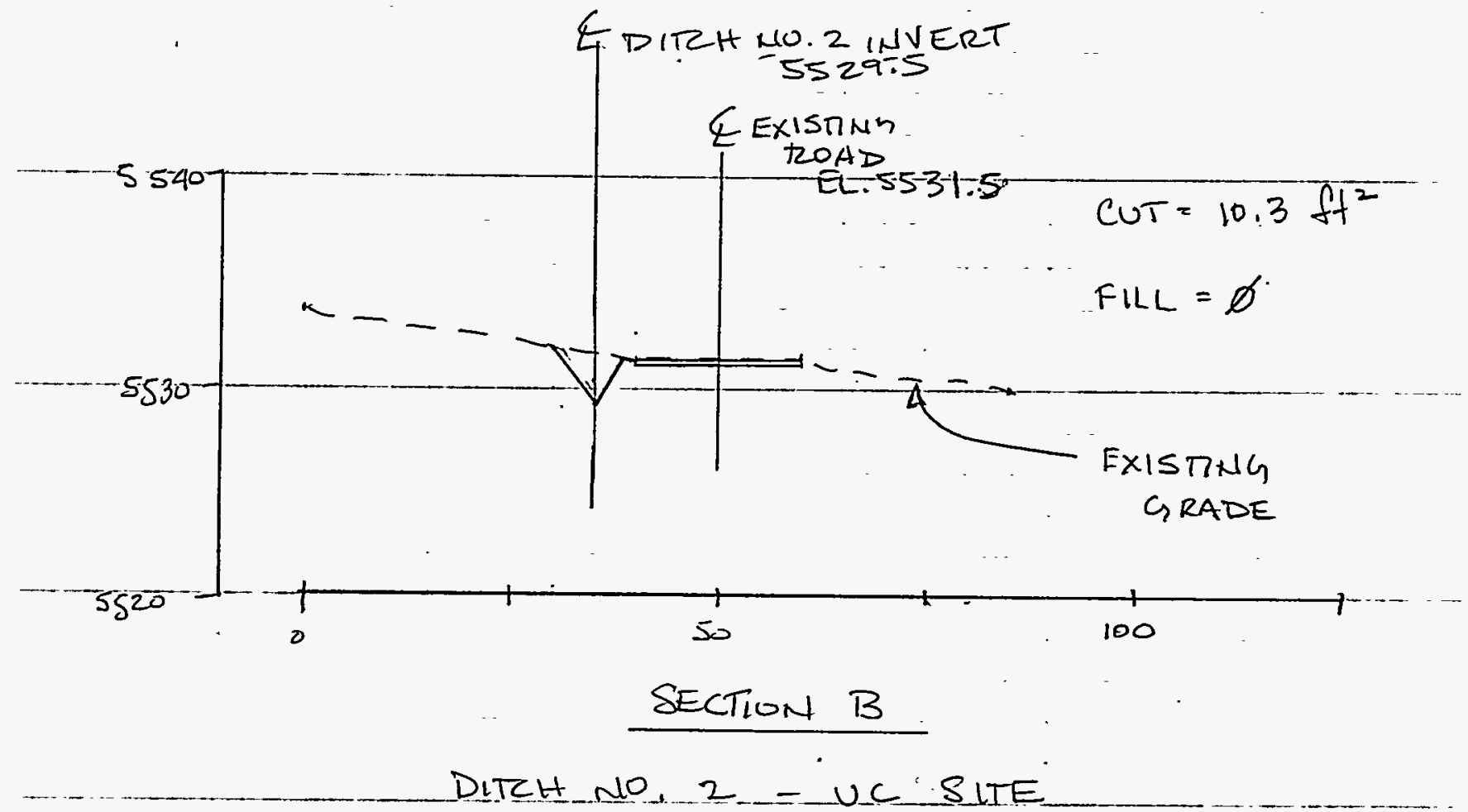




\section{Project UMTRA - SRK}

Feature TEMPORARY FACILITIES

Item : MATERIAL QUANTITIES
Contract No. $3885 \div 76$ Designed Kfe Checked AFS
Sheet 30

File No.

Date 2.2 .93

Date $2 / 16 / 93$
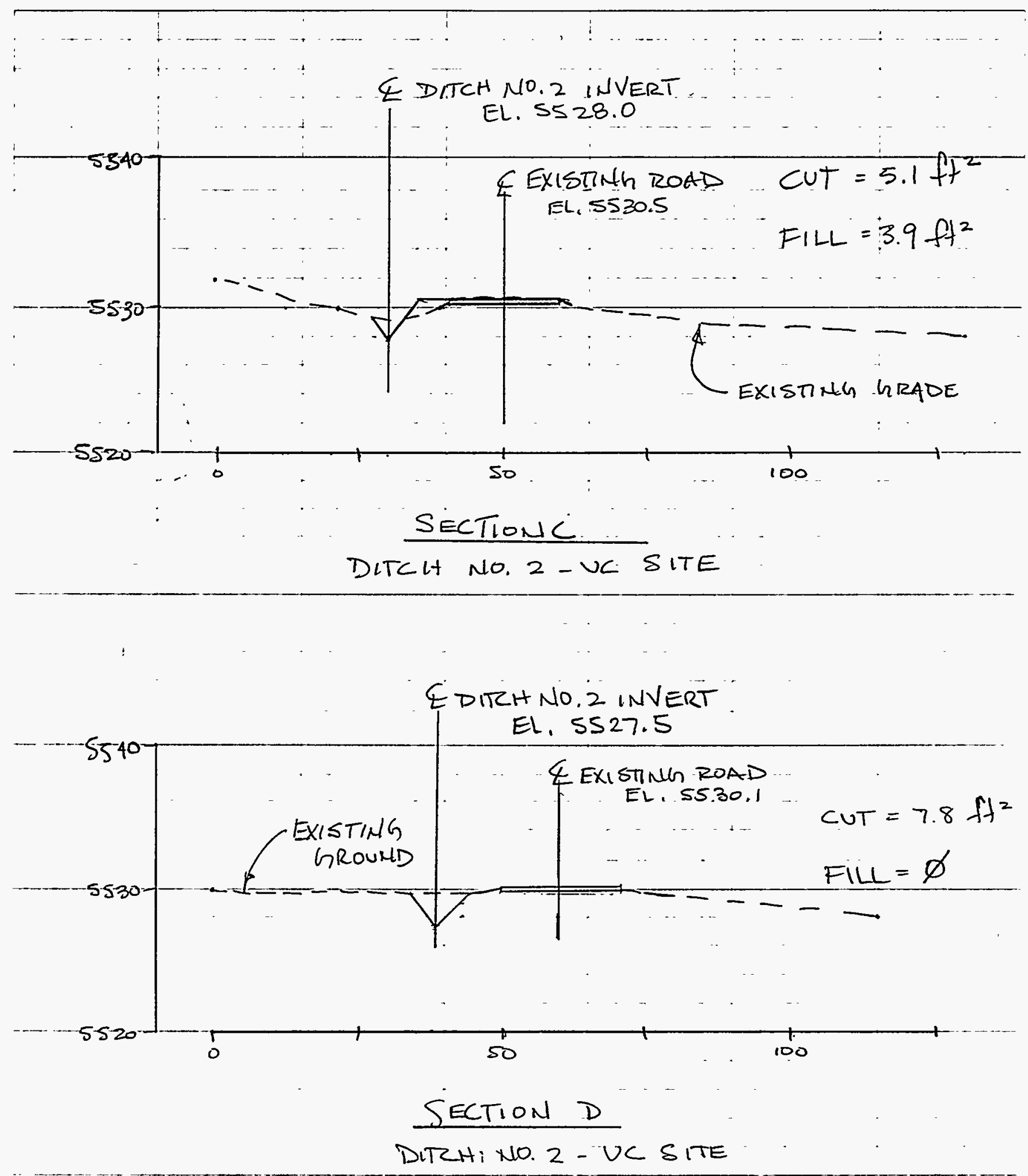
@1.9 MORRISON KNUDSEN CORPORATION

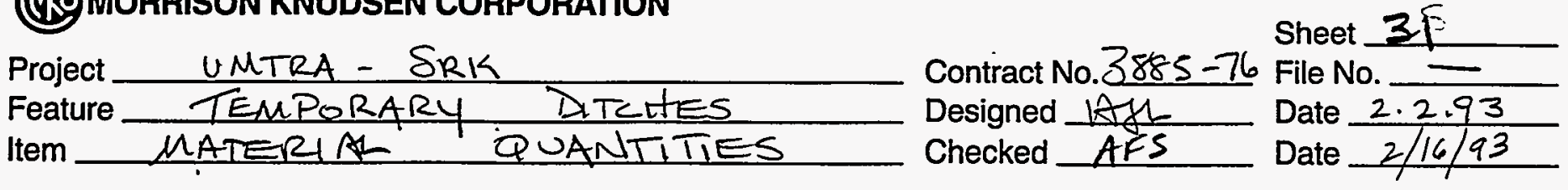

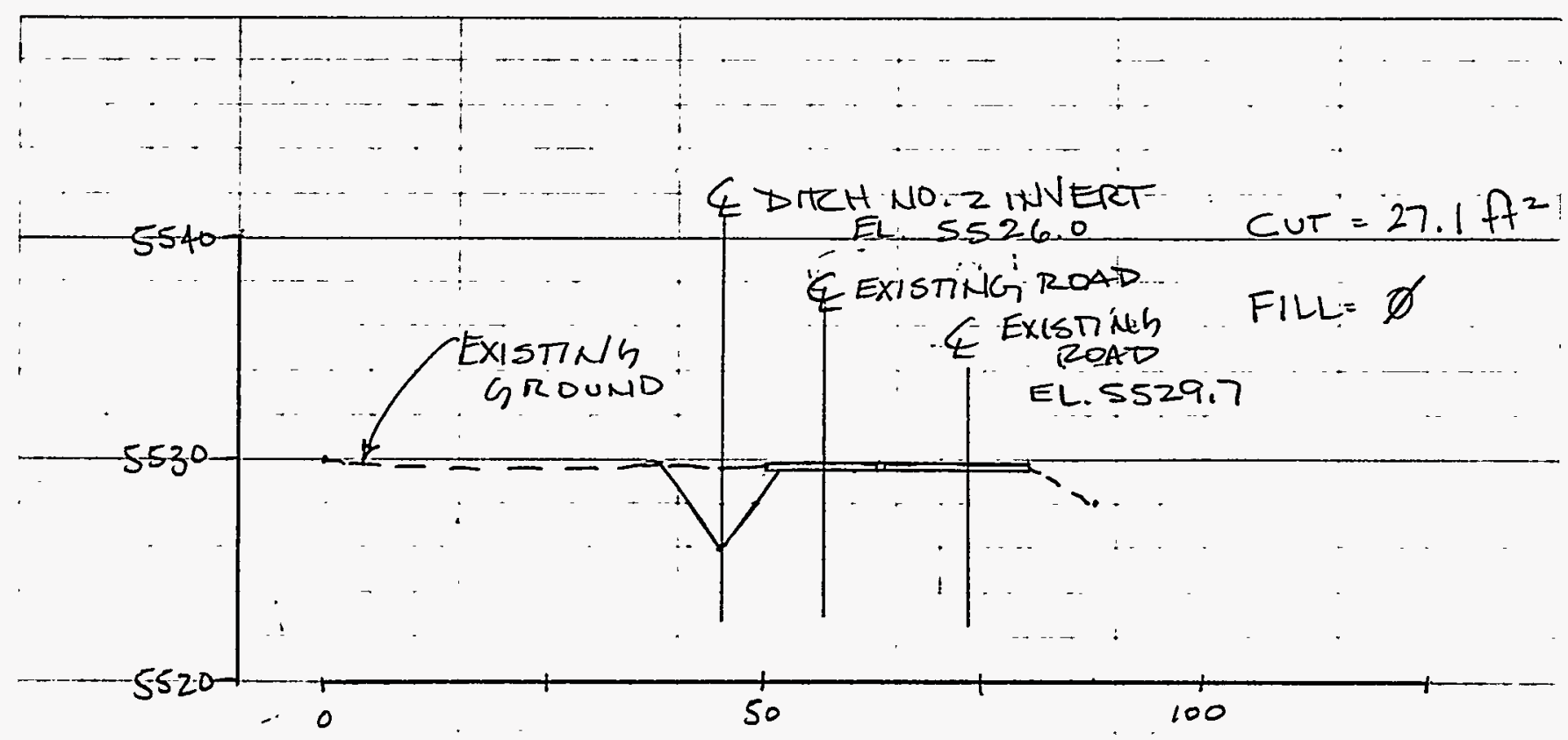

SECTION E

DITCH NO. 2 -VC SITE

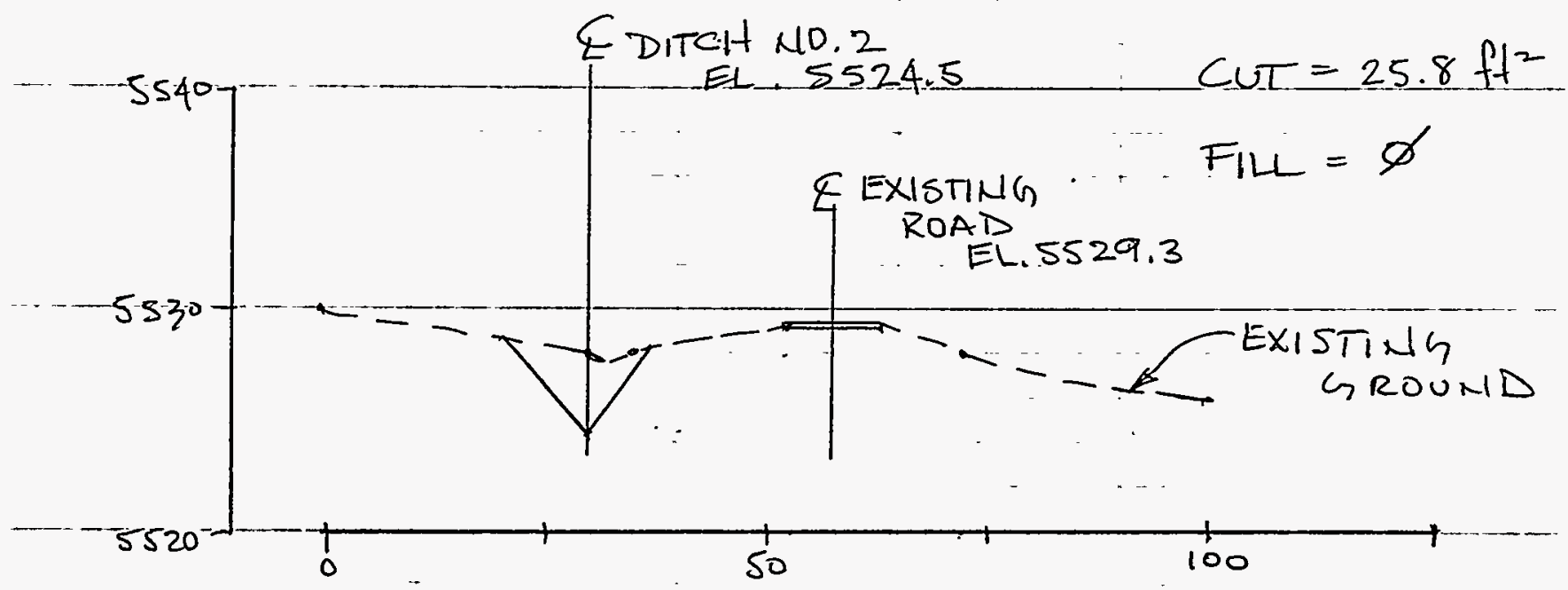

SECTION $F$

DIT LH NO. 2-UCSITE

ENG 375/91 
(40. 9MORRISON KNUDSEN CORPORATION

Project UMTRA - SRK

Feature TEMPORARY DITCHES

Item
Contract No.3885-26

Designed

Checked AFS
Sheet 32

File No.

Date $\frac{2 \cdot 2.93}{2 / 16 / 93}$

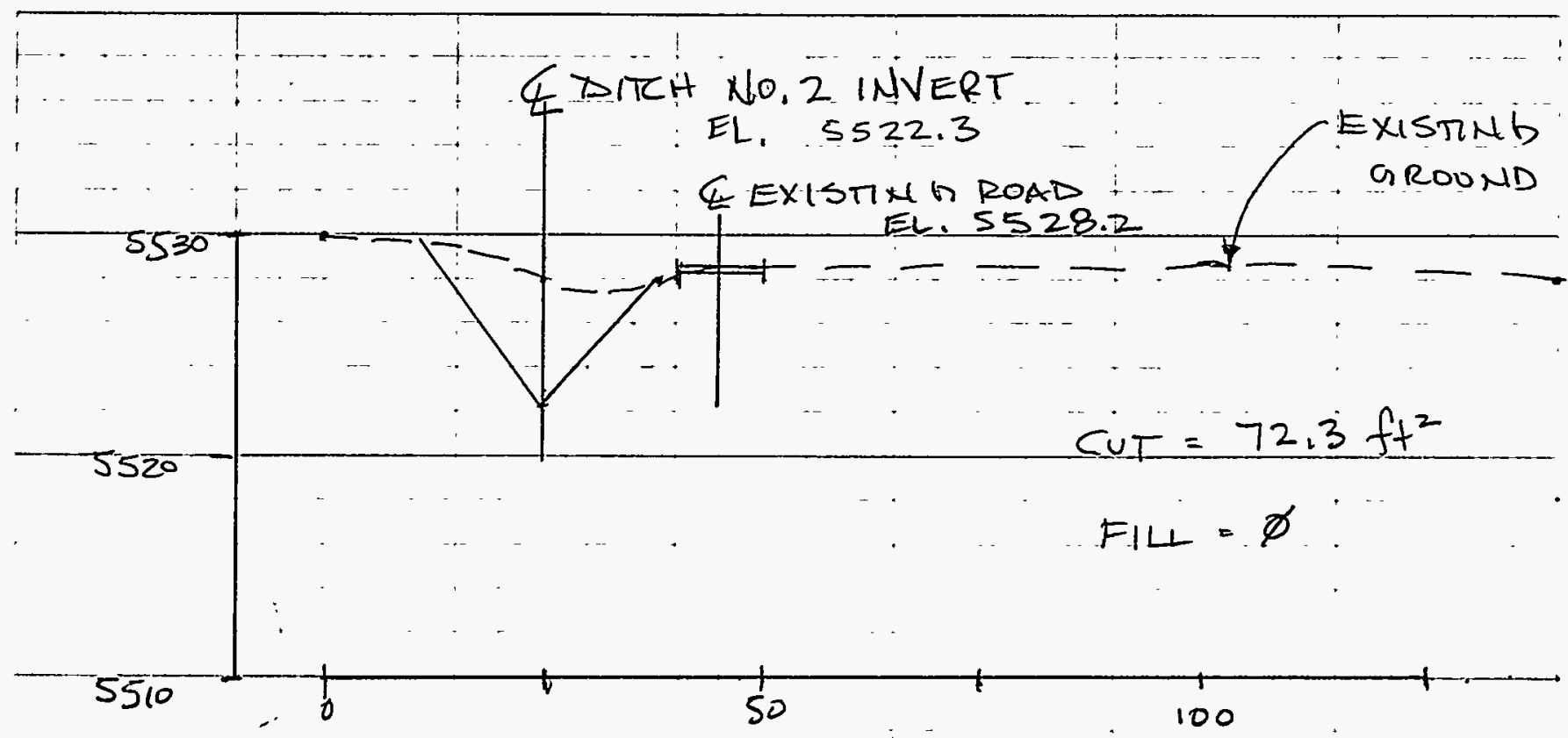

SECTION G

DITCH NOO.Z - UL SITE

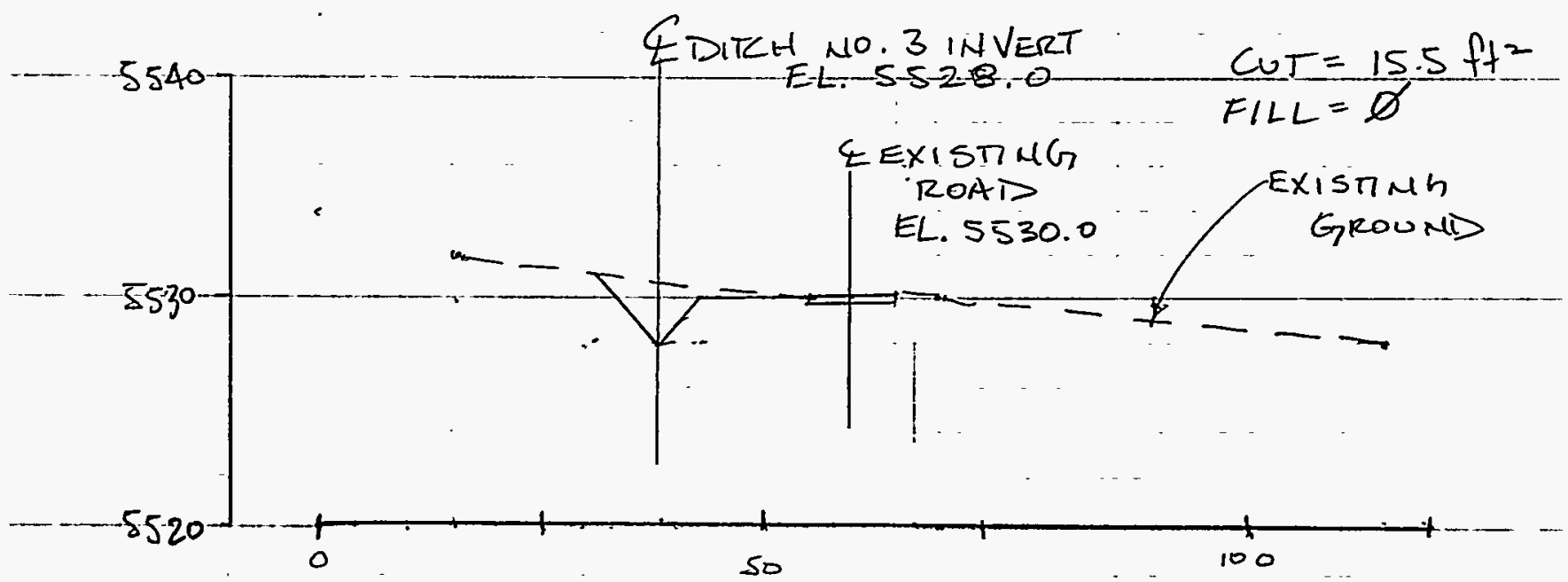

SECTION H

DITCH NO 3 - UK SITE

ENG 375/91 
(43 .MORRISON KNUDSEN CORPORATION

Project UMTRA - SRK

Feature TEMPORARY DITCHES

Item - MATERIAL L QuANTITIES
Sheet 33

Contract No. $3885-76$ File No.

Designed Hz

Date 2.2 .93

Checked AFS

Date $2 / 16 / 93$

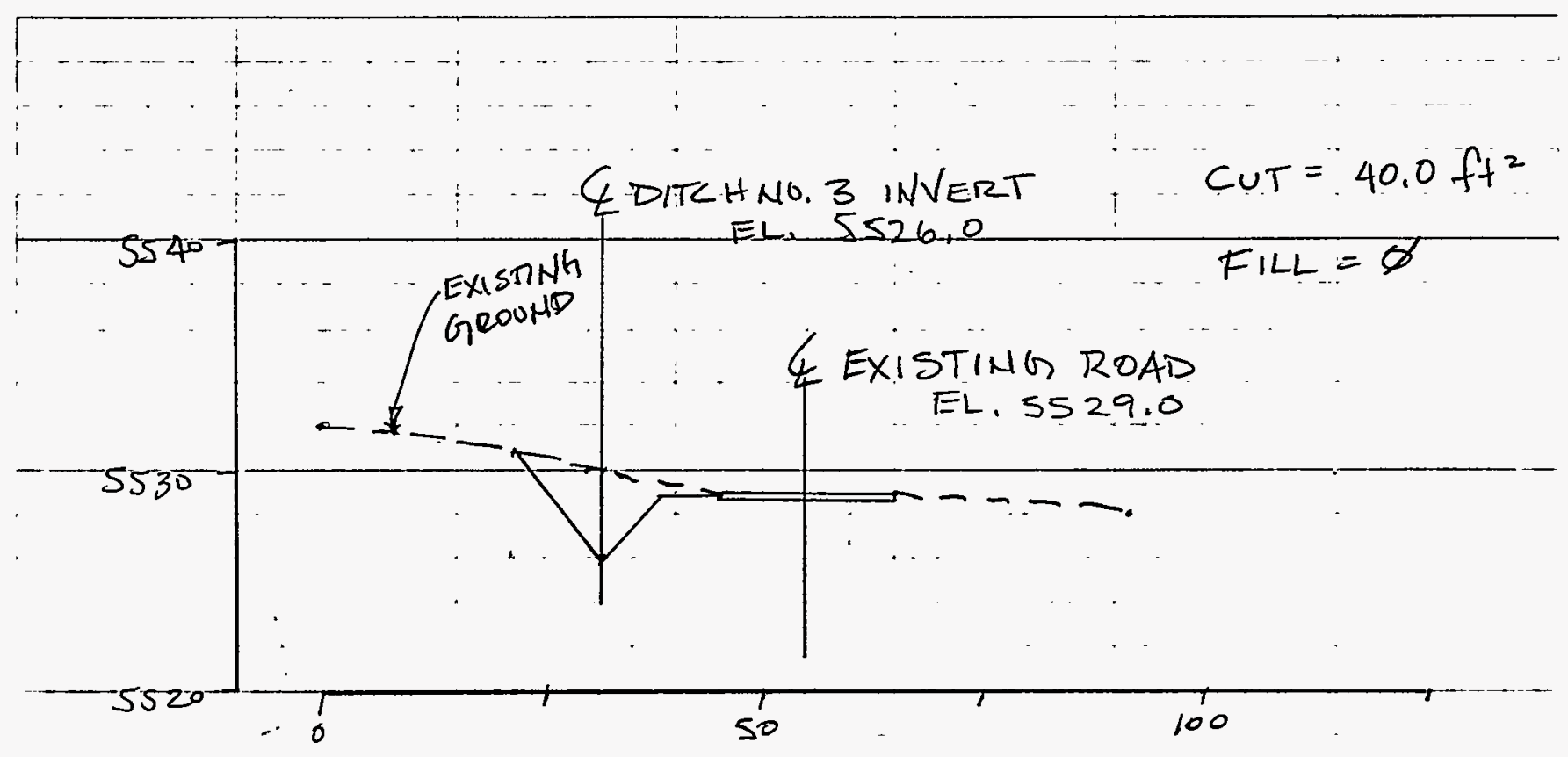

SECTION I

DIT EH NO. 3 - VC SITE

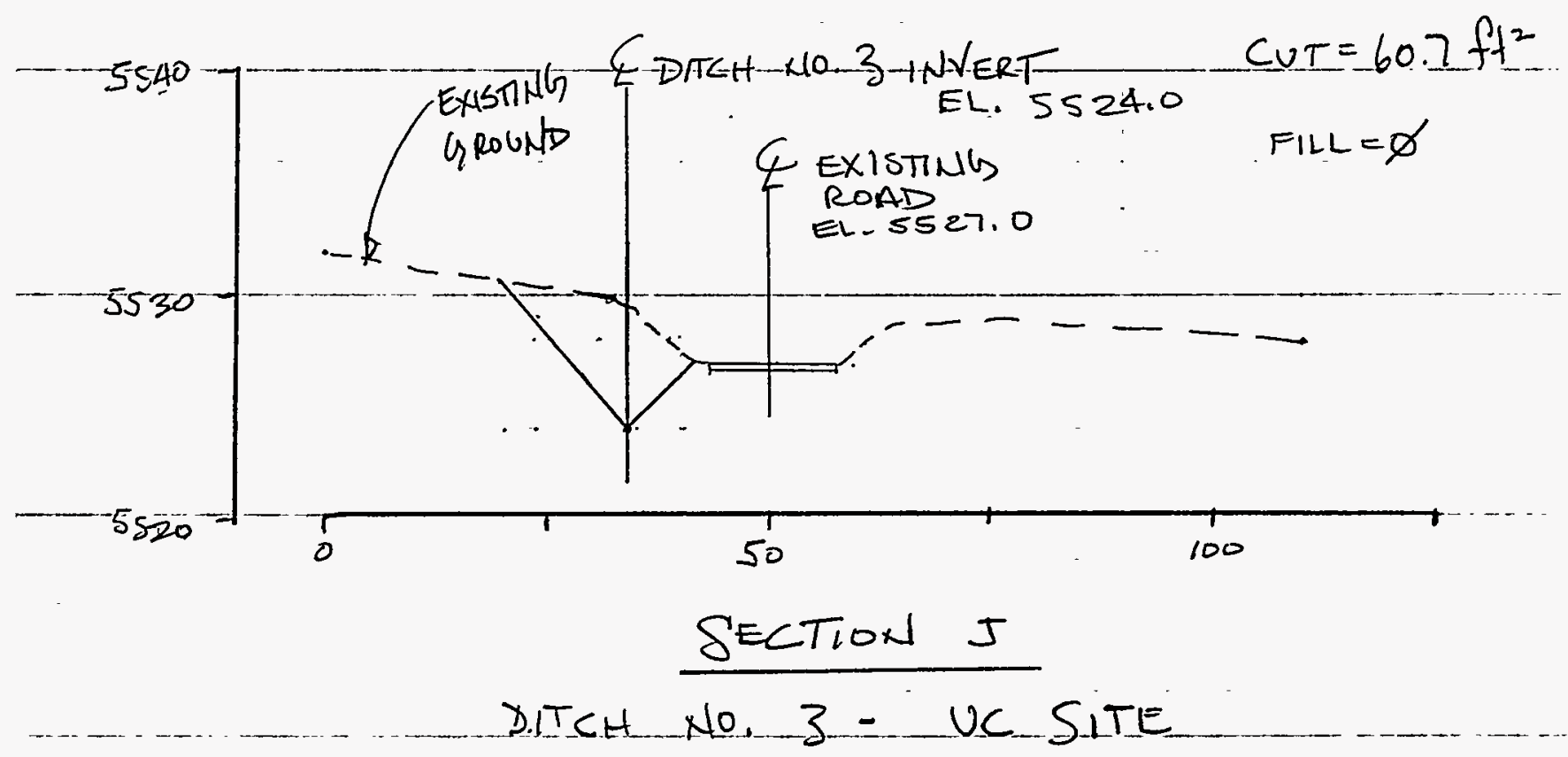

ENG 375/91 
$\therefore$

STS Project UMTRA - SRK

Feature EMPORARY FACILITIES Item MATERIAL QUANTITIES

Contract No. $3885-76$ File No.

Designed He

Checked A's

Date $\frac{2.5 .93}{2 / 16 / 93}$

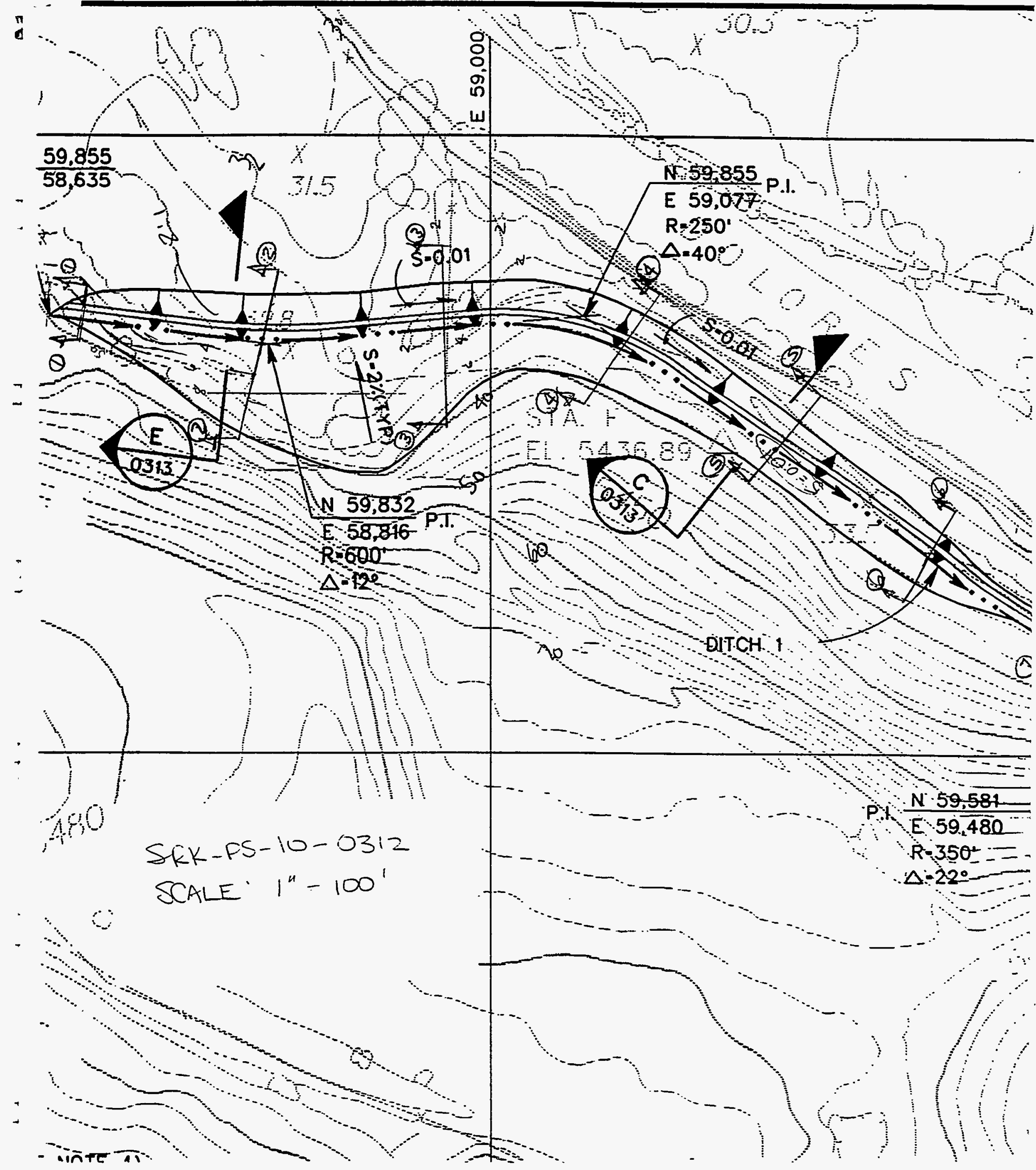


@17. MORRISON KNUDSEN CORPORATION

Project UMTRA - SRK

Feature TEMPORARY FACIUTIES

Item FMATERIIAL QUANTITIES
Contract No. $3885-76$ Designed $\frac{142}{A F S}$ Checked AFS
Sheet 36

File No.

Date $\frac{2.2 .93}{2 / 16 / 93}$

3. Dikes 1 and 2

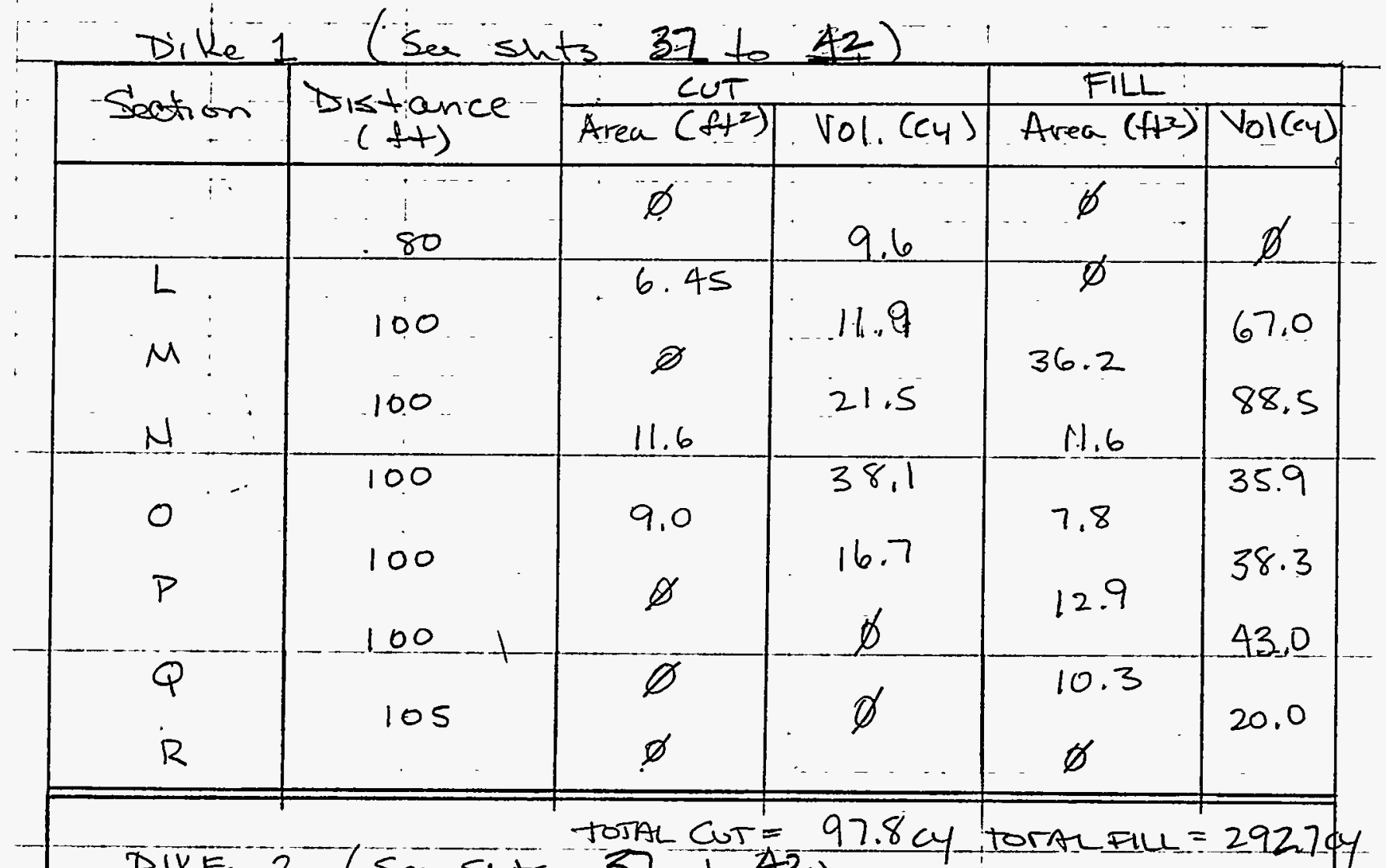

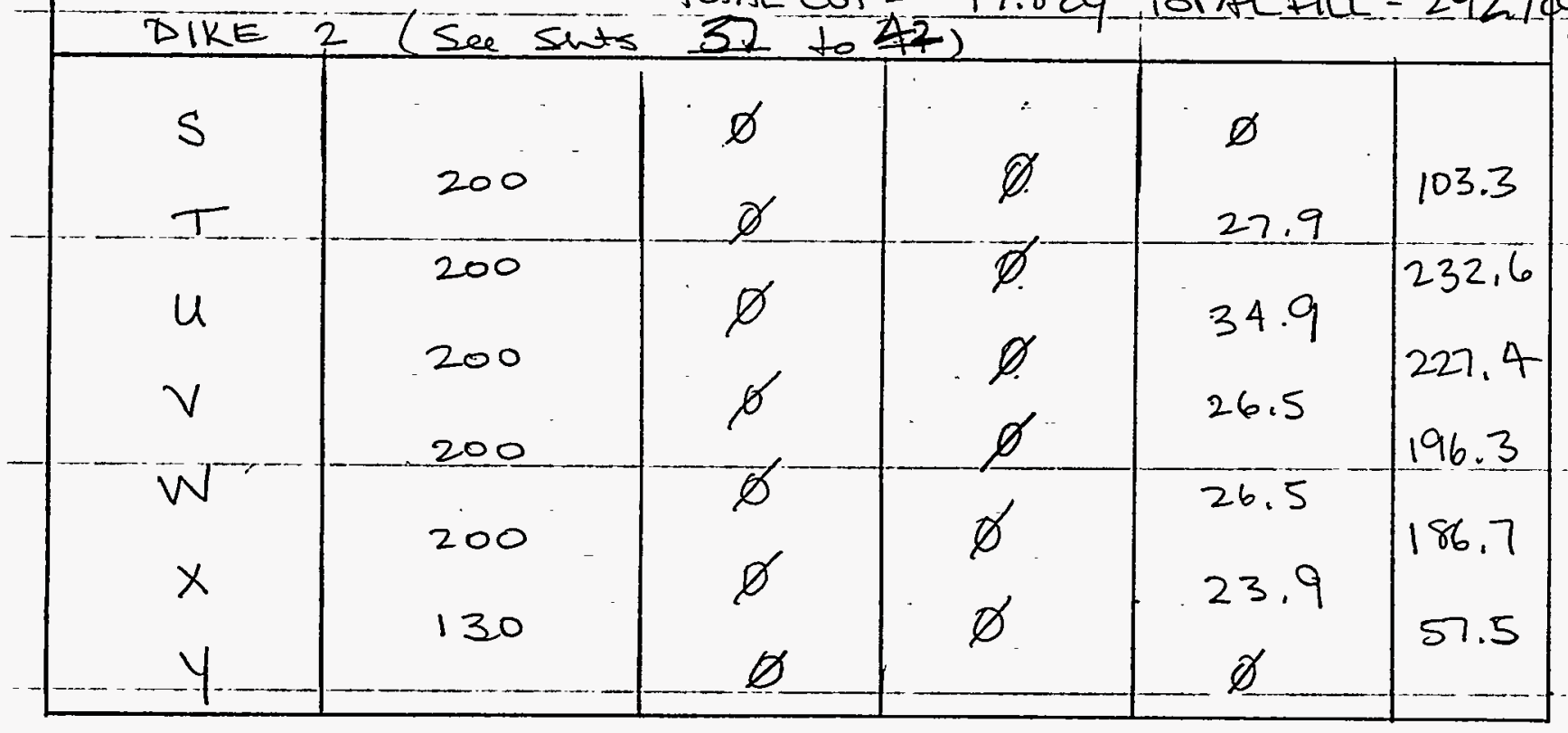

TOTAL CUT=

TOTAL FILL $=1003,8 \mathrm{CY}$

ENG 375/91 
(4. MORRISON KNUDSEN CORPORATION

Project UMTRA - SRK

Feature TEMTPORARY FACILITIES

Item MATERIAL QUANTITIES
Sheet 37

Contract No. $3885-76$ File No. -

Designed

Date 2.2 .93

Date $2 / 16 / 93$

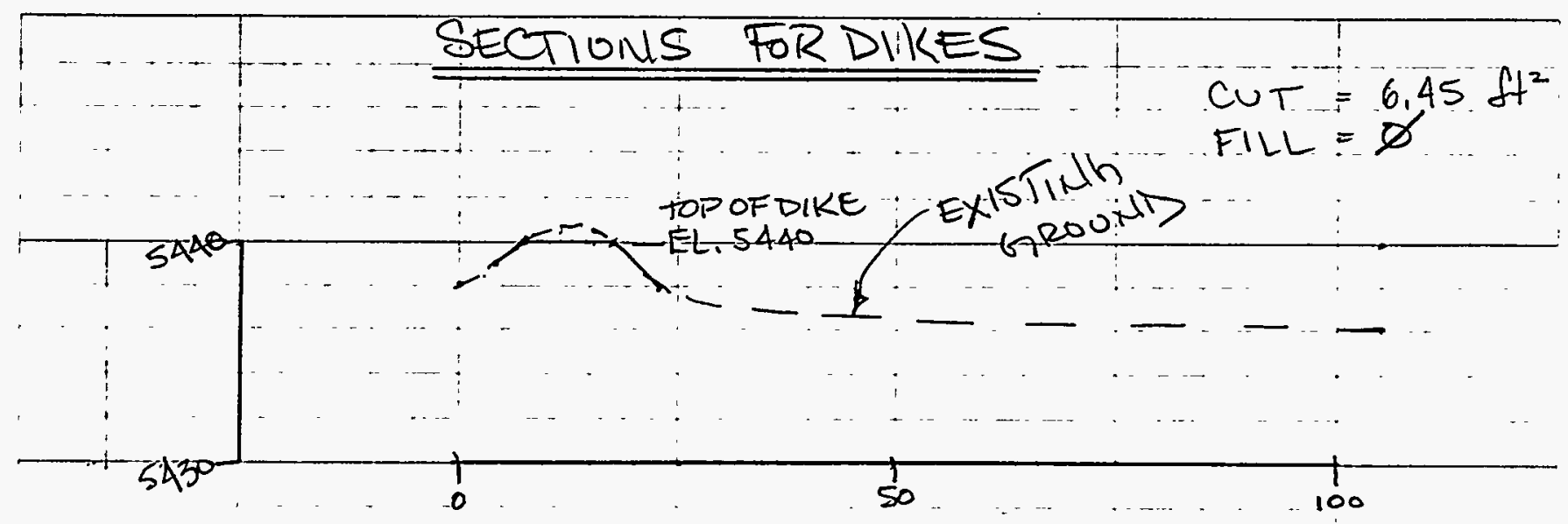

SECTION L

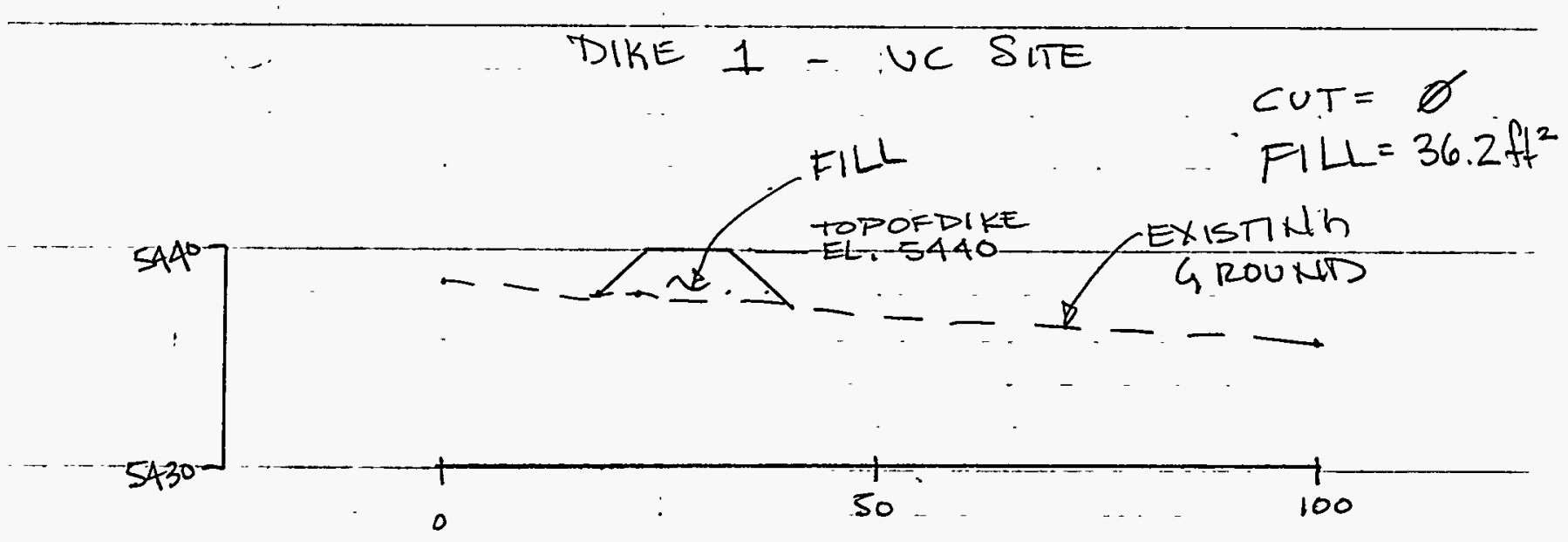

SECTION M

DIKE 1 - VC SITE

$$
\begin{aligned}
& \text { CUT }=11.6 \mathrm{ft}^{2} \\
& \text { FILL }=11.6 \mathrm{ft}^{2}
\end{aligned}
$$

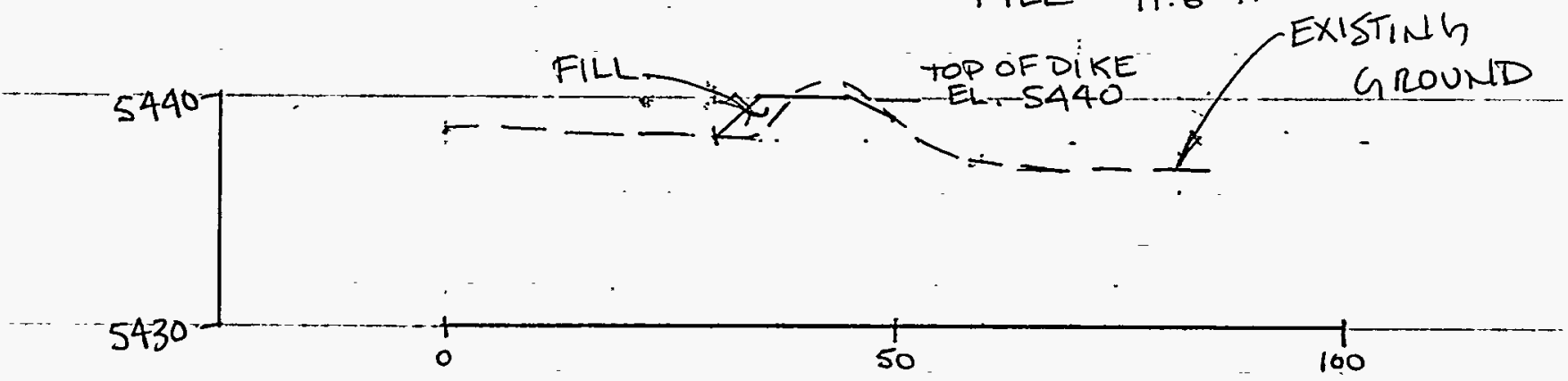

SECTION N

DIKE 1 - UL SITE

ENG 375/91 
(17. 0MORRISON KNUDSEN CORPORATION

Project UMTRA - SRK

Feature TEMPORARY FACILITIES

Item MATERIAL QUANTITIES
Contract No. $3885-76$ File No. Designed

Checked AFS
Date $\frac{2.2 .93}{2 / 16 / 93}$

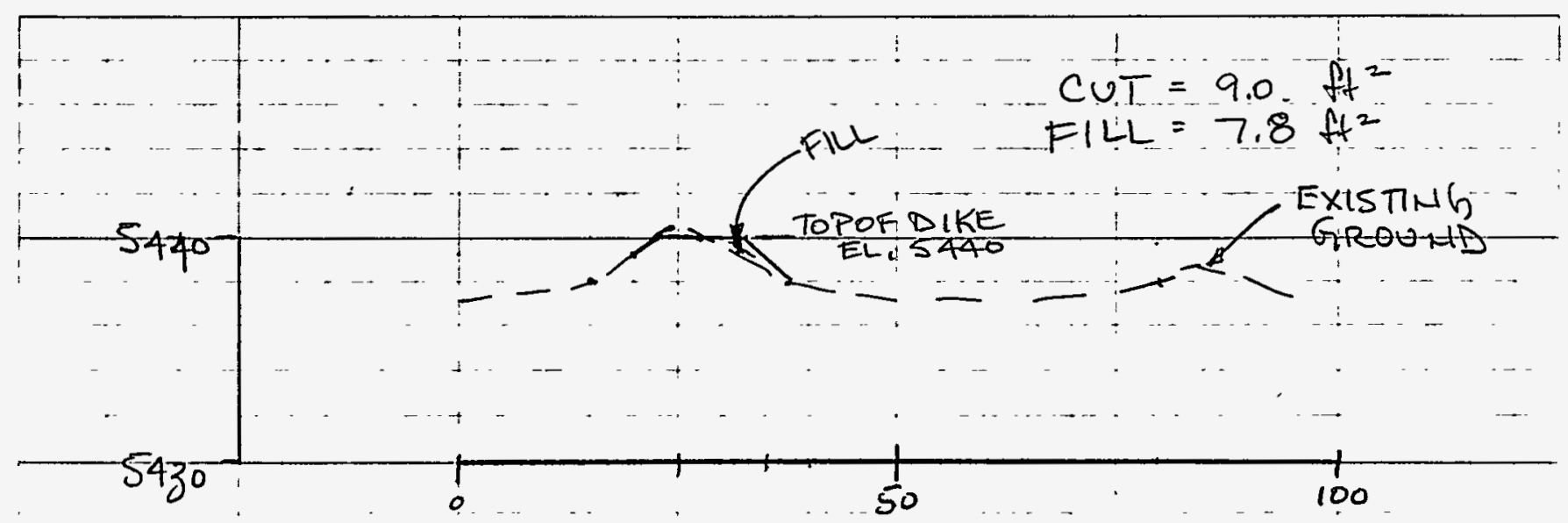

SECTION O

DIKE 1 - VC SITE

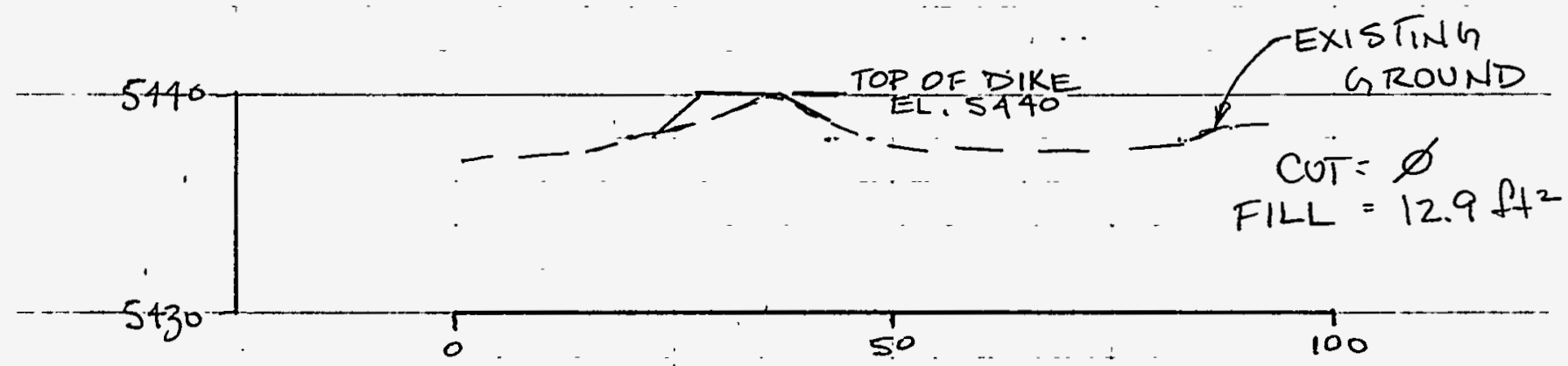

SECTION $P$

DIKE 1 - VC SITE

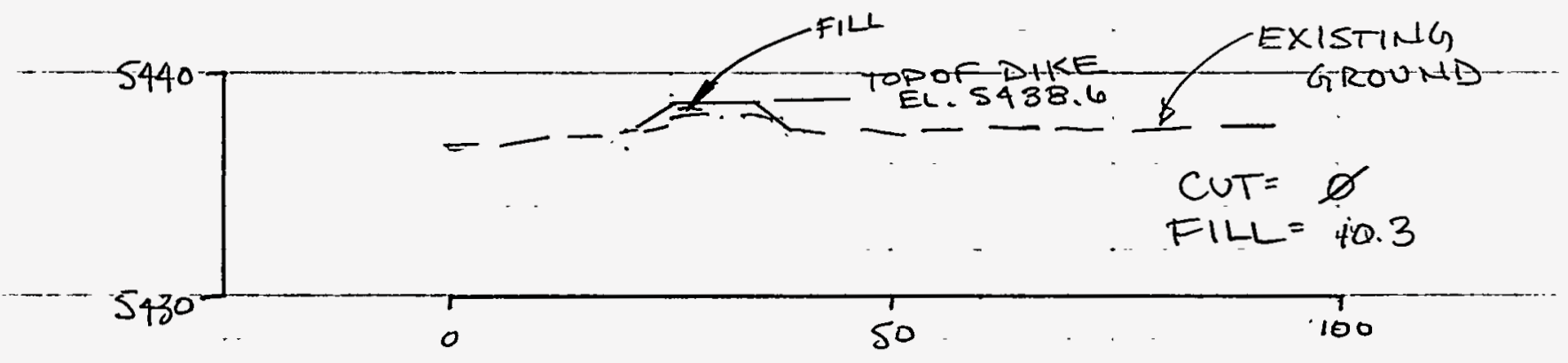

SECTION Q

DIKE 1 -VC SITE

ENG 375/91 
(4) MORRISON KNUDSEN CORPORATION

Project UMTRA - SRR

Sheet 39

Feature TEMPORARY FACILITIES

Contract No. $3885-76$ File No.

item MATERITL QUANTITIES

Designed H

Date $\frac{2 \cdot 2.93}{2 / 16 / 93}$

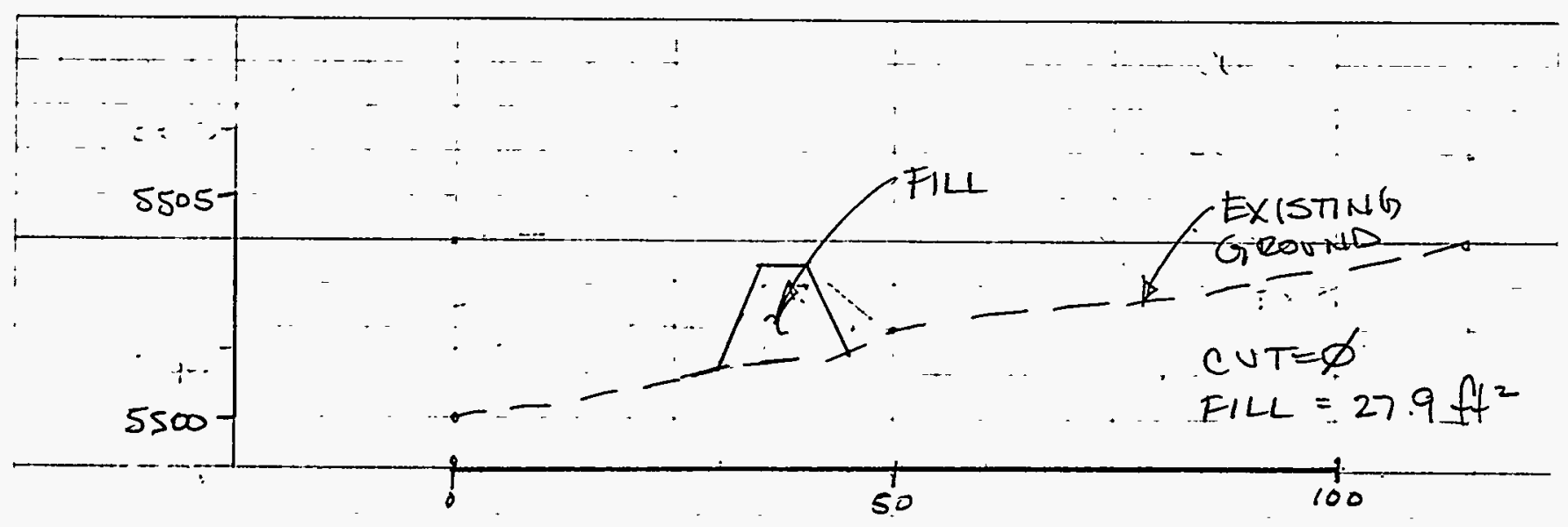

SECTION T

DIKE 2 - US SITE

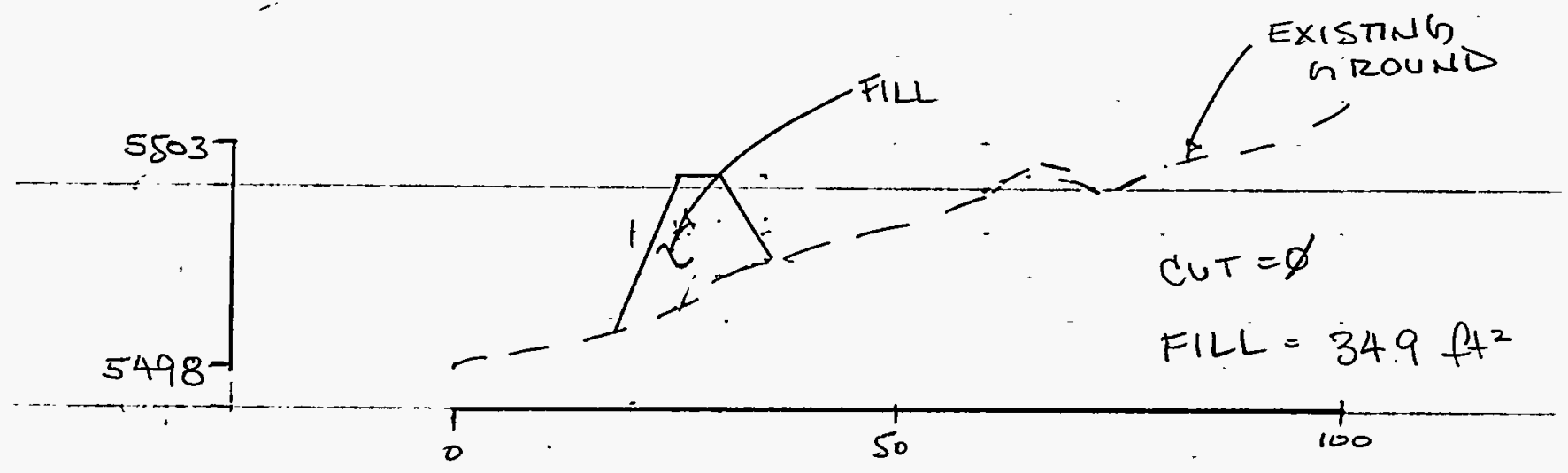

SECTION 4

DIKE 2 - VC SITE

$\left.\begin{array}{l}5497 \\ 5492\end{array}\right]$

$$
\begin{aligned}
& \text { CUT }=\not \varnothing \\
& \text { FILL }=26.5 \mathrm{~A}^{2}
\end{aligned}
$$

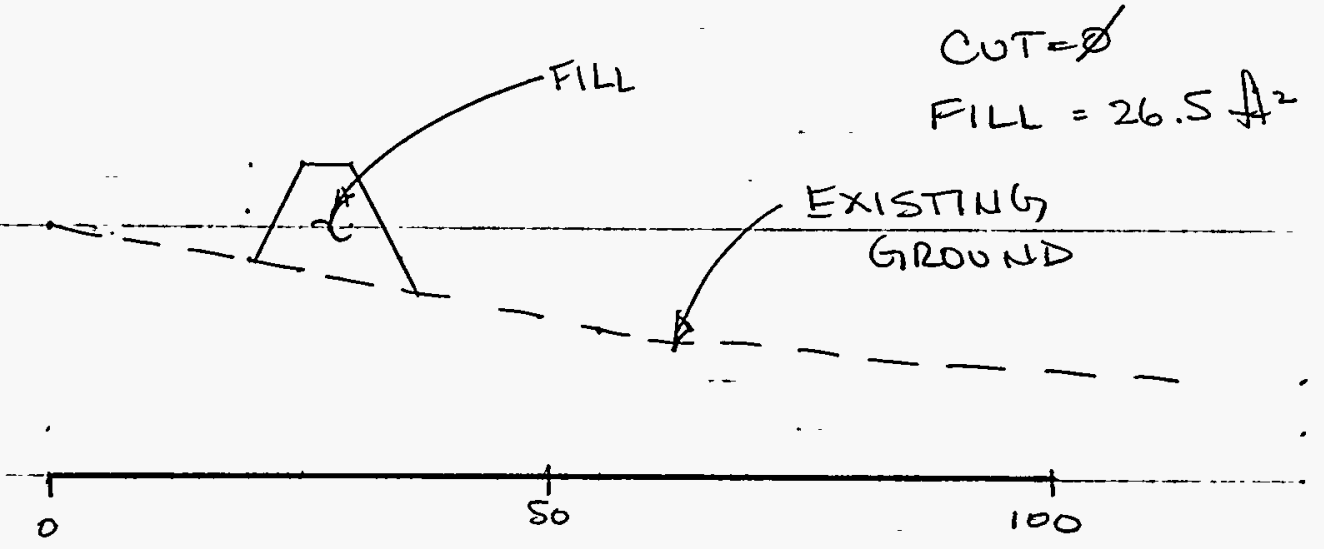

DIKE $\frac{\text { SECTION V }}{2-V C \text { SITE }}$

ENG 375/91 
(4. MORRISON KNUDSEN CORPORATION

Project UMTRA - SRK

Feature TEMPORARY FACILITIES

Item MAATERIAL" QUANTITIES
Contract No. $8885-76$

Designed

Checked A t es
Sheet 40 File No. Date $\frac{2 \cdot 2 \cdot 93}{2 / 16 / 93}$

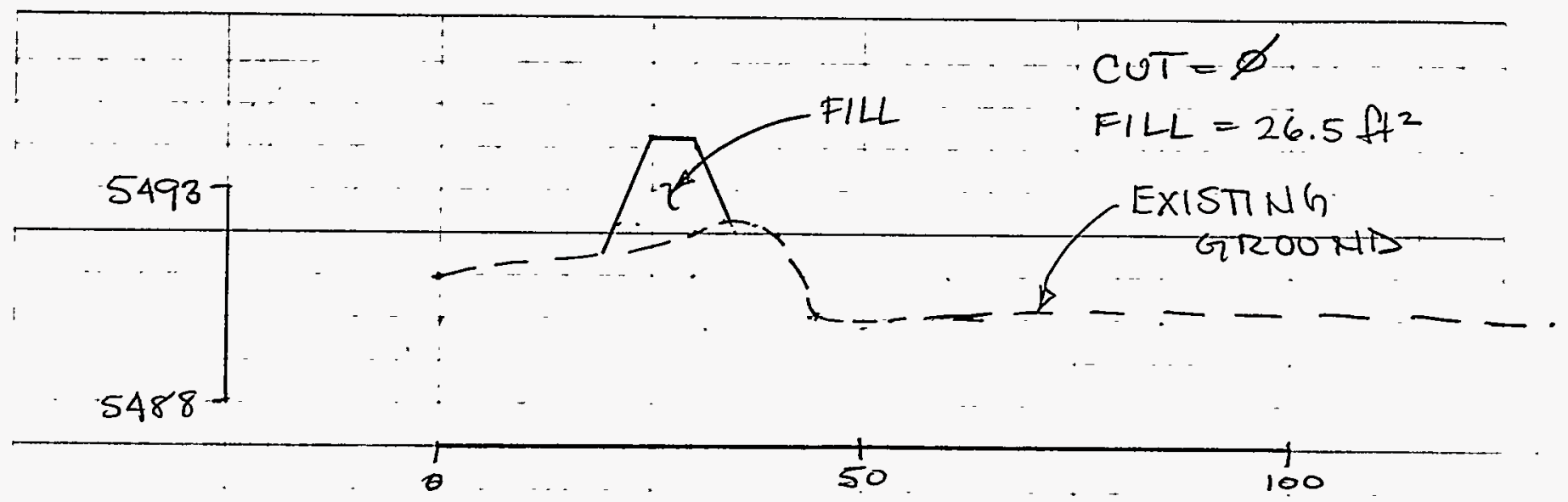

SECTION W.

DIKE 2 - UL SITE

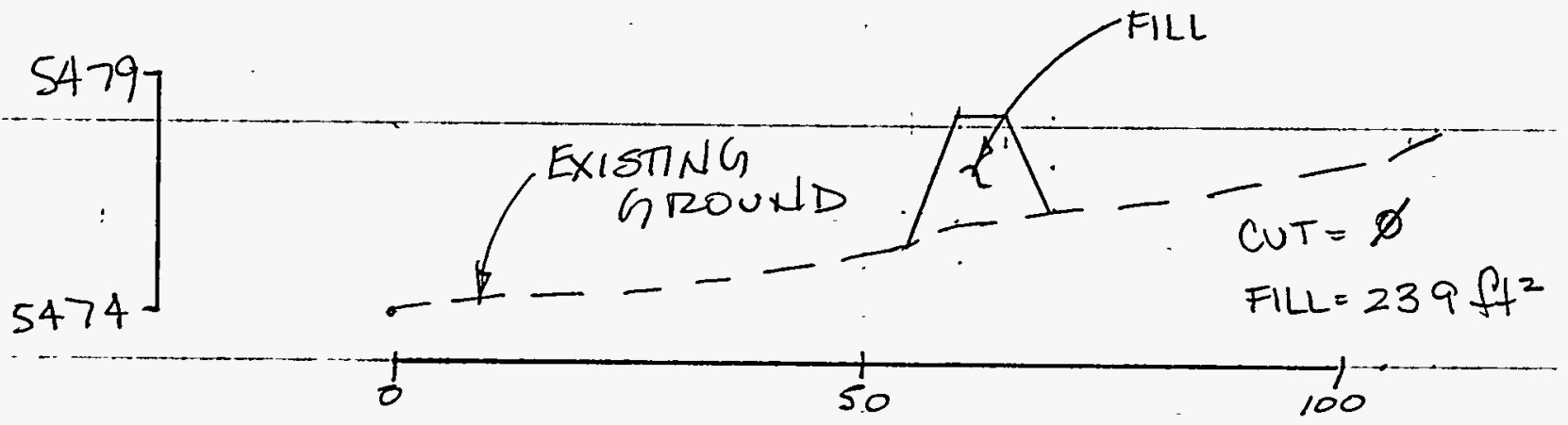

$\delta E C \pi D N \times$

DIKE 2 - VC SITE

ENG 375/91 

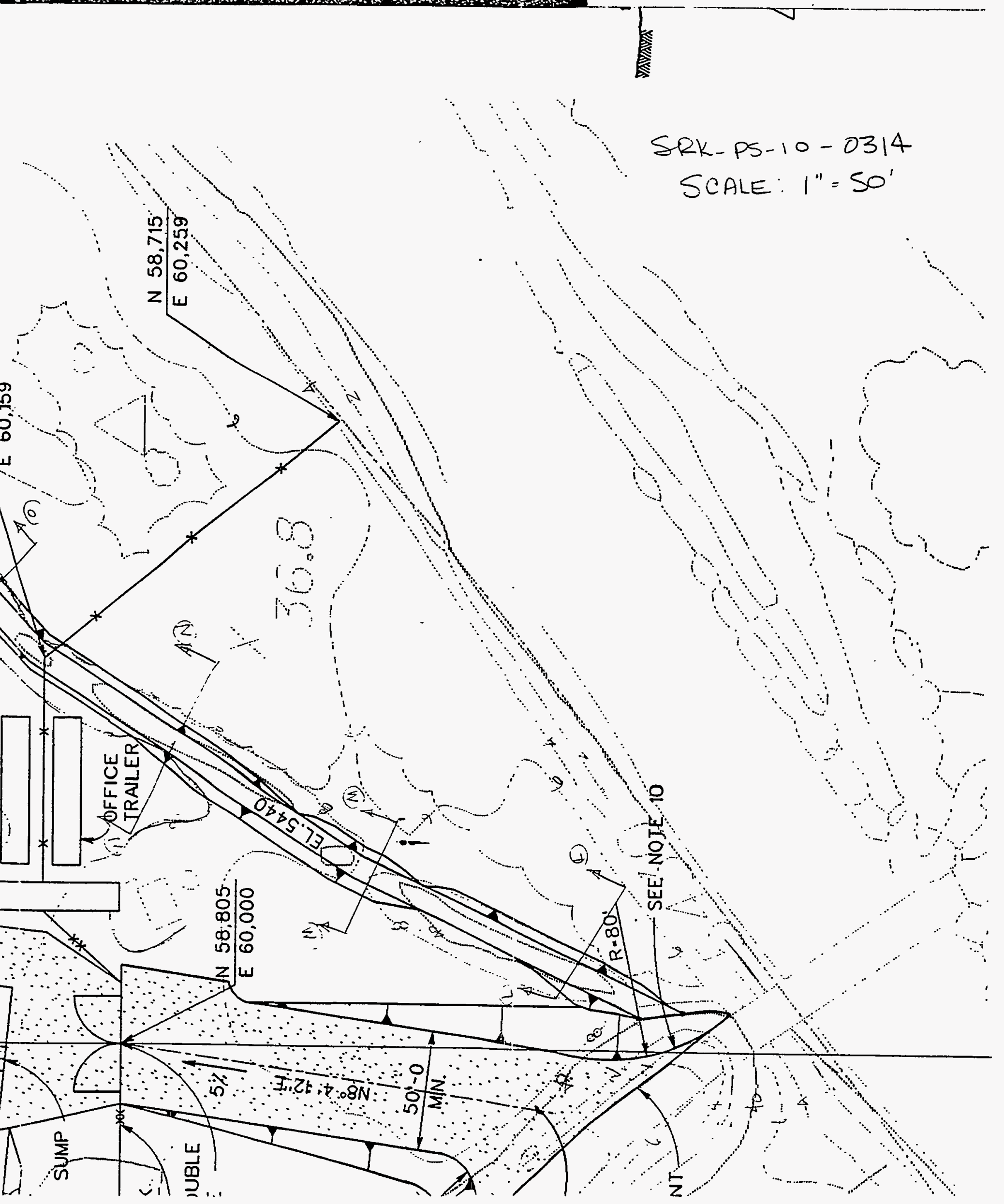
@i. MORRISON KNUDSEN CORPORATION

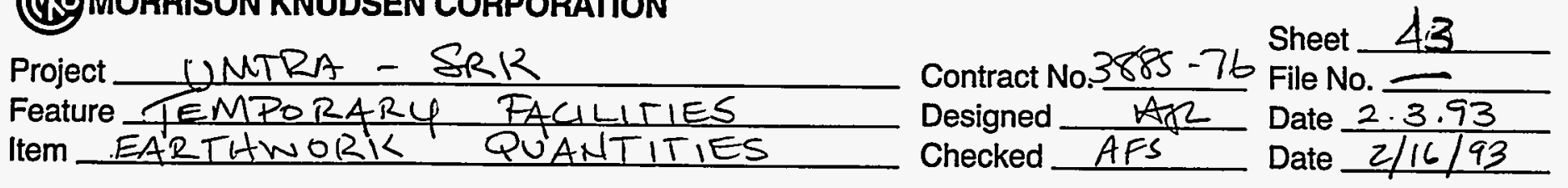

4.) ACLESS CONTTROL FACILITIES

a.) Recirculation basin

i) Approximate excavation quantity using. basin. dimensions

( See SRK - PS: 10-0314, SAt 9 and 49)

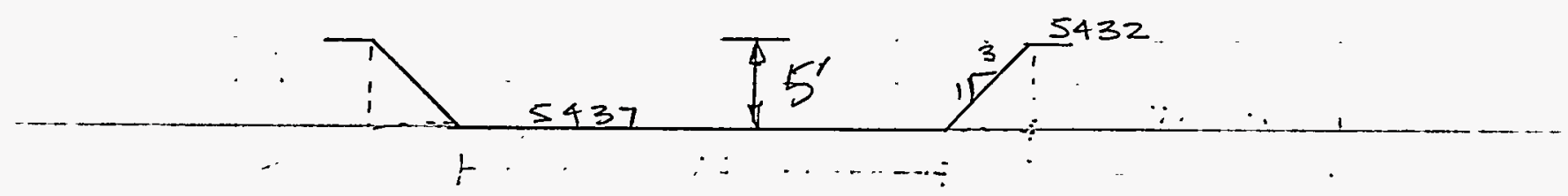

Basin Section (nits)

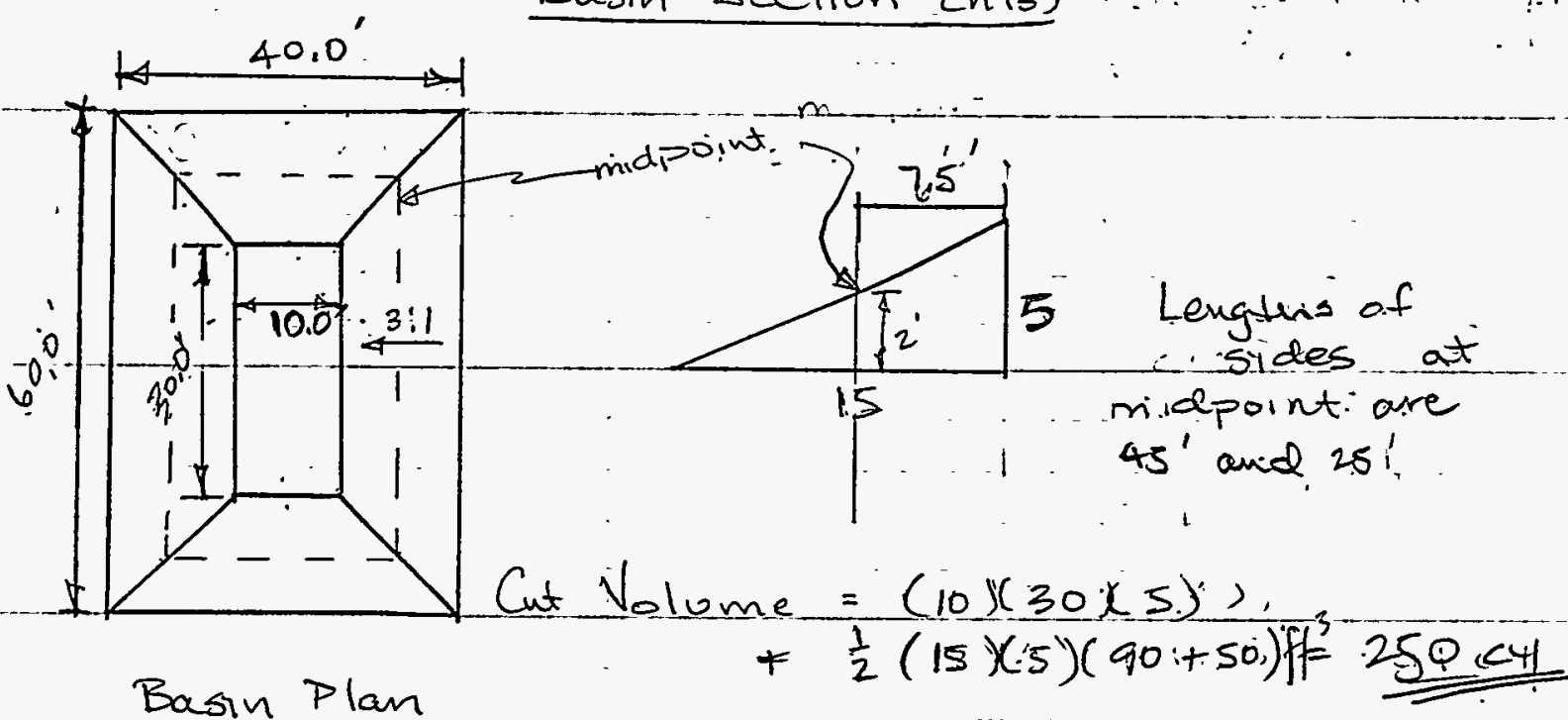
Basin Plan
$(n+5)$

$$
\begin{aligned}
& \text { Additional cut }=(3)(9)(50+20)+(5) \times 3)(50+20) f^{3} \\
& =110 \mathrm{cy} \text { : } \\
& \text { Fill Volume }=(1)(3)(50+30)+(5)(3)(50+30) f^{3} \\
& =5 \overline{3 c 4} \\
& \text { TOT AL CUT }=3.60 \mathrm{cY} \\
& \text { TOTAL FILL }=53 C 4
\end{aligned}
$$

ENG 375/91 
(46 )MORRISON KNUDSEN CORPORATION

Project UMTRA - SRK

Feature TEMAPORARY FACILITIES

Item MATERIAL QUANTITIES
Contract No .3885-76 Designed 172 Checked
Sheet 24

File No.

Date $2 \cdot 3.93$

Date $2 / 16 / 93$

D) ACCESS RAMP

Access ramp includes area to section $A A$, as shown on sit 49 .

i) Fill Volume

(See. Shuts 44 to 49)

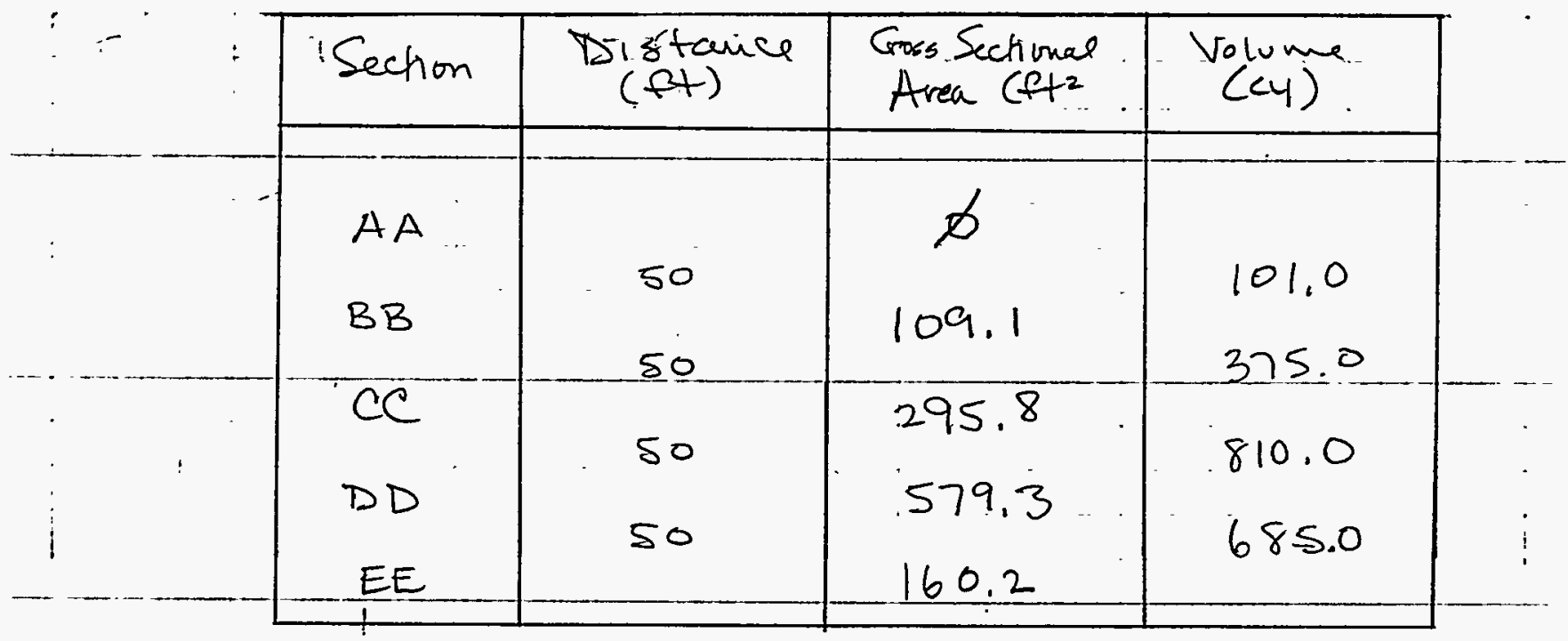

TOTAL FILL $=1971 \mathrm{CY}$

ii) Aggregate - $4^{4}$ thick layer (SRK-PS-10-0314)

Ramp plan area $=11,302,1 \mathrm{ft}^{2}$

Slope factor $=1.0012$

Volume $\left.=(11.302 .1) \times \frac{4}{12} \quad X_{1.0012}\right)\left(\frac{1}{27}\right)=140.0 \mathrm{c4}$

c) Length of county road, from SRR-ips f Ko, 03 16 : Sit li,

is approximately $915 \mathrm{ft}$

Earthwork Volume $=\left(915^{\prime}\right)(1)\left(20^{\prime}\right)\left(\frac{1}{27}\right)=67278 \mathrm{c4}$

Ag spegate... $(915)(0.5)(20)\left(\frac{1}{22}\right)=338.9<4$

ENG 375/91 
서MORRISON KNUDSEN CORPORATION

Project UMTRA - SRK

Sheet AS

Feature TEMPORARY FACILITIES

Contract No. $3885-76$ File No.

item Material quantities

Designed

Date 2.3 .93

Checked

Date $2 / 16 / 93$

d) DECON PAD AREA

Assume earthwork quantities are negligible, given the flat grade of the area..

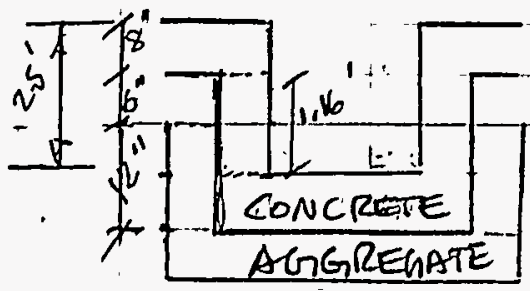

i) Aggregate

4" thick: : plain area $=5877.1$

Volume $(5877.1)\left(\frac{4}{12} \times \frac{1}{27}\right)=72.5 \mathrm{c4}$

Aggregate $6^{4}$ thick base:

volume for pad $=2\left[\left(25.3^{\circ}\right)(1.0005)\left(100^{\prime}\right)\left(0.5^{\prime}\right)\right]\left(\frac{1}{27}\right)=93.8 . \mathrm{cy}$ $3 \%$ slope-correction

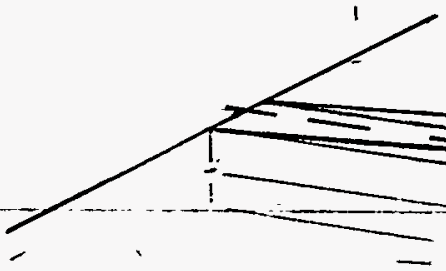

Fig $A$.
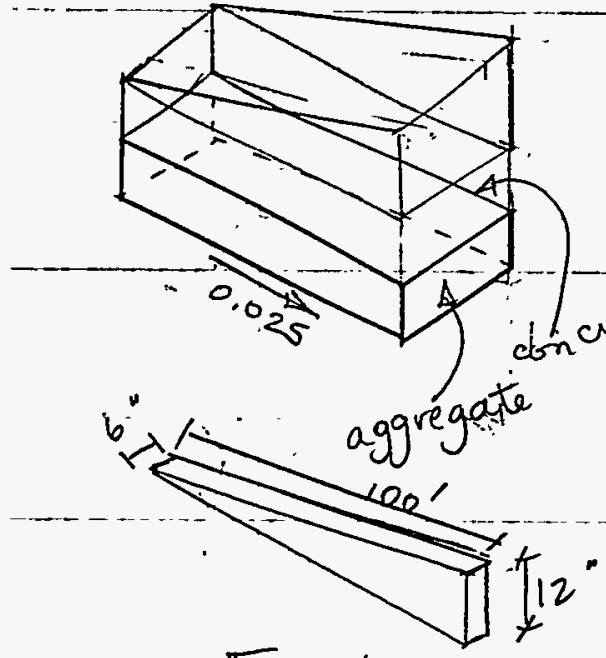

Aggregate: if or Sump

Vol, $=1 .(100)(10.3)(0.5)(1.000)\left(\frac{1}{27}\right)=19.1 . \mathrm{cy}$

From Fig : $2.5 \%$ slopecorrection

$V_{0 I_{2}}=\left(\frac{1}{2}\right)(100)(6.5)(1.0)\left(\frac{1}{27}\right)=0.92 \mathrm{cy}$

Fig B

Total aggregate volume $=113.8 \mathrm{cy}$

ENG 375/91 
CA. MORRISON KNUDSEN CORPORATION

Project UMTRA - SRK

Feature TEMPORARY FACILITIES

Item Material QuaNTITIES
Contract No. $3885-76$

Checked AF S
Sheet 46

File No.

Date 2.3 .93

Date $2 / 16 / 93$

ii) Concrete - 8" thick

Volume for $\left.p a d=2\left[(2.6)(1.0005)(100)^{\frac{8}{12}}\right)\right]\left(\frac{1}{27}\right)$

$=128.5 \mathrm{cy}$

Concrete for. Sump.

$$
\begin{aligned}
\text { Volume }= & (100)(9.3)\left(\frac{8}{12}\right)(1.000)\left(\frac{1}{27}\right)=\ldots \\
& +\frac{1}{2}(100)\left(\frac{3}{12}\right)\left(\frac{10}{12}\right)\left(\frac{1}{27}\right)=24.4 \mathrm{c4} \\
& \text { TOTAL CONCRETE }=152.9 \mathrm{cy}
\end{aligned}
$$

e) Membrane Liner for recirculation basin

i) Bottom Area $=(10 \times 3.0)=300156$

ii) Side slope

$$
\text { Width = } 15.8^{\prime}
$$

Perimeter length at midpoint

$$
\text { of side slope }=24\left(45^{\prime}+2 s\right)=140^{\prime}
$$

$$
\text { Area }=(158,(140)=2212.5 \mathrm{f}
$$

iii) Anchor trenches

$$
\begin{aligned}
& \text { Length. }=2(60 .+40)=200.6 \\
& \text { width }=2+1.8+0.5=4.3^{1} \\
& \text { Area }=(4.3)(200)=860 \text {.sf }
\end{aligned}
$$

Total Liner Area

$$
\begin{aligned}
& =300+2212+860.0 .1 \\
& =3.3725 f
\end{aligned}
$$

ENG 375/91 


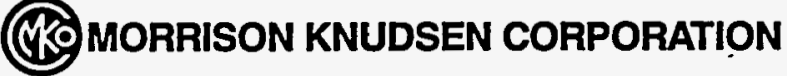

Project UMTRA - SRR

Feature TEMPORARY FACILITIES

Item MATERTAL QUANTITIES
Contract No.3885- 26

Designed AK

Checked AF S
Sheet -47

File No.

Date 2.3 .93

Date $2 / 16 / 93$

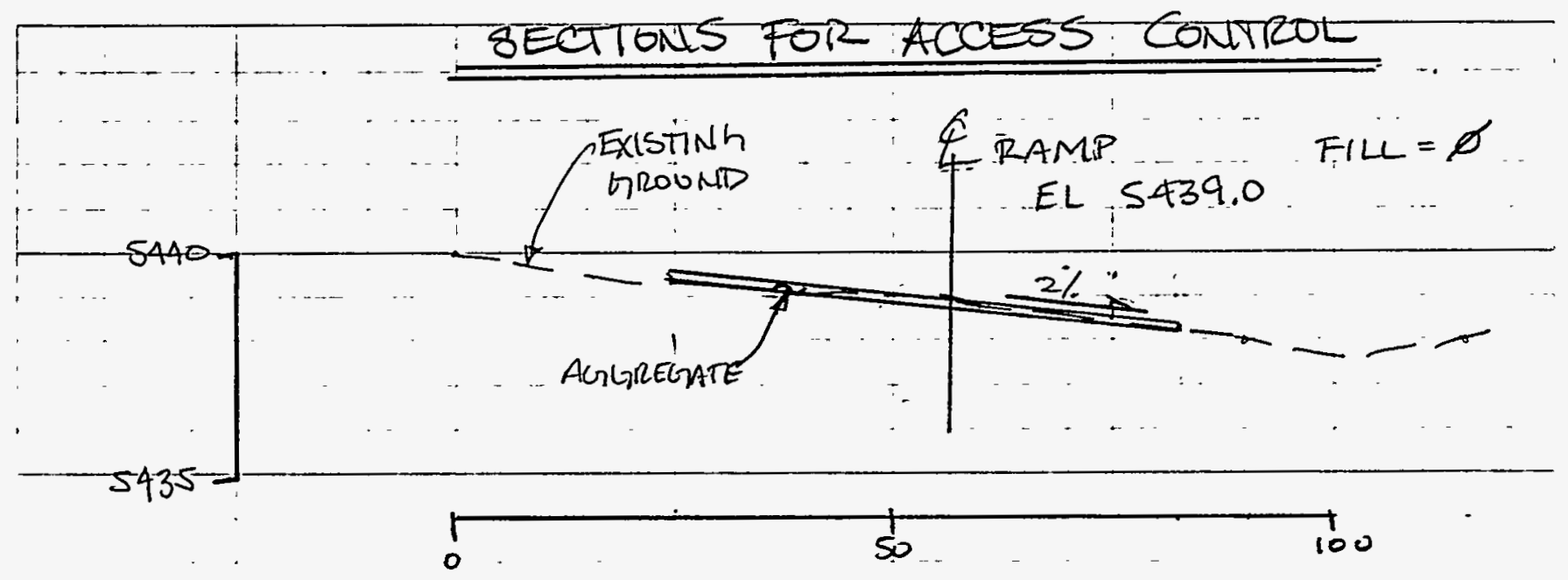

SECTION AA

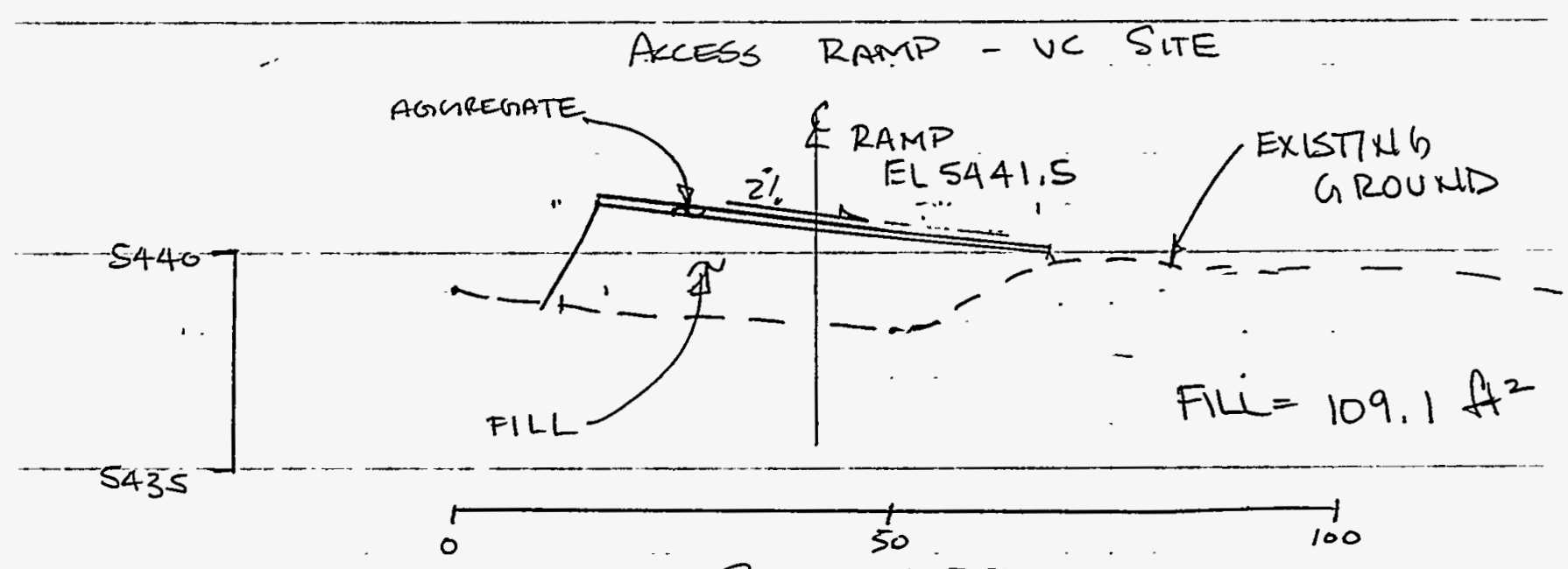

SECTION BB

ACCESS RAMP - VESITE

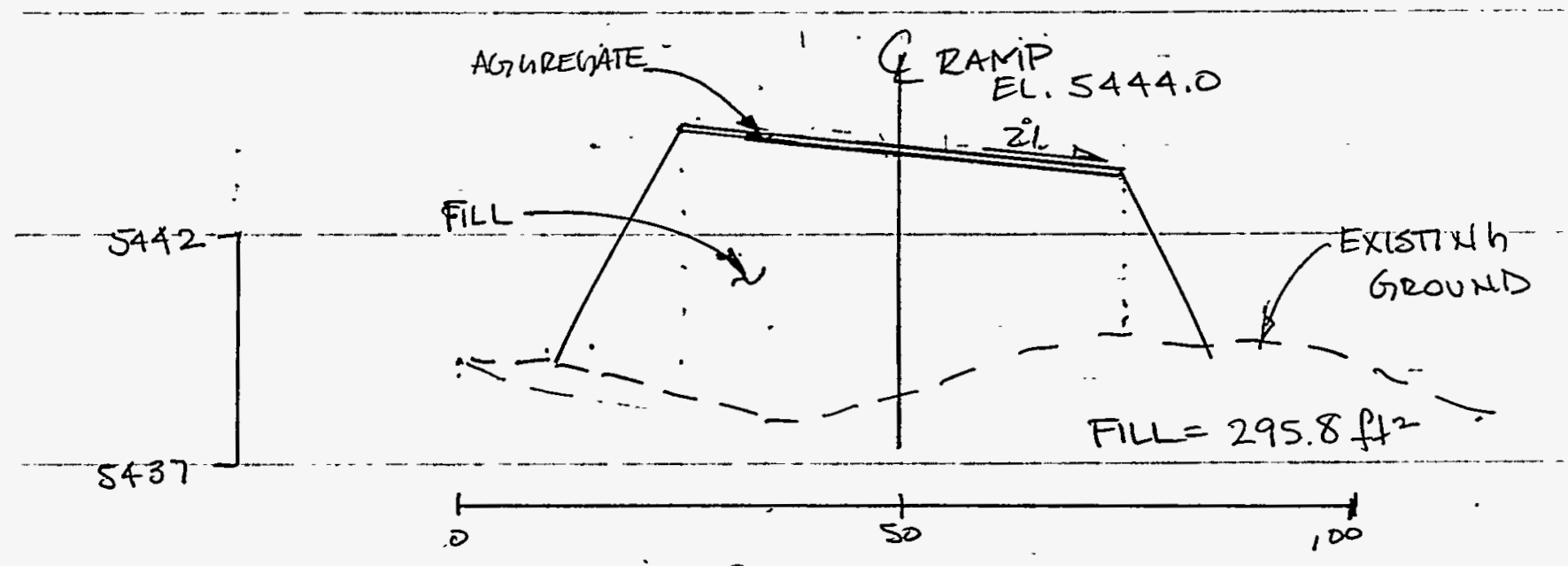

SECTION CC

ALES RAMP - UL SITE

ENG 375/91 
417. MORRISON KNUDSEN CORPORATION

Project UMTRA - SRK

Feature TEMPORARY FALIUTIES

Item

MaTERIAL QuANTITIES
Contract No. $3885-76$

Designed

Checked
Sheet 48

File No.

Date 2.3 .93

Date $2 / 16 / 93$
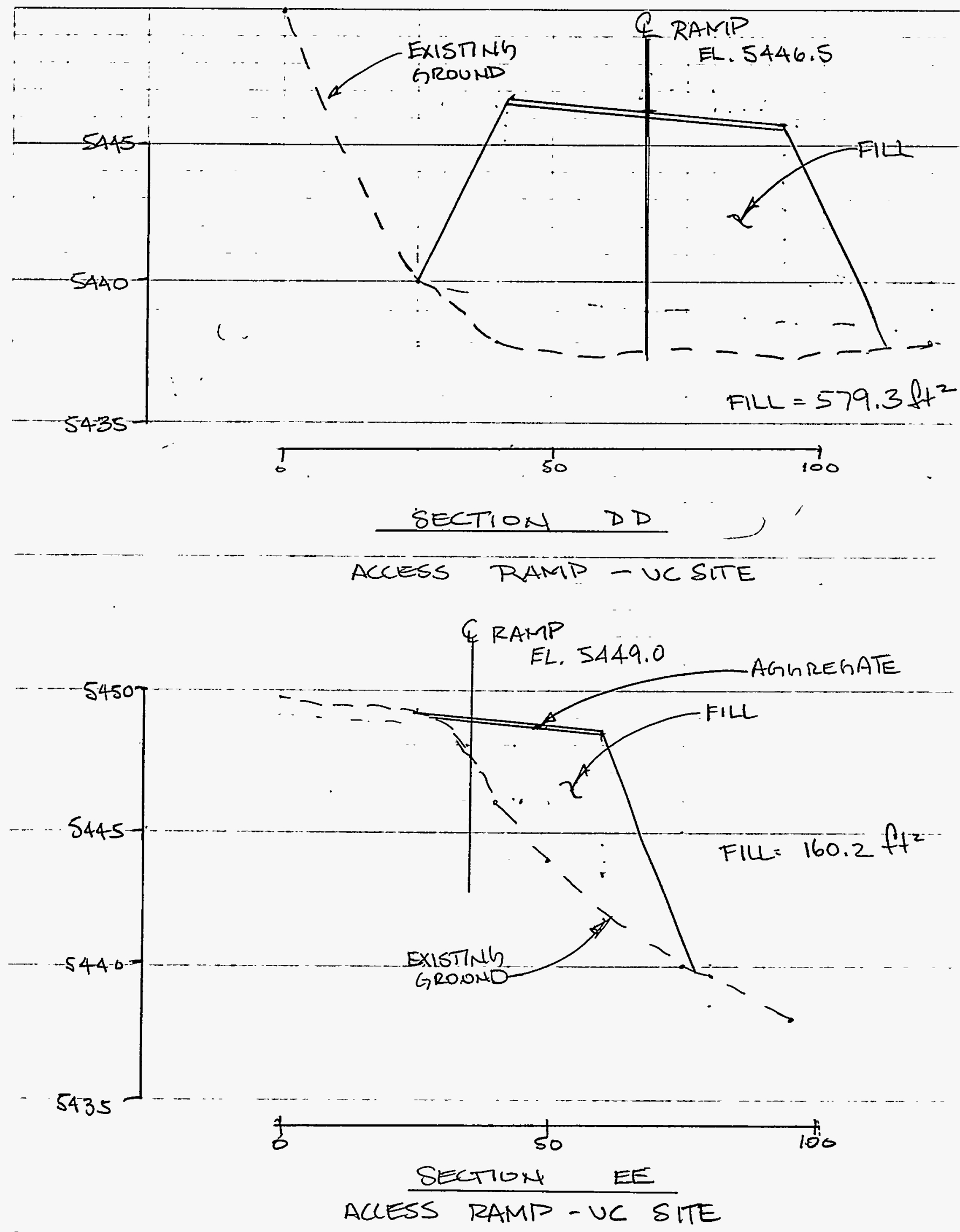

ENG 375/91 


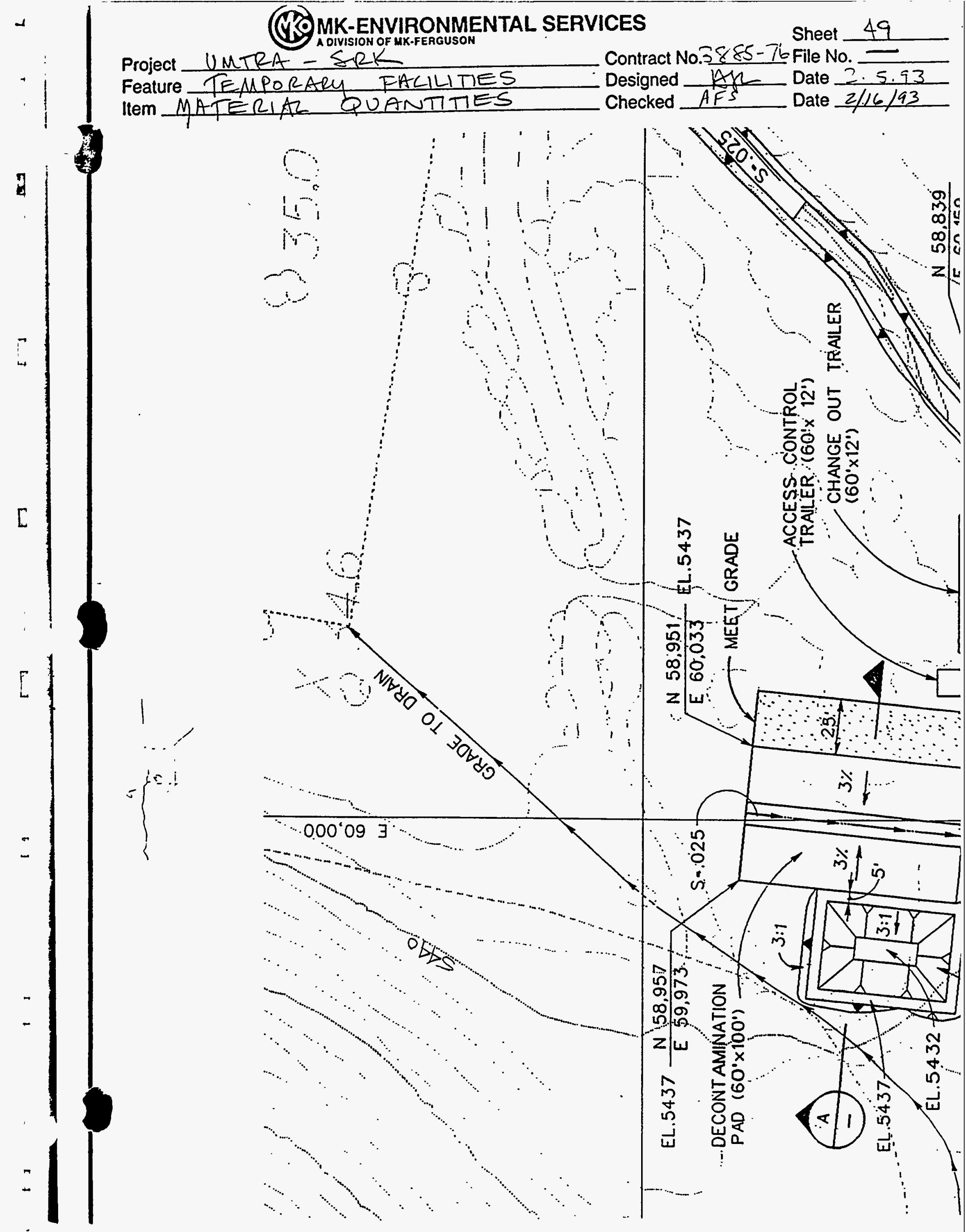




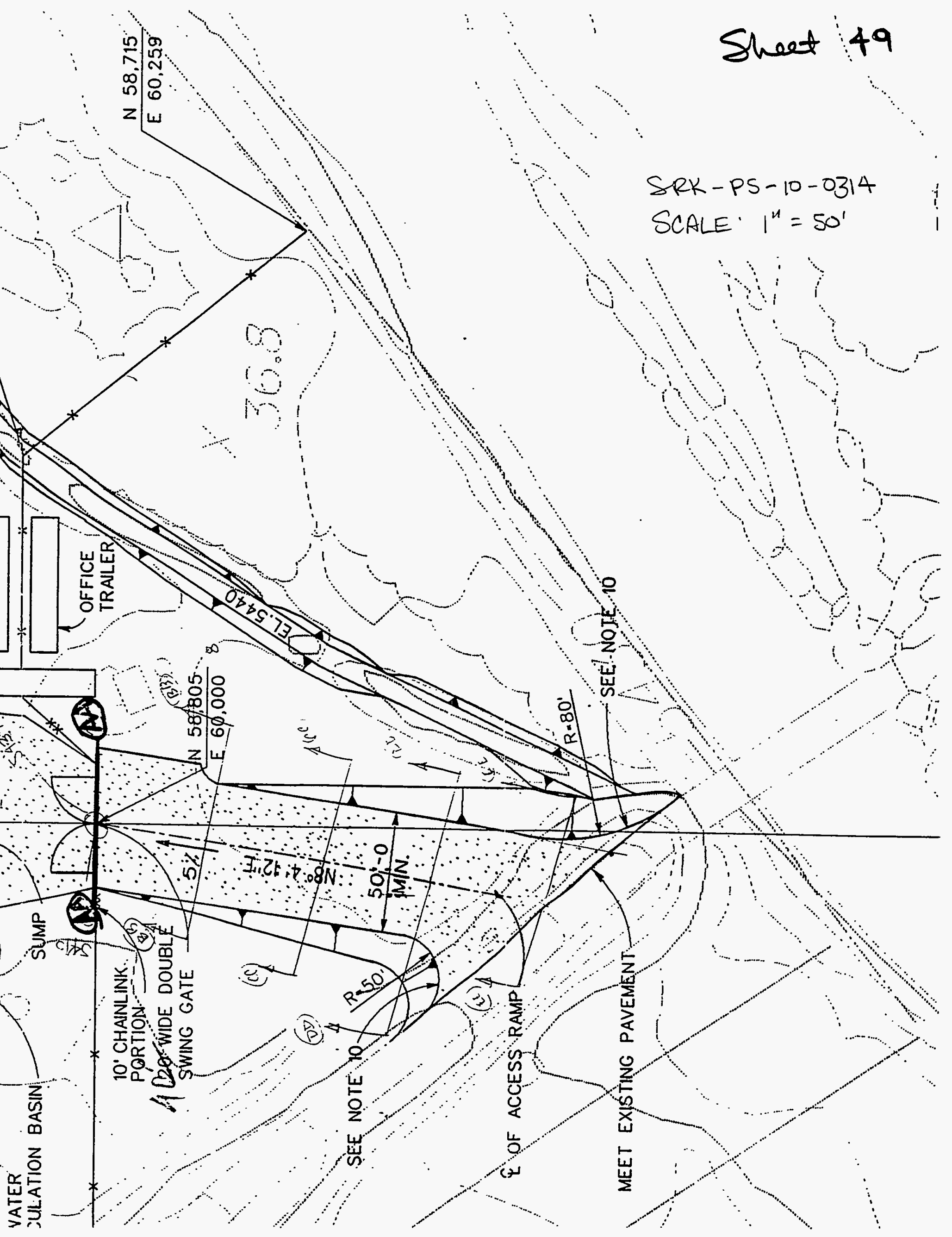


@40 .MORRISON KNUDSEN CORPORATION

$$
\begin{aligned}
& \text { Project UMTRA - SRK } \\
& \text { Sheet S50 } \\
& \text { Feature TEMPORARY FACILITIES } \\
& \text { Item MATERIAL QuANTITiES } \\
& \text { Designed } \\
& \text { File No. } \\
& \text { Date } \frac{4.27 .93}{4 / 30 / 93} \\
& \text { Checked JSR }
\end{aligned}
$$

Rev 2 BY JOR 811194 Qt: $205-9 / 13 / 45$

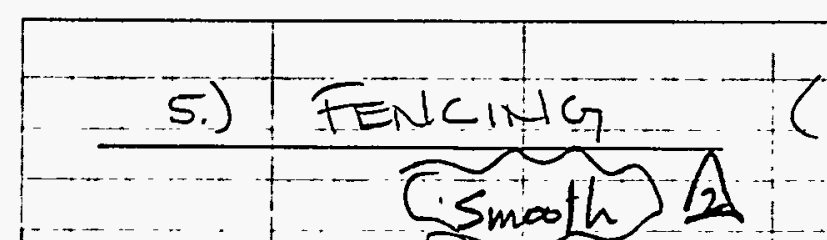

(Rife rte Duns SRK-PS-10-0310 and -0314 , shuts 6 and -9 )

a) Bare wire -

$$
\text { From spk-PS-10-0310, lengths }
$$

ineasured from N60,400 and ES8530i proceeding sow th and east ar el as

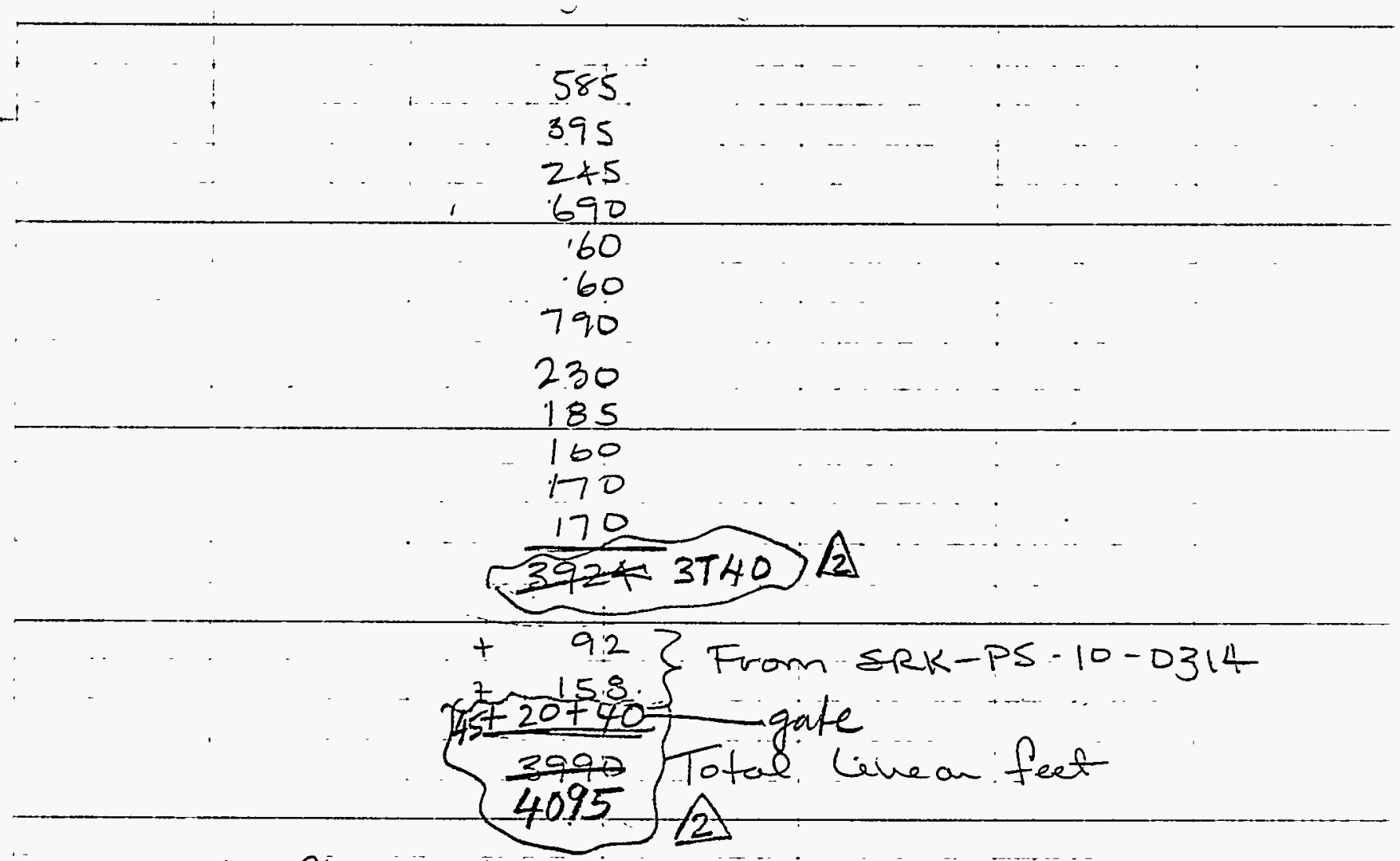

b.) Chain Link

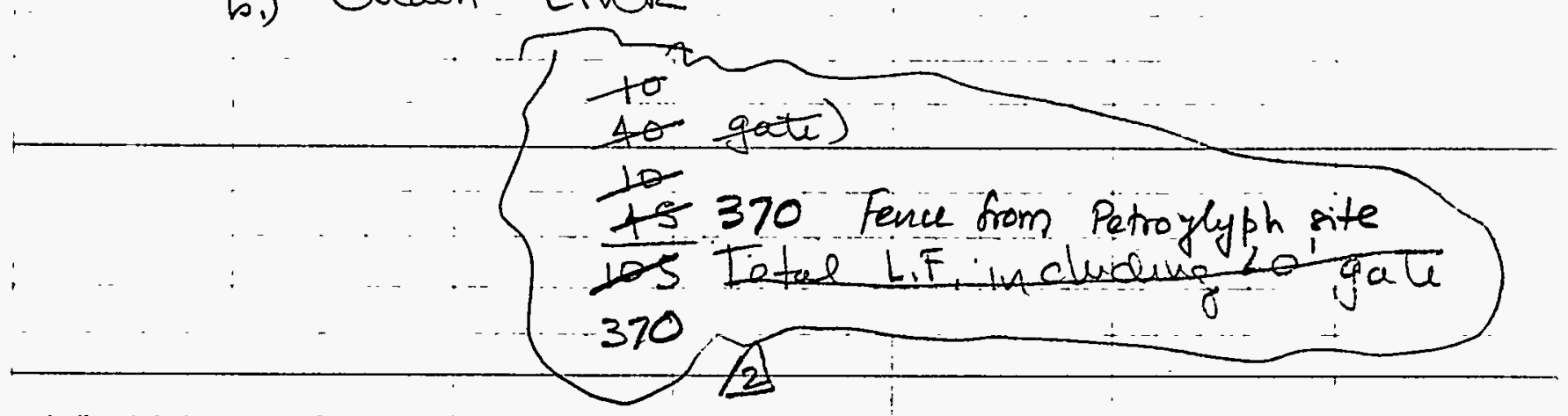

ENG 375/91 
4. MORRISON KNUDSEN CORPORATION

Project UMTRA - SRK Sheet SOA Feature Temporary Facilities Contract No. $3885-76$ File No. Designed JSR Date $8 / 12 / 94$ Date $9 / 13 / 95$

(c) Deer Fence

From sek-iss-10-03:12 (shit. 7),

Perimeter, of retention basin $=2(400+290)=1380^{\prime}$

from sRK-PS-10-03ily (sita),

Perimeter of recirculation basin $=2(50+70)=240^{\prime}$

Assume $200^{\prime} \times 200$ ' basin for construction water,

Perimeter of construction water

$$
\text { basin }=2(200+200)=800^{\prime}
$$

$\therefore$ Length of Deer. Fence e un site

$$
\begin{aligned}
& =1380+240+800 \\
& =2420 \mathrm{ft}
\end{aligned}
$$

ENG 375/91 
Sheet 51

Project UMTRA-Slick Rock Contract No. $3885-76$ File No.

Feature TAPORARY FACILITIES Designed $14 \mathrm{HL}$. Date 4.27193 Item MATERUAR-QSANTITIES Checked Date $4 / 30 / 93$

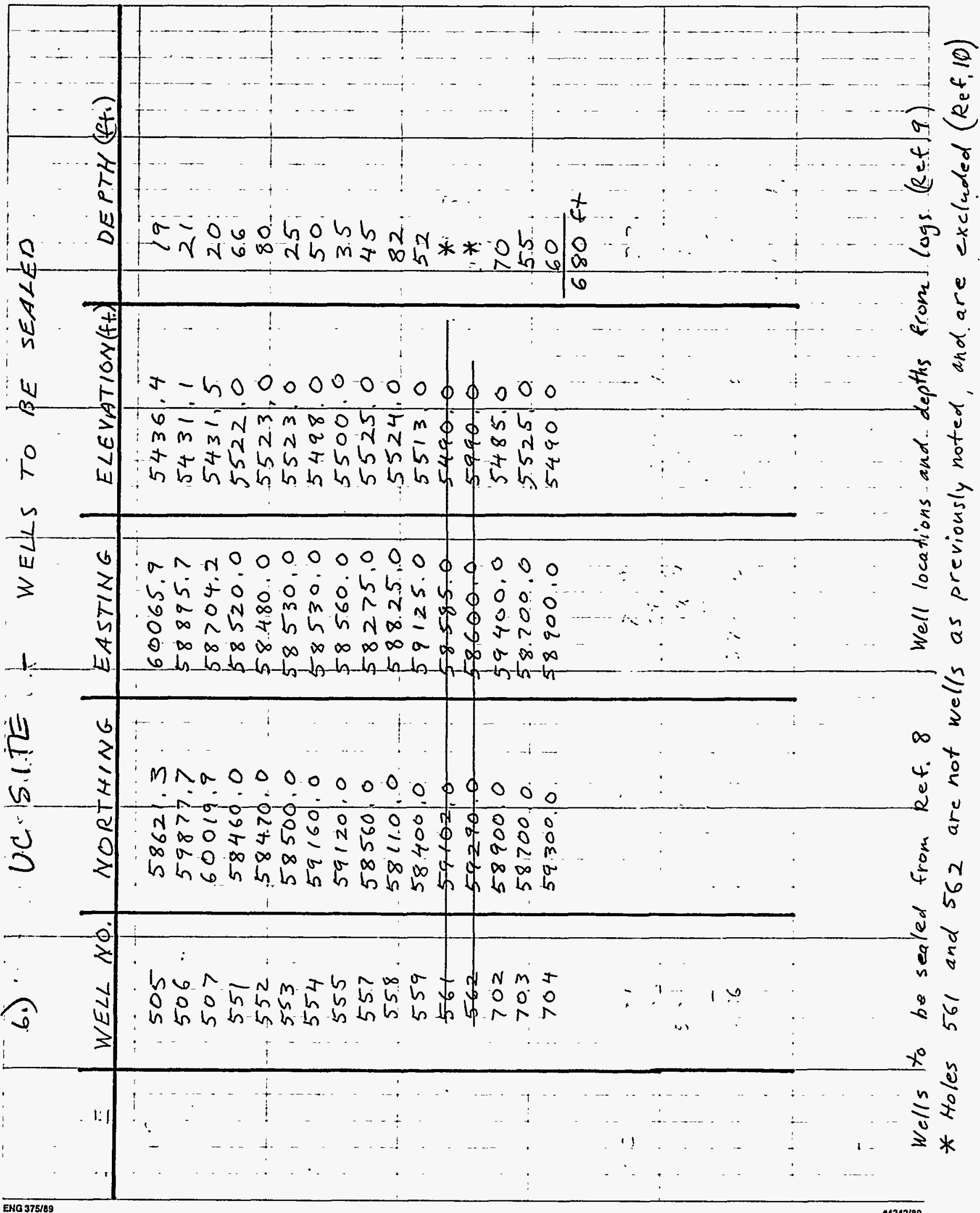


Contract No. 3885-76

Feature TEMPORARY FACILITIES

Item MATERIAL QUANTITIES
Designed HJL

Checked AFS
Sheet 52

File No.

Date 2-12-93

Date $2 / 16 / 93$

IV. CALCULATIONS - B. NC SITE 


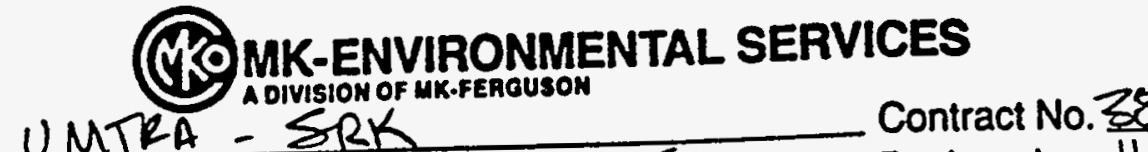

Feature TEMPORARY FACILITIES m MATERAR QuANTITES

Contract No. $3885-76$ File No.

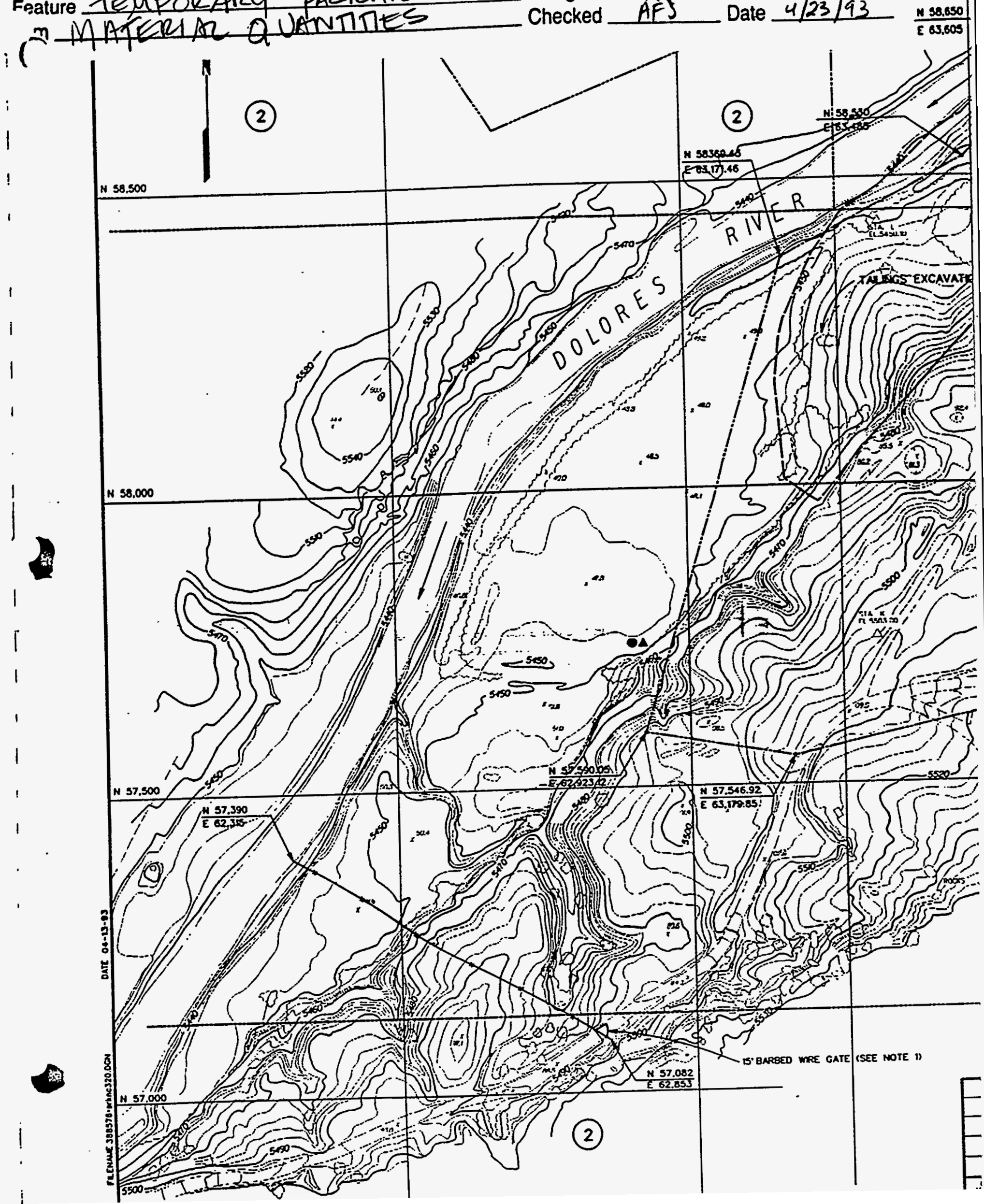




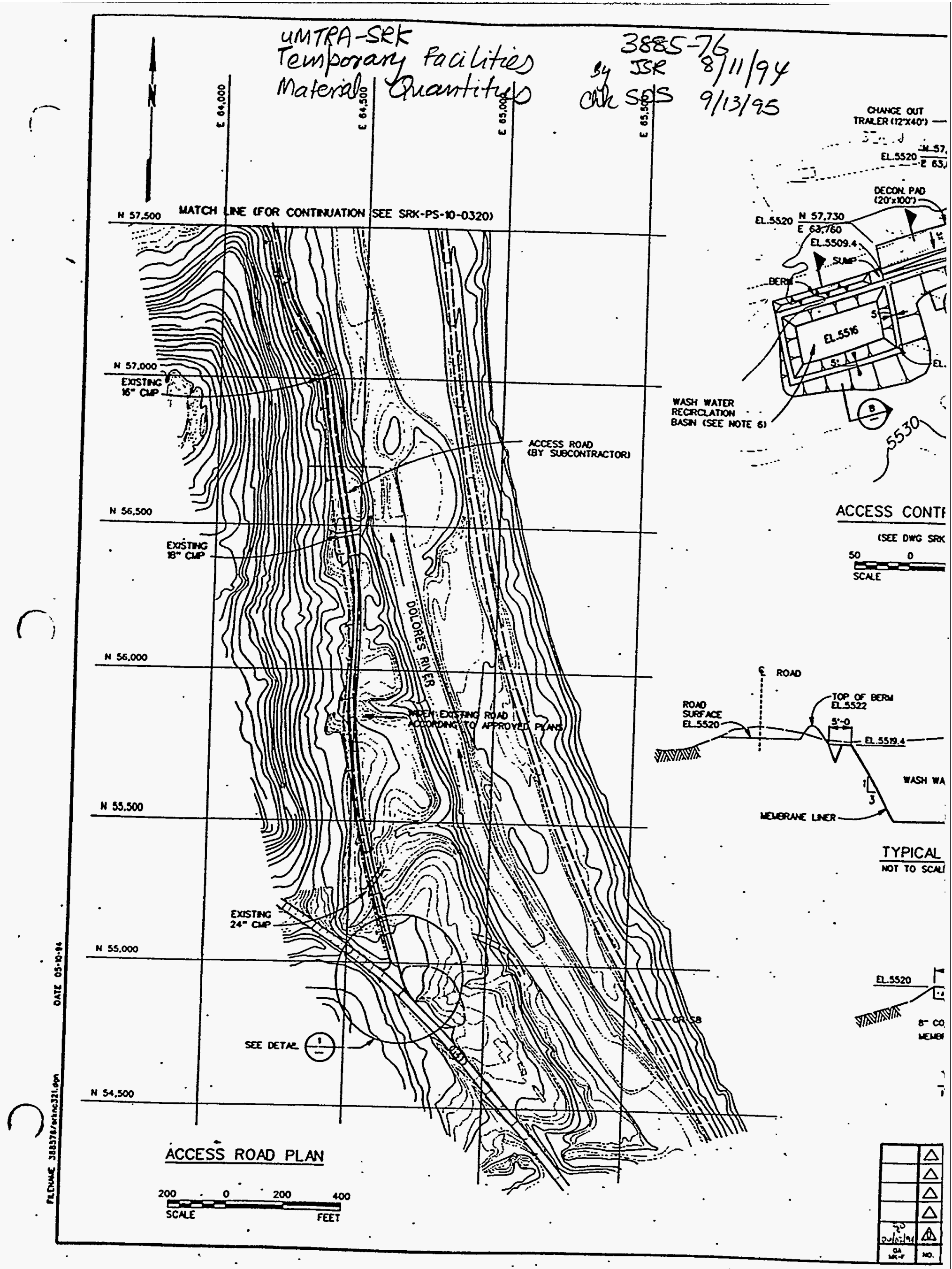




\section{(16) MKE.ENVIRONMENTAL SERVICES}

Project UNTTRA - SRIS

Feature TEMPORARY FACILITES Contract No. 3885-76 File No.

Designed 廿 Date $\overline{4.21 .53}$ in material quantities Checked Date $4 / 23 / 93$
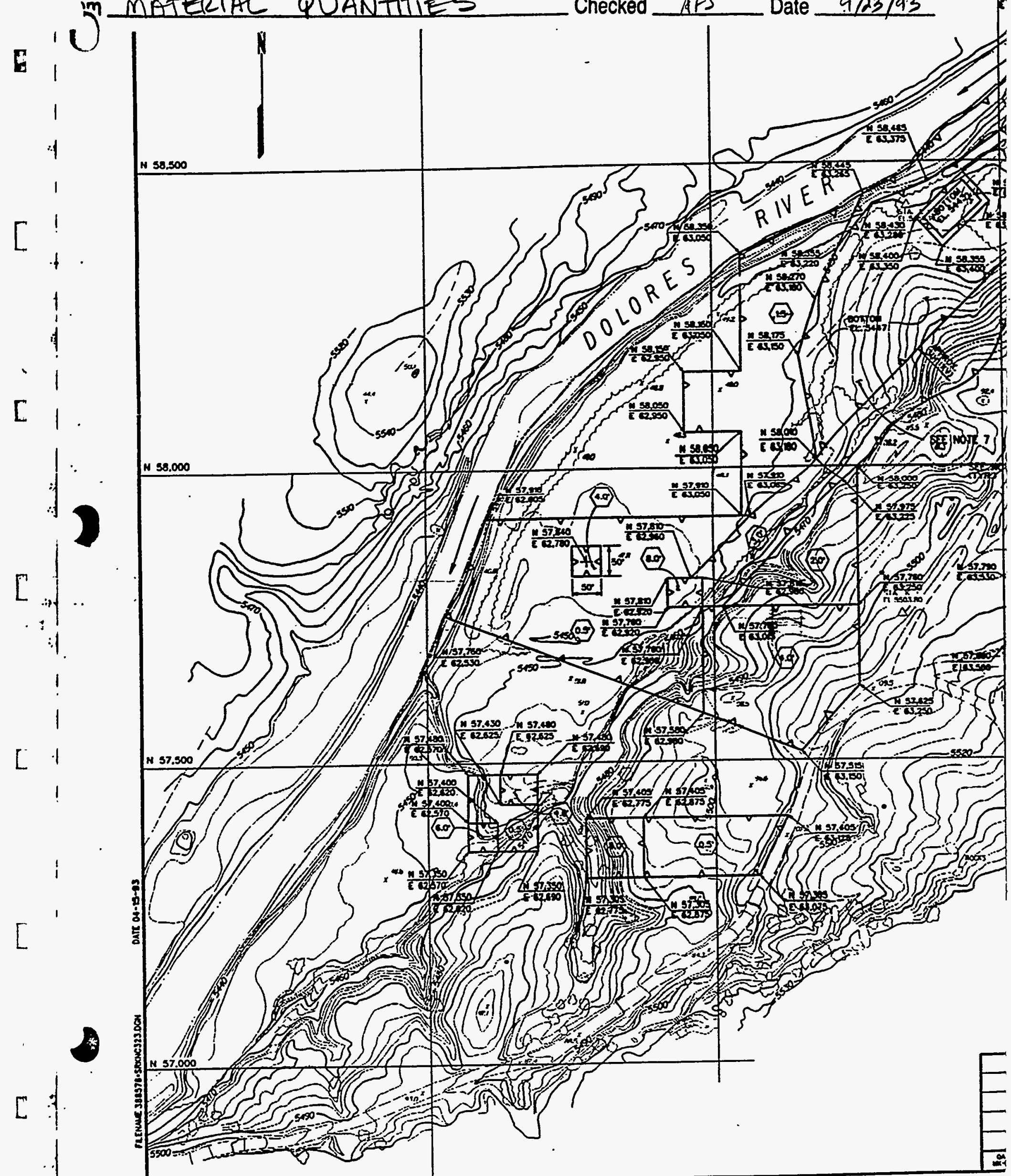


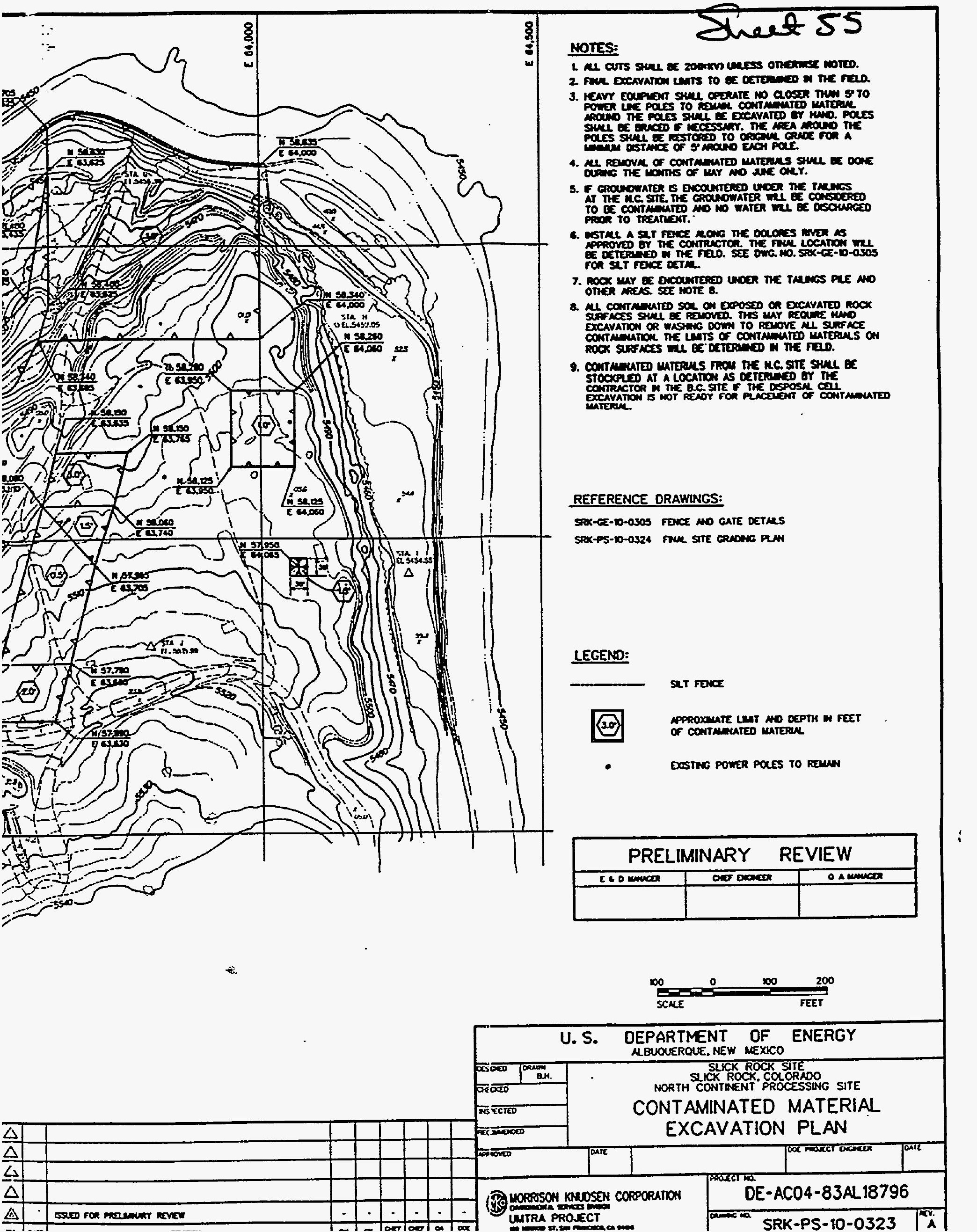




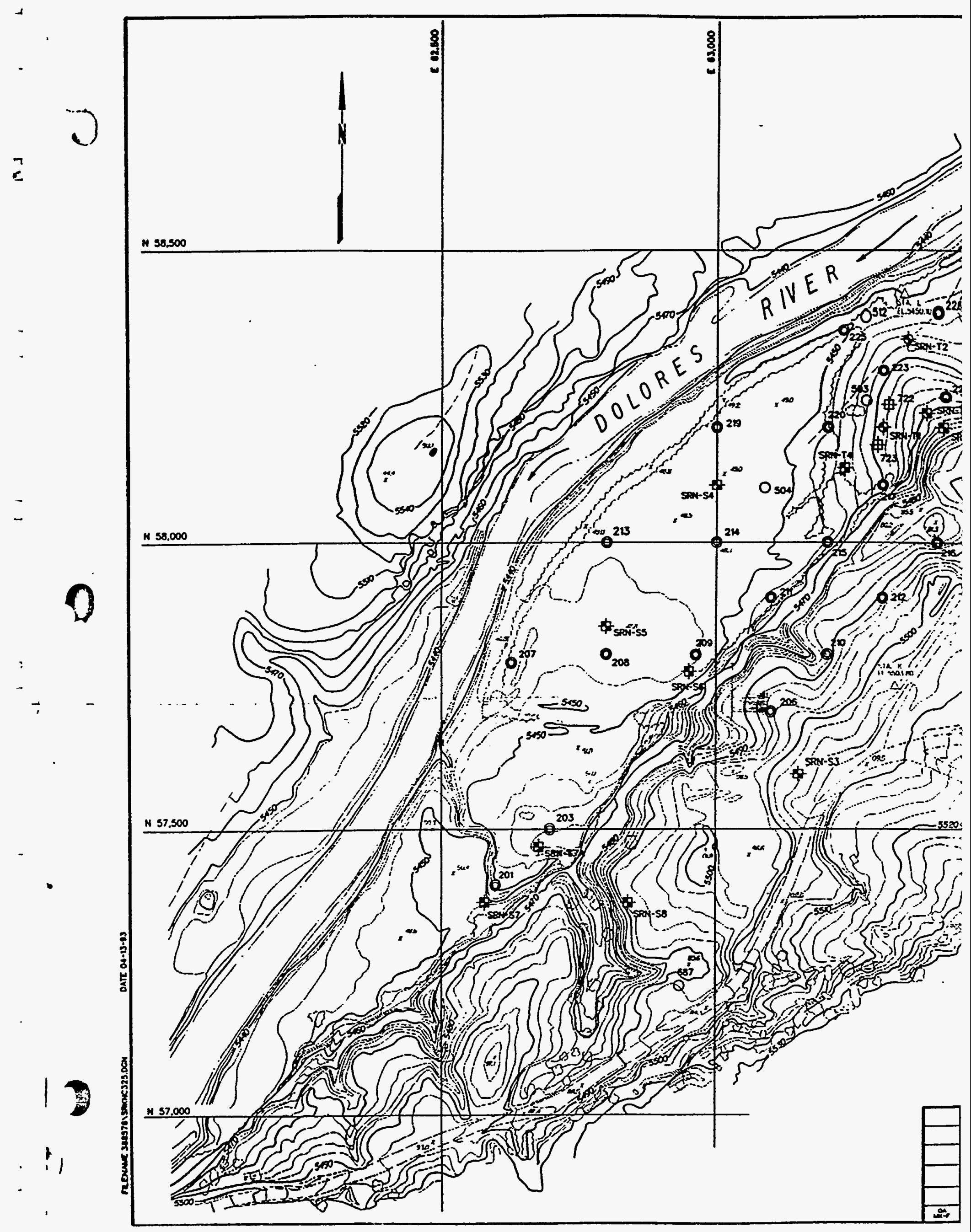




\section{(\%) MK-ENVIRONMENTAL SERVICES}

Project UANTRA - SRK

Feature TEMPORARY IACILITIES item material ojaritite

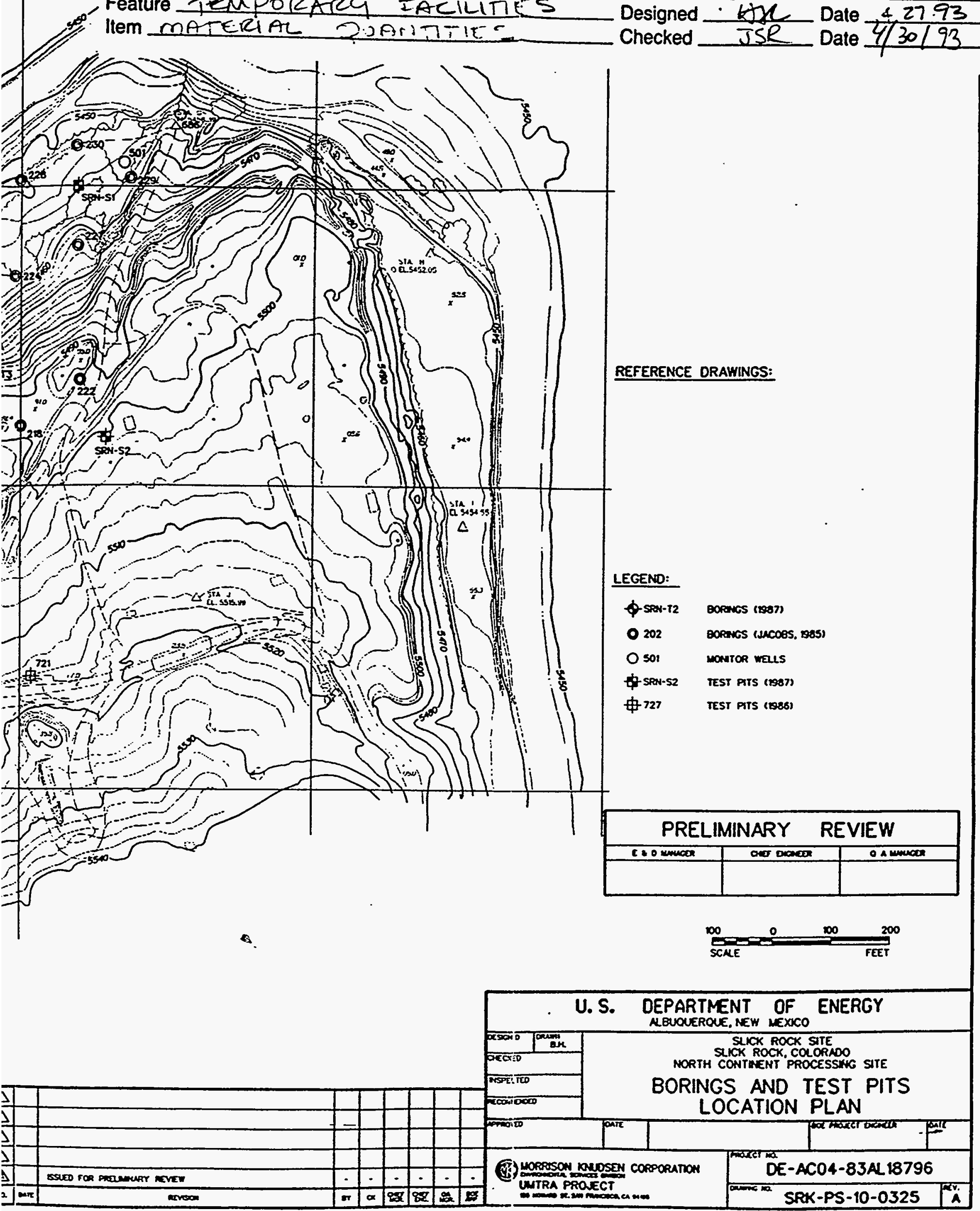


@19.9MORRISON KNUDSEN CORPORATION

$\begin{array}{ll}\text { Project UMTRA-SRK } & \text { Contract No. 3885-76 File No. } \frac{57 .}{12.93} \\ \text { Feature TEMPORARY FACILITIES } & \text { Designed } \\ \text { Item MATERIAL QUANTITIES } & \text { Checked AF5 }\end{array}$

Bev 2 by JSR $8 / 11 / 94$

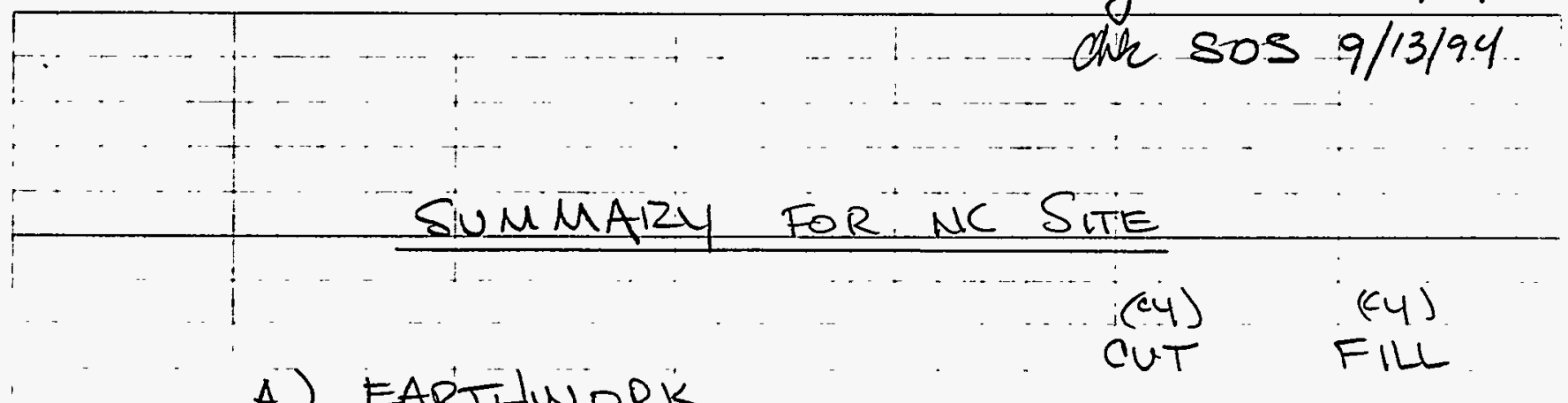

A.) EARTHWORK

1.) Recirculatian basin

- AND DEON PAD. 223.9

b.) otiter quantities.

1.) Concrete for decon pad $=50,0 \mathrm{c4}$

2.) Héntrave Liner = 81830 s.f.

31) Road Tmprovement - Leugth - Exation $1703,3 \mathrm{cy}$

$\therefore$ Aggregation $1703,3 \mathrm{cy}$

4i) Fencang

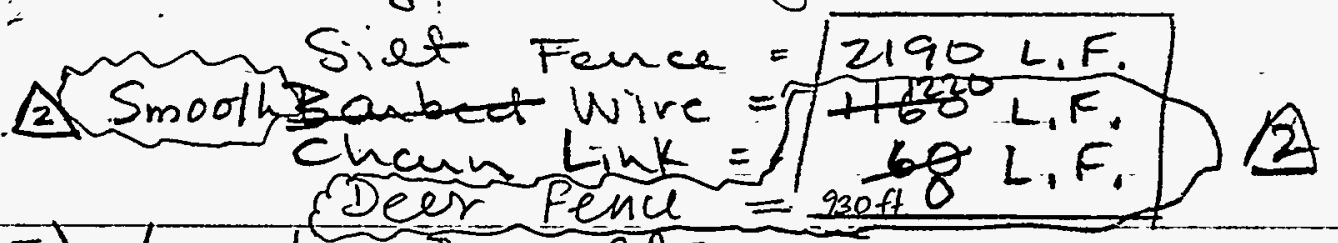

5.) Length of wels 1 be $110 \mathrm{ft}$.

ENG 375/91 
4.6.MORRISON KNUDSEN CORPORATION

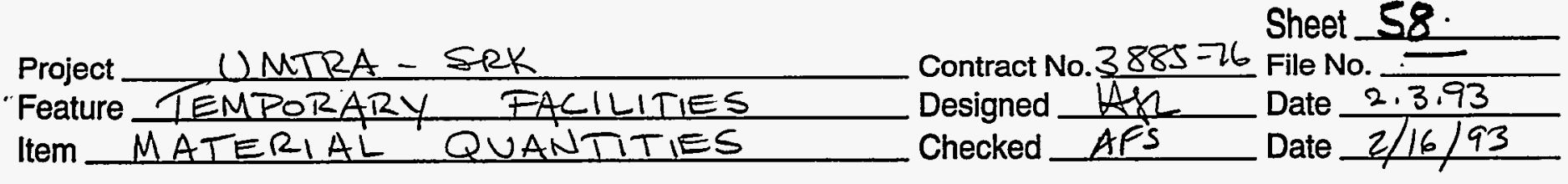

1.) Access Control Facilities

a.) Recirculation Bassn and decon prad cut rifill:

(See shts 61. to 65.)

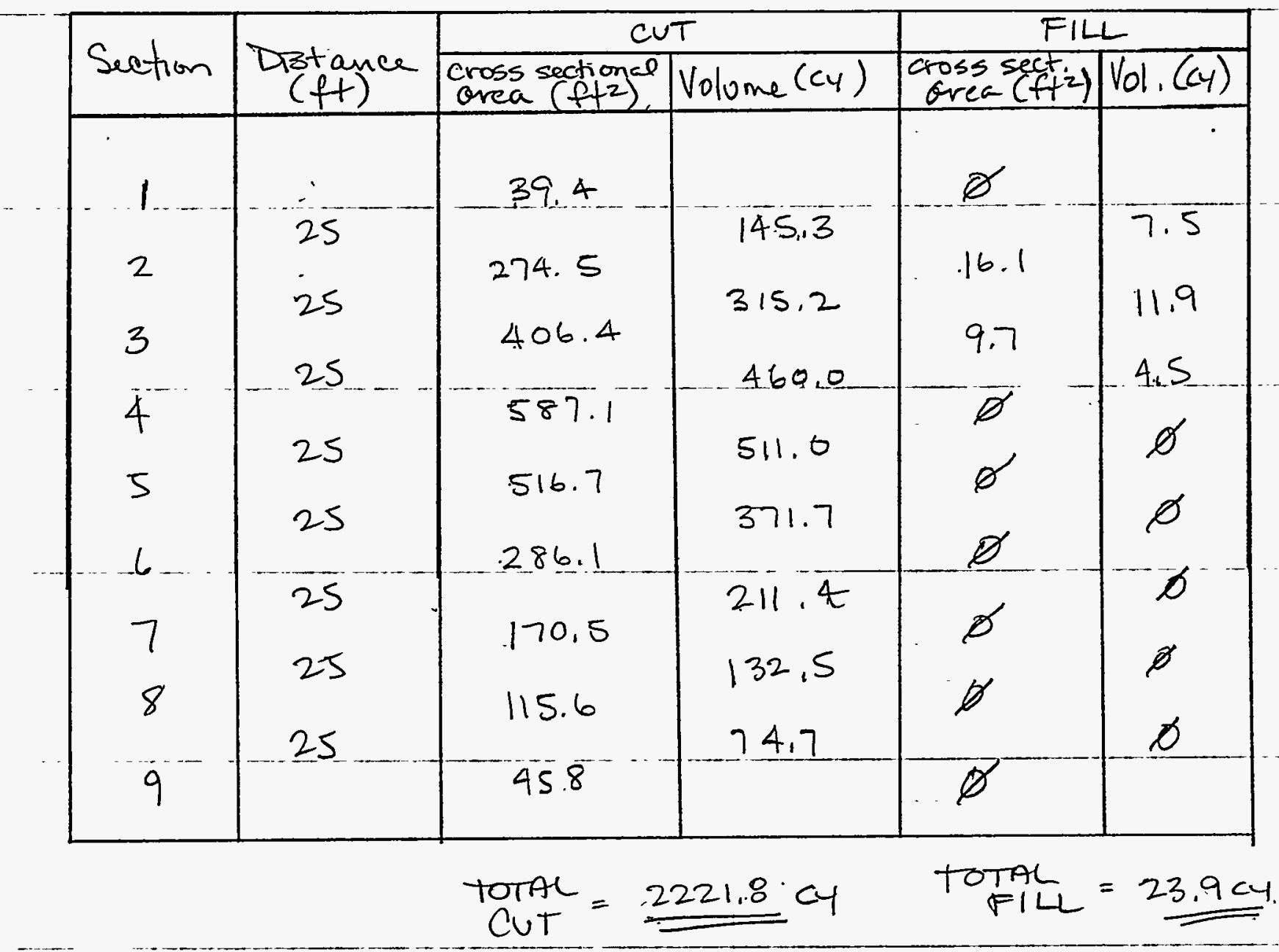

$b_{1}$ ) Concrete for decon pad

$$
\text { Volome }=\frac{(20 \times 100)(1.0005)\left(\frac{8}{12}\right)\left(\frac{1}{27}\right)=49.4 c 4}{\begin{array}{l}
30 . \text { slope } \\
\text { conection }
\end{array}}
$$

ENO $375 / 89$

$\$ 4242189$ 
@K MORRISON KNUDSEN CORPORATION

Project UMTRA - SRK

- Feature Temporary facilities

Item MATERIAL QUANTITIES
Sheet 59

Contract No. $3885-76$ File No.

Designed 482

Date 2.4 .93

Date $2 / 16 / 93$

c) Membrane Liner - recirculation basin and collection ditch

i) bottom area $=(30)(75)=2250 \mathrm{ft}^{2}$

ii)' sideslope length = (1: 1 :

$$
\sqrt{(5519.4-556)^{2}+\left[3(3.45]^{2}\right.}=10.8 \mathrm{ft}
$$

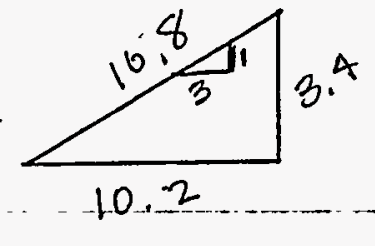

perimeter of basin at midpoint of sideslope =

$$
\begin{aligned}
2(75+5.1)+ & 2(30+5.1)=230.4 \mathrm{ft} \\
\text { Area } & =(230.4)(10.8)=2488.3 \mathrm{ft}^{2}
\end{aligned}
$$

iii) Arch on trenches (See SRK-PS-10-0315, int 10)

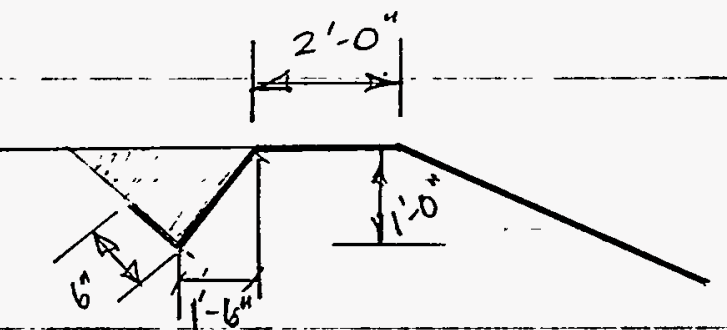

Anchor Trench Detail $(n+5)$

"Width" of liner for trench

$$
=(2)+(1.8)+(0.5)=4.31
$$

Length of finer $=2(75+10.8)$

$$
\begin{aligned}
& +2(30+10.2)+100=350.8 . \mathrm{ft}^{2} \\
& \text { Area }=(350.8)(4.3)=1508.4 \mathrm{ft}^{2}
\end{aligned}
$$

ENล375/69

$\$ 4242 / 89$ 
서MORRISON KNUDSEN CORPORATION

$$
\begin{aligned}
& \text { Project UNTRA - SRK } \\
& \text { Sheet } 60 \\
& \text { Feature TEMPORARY FACILITIES } \\
& \text { Contract No. } 3885-76 \text { File No. - } \\
& \text { ITEM MATERIAL QUANTITIES } \\
& \text { Designed } \frac{\mathrm{H} 2}{A F 5} \text { Date } \frac{2.4 .93}{2 / 16 / 93} \\
& =(2)(1.0005)(100)+\left(\frac{8}{12}\right)(100)=266.8 \mathrm{ff}
\end{aligned}
$$

v.) Area of liner between anchors trench $T$ and decor pad.

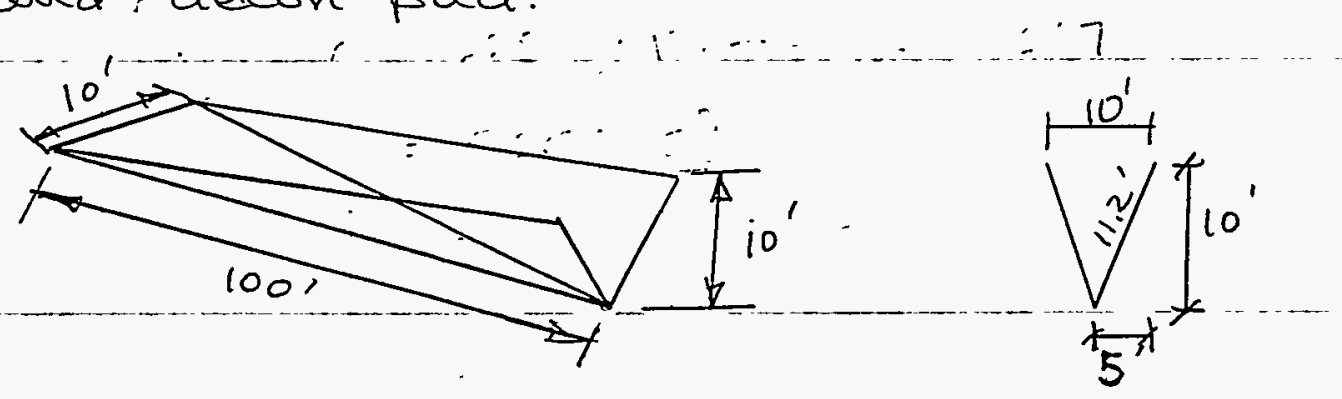

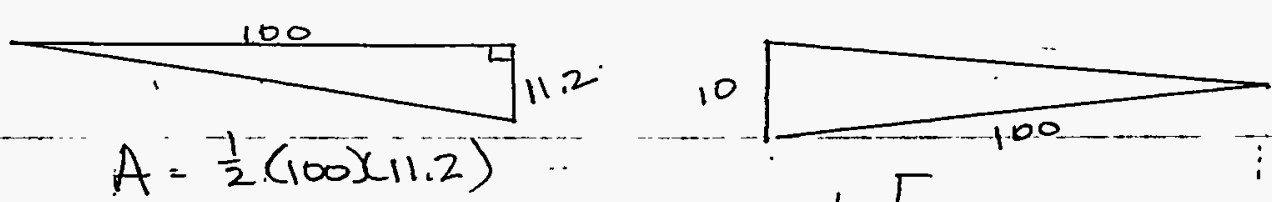

$$
\begin{aligned}
& A=\frac{1}{2} \cdot\left[(10)\left(100^{2}-10^{2}\right)^{1 / 2}\right] \\
& \text { Surface Ave }=2\left[\frac{1}{2}(100)(11.2)\right]+\frac{1}{2}\left[(10)\left(100^{2}-102\right)^{1 / 2}\right] \\
& =16117.5 \mathrm{ft} 2
\end{aligned}
$$

TOTAL LINER AREA $=2250+2488.3+1508.4$

$$
\begin{aligned}
& +266.8+1617.5 \\
& =8130.6 .8 t^{2} .
\end{aligned}
$$

2.) ALCESS ROAD

from srk-PS-10-0321, set 53 , approx length of road to be.

$$
\text { widened }=1840 \text { it }
$$

$$
\begin{aligned}
& \text { Width }=25 ; \text { Excavation }=(1840)(25)(1)\left(\frac{1}{27}\right) \\
& \text { Voloni }=1703.7 \mathrm{cy} \\
& \text { Aggregate: }=(1840)(0.5)(25)\left(\frac{1}{27}\right)=851.9 \mathrm{cy}
\end{aligned}
$$

ENG 375/.9

$14242 / 99$ 
CA MORRISON KNUDSEN CORPORATION

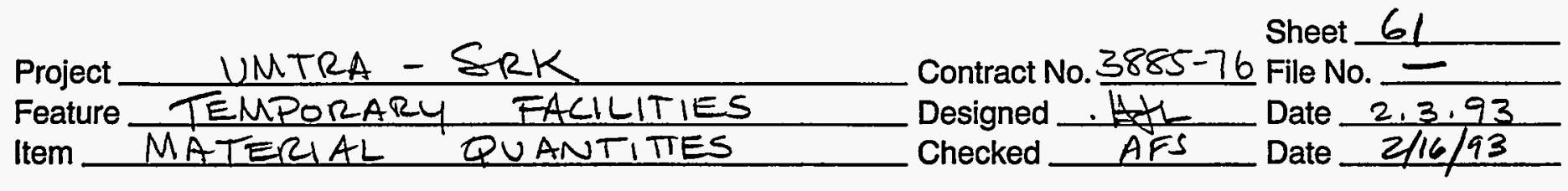

recirculation basin Sections

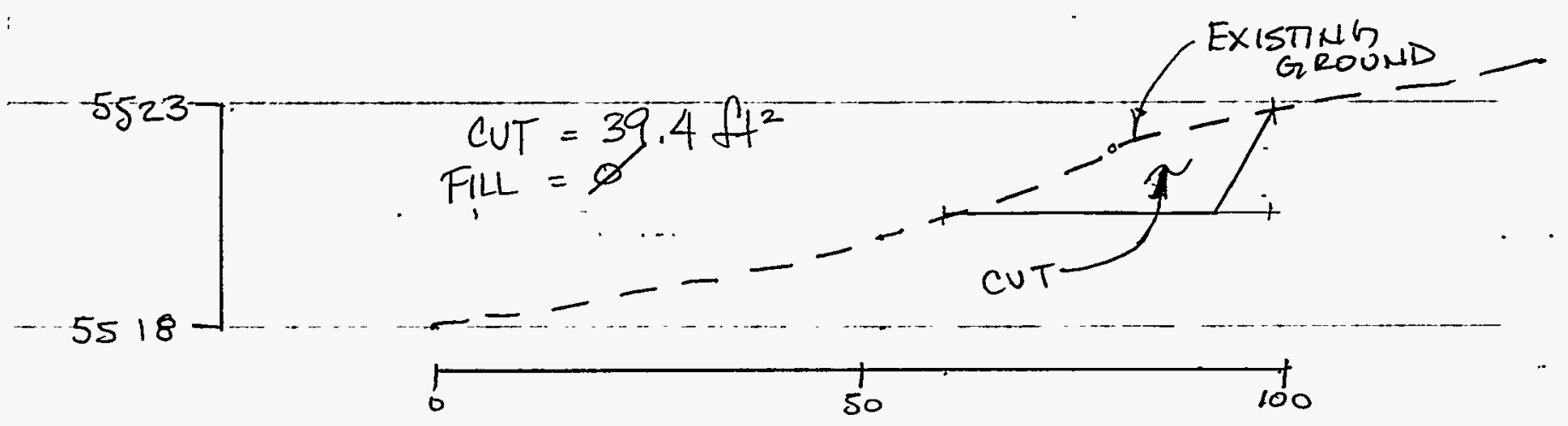

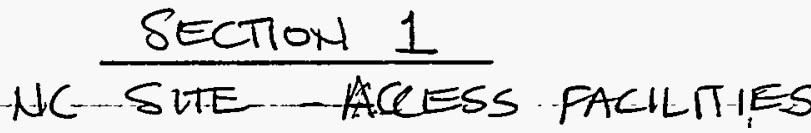

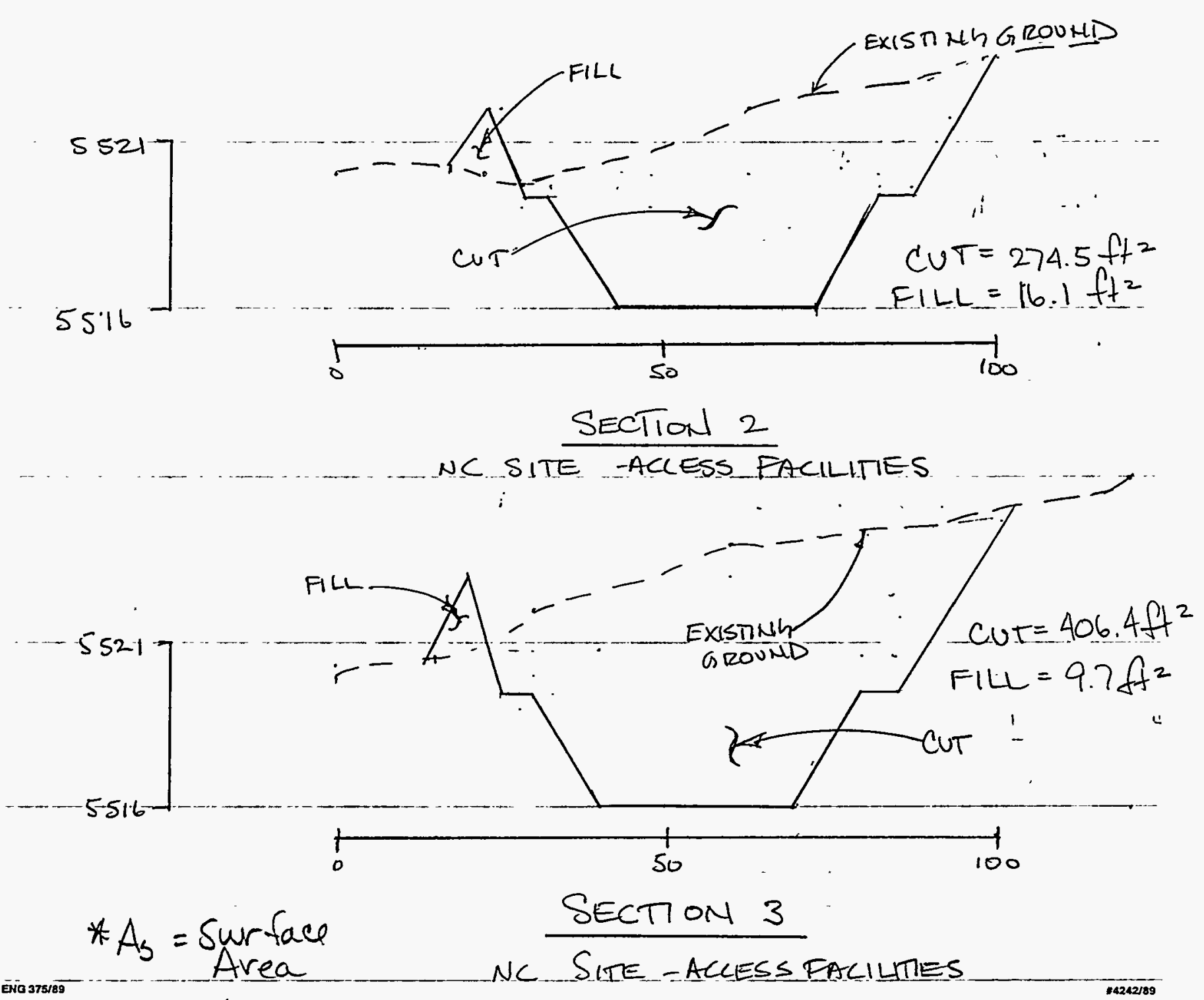


Project UMTRA - SRK - Feature TEMPORARY FACILITIES
Sheet 62 Designed . I L Checked
File No.

Date 2.3 .93

Date 2/16/93

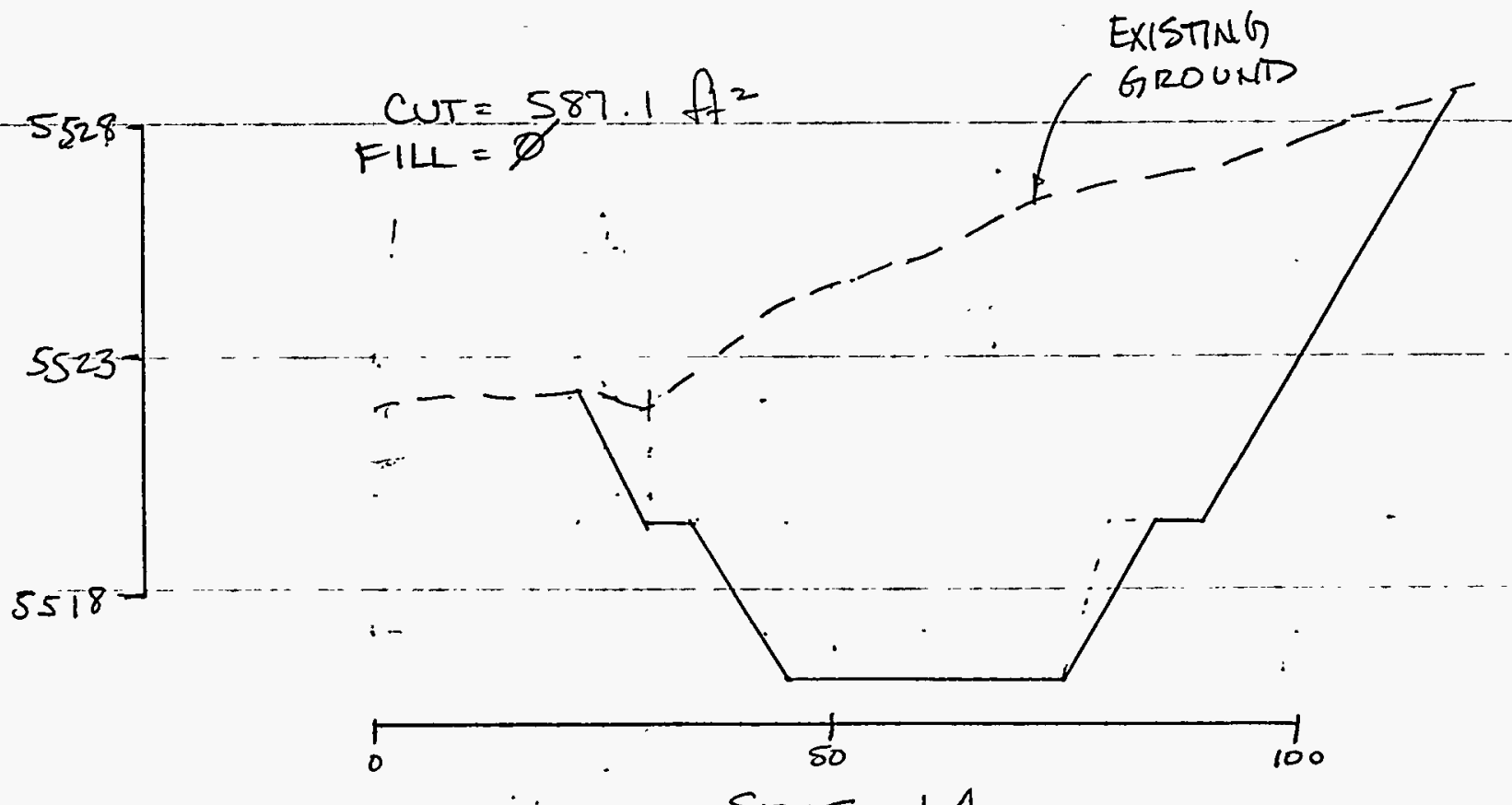

SECTION 4

NC SITE - ACLESS FACILITIES

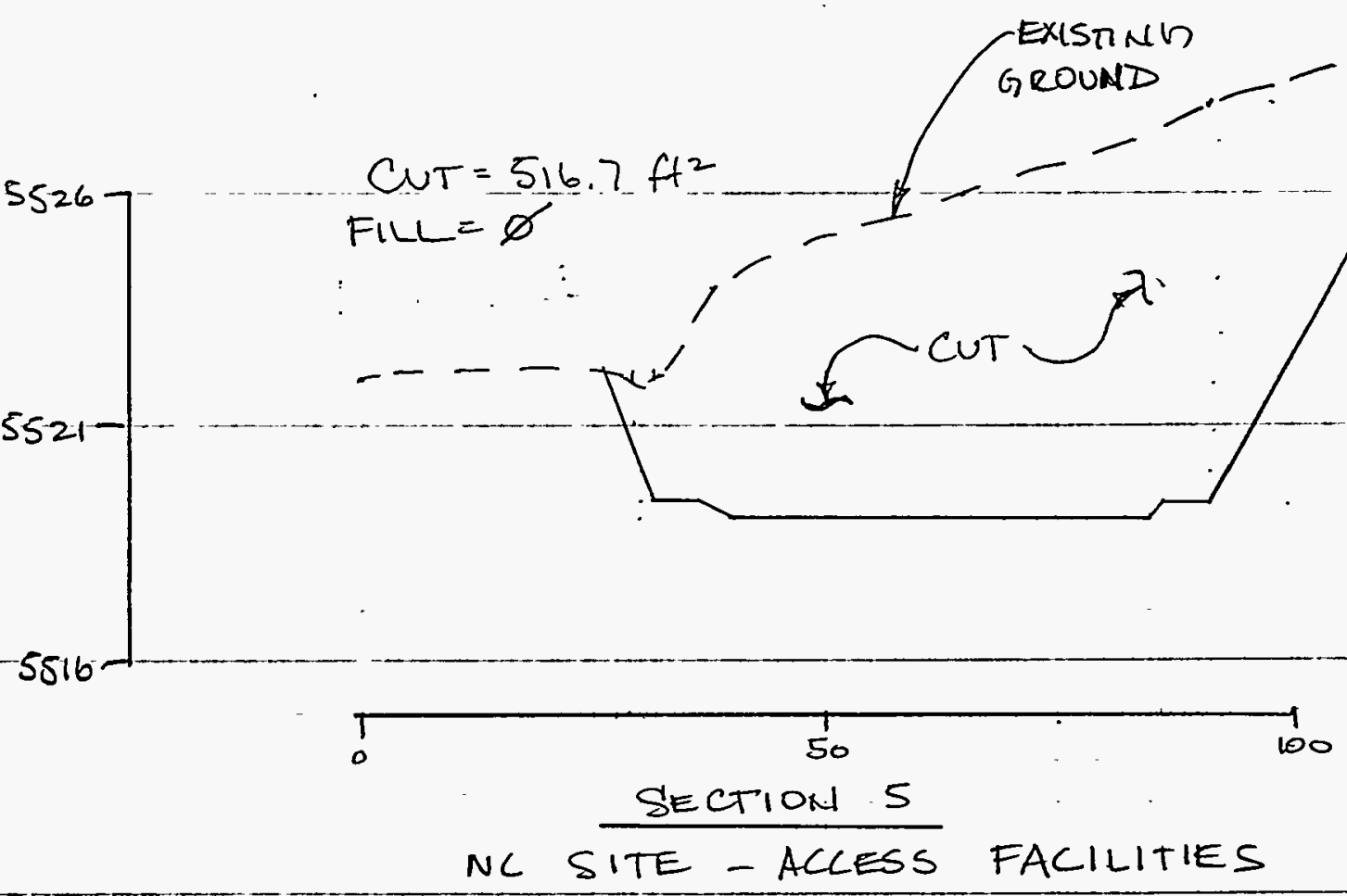


CAOMORISON KNUDSEN CORPORATION
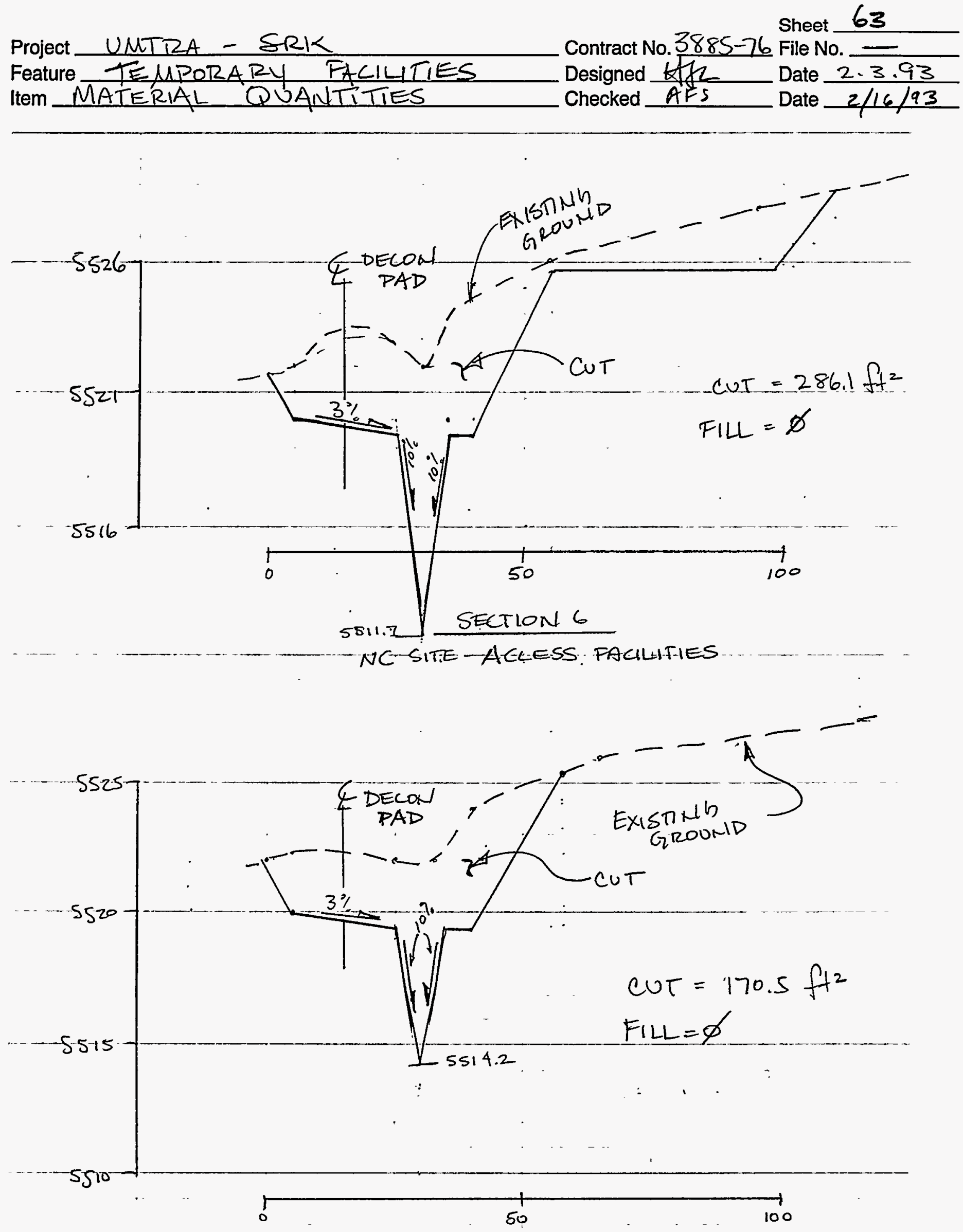

SECTION 7

NC SITE - ACCESS FACILITIES

ENG $375 / 89$ 
C.RMMORRISON KNUDSEN CORPORATION

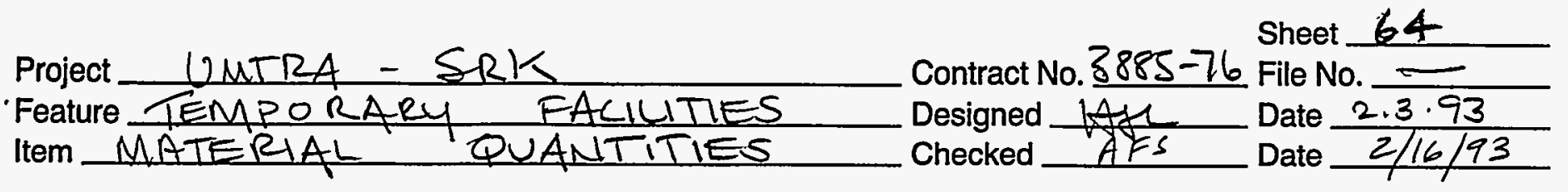
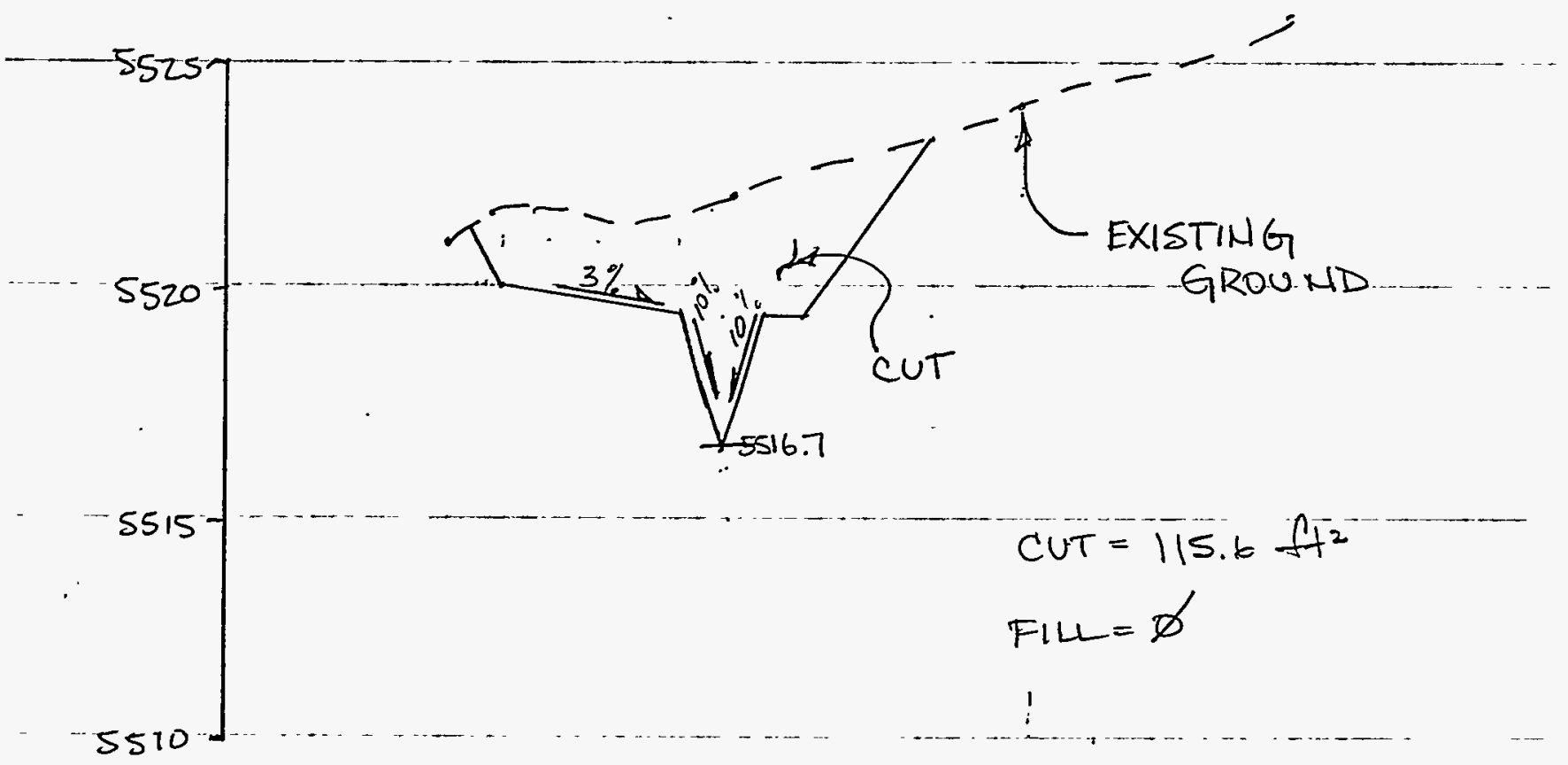

$$
\text { FIN }=\varnothing
$$

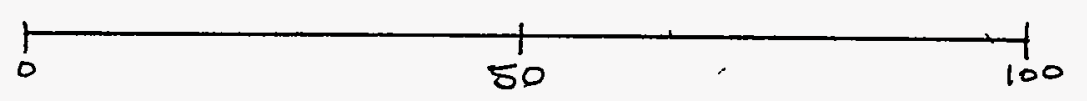

SECTION 8

NC SITE - ACCESS FACILITIES
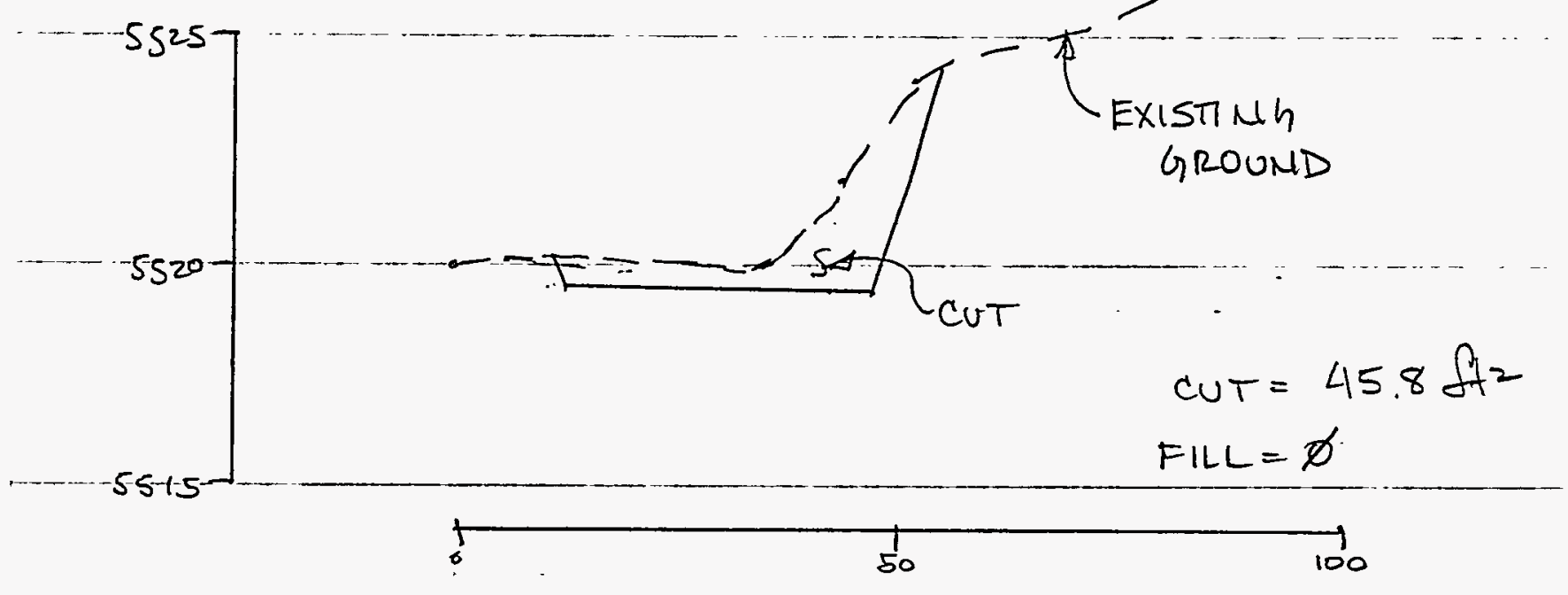

SECTION I 9

NC SITE - ACCESS FACILITIES

ENG $375 / 89$ 


\section{(3. MK-ENVIRONMENTAL SERVICES}

Project UMTRA - SRK Contract No.3885-76 File No.

Feature TEMPORARY FKCLILITES Designed AR Date $2 . \overline{5.93}$ Item MATERIAL QUANT:TTES Checked AFS Date $2 / 16 / 93$

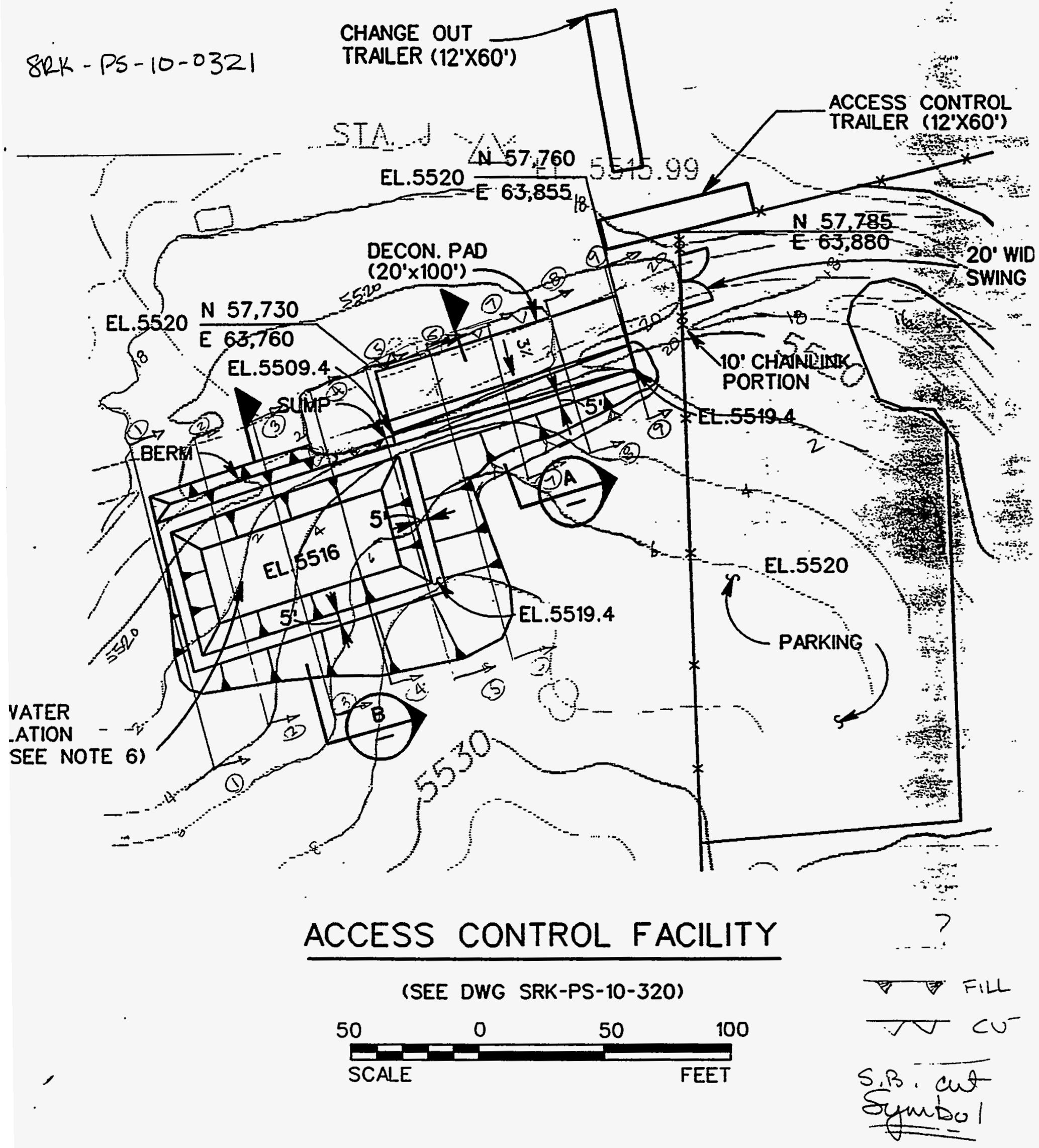




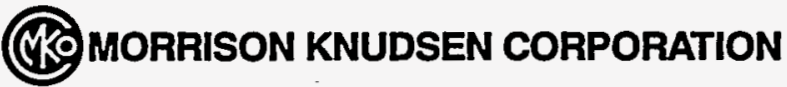

Project UMTRA - SRK

Sheet 66

Feature TEMPORARY FALILITIES

Contract No. $3885-76$ File No.

Item MATERIAL Q QUANTITIES

Designed H

Date $\overline{4.27 .93}$

Checked

Date $4 / 30 / 93$

Rev 2 BY JAR $8 / 1194$

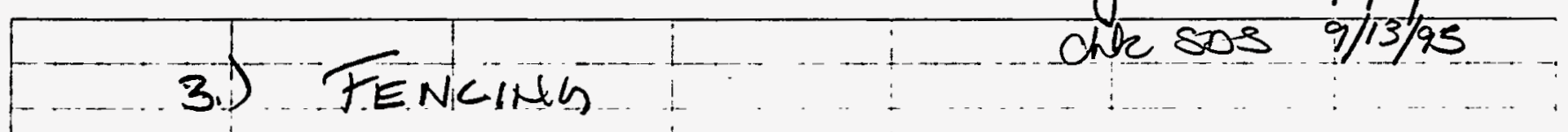

a.) Silt Fence

From SRK-PS - 10-0323 (Sheet 55) silt fence is approx. 2190 linear $f$

Smooth 2

b.) San od wine

From SRK-PS $-10-0320$ and - 0321

(Sheets $\frac{53}{3}$ and $\frac{54}{3}$ ) beginning from N57390 and E 62,315 and proceeding-

$$
\text { length }=610 \mathrm{ft} \text { inch ding }
$$

from N157,460 and E6,8,890 and proceeding North East:

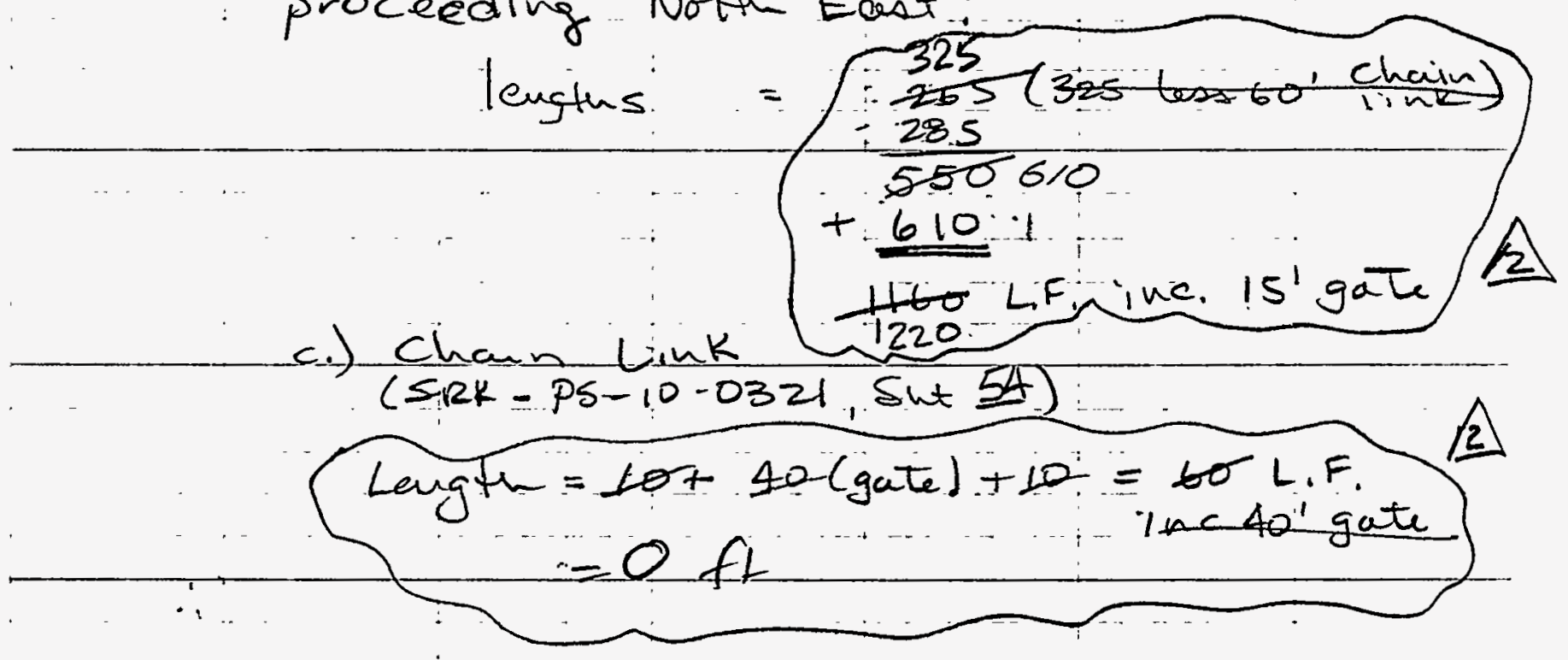

ENG 375/91 
상 MORRISON KNUDSEN CORPORATION

Sheet $66 \mathrm{~A}$

Project $4 M$ TA - SR

Contract No. $3885-76$ File No.

Feature Temporary Facilities

Designed JSR

Date $8 / 22 / 94$

Item Material quantities

Checked 80S Date $9 / 13 / 95$

(d) Deer Fences

Rev 02

From SRK-PS-10-0321 (st 65),

Perimeter of recirculation basin $=2(105+60) \mathrm{ft}$

$=330 \mathrm{ft}$

Assume a $150^{\prime} \times 150^{\prime}$ construction water basin.

Perimeter of construction water basing

$$
=2(100+100)=600^{\prime}
$$

Length of deer. Fence at NC site

$$
=330+600=930 \mathrm{ft}
$$

ENG 375/91 
Project UMTRA - SRK

Feature TEMPORARY FACILITES Item MATERIAL QuaNTTTIES
Contract No.3855-76 File No 67 Designed Checked
Date 4.27 .93 Date $\frac{4.30793}{43}$

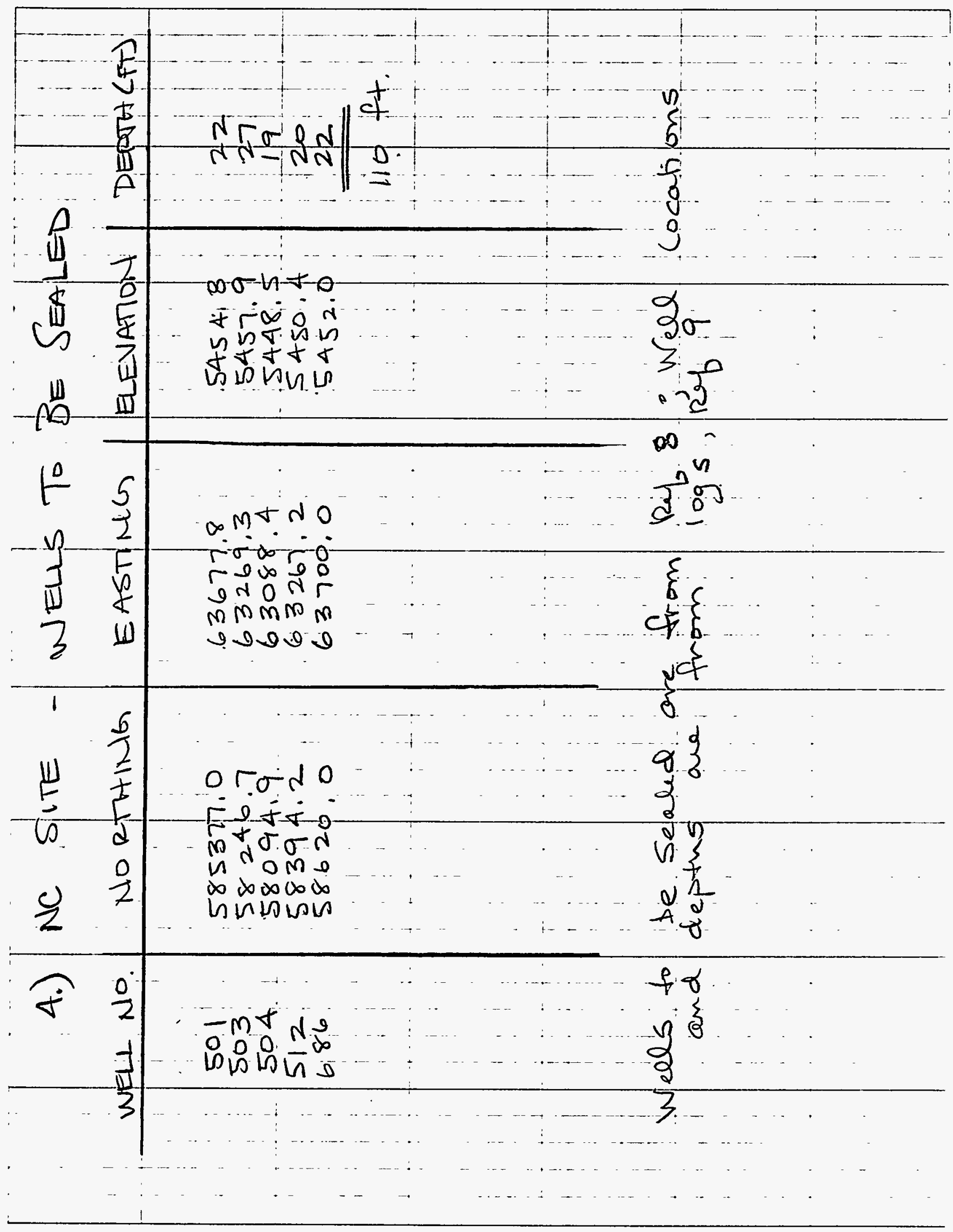


Contract No. 3885-76

Feature TEMPORARY FACILITIES

Designed HJL

Item MATERIAL QUANTITIES
Checked AFS
Sheet

File No.

Date 2-12-93

Date $2 / 16 / 93$

IV. CALCULATIONS - C. BC SITE 


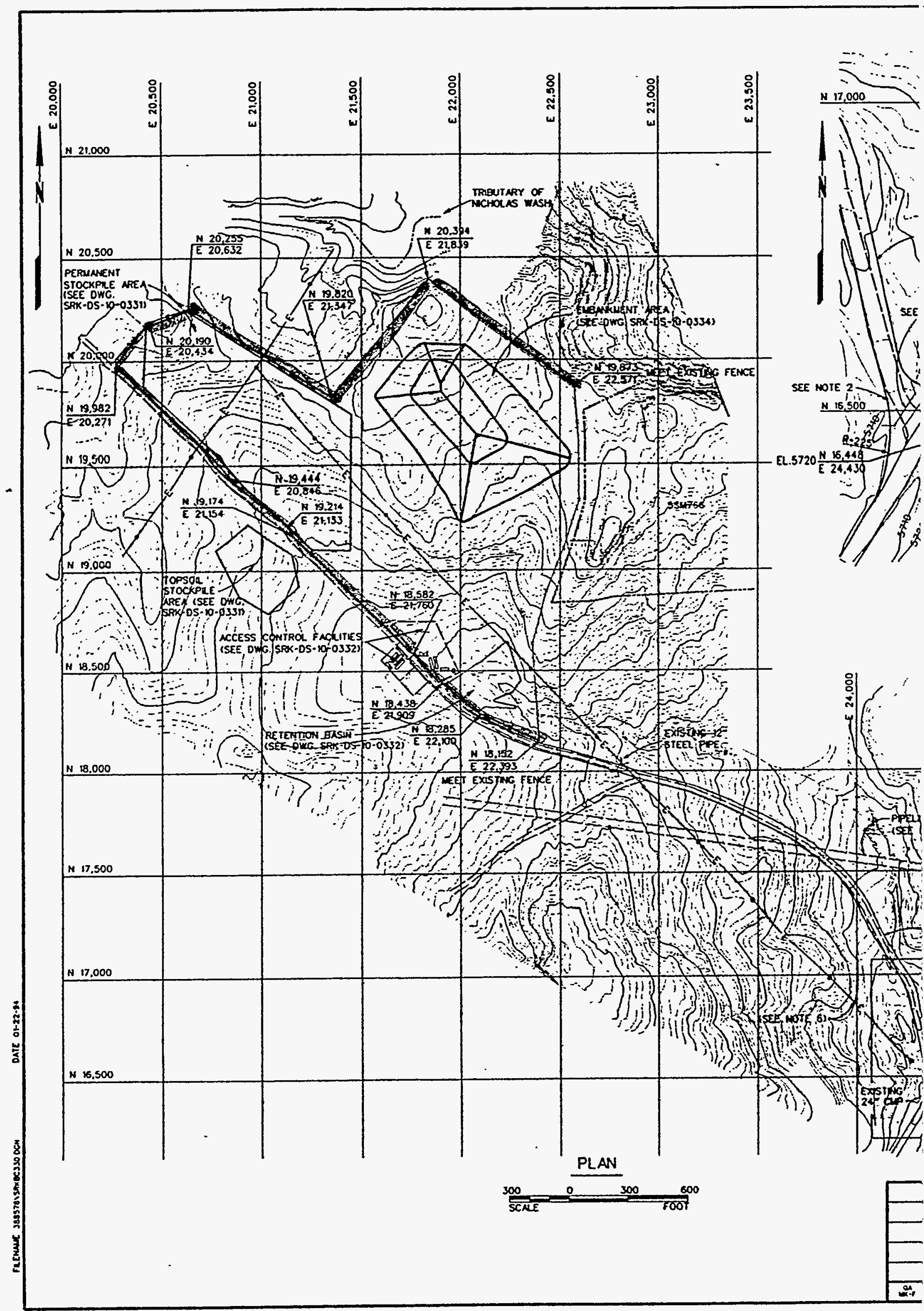




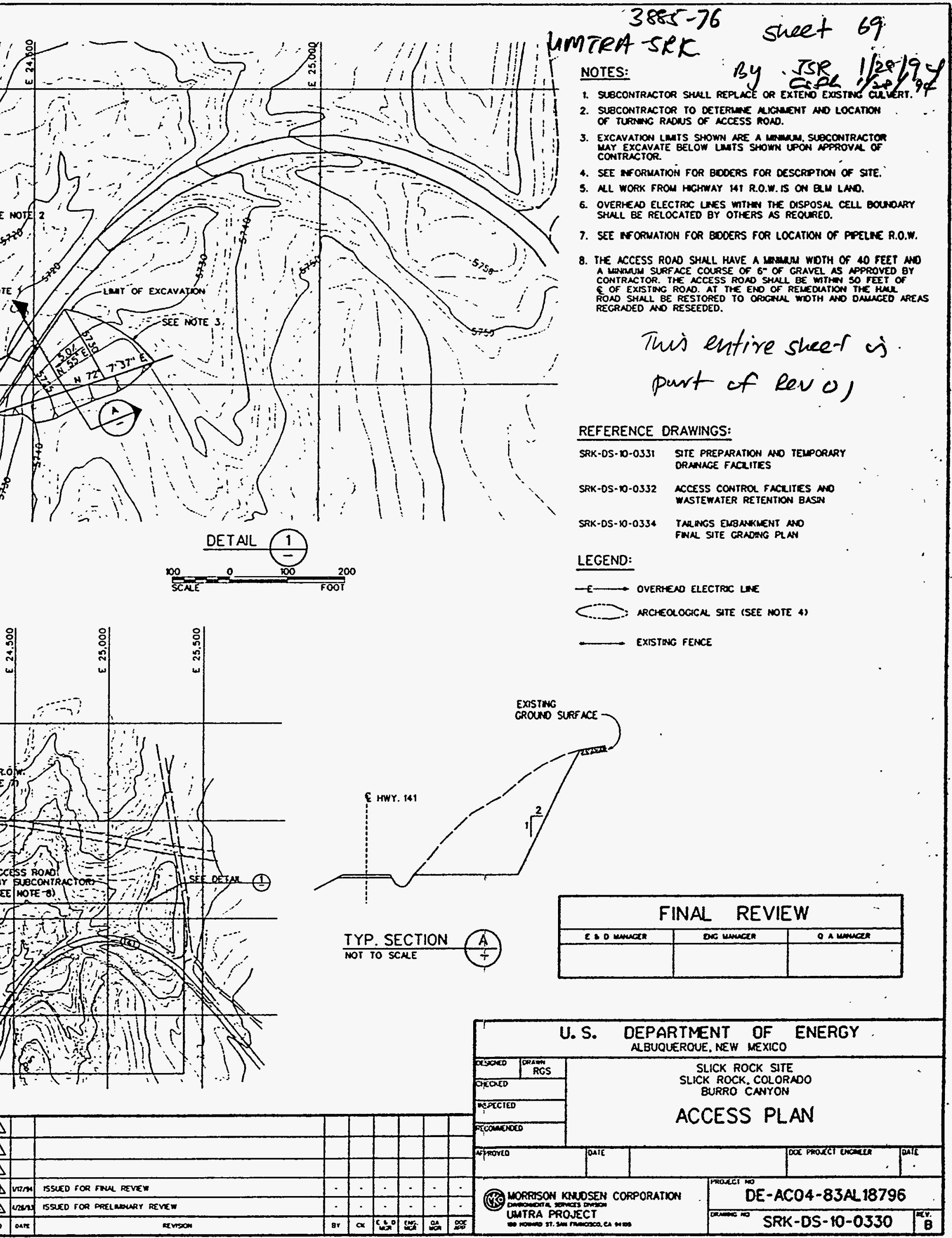




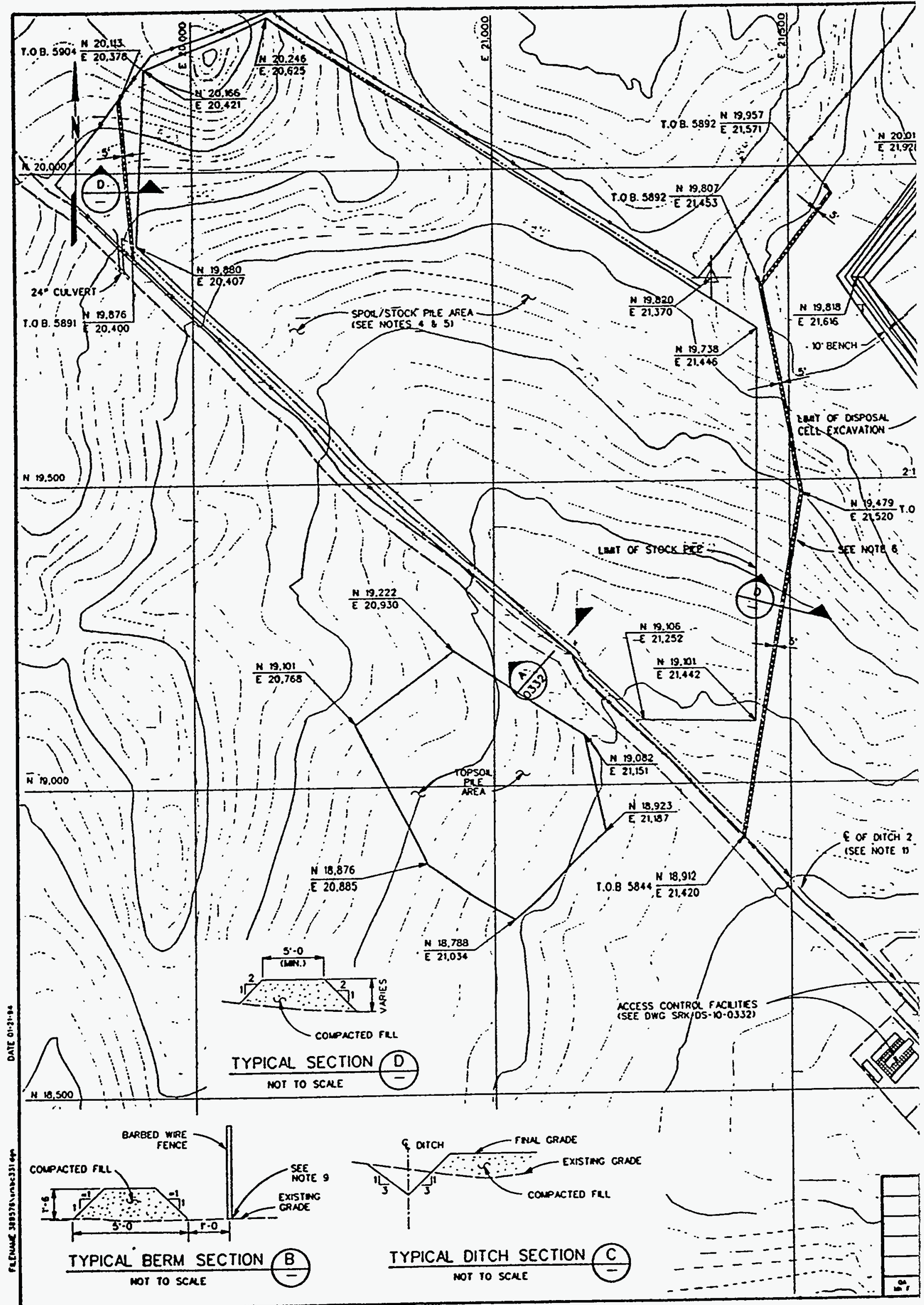




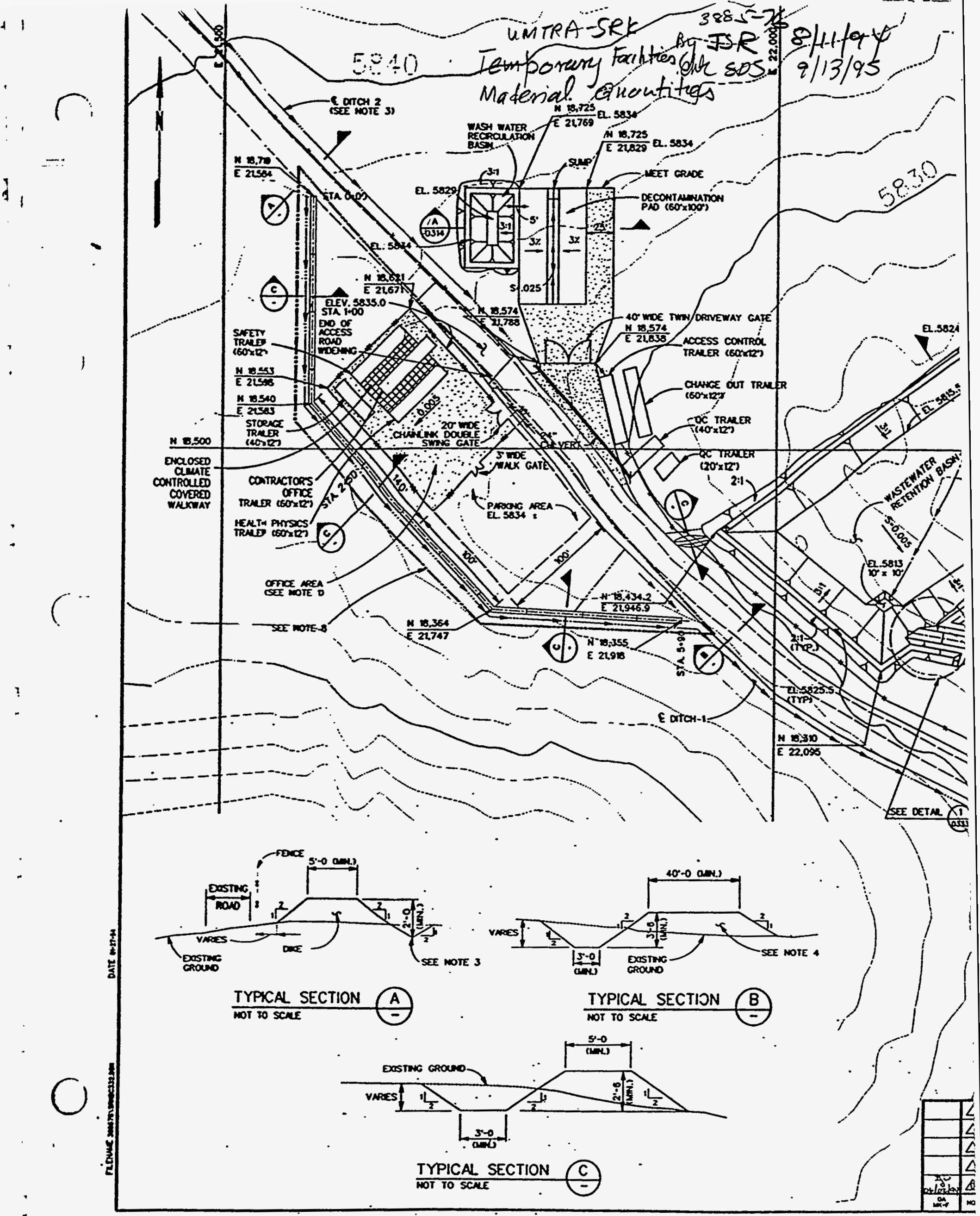




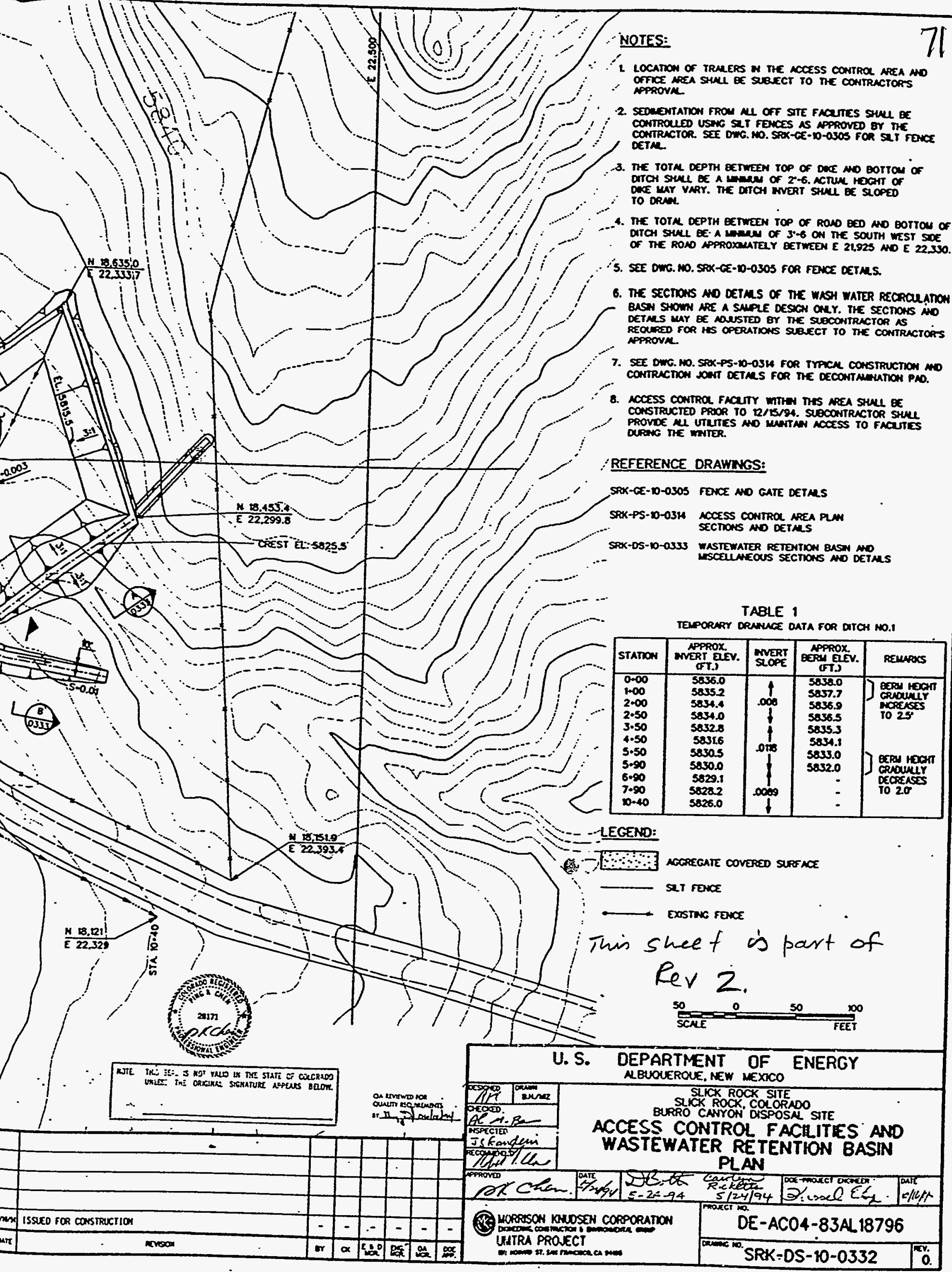




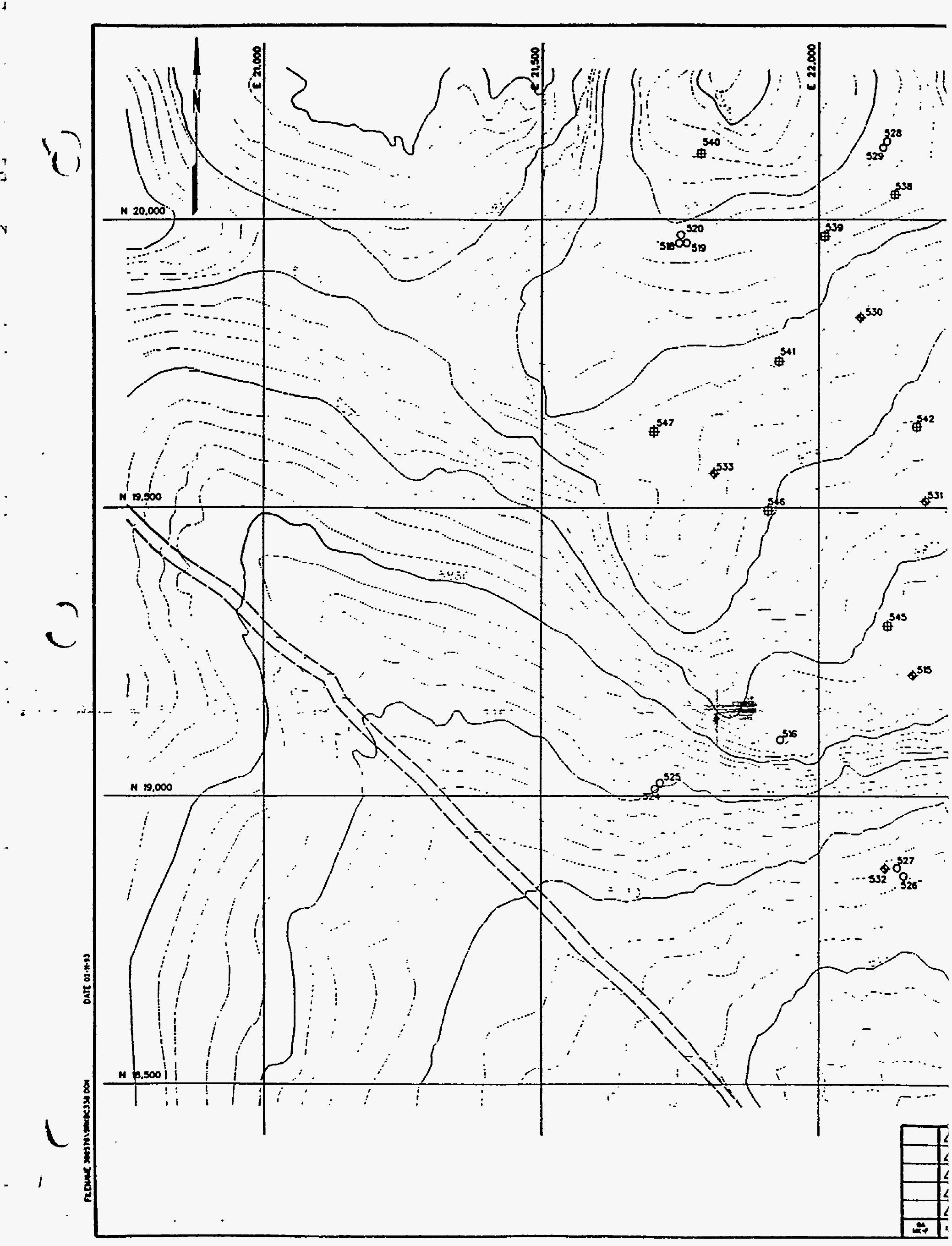


@MOMORRISON KNUDSEN CORPORATION

Project UMTRA - SPK
Feature TEMPORARY FALILITIES
Item MATERIAL QQNTTTIES

Sheet 74 Contract No.3885-76 File No. Designed.AKL Date 4.27 .93 Checked ISR Date $4 / 30 / 93$ $\frac{R B V \cdot 1}{D B W / 25 / 94 \quad B V}$

cur 2

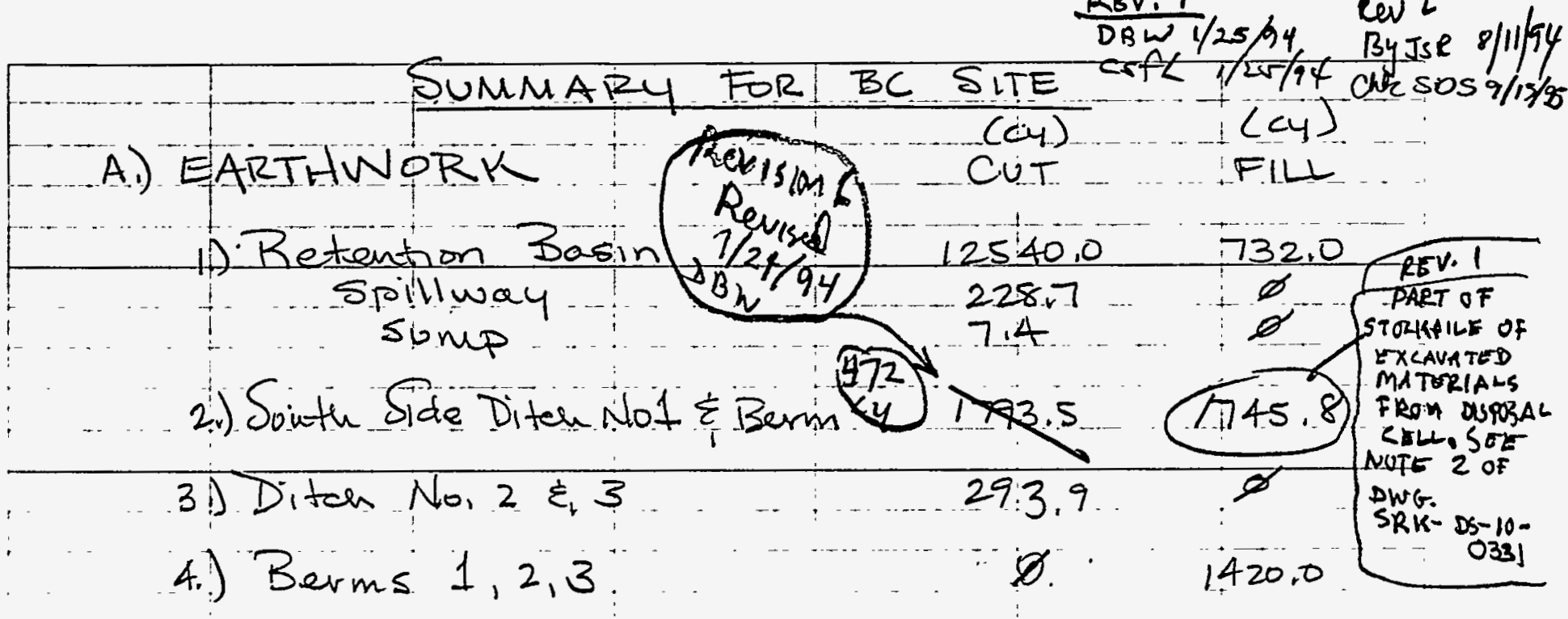

5) Access ramp, deconpad, basin 510.8 501.0

6.) Highwon 141 line of Site 6444.9 improveument

B.) OTHER QUANTITIES

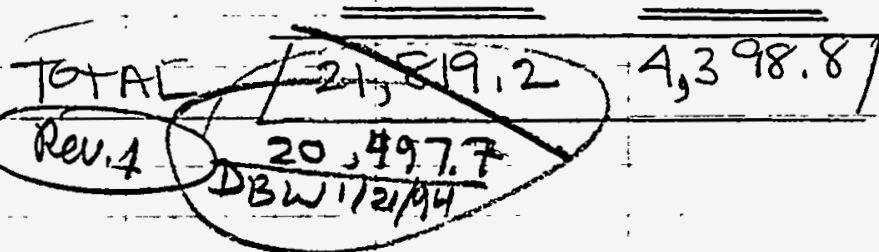

1.) $N$

$$
\begin{aligned}
& \text { MEMBraNe LiNER- BASIN - } 55,834.5 \text { s.f. } \\
& \text { MEMBRANE LINER - RECIRL, } 3,372.05 \text {. } \\
& 59.206 .5 \text { S.f. }
\end{aligned}
$$

2.) CONCRETE - DEUON PAD - $152.9 \mathrm{CY}$

3.) AGGRELATE - ACCESS ... - 386.944

$$
\text { FACILITIES - } 386,9 \angle 4
$$

4.) New Culverts - 190 L.F.

4.) New Culverts - FENCING - SILTFENCE-2HSOLF L.F.J

Deer Fence-2000' EHAIN LINK - 480555 L.F.

6.) T.OTAL WELLLENGTH - 1914 FT

7.) ROADWORK

LENGTHA
$3550 F T$
5 EXAVATION
AGGREGATE $2629.6 \mathrm{C4}$
26.44

ENG 375/91 
ACOMORRISON KNUDSEN CORPORATION

Project UMTRA - SRK

Feature TEMPORARY FACILITIES

lem MATERIal quantities
Sheet 75

Designed. Afr Date $2 . \overline{4.93}$ Checked AFS Date 2/16/93

1.) Retention Basin

a). Excavation Quantities

i) Basin (see shuts to to ot)

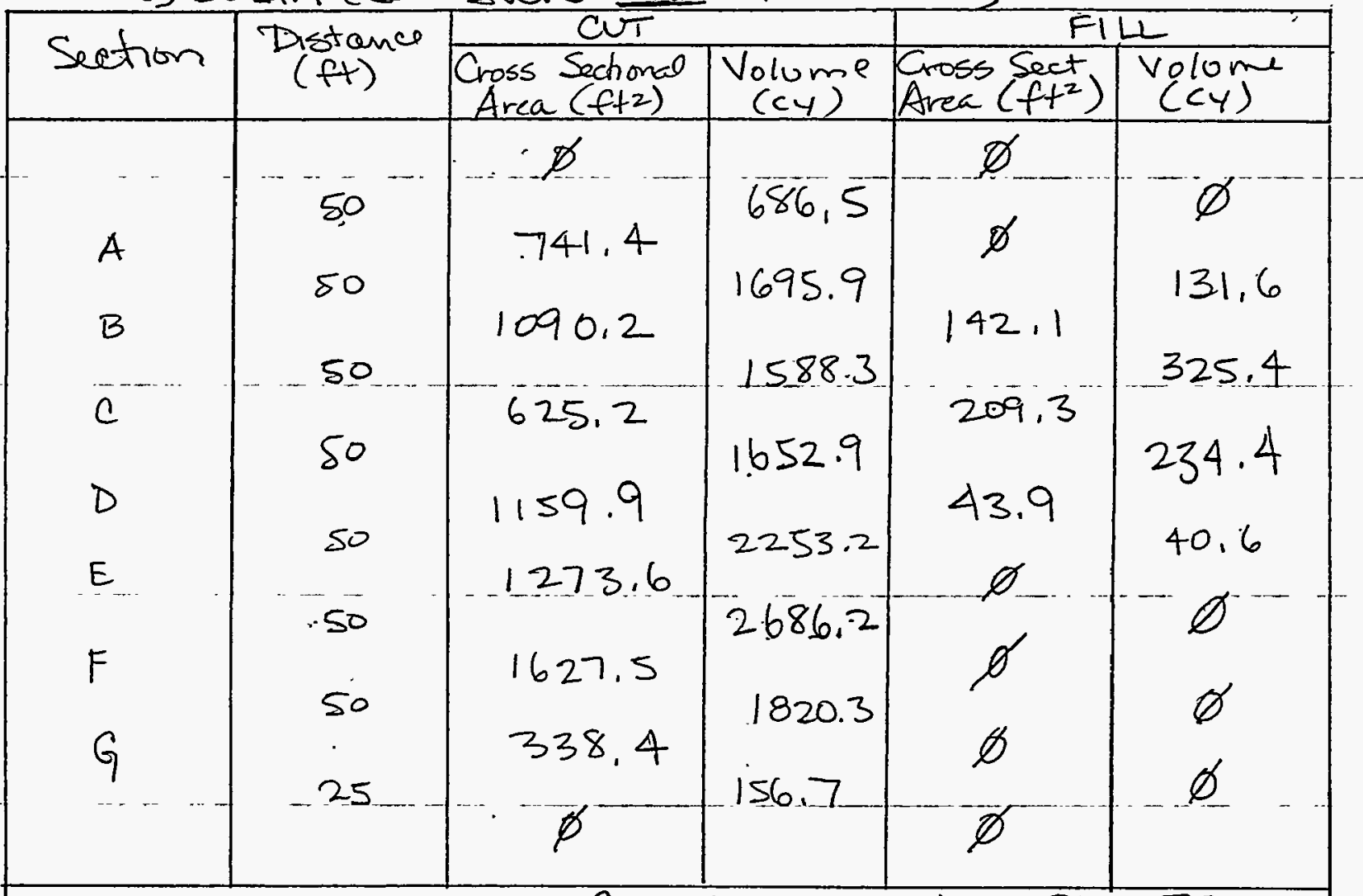

Total Cut $=12,540.0 \mathrm{cy}$ Total Ell $=732.0 \mathrm{~cm}$

ii) Spillway (See sits $80 \%$ ' 81 )

\begin{tabular}{|l|c|c|c|c|c|}
$H$ & so & 87.8 & 125.6 & $\varnothing$ & $\varnothing$ \\
\hline & 50 & -47.8 & 75.9 & $\varnothing$ & $\varnothing$ \\
\hline & 43 & 34.2 & 27.2 & $\varnothing$ & $\varnothing$ \\
\end{tabular}

Total Cut $=228,7.04$ Total Fill $=\varnothing$

iii) Approximate excavation for sump

Cut Volume $=(5815.0-\$ 813.0)(10)(10)\left(\frac{1}{27}\right)=7.4 \mathrm{c4}$

$74242 / 89$ 
(40. MORRISON KNUDSEN CORPORATION

Project UMTRA - SRK

Feature TEMPORARY FACILLTIES

Item MATERIAL QUANTTIEES
Contract No. 3885-76 File No.

Designed

Checked AFS

$$
\text { Date } \frac{4.21 \cdot 93}{4 / 23 / 93}
$$

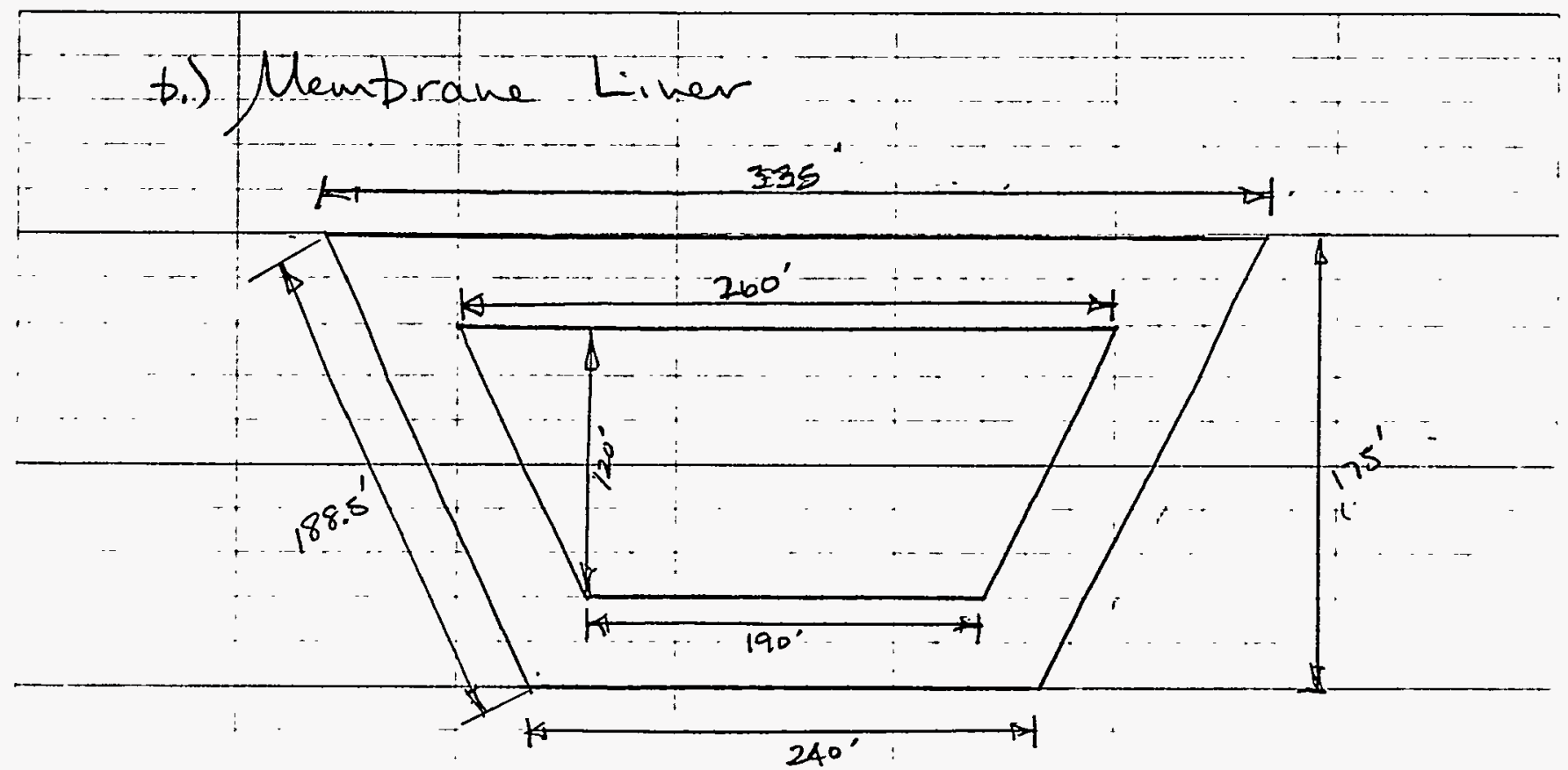

COLL DIMENSIONSS ARE SCALES OFF DRAWING)

i) Bottom Area (neglect bottom slope)

$$
\text { Area }=120(190+35)=27.000 \mathrm{sf} \text {. }
$$

Subtract Sone Area $=(22)(22)=484 \mathrm{sf}$

Area $=26,5165,5$

ii) Sump surface area

Area $=(10 \times 10)+(6.3)(4)(16)$

$$
=503.2 \text { sf. }
$$

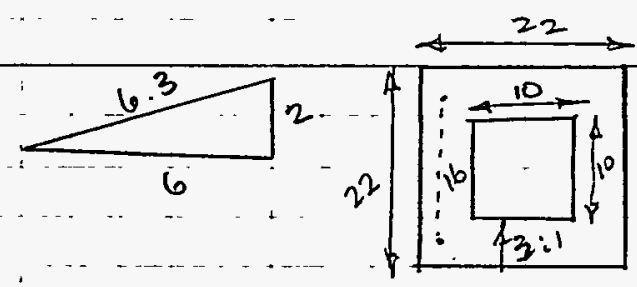

iii) Sideslopes

Outside Area $=(125)(240+47.55)=50,312.55, f,-$

Sideslope Area $-(1,05.4)(5,0,312,5,-27000)=24,571,45 f$.

$$
\begin{aligned}
& \text { Five faitor Archer Trend Area }=i(4,3)(240+370+188.5+188.5) \\
& \text { Total Area }=(26,516+503,2+24,571,4+4243,9)=55,834.558 .
\end{aligned}
$$

ENG 375/91 
Project UMTRA - SRK Feature TEMPORARY FACILITIES Item MATERIAL QUANTITIES
Contract No.3885-76 File No. Designed Checked
Date 2.4 .93

Date $2 / 16 / 93$

\section{SECTIONS FOR RET, BASIN}

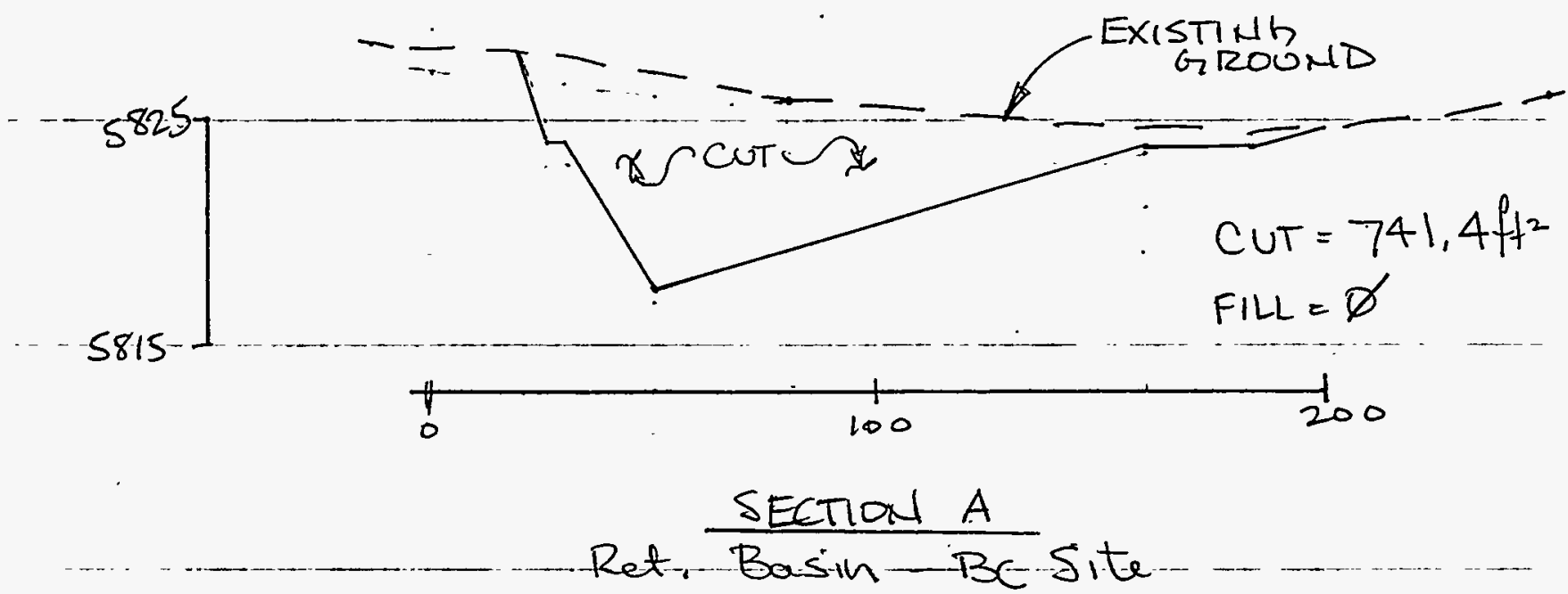

58.14

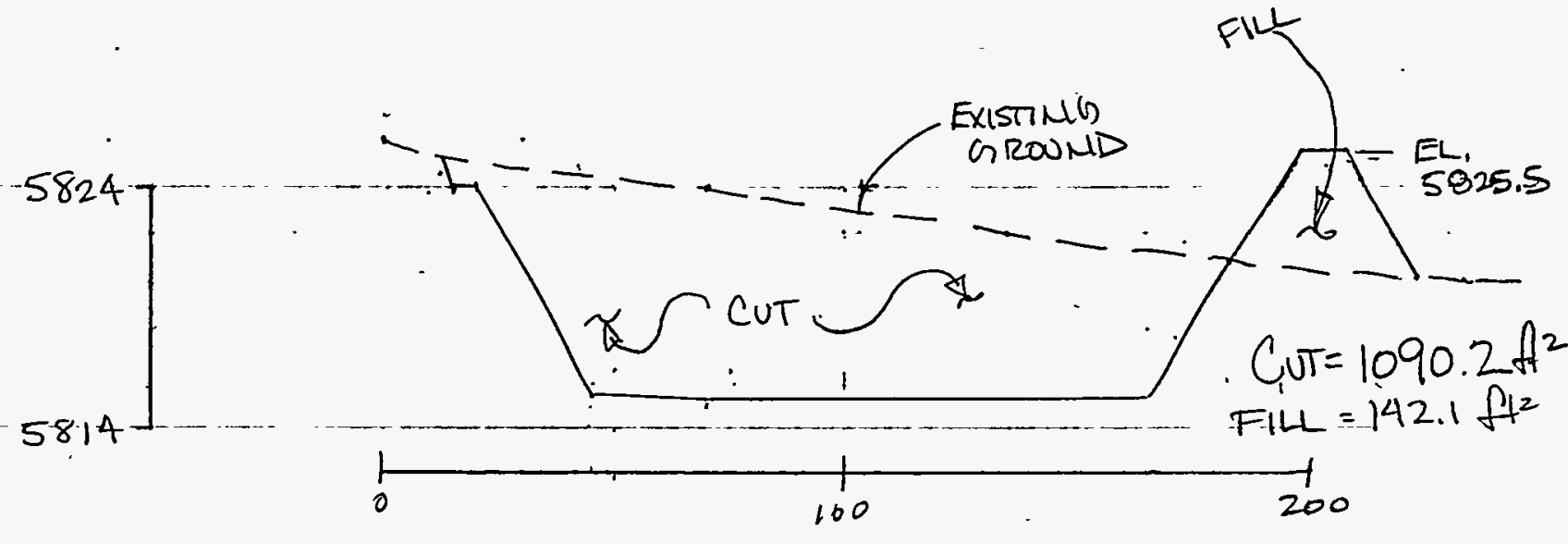

Ret BECTIONI B

$\left[\begin{array}{r}5524 \\ \ldots 514\end{array}\right]$

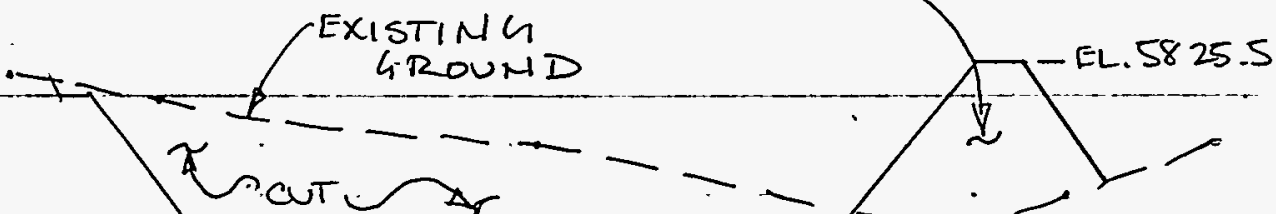


(10 9MORRISON KNUDSEN CORPORATION

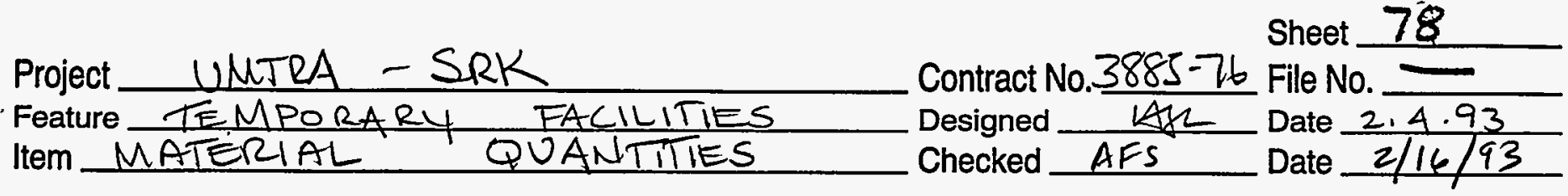

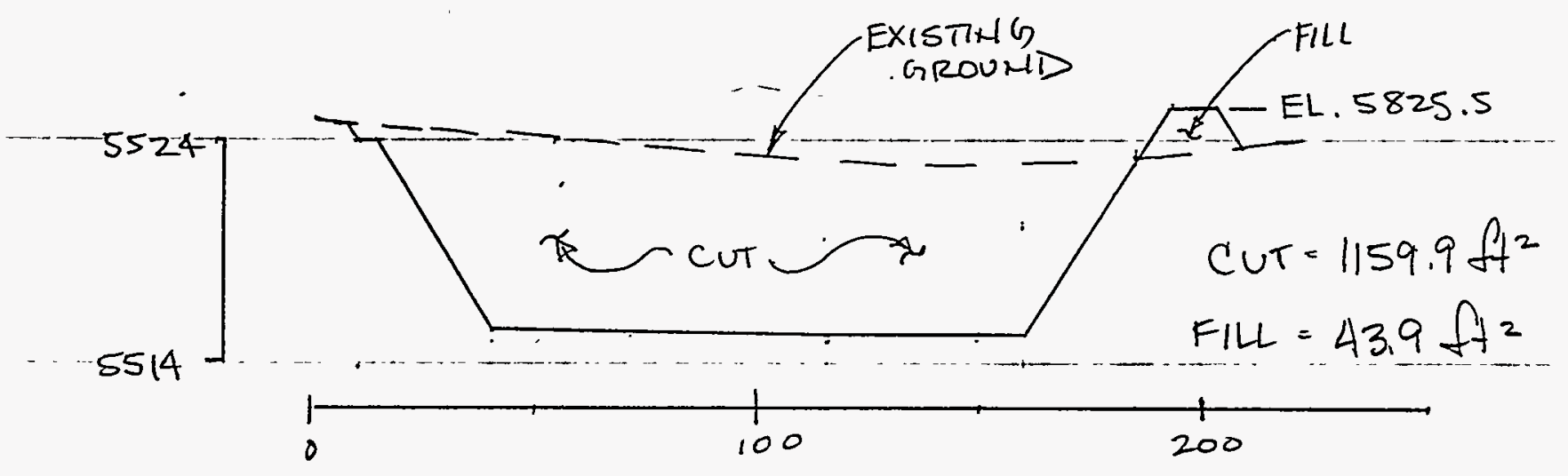

SECTION D

Ret. Basin - BC Site
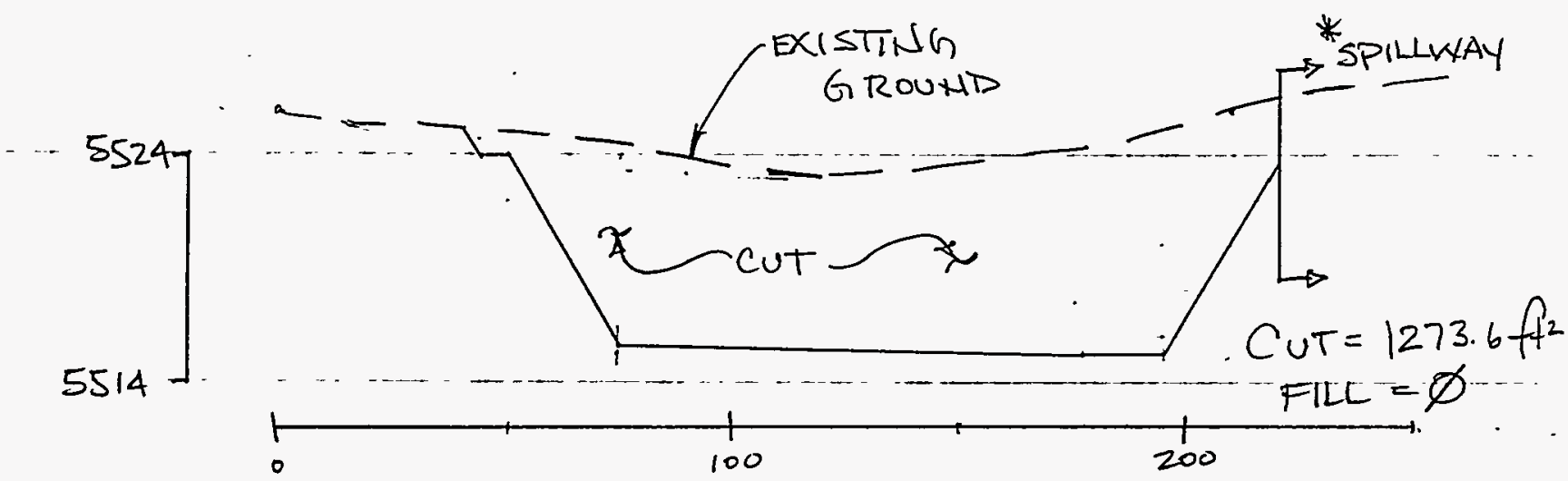

SECTIONS E

Ret. Basin-BC-Siti

* spillway calculated separately

ENG $375 / \overline{9}$

$14242 / 89$ 
(4. MORRISON KNUDSEN CORPORATION
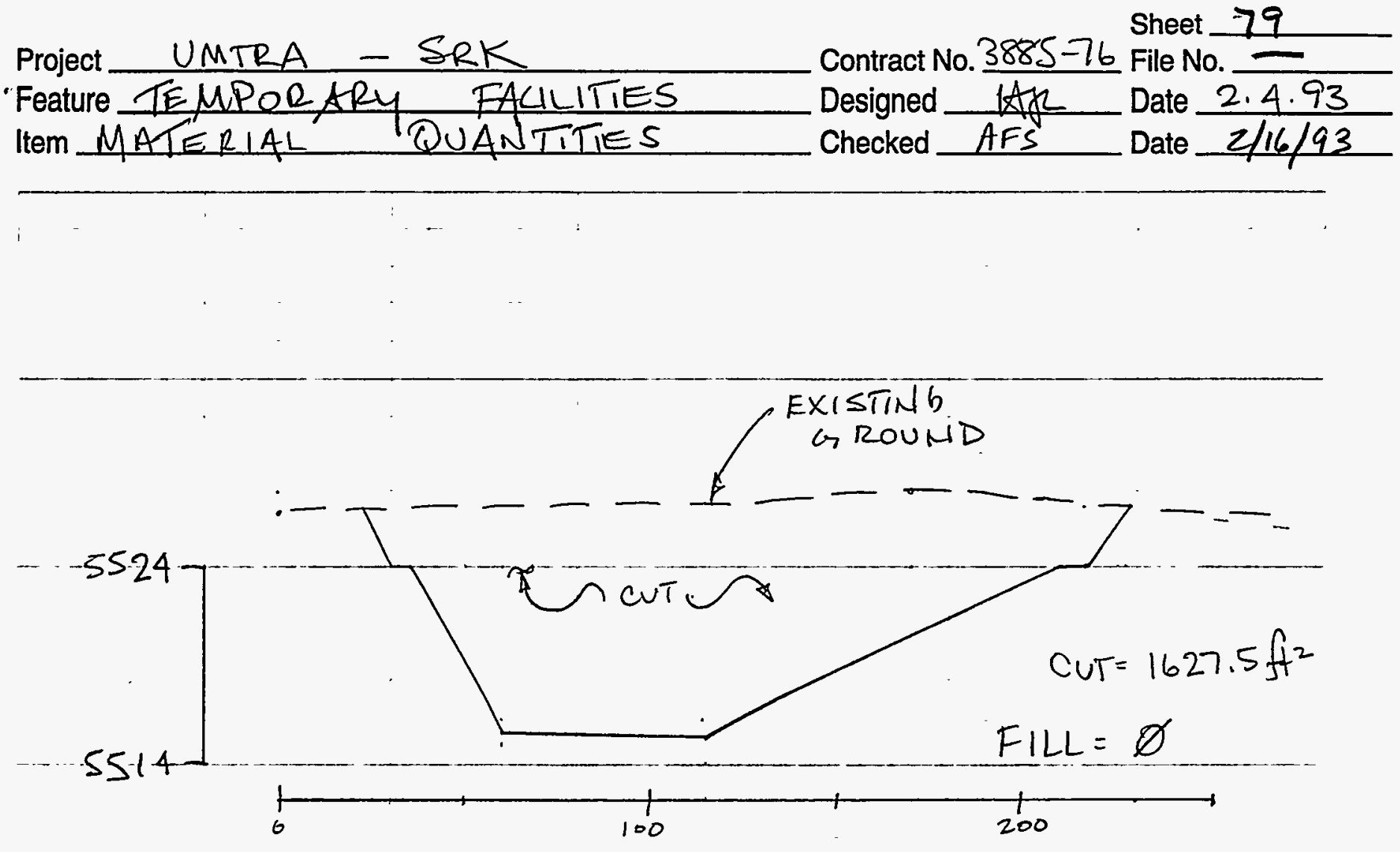

SECTION F

Ret. Basin - BC Site
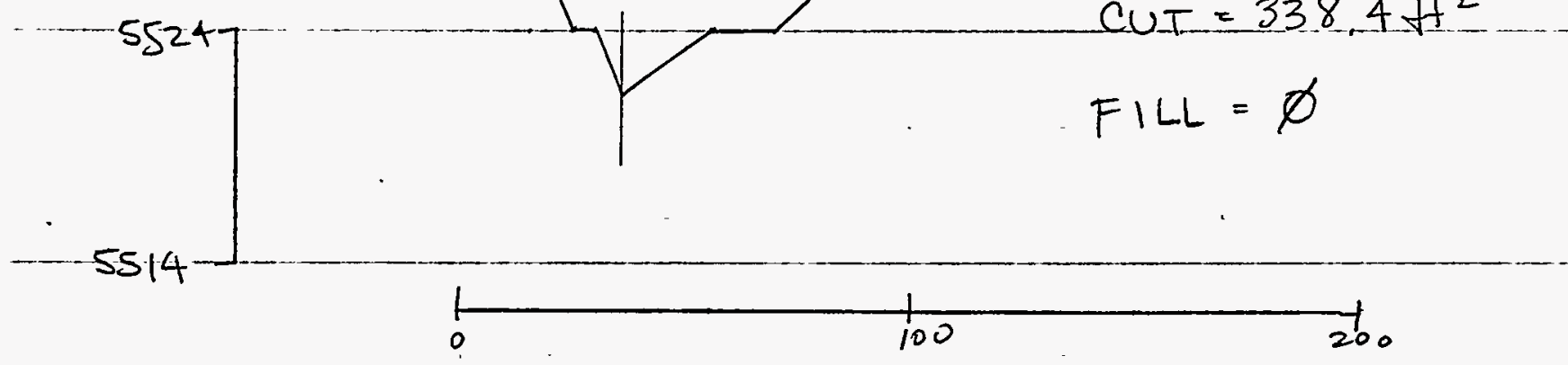

$\frac{\text { SECTION G }}{\text { Ret. Basin-BESte }}$

EMO 375/89

$14242 / 89$ 
@ MORRISON KNUDSEN CORPORATION

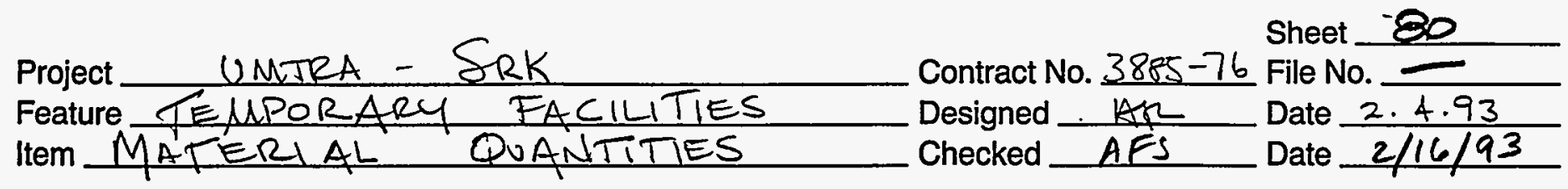
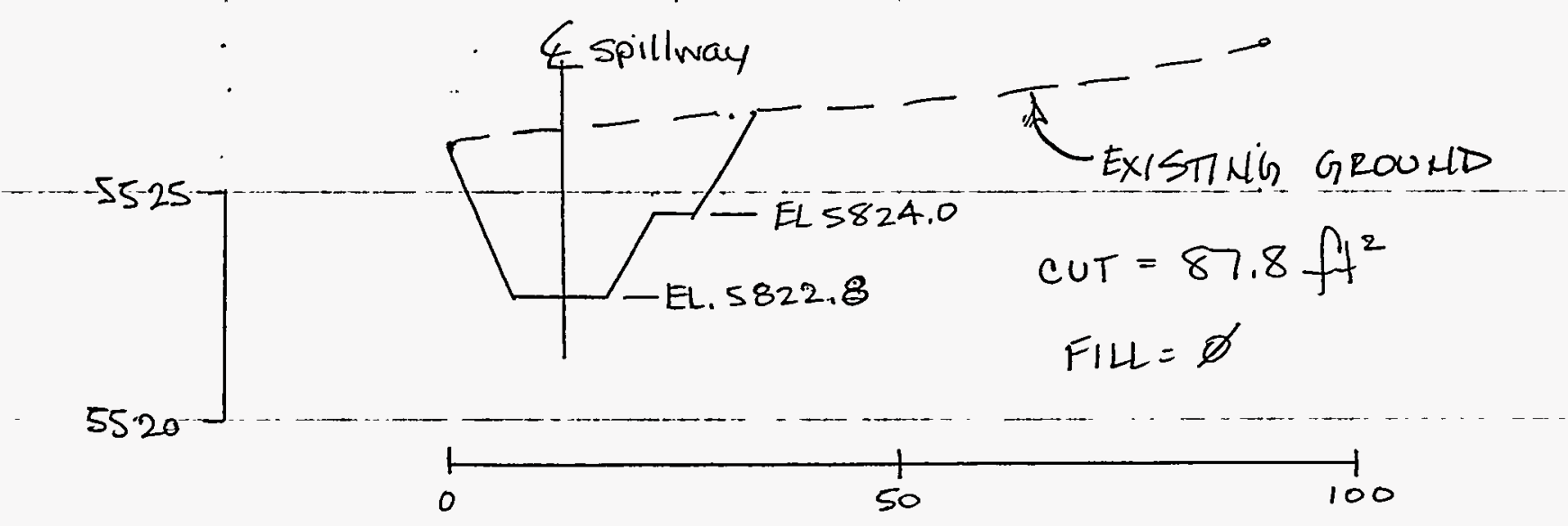

$$
\frac{\text { SECTION. H }}{\text { Spill way }=\text { BC Site }}
$$

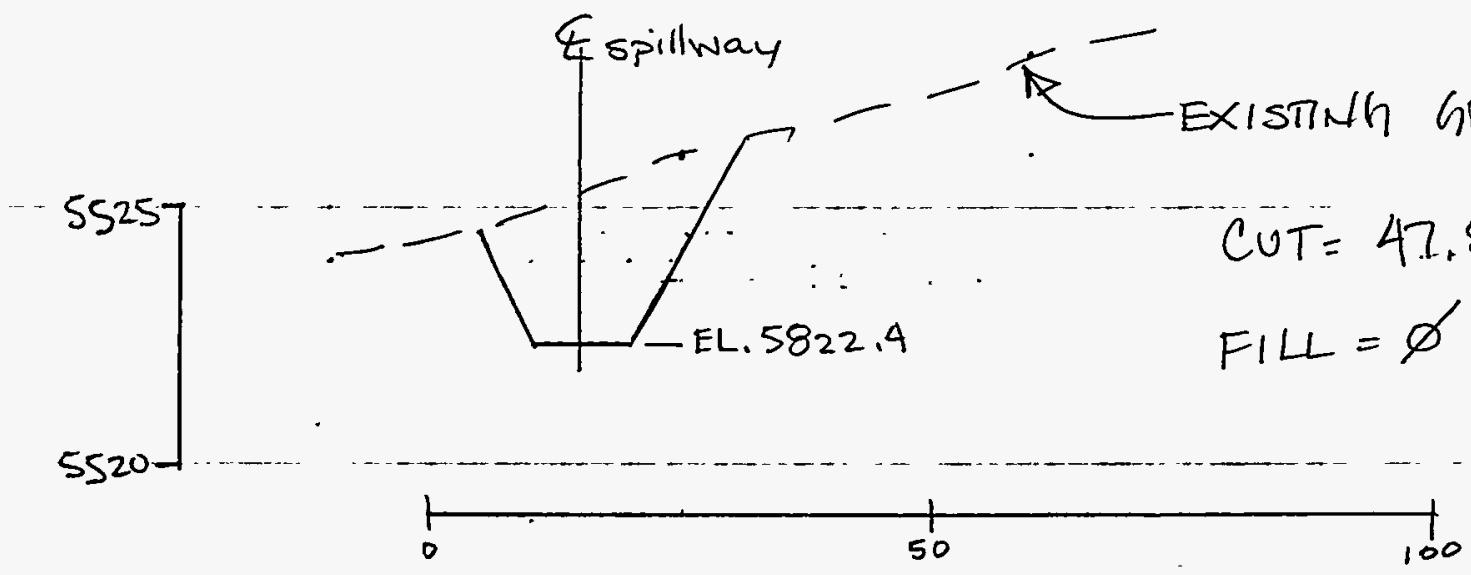

SECTION

Spillway- $B C$ Site

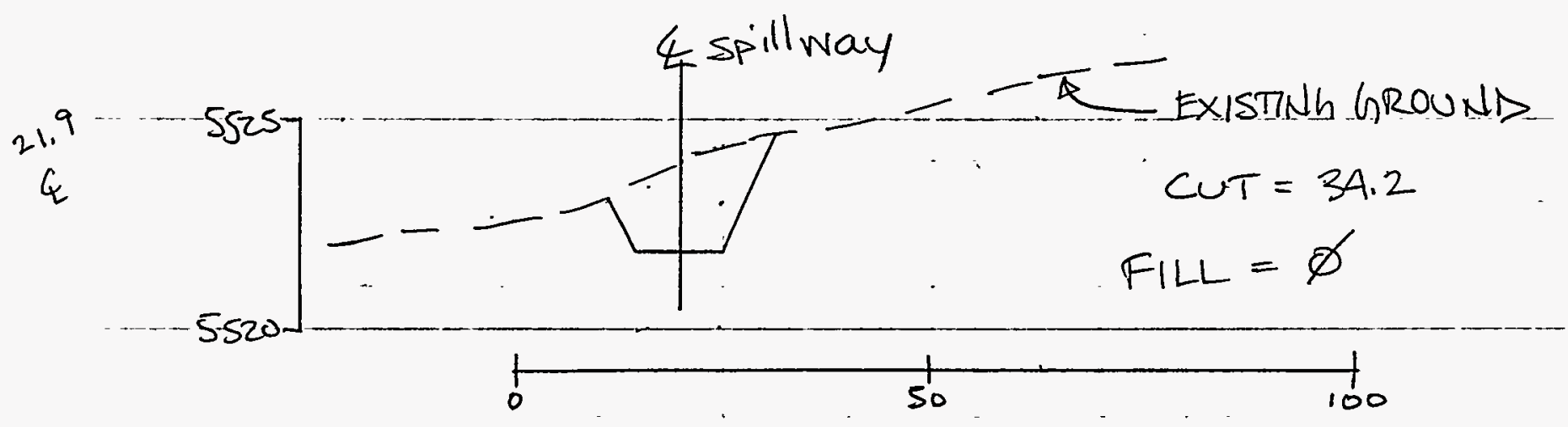

$\frac{\text { SECTION I }}{\text { Spillway - BC Site }}$

ENC $375 / 89$ 


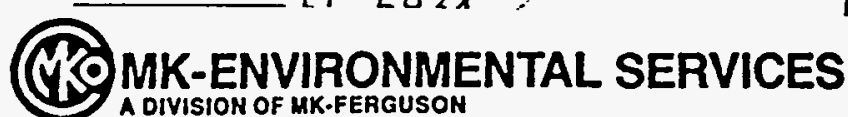

$=$ Project UMTRA - SRK

Feature TEMPORARY FacILITIES

'tem material quantities
Contract No.3885.76

Designed Hy Checked
Sheet 81 .

File No.

Date 4.21 .93

Date $4 / 23 / 93$
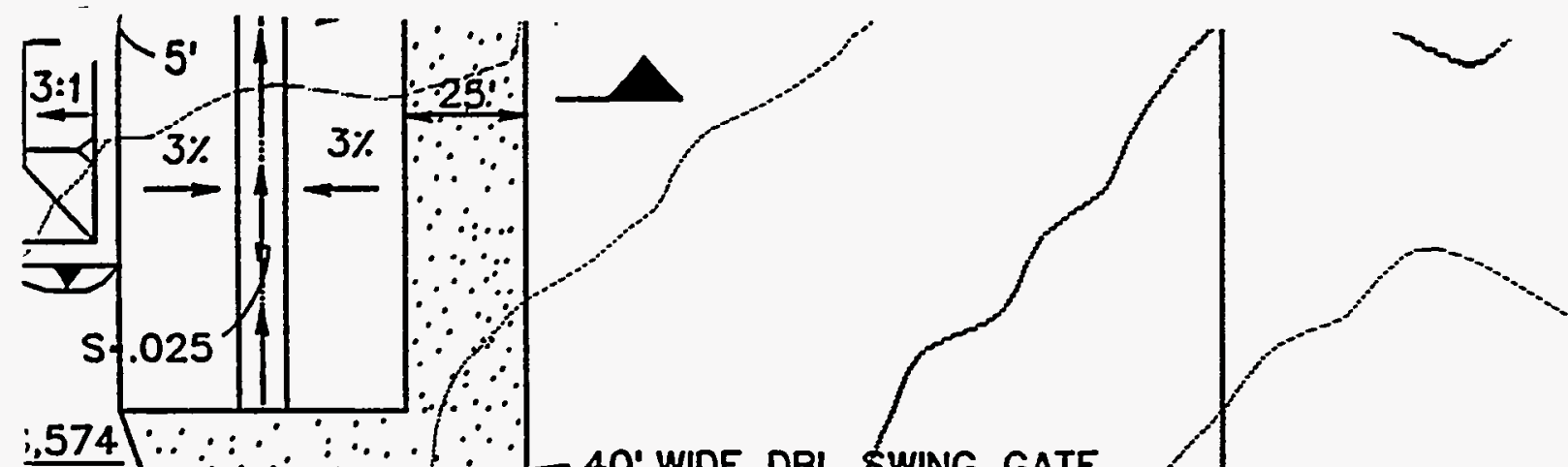

1,788 40' WIDE DBL SWING GATE N 18,574

E 21,838 ACCESS CONTROL

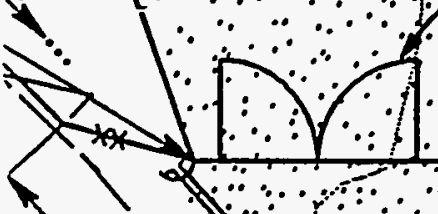

150.

0.)

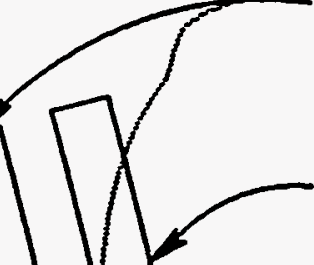

TRALER (60!':x'2')

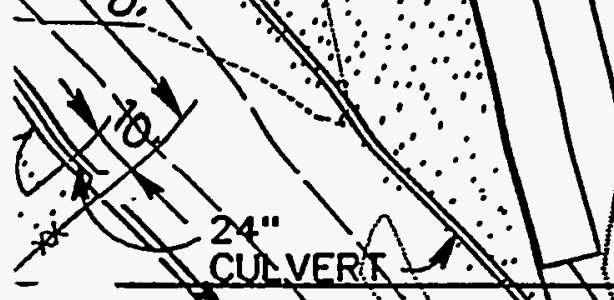

CHANGE OUT TRALLR $\left(60^{\prime} \times 60^{2}\right)$

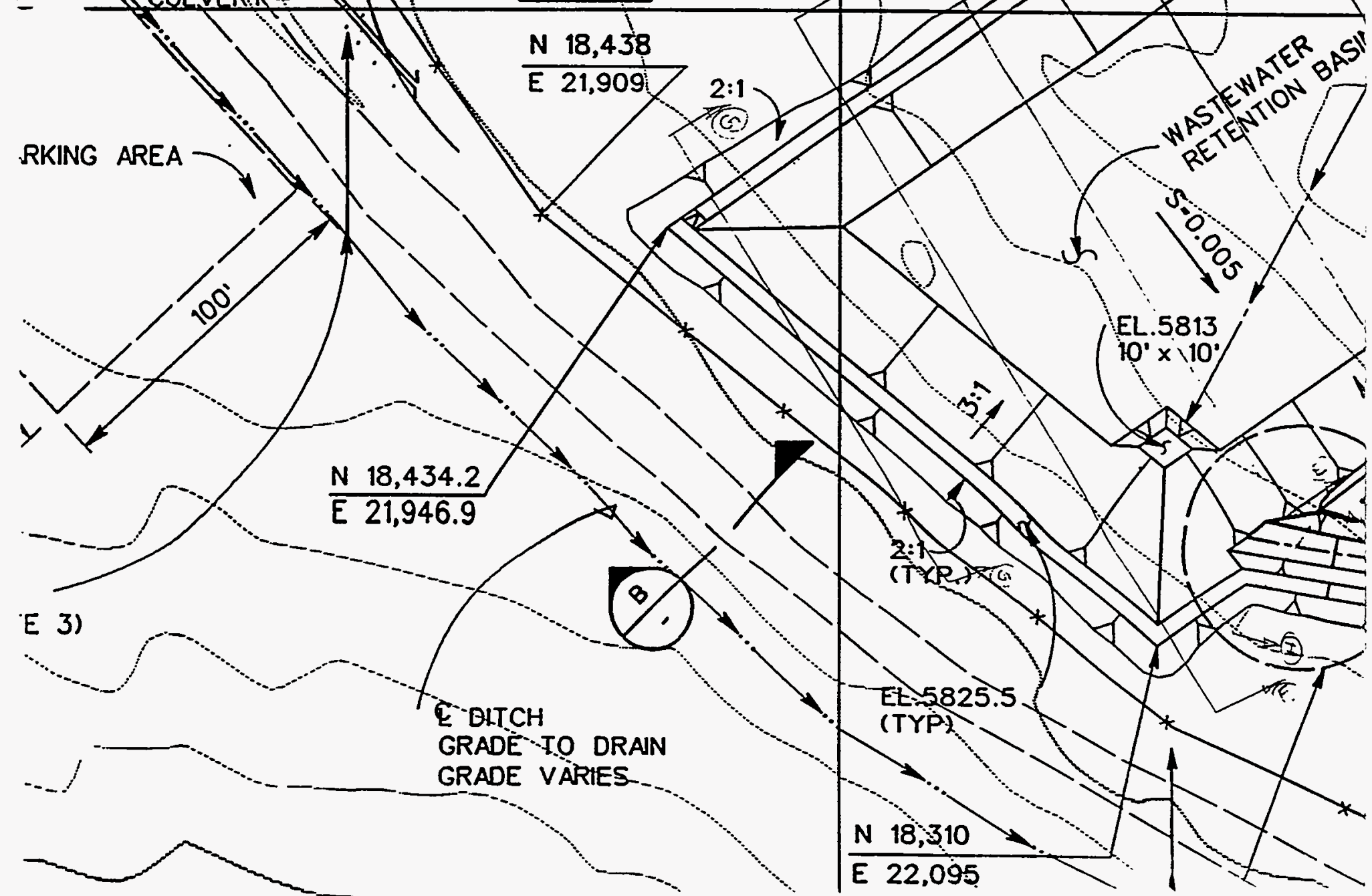


@17. MORRISON KNUDSEN CORPORATION

Project_UMTRA - SRK

Feature TEMPORARY FACILITIES
Sheet 82

Contract No. $3885-76$ File No.

Designed

Date 3.22 .93

Date $4-5-93$

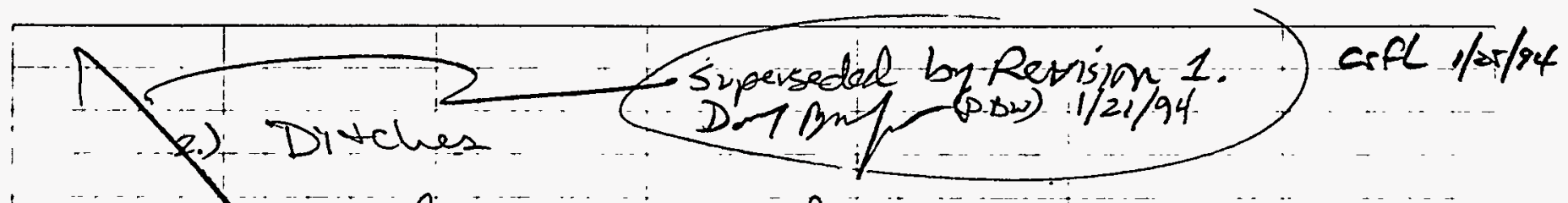

a) Cut and Fill for dizen and berm on south side of pile

(See sheets 83-86).

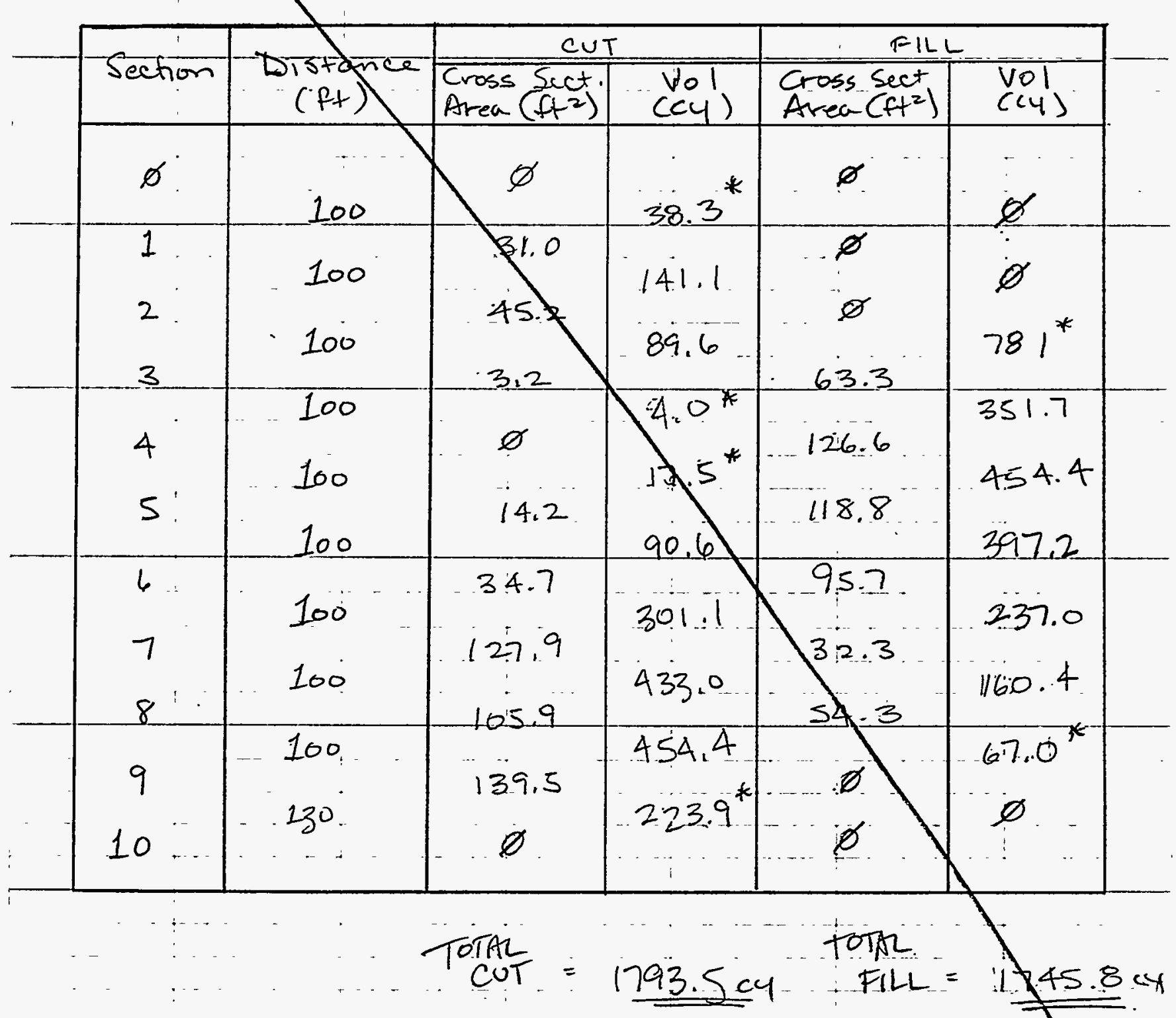

* Pyramid volume formula used

ENG $375 / 91$ 
상 MORRISON KNUDSEN CORPORATION

Sheet $82 A$

Project UMTRA S $S R K$

Contract No. $3885-76$

File No

Feature TEMP FACILITIES

Designed DBW

Date $1 / 13 / 94$.

- Item MATERIAC QUANIITTES

Checked EfL

Date $1 / 2 r / 9 x$

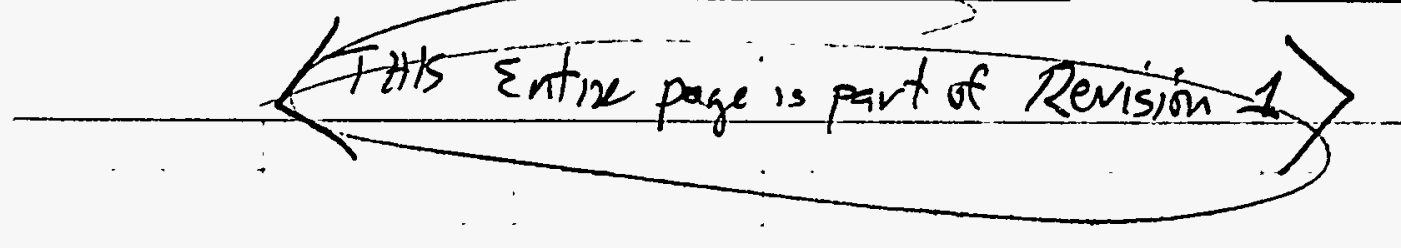

2. DITCHES - SOLTH SIDE

- CUT volumes -

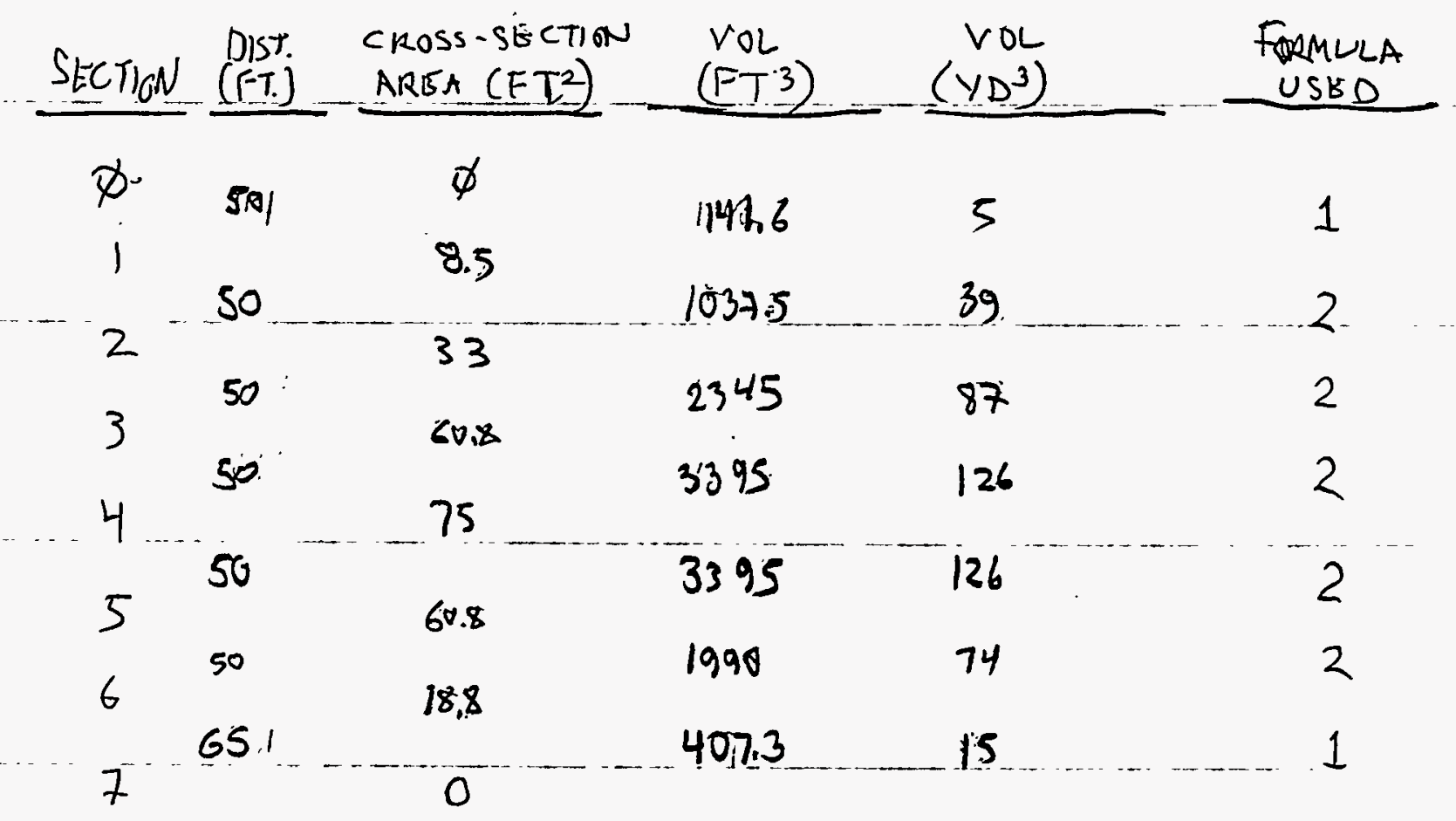

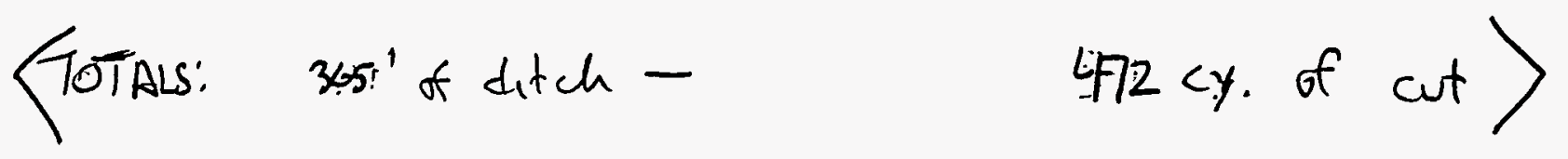

FORMULA's

1. Pyramid: $($ Area $/ 3) \times$ Distance $=V G$

2. Prism: $\left(\right.$ Area $\left._{1}+A_{\text {Area }}\right) / 2 \times$ Distance $=N / 6 l$

ENG 375/91 
@M MORISON KNUDSEN CORPORATION

Project UMTRA-SRK

Feature TEMP. FACLLITIES

Item MATERIAC QUDATITES
Contract No. $3885-76$

Designed $D \mathrm{BW}$

Checked Cafe

Sheet $83 \mathrm{~A}$

File No.

Date $1 / 12 / 94$

Date $1 / 2 r / 94$

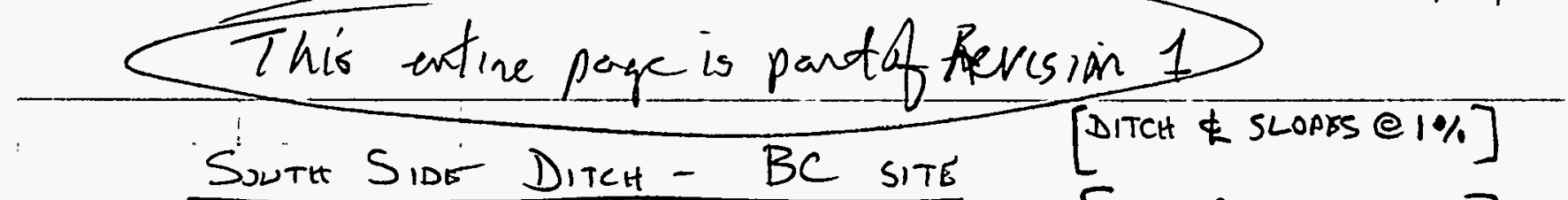

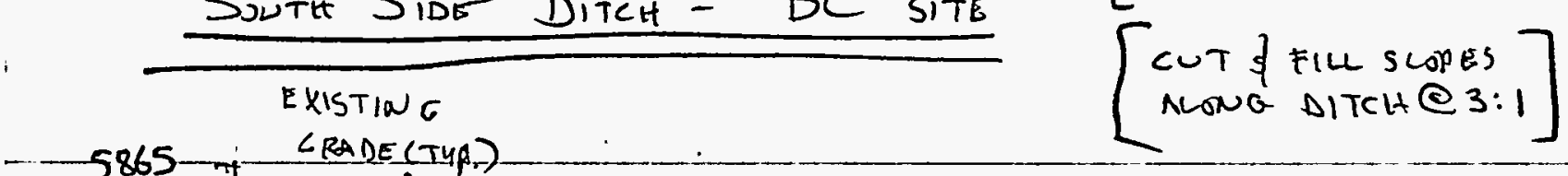

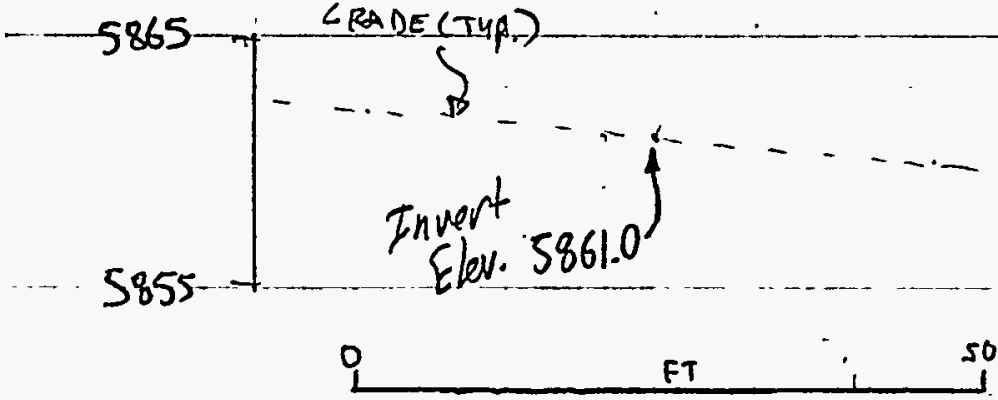

$\left[\begin{array}{cc}\operatorname{SECT} T \operatorname{Ln} & - \\ \text { nO } & \operatorname{CUT}\end{array}\right]$

$\left.\begin{array}{l}5865^{\prime} \\ 5055^{\prime}\end{array}\right]$
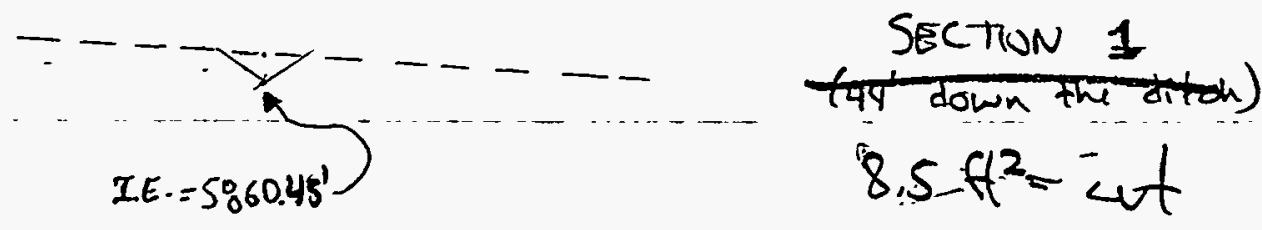

6

FT

50

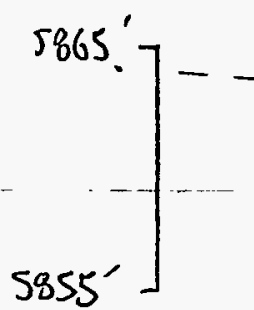

SECTION 2

$33+t^{2}=$ cut

I. $E=5859.9$ )

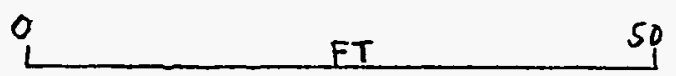

ENG 375/91 


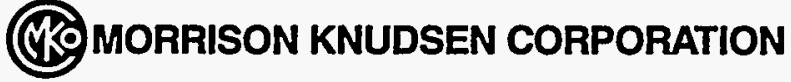

Project UMTRA - SRK

Feature TEMPORARY FACILITIES

Item MATERIAL QUANTITIES
Sheet 83

Contract No. $3885-76$ File No. -

Designed 182 Date $\frac{3.22 .93}{3.52}$

Checked

By w

Date $4 \cdot 5 \cdot 93$
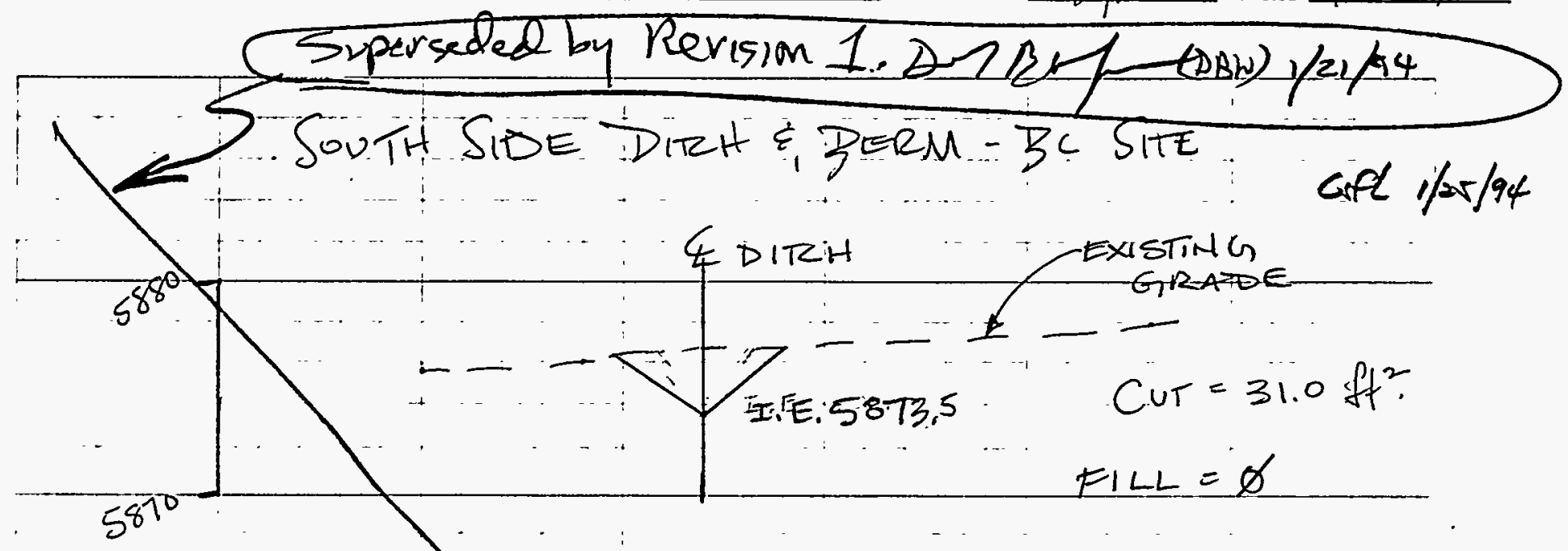

$s$
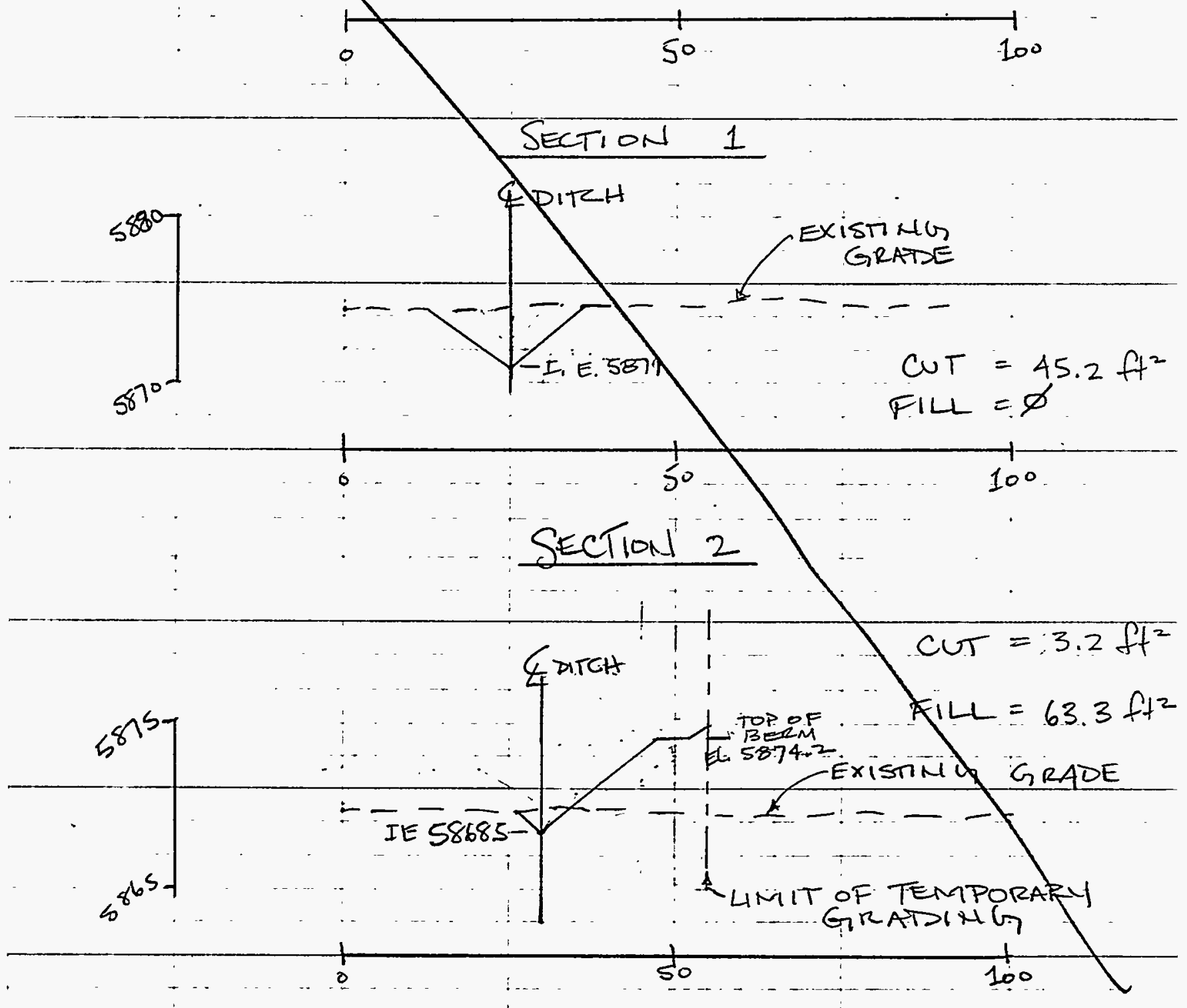

ENG 375/91 
(4. )MORRISON KNUDSEN CORPORATION

Project UM TRA - SRK

Feature TEMP FACILITIES Item
Contract No. 3885-76

Designed $D B W$

Checked CsfL
Sheet 244

File No.

Date $1 / 12 / 94$

Date $1 / 21 / 94$

This entire sheet is part of Revision 1
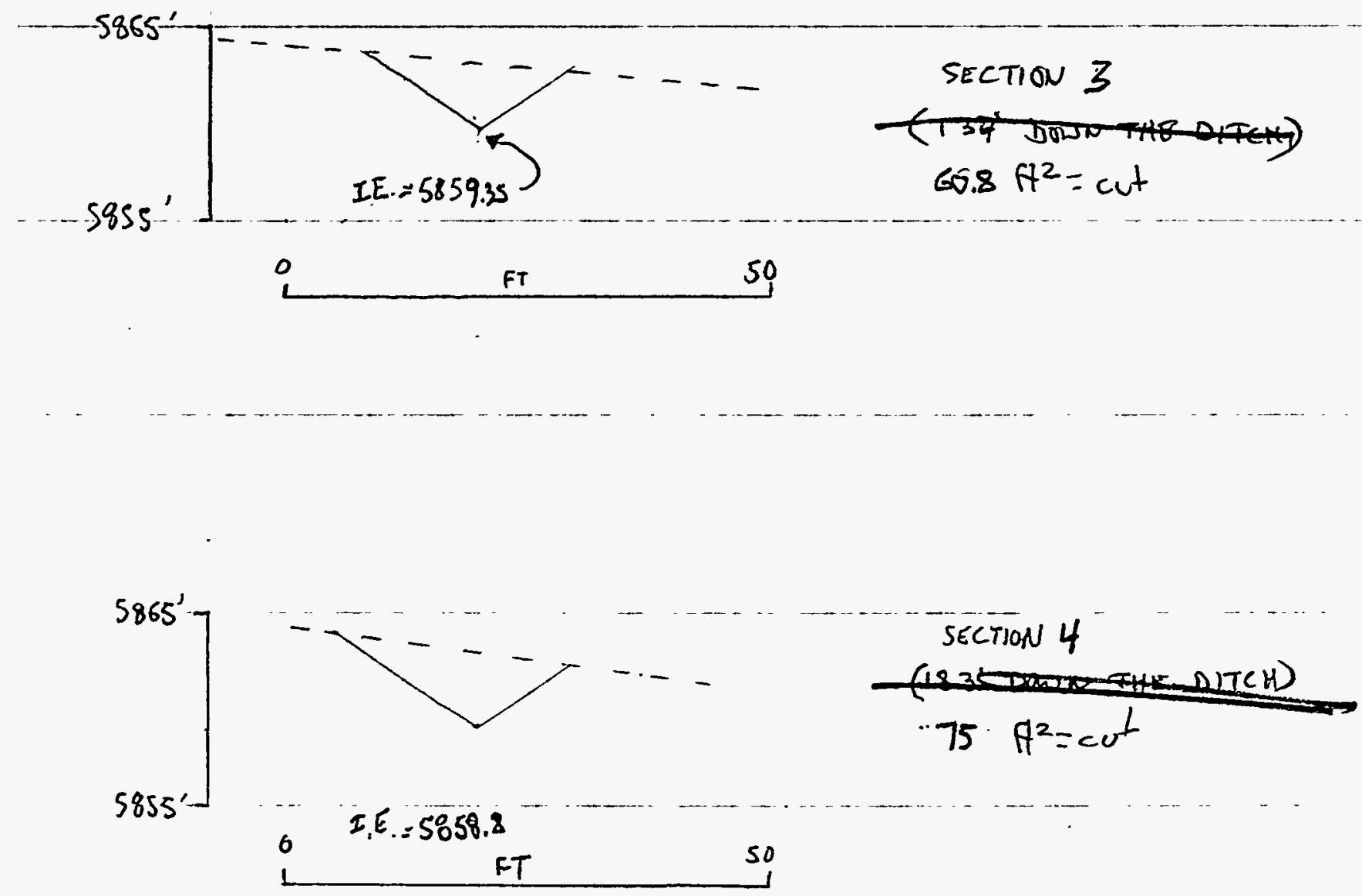

SECTION 4

(1) $3=0$ ITCH)

$75 \cdot R^{2}=c u t$

$\left.\begin{array}{c}-5865^{\prime} \\ -5855\end{array}\right]$

SECTION 5

(CT) DOWN THE DITS)

$60.8 \cdot H^{2}=$ cut

ENG 375/91 
Project UMTRA - SRK

Feature TEMAPORARY EACILITIES

Item MATERIAL QUANTITIES

Contract No.3885-76 File No -

Designed Ay

Checked BUW
Date 3.22 .93

Date 9.5 .93

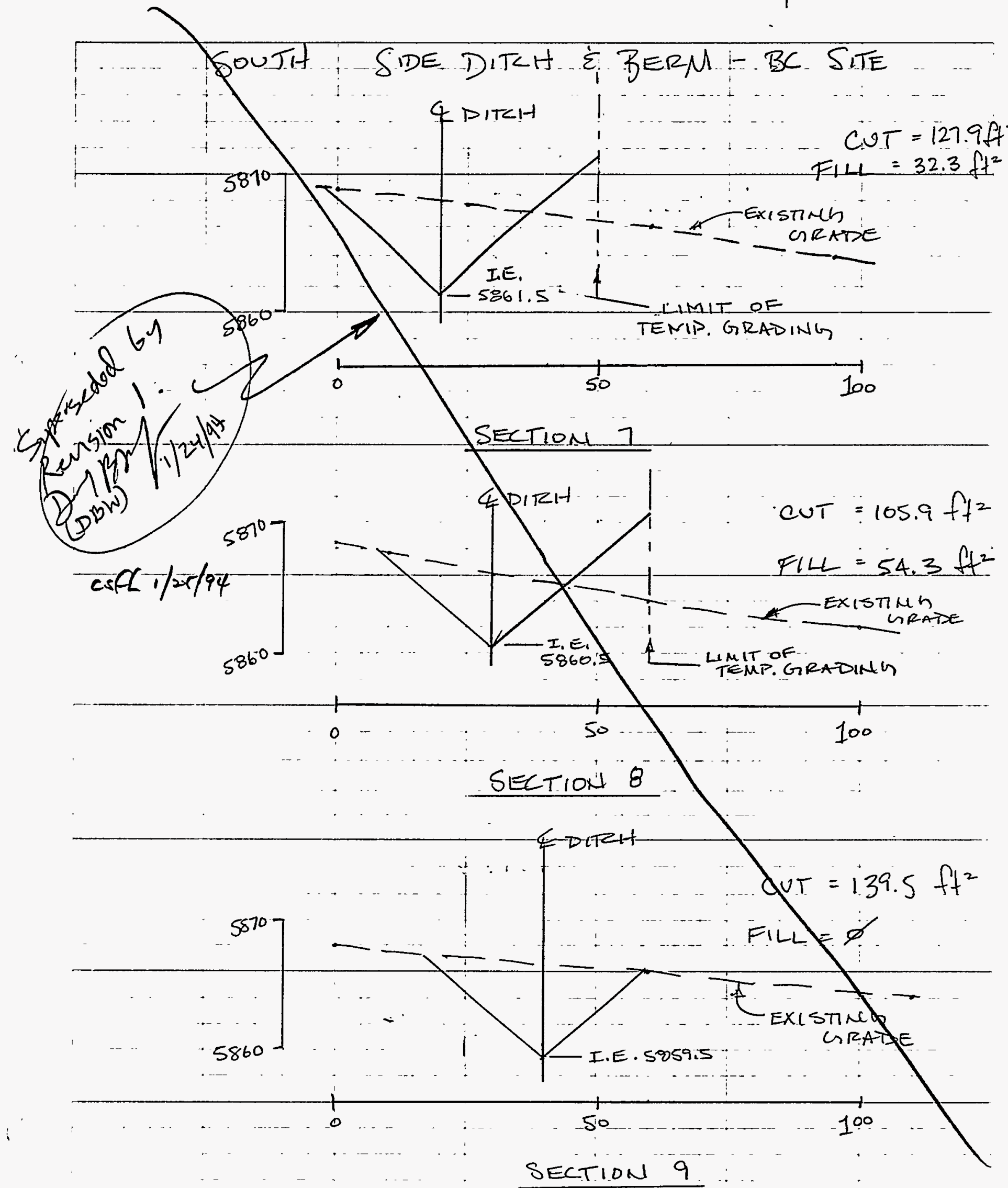


@16 .MORRISON KNUDSEN CORPORATION

Project UM TRA - SRK

Feature TEMP FACILITIES Item
MATERIAL
Contract No. $3885-76$

Designed DBW

Checked SFL
Sheet $85 \mathrm{~A}$

File No.

Date $1 / 12 / 9.4$

Date $1 / 20 / 94$

This entire shat is port of Revision 1
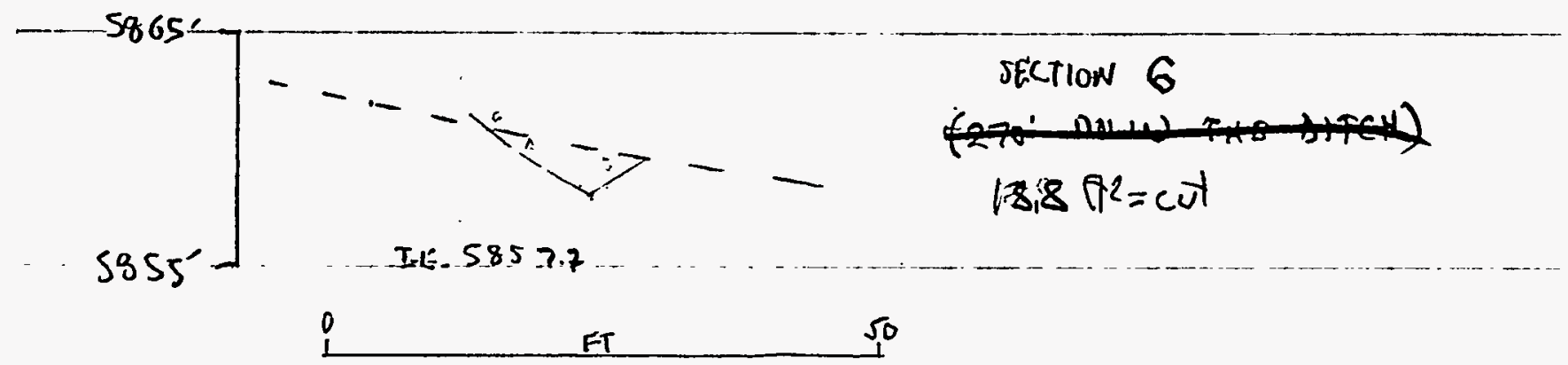

$\left.\begin{array}{l}5805 \\ 5850\end{array}\right]$

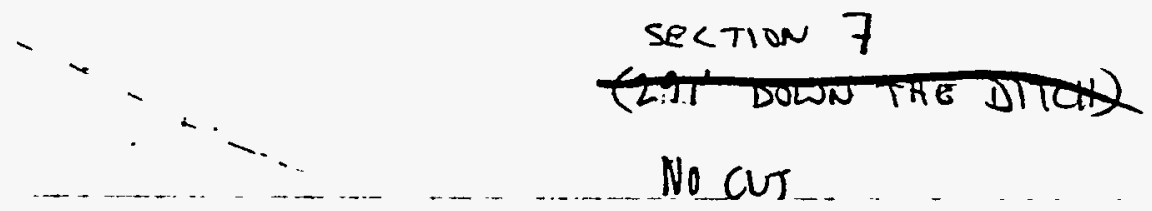

$I \cdot E .=585: 7.0^{\circ}$

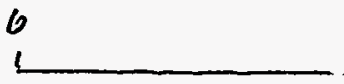

so

ENG 375/91 


\section{(1.) MK-ENUIRONMENTAL SERVICES}

(1) Shet 86

DEs Projeg UMTRA-SRK
CAVA Feature JTEMFORARY FACILITIES

Contract No. $3885-76$ File No.

W. llem MATERIAL Q JF: TIEIES Designed

Date 3.23 .93
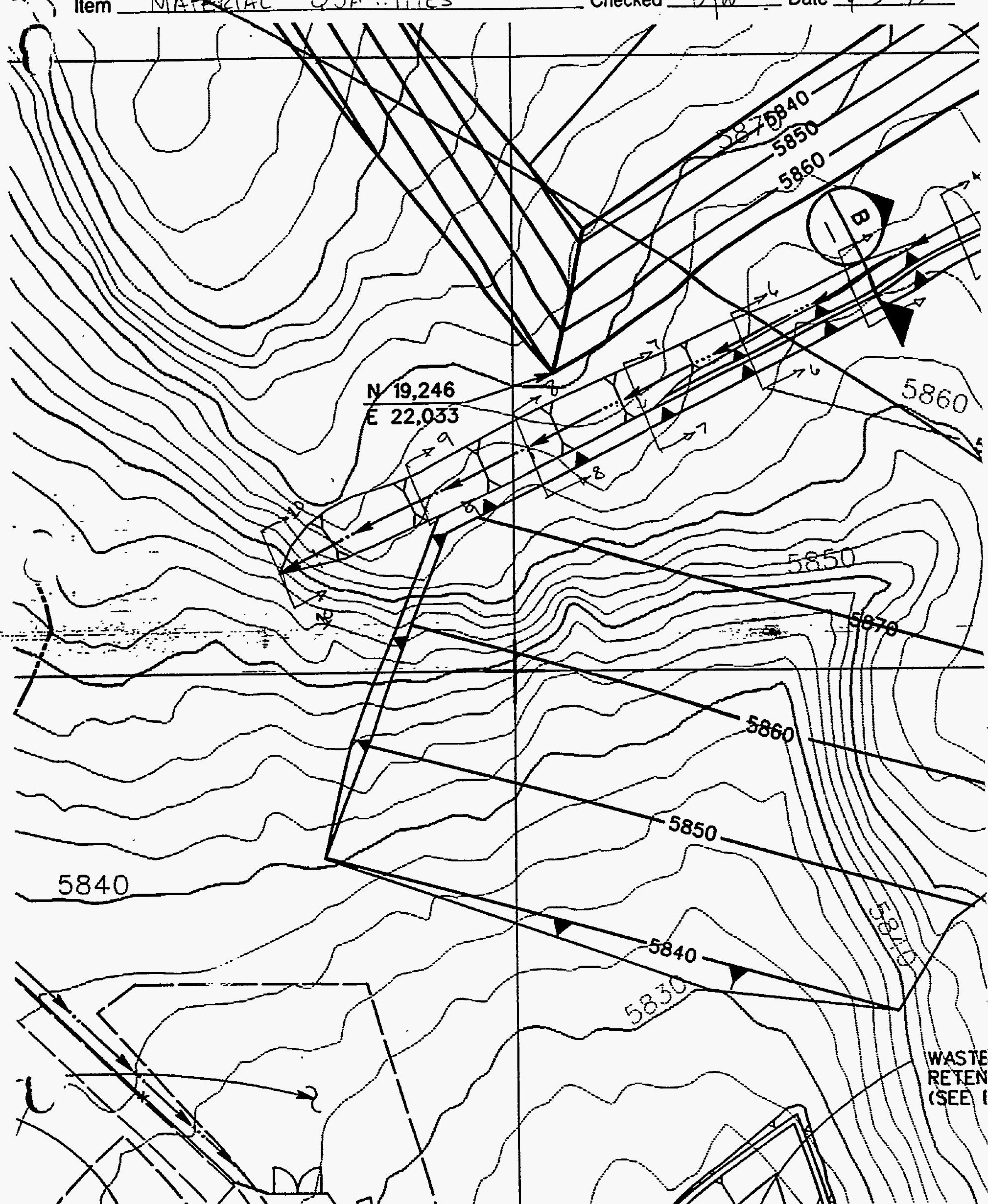


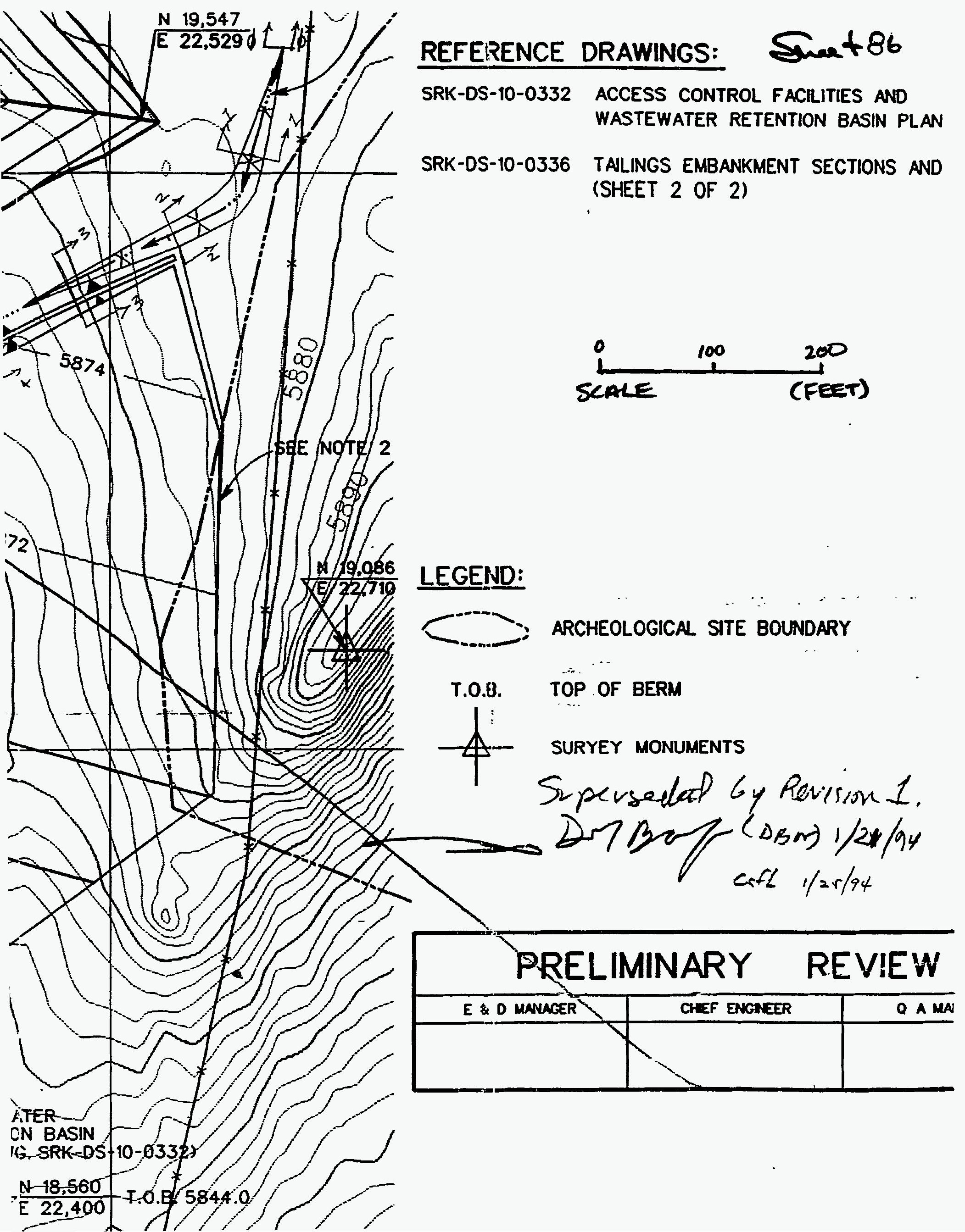




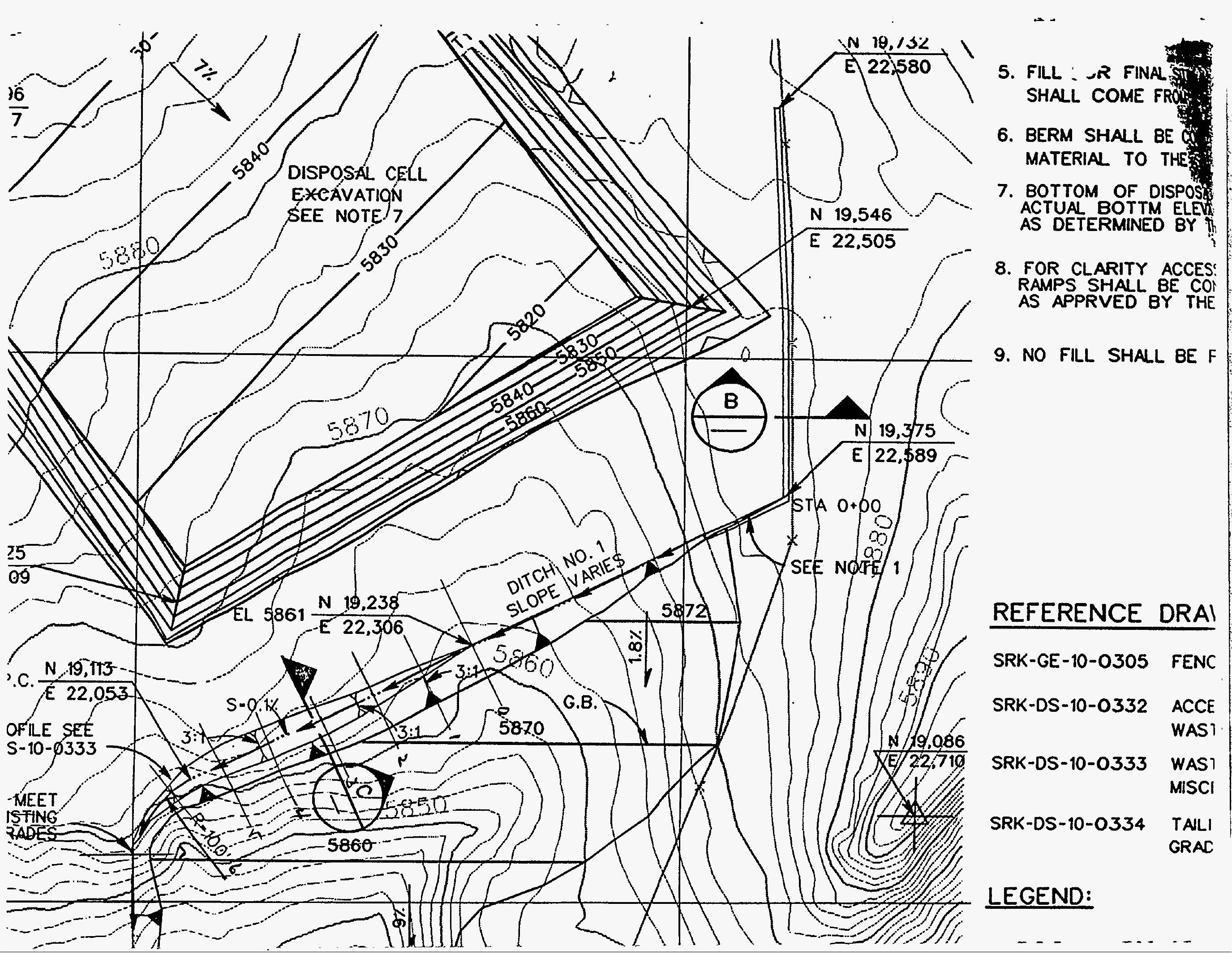




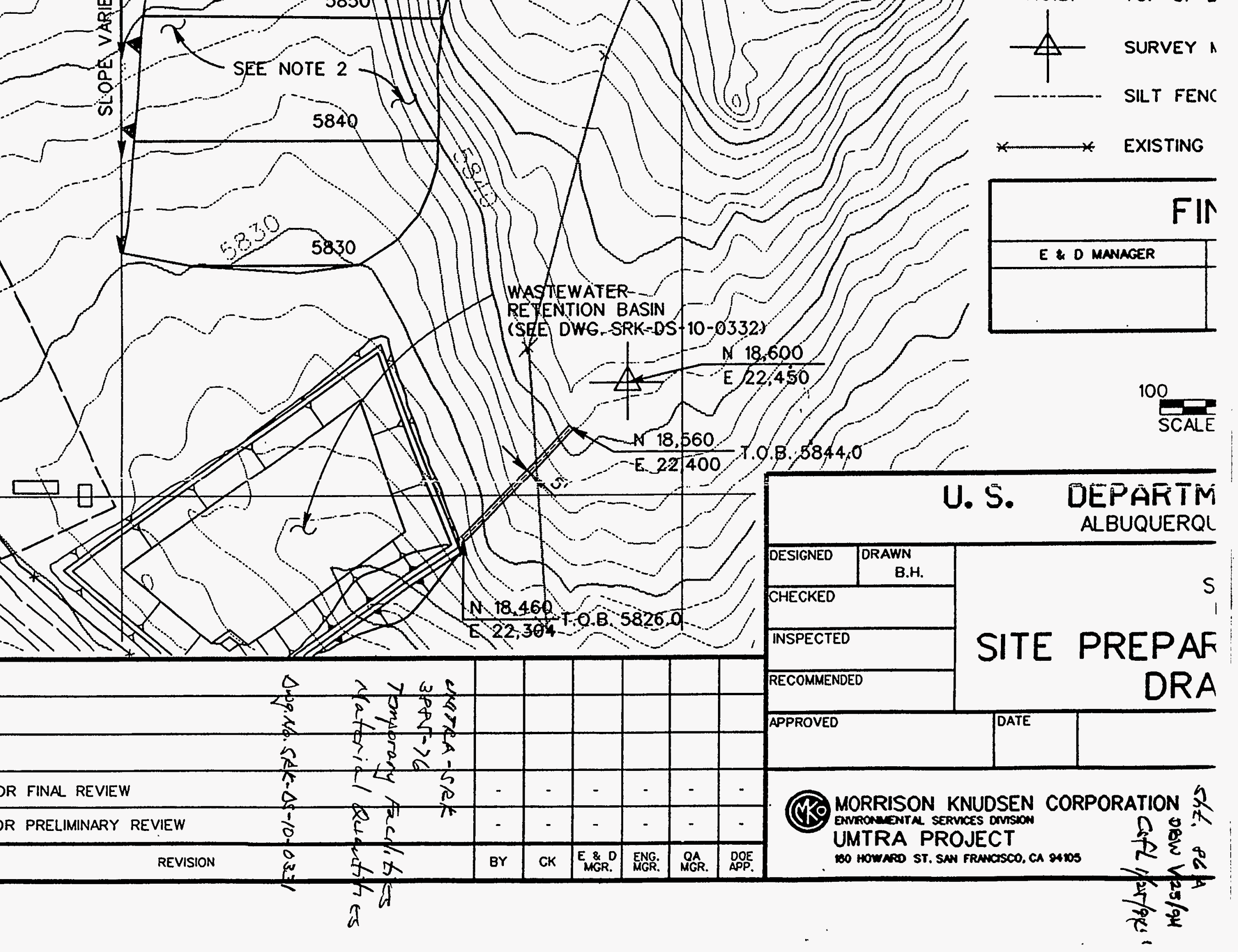


5810

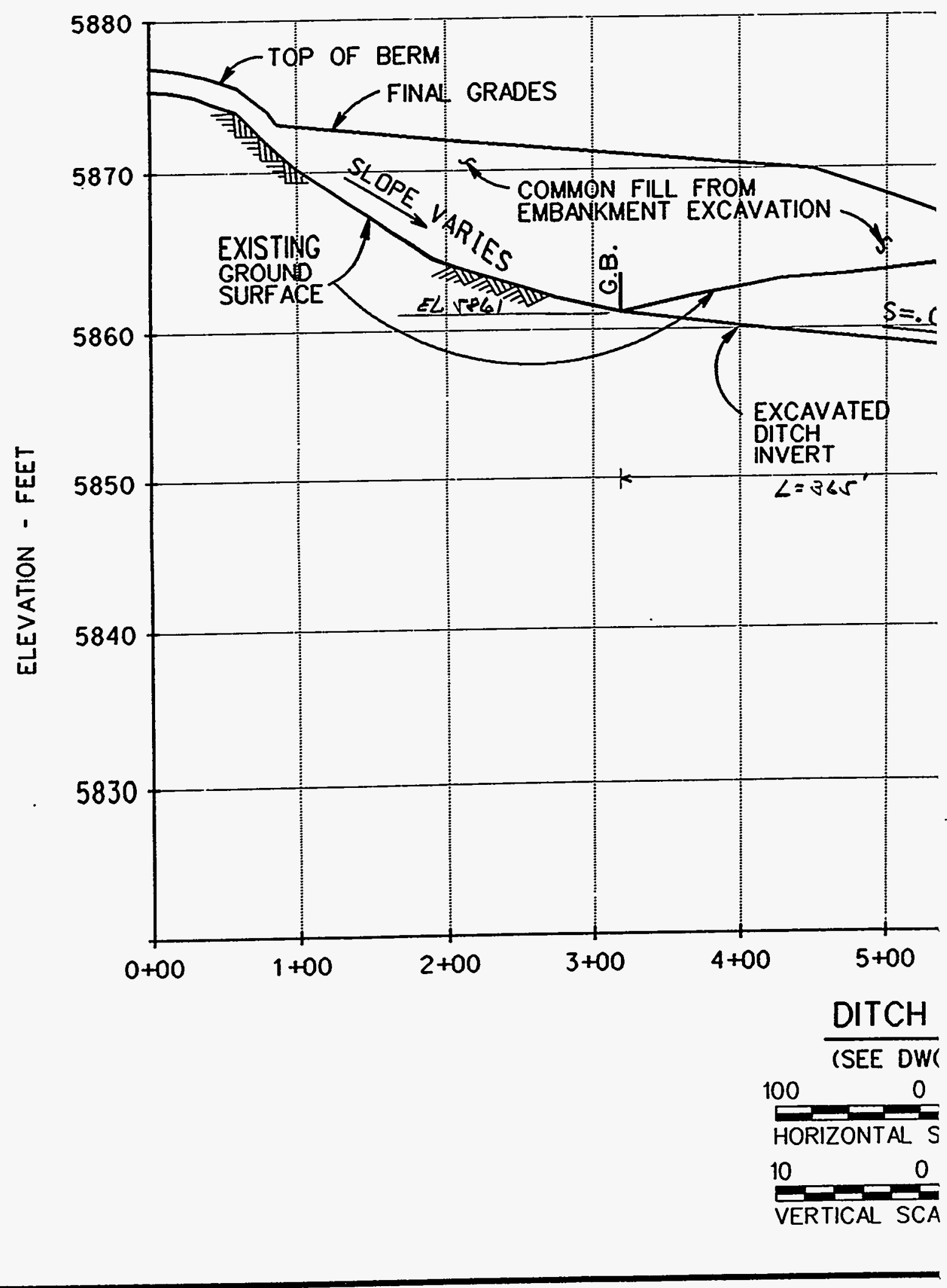




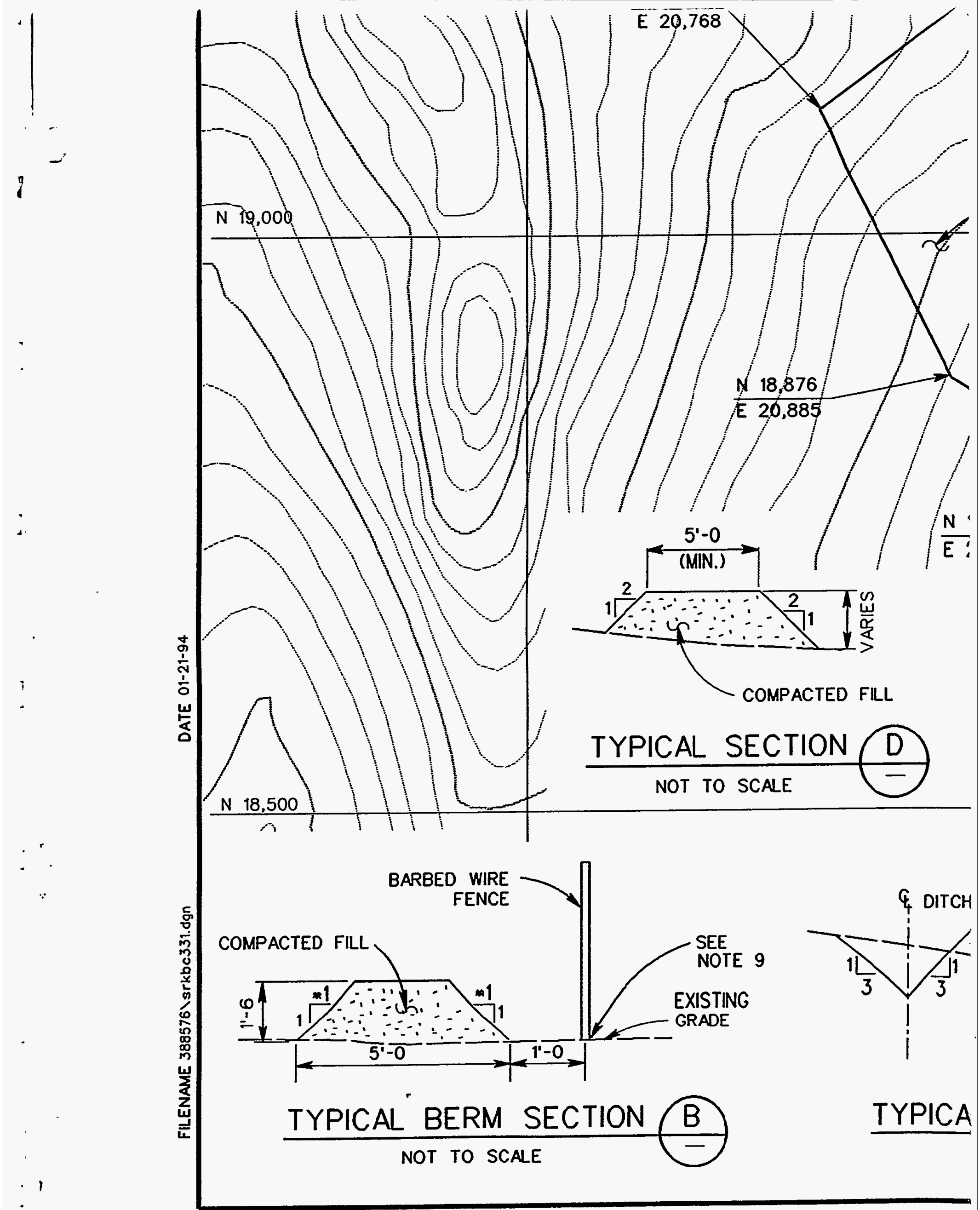




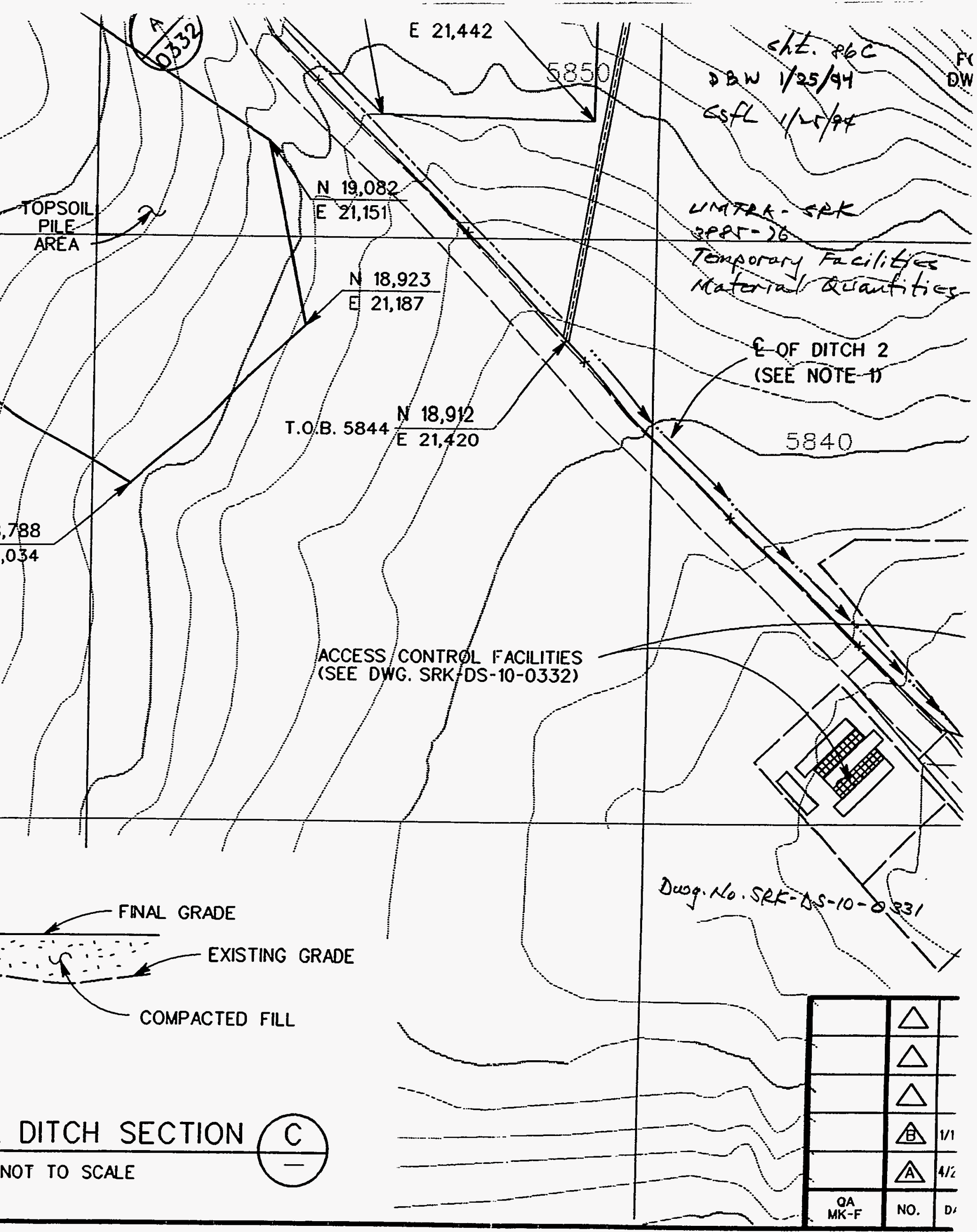


(4. MORRISON KNUDSEN CORPORATION

Project UNATRA - SRK

Feature TEMPORARY FACILITIES Item
Contract No. $3885-76$ Designed 472 Checked fy W
Sheet 87

File No.

Date $\overline{3.23 .93}$

Date $\frac{3.5 .93}{4 \cdot 93}$

b) Ditch 2 and 3

Titan 2 is iscatid on the north ste of thercessd on Drawing SRK-PS $=10-0331$ (St 70) and Ditch 3 - is located ... on the south side of the access road on drawing Sek-sio-0332 (Shit II).

Cross Sectors from SRK-DS-10-0332 are shown: below:

(Note: Length of Ditch 3 is $580 \mathrm{ft}$ - see she II)

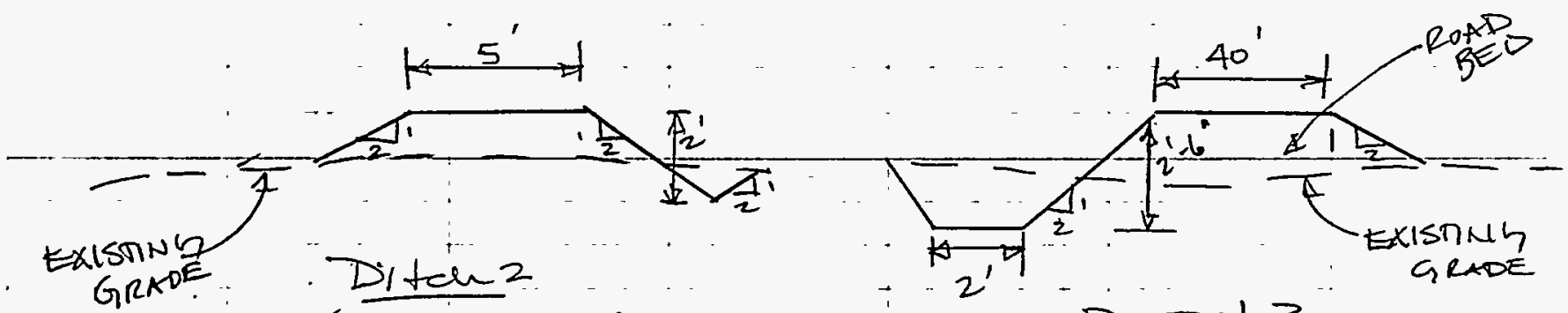

(NOT TO SCALE)

DIT EH 3

(NOT TO SCALE)

The berm for ditch 2 is included in the next section. Excavation quantities for ditch 2 will be negujible and are not calculates.

For ditch 3 assume 3 different road elevations calculate cut/fill volumes plot ilevatsuns vs.

voluone and find a bal once, as follows:

i) Assume road is $6^{\text {" above }}$ existilley irade

Using a trjekal cross section, . Cut volume $=[(2 \times 2)+(2)(4)]^{\prime} 580^{\prime}\left(\frac{1}{27}\right)=38 . \mathrm{cy}$ and fill volume $=\frac{1}{2}[40+42](0.5)(580)\left(\frac{1}{2 i}\right)=440 \mathrm{ky}$

ENG 375/91 
@160RRISON KNUDSEN CORPORATION

Project UMTRA - SRK

Sheet 88

Feature TEMPORARY FACILITES

Contract No :3885-76 File No.

MATERIAL QuANTITIES

Designed

Date 3.23 .93

Date $4-5-93$

i) Assure, road tacet's made

$$
\begin{aligned}
& \text { Cut volume }=[2.5)(2)+(2.5)(5)] 580\left(\frac{1}{22}\right) \\
& \text { Fill volume }=\varnothing .
\end{aligned}
$$

iii) Assume road is $3^{\text {"above grade }}$

$$
\begin{gathered}
\text { Cut } V_{01}=[(2.25)(2)+(2.25)(4.5)]=380\left(\frac{1}{27}\right) \\
\text { Fill } V_{0} 1 .=\frac{1}{2}\left[(40+41)(0.25)(580)\left(\frac{1}{22}\right)=218 \mathrm{cy}\right.
\end{gathered}
$$

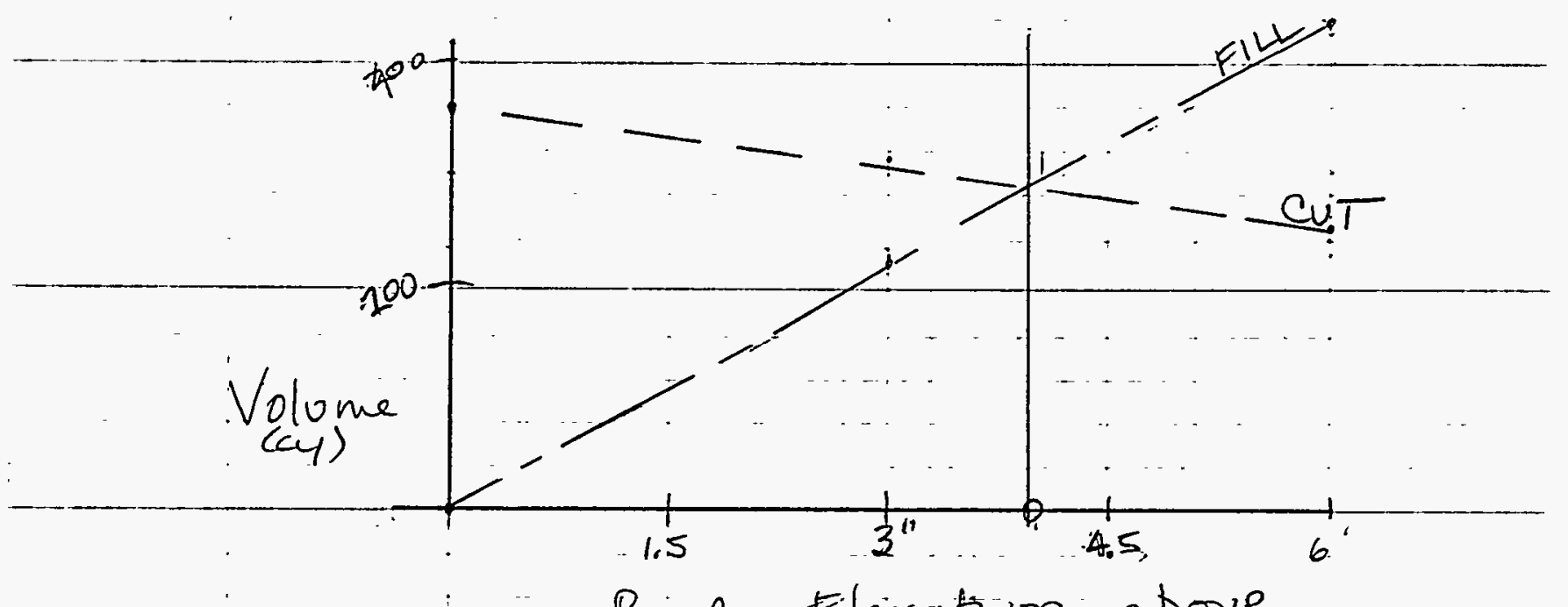

Road Elevation above grade (inches)

Rode -is it" above grade

check: Cut Volume $=[(2) 2.2)+(2 \times 2)(21)=4](580)\left(\frac{1}{27}\right)$ Fill Volume $=\frac{1}{2}[4 a+4]\left(\frac{4}{12}\right)(580)\left(\frac{1}{22}\right) \underline{=}$

This fill volume is riücluded with 290.004 total road excavation valine with as calculated on Shut 89

ENG 375/91 
MA MORRISON KNUDSEN CORPORATION

Project UMTRA - SRK

Feature TEMPORARY FACILITLES Item MATERAL QUANTITIES
Contract No $3885-76$ File No.

Designed Date 2.4 .93

Checked AFS

Date $2 / 16 / 93$

3.) Barms

$\therefore$ Fill Quantity -

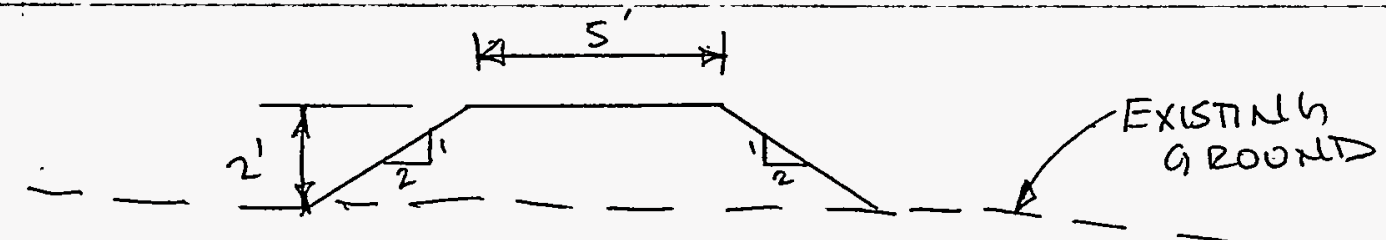

$$
\text { (nits - Typical Section }
$$

Includes three terms shown on SRR-DS-10-0331(SiA70) and the term along the road, cross section

shown ion SRK-DS-10-0332 (some as above) (Bht 71)

$$
\begin{aligned}
\text { Cross Sectional Area } & =(2)(4)+(5)(-2) \\
& =18 \text { sf. }
\end{aligned}
$$

Total Berm Length $=140^{\circ}+1000^{\prime}+240^{\circ}+750^{\prime}$$$
=2730^{\prime \prime}
$$

Fill Volume $=(2130) \times(18)\left(\frac{1}{27}\right)=1.420, \overline{0} \cdot \mathrm{cy}$

i

4.) Access Control Facilities a) Access Road length to be

SRK-DS-10-0330, ShE 69,

Approx. Length $=3550 \mathrm{ft}$

Excavation Volume $=(3550)(40)(1)\left(\frac{1}{22}\right)=5259,3 \mathrm{cy}$

Aggregate $=(3550)(0.5)(40)\left(\frac{1}{27}\right)=2629.6 \mathrm{c4}$

$-7242189$

m 
4.7. MORRISON KNUDSEN CORPORATION
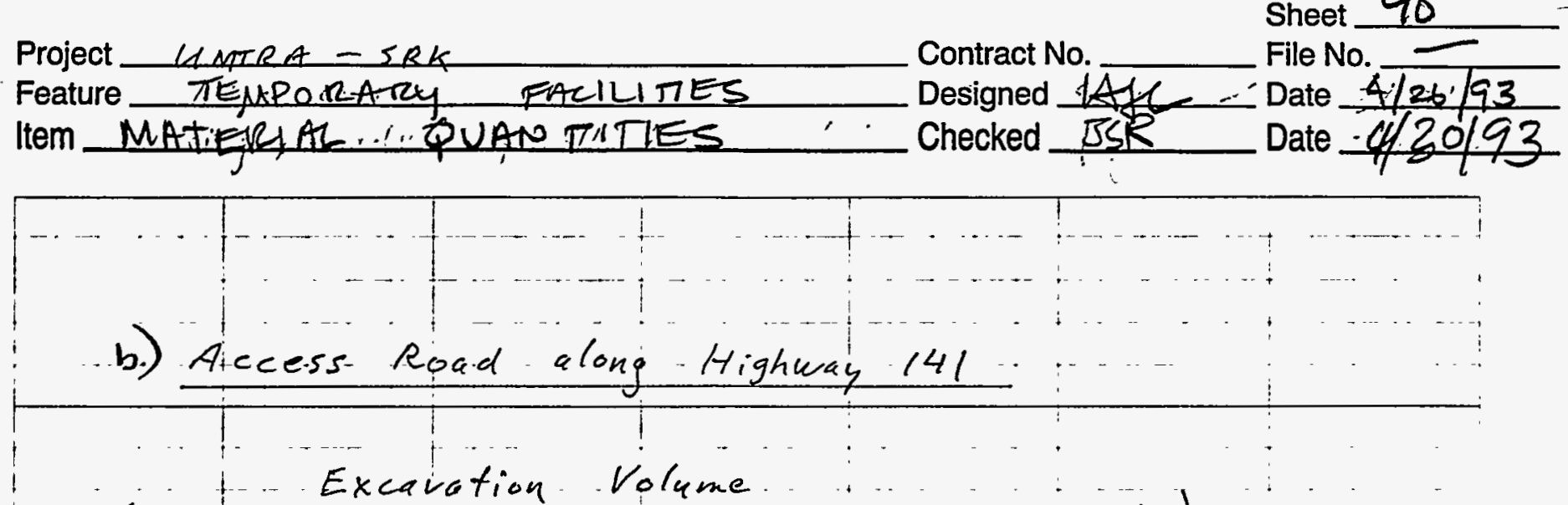

(from SRK - PS - $10-0.330$; see following sheets)

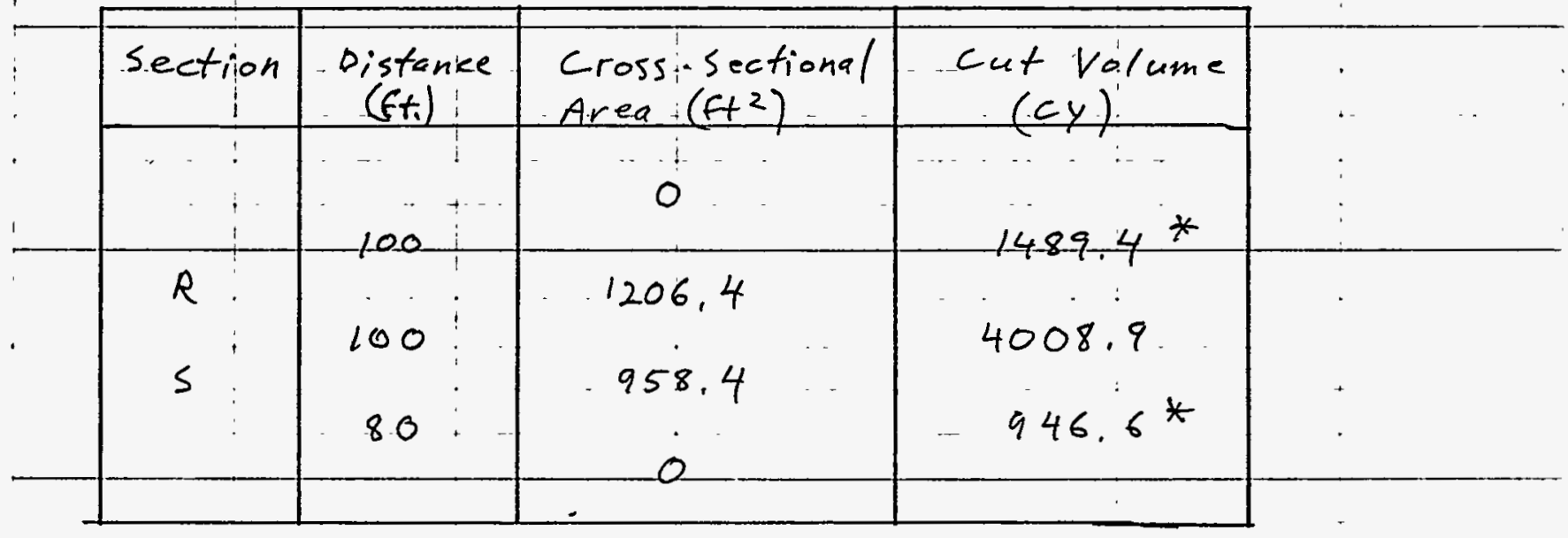

TOTAL CUT: $6444.9<y$

* by pyramid volume formula

ENG 375/49

$\$ 4242 / 89$ 
@i. MORRISON KNUDSEN CORPORATION

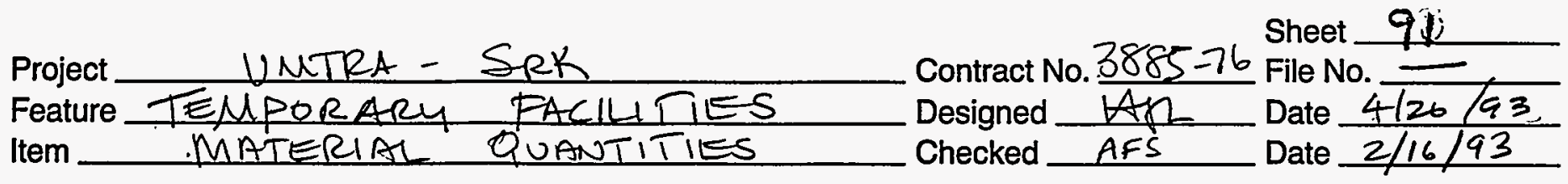

HWY 141 SECTIONS

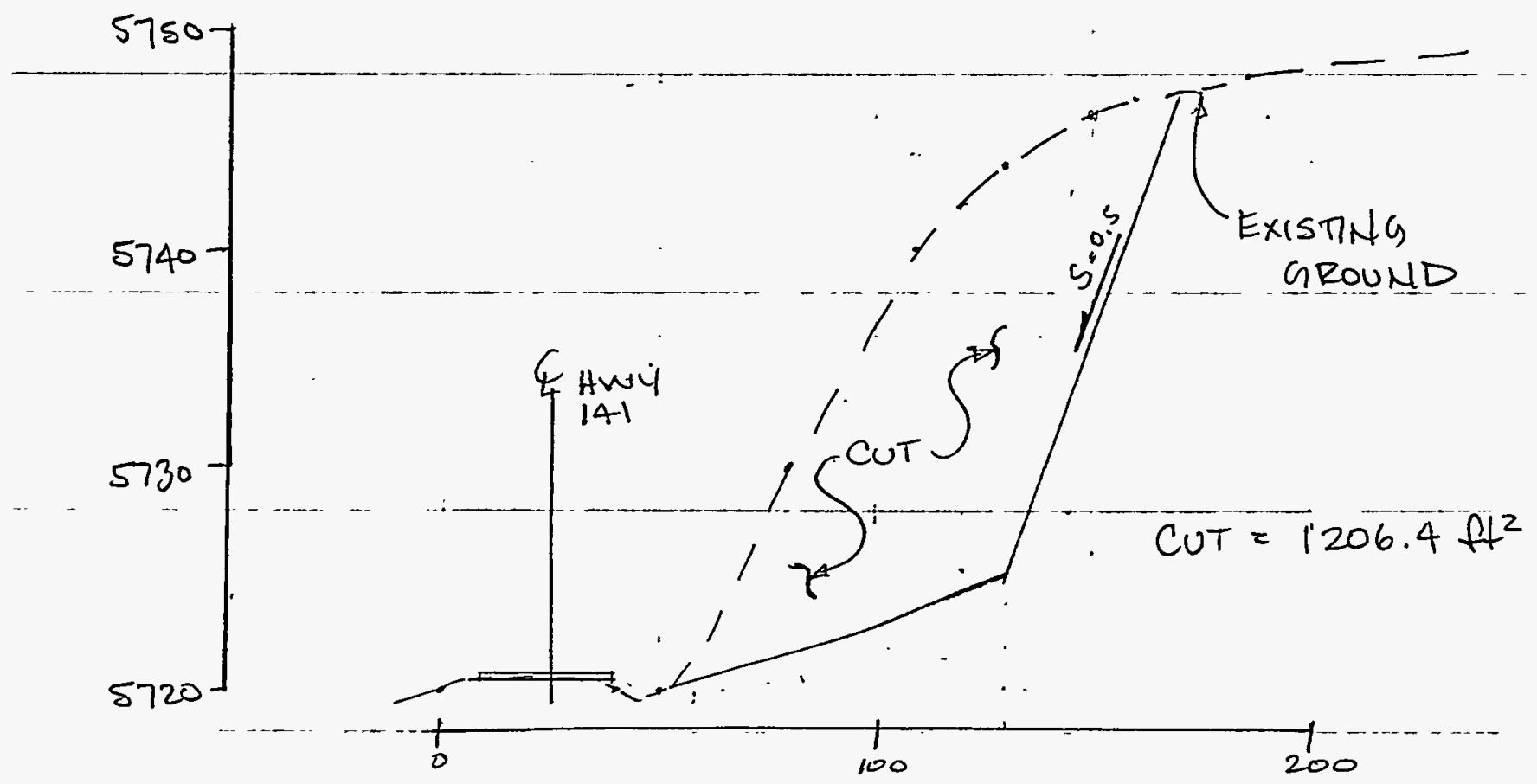

$\frac{\text { SECTION R }}{\text { HWY } 141-B C \text { STE }}$

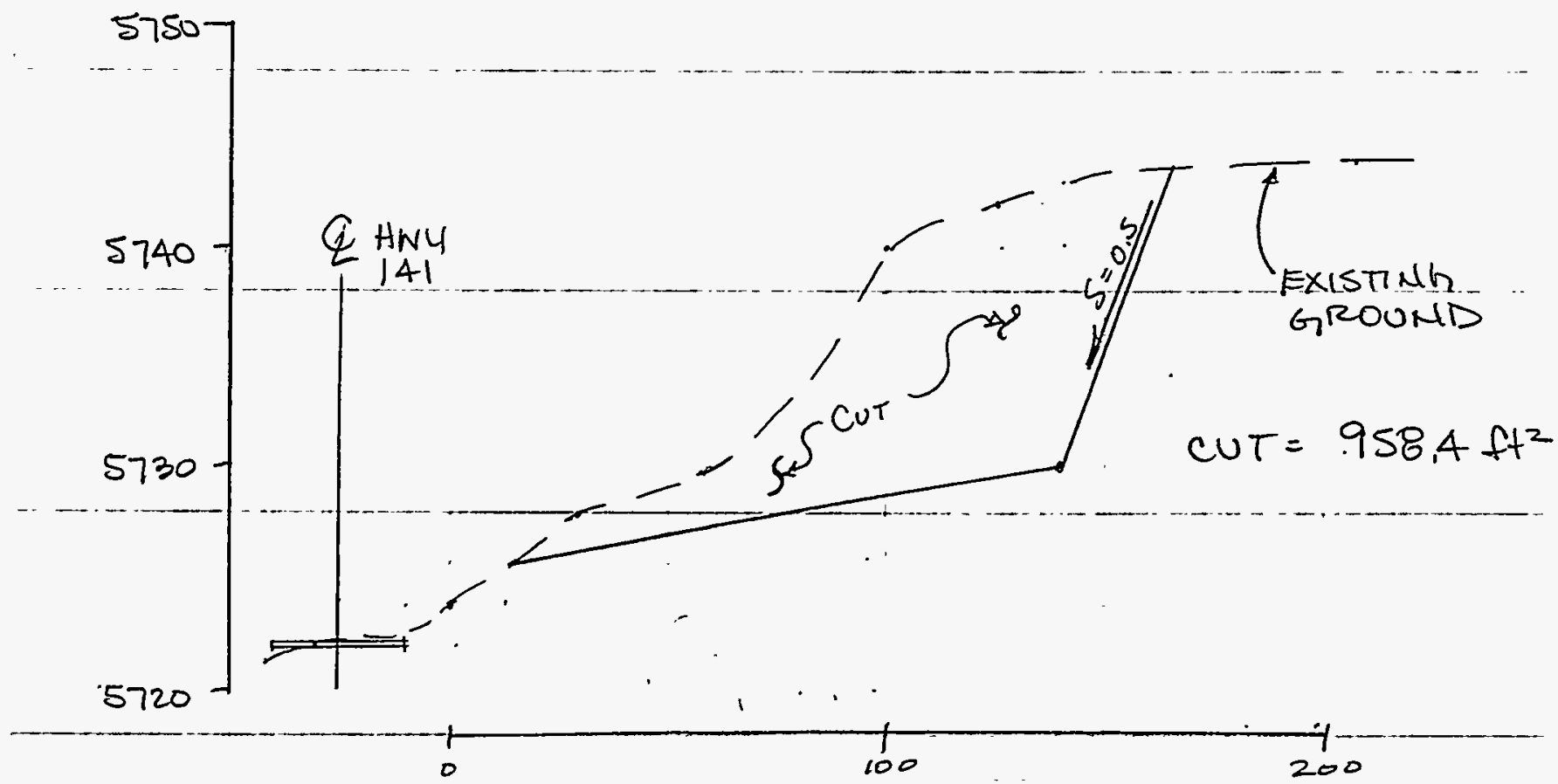

SECTION 5

HWY 141 - BC SITE

ENG 375169

14242189 
Project UNITRA - SLICKROCK Contract No. 3885-76 File No.

Feature TEMPORARY FALILITIES ITEM MATERIAL QUANTITES. Checked Date $\frac{4126193}{4(3993}$

N $17 \% 000$
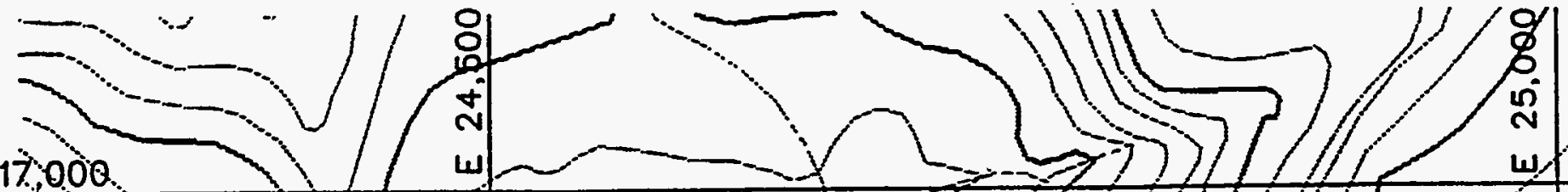

SEE NOTE

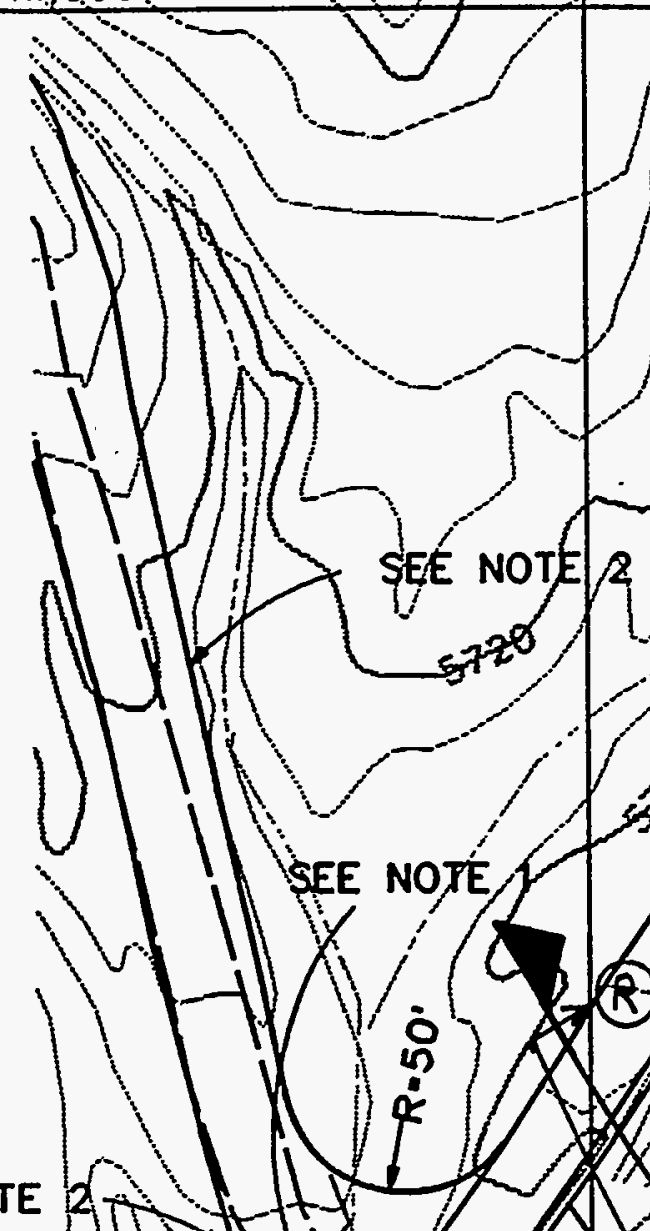

$5720 \frac{\mathrm{N}}{\mathrm{E}} 16.44 \mathrm{2}$

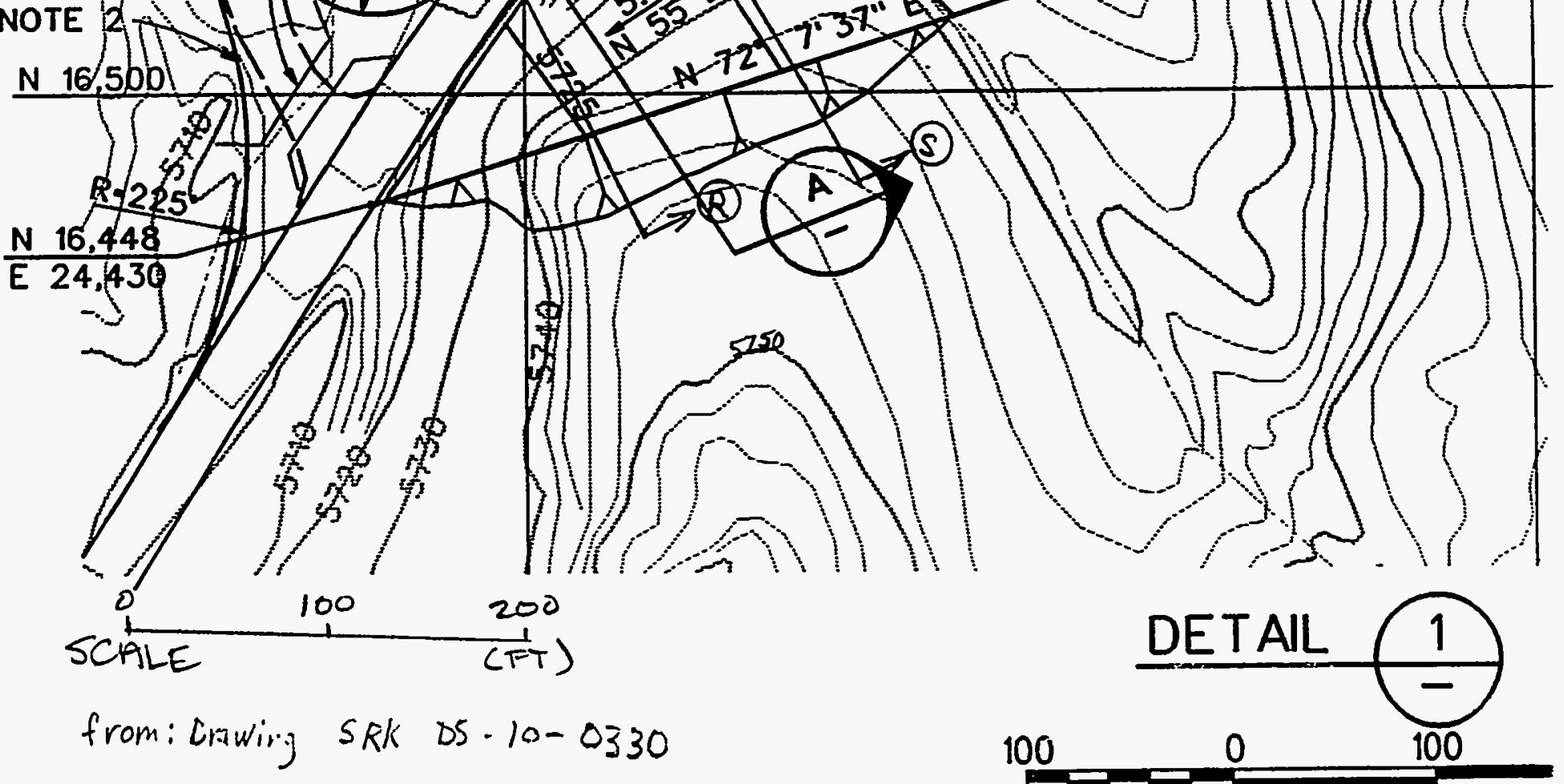


@ MORRISON KNUDSEN CORPORATION

Project UNJRA - SRK

- Feature TEMPORARY FACILITES
Sheet 9.3

Contract No. $3885-76$ File No.

Designed

LAY 2

Date 2.4 .93

Date $2 / 16 / 93$

c.) Excavation On Quantifies (see shuts 9.5 t0.9.7) for basin and decor $P$ ad

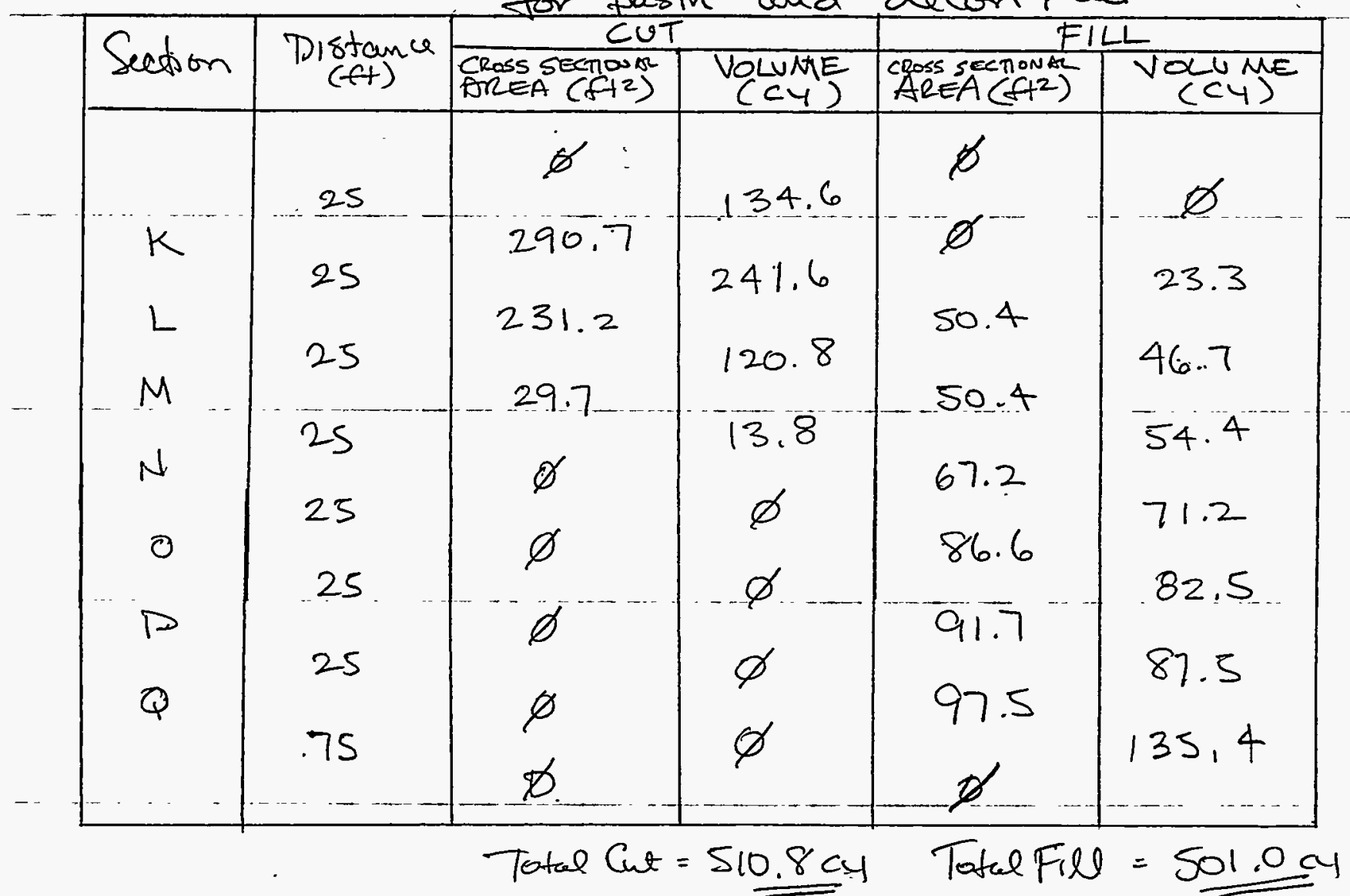

d) Membrane Liner

i) Bottom Area $=(10)(30)=3005 \mathrm{f}$

ii) Sideslopes

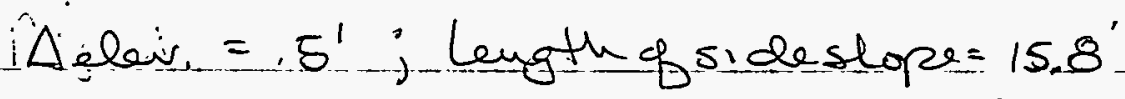

$$
\begin{aligned}
& \text { Perinuter at midpoint }=2(10 * 15.0+30+15.0) \\
& =140.0 \\
& \text { Area }=(140)(1.58)=1 \quad 2212 \text {. sf. }
\end{aligned}
$$

iii) Anchor trenches

Perimeter Length $=2(10+4(15.0)+30 i)=2.00 .01$ Area $=(200 \times(4.3)=860.05 f$

ENG 375/89

$\$ 4242189$ 
@MORRISON KNUDSEN CORPORATION

Project UMTRA - SRK

Sheet 94

Feature TEMPORARY FACILITIES

Contract No .3885.76

File No.

Item MATERIAL QUANTITIES Designed An

Date 2.4 .93

Date $2 / 16 / 93$

Total. Meunbrame Liner $=3372,0 \mathrm{sf}$

Concrete -

- Same design as VC site, see shut 46.

Volume for pad $=128.5<4$

Volume for sump $=24.4 \mathrm{c4}$

TOTAL CONCRETE $=152.9 . \mathrm{cy}$

f.) Aggregate (4" thick)

i) Recon Pad - see sit 45

Area Pad: = 1.i3.8.

Area around pad $=\left(\frac{4}{12}\right)\left(8(25)\left(\frac{1}{27}\right)=100.3 c 4\right.$

ii) Office Area

see sit 97.

Surface area $=(14601100)=14,000$ sf

Volume $=\left(\frac{4}{12}\right)(14,000)\left(\frac{1}{27}\right)=172.8 .04$.

Total Aggregate $=386.9 \mathrm{cy}$

5.) NEW CULVERTS

From SRK-DS-10-0332, Sit II two new culverts will be installed on en ch side. of the road at access entrances and a third under the road at ld NW conner of the stock pile:

New Culvert length $=130+35+25=190 \mathrm{FT}$.

ENG 375/89

$14242 / 89$ 
MOAORRISON KNUDSEN CORPORATION

Project UMTRA - SRK Feature TEMPORARY FACILITIES Item MATERIAL QuANTITIES
Sheet 95

Designed $\frac{38857}{42}$ Date 2.4 .93

Checked AFS Date $2 / 16 / 93$
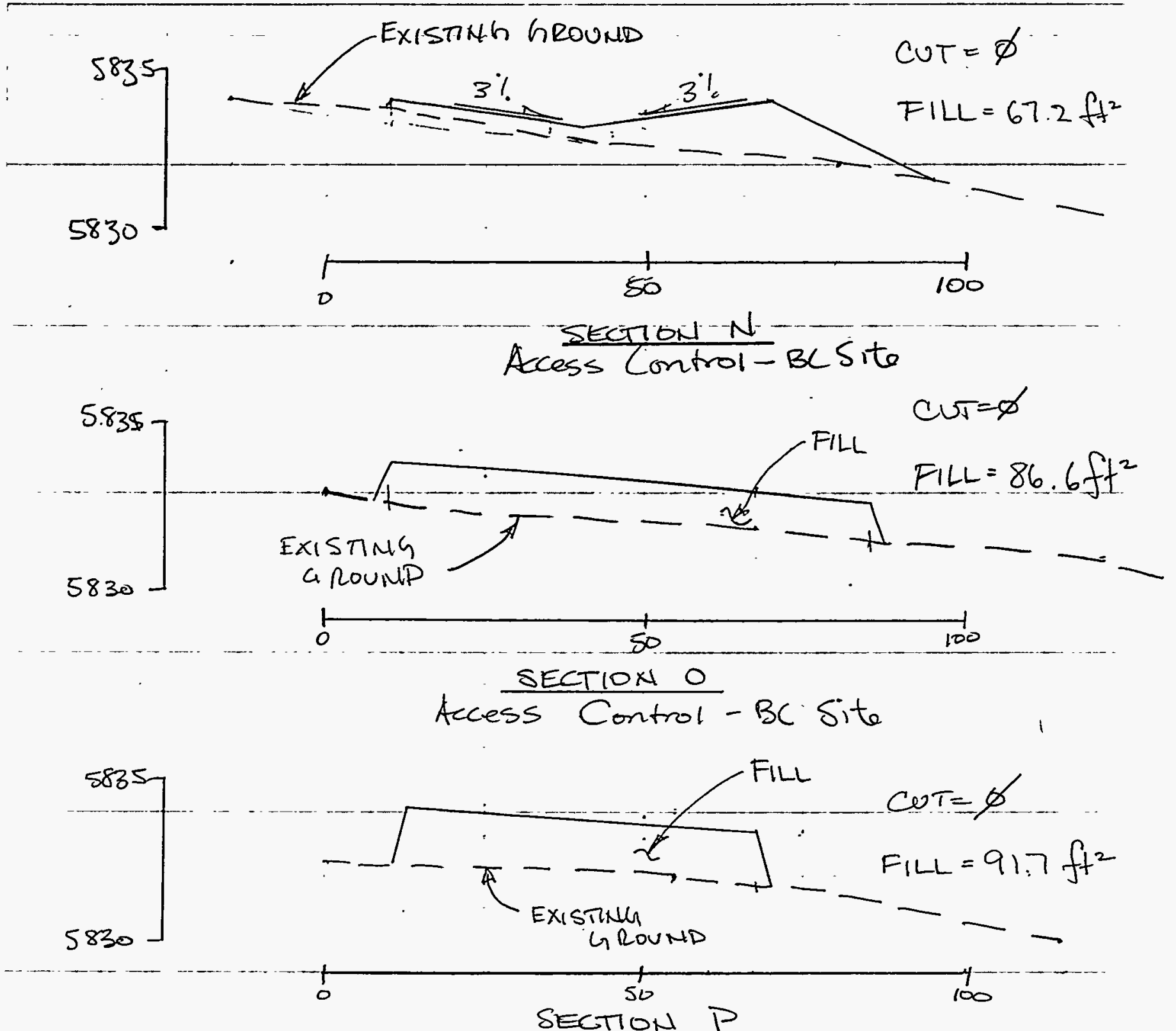

Access Control - BC Site

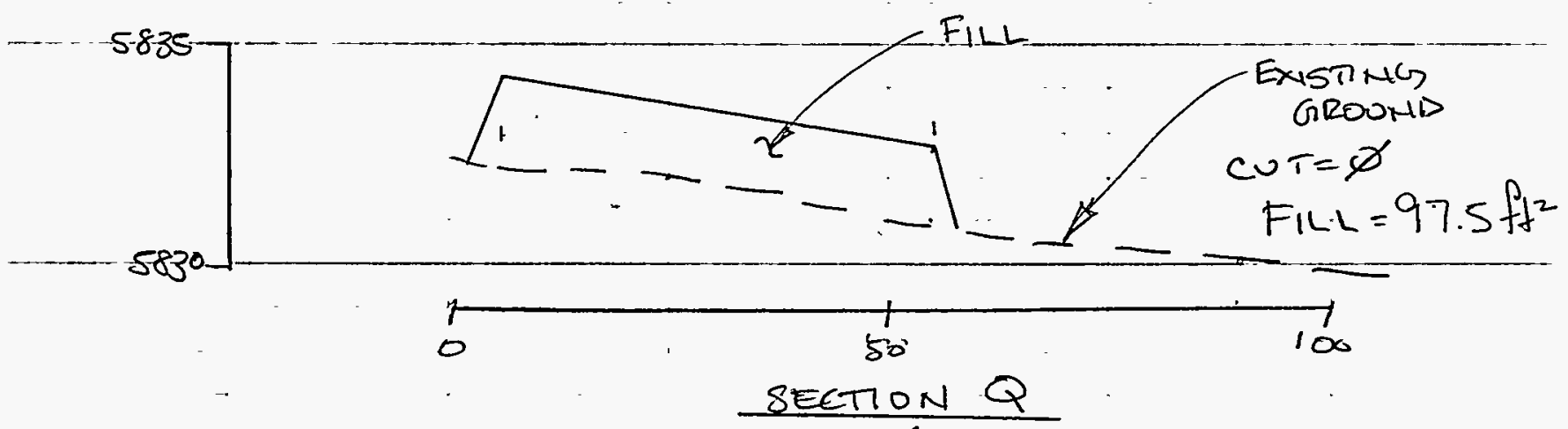

Access Control-BCSito

ENG 375/8

$4242 / 89$ 
(4. )MORRISON KNUDSEN CORPORATION

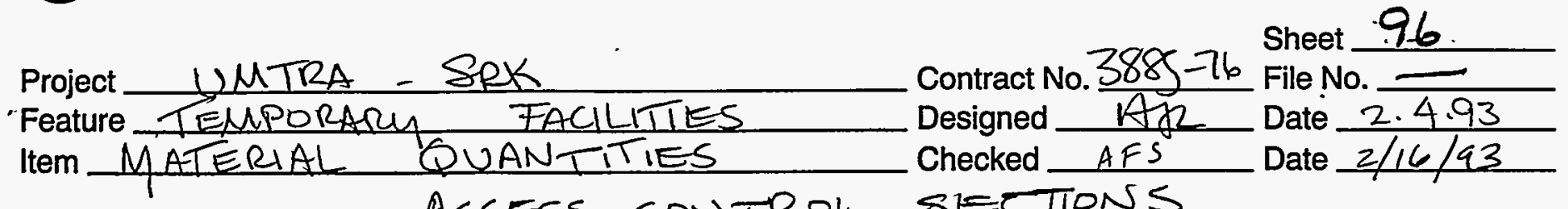

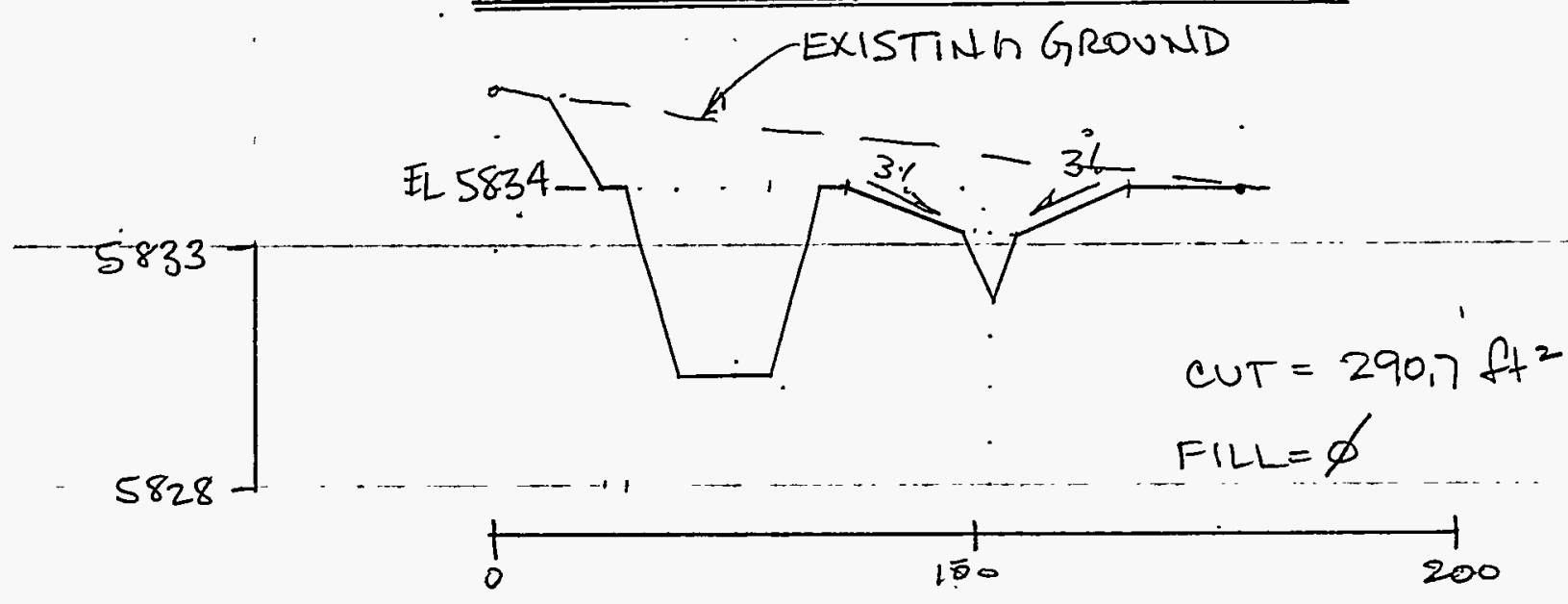

SECTION K

Access Control - BC Site

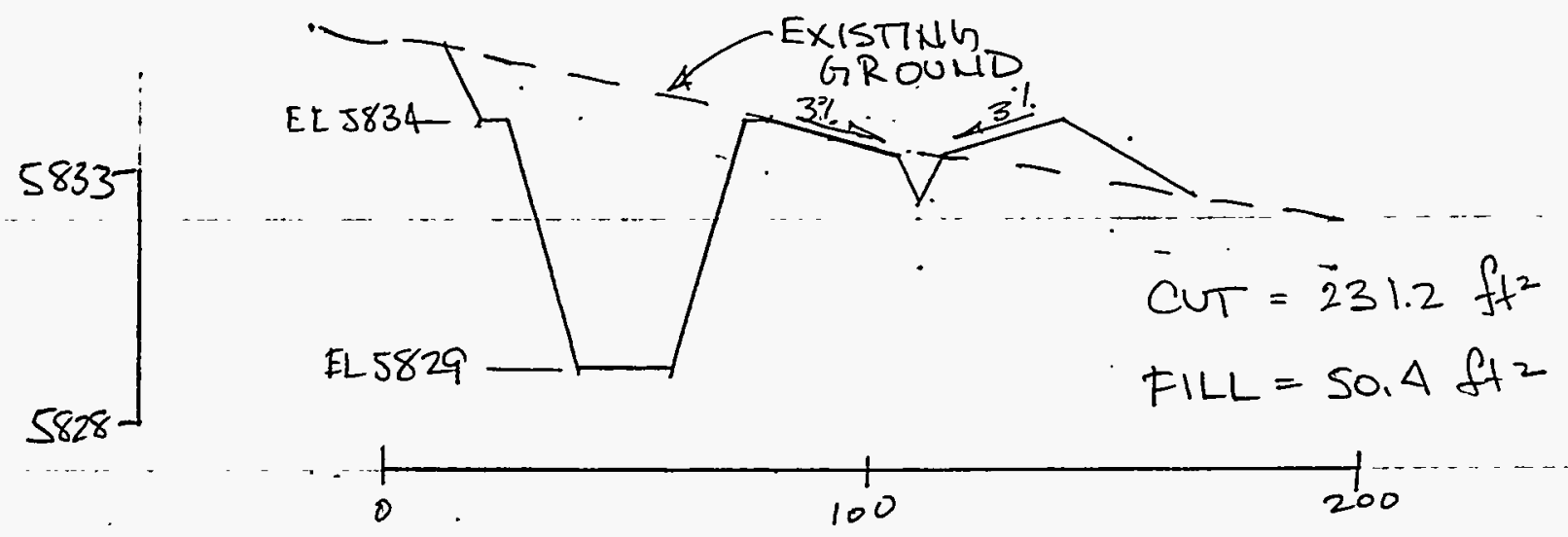

SECTION L

Access Control - BCSite

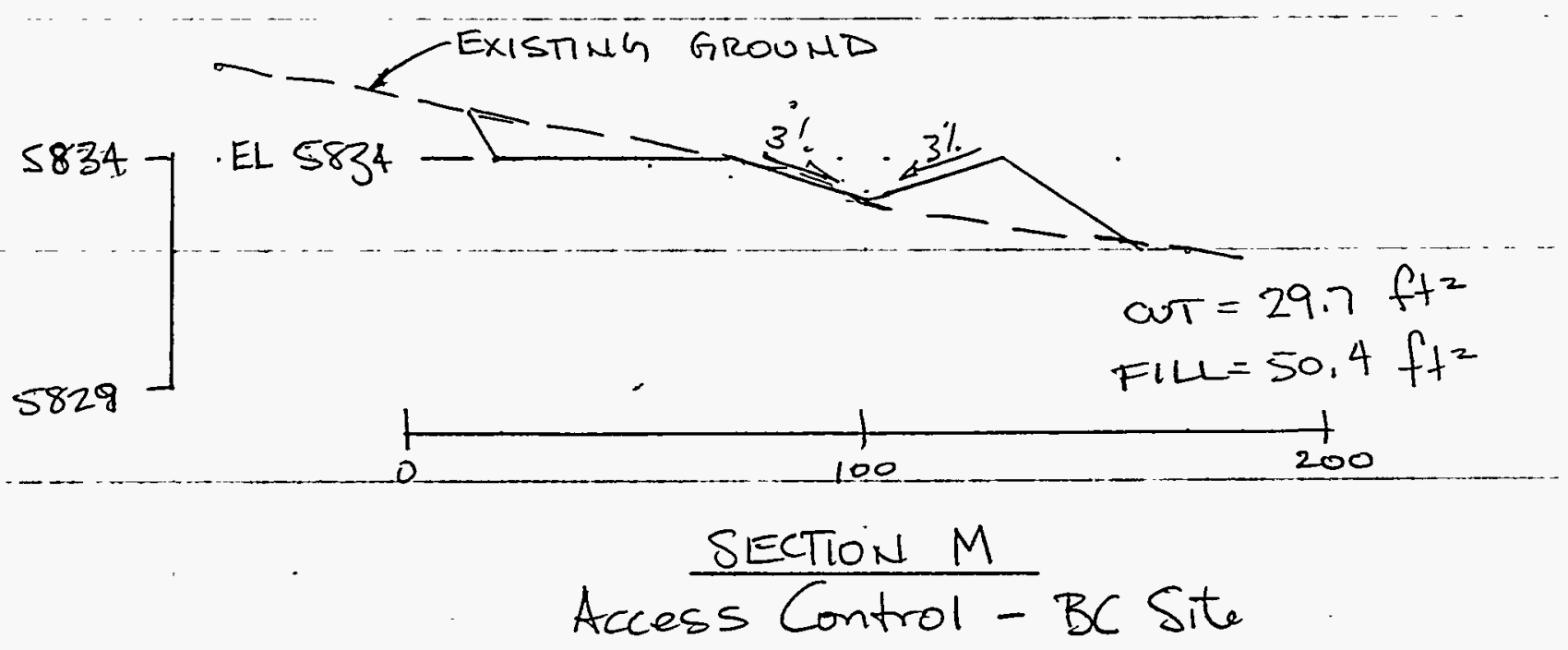

ENG 375/89

$14242 / 89$ 


\section{(28)MK-ENURBOMMENTAL SERVICES}

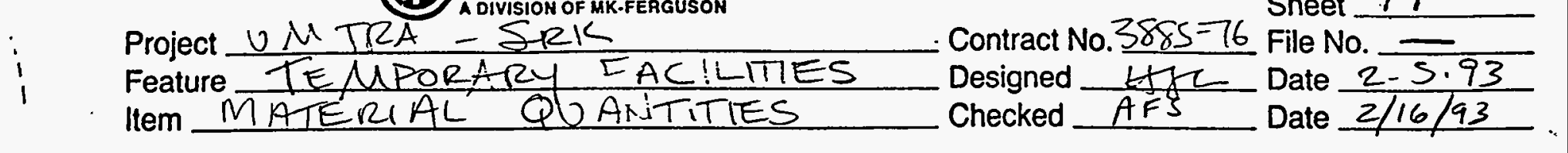

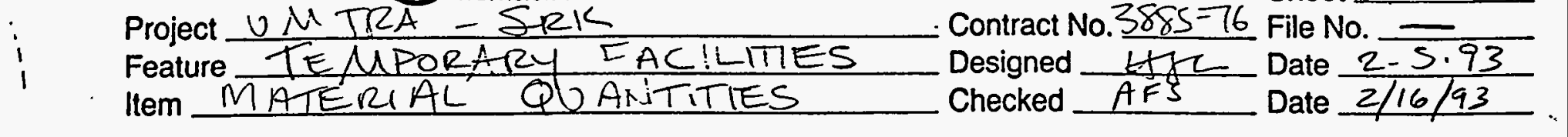

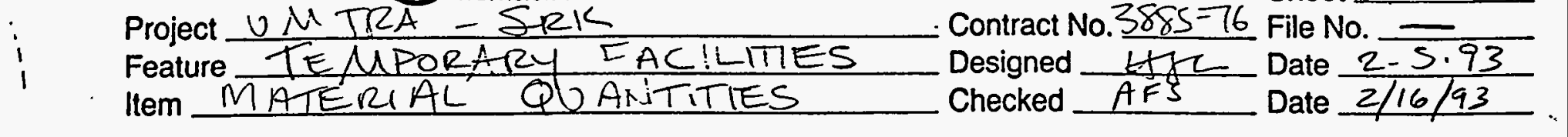

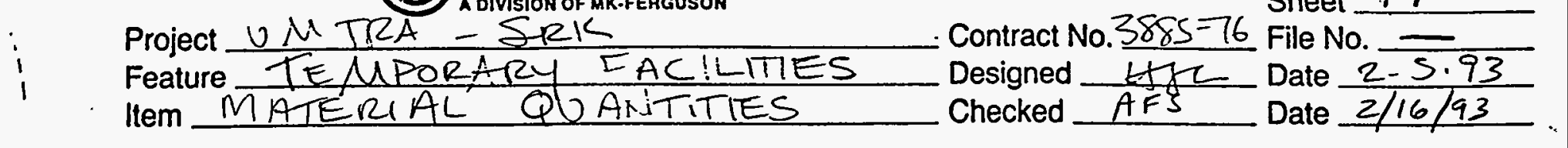

Sheet 97

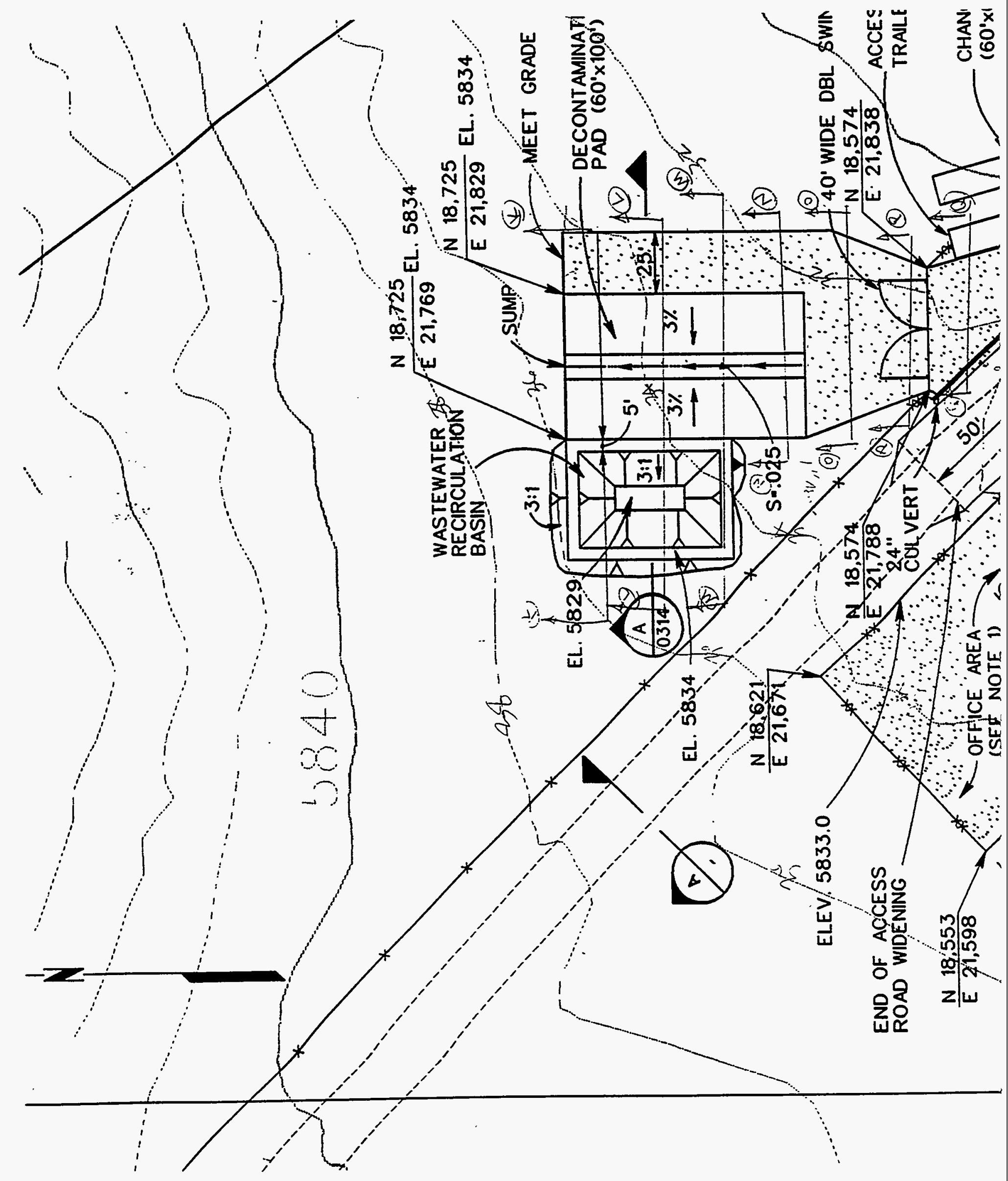




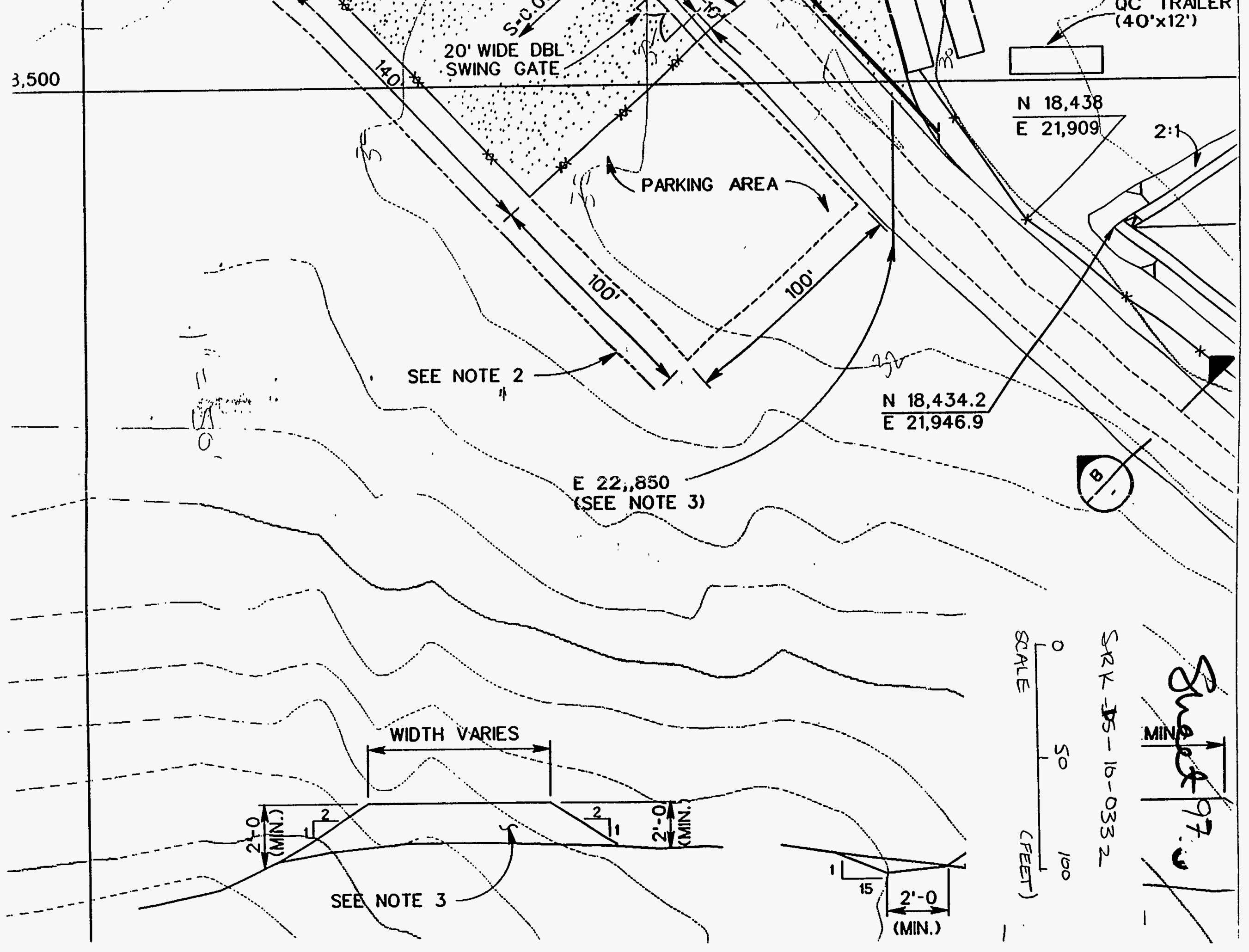


4130 MORRISON KNUDSEN CORPORATION

Project UMTRA - SRK.

Feature

Item

Sheet 98

Contract No. 3885-76 File No. -

Designed HR

Date 4.07 .9 .3

Checked IJSR

Date

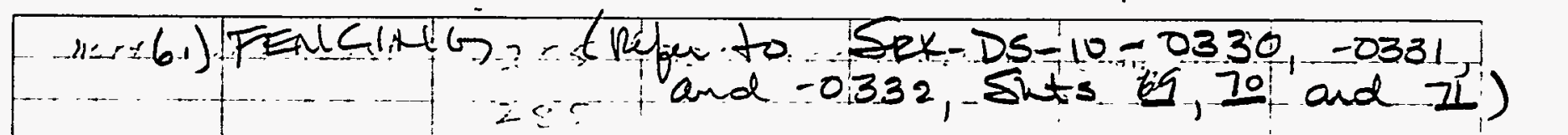

a) Aet Fence

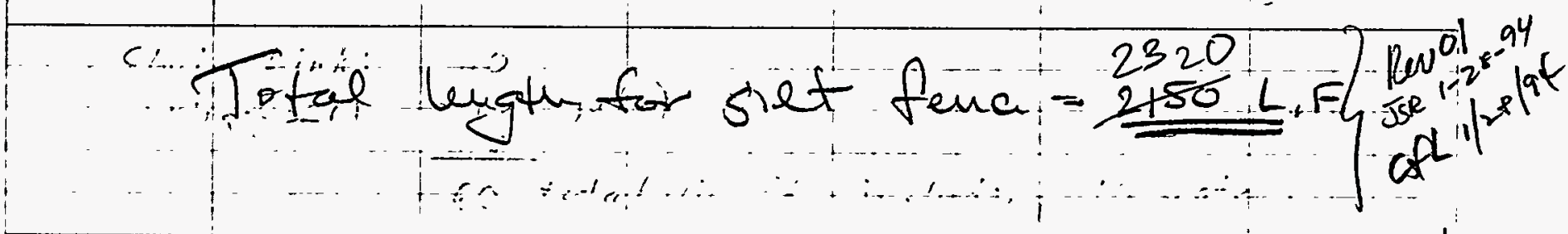

ReV 2 By JSR 8/11FY

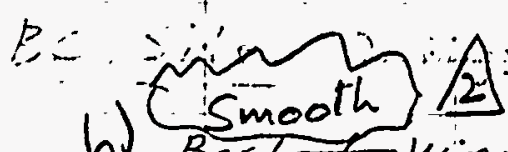

ave sos $q / 13 / 95$

b) Bore! beginning of point N12,909 and E 20,330 and proceeding counter clockwise:

$$
\begin{aligned}
& \left.\begin{array}{l}
\text { Q20 } \\
950 \\
15 \\
80
\end{array}\right\} \text { Drawing - } 0330 \\
& 245 \text { drawing } 0332
\end{aligned}
$$

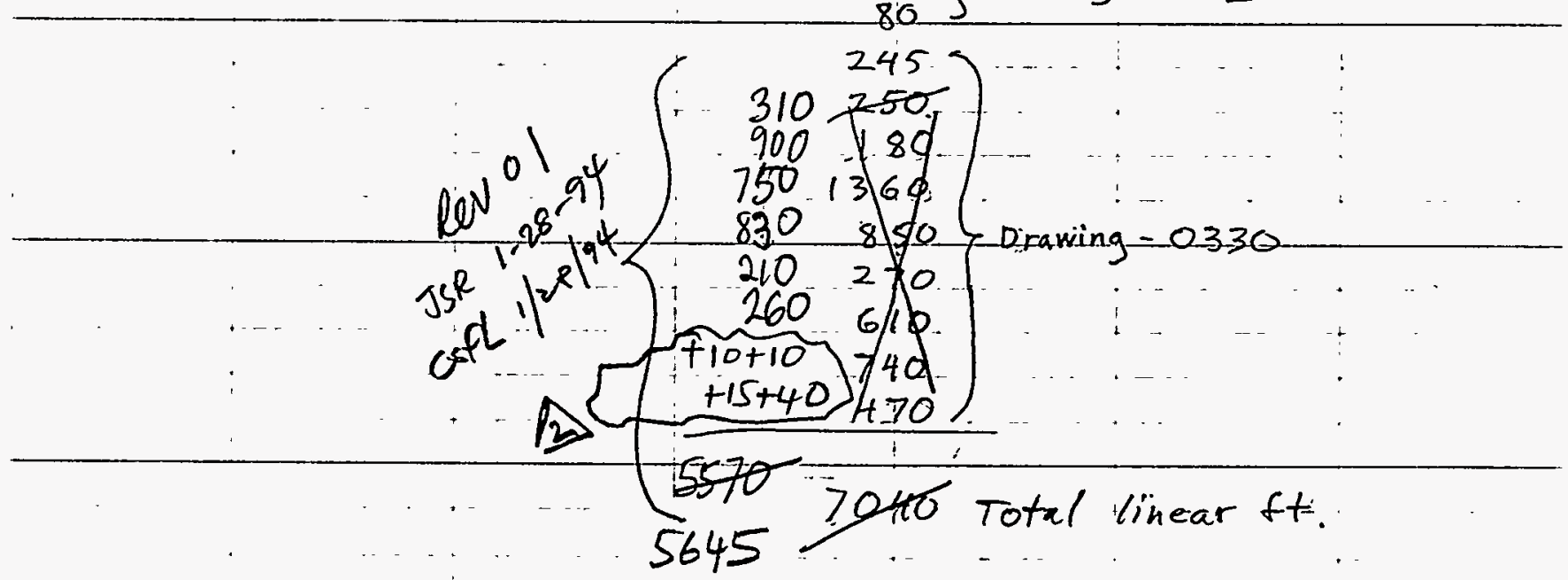

c) Chain Link: (Drawing -0.332$)$

$$
\text { Office Area: }(2 \times 140)+(2 \times 100)=480 \text { (incl. } 20^{\prime} \text { gate) }
$$

Deon Pad Access:

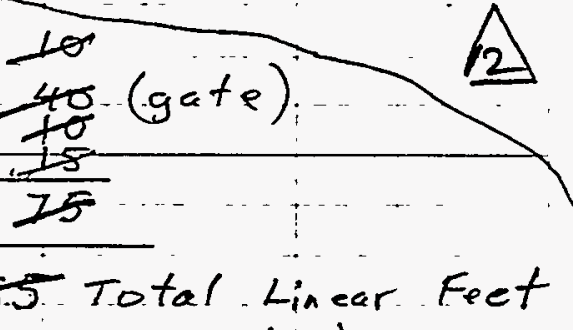

ENG 375/49

$\lcm{4242 / 89}$ 
(16 )MORRISON KNUDSEN CORPORATION

Project $\frac{\text { UMTREA-SRK }}{\text { Temporary facilities }}$
Itemeterial Quantities

Sheet $98 \mathrm{~A}$

Contract No. $3855-76$ File No.

Item Material Quant

Designed J5R

Date $\varepsilon / 22 / 94$

Checked SOS Date $2 / 13 / 95$

(d) Deer Fence

From ȘRK-DSti-0332, sht.71,

$$
\begin{aligned}
\text { Perimeter of retention basin } & =(340+190+240+190) \\
& =960 \mathrm{ft} .
\end{aligned}
$$

Perimeter of recinatation basin $=2(50+70)$

$$
=240 \mathrm{ft}
$$

Assume 200'x200' construction water pond, Perimeter of construction water

$$
\text { pond }=2(200+200)=800^{\prime}
$$

Total Length of Deer Fence $=(960+240+800)$

$$
=2000 \mathrm{ft}
$$

ENG 375/91 
Project UMTRA - Slickrock Feature THEMPQRAR FACHLITES Item MATERIAZ SOANTITES

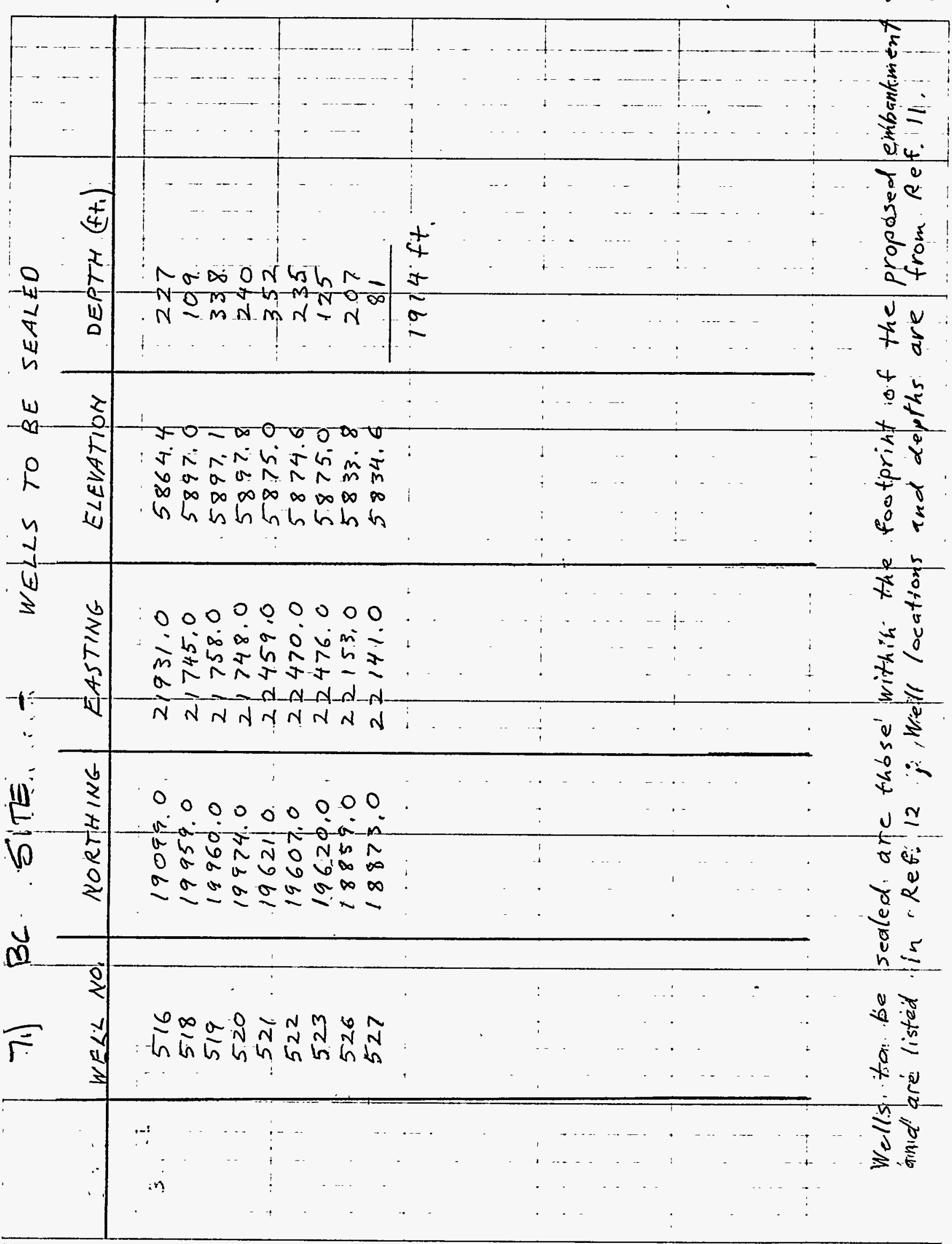

Contract No. $3885-76$ File No.

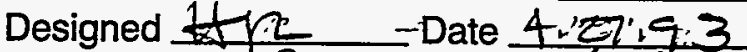
Checked 
The following monitor wells should be ABANDONED, based on the revised (Option B) design plans (1/6/93):

Monitor well

$\mathbf{5 1 6}$
$\mathbf{5 1 8}$
$\mathbf{5 1 9}$
$\mathbf{5 2 0}$
$\mathbf{5 2 1}$
$\mathbf{5 2 2}$
$\mathbf{5 2 3}$
$\mathbf{5 2 6}$
$\mathbf{5 2 7}$

516

518

519

520

521

522

523

527 stratigraphic unit

Middle Burro Canyon

Upper Burro Canyon

Lower Burro Canyon

Middle Burro Canyon

Lower Burro Canyon

Middle Burro Canyon

Upper Burro Canyon

Middle Burro Canyon

Upper Burro Canyon

The following monitor wells should REMAIN IN-PLACE at the proposed Burro Canyon disposal site:

Monltor well

$$
\begin{array}{r}
512 . \\
524 \\
525 \\
\therefore 528 \\
529 \\
551 \\
552
\end{array}
$$

\section{Stratigraphic unit}

$$
\begin{aligned}
& \text { Lower Burro Canyon } \\
& \text { Lower Burro Canyon } \\
& \text { Mlddle Burro Canyon } \\
& \text { H1ddle Burro Canyon } \\
& \text { Upper Burro Canyon } \\
& \text { Upper Burro Canyon } \\
& \text { Upper Burro Canyon }
\end{aligned}
$$

If you have any questions, comments, or concerns please advise.

RECEIVED-MKE

MAR 181993

UMTRA.S.F.
UMTRA-SRK

TEMP. FocILITES MAT. QUANTTTES 
CONTRACT NO. $\underline{3885-76}$ DISCIPLINE CIVIL

PROJECT

\section{UMTRA - SLICK ROCK}

\section{FEATURE}

\section{EMBANKMENT QUANTITIES}

\section{ITEM}

\section{SOURCES OF DATA}

Reference drawings \#1-5

\section{SOURCES OF FORMULAE \& REFERENCES}

1-5: MKES, 199ß4 UMTRA PROJECT, SLICK ROCK SITE DRAWINGS:
1. SRK-DS-10-0331 - Site Preparation and Temporary Drainage Facilities.
2. SRK-DS-10-0334 - Tailings Embankment and Final Site Grading Plan
3. SRK-DS-10-0335 - Tailings Embankment Sections and Details (Sheet 1 of 2)
4. SRK-DS-10-0336 - Tailings Embankment Sections and Details (Sheet 2 of 2)
5. SRK_DS-10-0337 - Tailings Embankment Erosion Protection

6. Intergraph Corp., 1992. Inroads Civil Engineering Site and Highway Design Software, Vers. 4.01.01.00.

7. niMTRA_SRK, site Restoration-Earthwork Quantities and seeding Cak No.11-330-01-11 PRELIMINARY CALC. $\square$ ' FINAL CALC. 区, SUPERSEDES CALC. NO.

\begin{tabular}{|c|c|c|c|c|c|c|c|}
\hline & & & & 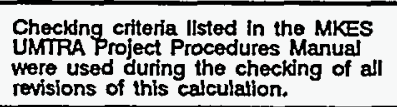 & & & \\
\hline 02 & See sheet IA. & D.S.Still & $8 / 29 / 95$ & & & & $19 / 13 / \pi$ \\
\hline 1 & See sheet $1 A$ & J.S.Randeui & $1 / 26 / 84$ & C. Cisardo & $1 / 20 / 94$ & PQPais & lishe \\
\hline$\theta$ & & $H$ & & & & & $4 / 27 / 5$ \\
\hline $\begin{array}{l}\text { REV. } \\
\text { NO }\end{array}$ & REVISION & $\begin{array}{l}\text { CALCULATION } \\
\text { BY }\end{array}$ & DATE & $\begin{array}{c}\text { CHECKED } \\
\text { BY }\end{array}$ & DATE & $\begin{array}{c}\text { APPROVED } \\
\text { BY }\end{array}$ & DATE \\
\hline
\end{tabular}


PURPOSE

Rev. OI

PROCEDURE

1

: EMBANKMENT. EXXCAVATIỌN

APRON EXCAVATION

COPIES OF REFERENCE DRAWINGS

Riprap $A, B$ and bedding in fival gradc

Volume of permanent stockpile
APPENDIX A

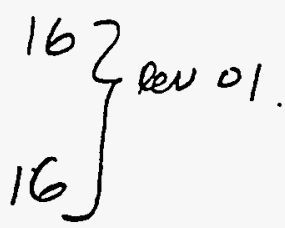


Contract No. $3885-76$

Designed AFS

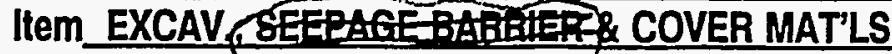

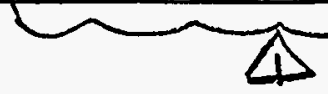

Checked

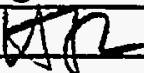

Rev I

SUMMARY OF QUANTITIES

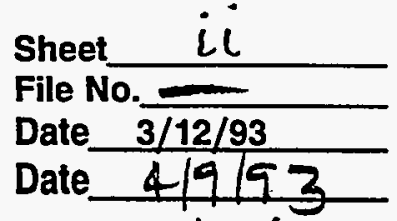

By: JSR 1/25/94 asfl. $1 / 2+1 / 74$
ITEM EMBANKMENT EXCAVATION (SHEET 3) (excluding topsoil)
Topsoil in embankment area APRON EXCAVATION (SHEET 11)

RIPRAP TYPE A IN COVER (SHEET 12)

RIPRAP TYPE B IN COVER (SHEET 12)

RIPRAP TYPE C

\author{
IN APRON (SHT. 12) \\ IN COVER \\ TOTAL
}

BEDDING MATERIAL IN APRON (SHT. 12)

IN COVER

TOTAL $\left.\begin{array}{l}\text { QUANTITY } 526,950 \mathrm{cy} \\ 42,70000 \mathrm{cy}\end{array}\right]\left[\begin{array}{l}\text { lev } 01 \\ (\sin +3)\end{array}\right.$ 3300 cy

$2800 \mathrm{cy}$

$12,930 \mathrm{cy}$

7370 cy

$1040 \mathrm{cy}$

$8410 \mathrm{cy}$

$980 \mathrm{cy}$

$9430 \mathrm{cy}$

$10,410 \mathrm{cy}$

FROST PROTECTION MATERIAL IN COVER (SHT. 15)

$.36,740 \mathrm{cy}$

RADON BARRIER MATERIAL IN COVER (SHT. 15) 26,2003 hev 02
35,170 cy (sht 15 ) Checked:
-ByDss. ND Riprap A in final grade $510 \mathrm{cy}]_{i .01}^{- \text {By }}$ Riprap $B$ in final grade Bedding material in final grade Volume of material to be placed in the permanent stock pile 


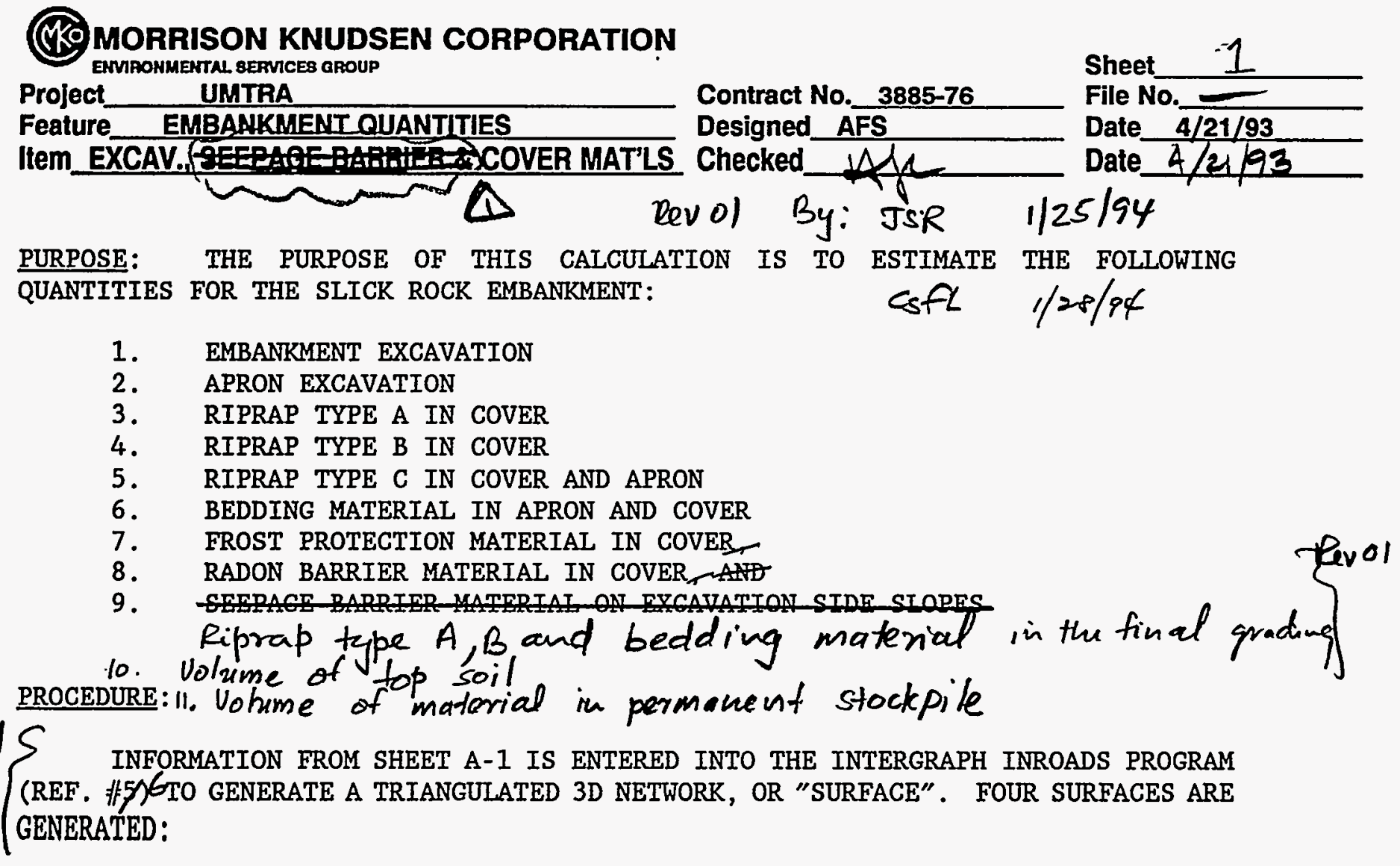

(1) TOP OF EMBANKMENT AND FINAL SITE GRADING (SEE SHEET 13),

(2) TOP OF RADON BARRIER MATERIAL (SEE SHEET 14),

(3) BOTTOM OF EXCAVATION BELOW EMBANKMENT (SEE SHEET 4), AND

(4) BOTTOM OF APRON EXCAVATION

A COMPARISON OF THE INROADS CONTOURS DISPLAYED ON SHEETS 4 AND 13 WITH THE CONTOURS SHOWN ON SHEETS A-1 AND A-2, RESPECTIVELY SHOW THAT THE INROADS SURFACES ACCURATELY REPRESENT THE DESIGN.

FROM THE LIST ABOVE, QUANTITIES ARE CALCULATED AS FOLLOWS:

1. THE INROADS TRIANGLE METHOD OF COMPUTING VOLUMES TO COMPARE THE BOTTOM-OF-EXCAVATION SURFACE WITH THE EXISTING GROUND SURFACE. FOR CHECKING PURPOSES, CROSS-SECTIONS ARE TAKEN THROUGH THE EXCAVATION SURFACE. INROADS PRODUCES BOTH A DRAWING OF EACH CROSS-SECTION AND A LIST OF CROSS-SECTIONAL AREAS. Since 6" of topsoil will be stockpiled 3 Rew o I

2. THE APRON SECTION IS PLACED EVERY 10 FEET ALONG THE EMBANKMENT TOE. INROADS USES A CROSS-SECTIONAL AREA METHOD TO COMPUTE THE TOTAL EXCAVATION. AS AN APPROXIMATE CHECK, THE LENGTH OF THE ALIGNMENT IN CUT ALONG THE EMBANKMENT TOE IS MULTIPLIED BY THE AREA OF EXCAVATION IN THE TYPICAL APRON SECTION.

3. INROADS IS USED TO COMPUTE THE SURFACE AREA OF THE TOP-OF-EMBANKMENT SURFACE WITHIN THE LIMIT OF TYPE A RIPRAP. VOLUME $=$ S.A. $x$ THICKNESS .

4. INROADS COMPUTES THE SURFACE AREA OF THE TOP-OF-EMBANKMENT SURFACE WITHIN TYPE B RIPRAP LIMITS. VOLUME $=$ S.A. $\times$ THICKNESS. 
MORRISON KNUDSEN CORPORATION

ENVRONMENTAL SERVICES GROUP

Project UMTRA

Contract No. $\quad 3885-76$

Sheet / A

Feature EMBANKMENT QUANTITIES

Designed JSR

File No.

Item EXCAVATION AND COVER MAT'LS

Checked CreeL

Date. 1/26/94

PURPOSE OF REV 01

1. Excavation for the disposal cell sideslopes has changed from $2.5 \mathrm{H}: 1 \mathrm{~V}$ to $1 \mathrm{H}: 1 \mathrm{~V}$. Therefore the excavation quantity has been revised.

2. There is a erosion protection layer in the west of the disposal cell. Riprap Type A, B and bedding quantities have been calculated for this area.

3. The topsoil in the BC site has to be stockpiled for later use as the top soil in the seeding areas.

4. Permanent stockpile volume from the embankment excavation and the volume of the design stockpile were also calculated.

Sheets $i, i i, 1,2$ and 15 have been revised.

Sheets $3-10,13$ and $A-1$ thru $A-5$ have been replaced with new sheets.

Chests $1 A$ and 16 were added.

Purpose of Rev 02

1. The source of the radon barrier material is no longer the Disappointment Valley borrow area. Radon barrier material is now coming from the required excavation at BC disposal site.

2. Based on calculations 11-340-01-00 and 11-340-02-00, the required radon barrier thickness has been changed from $2^{\prime}$ to $1.5^{\prime}$.

Sheets ii, 11, and 15 have been revised.

Sheets $A-3$ and $A-4$ have been replaced with new sheets.

Rev 02

Checked: Pig DSS

$9 / 12 / 95 \quad 8 / 29 / 25$ 
MORRISON KNUDSEN CORPORATION

ENMRONMENTAL SERVICES GROUP

Project

UMBRA

Contract No. $3885-76$

Sheet

2

Feature

EMBANKMENT QUANTITIES

Designed AFS

File No.

Item_EXCAV..(SEEPA COVER MAT LS

Checked

Date $\frac{3 / 12 / 93}{4 / 9 / 93}$

5. A TYPICAL CROSS-SECTION THROUGH THE EMBANKMENT TOE AND APRON GIVES

$1 / 28 / 94$ THE CROSS-SECTIONAL AREA OF RIPRAP TYPE C. THIS IS MULTIPLIED BY THE LENGTH OF THE EMBANKMENT TOE.

6. INROADS COMPUTES THE SURFACE AREA OF THE TOP-OF-RADON BARRIER SURFACE. VOLUME $=$ S.A. $\times$ THICKNESS. A SMALL VOLUME IS ADDED FOR THE AMOUNT OF BEDDING WHICH EXTENDS BEYOND THE RADON BARRIER TOE.

7. SURFACE AREA $\times$ THICKNESS IS USED TO COMPUTE THE VOLUME OF FROST PROTECTION MATERIAL IN THE COVER. THE TOP-OF-RADON BARRIER INROADS SURFACE IS USED, AND THE VOLUME OF FROST PROTECTION BEYOND THE RADON BARRIER LIMITS IS ADDED.

8. INROADS COMPUTES THE SURFACE AREA OF THE TOP-OF-RADON BARRIER SURFACE. VOLUME $=$ SA. $\times$ THICKNESS. A SMALL VOLUME IS SUBTRACTED, BECAUSE THE SURFACE AREA IS TAKEN FROM THE TOP OF THE RADON BARRIER LAYER, NOT THE BOTTOM.

9. THE SURFACE AREASOF THE materials are multiplied by thicknesses to get quantities. ALL INROADS SURFACES HAVE BEEN GIVEN FILE NAMES WITH THE EXTENSION ". tEn" AND STORED ON THE MKES-SFO CAD SERVER IN THE DIRECTORY D:\388576\.

10. Volume of topsoil in the BC site is calculated by multiplying the disturbed area by 6". It is assumed only b" of topsoil is needed to be stockpiled separately to be used as "Disturbed areas include the pile area, finish grade, access control areas and retention basin area.

11. Volume of material in permanent stockpile is excavated material from embankment excluding topsoil minus material needed in finish grading. and frost barrier. 
Sheet $\}$

File No.

Date $1 / 26 / 94$

Date

EMBANKMENT EXCAVATION:

THE INROADS SURFACE FOR THE EXCAVATION IS CALLED "TOTALEXCA.TTN". THE CONTOURS OF THIS SURFACE ARE DISPLAYED ON SHEET 4. THE BOTTOM OF THE TRENCH SLOPES +7\% TO THE NORTHWEST, AND THE SIDE SLOPES ARE 1H:1V (SEE REF. DWG. 非)! SHEETS 5-9 SHOW SECTIONS THROUGH THE EXAVATION. THE CROSS-SECTIONAL AREA OF THE EXCAVATION IS WRITTEN ON EACH SECTION.

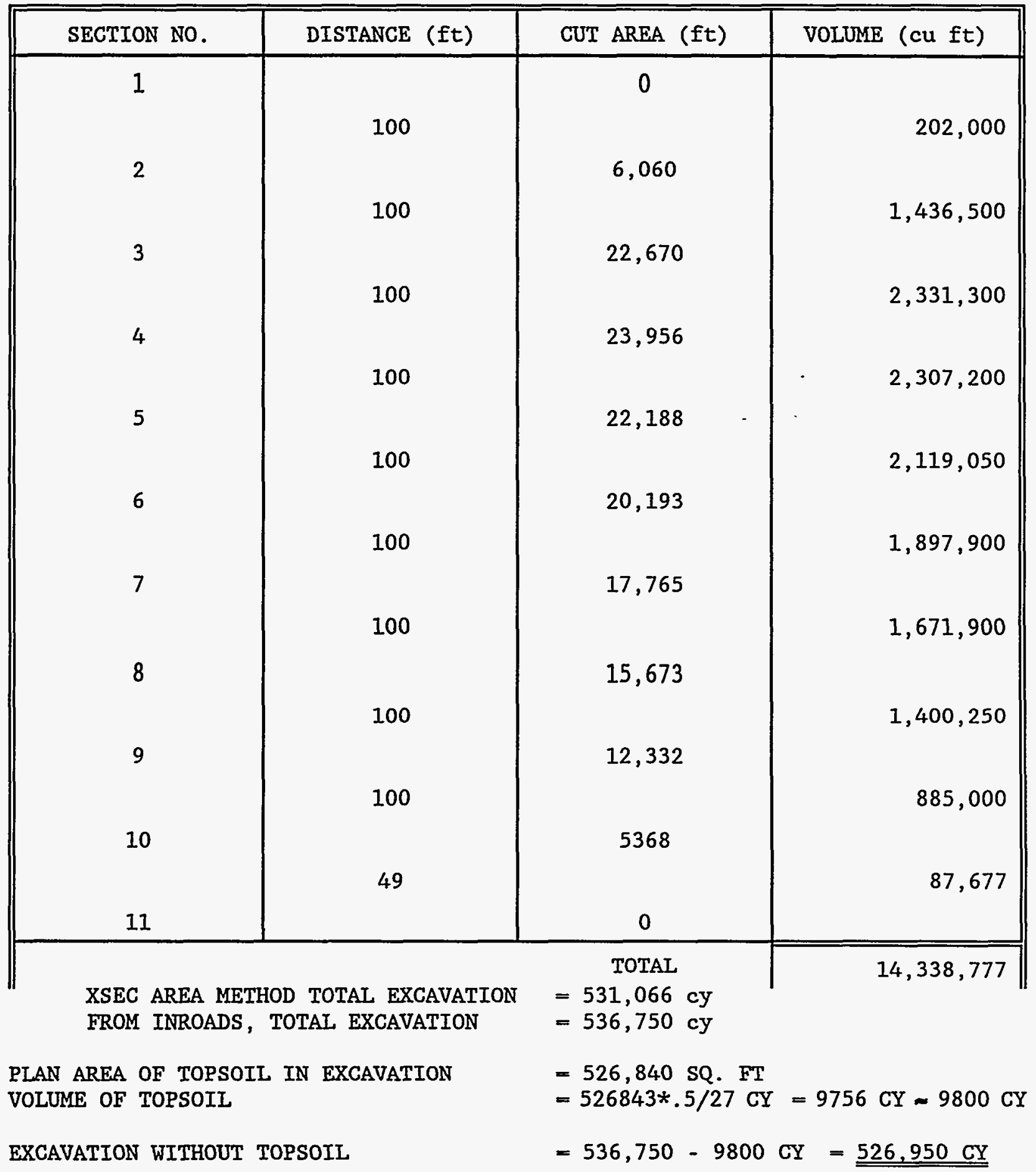


Project UMIRA- SRK

Feolure ELBAKLENT QUANIITSS

Item

EXCAVATION

AND COVER MATERTALS

Contract No. $3885-76$

Designed

Checked

$\sqrt{S R}$

JSR

Sheet 5

File No.

Date 01/25/94

This entive sheet is

part of ReV ol

SECTION I

Excavation Area $=0$

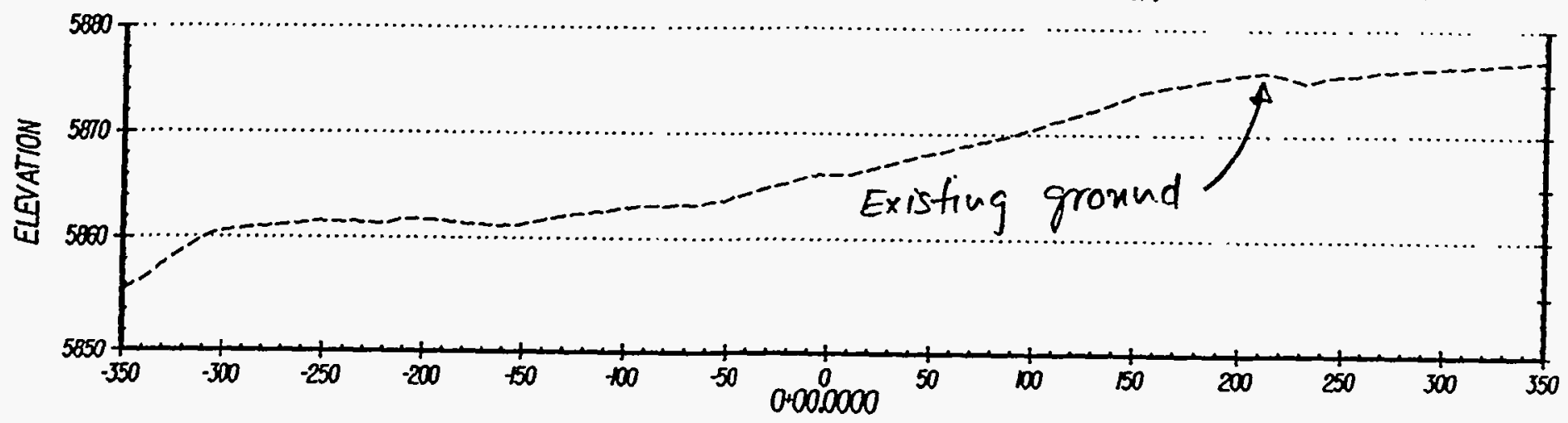

SECTION 2

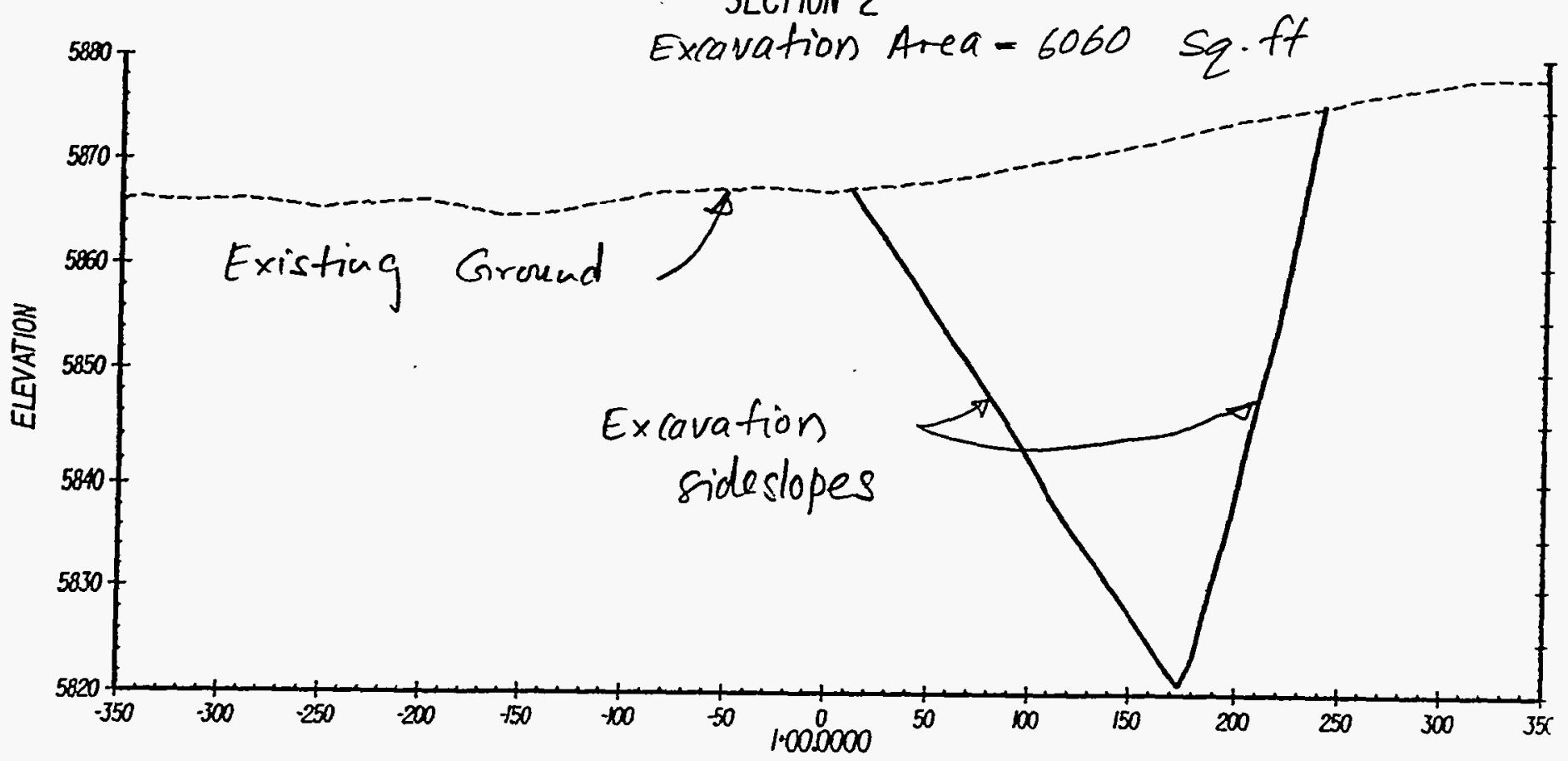


Project UMTRA- SRK

Feoture ELBAKKLENT OUNTIITES

Item EXCAVATHON

COVER MATERIALS

Contract No. $3885-76$

Sheet 6

File No.

Designed

Checked

CSTL

Date

Date

01/25/94

This entire sheet is port of Revol

SECTION 3

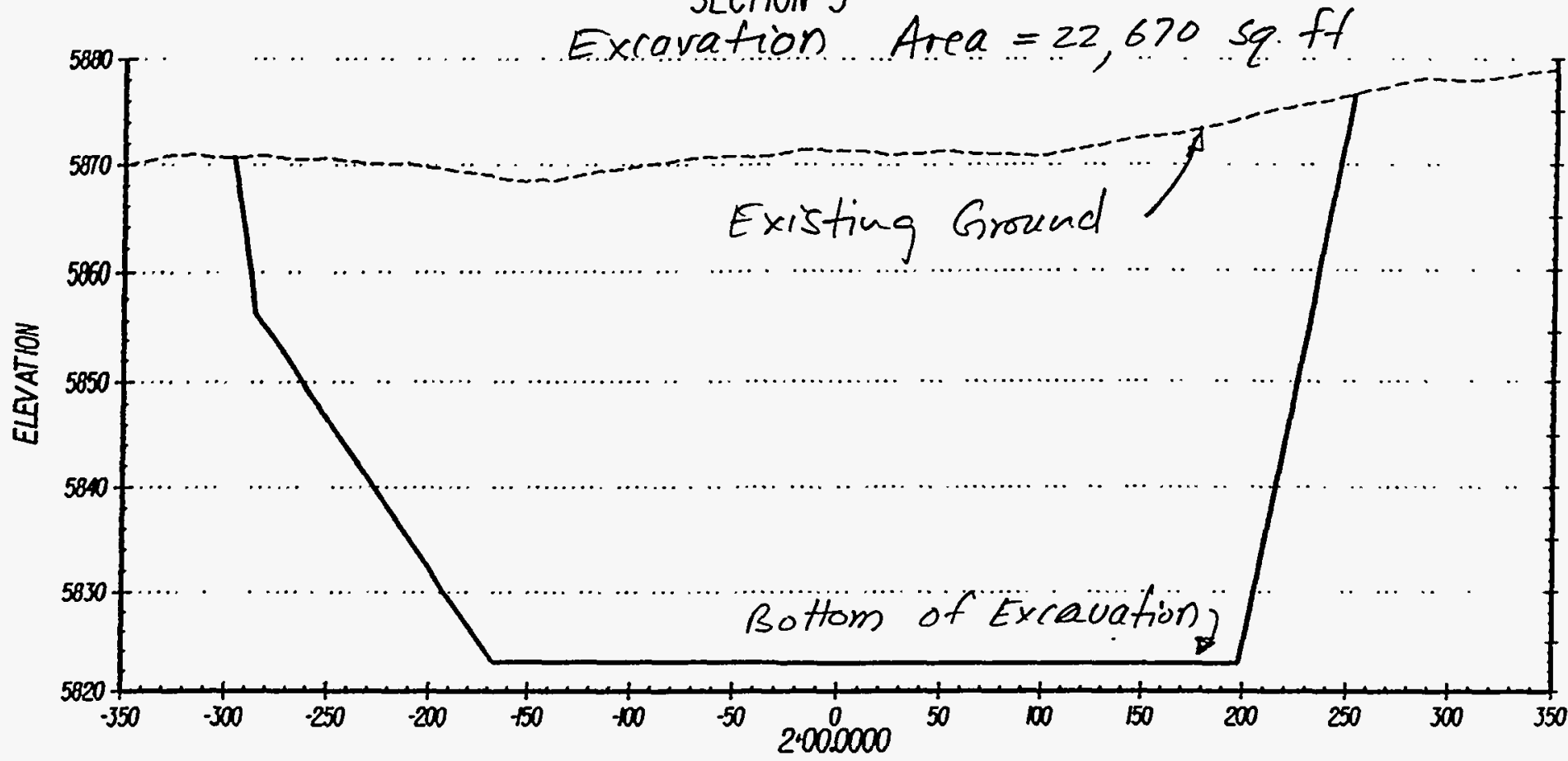

SECTION 4

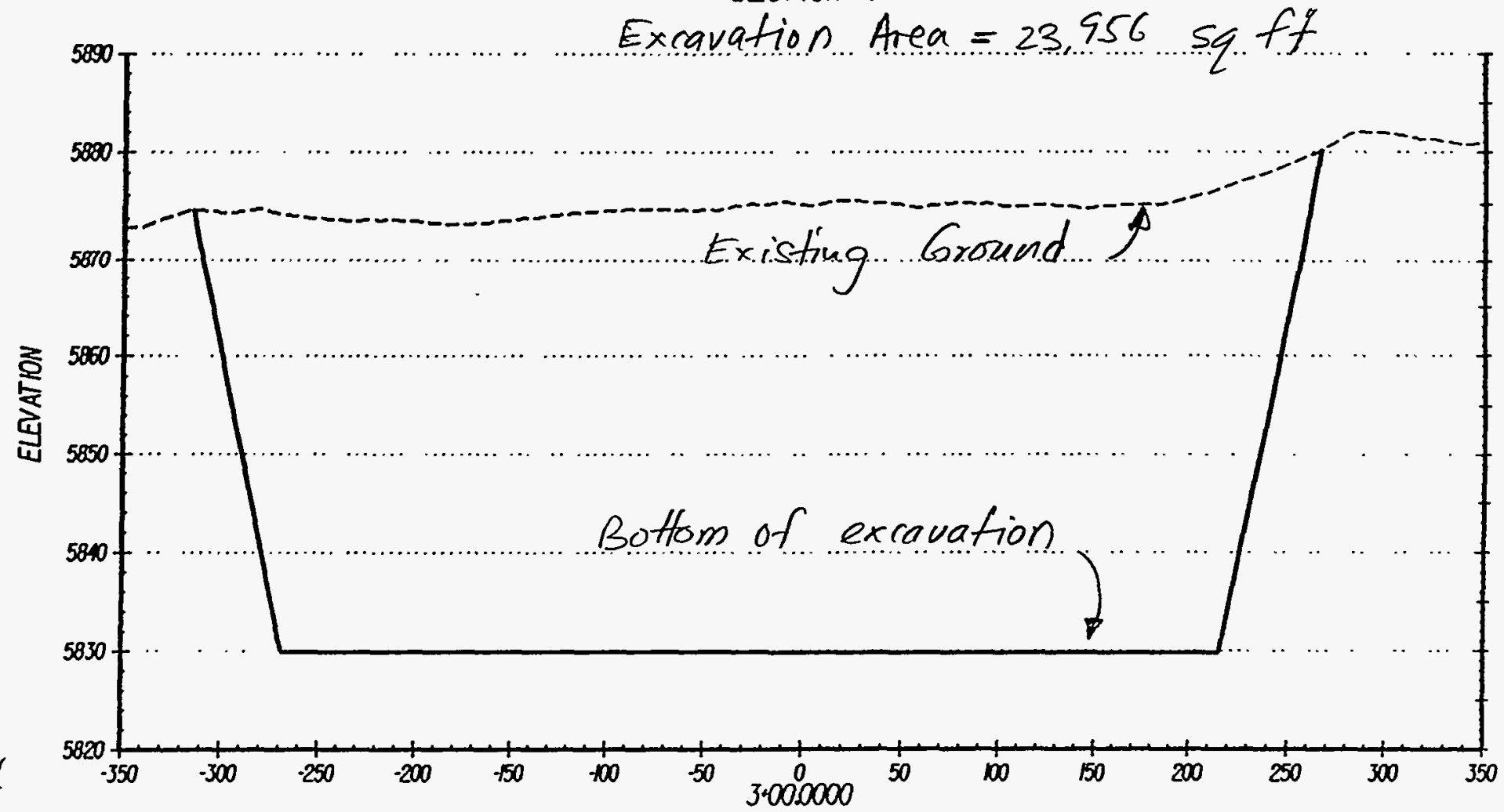


Project

Feoture EMBAKLENT QUANTITES

Item EXCAVATION,

AND COVER MATERIALS
Controct No. 3885-76

Designed

Checked CrLC
Sheet 7

File No.

Date

Dote

$01 / 25 / 94$

$1 / 2+194$

This entrre sheet is

rayt of Rovis

SECTION 5
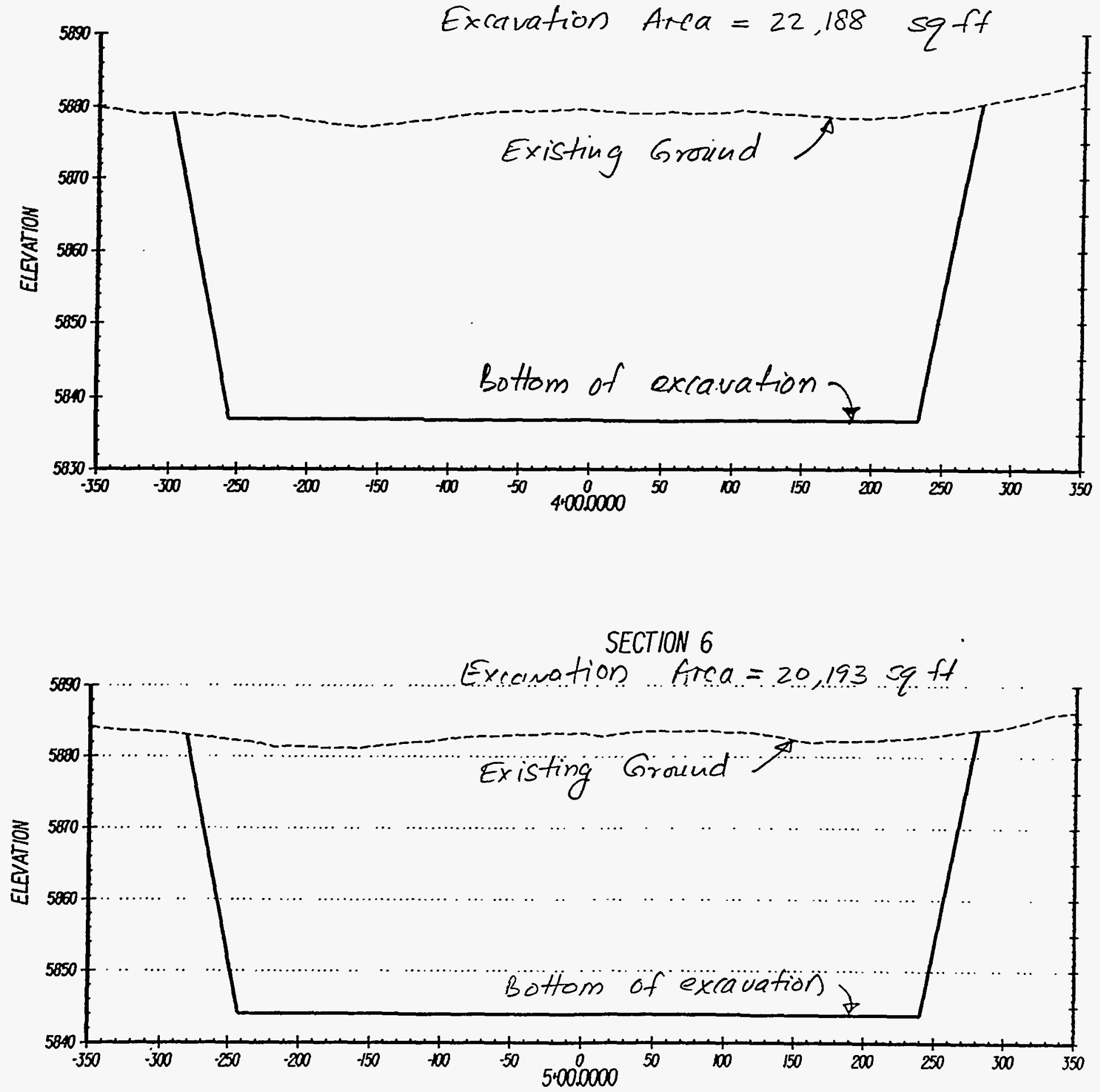


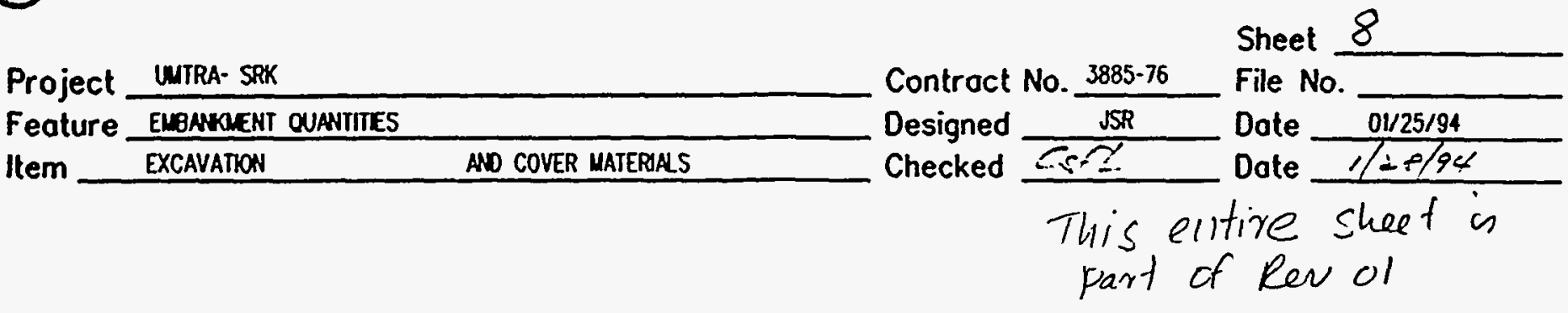

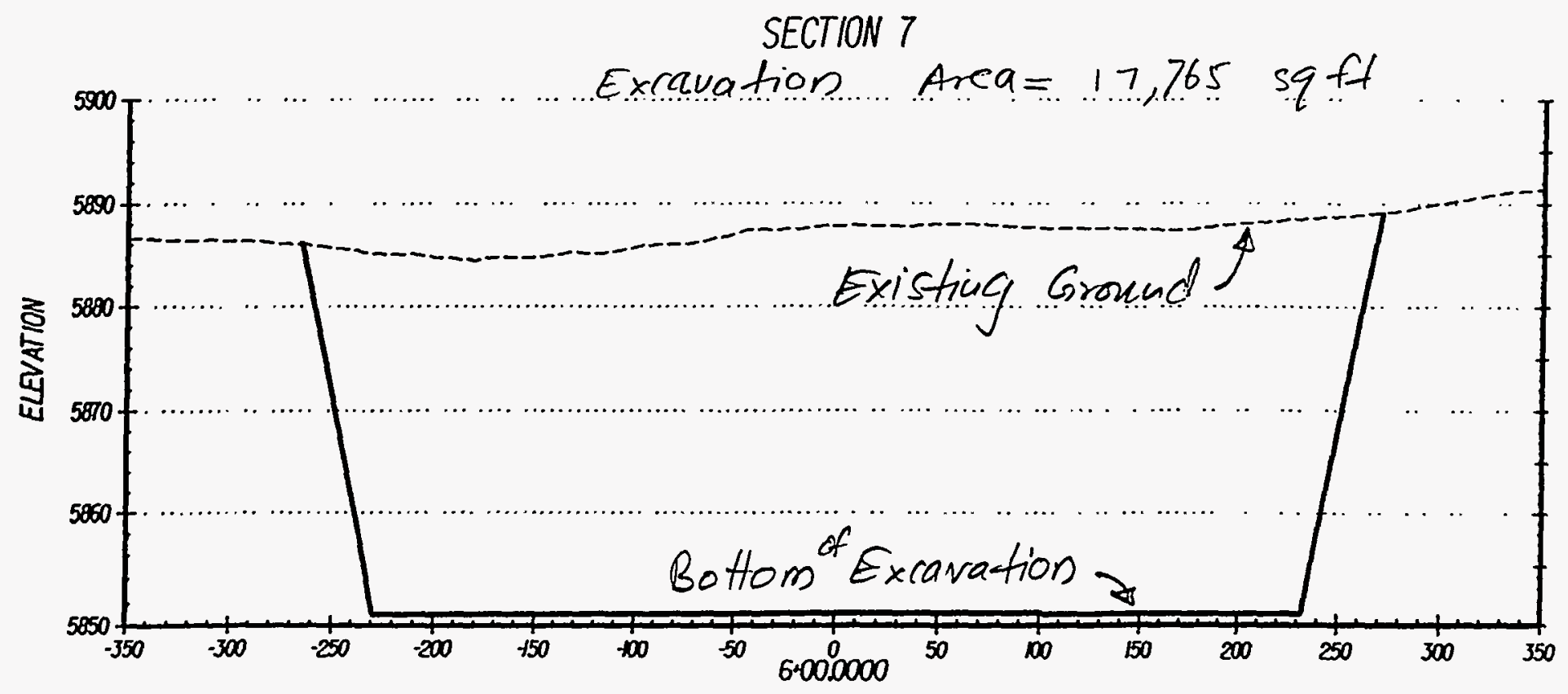

SECTION 8

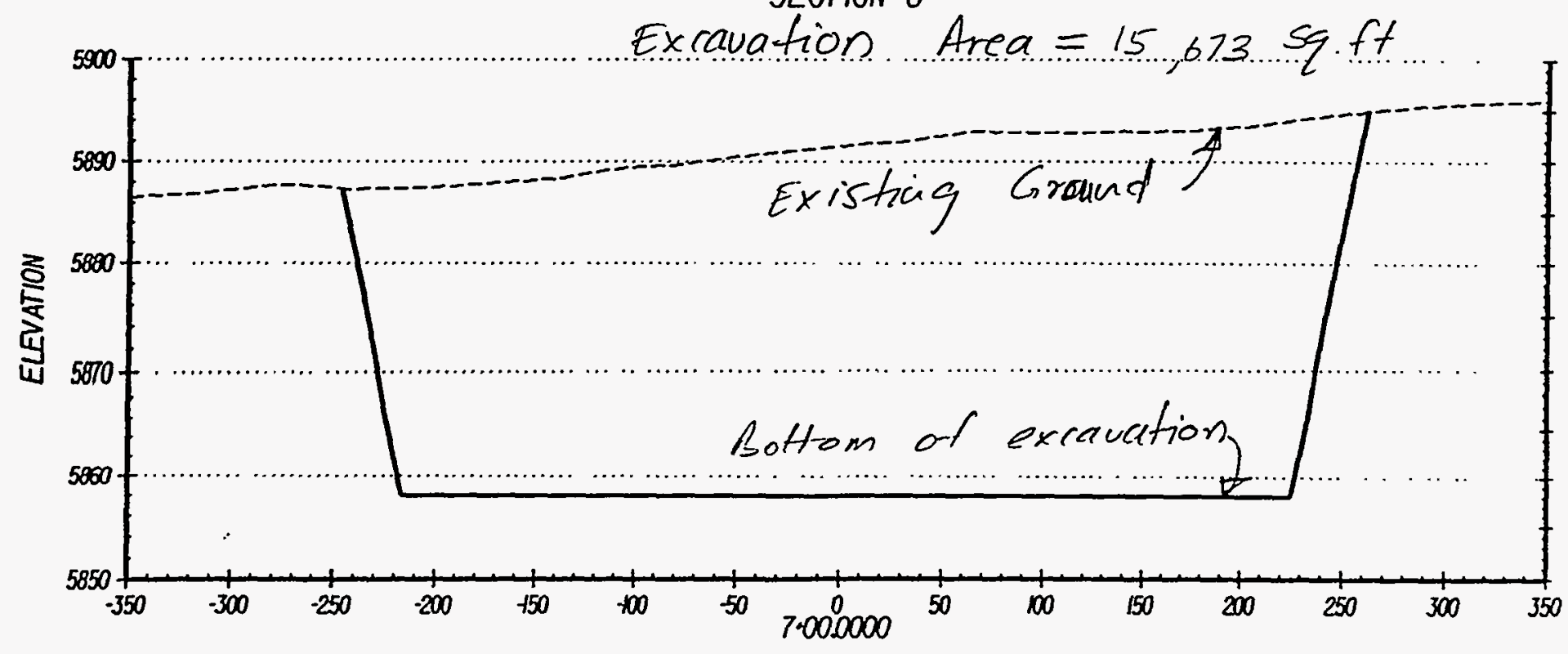


Project UUTRA- SRK

Feoture ELBAAKLENT QUANTITES

Item EXCAVATION AND COVER MATERIALS
Contract No. 3885-76

Designed Checked

Sheet 9 File No.

Date $\frac{\frac{01 / 25 / 94}{1 / 28 / 94}}{\text { Sheet is }}$

SECTION 9

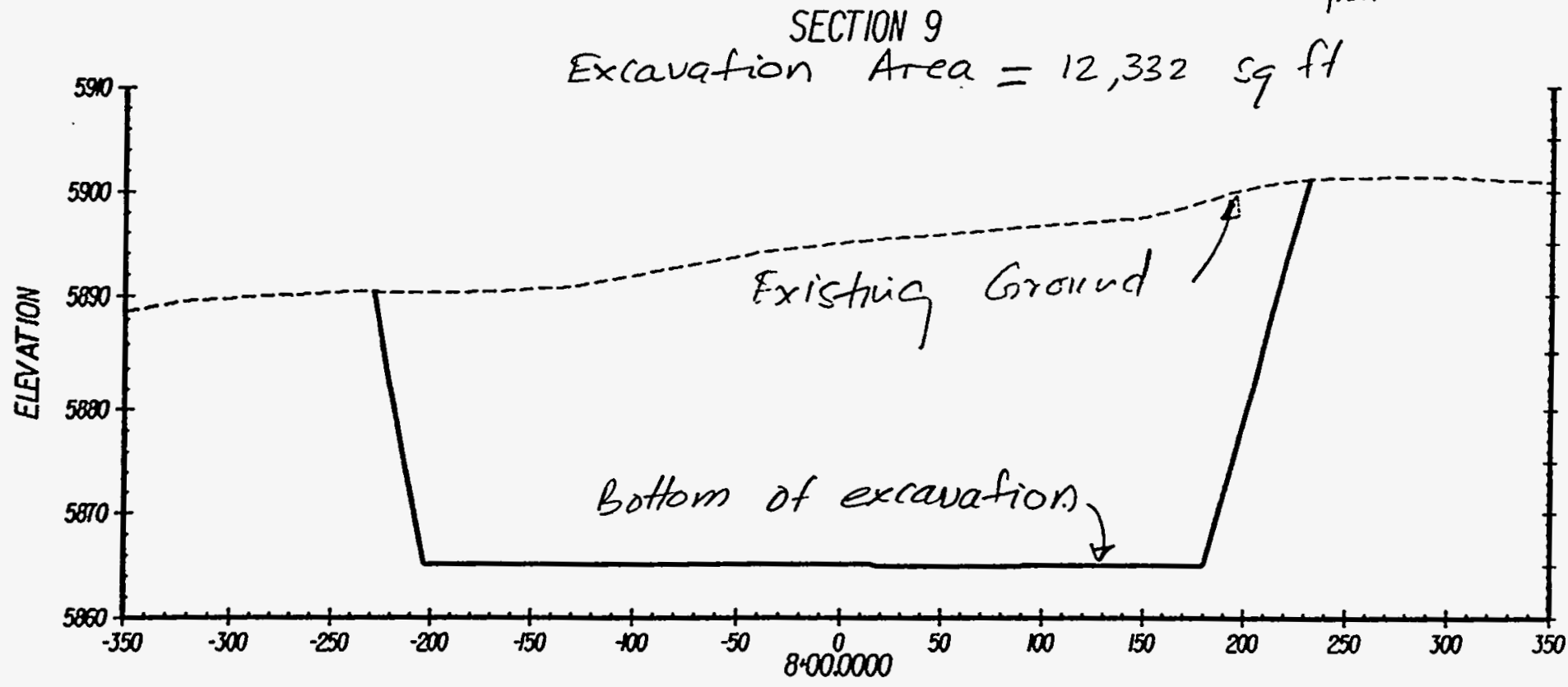

SECTION 10

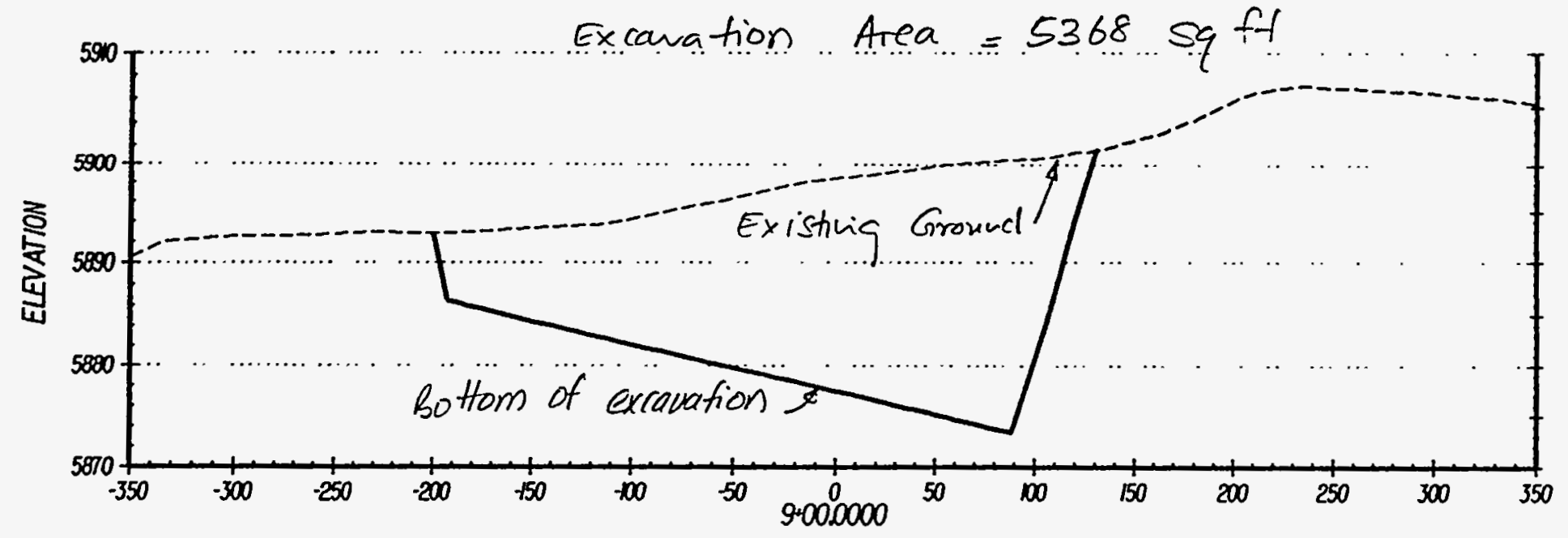


Project UMTRA-SRK

Feoture EMBANKMENT QUANTITIES

Item EXCAVATION. ( COVER MAT'LS Checked

Sheet

11

Controct No. $\frac{3885-76}{\text { AFS File No. }}$ Dote $3 / 12 / 93$
Designed

APRON EXCAVATION:

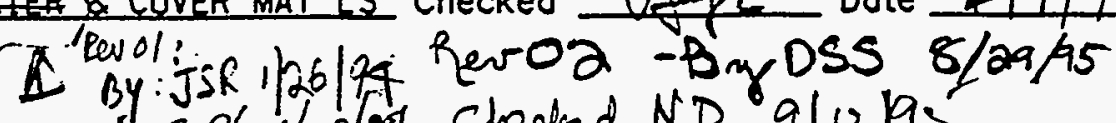

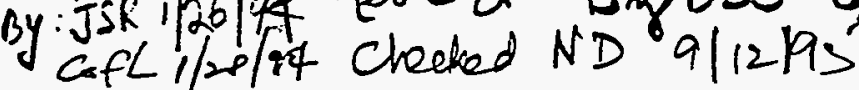

SHEET 10 SHOWS AN ALIGNMENT ALONG THE TOE OF THE EMBANKMENT SIDE SLOPES. THE TOTAL LENGTH OF THE ALIGNMENT. INCLUDING CHANGES IN ELEVATION, IS $2724 \mathrm{ft}$.. THE TYPICAL APRON SECTION IS SHOHN BELOH. INROADS IS USED TO PLACE THE BOTTOM OF "EXCAVATION" EVERY $10 \mathrm{ft}$ ALONG THE ALIGNMENT IN ORDER TO GENERATE THE ACTUAL EXCAVATION QUANTITY. ( THE TYPICAL EXCAVATION $X$ APRON LENGTH CANNOT BE USED, BECAUSE IN SOME AREAS THE APRON IS IN FiLL.)

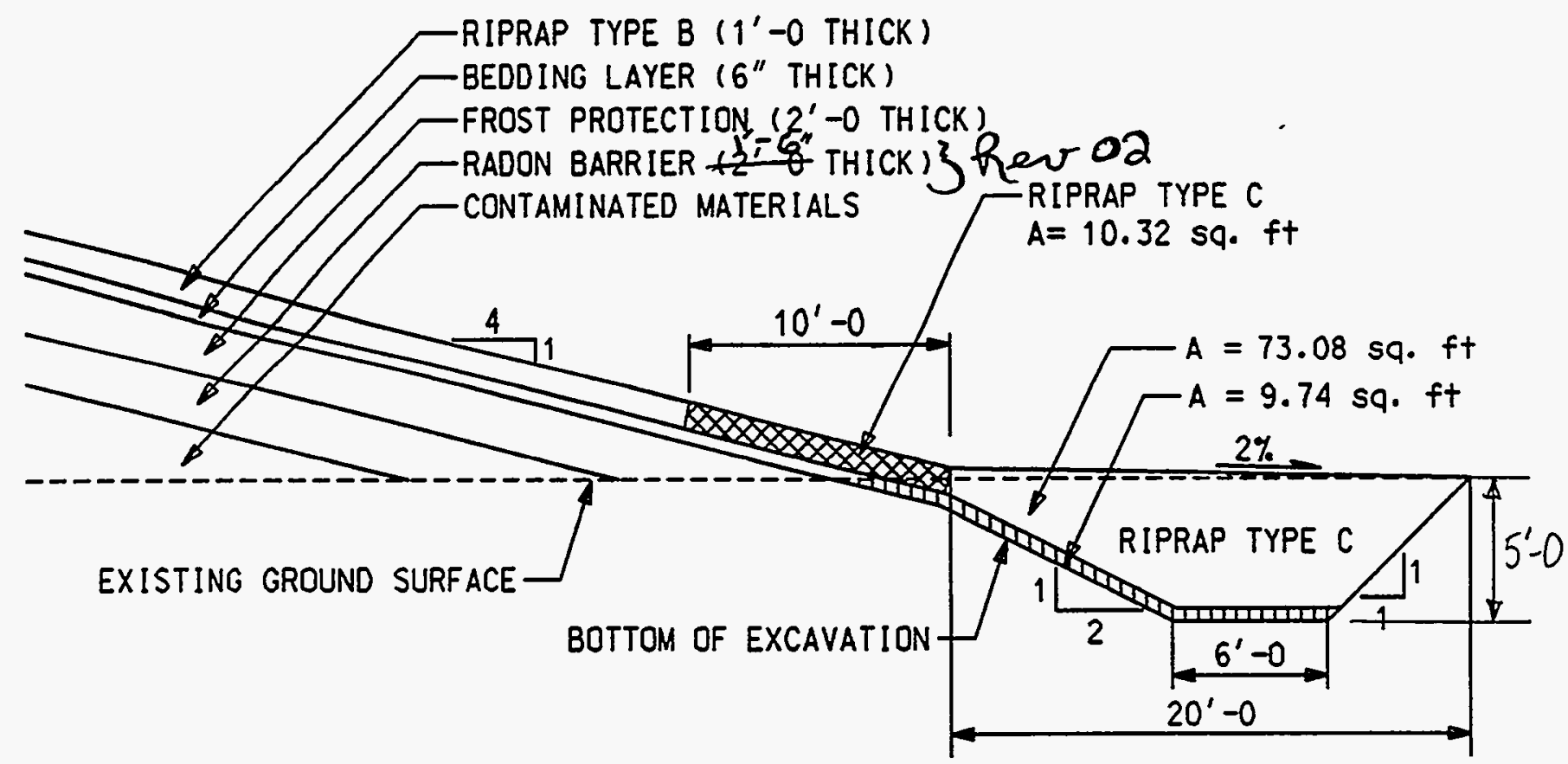

FROM INRDADS, TOTAL APRON EXCAVATION $=\underline{\underline{3304 \mathrm{cy}}}$

FOR USE IN RIPRAP TYPE C VOLUME CALC., SHEET 12:

SECTION THROUGH COVER MATERIALS AT EMBANKMENT TOE (APRON NOT SHOWN)

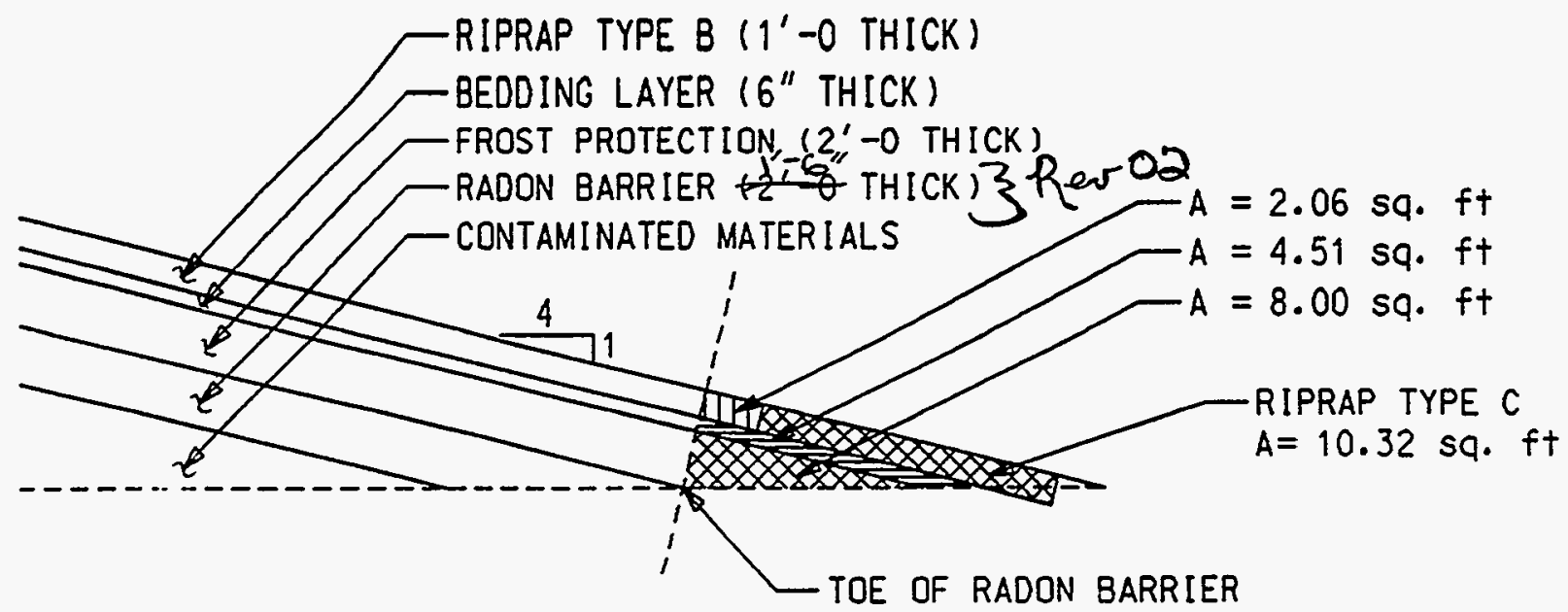


RIPRAP TYPE A IN COVER:

SHEET 13 SHOWS THE INROADS CONTOURS USED TO COMPUTE THE SURFACE AREA OF RIPRAP. FROM INROADS, THE SURFACE AREA OF RIPRAP TYPE A (SEE SHEET 10) IS 113,560 $\mathrm{ft}^{2}$. FROM SHEET A-3, THE THICKNESS OF RIPRAP TYPE A IS 8 in.

$$
\text { VOLUME }=113,560 \times 8 / 12=75,707 \mathrm{ft}^{3}=\underline{\underline{2800} \mathrm{cy}} \approx \text { RIPRAP TYPE A }
$$

\section{RIPRAP TYPE B IN GOVER:}

FROM INROADS, THE SURFACE AREA OF THE TOP-OF-EMBANKMENT SURFACE WITHIN RIPRAP TYPE B LIMITS IS $349,050 \mathrm{ft}^{2}$. FROM SHEET A-3, THE THICKNESS OF RIPRAP TYPE B IS $1 \mathrm{ft}$.

$$
\text { VOLUME }=349,050 \times 1=349,050 \mathrm{ft}^{3}=12,930 \mathrm{cY} \approx \text { RIPRAP TYPE B }
$$

\section{RIPRAP TYPE $G$ IN GOVER AND APRON:}

FROM SHEET 11, THE TOTAL LENGTH OF EMBANKMENT TOE IS $2724 \mathrm{ft.}$ SHEET 11 ALSO SHOWS THAT THE AREA OF RIPRAP TYPE C IN THE TYPICAL APRON SECTION IS $73.08 \mathrm{ft}^{2}$.

$$
\text { VOLUME }=2724 \times 73.08=199,070 \mathrm{ft}^{3}=\underline{7370 \mathrm{cy}} \Rightarrow \text { RIPRAP TYPE C IN APRON }
$$

SHT. 11 SHOWS THAT THE AREA OF RIPRAP TYPE C ON THE COVER IS $10.32 \mathrm{ft}^{2}$.

$$
\begin{gathered}
\text { VOLUME }=2724 \times 10.32=28,112 \mathrm{ft}^{3}=1040 \mathrm{cY} \text { RIPRAP TYPE C IN COVER } \\
\text { TOTAL VOLUME RIPRAP TYPE } \mathrm{C}=7370+1040=\underline{8410 \mathrm{cY}}
\end{gathered}
$$

BEDDING MATERIAL IN APRON AND COVER:

SHEET 11 SHOWS THAT THE TYPICAL APRON SECTION HAS $9.74 \mathrm{ft}^{2}$ OF BEDDING-MATERIAL. FROM SHEET 11, THE TOTAL LENGTH OF APRON IS $2724 \mathrm{ft}$.

$$
\text { VOLUME }=2724 \times 9.74=26,532 \mathrm{ft}^{3}=980 \mathrm{cy} \text { BEDDING IN APRON }
$$

SHEET 14 SHOWS THE INROADS CONTOURS FOR THE TOP-OF-RADON BARRIER SURFACE. THE TOTAL SURFACE AREA OF THIS SURFACE IS $485,370 \mathrm{ft}^{2}$.

REF. DWG. 非 SHOWS THAT THE BEDDING LAYER IS 6 in. THICK.

$$
\text { VOLUME }=485,370 \times .5=242,685 \mathrm{ft}^{3}
$$

THE LENGTH OF THE RADON BARRIER TOE, INCLUDING CHANGES IN ELEVATION, IS 2642 ft. THE SECTION THROUGH THE BEDDING TOE ON SHEET 11 SHOWS THAT S.A. RADON BARRIER $x$ THICKNESS DOES NOT INCLUDE A TYPICAL AREA OF $4.51 \mathrm{ft}^{2}$ OF BEDDING.

TOTAL VOLUME $=242,685+4.51 \times 2642=254,600 \mathrm{ft}^{3}=9430 \mathrm{cy}$ BEDDING IN COVER TOTAL VOLUME BEDDING $=9430+983=10,410 \mathrm{cY}$ 


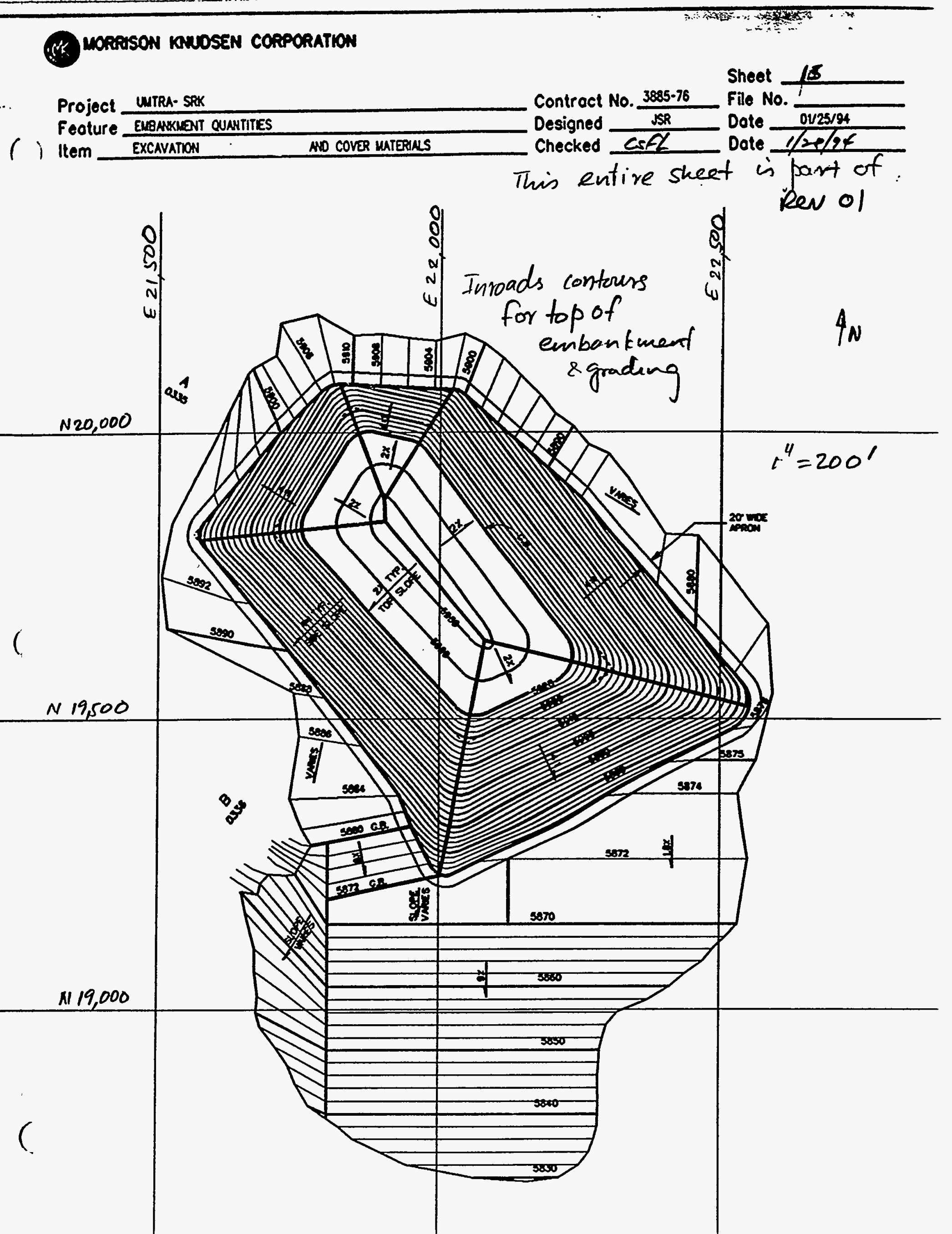




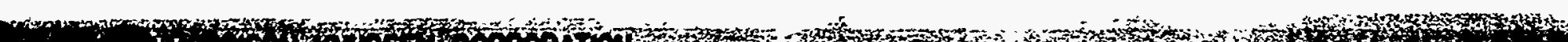

Project UMTRA-SRK

Contract No. $3885-76$

File No.

Feoture emaankment guantiLies

\section{Designed}

Date $3 / 12 / 93$

$(6, y)$

Item EXCAVATION SEETT OAVER MATERIALS II By ISR $1 / 26 / 94$

Dote $4 \operatorname{la} 193$

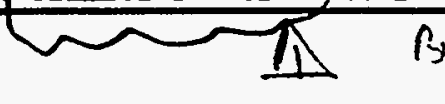
Checked
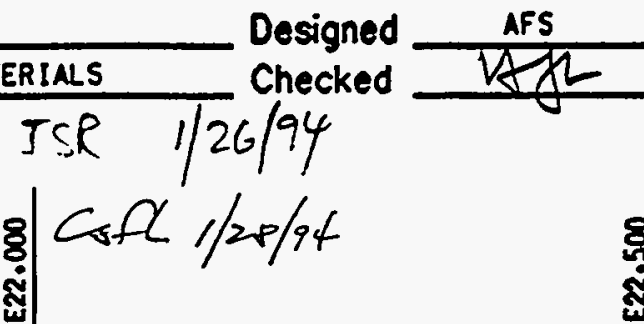

్ㅗㅀ

N 20.000

N 19.500

TOTAL SURAACE AREA $=485.370$ sq.

$\mathrm{ft}$

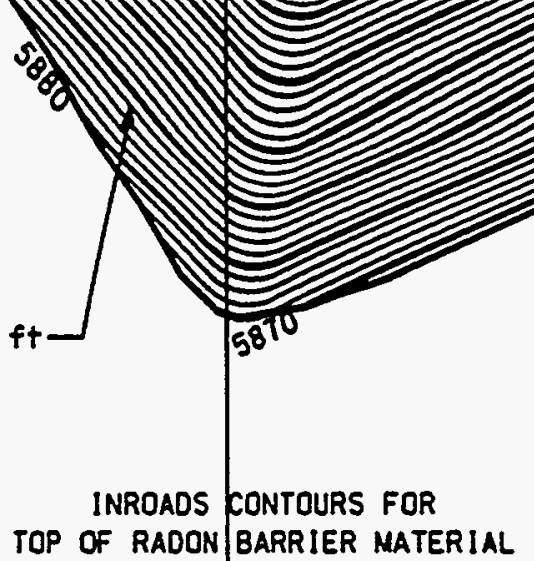

N 19.000

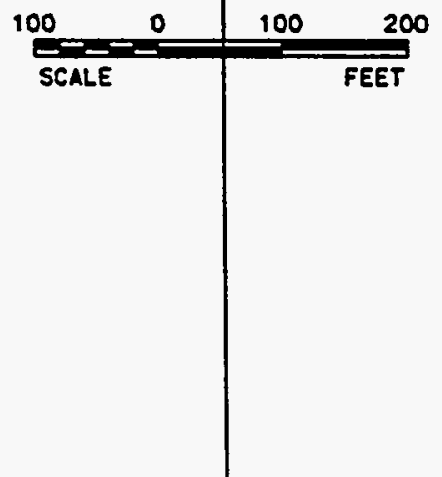


Project UMTRA

Feature EMBANKMENT QUANTITIES

Contract No. $\quad 3885-76$

Sheet 15

File No.

Item EXCAV. ST COVER MAT'LS

FROST PROTECTION MATERIAL IN COVER:

FROM SHEET 14, S.A. TOP OF RADON BARRIER $=485,370 \mathrm{ft}^{2}$. REF. DWG. 非 SHOWS THAT THE FROST PROTECTION LAYER IS 2 ft THICK.

$$
\text { VOLUME }=485,370 \times 2=970,740 \mathrm{ft}^{3}
$$

FROM SHEET 12, LENGTH OF RADON BARRIER TOE = $2642 \mathrm{ft.}$ ON SHEET 11 THE SECTION THROUGH THE FROST PROTECTION TOE SHOWS THAT $8.00 \mathrm{ft}^{2}$ OF FROST PROTECTION MATERIAL EXTENDS BEYOND THE RADON BARRIER TOE.

TOTAL VOLUME $=970,740+2642 \times 8.00=991,876 \mathrm{ft}^{3}=\underline{36,740 \mathrm{cy}}$ FROST PROTECTION

\section{RADON BARRIER MATERIAL IN COVER:}

THE RADON BARRIER SURFACE AREA ON SHEET 14 IS MEASURED WITHIN THE LIMITS OF THE Cheoked: TOE OF RADON BARRIER SHOWN ON THE SECTION ON SHEET 11 . THEREFORE, AN OVERLAP ND9/12 955
VOLUME OF (8.00 $\mathrm{ft}^{2} \times$ LENGTH OF R.B. TOE) IS INCLUDED IN THE VOLUME S.A. $\times$.

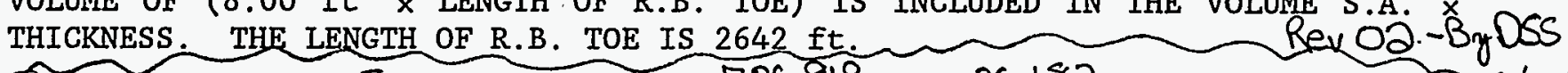

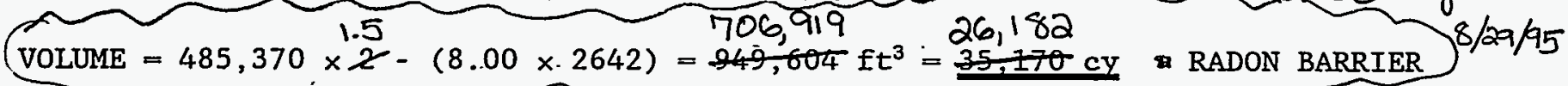
SEEPAGE BARRIER MATERIAL ON EXCAVATION SIDE SLOPES:

THE EXCAVATION PLAN IS SHOWN ON SHEET 4. FROM INROADS, THE SURFACE AREA OF THE EXCAVATIOK SIDE SLOPES IS $214,210 \mathrm{ft}^{2}$. THE SECTIONS ON REF. DWGS. 非 3 AND 非 4 SHOW THAT THE SEEPAGE BARRIER MATERIAL WILL BE PLACED HORIZONTALLY, 8 ft WIDE. THE SKETCH BELOW SHQWS THAT THE MATERIAL WILL THEN BE $2.9711 \mathrm{ft}$ THICK.

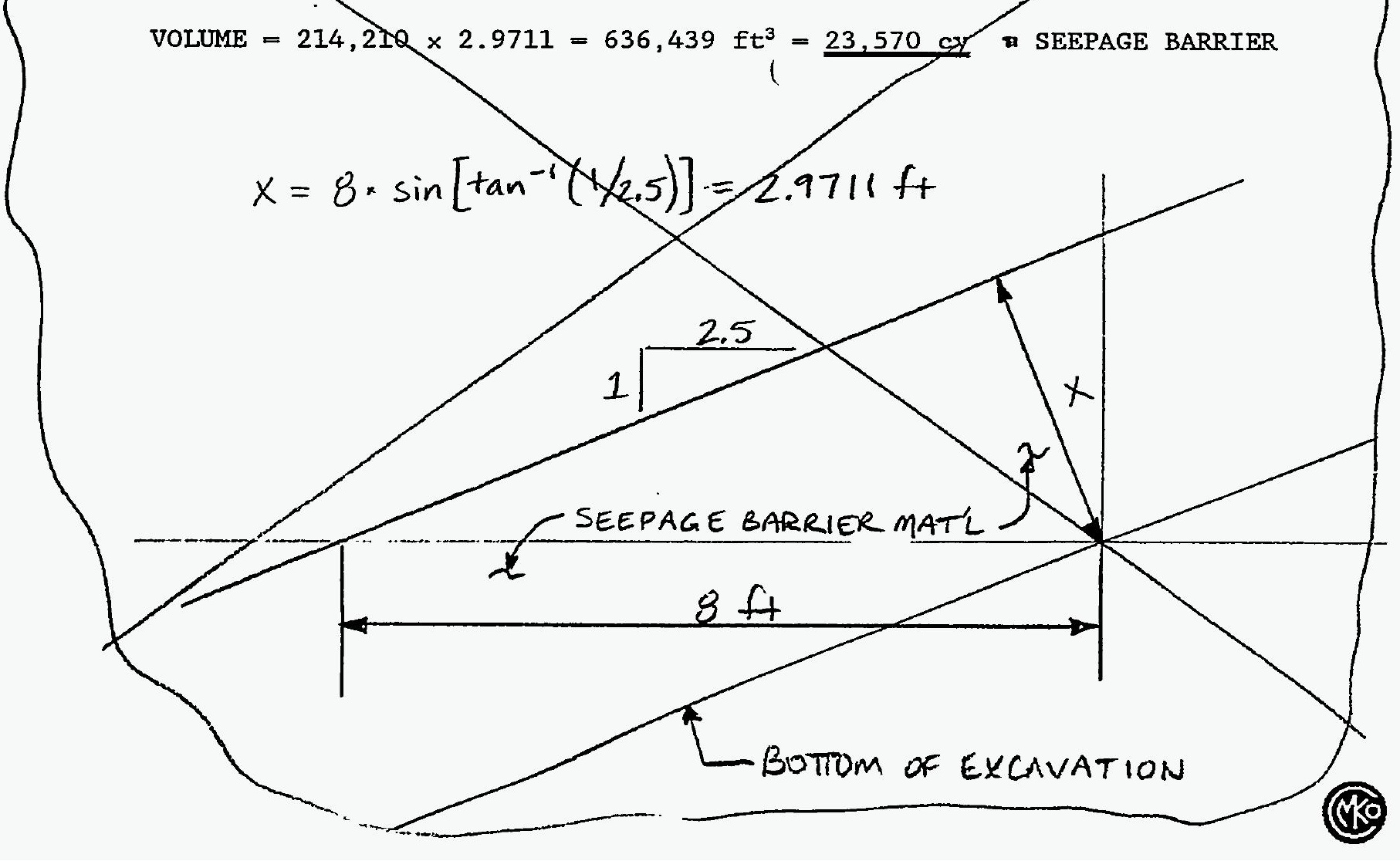




\section{RIPRAP A, B AND BEDDING MATERIAL WEST OF DISPOSAL CELL}

Riprap A west of cell

Plan area of Riprap $A$ (From sheet $A-5$ )

$=13,750 \mathrm{sq} . \mathrm{ft}$

Thickness of Riprap $A$ (From sec. $A$, sht $A-5$ and sec. $C$, sht $A-3$ ) = $1 \mathrm{ft}$

Volume of Riprap $A$

$=509 \mathrm{cy} \approx 510$

cy

Riprap B west of cel1

Plan area of Riprap B (From Sheet A-5)

$=23,625 \mathrm{sq}$.

$\mathrm{ft}$

Thickness of Riprap B (From section A, sht A-5)

$=1 \mathrm{ft}$

Volume of Riprap B

$=875 \mathrm{cy}$

Bedding west of cell

PTan area of bedding material (From Sheet A-5)

$=37,375 \mathrm{sq} . \mathrm{ft}$

Thickness of bedding material (From section $A$, sht A-5)

$=6$ in

Volume of bedding material

$$
=692 \mathrm{cy} \approx 700 \mathrm{cy}
$$

\section{STOCKPILES}

Temporary topsoil stockpile

Total disturbed area (See item 10 of sht 2)

$=1,343,250 \mathrm{sq} . \mathrm{ft}$

Thickness of topsoil

$=6$ in

Volume of temporary topsoil stockpile

$=24,875 \mathrm{cy}$

Volume of topsoil neeeded for finish grading (from sht $i i i$, Ref 7 ) $=15,100 \mathrm{cy}$

\section{Permanent stockpile}

Volume of materials from 1:1 excavation (from sht 3) $=526,950 \mathrm{cy}$

Volume of material from apron excavation (from sht $11=3,300 \mathrm{cy}$

Total excavated material from embankment $\quad=526,950+3300=530,250 \mathrm{cy}$

Material needed for frost barrier (from sht 15) $=36,750 \mathrm{cy}$

Material needed for final grading (from sht iii, Ref. 7) $=117,000 \mathrm{cy}$

Volume of excess material $=530,250-36750-117,000 \mathrm{cy}$

$=376,500 \mathrm{cy}$

Volume of design stockpile (sht A-5) (from inroads)

$=413,200 \mathrm{cy}$

Therefore design stockpile has a 10\% contingency. 


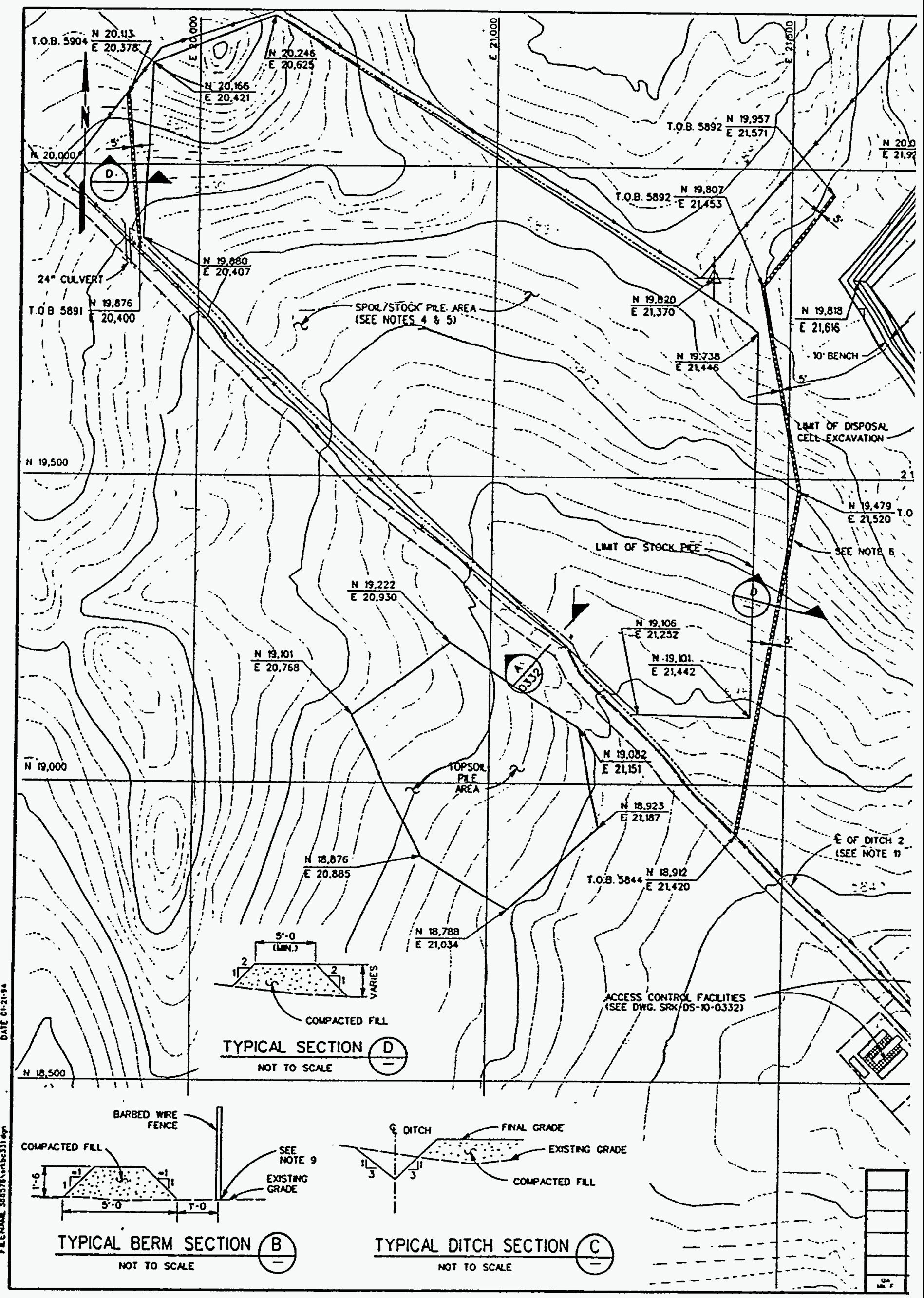




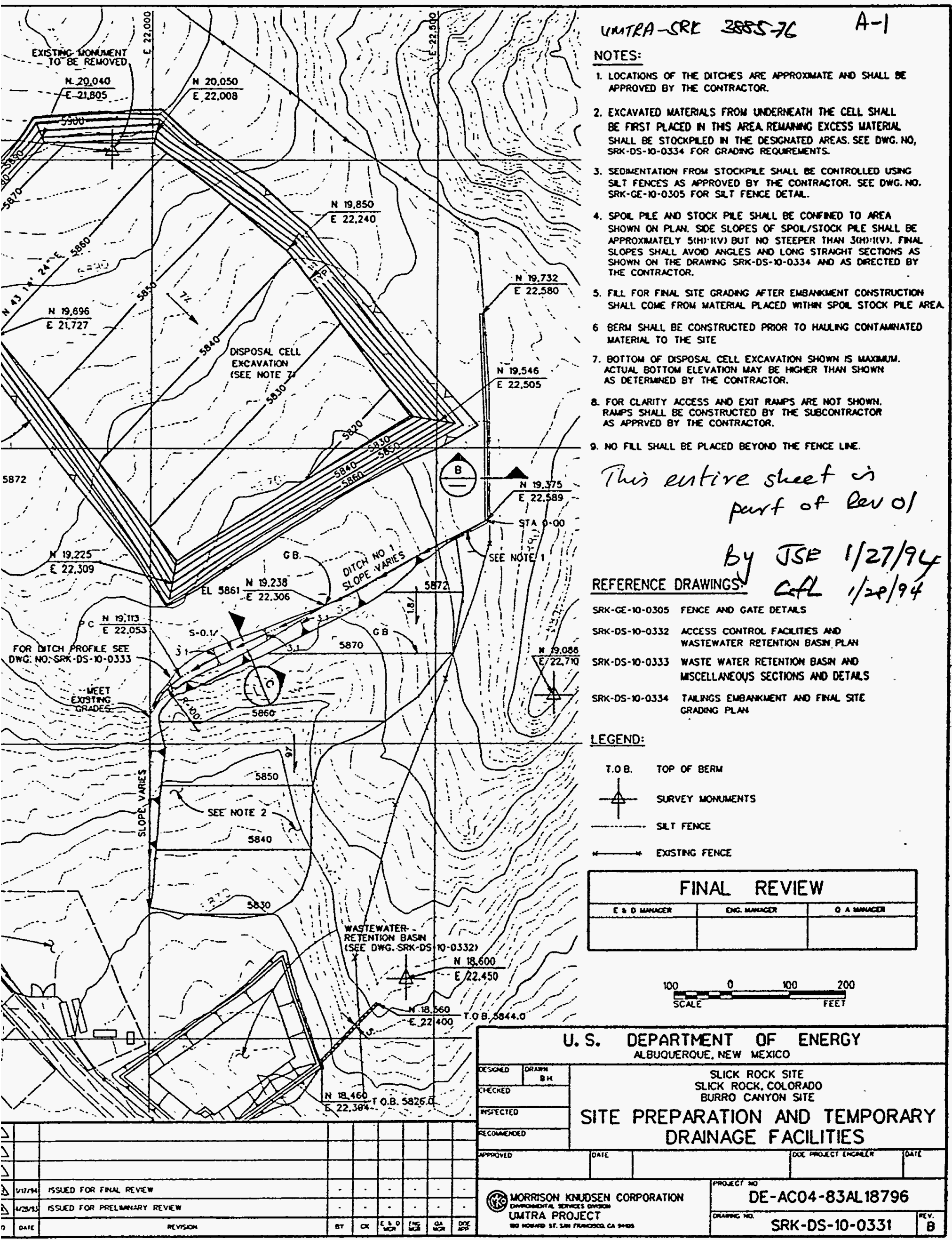




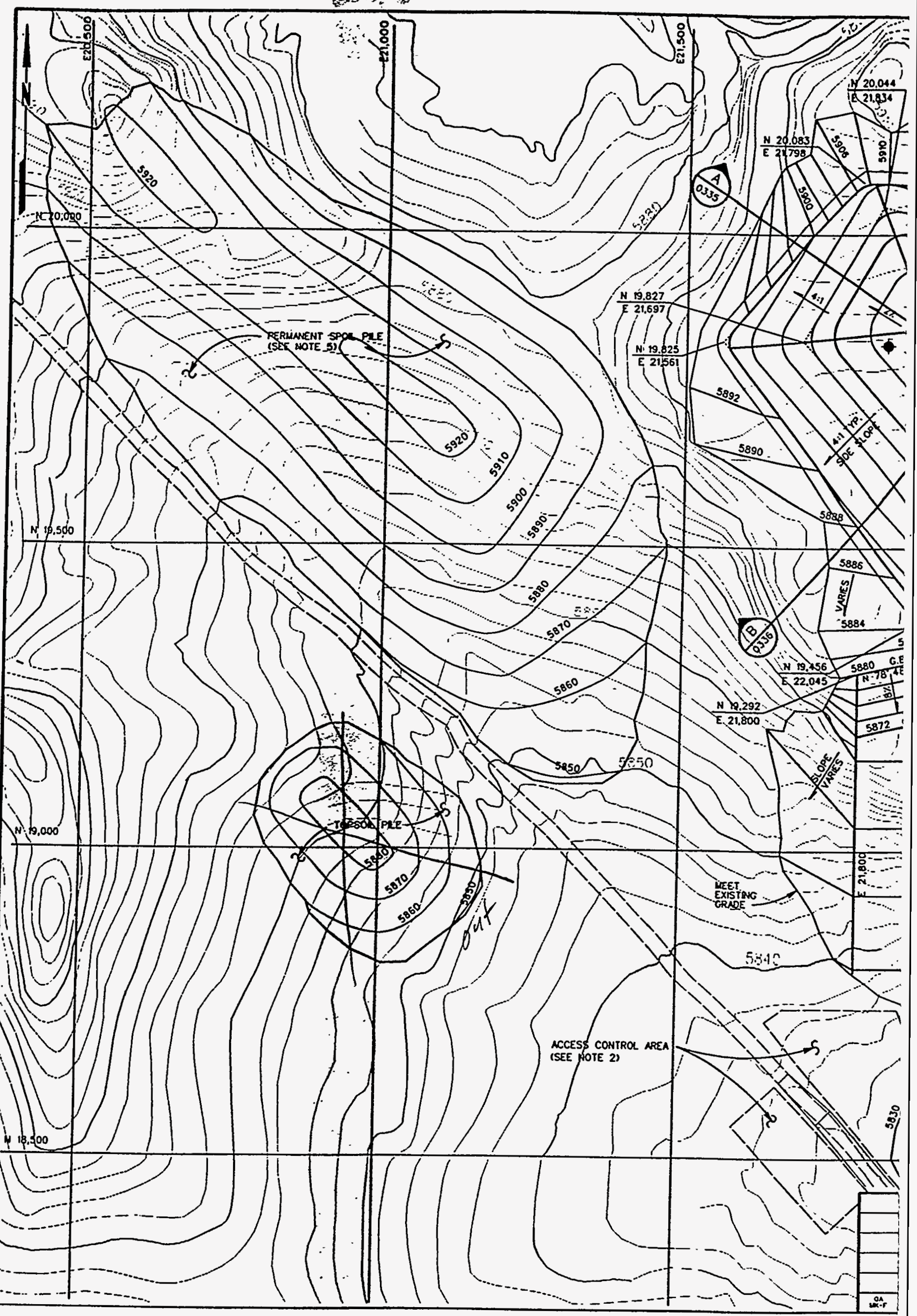




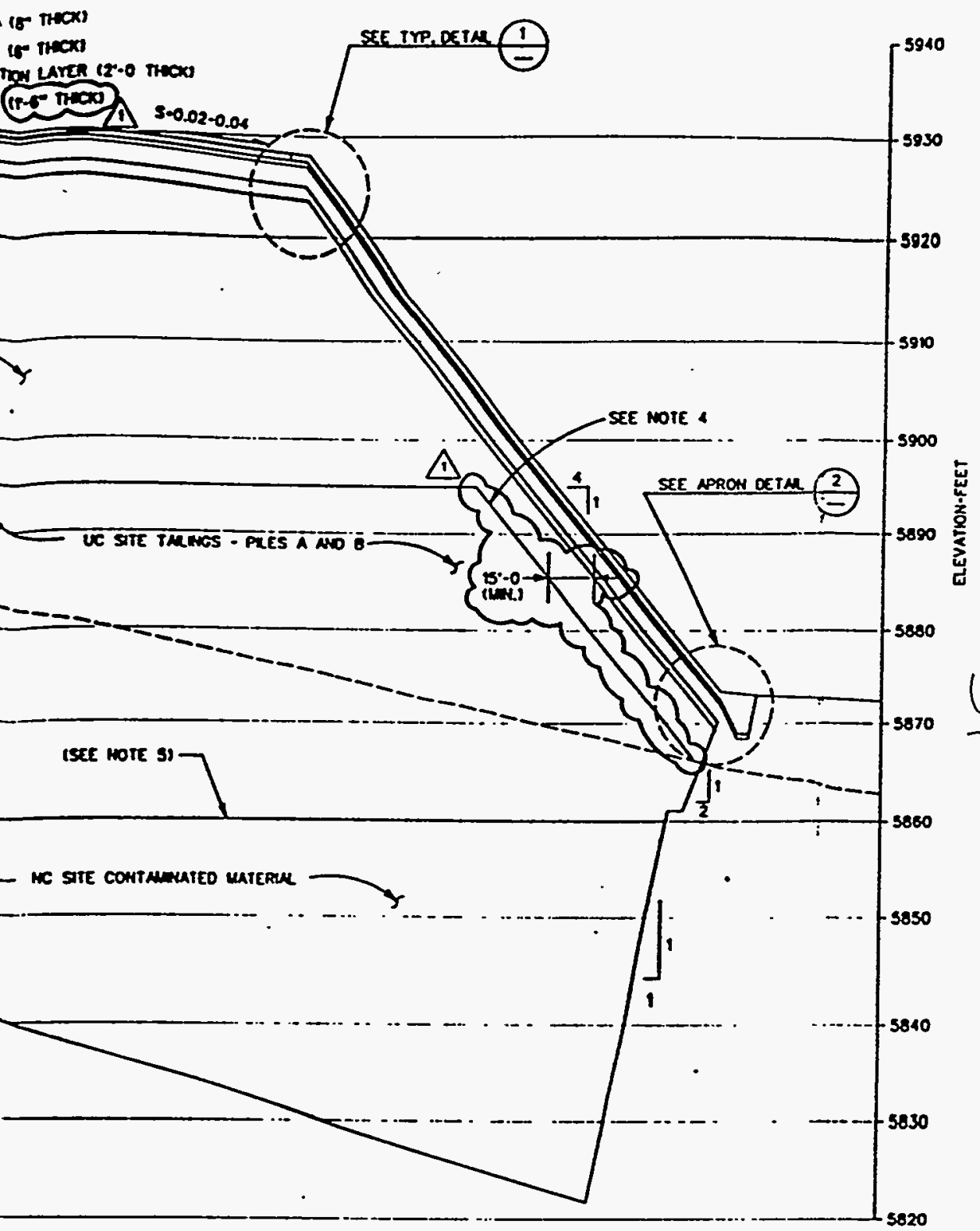

NOTES:

$A-3$

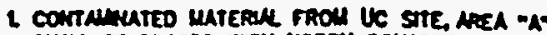

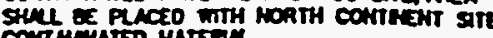
Cortminuted materale.

2. PLE HECOH YARES DEPEDANO LPOH THE VOLUNE of

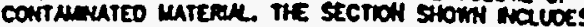
contweency.

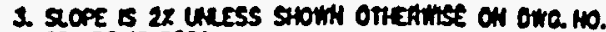

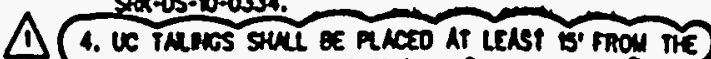
RHOOH GLARER 15 SHOWH.

3. LNTS OF CONTAINATED MATERALS AAE APROXNuTE.

6. DEPTH OF BEHCH TO BE OETERANEO N THE FRZD.

7. oorton of orsposa cell excayation shown is

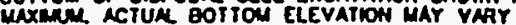
AS DETERMANEO BY THE COMTRACTOR.

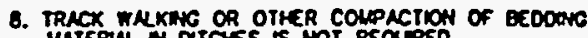
MATERTN W DTOCES IS NOT REOUREO.

\section{REFERENCE DRAWINGS:}

SRK-OS-10-0334 TARAKS EMGANKLENT MO FRK STIE CRAOHG PLAH

UMTRA-SRK

This entive sheet is part of

Revision $\mathrm{O2}_{2}$

checked:

$$
\text { - By DSU 8/29/95 }
$$
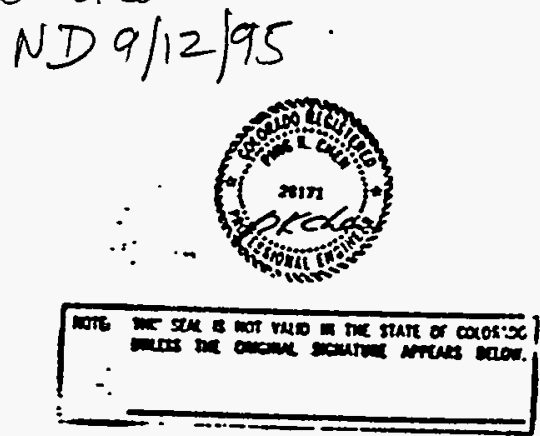

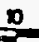

FEET
PE C

$\frac{1}{1}$

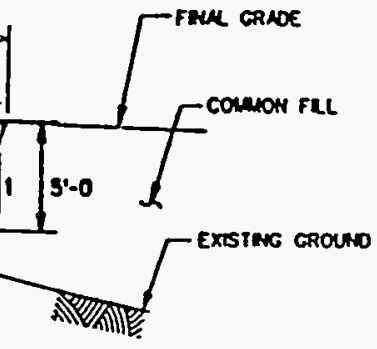

RPPAP ITPE A IT-O THC BEDOANG LAYER (G" THICK) FNAL CRAOE

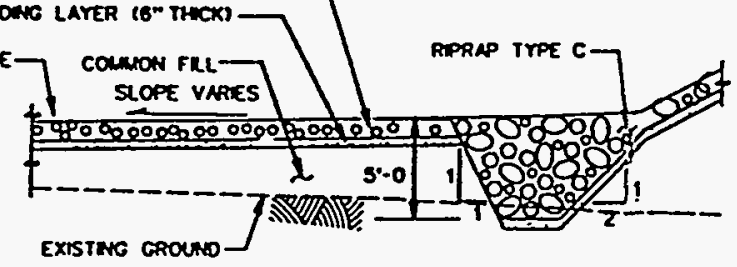

SECTION C HOT TO SCNE 0337
OA REVIEWED FOR OUN TIY REOUTREMENTS BY: G. DQYLE 6/2/94
NOW FLL

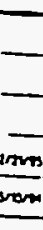

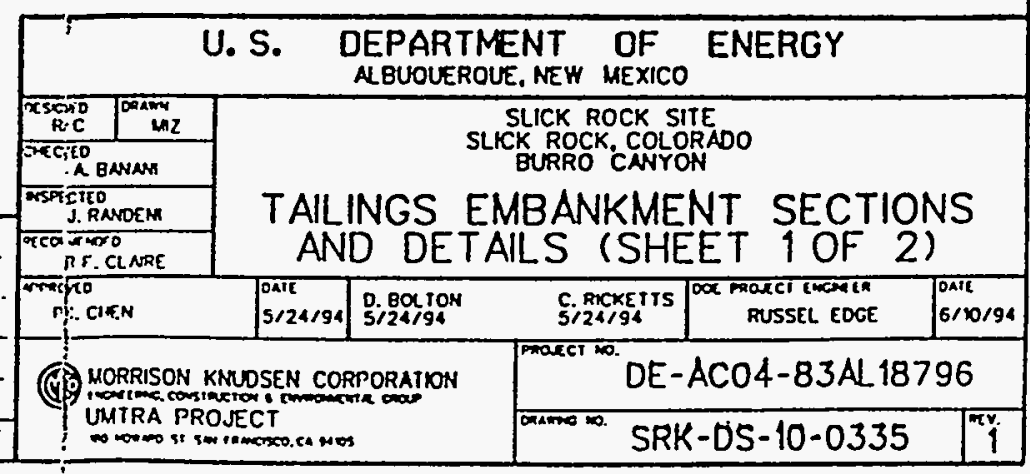




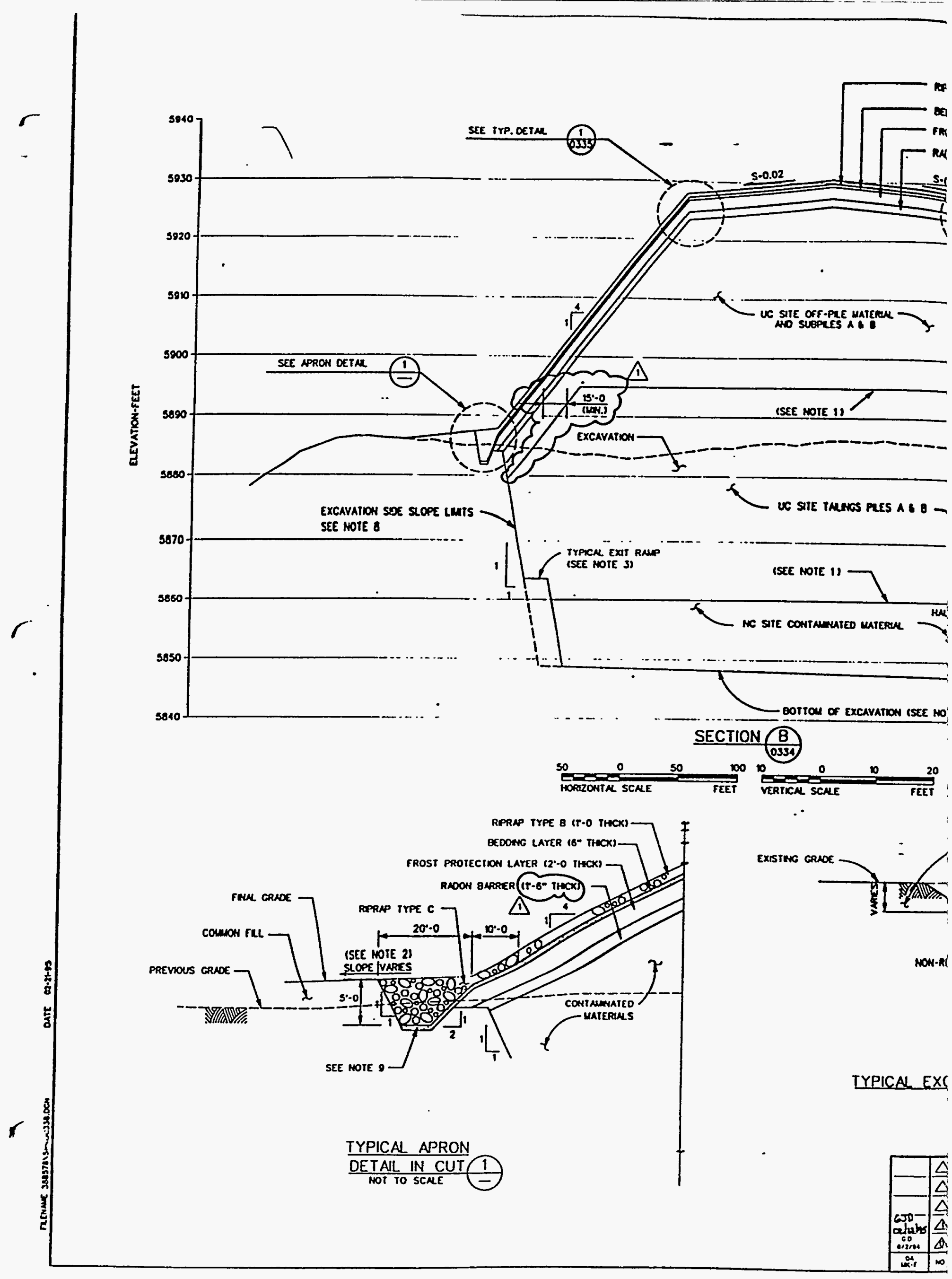




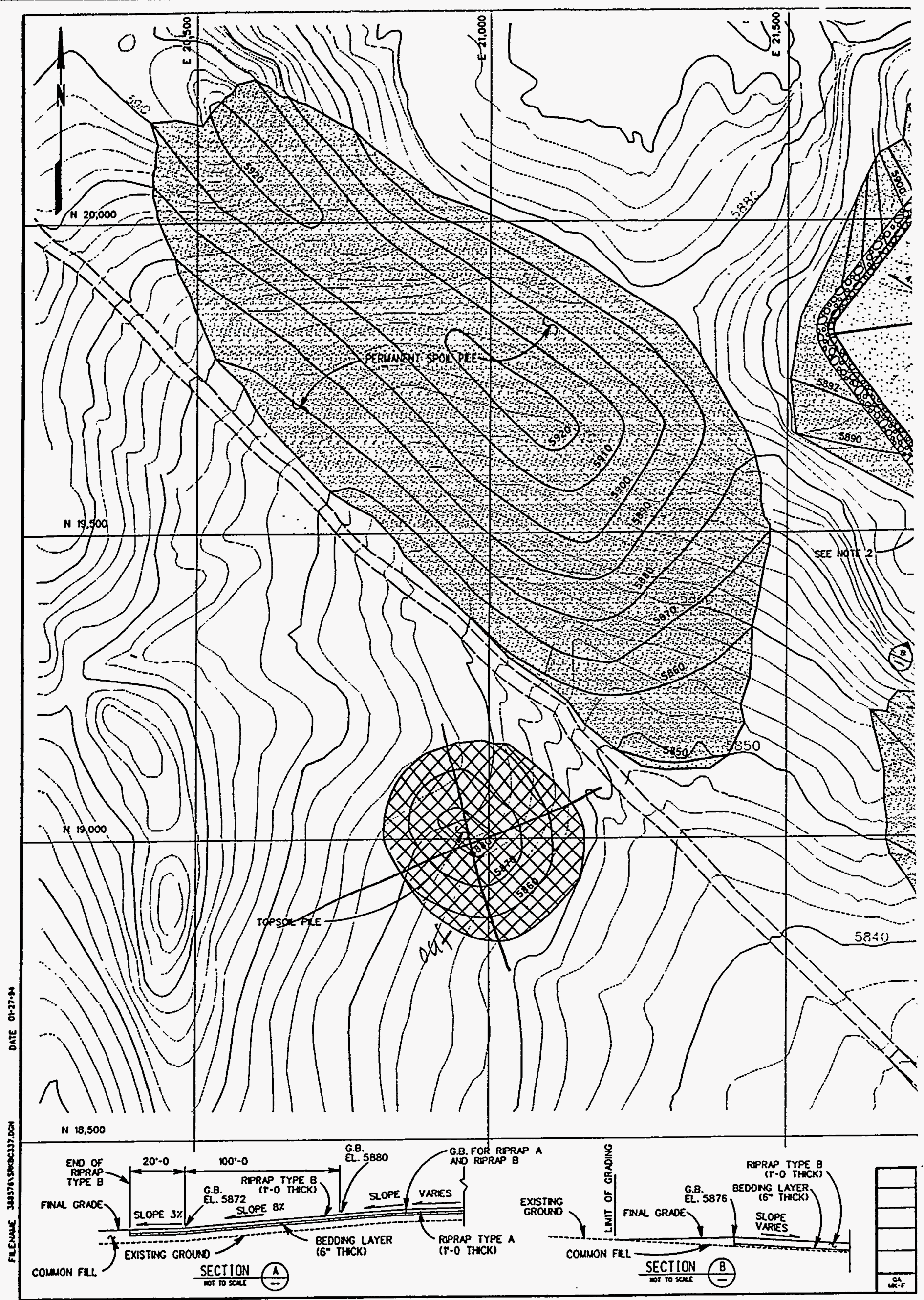




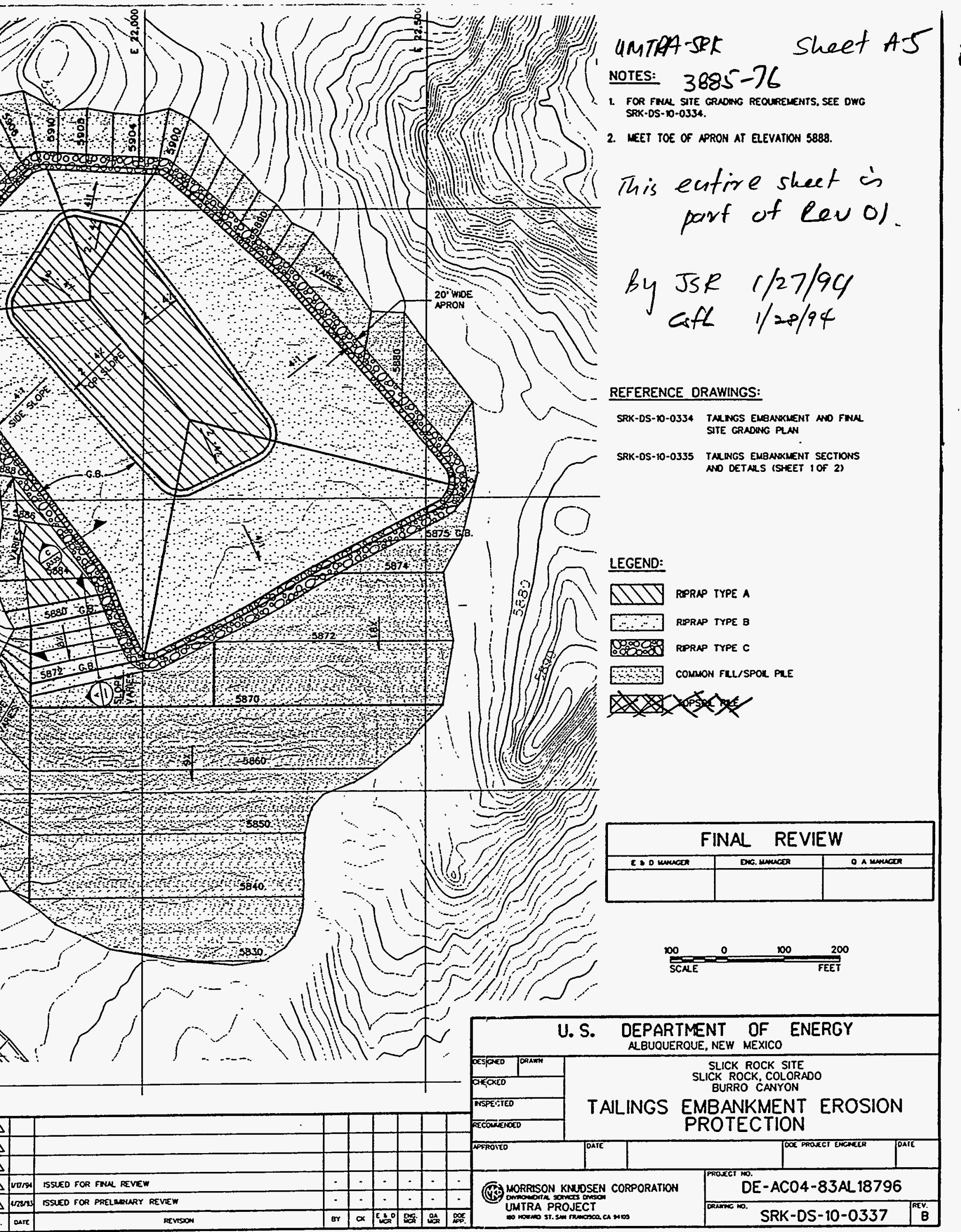


MKES DOC. No. 3885-SRK-C-01-02573-00

MORAISON KNUDSEN CORPORATION

ENVRONMENTAL 8ERMICES DIMSION
UMTRA PROJECT CALCULATION COVER SHEET CALC. NO. 11-333-02-00

CONTRACT NO. $3885-76$

DISCIPLINE CIVIL NO. OF SHEETS 1.7 PROJECT

\section{UMTRA - SLICK ROCK}

\section{FEATURE}

\section{BC SITE - EXCAVATION QUANTITIES}

ITEM

RIPPABLE AND UNRIPPABLE MATERIALS

\section{SOURCES OF DATA}

Ref. 1 (Topography and excavation plan)

MKES Dwg No. SRK-DS-10-0331, Revision B.

Ref. 2 (Pre-1993 boring and test pit logs)

MKES DOC. No. 3885-SRK-01-00338-01, 041493

(Information for Bidders, Volume III).

Ref. 3 (1993 Boring logs)

MKES Doc. No. 3885-SRK-X-01-02554-00 (Boring Logs).

Ref. 4 (1993 Test Pit logs)

MKES Doc. No. 3885-SRK-X-01-02541-00 (Test Pit Logs).

Ref. 5 (Locations and elevations of test pits and 1993 borings)

MKES Doc. No. 3885-SRK-Z-09-02538-00 (Facsimile Record).

SOURCES OF FORMULAE \& REFERENCES

1. Formulae for rectangles are common knowledge and are not referenced.

PRELIMINARY CALC.

FINAL CALC. $\bigotimes$ SUPERSEDES CALC. NO. NA

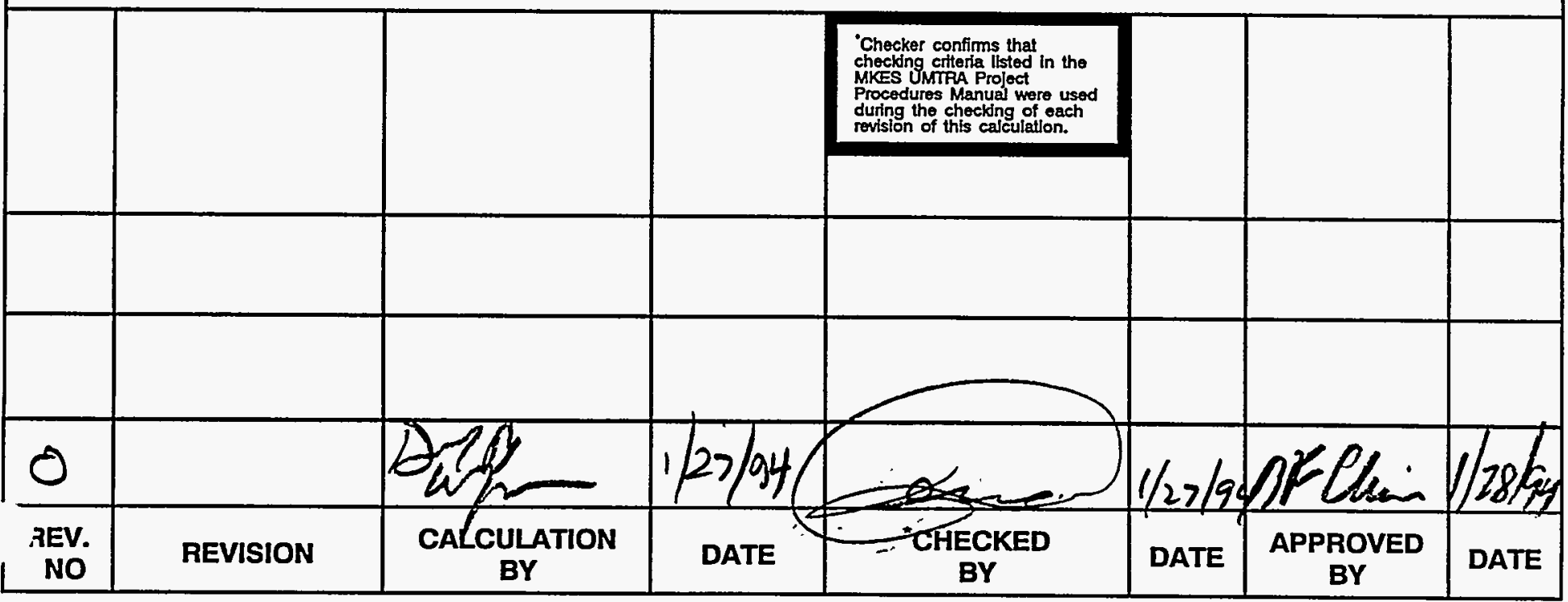




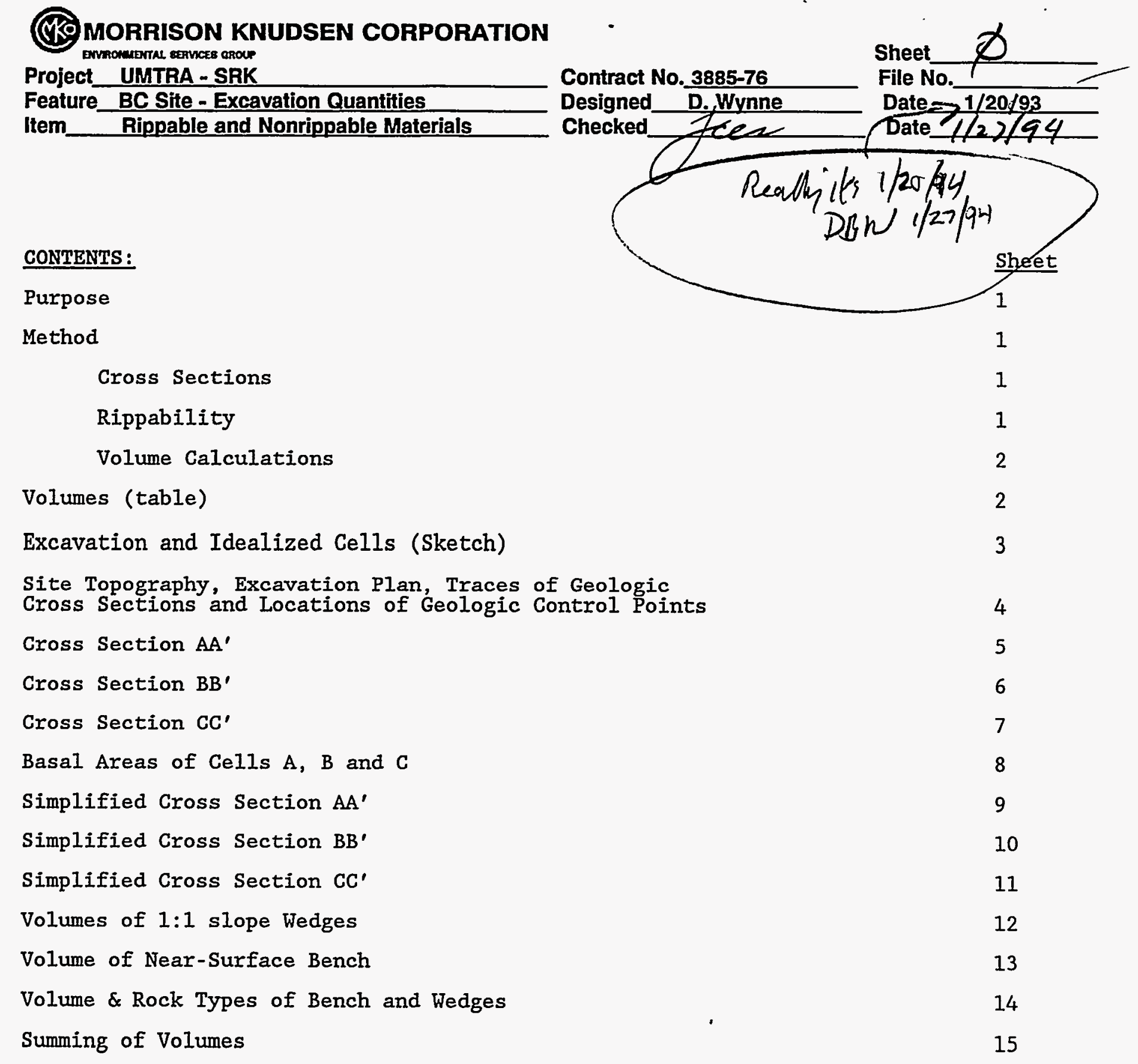


Project UMTRA - SRK

Feature BC Site - Excavation Quantities

Item Rippable and Nonrippable Materials
Contract No. 3885-76

Designed

Checked
D. Wynpe

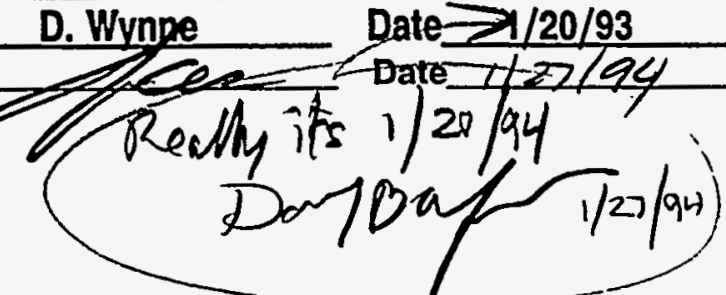

PURPOSE:

This calculation provides an estimate of the in-place volumes of non-rippabte material and rippable material to be encountered during excavation of the disposal cell at the Burrow Canyon site. This calculation. does not address the efficiency of actually separating rippable and unrippable or suitable and unsuitable materials.

\section{METHOD:}

\section{Cross Sections}

Three cross-sections were made for the purpose of determining volumes of materials. The three sections ( $\mathrm{AA}^{\prime}, \mathrm{BB}^{\prime}$ and $\mathrm{CC}^{\prime}$ ) are parallel to one another and trend along the long axis of the disposal cell, from northwest to southeast. Cross sections incorporate all boring and test pit data from locations within and close to the excavation. Lithologic correlation amongst pits and borings are made assuming that geologic strata generally dip gently to the southeast.

Each geologic cross sections was then modified, to simplify calculation of volumes.

Rippability:

Sandstone was assumed to be unrippable for the following reasons:

- It could not be penetrated by Standard Penetration Testing or by a truckmounted auger drill.

- It caused refusal in most of the test pit excavations (both 1990 and 1993).

- Both unweathered and weathered pieces of sandstone were barely scratcheable with the point of a knife.

- Sandstone pieces observed in outcrop on several sides of the site 'mesa' were observed to be as thick as 8 feet.

Mudstone and shale were assumed to be rippable for the following reasons:

- They could be penetrated by the backhoes used in both 1990 and 1993 test pit excavation.

- They were relatively brittle and sometimes easy to fracture or crush, even where closely-spaced horizontal joints were not apparent.

Siltstone was or was not assumed to be rippable in the following situations:

- Where it was dark colored and stratigraphically equivalent to mudstone or shale, it was assumed to be a facie variant of mudstone or shale and therefor rippable.

- Where it was light colored and occurred at the base of a sandstone it was assumed to be a subfacie of sandstone and therefor unrippable.

Sandstones were designated as either the bottom or the top unit. Mudstones/shales were designated as either the bottom, top or isolated unit. The bottom and top unit of each lithology are present on each cross section. The isolated mudstone unit is shown only in boring 607 on cross section $\mathrm{CC}^{\prime}$. 
Contract No. $3885-76$ Designed Checked

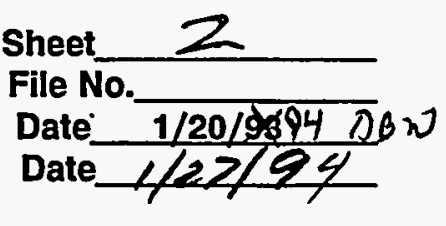

\section{Volume Calculations:}

Volumes of material to be excavated were calculated in three steps. The steps were:

1. Constructing three parallel, idealized cells to determine the area directly above the floor of the disposal cell (volume to be excavated outward of the floor area, due to the 1:1 slope and top bench, are calculated in steps 5 and 6 ).

2. Constructing three simplified cross sections from the more detailed sections, and determining the average thickness of each geologic unit within each of the simplified sections.

3. Multiplying the average thickness (of each geologic unit within each simplified cross section) by the basal area of the corresponding idealized cell, to get the volume of each unit within each cell, and adding those volumes.

4. Estimating the volume of material to be excavated outside the basal footprint of the disposal cell, due to sloped back, rather than vertical sides (the 'Wedge' volumes).

5. Estimating the volume of material to be excavated at the top of the side slopes for construction of a 10-foot wide bench, estimated to be 5 feet below previous grade, with a back slope of 2:1 up to preexisisting grade (the "bench' volume).

6. Adding together the Wedge and Bench volumes and assigning geologic units to these volumes (done by arbitrary assignment, after construction of an idealized average side wall cross section).

7. Adding together the volumes from the three idealized cells (step 3 ) and the volumes from the Wedge and Bench (step 6).

Haul ramps are neglected in the calculation.

Volumes

(from sheet 16)

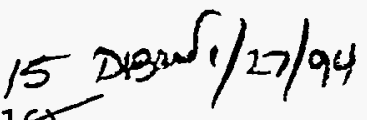

\begin{tabular}{||l|l|}
\hline \hline Material & Cubic Yards (in place) \\
\hline \hline Rippable (soil, mudstone) & 324,872 \\
\hline Nonrippable (sandstone) & 205,067 \\
\hline \hline Total & 529,939 \\
\hline
\end{tabular}




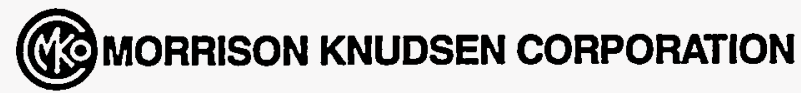

Project UM TRA-SRK

Feature $B C$ Site -Excavation Quantities

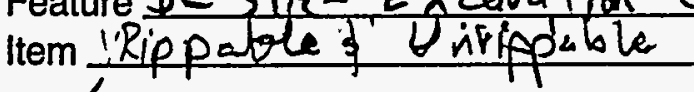

Contract No. 3885

Designed $\mathrm{BBW}$

Excavation and Idealized cells

Checked free ce

Sheet 3

File No.

Date $\frac{12 / 30 / 93}{1 / 18 / 94}$

Exhaustion and Indie

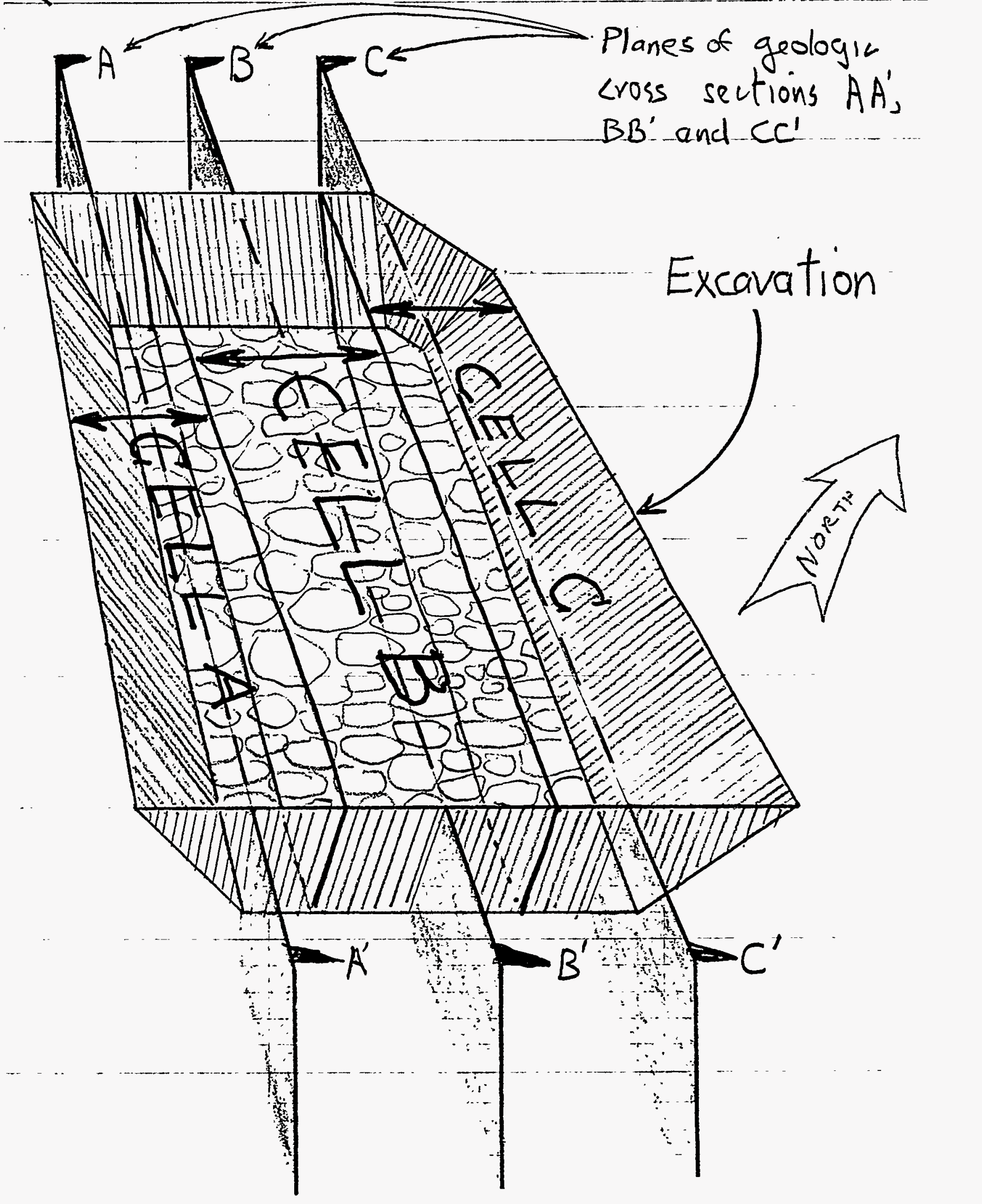

ENG 375/91 


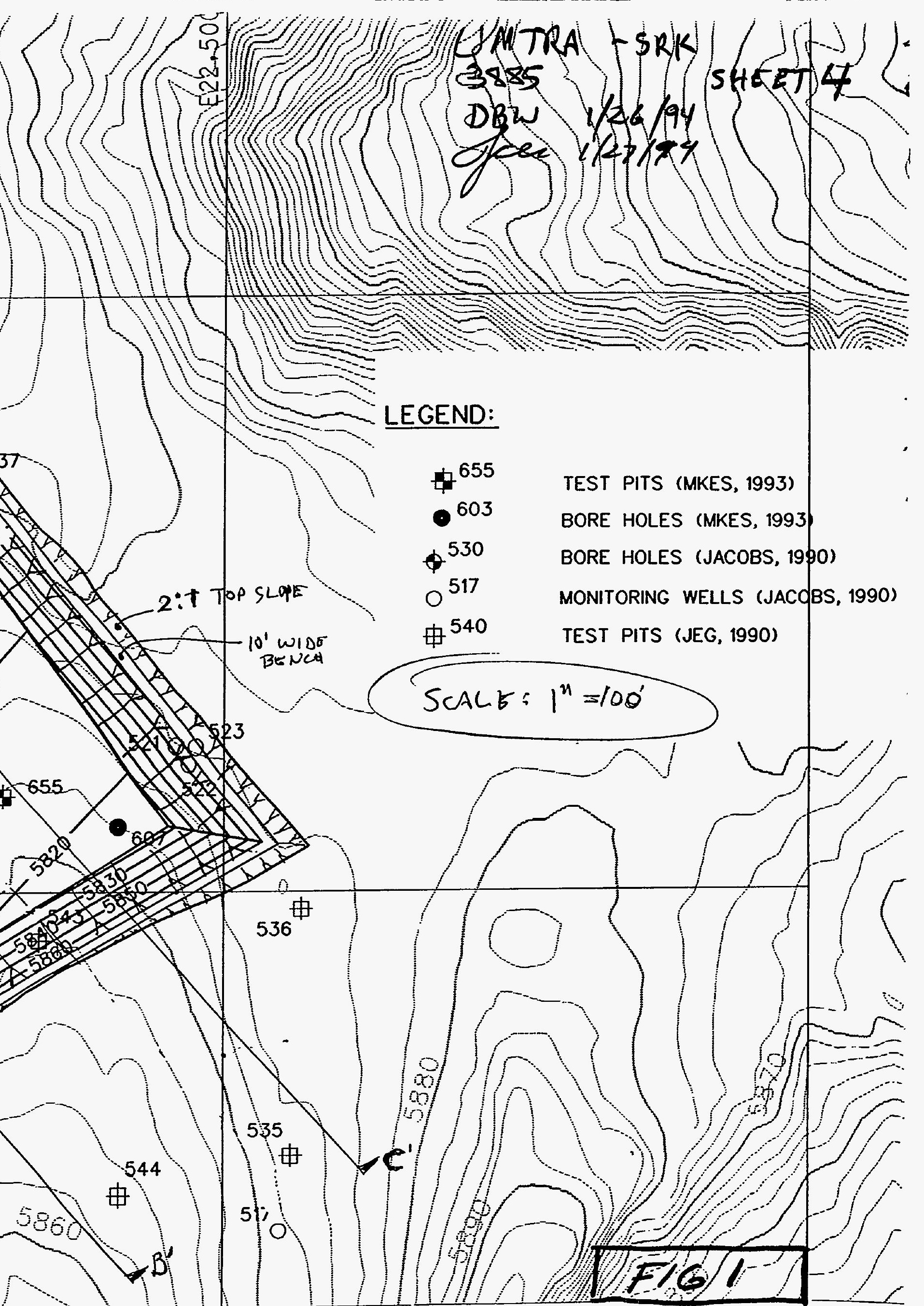




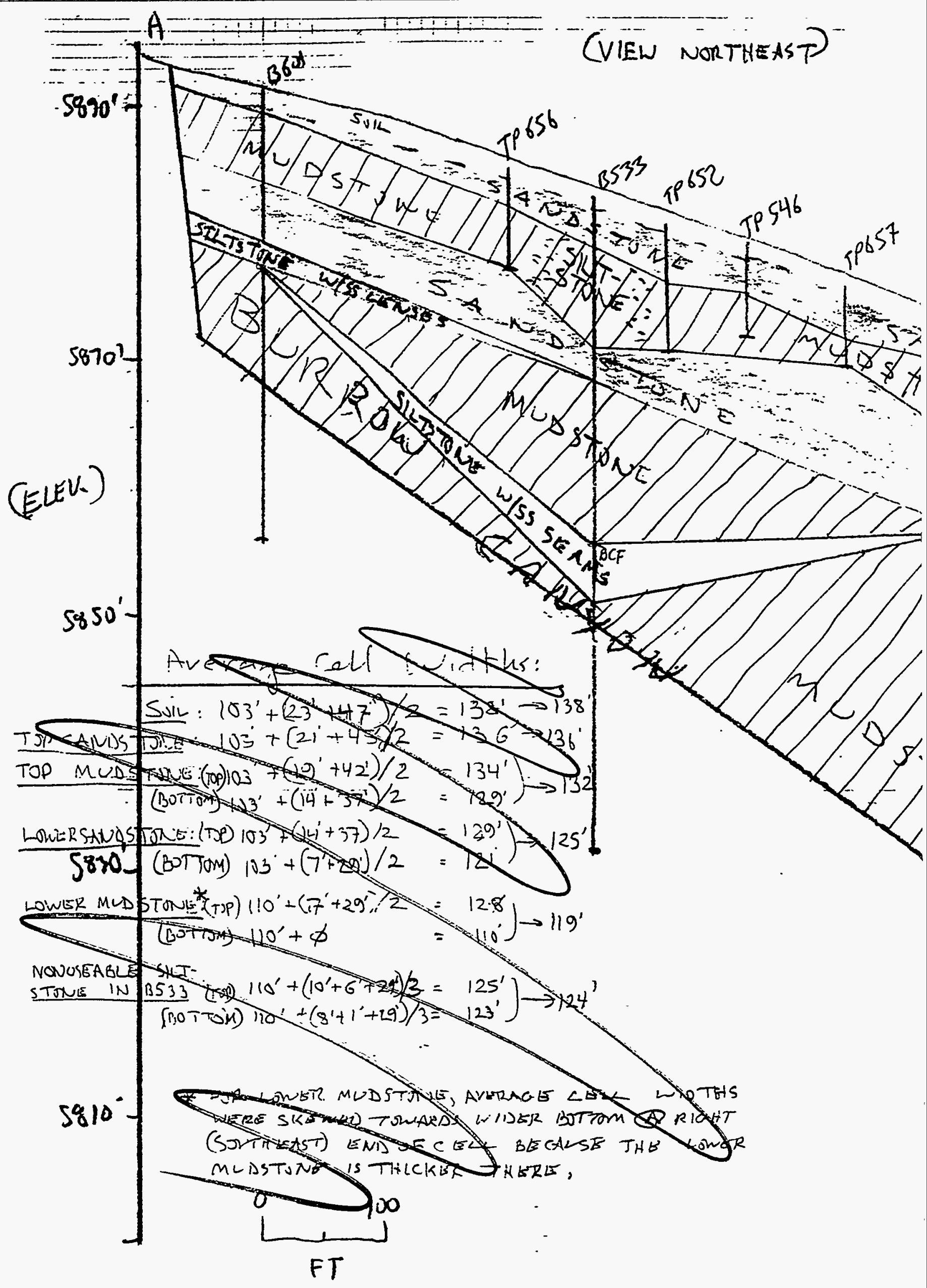



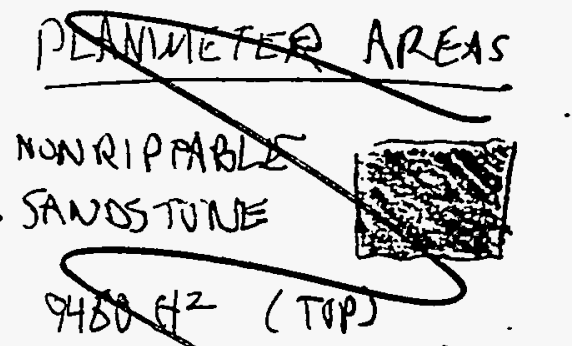

$4845 \mathrm{ft}^{2}$ (BOTTOM)

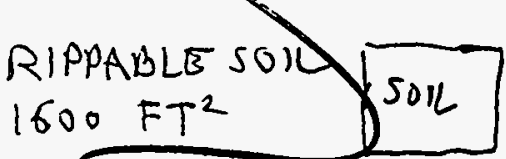

RIPPABL

(USEABLES MUASTUNE \SHALE)

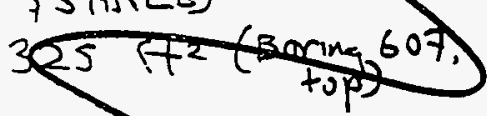
4990 (TOP) $8350 \mathrm{H}^{2}$ (bottom)

Rिए। UNUSA

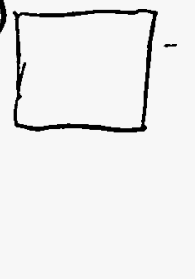


CA. MORRISON KNUDSEN CORPORATION

Project UMTRA - SRK

Feature BC Site- Excavation Quantities

Contract No. 3885

Sheet 8

Item Ropable \& Nonripp-ble

Designed D W

File No.
Date $\frac{1 / 26 / 94}{1 / 22 / 94}$

Checked

$\angle$ Basal Areas, Cell $A, B$

The Footprint Area of each all $\left(H^{2}\right)$ is:
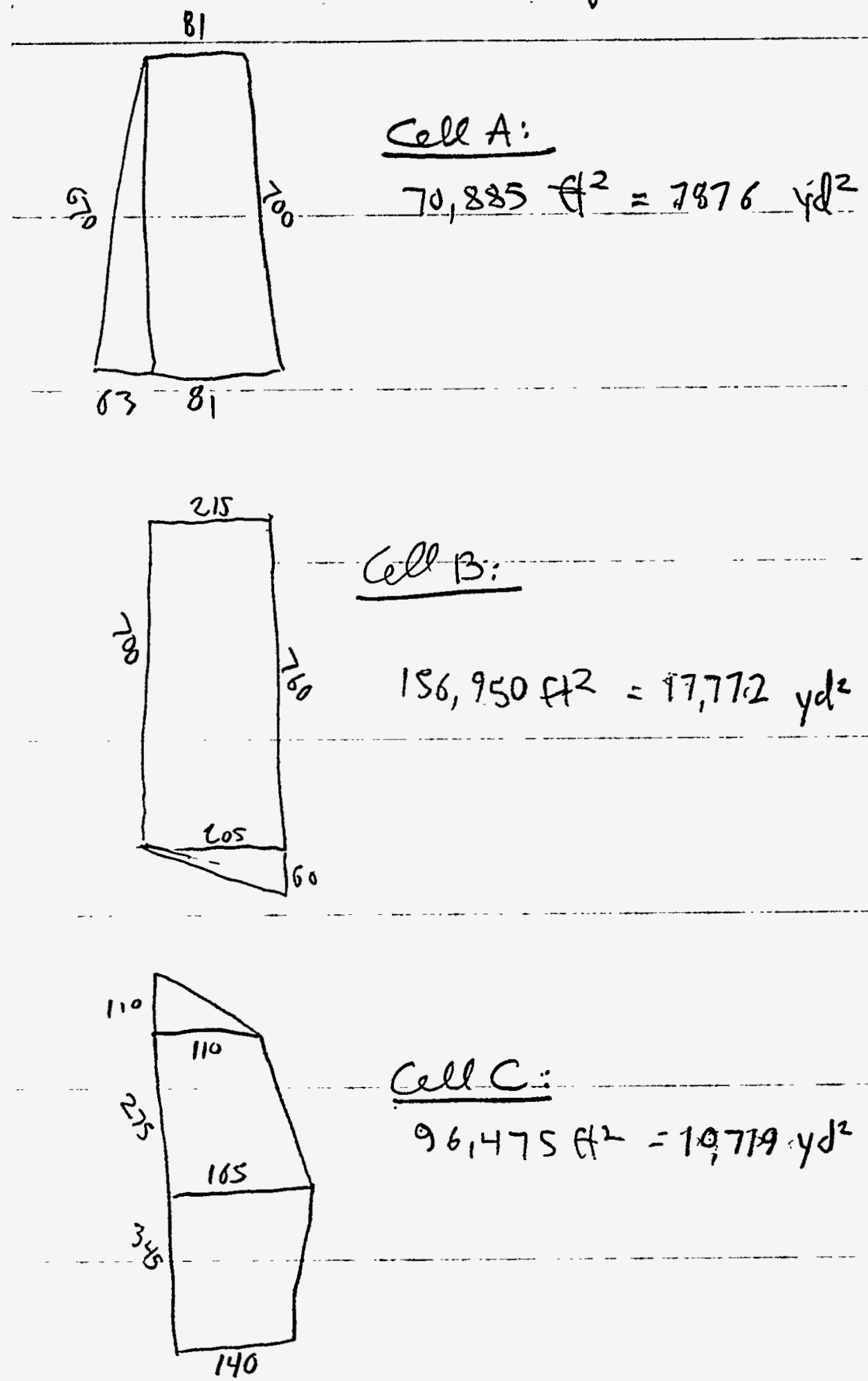

Cell:

$$
96,475 \mathrm{ft}^{2}=10,779 \cdot y \mathrm{~d}^{2}
$$

ENG 375/91 
(16) MORRISON KNUDSEN CORPORATION

Project UM TKA -SRK

Feature $B C$ Site $5 \times$ cerstim Quantities

Sheet 9

Contract No. 3885

File No. Item Ropable of Nonrippable

Checked

Date $1 / 26 / 94$

Simplified Cross Section $\Delta A^{\prime}$ '

Cross-Section $A A^{\prime}$ (simplified)

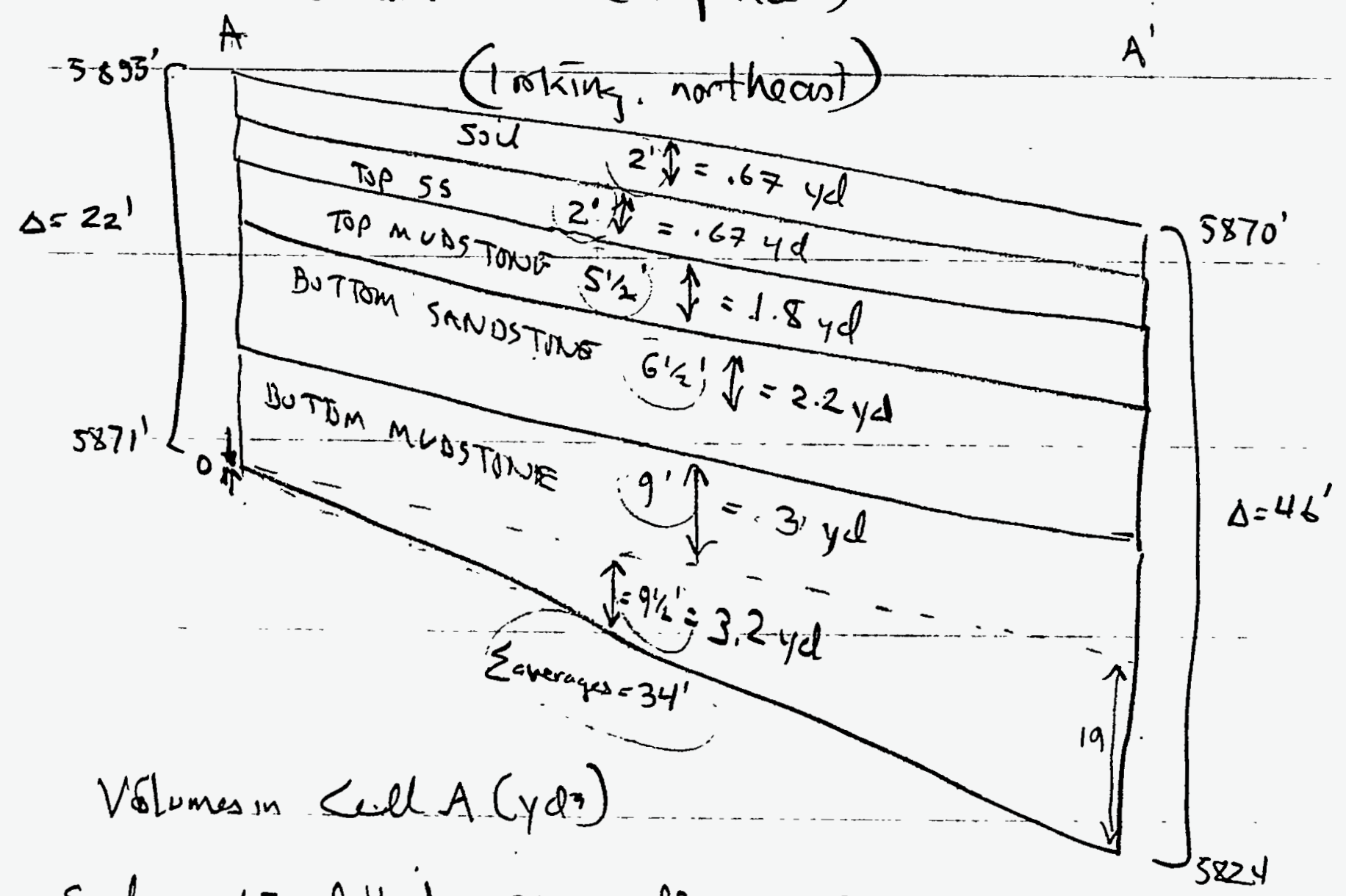

Sol: 167 yd thick $\times 7876 y^{2}=5277$

Tops: .67

Top Mast: 1.8

$\times 7876=5277$

Bott. SS: 2.2

BHt MD S 3.0

3.2

$\times 7876 \approx 14177$

$\times 7876=17327$

$\left.\begin{array}{ll}7876 & =23,628 \\ 7876 & =25,203\end{array}\right) \rightarrow 48,331$

ENG 375/91 
A39 MORRISON KNUDSEN CORPORATION

Project UMTRA- SRK

Sheet 10

Feature BC Sle- Exexratim Duantifices

Contract No. 3885

File No.

Item Ruppable f Unrippable

Designed $D$

Date $1 \longdiv { 2 6 / 9 4 }$

$\angle$ Simplified Cooss-Section $B B^{\prime}$

Cross Section BB' (simpliEvid)

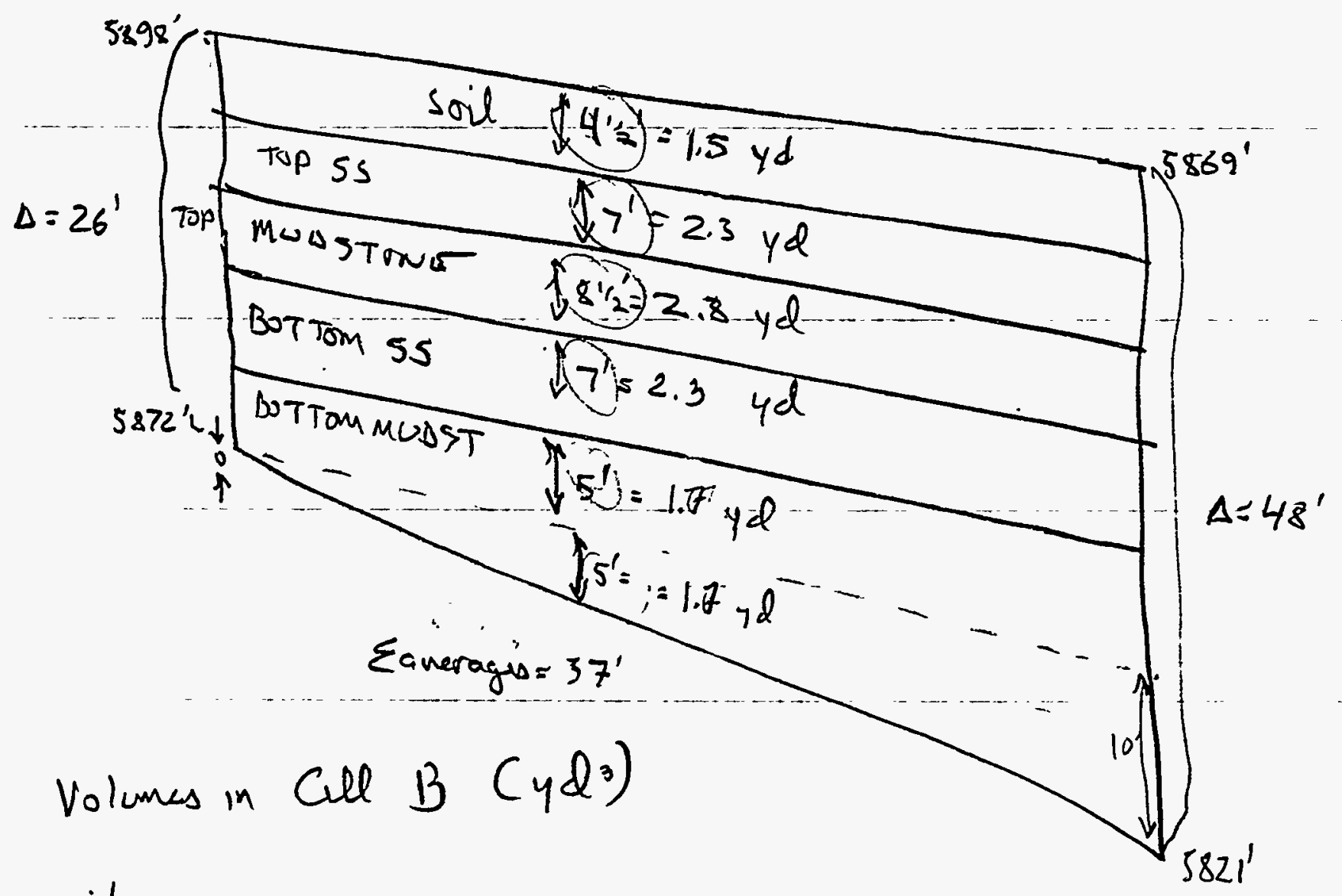

Soil : 1.5 ydthick $x 17,772$ ydz $=26,658$ cy

Topss $2.3 \times 17,772=40,876$

tppedt $2,8 \times 17,772=49,762$

Bott.55 $2,3 \times 17,772=40,876$

Bbtt.mdst $3.9 \times 17,772=60,425$

Cell subtotul= $218,597 \ldots 4$.

ENG 375/91 
(192) MORRISON KNUDSEN CORPORATION

Project UATRA-SRK

Feature BCSite- Ex cavation Quantites

Contract No. 38

Sheet 11

Item Rippable \& Unvippable

Designed DWW Date $1 / 26 / 94$

Simplifed Cross Section $C C^{\prime}$

cross-Section C' (simplifiéf)

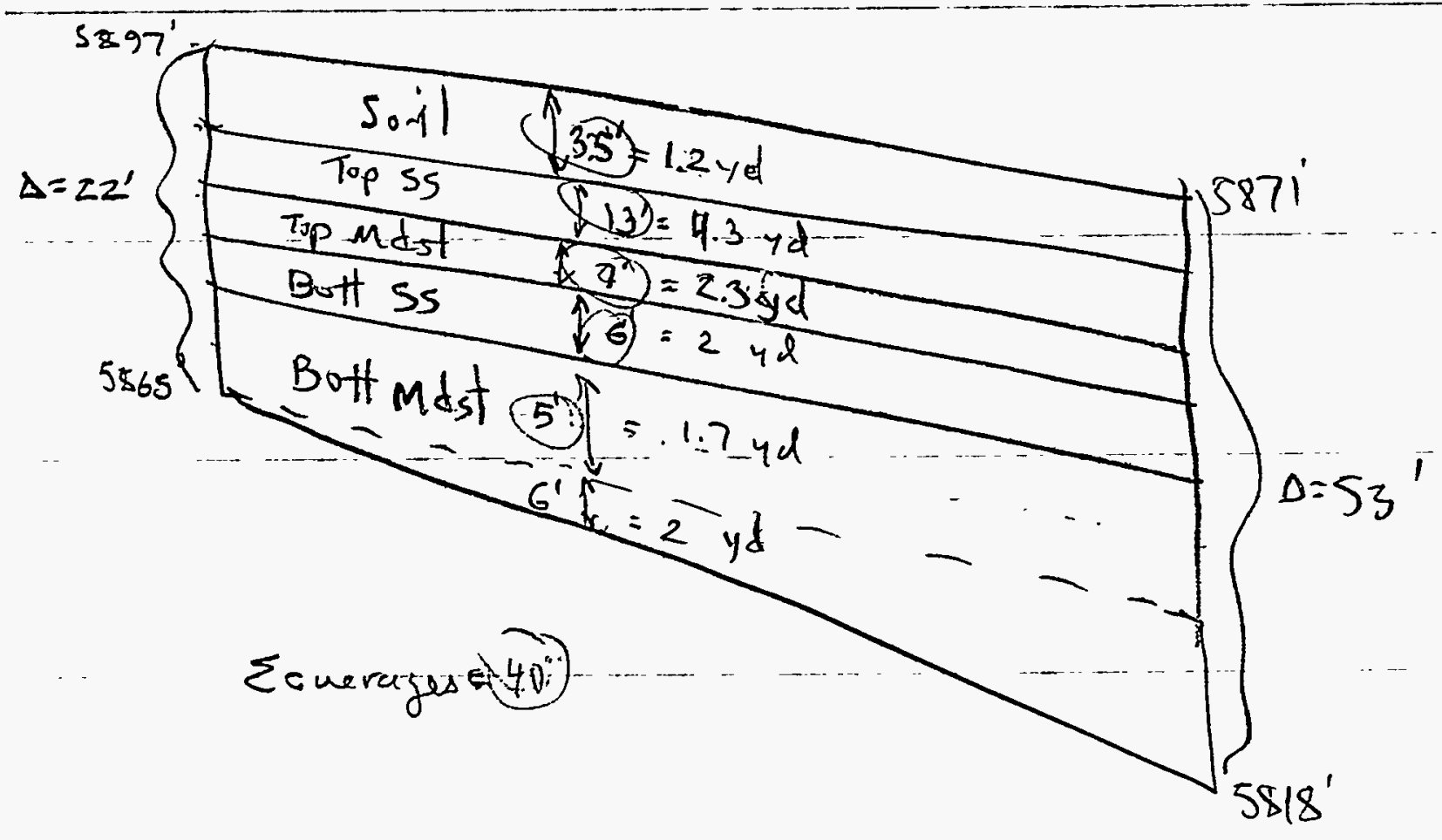

Volumesin CUIC (yds)

Sorl : $1.24 d$ thick 10,719 ydz $=12,863 \quad c y$

Topss: 4.3

$\times 10,719=46,092$

Tppondst, 2.3

$\times 10,719=24,654$

Bottiss': 2

$\times 10,719=21,438$

Bott indst: 3.7

$\times 10,719=39,660$

Cll Subtial = $\quad 144707 \mathrm{cy}$

| ENG 375/91 
@MORRISON KNUDSEN CORPORATION

Project UMTRA - SRK

Feature $\overline{B C}$ Sike $-\overline{5}$ xeavation Quantity

Contract No. $3 \$ 5$

Sheet 12

item Ropable \& Norippatile

Designed $D B W$ Date $\perp / 26 / 94$

〈Vowme of 1.1 slope Wedges"
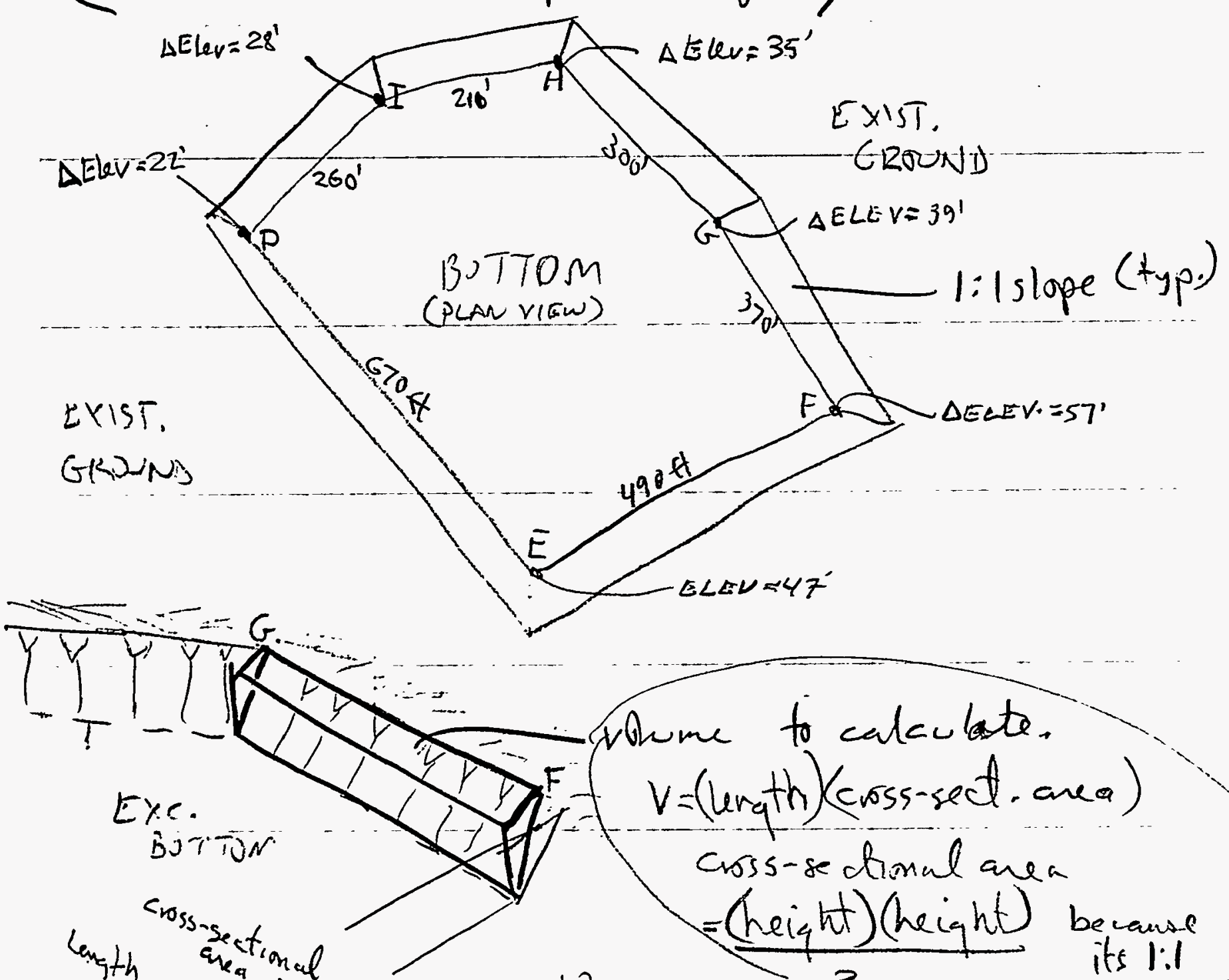

$$
\begin{aligned}
& \text { row me to calculate. } \\
& V=(\text { length }) \text { (coss-sect area) }
\end{aligned}
$$

cross-sectional area $=($ height) (height) be cause

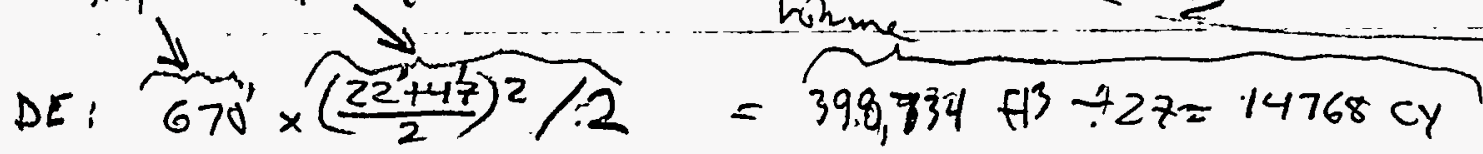

FF: $490^{\prime} \times\left(\frac{47^{\prime}+57^{\prime}}{2}\right)^{2} / 2=662,480 \mathrm{fB} \div 27=24,536 \mathrm{cy}$

$F G: 370^{\prime} \times\left(\frac{39^{\prime}+57^{\prime}}{2}\right)^{2} / 2=426,240+f^{3} \div 27=15,787 \mathrm{Cy}$

Gl: $300^{\prime} \times\left(\frac{35^{\prime}+39^{\prime}}{2}\right)^{2} / 2=205,350 \div 3 \div 27=7606 \mathrm{cy}$

HI: $210^{\prime} \times\left(\frac{28^{\prime}+35^{\prime}}{2}\right)^{2} / 2=104,186 \mathrm{~A}^{3} \div 27=3859 \mathrm{cy}$

ID: $260^{\prime} \times\left(\frac{221+25^{\prime}}{2}\right)^{2} / 2=81,250 \mathrm{HB}^{3} \div \frac{27}{}=\frac{3009 \mathrm{cy}}{69,565 \mathrm{cy}}$
est id total vol., slope "wedges"

ENG 375/91 
@ MORRISON KNUDSEN CORPORATION

Project UMT $A A-S R K$

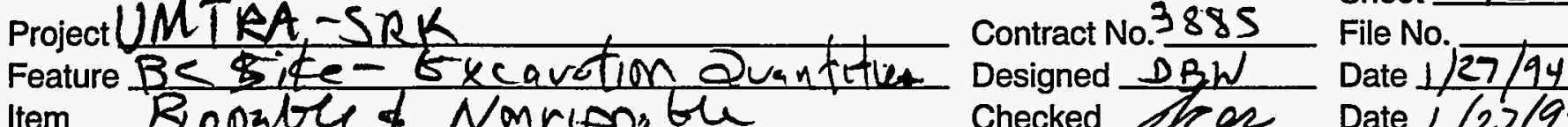

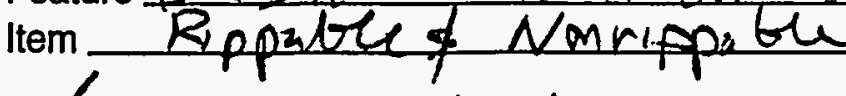

Checked Aten Date +/27/94

$\left\langle V_{0}\right.$. \& rock ty of wedges

3

From shat 12 vol. it 'madge areas $=69,565 \mathrm{cy}$

Idealized Average section

Arbitrary Assignment:

Bott

Mast

Soil $=16 \% \times 69,565$

$c y=11130 \mathrm{cy}$

Tops $=3100 \times 69,565$

$c y=21,565 c y$

TopMast $=23 \% \times 69,565$

$c y=16,000 \mathrm{cy}$

Bot $S 5=1570 \times 69,565$

$c y=10,435 c y$

Butt Mast $=15 \% \times 69,565$

$c y=10,435 \mathrm{cx}$

$\Sigma=69,565$

ENG 375/91 
@17. MORRISON KNUDSEN CORPORATION

Project UMTRA-SRK

Feature BC Sike- $5 \times$ ceration Qvantities

Contract No. 3885 File No.

ltem Rippable f Nonrippatale

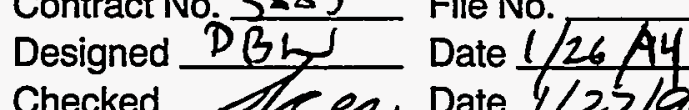

$\langle$ Vol, of neor-sifface buch

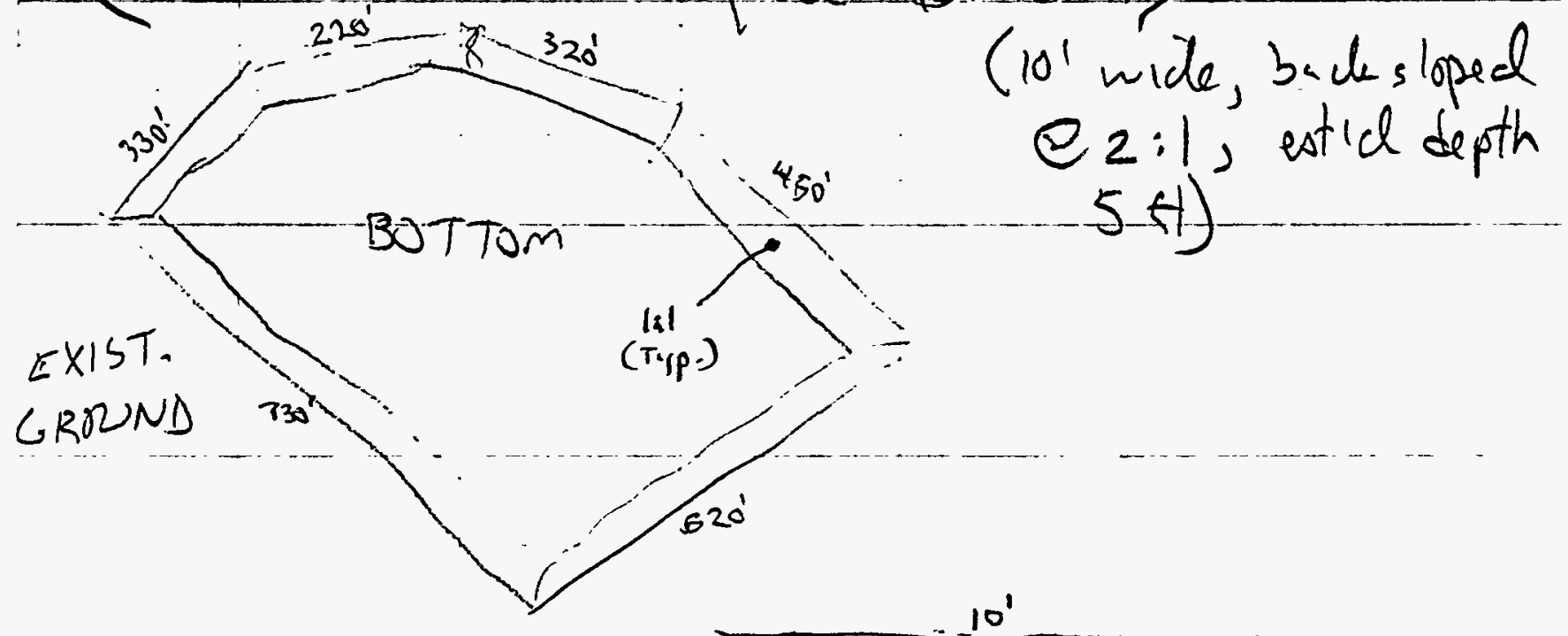

Pevinatere $2670^{\circ}$

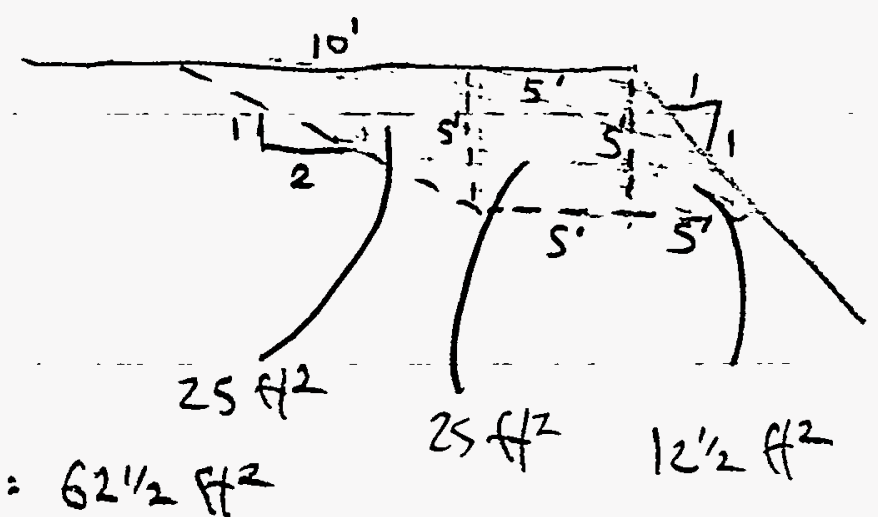

$\sum A: 621 / 2 H^{2}$

Vol of near-ste bench $=2670^{\prime}$ length $\times 62^{\prime \prime} 2 t^{2}$ :cross $\begin{gathered}\text { sectiond } \\ \text { cuec }\end{gathered}$

$$
=2670 \times 62^{1 / 2}
$$

$=166,875 \mathrm{ft}^{3}=6181<y$

AssLME of 618$) \mathrm{CY})(5000 \mathrm{cy}=\mathrm{soll}$

TO TALS, RUEDGE - BENCH

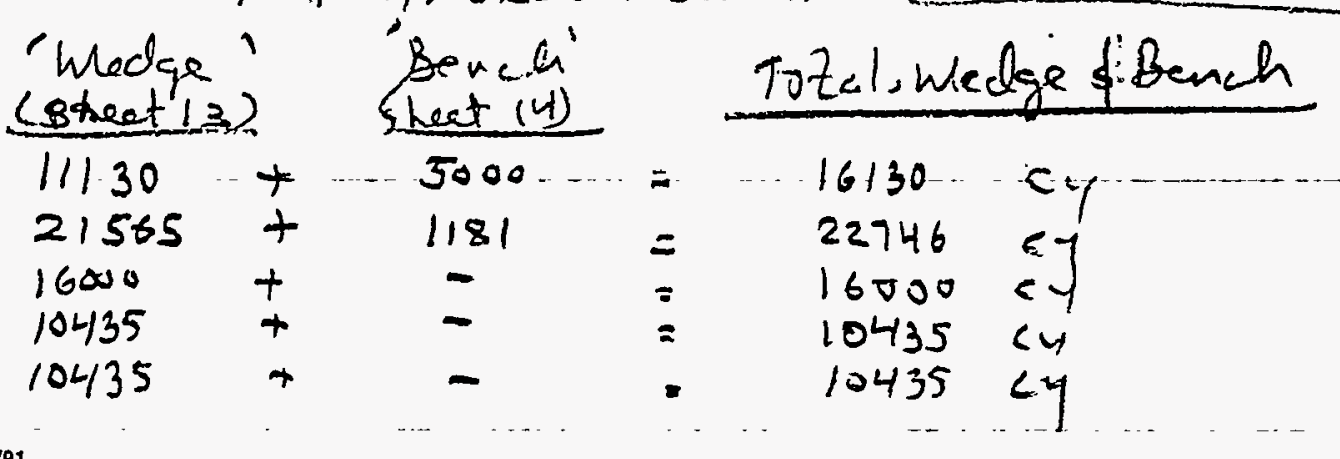

ENG 375/91 
Project UMTRA SRK

Feature BC Site $\overline{5}$ xeavation Quantitio

Item Rippable of Won rippa dele

Summina of Voungos
Contract No. 3885

Designed $\mathrm{BN}$

Checked
Sheet 15

File No.

Date $\sqrt{27 / 94}$

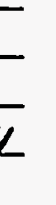


MORRISON KNUDSEN CORPORATION

ENMRONMENTAL 8ERMCES DVIBION
UMTRA PROJECT

CALCULATION COVER SHEET

CALC. NO. 11-330-01-00 of

CONTRACT NO. $\underline{3885-76}$ DISCIPLINE CIVIL PROJECT

NO. OF SHEETS 32 (includes Cover sheet)

\section{UMTRA - SLICK ROCK}

\section{FEATURE}

\section{SITE RESTORATION}

\section{ITEM}

\section{EARTHWORK QUANTITIES AND SEEDING}

\section{SOURCES OF DATA}

\section{REFERENCES 1-9 AND 11-17 (SEE SHEET ii)}

\section{SOURCES OF FORMULAE \& REFERENCES}

SEE SHEET ii.

PRELIMINARY CALC. $\square \quad$ FINAL CALC. $\bigotimes$ SUPERSEDES CALC. NO.

\begin{tabular}{|c|c|c|c|c|c|c|c|}
\hline \multirow[b]{2}{*}{03} & \multirow[b]{2}{*}{ See Sheet ii $(a)$} & \multirow{2}{*}{ Areayangy } & \multirow{2}{*}{$9 / 7 / 95$} & \multirow{2}{*}{ 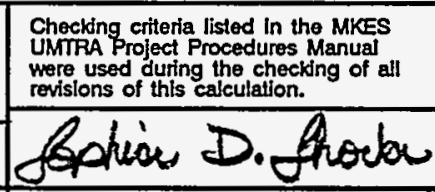 } & \multirow{2}{*}{$9 / 13 / 95$} & \multirow{2}{*}{ PHC Su } & \multirow{2}{*}{$9(1,4)$} \\
\hline & & & & & & & \\
\hline 02 & See sint IA & 3.5.Randeris & $8 / 22194$ & Ali M. Banani & $8 / 29 / 94$ & & Q/29) \\
\hline 01 & See sut IA & JS Randeri & $1 / 2 / 34$ & Ali M-Banani & & & \\
\hline 00 & & Afteums & $4 / 21 / 23$ & J.S Randeui & $14)^{2}$ & & 4has \\
\hline $\begin{array}{l}\text { REV. } \\
\text { NO }\end{array}$ & REVISION & $\begin{array}{c}\text { CALCULATION } \\
\text { BY }\end{array}$ & DATE & $\begin{array}{c}\text { CHECKED } \\
\text { BY }\end{array}$ & DATE & $\begin{array}{c}\text { APPROVED } \\
\text { BY }\end{array}$ & DATE \\
\hline
\end{tabular}


Project

UMTRA

Feature

SITE RESTORATION

EARTHWORK QUANTITIES
Contract No. $3885-76$

Designed AFS

Checked_JSR

Sheet $\frac{i}{\text { File No. } \frac{i}{4 / 21 / 93}}$
Date $\frac{4 / 23 / 93}{4 / 23}$

TABLE OF CONTENTS

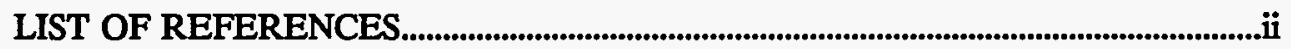

SUMMARY OF QUANTITIES.........................................................................................

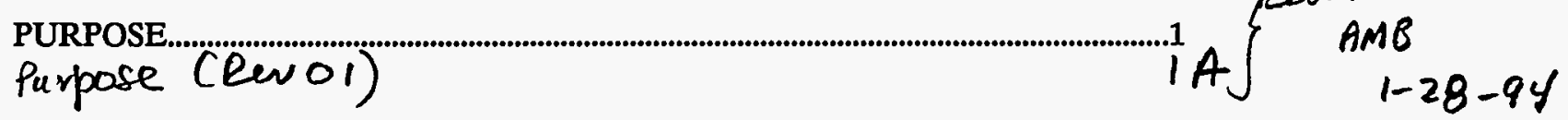

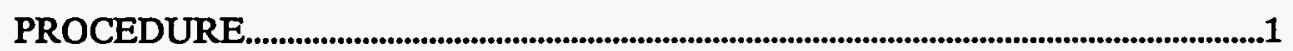

CALCULATIONS:

COMMON BACKFILL - UC, NC AND BC SITES.............................................2

EXCAVATION FOR GRADING/CONTOURING - ALL SITES................18

SEEDING ACREAGE - ALL SITES.............................................................22 
REFERENCES

1. MKES, 1988. UMTRA-SRK, "Site Restoration - Earthwork Quantities." MKE Calc. No. 11-250-01.

2. MKES, 1988. UMTRA-SRK, "NC Site and UC Site Contaminated Materials - Excavation Quantities," MKE Calc. No. 11-212-01-02

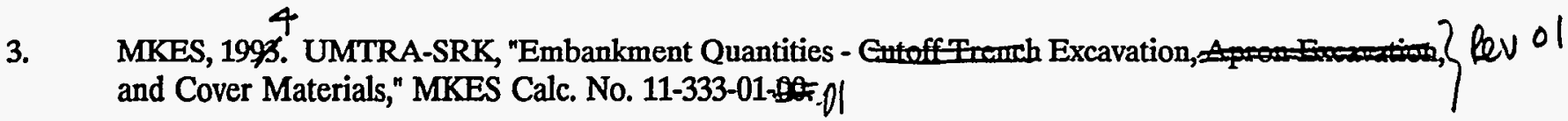

4. Intergraph Corp., 1992. Inroads civil engineering and highway design software, Version 4.01.01.01.

5. MKES, 1993. UMTRA-SRK, Union Carbide Processing Site, Final Site Grading Plan, MKES Dwg. No. SRK-PS-10-0317.

6. MKES, 1993. UMTRA-SRK, UC Site, Contaminated Material Excavation Plan, MKES Dwg. No. SRK-PS-10-0316.

7. MKES, 1993. UMTRA-SRK, UC Site, Temporary Site Drainage Plan, MKES Dwg. No. SRK-PS-10-0312.

8. MKES, 1993. UMTRA-SRK, UC Site, Wastewater Retention Basin Sections and Details, MKES Dwg. No. SRK-PS-10-0315.

9. MKES, 1993. UMTRA-SRK, North Continent Processing Site, Final Site Grading Plan, MKES Dwg. No. SRK-PS-10-0324.

10. MKES, 1993. UMTRA-SRK, NC Processing Site, Contaminated Material Excavation Plan, MKES Dwg. No. SRK-PS-10-0323.

11. MKES, 1993. UMTRA-SRK, NC Site, Existing Untilities and Demolition Plan, MKES Dwg. No. SRK-PS-10-0322.

12. MKES, 1993. UMTRA-SRK, Burro Canyon Site, Access Control Facilities and Wastewater Retention Basin Plan, MKES Dwg. No. SRK-DS-10-0332.

13. MKES, 1998. UMTRA-SRK, BC Site, Tailings Embankment and Final Site Grading Plan, MKES Dwg. No. SRK-DS-10-0334.

14. MKES, 1993. UMTRA-SRK, Borrow Area Site Plan, MKES Dwg. No. SRK-GE-10-0304.

15. MKES, 1993. UMTRA-SRK, Tailings Embankment Erosion Protection, MKES Dwg.

No. SRK-DS-10-0337.

16. MKES, 1995. UMTRA-SRK, BC Site, Site Preparation and Temporary Drainage Facilities, MKES Dwg No. SRK-DS-10-0331.

17. MKES, 199\%. UMTRA-SRK, BC Site, Access Plan, MKES Dwg. No. SRK-DS-10-0330. 
(O) MK-ENVIRONMENTAL SERVICES

Project UMTRA - SRK

Sheet ii (a)

Contract No. $3885-76$. File No.

Feature SITE RESTORATION

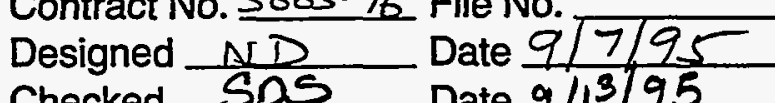

Item EARTHWORK QUANTITIES

Checked

Date $9 / 13 / 95$

Purpose of Revision 03:

Disappointment Valley Borrow Site is no longer used. for borrow material. The excavation of the

disposal cell at the $\overline{B C}$ STe provides clean material.

All references to Disappointment valley are hence taken out.

Relevant sheets affected:

sheet iii - Delete Disappointment valley references re -total the quantities

sheet 20 - Delete Disappoint valley volume \& adjust the total volume of excavation.

sheet 25 - Delete Disappointment valley references \& adjust the total seeding area.

ENG 375/89 (ENV.)

*4242/89 
Project

Feature

Item
UMTRA

SITE RESTORATION

EARTHWORK QUANTITIES
SUMMARY OF QUANTITIES

Contract No. $3885-76$

Designed AFS

Checked JSR
Rev 01
Sheet $\frac{\text { iii : }}{\text { File No. }}$
Date $4 / 21 / 93$
Date $4 / 23 / 53$

$1 / 27 / 54$

$A M B \quad 1-28-94$

1. COMMON FILL Rev 2 By JSR $8 / 22 / 94$
AMB 8129194

UC site 20,700 cy (sheet 7)

NC Site

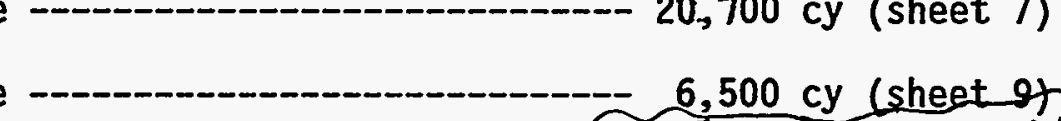

BC Site 117,000 84,100 cy (sht 19) Rev ol

Topsoil needed at $B C$ site

2. EXCAVATION FOR GRADING/CONTOURING 15,100 cy $\operatorname{csh} t$

14)

UC Site 23,500 cy (sheet 18)

NC Site 11,100 cy (sheet 18)

$\mathrm{BC}$ Site

$$
0 \text { cy (sheet 19) }
$$

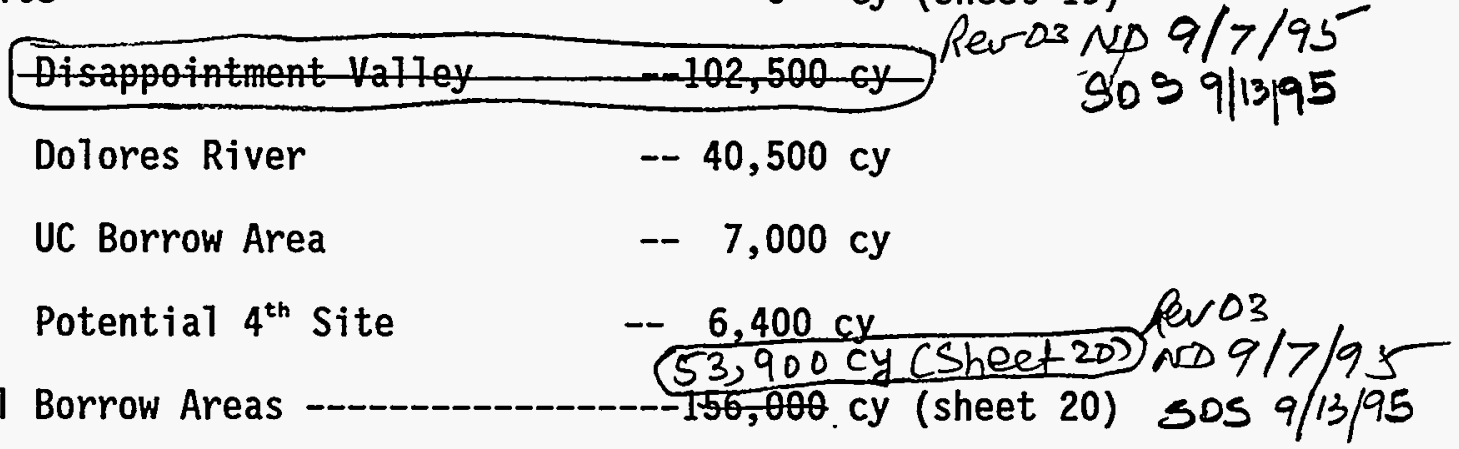

\section{SEEDING}

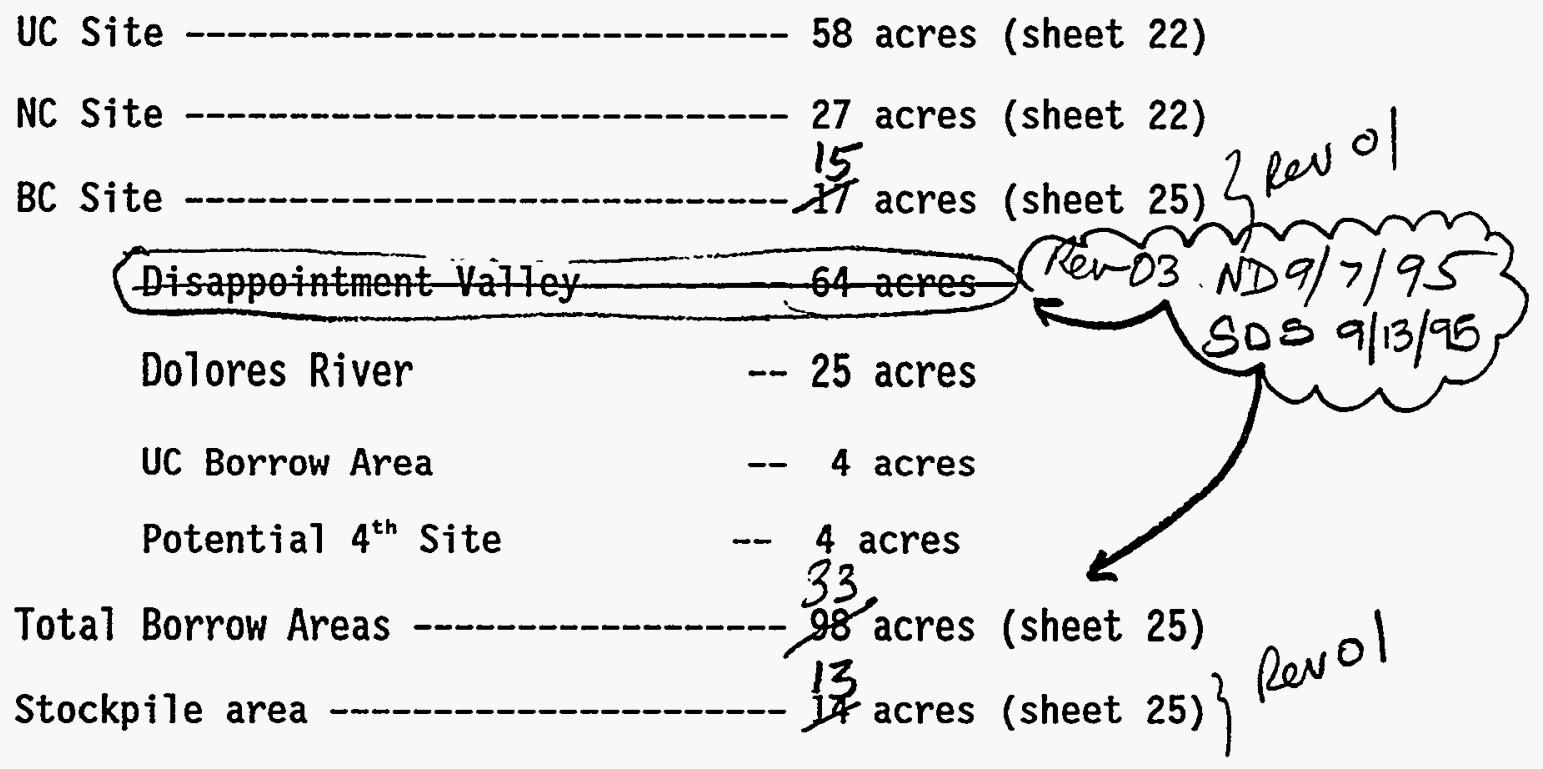




\section{PURPOSE:}

The purpose of this calculation is to determine:

1. The quantity of fill required for site restoration at the UC, NC and BC sites.

2. The quantities of materials excavated for grading and contouring at all sites, including borrow areas, and

3. The required seeding acreage at all sites.

\section{PROCEDURE:}

1. According to Ref. 1, common backfill will be used in certain areas to fill excavations that are below adjacent grade to match adjacent grades. Information from references 2 and 4 (contaminated material excavations) is used to determine the amount of backfill required at the UC and NC sites. For the BC site, an InRoads (Ref. 4) surface has been generated for the final grading contours around the embankment (See Ref. 3). InRoads computes the volume of fill between the desgin surface and the existing ground surface for the area outside the embankment and apron.

2. Rough estimates of the depths of grading are used to determine the approximate amount of grading required at each site.

3. According to Ref. 1, seeding is required over all graded, excavated, or similarly disturbed soil surfaces. The acreage of disturbed areas shown in references 5,6 , and 9 is measured using the CADD drawings and the Intergraph Microstation "measure area" command. Intergraph uses the coordinates of points along the perimeter of an area to compute the acreage within the perimeter defined by those points. 
AM MORRISON KNUDSEN CORPORATION

Project UMTRA - SRK

Sheet IA

Feature Site Restoration.

Contract No. $\frac{3885-76}{5}$ File No.

Item Earthwork Quantities \& Seeding Designed $\frac{J S R}{A M B}$ Date $\frac{1 / 27 / 94}{1-28-94}$

Rev $2:$ By J SR $8 / 22 \%$

Purpose of Rev of

$A M B$ 8/29/64

1. Final $n$ gradin in BC disposal site has changed. Sheet 14 has been revised. Sheets is 16 and 17 has been replaced with new sheets suet 19 has been revised.

2. Permanent. stockpile has been changed Therefore seeding areas of stock pile is revised. Seedling area of final grade is also revised. Sheet 22 and 25 has been revised. Sheets 23 and 26 has been replaced with new sheets.

Purpose of lev-2

1. During cell foundation excavation, material will be placed directly on to the finished grading area as shewn on dug SRK DS-10-0331. The rest will be placed in the stockpile. The se quantities are codculted ain sit 19 , Shits iii, iA, 19 are pressed.

ENG 375/91 
@ MORRISON KNUDSEN CORPORATION

Project UMTRA-SRK

Feature SITE RESTORATON

Item EARTHWORK QUANTITIES
Sheet 2

Contract No. 3885-76 File No.

Designed AFS Date

Checked

Date

COMMON BACKFILL

LC SITE:

THE area marked (1) ON SHeET 3 is an area of CONTAMINATED MATERIAL EXCAVATION WHICH MUST BE BACK-

- FILLED to the final Grade. THE quantITY of backFILL Equals the quantity of excavated contaminated material.

Contaminated material excavation quantities are calculated in Reference 2.

Area \# 1 corresponds to subarea \# IIX from ref. 2 ,

SHEET 45 A QUANTITY TO BE REMOVED $=867 \mathrm{cy}$ (REF, 2, SHT, 42B)

Volume in AREA "A" (ON SHEET 4) CORRESPONDS to SUB-AREAS

XXIII + ZXII + ZXIV IN REF, 2, SHEETS $42 D \& 42 E$

$\therefore V_{\text {al }} A=(13,292 \times 5+2644 \times 10+8300 \times 2) / 27=4056 \mathrm{cy}$

SECTION THROUGH RETENTION BASIN (SEE SHEET .6)

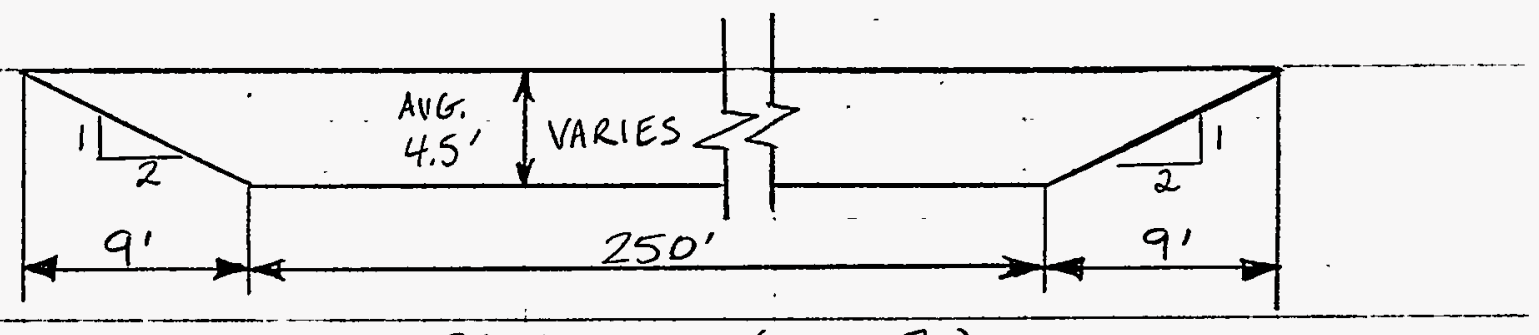

RETENTION BASIN IS 360' LONG (REF, 7), WITH THE LAST $\sim 9 \mathrm{ft}$ SLOPING UP $2 H: 1 V$ TO MEET FINAL GRADE.

$$
\begin{aligned}
\text { AREA of CROSS-SECTION } & =4.5 \times 250+2\left(\frac{1}{2} \times 4.5 \times 9\right) \\
& =1165.5 \mathrm{ft}^{2}
\end{aligned}
$$

EMO 375/49

$\$ 4242 / 89$ 


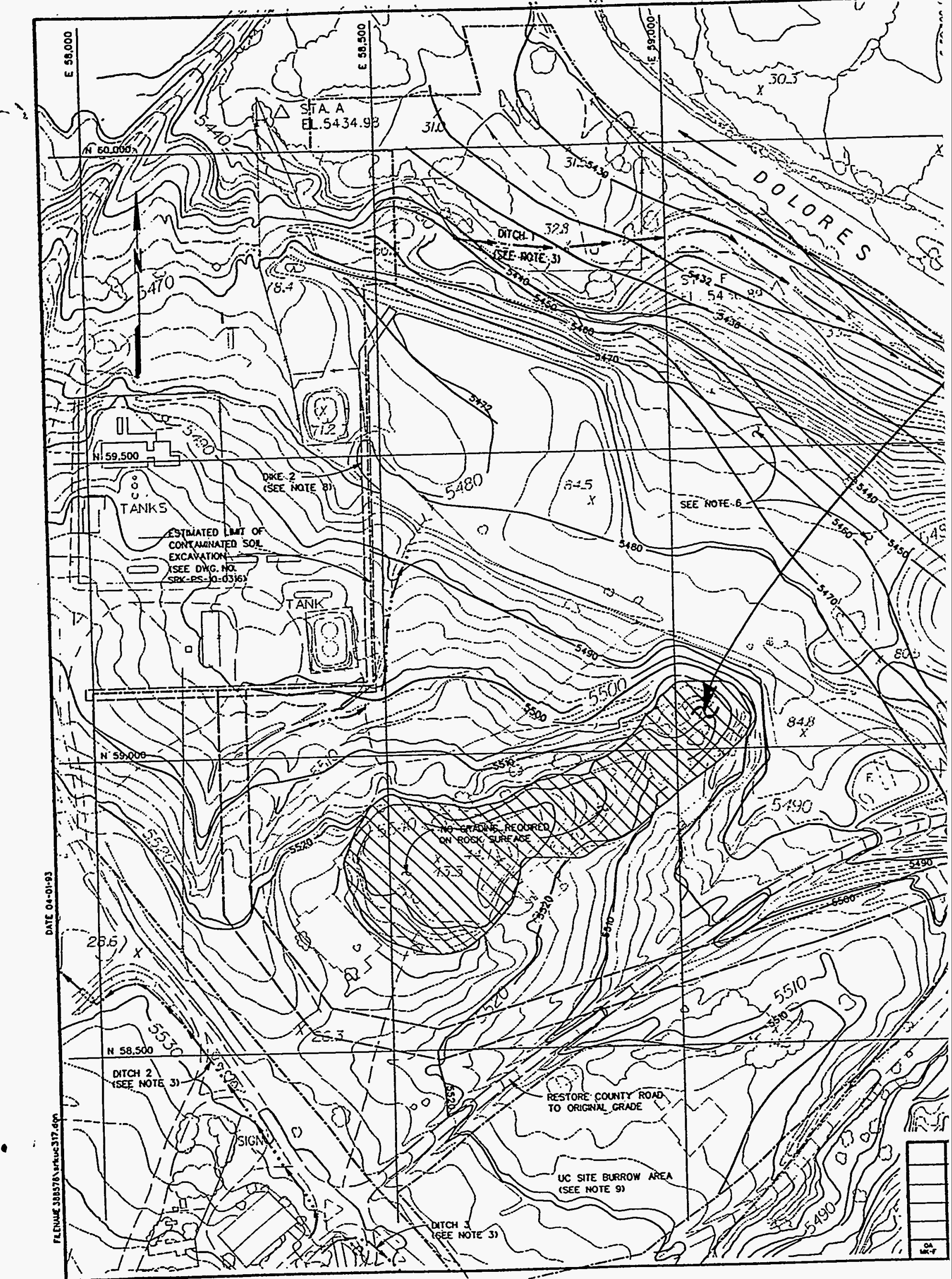




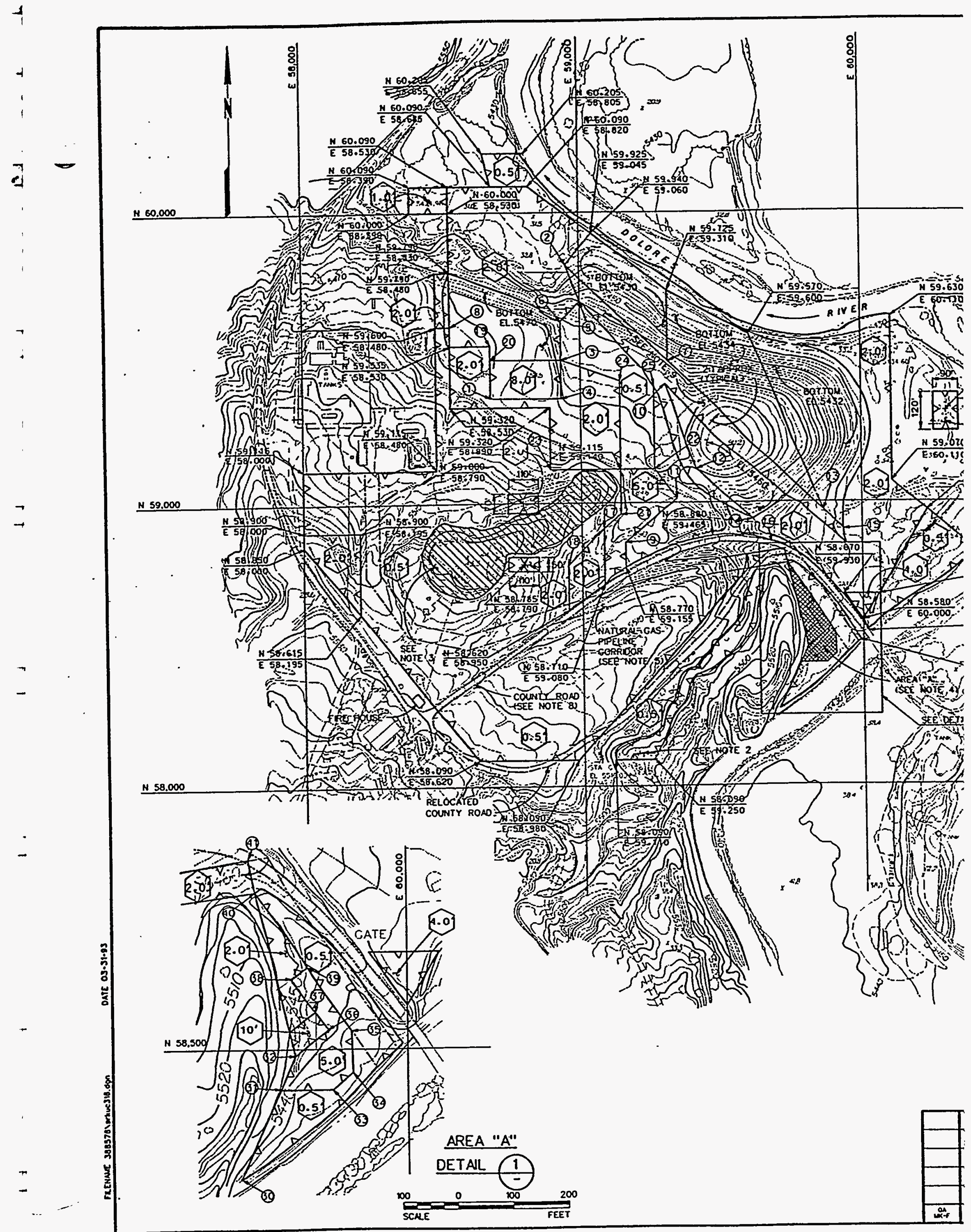


TABLE 1

LOCATKON PONT COORDONATES

\begin{tabular}{|c|c|c|}
\hline LOCATION & $\begin{array}{l}\text { MORTH } \\
\text { COOROENATE }\end{array}$ & $\begin{array}{c}\text { EAST } \\
\text { COORONATE }\end{array}$ \\
\hline 1 & N 59.350 & E 58.675 \\
\hline 2 & N 59.815 & E 58.950 \\
\hline 3 & I 59.485 & $E 58.930$ \\
\hline 4 & N 59.350 & $E 58.930$ \\
\hline 5 & K 59.690 & E 59.000 \\
\hline 6 & N 59.620 & E 58.930 \\
\hline 7 & N59.480 & E 59.310 \\
\hline 8 & K 59.600 & E 58.530 \\
\hline 9 & N 58.860 & E 59.155 \\
\hline 10 & K 59.400 & E 59.300 \\
\hline 18 & N 59.015 & E 59.340 \\
\hline 12 & N 59.115 & E 59.410 \\
\hline 13 & N58.970 & E 59.860 \\
\hline 14 & $N 58.940$ & E 59.610 \\
\hline 15 & 458.895 & E 59.940 \\
\hline 16 & N 58.855 & E 59.675 \\
\hline 17 & N 58.910 & $E 59.080$ \\
\hline 18 & N 58.780 & E 58.950 \\
\hline 19 & N 59.535 & E 58.675 \\
\hline 20 & N 59.485 & E 58.675 \\
\hline 21 & N 58.960 & E 59.140 \\
\hline 22 & N 59.115 & E 59.260 \\
\hline 23 & N59.115 & $E 58.890$ \\
\hline 24 & $N 59.410$ & E $59.1: 0$ \\
\hline 25 & N 59.395 & E 59.260 \\
\hline 30 & N 58.269 & E 59.701 \\
\hline 31 & H58.429 & E 59.774 \\
\hline 32 & N 58.467 & E 59.801 \\
\hline 33 & M 58.429 & E 59.865 \\
\hline 34 & N 58.459 & $E 59.900$ \\
\hline 35 & N 58.532 & E 59.899 \\
\hline 36 & $N 58.534$ & E 59.859 \\
\hline 37 & N 58.566 & E 59.260 \\
\hline 38 & N 58.625 & E 59.794 \\
\hline 39 & N 58.644 & E 59.823 \\
\hline 10 & N 58.782 & E 59.730 \\
\hline 41 & H 58.831 & E 59.719 \\
\hline
\end{tabular}

NOTES: REFERENCE \#6

1. ML CUIS SHRL BE $2(H): x V)$ LVESSS OTKERMISE

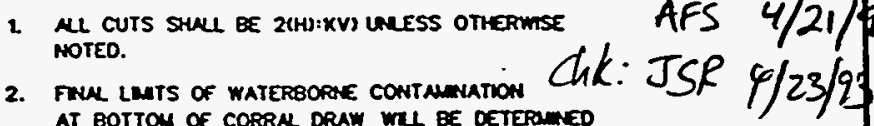
AT BOTTON OF CORRN DRAW WLL BE DETERamEeo N THE FEID.

3. NLL CONTAMRATED SOL ON EXPOSED OR EXCAVATEO ROCK SURFACES SHNL QE REWOVED. THES MAY REOURE HANO EXCAVATIOA OR WASHANG DOWN TO REMOVE ALL SURFACE CONTAMANATON. THE LMTTS OF CONTMUNATED WATERALS ON ROCK SURA ACES WhL $8 E$ DETERIMEO N THE FELD.

4. Contanatation materan from area "A" SHNLL BE PLACED W TTE OXSPOSA EMBAHKMENT WTH TALNICS PRBOR TO OTHER OFF-PLE CONTAMANATED SOLS.

5. EXCAVATIONS N THE MATRRA GAS PPELNE CORRDOOR (SO. ROW) WLL BE WSPECTED BY A PEPPRESONTATIVE OF THE RM.M.G. (ROCKY MOUNTAN MATURAL GAS) COMPANY. THE PPPELAE WRL BE TMKEN OUT OF SERVICE DURAKC THE EXCAVATION BUT maY CONTAN GR. SUBCONTRACTOR SHULL SUEMT PLAN FOR EXCAVATION MITHA THE GASLNE R.O.W. WHCH SHUL MCLUDE PROTECTION MO SUPPORT Of THE PPELNE DURANG EXCAVATION.

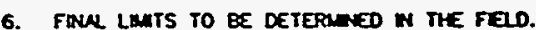

7. EXCAVATIONS SHULL MOT DAMACE OR EROANGER THE PETROG YPMS LOCATED ON THE CUFF FACE, WEST OF THE COUNTY RON BRTOCE.

8. EXISTNG COUNTY RON SHNL BE DETOURED TO LOCATION SHOWN. UNTR AREA SOUTH OF FENCE IS REMEDOATED MNO DELOLITION DEBRIS IS REMOVED. COUNTY ROND SHML THEN BE FULLY RESTORED TO ORIGNN LOCATION.

9. ROCK MAY BE ENCOUNTERED UNOER TMUNCS PUE $A$ * $B$ NO OTHER NREAS. SEE HOTE 3.

\section{REFERENCE DRAWINGS:}

\section{LEGEND:}

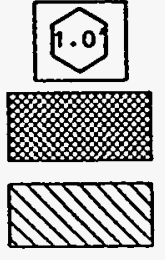

(1)

\author{
APPROXWUTE LUNT MSO DEPTH \\ $N$ FEET OF WADELOWN \\ CONTAURUTED MATERAN \\ AREA "A-
}

EXPOSED ROCK OUTCROP

LOCATION PONTS SEE TABLE, FOR COORONATES

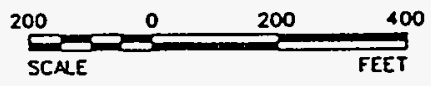

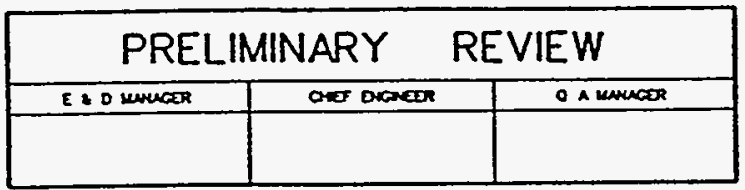

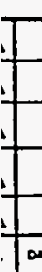

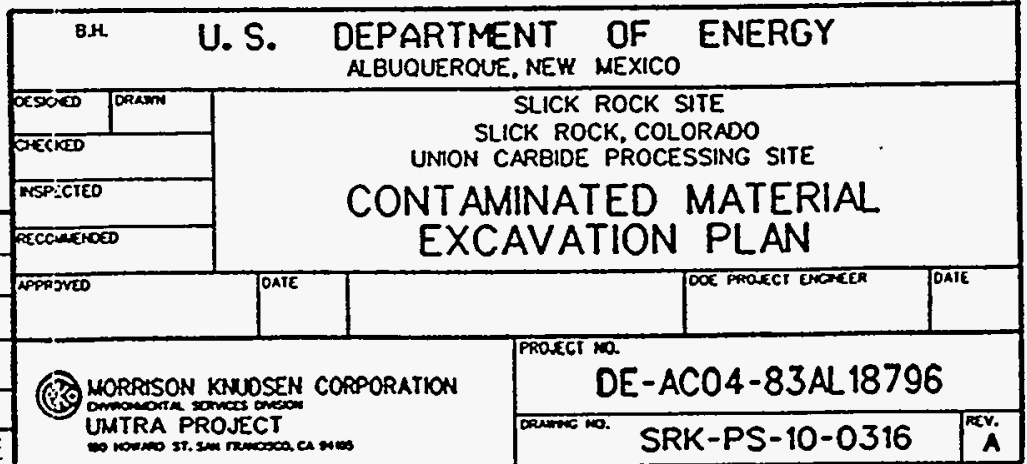



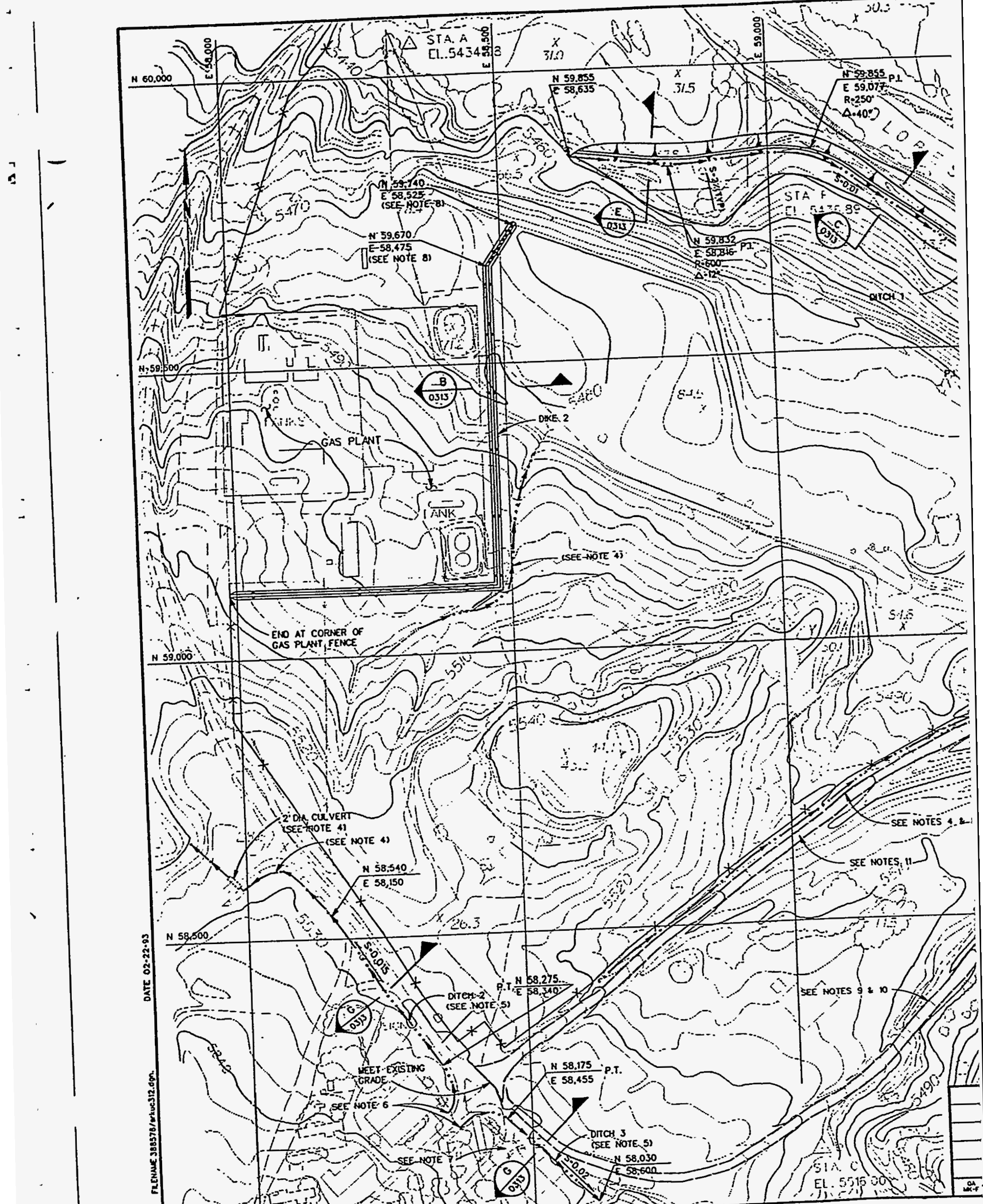
REF. \#T

SHEET 5
咅
:ํํㅇ
이 NOTES:
by AFS 4/21/93

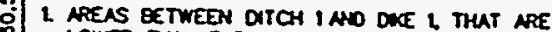
LOWER THW ELEVATION 5434.0 SHWL BE BAOKFUED NO CRWED TO DRAN NTO THE RETENTION BNSWI.

2. THE RETENTION BASW BERM, ON THE RAVER SDE. EETWEEN DTCH I NO ONE T, SHML DE AT DITS

3. SEOCONTRACTOR SHUL PROVDE FOR POSITVE DRANUCE TOWAFD THE RETENTIOH BNSW OR MAKE PROVISTOWS FOR REMOVINTS WAIER FROW POWO AREA

4. SUBCONTRACTOR SHALL PROVIOE FOR NO MUNTAN POSTTVE DRAMHAE.

5. MONG DOSTING COUNTY ROND.BOTTON OF DRUNS PATH SHULL BE MN. $T$ BELOW TOP OF THE RONO.

6. SUACONTRACTOR SHNLL GRADE RONO TO PROVDEE - POSITIVE ORAWHAE TOWARD TELPORARY DTCEES. EEGN LOCATION OF TOPORURY DICEES AT pPpoxuate locations as SHOWN. Exact locations SHML FE DETERIANED W THE FELD BY THE ISUECONTRUCTOR.

7. ROLA SHWi- EE TEMPORARYY CLOSEO TO NLOW FOR

- TELPORART ditca cRossang.

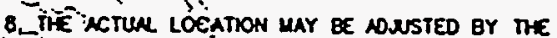
contractor to. betTER fit the fELD cohoition.

9. EXISTING COUNTY. ROND CR-SB SHNL EE DETOARED TO -LOCATION SHOWN LNTI AREA SOUTH OF THE FENCE

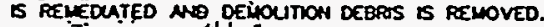

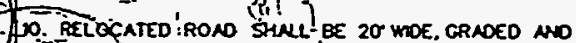

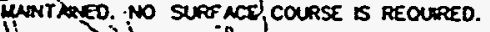

11. AFTER ARE SOUTH OF' FEACE IS RELEOUATED CONTY RONO CR-SB FULL BE'RECONSTRUCTED NONG THE

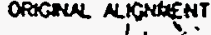

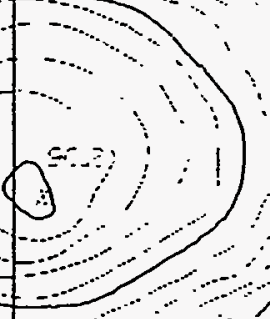

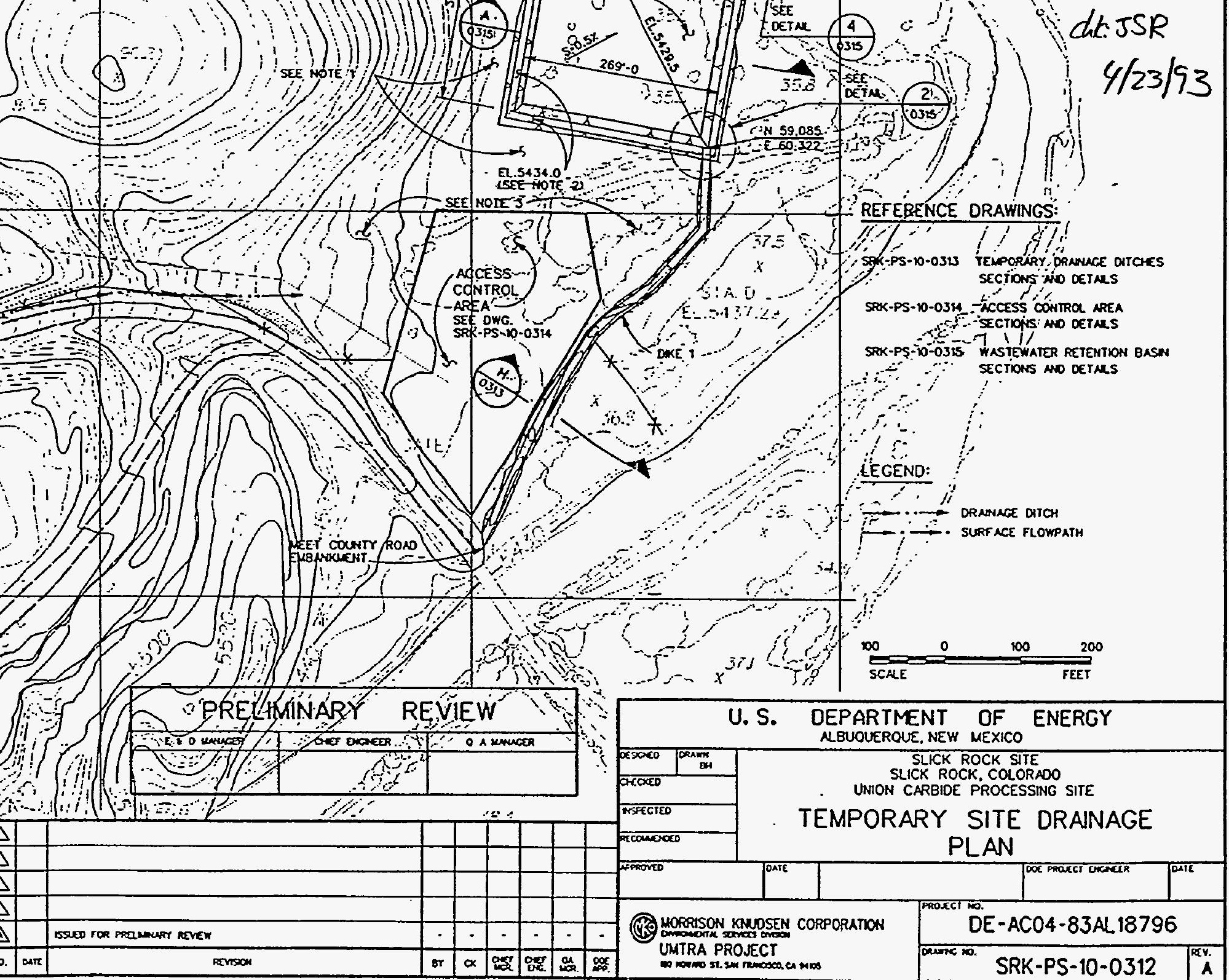



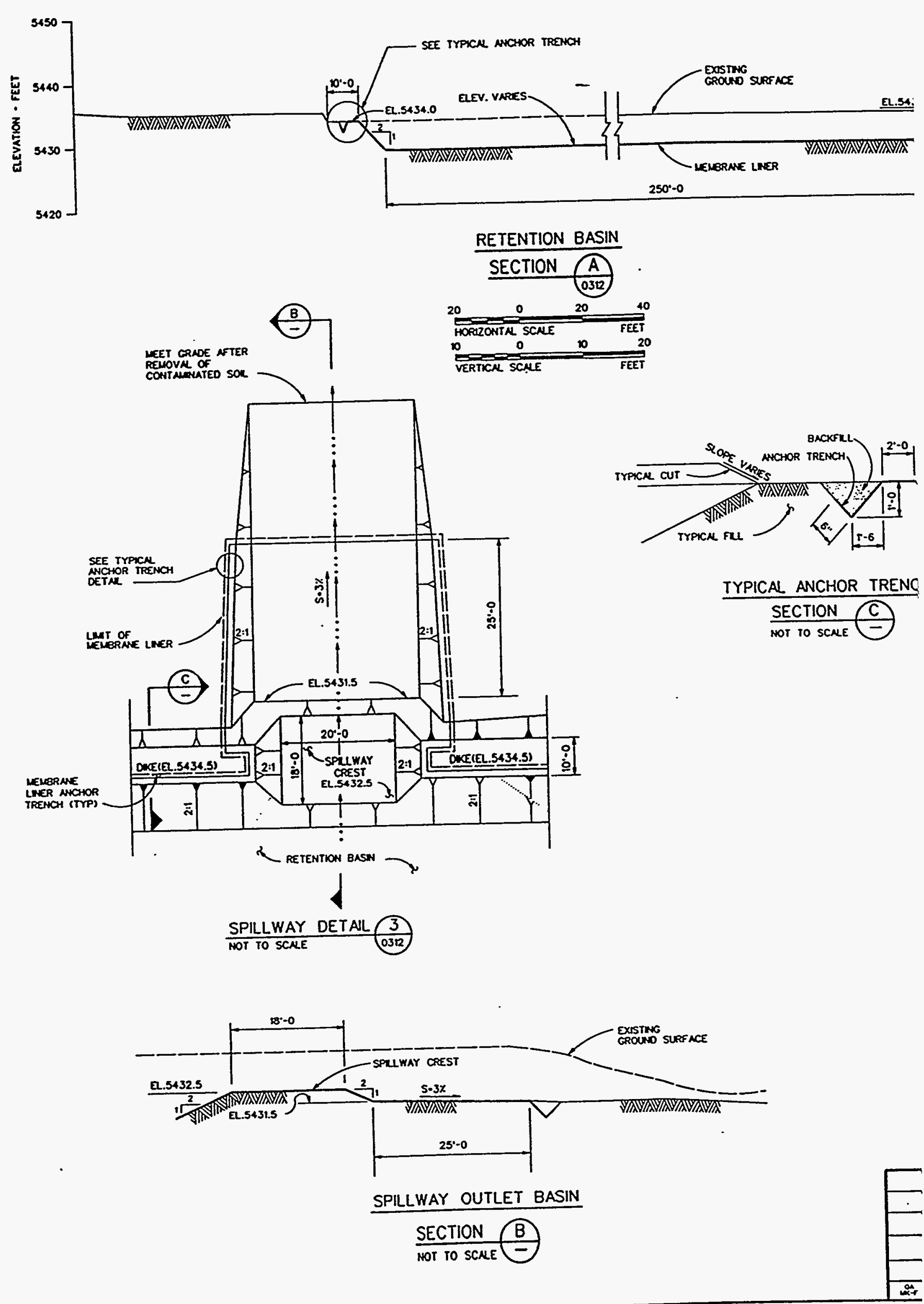
@9 .MORRISON KNUDSEN CORPORATION

Project UMTRA-SRK

Feature SITE RESTORATION Item EARTHWORK QUANTITIES
Contract No. 3885-76 File No.

Designed AFS Checked ISR
Date

Date

(COMMON BACKFILL - UL SITE, CONTD)

VOLUME OF TRAPEZOID-LIKE SHAPES IN LAST $9 \mathrm{ft}$ of THE

ENDS OF THE RETENTION BASIN:

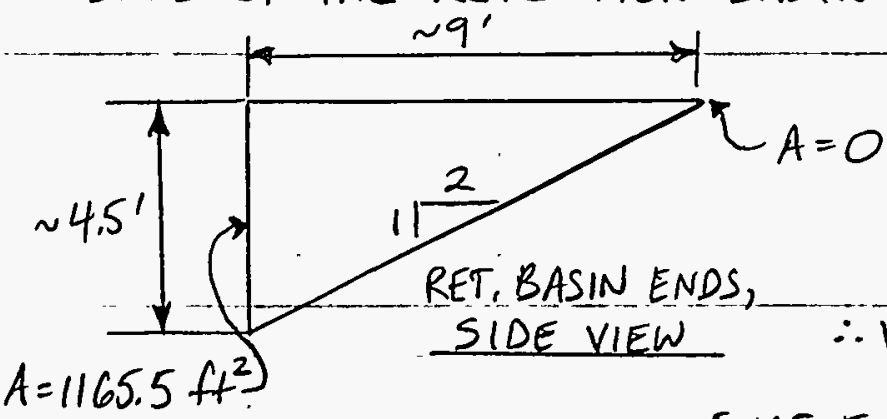

$$
[1165.5 \times(360-18)+2(3496.5)] / 27=15,022 \mathrm{~g}
$$

EXCAVATIONS IN THE PIPELINE CORRIDOR CORRESPOND TO SUBAREAS

XII + XXV + XXIII in REF. 2 , SHTS $420242 E \rightarrow \frac{1}{2}(14,820+6840+17,135) / 27=718 \mathrm{cy}$

$\therefore$ VOLUME OF BACKFILL \& UL SITE $=867+4056+15,022+718 \cong 20,700 \mathrm{cy}$

NC SITE:

The areas marked (2) Through (7) and (X) ON Sheet 8 . are areas of contaminated material excavation. They CORRESPOND TO SUBAREAS LABELED ON SHEET. 13 E OF RES. 2. THE QUANTITY of baCKFILL REQUIRED EquALS THE quANTITY of excavated contaminated material, calculated in Ref 2.

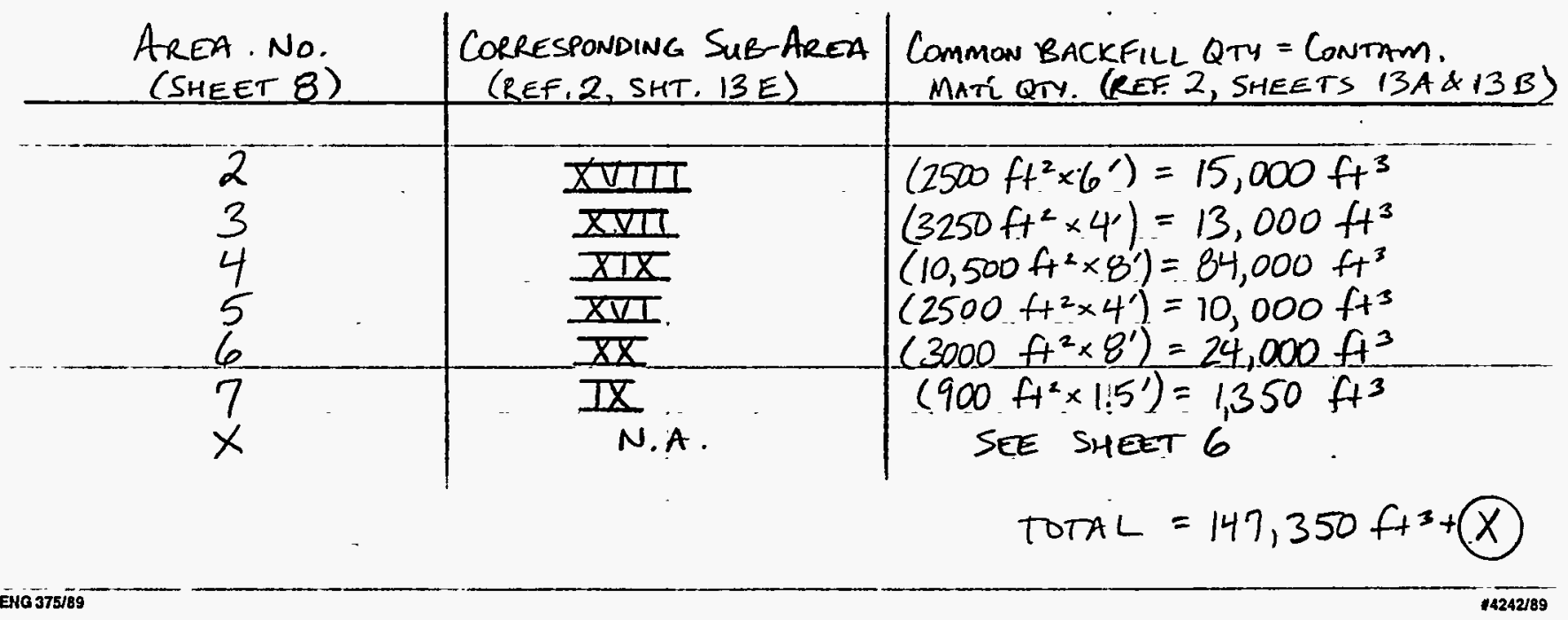




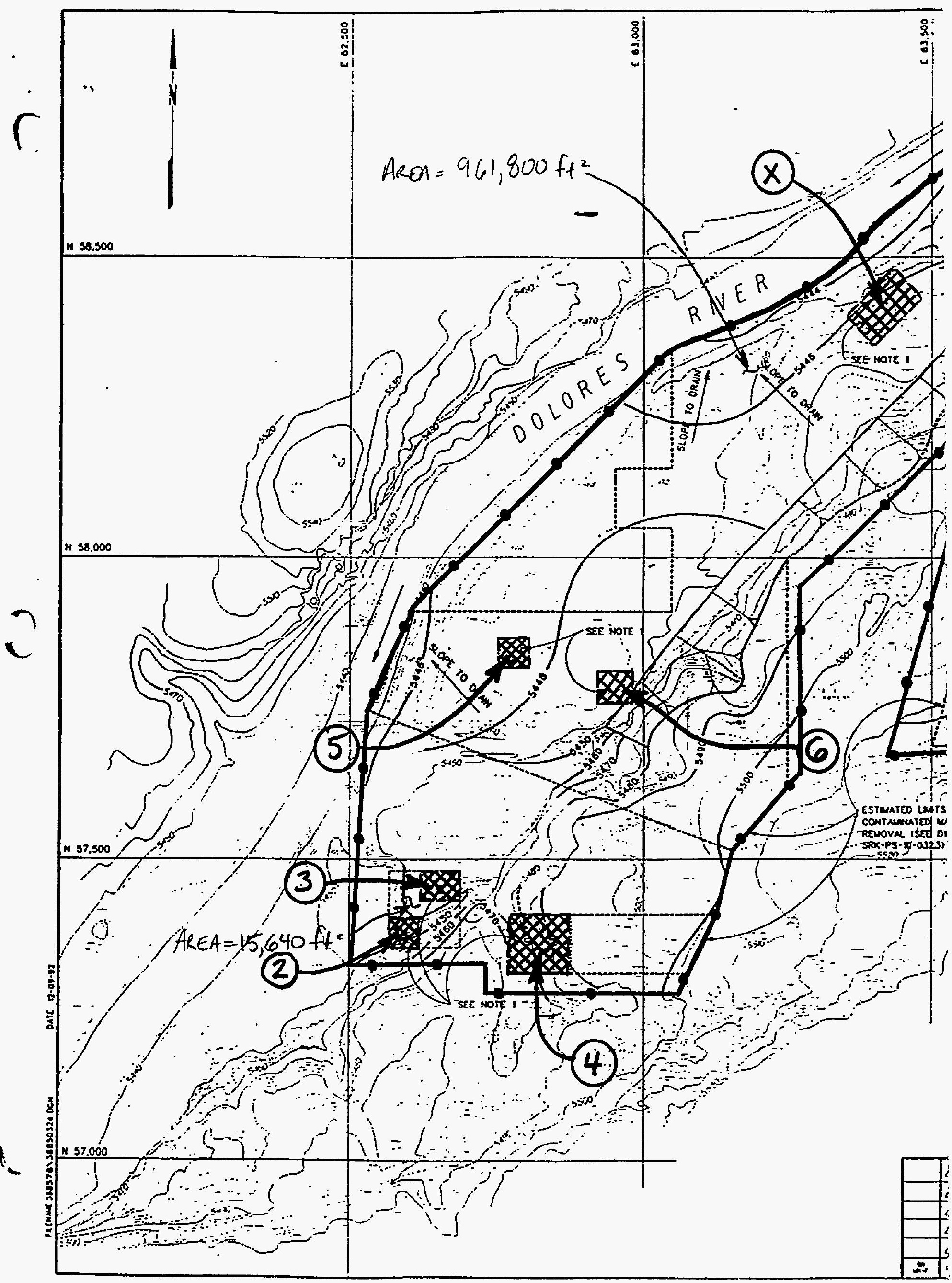




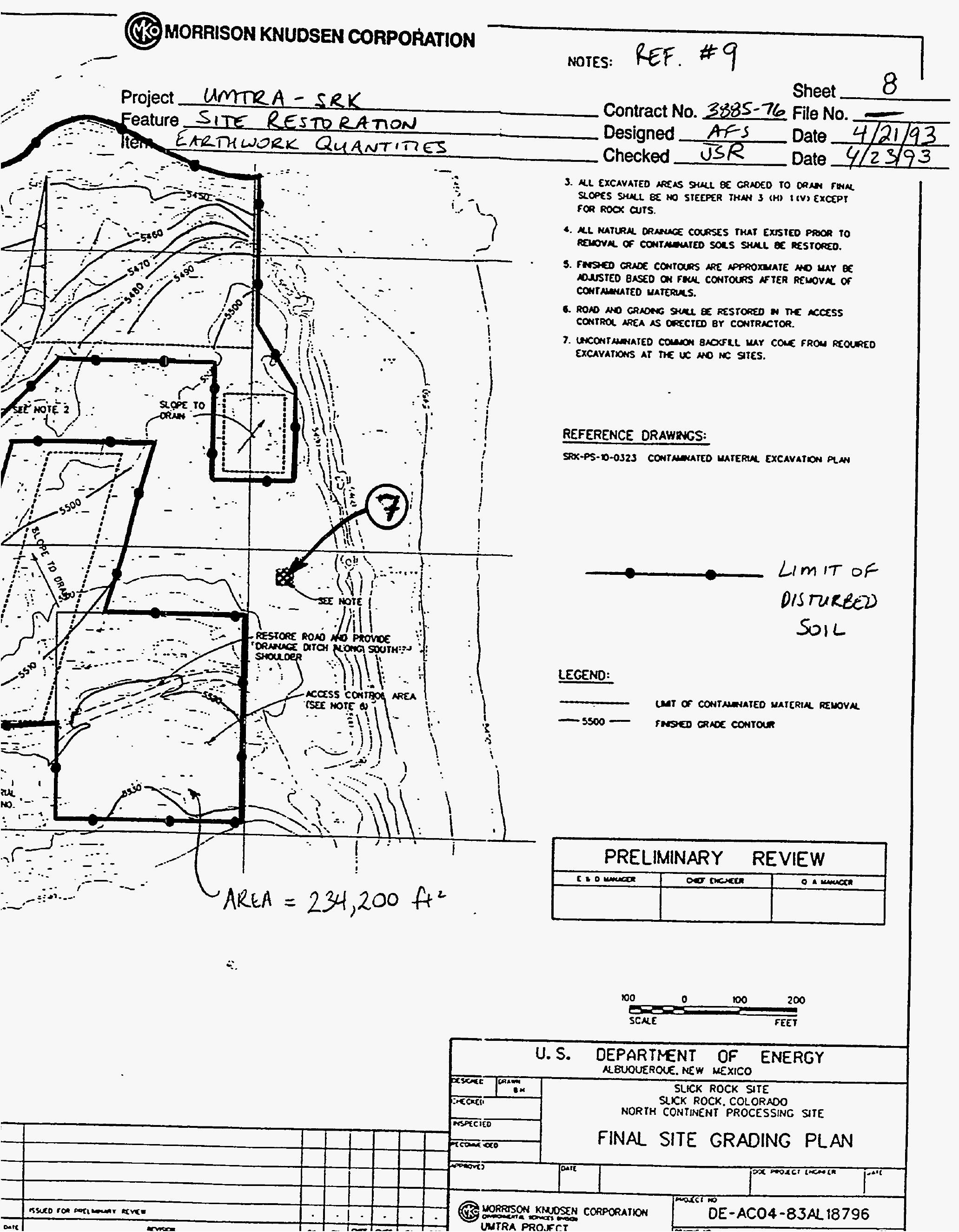


CR MORRISON KNUDSEN CORPORATION

Project UMTRA - SRK

'Feature SITE RESTORATION Item EARTHWORK QUANTTIES
Sheet 9 Contract No. $\frac{3885-76}{3}$ File No.

Designed AFS

Date

Date

(COMMON BACKFILL - NC SITE, CONTO)

Area of $x=70^{\prime} \times 110^{\prime}=7,700 \mathrm{ft}^{2}$ (SCALED Off sheet 8)

Bottom of excavation of area $X=5443 \mathrm{ft}$ (SEe SHeEt: 10 );

AVG. FINAL GRADE aT area $X=5446.5 \mathrm{ft}$ (SEE SHEET 8 );

AVG. DEPTH OF BACKFILL O AREA $X=5446.5-5443=3.5 \mathrm{ft}$.

Volume $=7,700 \mathrm{ft}^{2} \times 3.5=26,950 \mathrm{ft}^{3}$

$\rightarrow$ VOLUME OF BACKFILL IN EXCL VAPED AREAS $=(147,350+26,950) / 27$

$=6456 \mathrm{cy}$

The nC site also has 5 corrugated steel pipe manholes

AND 3 FORMER OUTHOUSES OR CESSPOOLS WHICH MUST BE

REMOVED. THE HOLES MUST BE BACKEILLED (SOE REF 11, OR

SHEET 11)

The dimensions of the cist. manholes are approximately 18 "DA, $3 \mathrm{fH}$ TO $4 \mathrm{ft}$ DEEP.

$$
\text { VoLUME C.S.P. MH. }=\pi\left(\frac{9}{12}\right)^{2} \times 4=7 \mathrm{fH}^{3}
$$

- THE DIMENSIONS OE THE CESS POOLS ARE APPROXIMATELY

$10 \mathrm{ft} \times 6 \mathrm{ft} \times 6 \mathrm{ft}$ DEEP

VOLUME CESSPOOL $=10 \times 6 \times 6=360 \mathrm{ft}^{3}$

$$
\begin{aligned}
\rightarrow \text { VOLUmE of MANHOLES \& CESSPOOLS } & =\left(5 \times 7 \mathrm{ft}^{3}+3 \times 360 \mathrm{ft}^{3}\right) / 27 \mathrm{ft} / \mathrm{cy} \\
& =41 \mathrm{cy}
\end{aligned}
$$

$\therefore$ TOTAL BACKFILL Q NC SITE $=6456+41=6497 \mathrm{cy}$

$$
\cong 6500 \mathrm{cy}
$$

ENC $3 \overline{75 / 89}$

$14242 / 89$ 


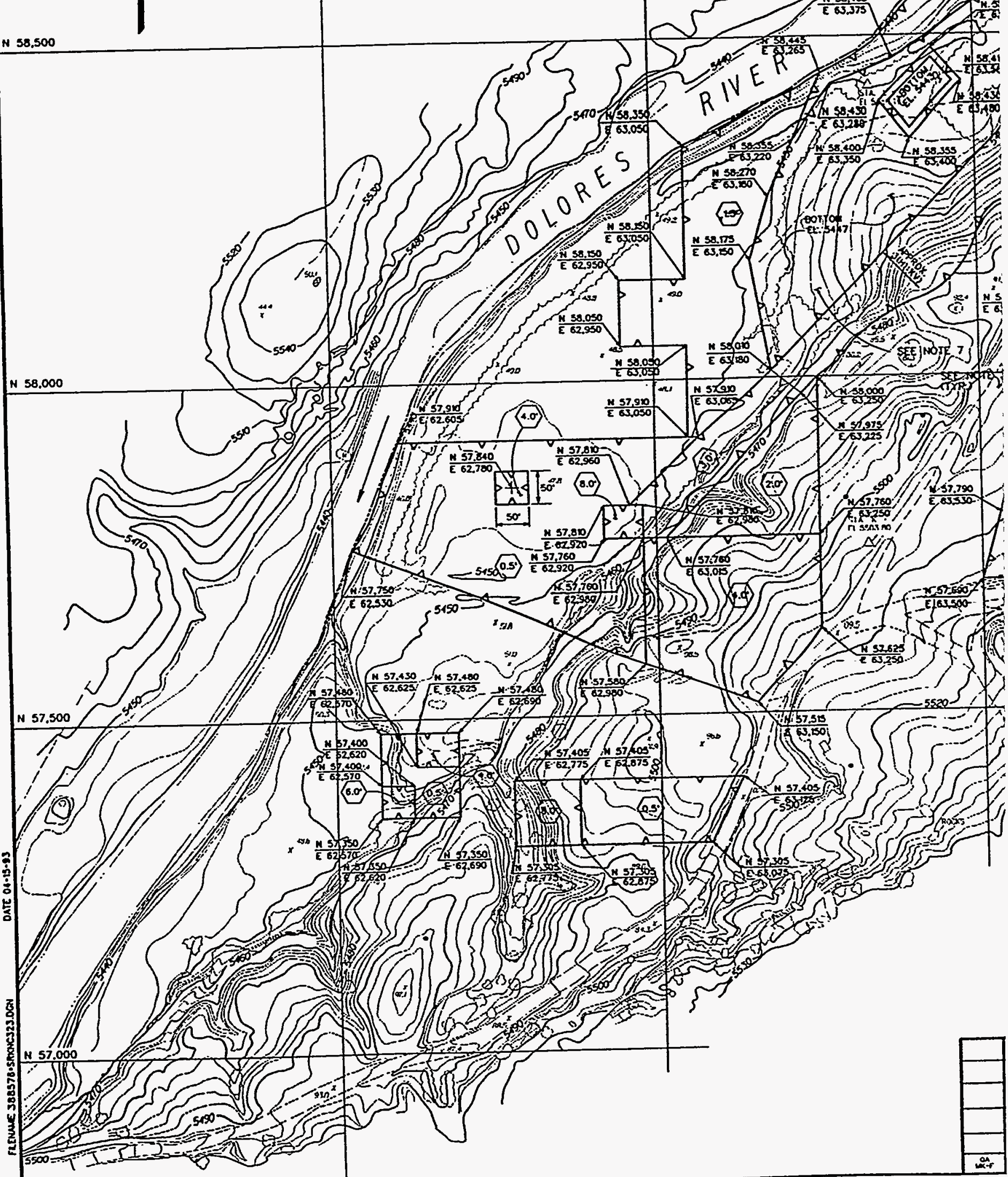




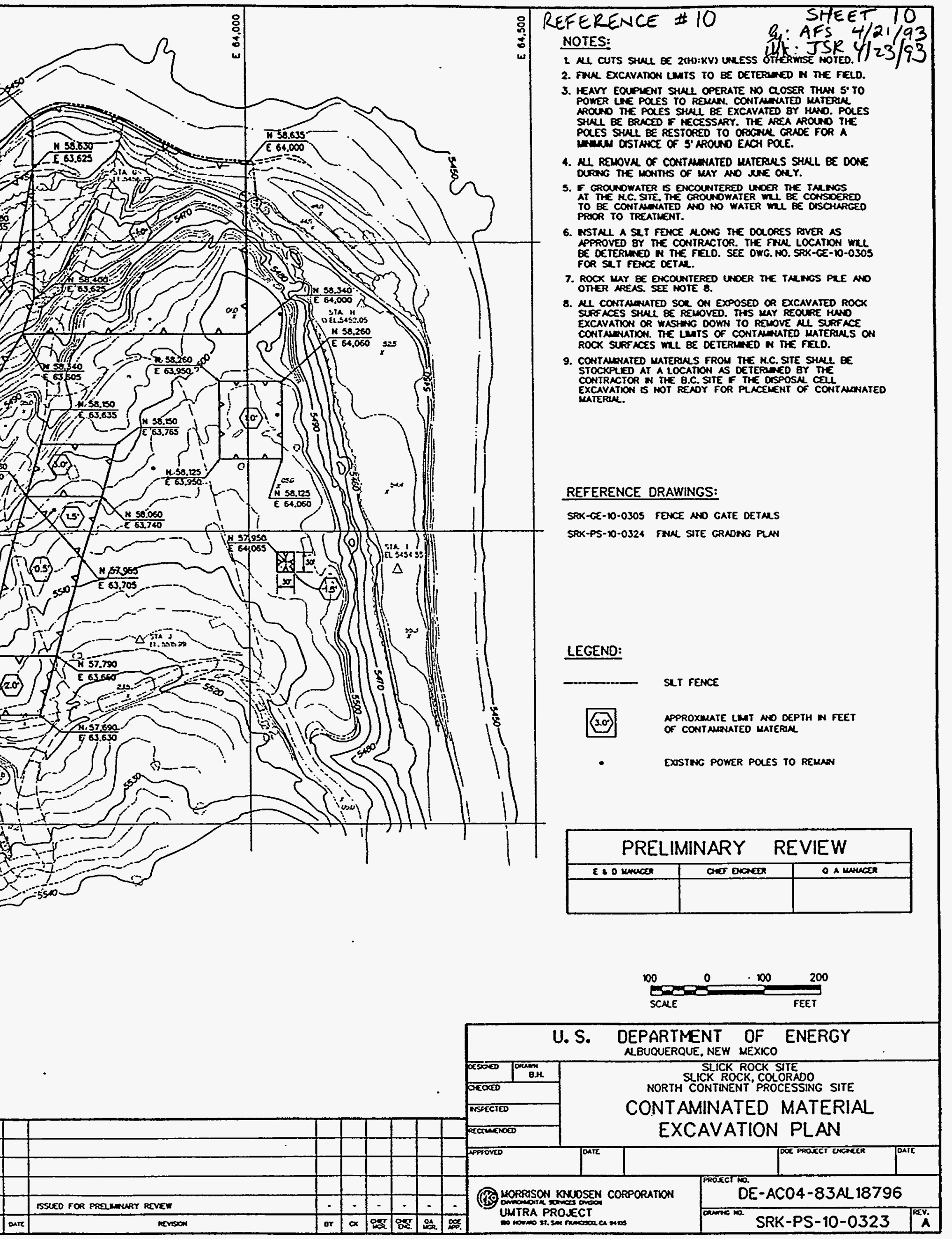




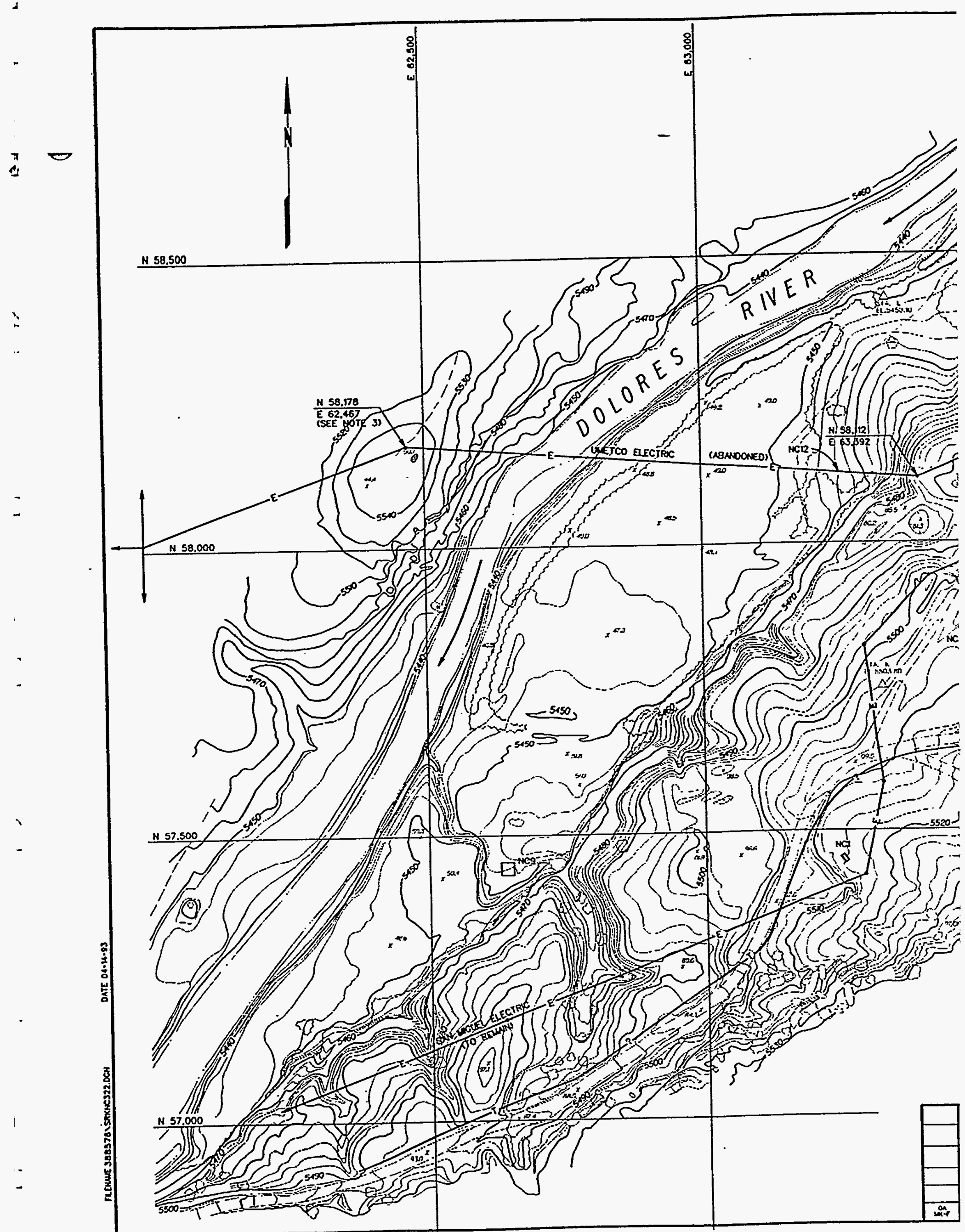




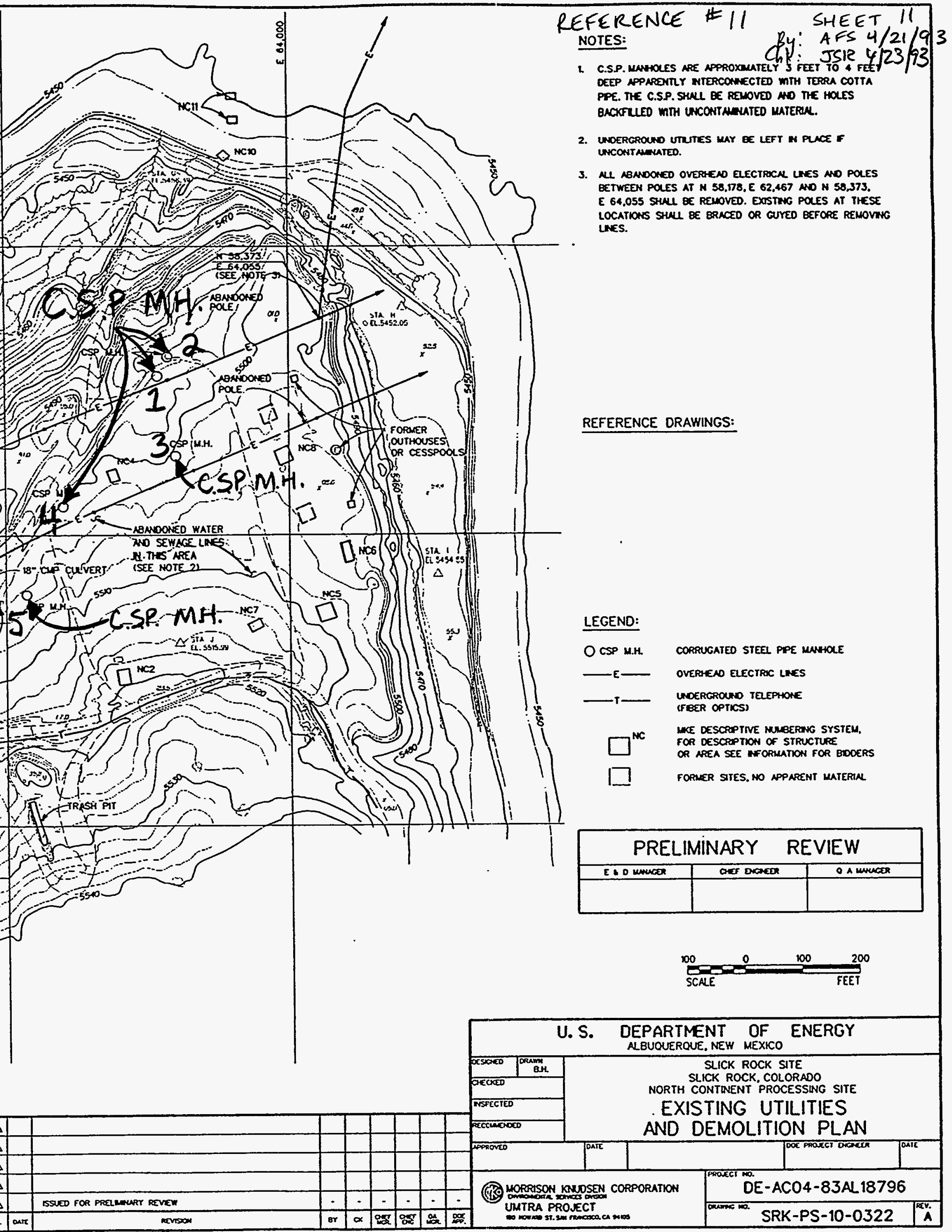


@MORRISON KNUDSEN CORPORATION

Project UMTRA-SRK

- Feature SITE RESTORATION Item EARTHWORK QUANTITES
Sheet 12

Contract No. $\frac{3885-76}{A \text { File No. } \frac{\bar{T}}{4 / 21 / 93}}$

Checked Date $4 / 23 / 93$

(common FILL, conTos)

BC SITE:

SHEET 13 (REF. \#12) SHOWS THE RETENTION BASIN AT THE BC SITE. It mUST BE BACKFILLED:

The average depth to Bottom is

$\frac{5824+5825.5}{2}-5815.5=9.25 \mathrm{ft}$ (SEE SHT. 13)

VOLUME IN MIDDLE $=(190 \times 120+35 \times 120)(9.25)=249,750 \mathrm{ft}^{3}$

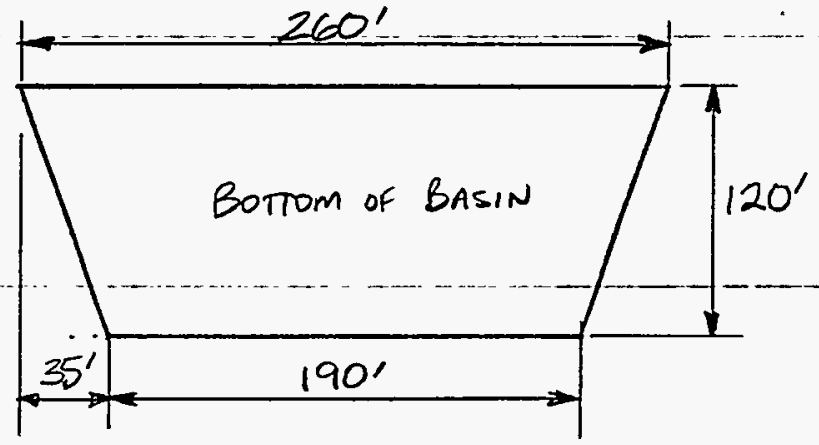

LENGTH OF SIDE SLOPES $=3(9.25)=27.75 \mathrm{ft}$

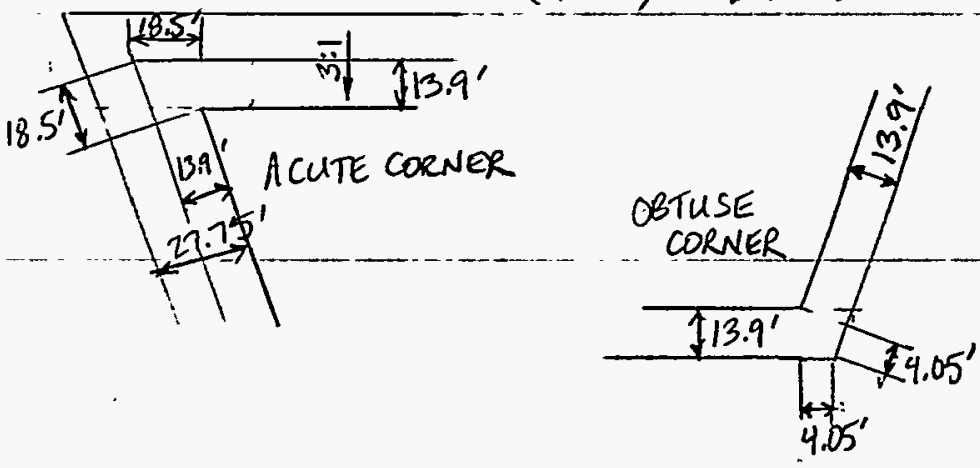

PERIMETER AROUND MIDPOINT OF SIDE SLOPES

$$
\begin{aligned}
P & =260+2(18.5)+2(120+18.5+4.05)+190+2(4.05) \\
& =780.2 \mathrm{ft}
\end{aligned}
$$

AREA OF SIDESLOPE SETON $11^{3} / 9.25^{\prime}-A=\frac{1}{2}(3)\left(9.25^{2}\right)=128 \mathrm{ft}^{2}$

TOTAL VOLUME TO BACKFiLL $=249,750+780.2 \times 128=349,616 \mathrm{ft}^{2}=12,949 \mathrm{cy}$

Eพัติว75/89

$44242 / 89$ 


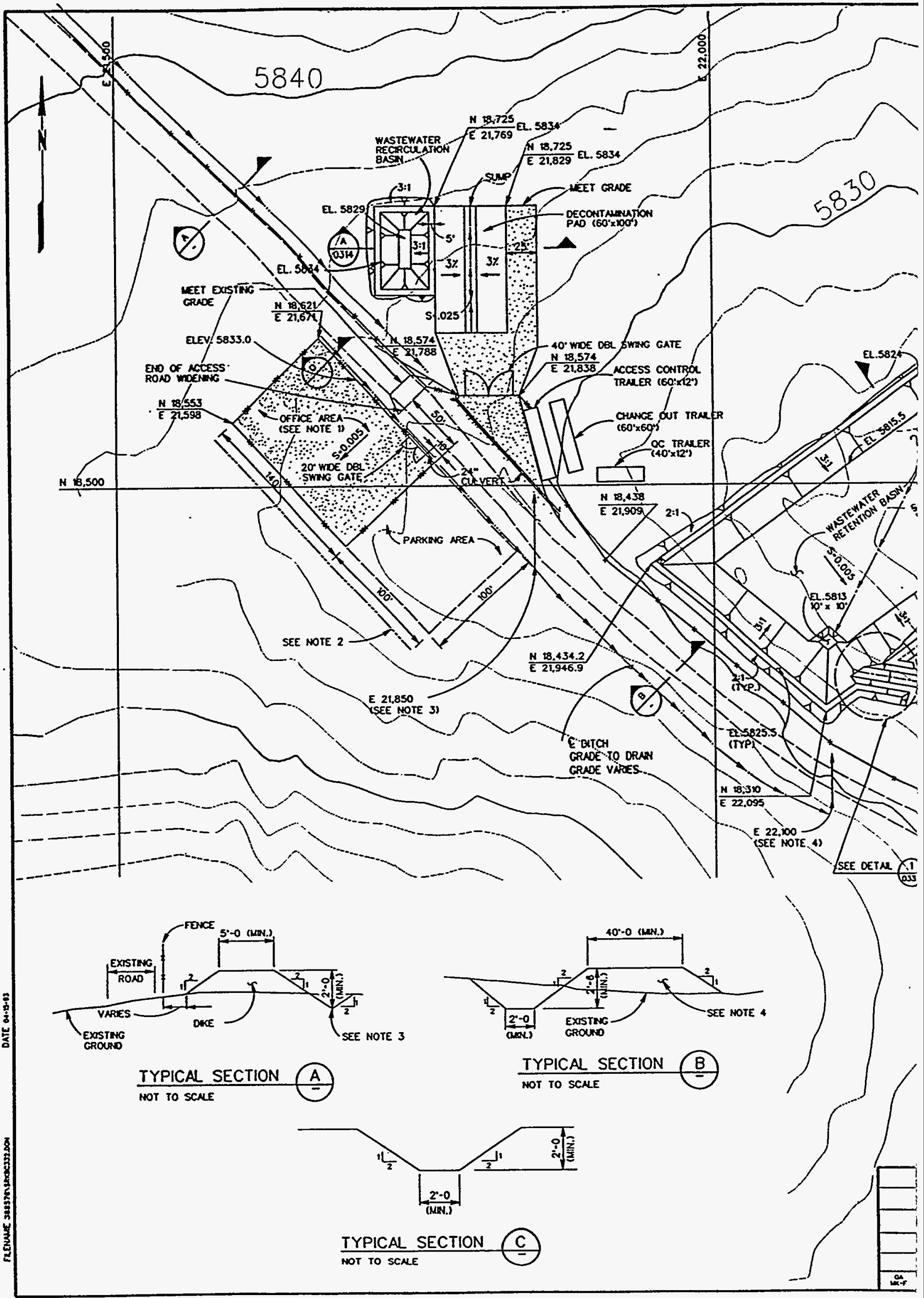




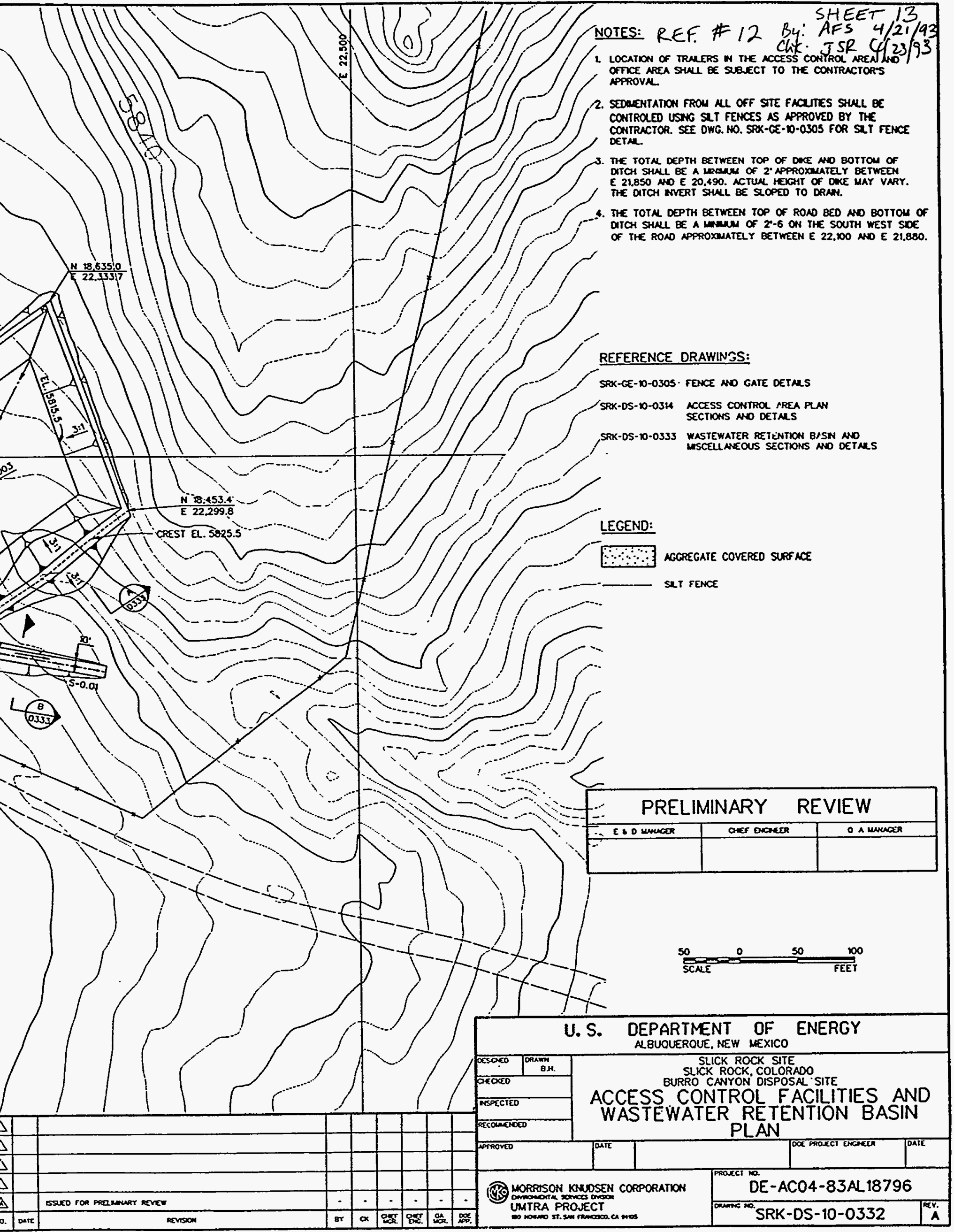


@i.MORORISON KNUDSEN CORPORATION

Project UMTRA - SRK

"Feature SITE RESTORATION Item EARTHWORK QUANTITIES
Contract No. $3985-76$

Designed AFS Checked J $J ! R$

Rev of By

SR

$A M B$
Sheet

Date $4 / 3 / 93$

Date $4 / 23 / 93$

(COMMON FILL, CONTD).

BC SITE

SHEET: 15 SHOW' the CONTOURS OF THE INROADS-gENERATED

SURFACE THAT REPRESENTS THE EMBANKMENT AND FINAL SITE

GRADING. A cOMPARISON OF these cONTOURS AND the cONTOURS

SHOWN ON SHEET 16 (REF, DUG B).

InRoAds IS USED to compute the fill volume between

the dESIGN SURFACE AND tHE EXISTING GROUND SURFACE FOR

THE AREAS OUTSIDE THE APRON. THE RESULTING VOLUME IS:

$$
\left.\begin{array}{l}
103,880 \mathrm{cy}, \text { SAY } 103,900 \\
V_{F}=135,1+35 \mathrm{cy}, 000 \mathrm{y}
\end{array}\right\} \text { Reval }
$$

AS A CHECK, SHEET 17 DEFINES 3 SEPARATE AREAS OF GRADING/CONTOURING.

AREA $1\left(\sim 65,900 \mathrm{ft}^{2}\right)$ REQUIRES AN AVG. OF ABOUT $2 \mathrm{ft}$ OF FILL AREA $2\left({ }^{3} 390,380\right.$

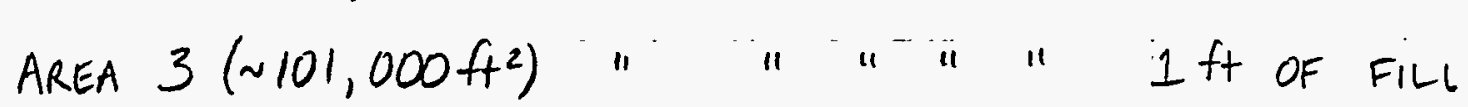

$$
\begin{aligned}
\text { TOTAL VOLUME } & =(65,900 \times 2)+(490,380 \times 6 \\
& =3,5,000 \times 9)+(101,000 \times 1) \\
& \approx 1,57,800 \mathrm{ft}^{3} \\
& =1,000 \mathrm{cy} \quad(2 \% \text { DIFFERENCE })
\end{aligned}
$$

$\operatorname{ReV}$ (exdudes topsoil)

$\therefore$ TOTAL COMMON FILL REQUIRED, AT. BC SITE:

$$
12,949+135,103,13=1168,069 \cong 117,000 \mathrm{cy}
$$

- Since $6 "$ of topsoil is removed from access control, retention basin and tiunsh grading areas, that 6 " has to be replaced.

Area that needed topsoil replaccucent $=816,400 \mathrm{ff}^{2}$.

vol. of material needed for

ENG $375 / 199$

$$
\equiv 816400 \times \frac{1}{2} \times \frac{1}{27} C Y=15,119 \mathrm{CY}
$$

44242389 
Project UWTRA- SFK

Feoture Site Restoration

Item Earthwork Quantitios

This entire sheet is part of ReV OI

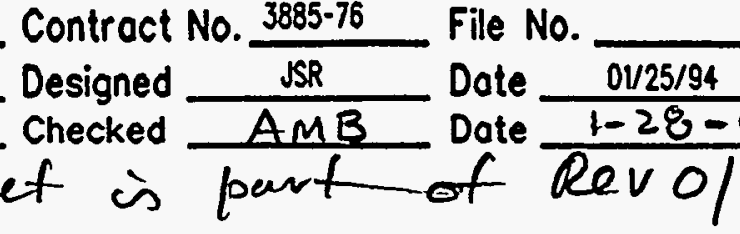

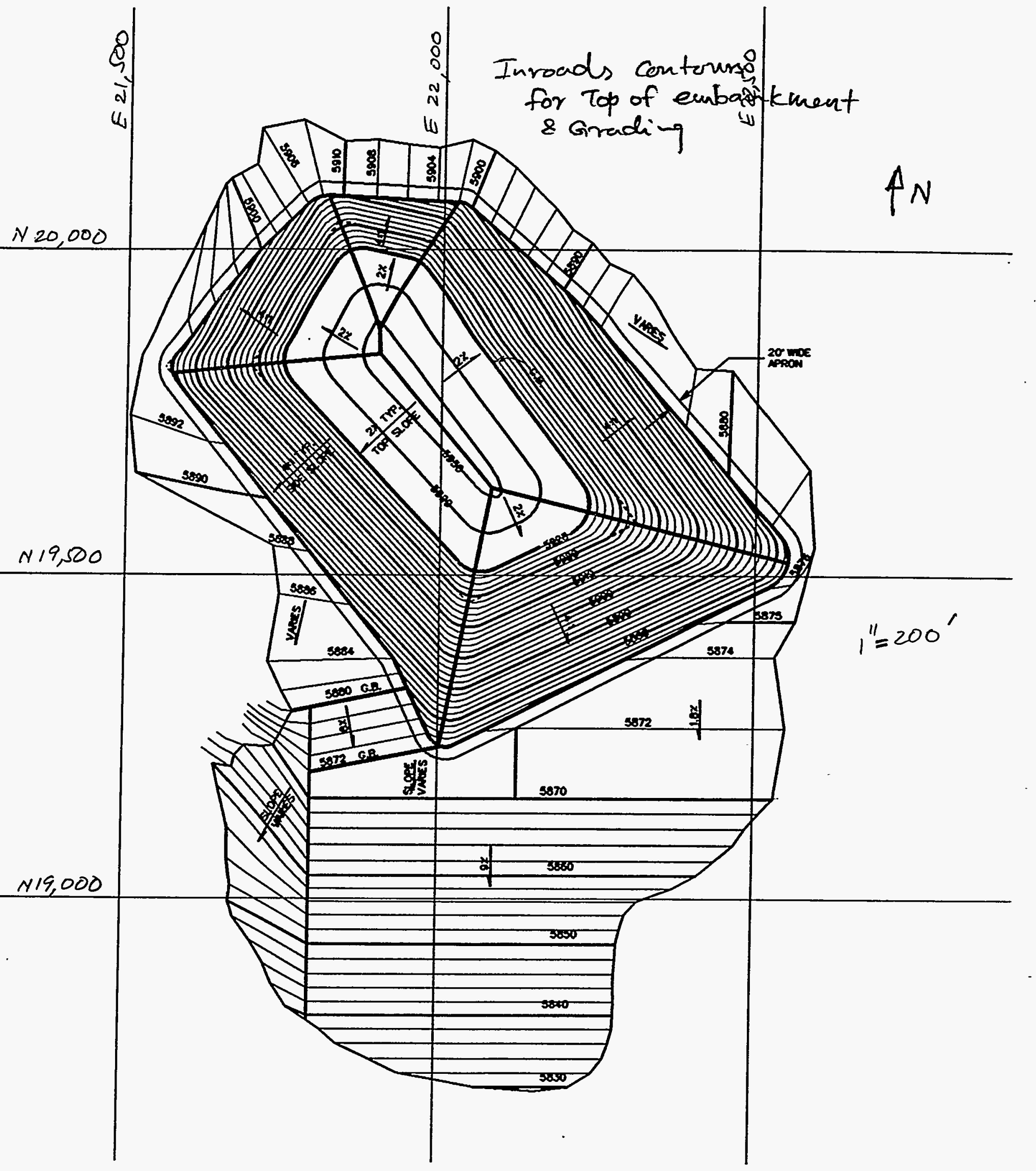




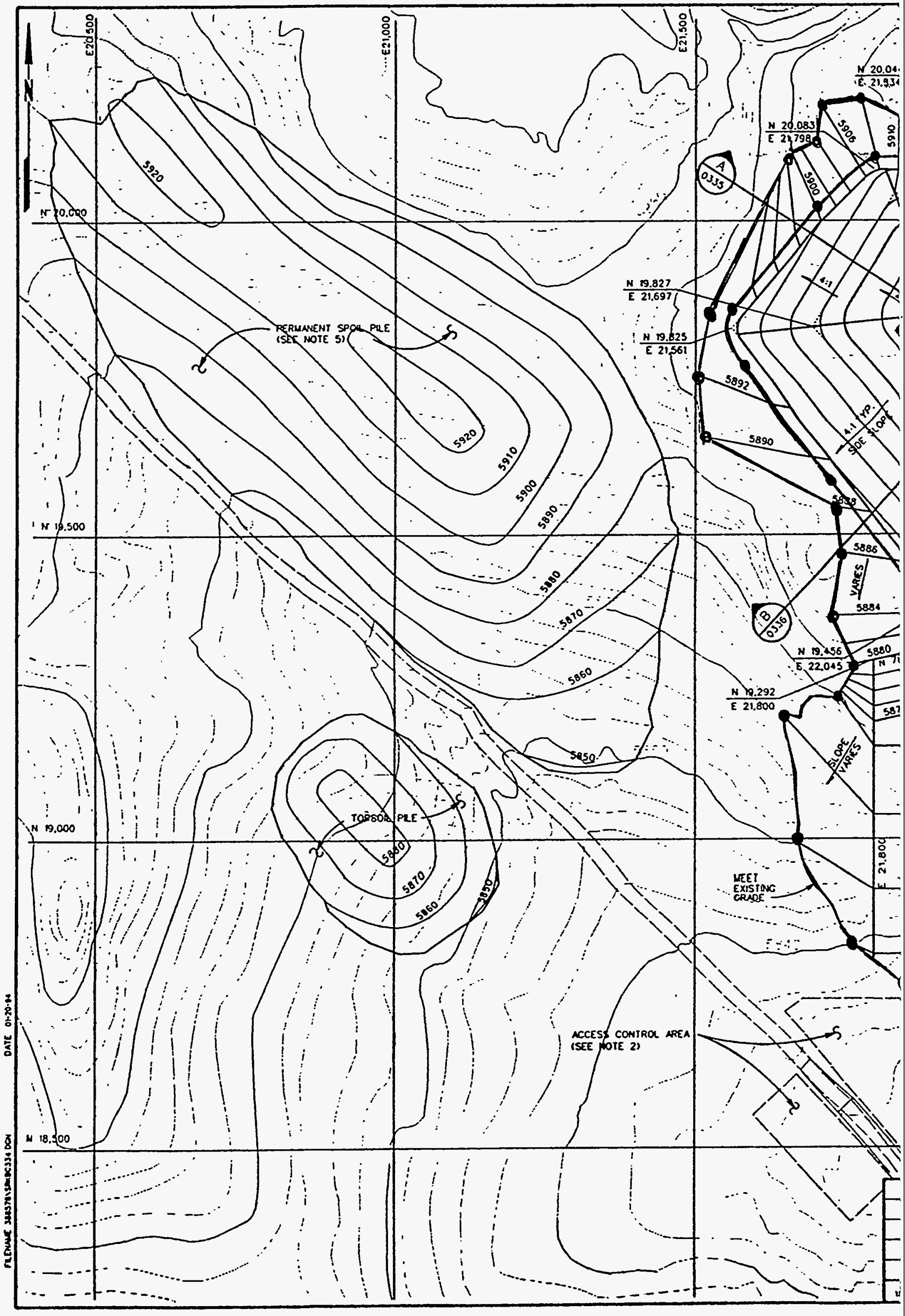




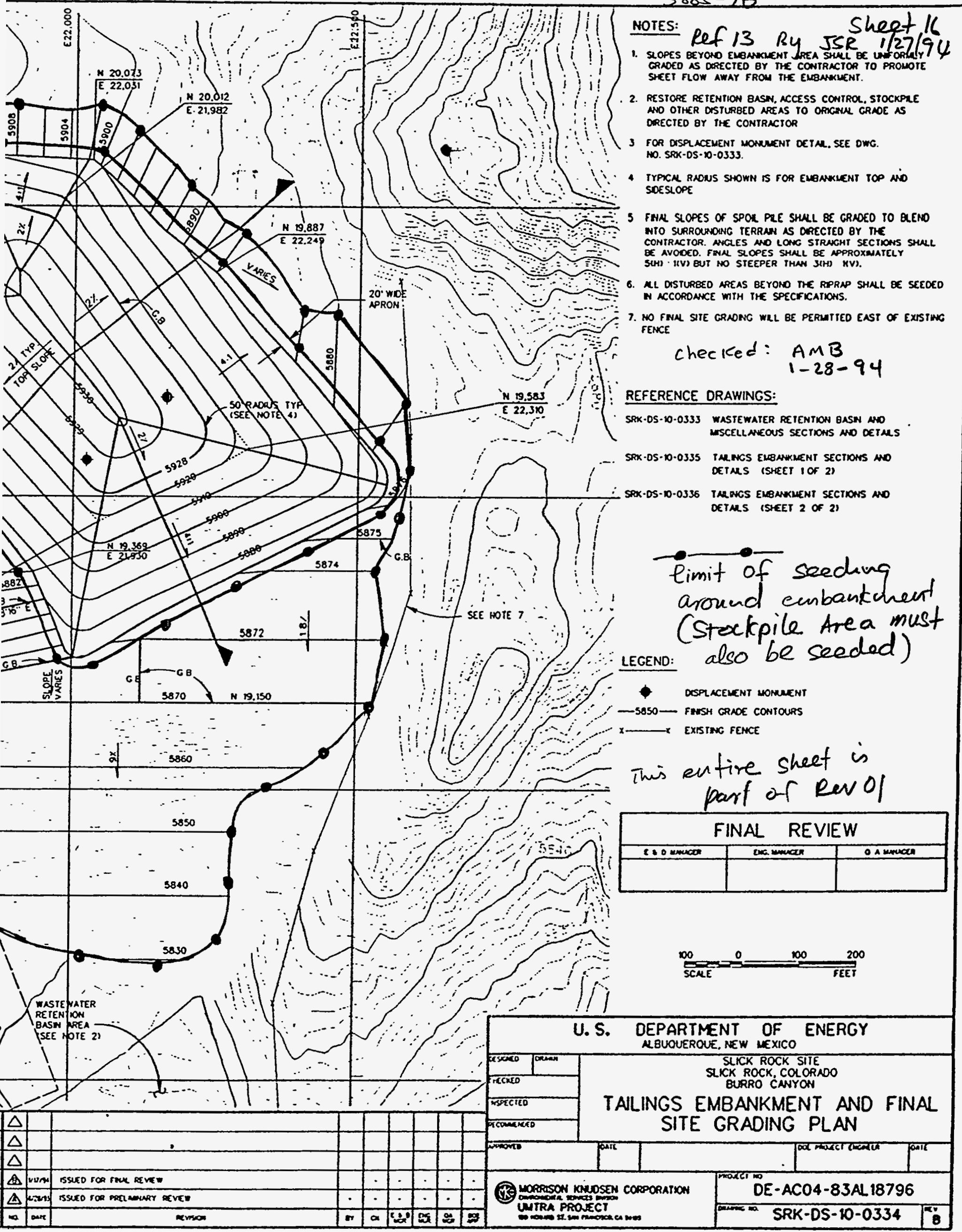


(40 .MORRISON KNUDSEN CORPORATION

Project UMTRA-SRK

Feature SITE RESTORATION

Item EARTHWORK QUANTITIES
Sheet 18

Contract No. 3885-76 File No.

Designed AFS

Checked

Date

Date

EXCAVATION FOR GRADING/CONTOURING

UL SITE AND NC SITES

FROM SHEETS $3 \& 4$ (UL SITE). AND 8 (NC SITE), THE

total areas of GRADED, EXcAVATED, or otTER WISE DISTURBED

-.. SOIL SURFACES AT THE UL \& NC SITES ARE:

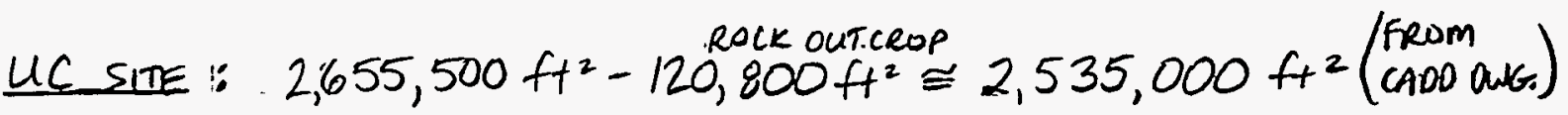

NOTE: SHEET 3 SHOWS NO GRADING IN CORRAL DRAW, AND

INDICATES THAT NO GRADING IS REQUIRED ON ROCK SURFACE.

NC SIRE: $961,800+234,200=1,196,000 \mathrm{ft}^{2}$

(MEASURED BY PLANIMETER ON SHEET 4 )

From SHEETS $3 \& 4$ IT IS ESTIMATED THAT ON THE AVERAGE APPROXIMATELY HALF OF THE DISTURBED AREAS WILL REQUIRE EXCAVATION FOR REgRADING, aNd that thE aVERAGE DEPTH OF EXCAVATION WILL BE APPROX $0.5 \mathrm{ft}$ AT BOTH TE UL AND NC SITES.

$\therefore$ VOLUME GRADING EXCAV. $=\frac{1}{2}$ (DISTURBED AREA) $\left(0.5^{\prime}\right)$

\begin{tabular}{c|c|c} 
SITE & DISTURBED SOIL AREA & VOLUME (wy) \\
\hline UT & $2,534,000 \mathrm{ft}^{2}$ & $23,470 \cong 23,500 \mathrm{cy}$ \\
NC & $1,196,000 \mathrm{ft}^{2}$ & $11,074 \cong 11,100 \mathrm{cy}$ \\
\hline
\end{tabular}

ENG 375/s

$\$ 4242 / 89$ 
ROM MORRISON KNUDSEN CORPORATION

Project UMTRA - SRK

Feature SITE RESTORATION

Item EARTHWDRK QUANTITIES
Contract No. $3895-76$

Designed AFS

Checked

Sheet 19

File No.

Date $4 / 21 / 93$

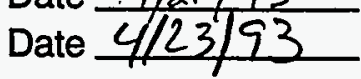

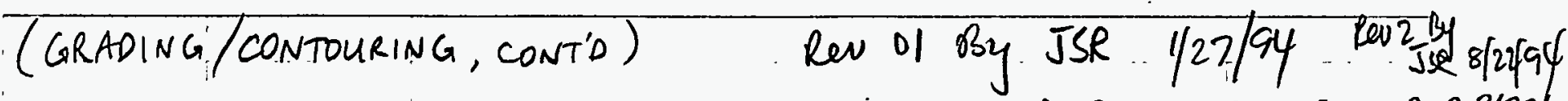

$B C$ SITE.

AM $1-28-94 \quad A M B 8 / 29 / 94$.

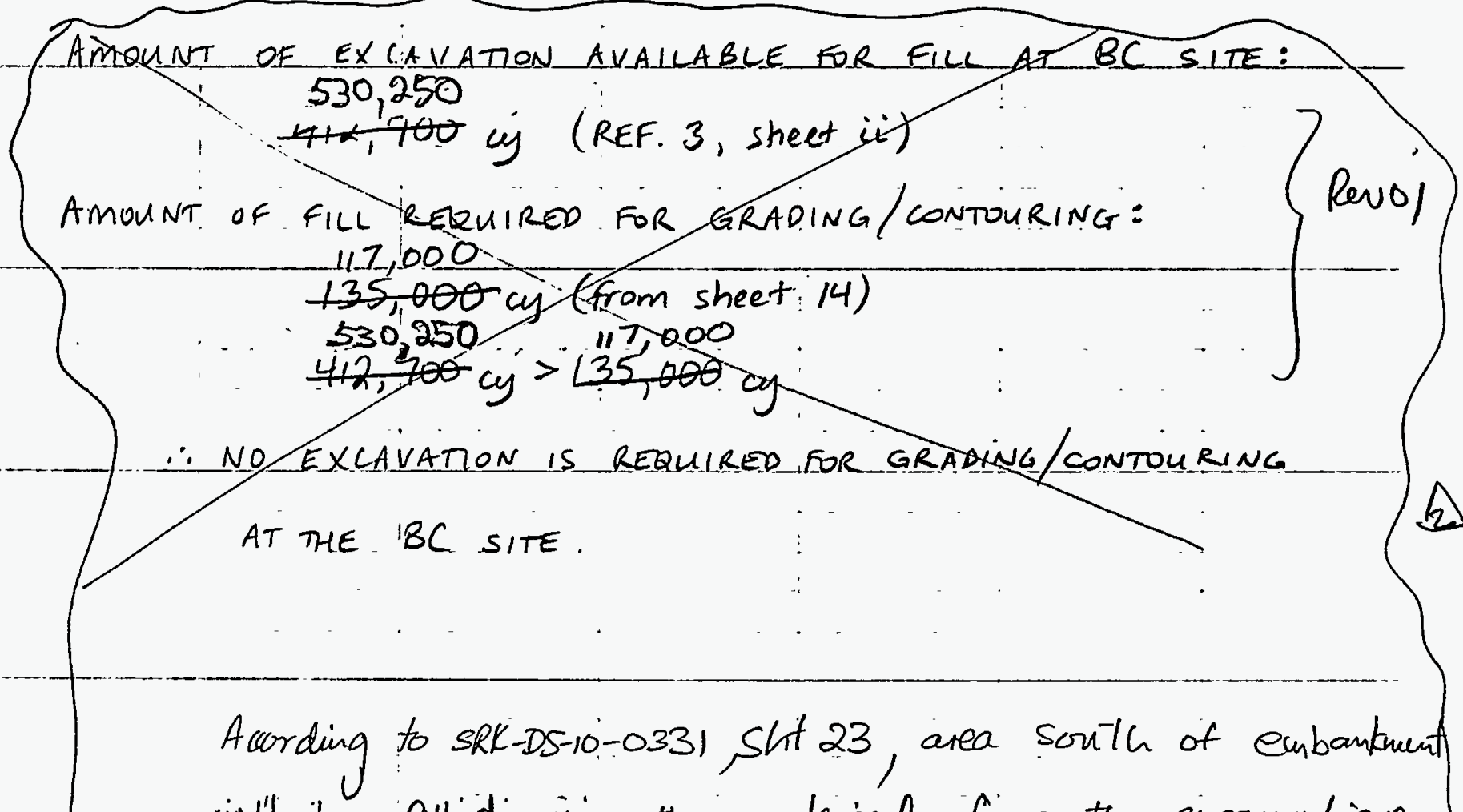
will be filled using the material from the excavation.

Amount of material required for fill $=48,000 \mathrm{CY}$. s using In roads program)

from sit. 14 , total common fill required $=117,000 \mathrm{CY}$. (including $48,000 \mathrm{CY}$ )

$\therefore$ Material required double handing... $=69,000 \mathrm{cY}$

Amount of litopsisl to be replaced $=15,100 \mathrm{cY}(5 \mathrm{~s}+14)$

$\therefore$ Material requited from stockpile $=84,100 \mathrm{CY}$

ENG $375 / 89$

2 
MORRISON KNUDSEN CORPORATION

Project UMTRA - SRK

Feature SITE RESTORATION

Item EARTHWORK QUANTITES
Contract No. $3885-76$

Designed AFS

Checked JSR
Sheet 20

Date $\frac{\overline{4} / 21 / 93}{4 / 23 / 93}$

Date $4 / 23 / 93$

(GRADING /CONTOURING, CONTD)

BORROW AREAS:

THE AREAS OF THE UL BORROW AREA, DELORES RIVER

BORROW AREA, AND POTENTIAL THIRD BORROW AREA ARE

Scaled ofF SHEET 21. It is estimated that an area

EQUIVALENT TO THE TOTAL BORROW AREA AT EACH BORROW

SITE WILL REQUIRE EXCAIATION fOR REgRADING PURPOSES.

It is assumed that die average depth. of excavation FOR REGRADING WILL BE $1.0 \mathrm{ft}$.

BORROW AREAS:

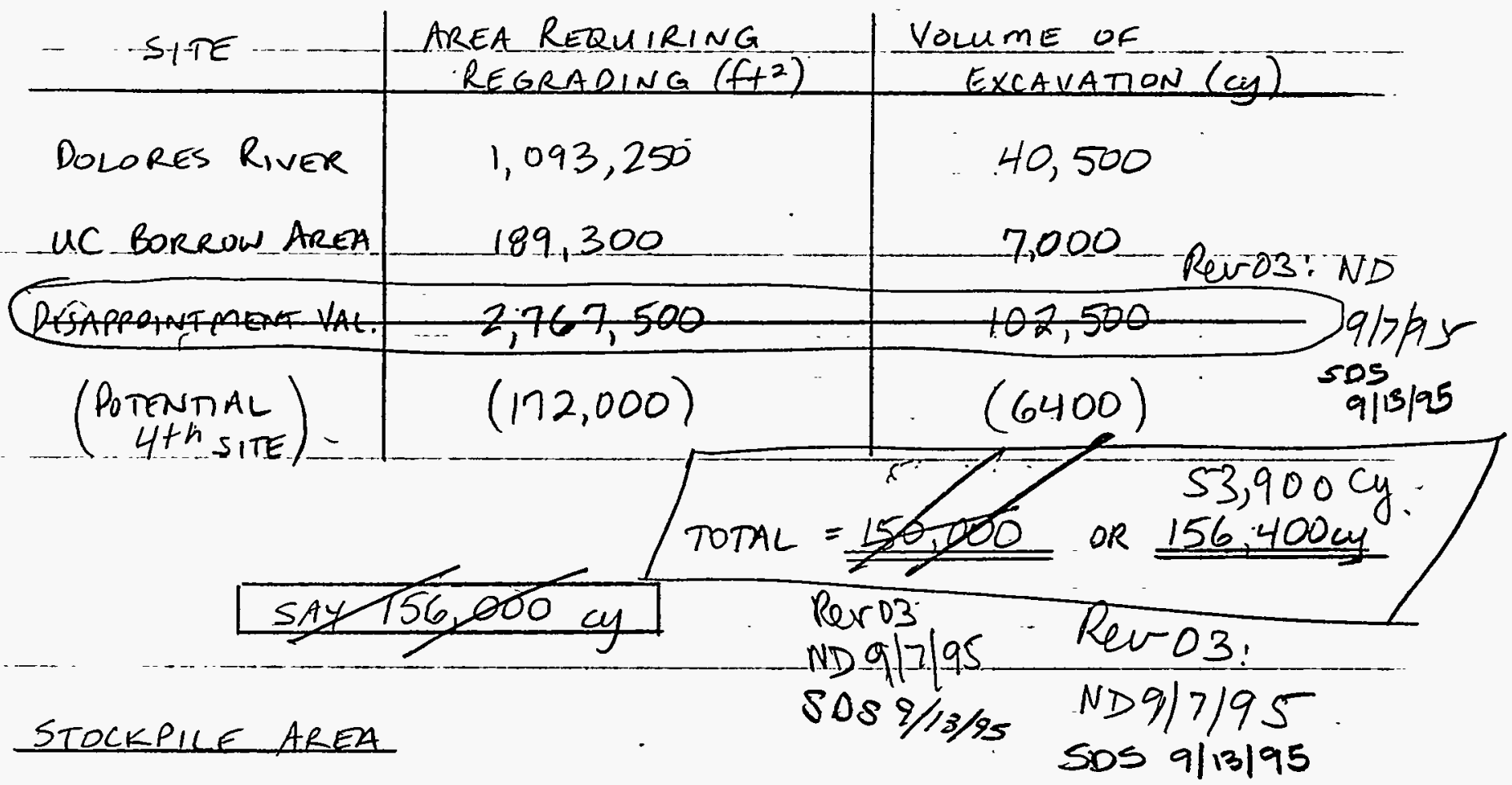

SHEET 16 SHOWS A PLAN OF THE STOCKPILE. IT is

ESTIMATED THAT NO EXCAVATION FOR REGRADING WILL

bE REQUIRED IN THIS AREA (IT WILL REQUIRE ONLY SEEDING).

ENC $37518 \overline{9}$

$\frac{14242 / 89}{1.48}$ 


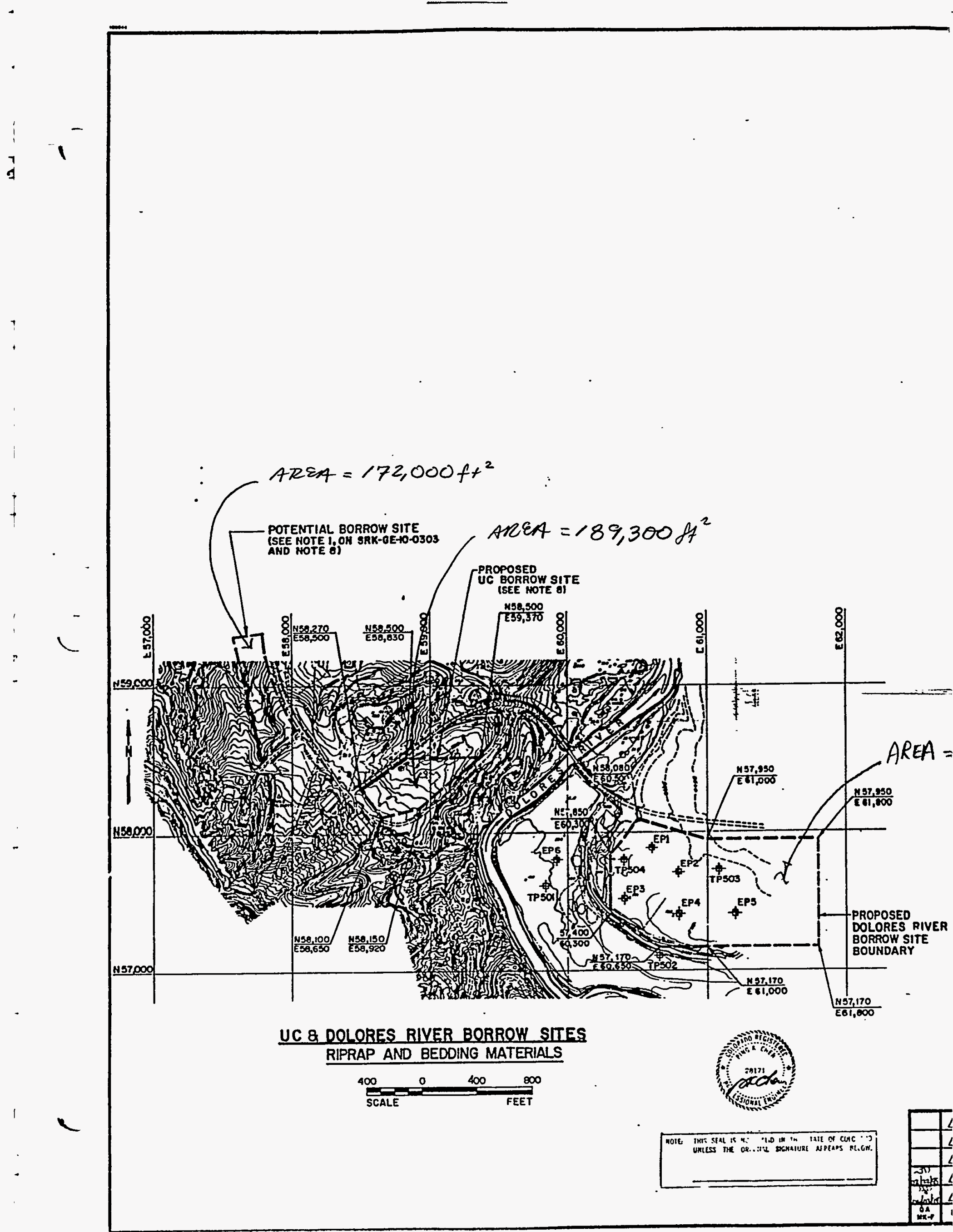




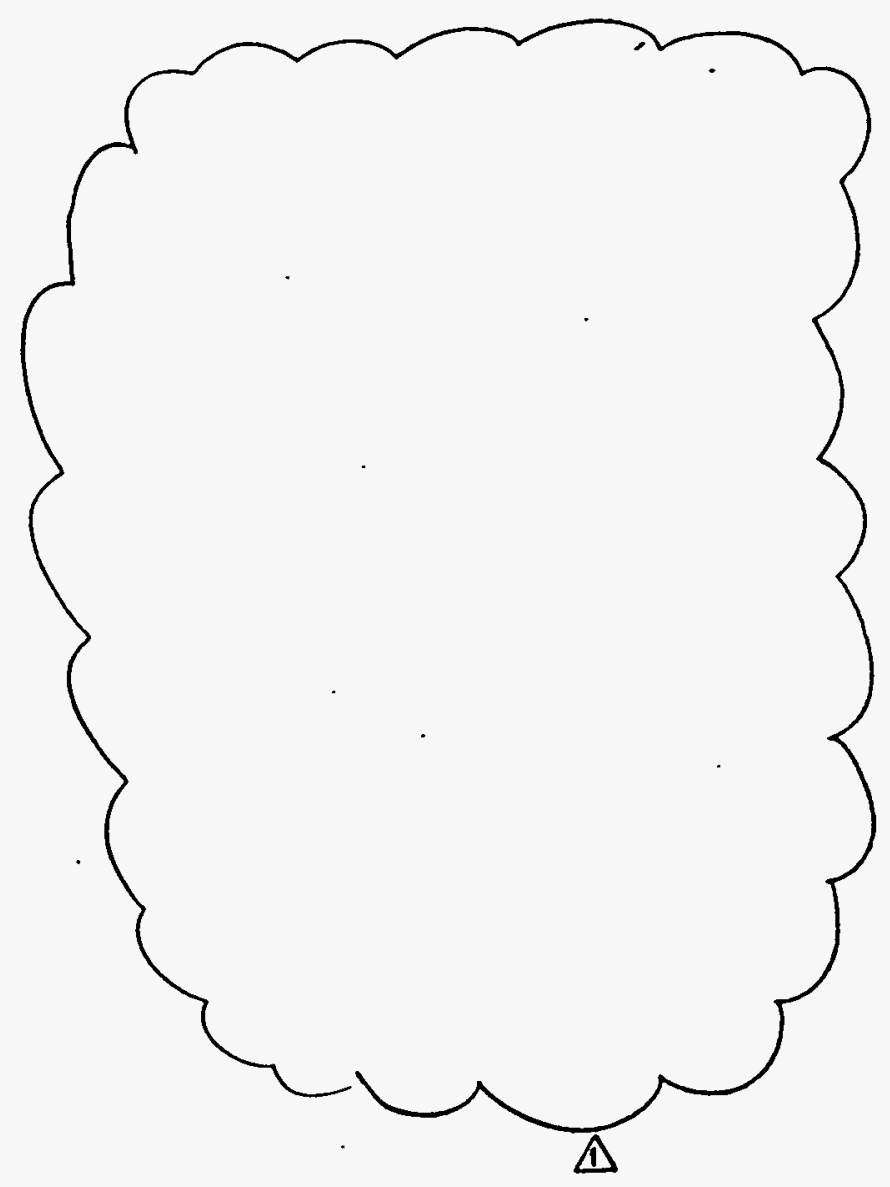
REFERENCE 4 SHEET 21
UMVTAA-SRK

NOTES :

1. RADON BARRIER MATERIAL SHALL BE OBTAINED FROM THE QURRO CAMYOH DISPOSAL SITE AS SPECIFIED.

2 ASSUMED COCFOAUTE STSTEN IS TAKDN FROM THE SURVEY CONOUCTED BY $M$ H STTH RLS. 10738 , ESTABUSH HORZONTML AHO VERTCAL CONTRO FOR HTE SUOK ROCK SIES.

3. AT THE DOLORES AIVER BORROW SITE

THE TOPSOIL SHALL BE STRLPPED ANO STOCKPIED WITHA THE SITE BOUNOARIES.

4. AFTER REMOVAL OF MATERIAL. THE BORROW SITE SHALL BE REORAOED TO ORAIM. FIHAL SLOPES SHALL WOT DE STEEPER THAH FIVE (5) HORIZONTAL TO I VERTICAL. THE FIKAL GRADE AT THE DOLORES RIVER SITE SHALL DRAIM TOWARD THE RIVER.

5. AFTER FINAL GRAOINO AT THE DOLORES aIVER SITE, TOPSOIL SHALL BE REPLACED IN UNIFORM THICKNESS AND SEEDED.

THE DOLORES RIVER SITE SHALL BE PLANTED WITH

COMMERCIAL CRESTED-WHEAT SEED IN ACCORDANCE WITH SUPPLIERS RECOMMENOATION.

6. A POWER LIME CROSSES THE DOLORES RIVER BORROW AREA. EXCAVATIOHS SHALL BE AT LEAST 20 FEET AWAY FrOM THE BASE OF THE UTILITY POLES.

7. EXISTING ROADS ADJACENT TO THE BORROW AREAS SHALL DE MAINTAINED AND KEPT OPEN TO THE PUBLIC.

- uc borrow area contans large cobbles and gravel that MAY BE COMBINEO WITH MATERIAL FAOM THE DOLORES RIVER BOAROW STE TO PROOUCE THE REOUIRED RIPRAP SIZE. SEE DHG. NO. SRK-PS-10-O310 FOR BOAIKO ANO TEST PIT LOCATIONS.

9. MO MORE THAN 3,500 CY MAY BE REMOVEO FROM THE UC SITE FOR USE AS MIPRAP AND BEDOWO MATERIAL.

A

$$
\begin{aligned}
& \text { Rev03 } \\
& \text { ND } 9 / 7 / 95 \\
& \text { SDS } 9 / 13 / 25
\end{aligned}
$$

\section{REFERENCE DRAWINGS:}

SRK-OE- $10-0303$ PROCESSING SITES, DISPOSAL SITE ANO BORROW STTES
LOCATIOH MAP
SRK-PS-DO-O3IB GORINOS AND TEST PITS LOCATION PLAN

$1,093,250-\mathrm{FH}^{2}$

SRK-PS-10-O3IB. BORINOS AND TEST PITS LOCATION PLAN

\section{LEGEND:}

\begin{tabular}{|c|c|c|c|}
\hline U. & $\begin{array}{l}\text { S. DEPARTME } \\
\text { ALBUQUERQUE }\end{array}$ & $\begin{array}{l}\text { NT OF ENERGY } \\
\text { NEW MEXICO }\end{array}$ & \\
\hline 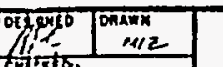 & & $\begin{array}{l}\text { SLICK ROCK SIIE } \\
\text { CK ROCK, COLORADO }\end{array}$ & \\
\hline 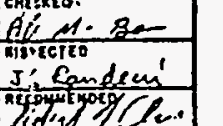 & $\begin{array}{r}\text { PROPOSED } \\
\text { SI }\end{array}$ & $\begin{array}{l}\text { BORROW SOURCES } \\
\text { TE PLANS }\end{array}$ & \\
\hline atchan & 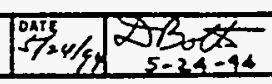 & 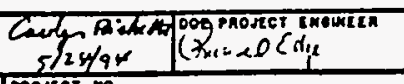 & 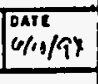 \\
\hline (19) HORRISON KN & IN CORPORATIOH & $D E-A C O 4-83 A L 1879$ & \\
\hline UMTRA PRO & & 304 & net. \\
\hline
\end{tabular}
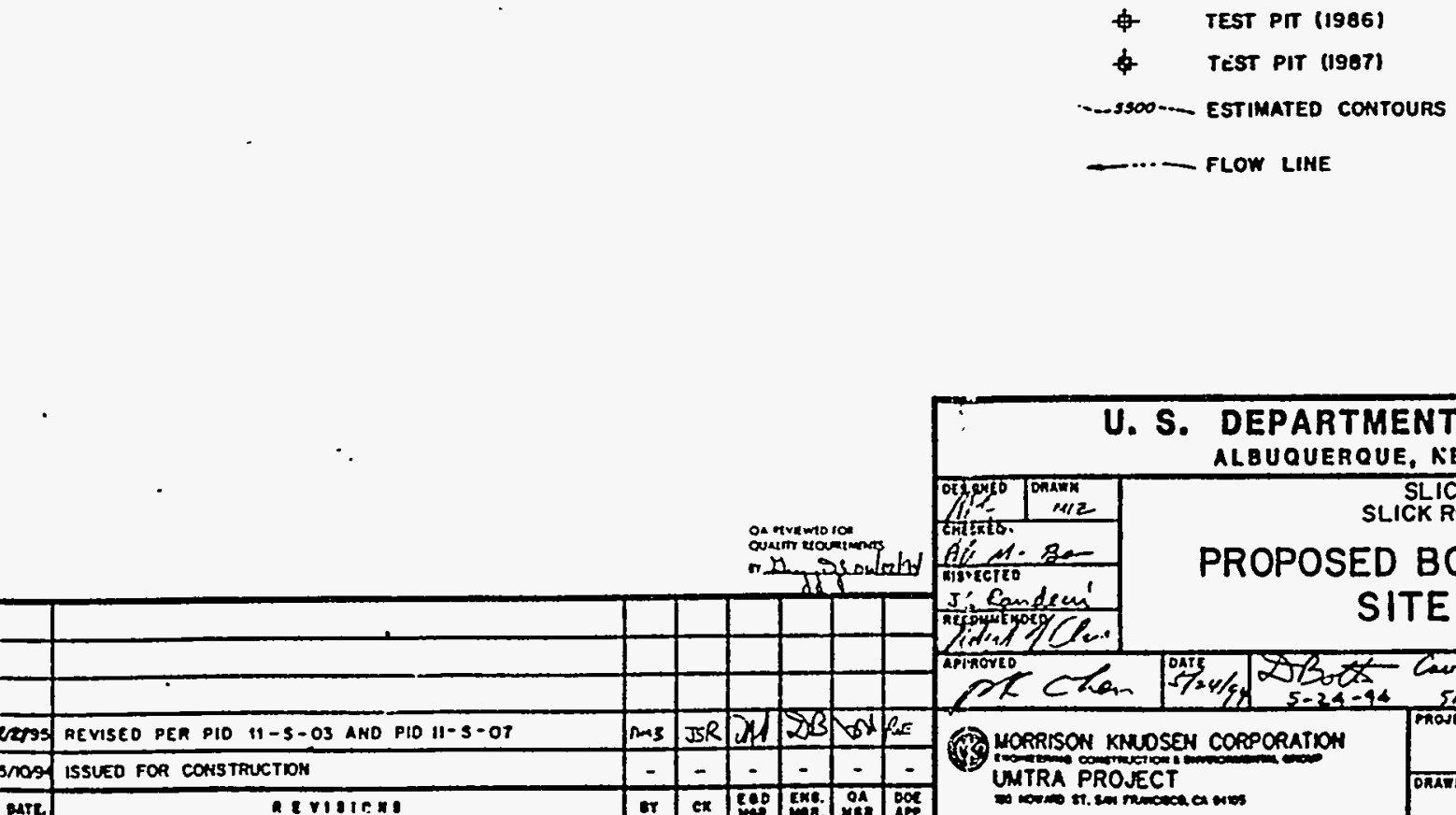
@i MORRISON KNUDSEN CORPORATION

Project UMTRA - SRK

Feature SITE RESTORATION

Item EARTHWORK QUANTITES
Contract No. 3885

Designed AFS

Checked JSR
Sheet

File No.

Date $\frac{4 / 21 / 93}{4 / 23 / 93}$

Date $4 / 23 / 93$

SEEDING ACREAGE

UL SITE

As STATED ON SHEET 1, ALL GRADED, EXCAVATED, OR OTMERWISE DISTURBED SOIL AREAS WILL REQUIRE SEEDING.

DISTuRBED AREA $=2,535,000 \mathrm{ft}^{2}$ (EROM SHEET 18)

$\therefore U C$ SITE. SEEDING AREA $=58$ acres

NC SITE

$$
\begin{aligned}
& \text { DISTURBED AREA }=1,196,000 \mathrm{ft}^{2} \text { (FROM SHEET 18) } \\
& \therefore \text { NC SITE SEEDING AREA }=27 \text { acres }
\end{aligned}
$$

$B C$ SITE

ShEeT 27 (REF. 15) Shows the location of rip rap in the BC site finAl GRADING PLAN. WHERE RIPRAP IS NOT USED ONER DISTIRBED SOIL, IHE AREA mUST be SEEDED. LIMITS of SEDING ARE PRAWN ON SHEET 16. THE "SURFACE AREA" OPTION OF THE INROADS (REF, 4 ) SOFTWARE is used to COMPUTE THE SEEDING AREA OF THE SURFACE SHOWN ON SHAT 15 SEEDING

SHEET 15, WITHIN THE LIMITS DRAWN ON SHEET 16. BY: SR

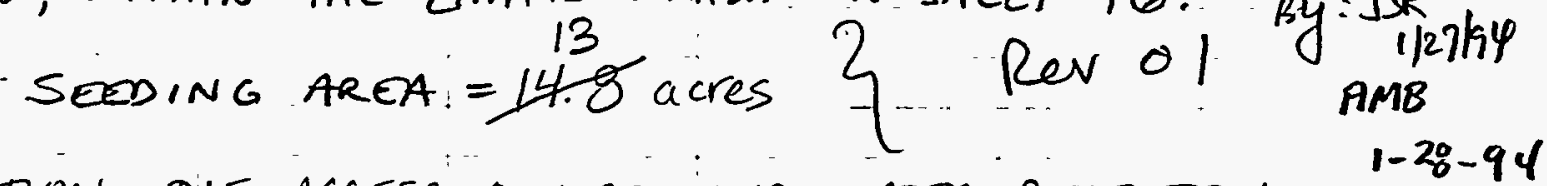

In ADDITION, DLE ACCESS CONTRQL ANO WATER RETENTION basin areas must bE SEeDED. THESE areAs are. SHOWN ON SHEETS 23,24 , AND 13.

ENO375/89

$14242 / 89$ 


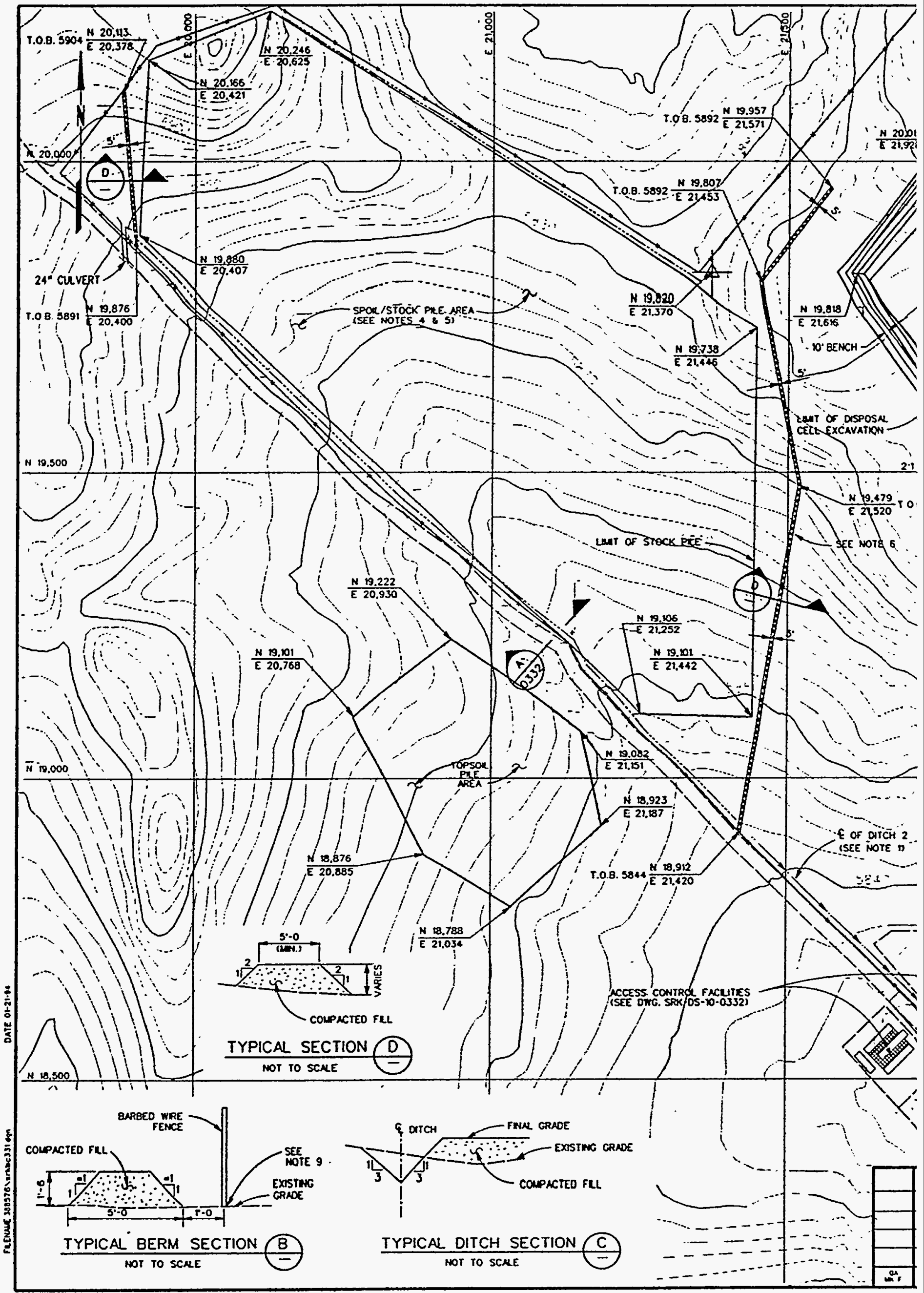




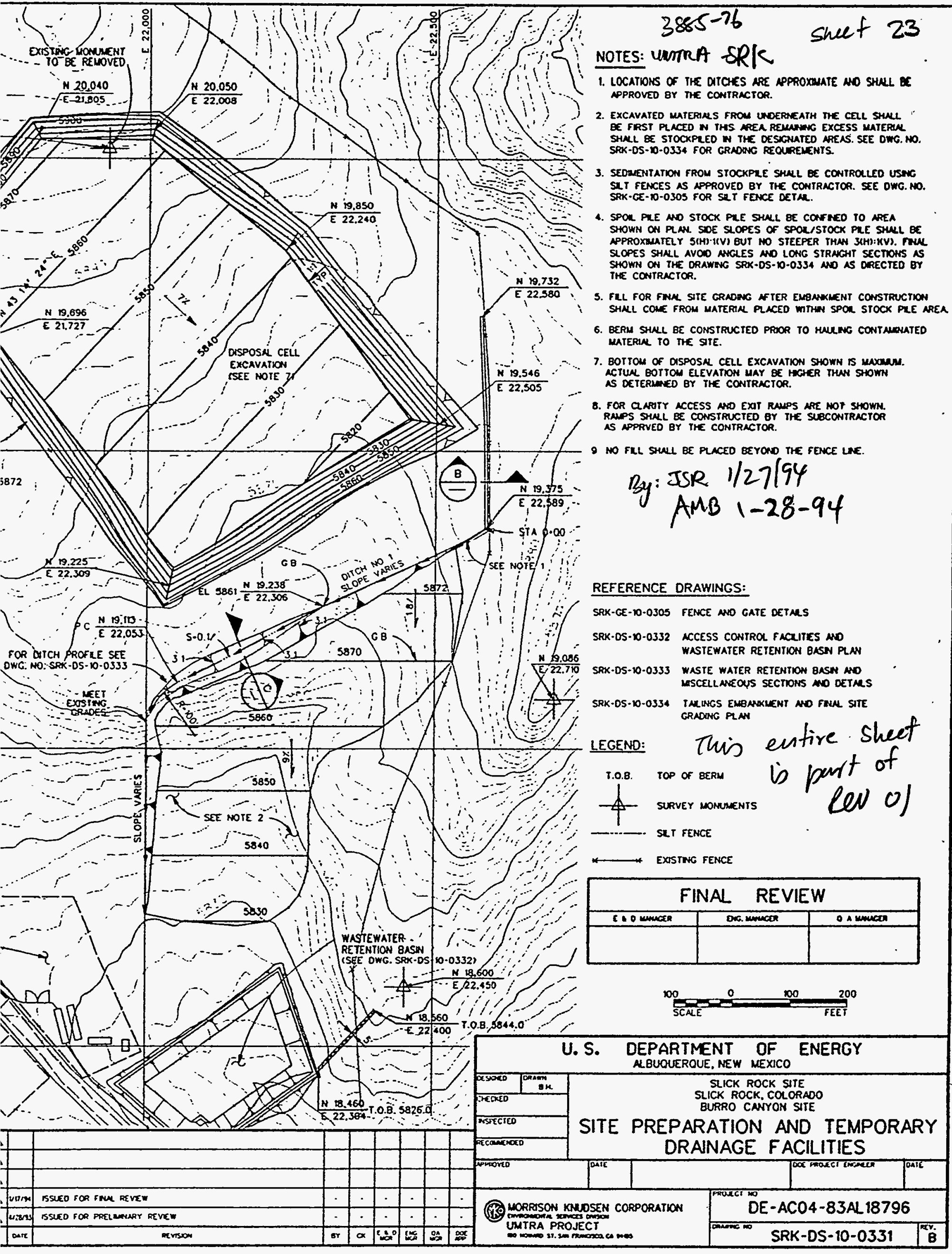




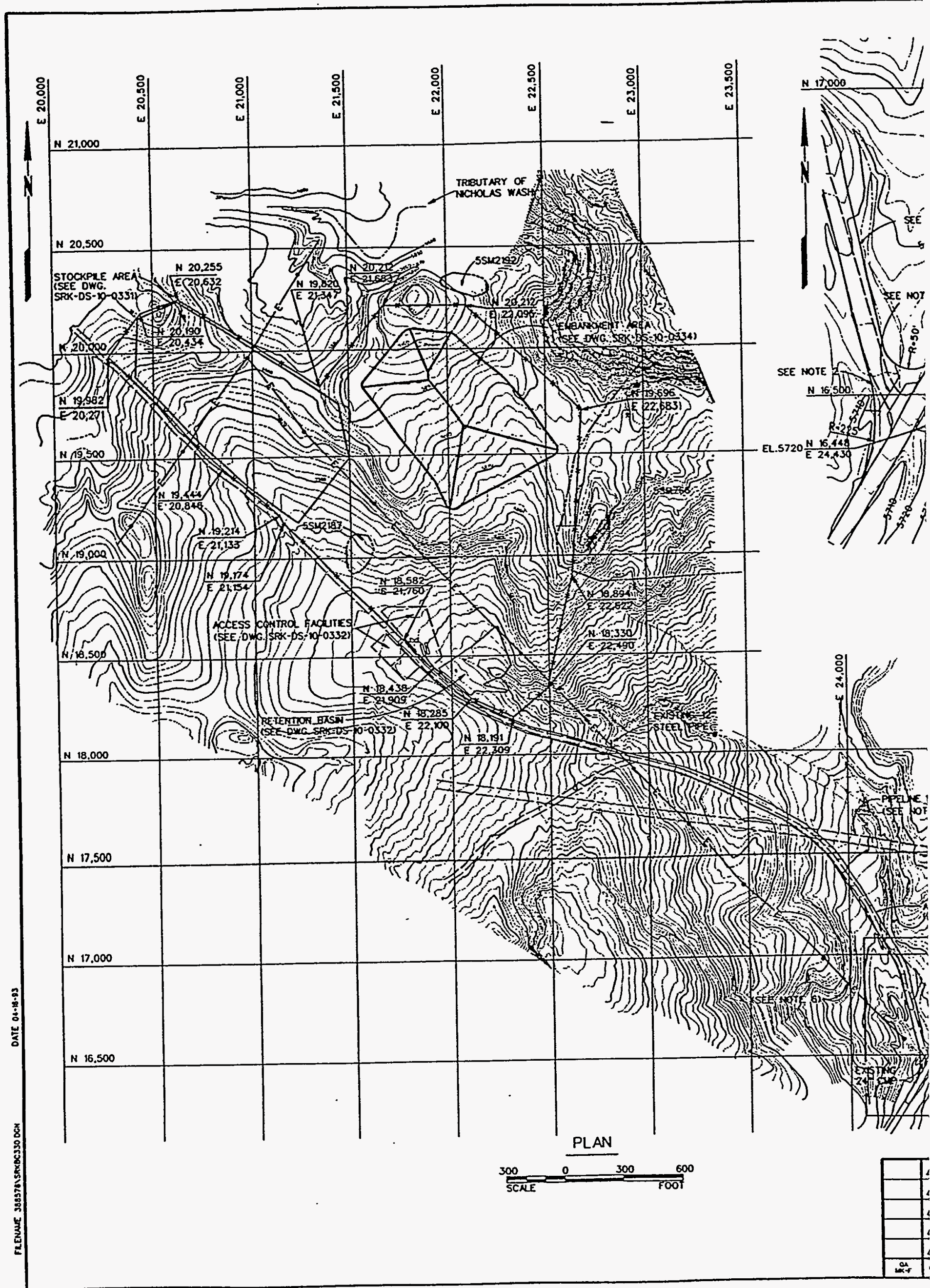


4.0. MORRISON KNUDSEN CORPORATION

Project UMTRA-SRK

Feature SITE RESTORATION Item
Contract No. 3885-76

Designed AFS

Checked ISR
Sheet 25

File No.

Date $\frac{4 / 21 / 93}{4 / 23 / 93}$

AREA OF ACCESS CONTROL FACILITiES: $68,500 \mathrm{ft}^{2}=1.6$ acres

(SCALED OFf SHEET 23).

AREA OF WATER RETENTION BASIN: $32,000 \mathrm{ft}^{2}=0.7$ acres

(SCALED off SHET 24)

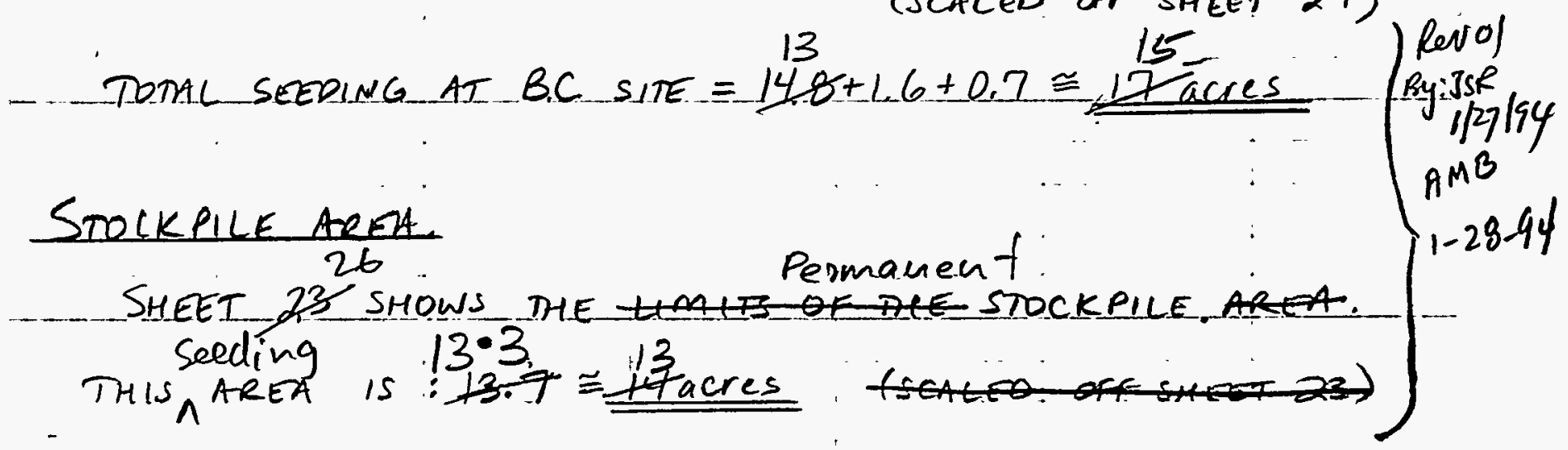

BORROW AREAS

THe seeding acreage is equal to the area disturbed

For GRADING/CONTOURING at TMESE SITES. From

SHEET 20, the AREAS ARE:

SITE DISTURBED SOIL AREA $\equiv$ SEEDING AREA

DELORES RIVER. 1,093,250 $\mathrm{ft}^{2} . \cdots .25$ acres

LC BORROW AREA $189,300 \mathrm{ft}^{2}: 4$ acres

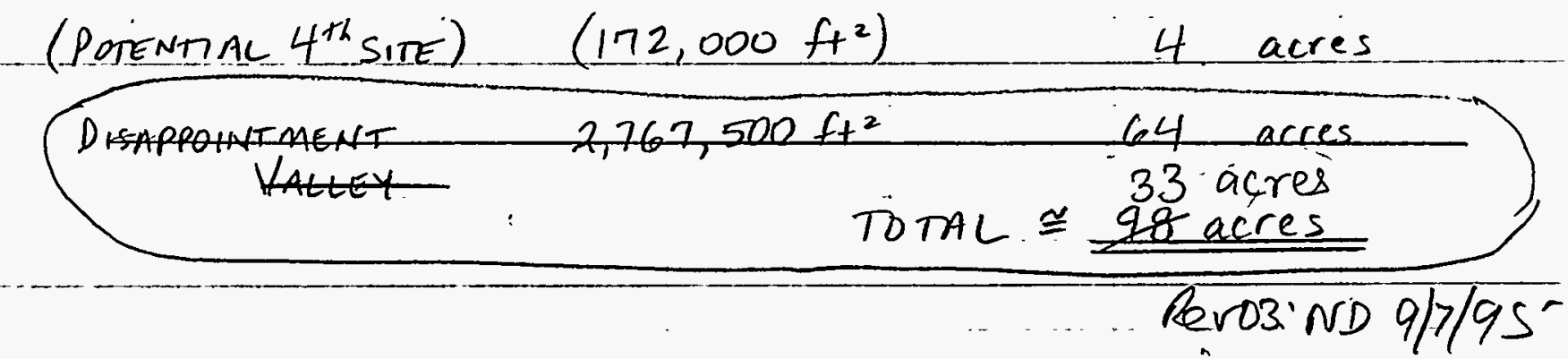

Sos $9 / 13 / 95$

ENG 375/69

$\$ 4242 / 89$ 
CONTRACT NO. $\underline{3885}$ DISCIPLINE CIVIL

\section{PROJECT}

\section{UMTRA - SRK}

\section{FEATURE}

\section{BID SCHEDULE}

\section{ITEM}

\section{QUANTITIES AND MATERIAL BALANCE}

\section{SOURCES OF DATA}

(SEE SHEET ii)

\section{SOURCES OF FORMULAE \& REFERENCES}

(SEE SHEET ii)

\begin{tabular}{l} 
PRELIMINARY CALC. $\square$ \\
\hline
\end{tabular}


Contract No. $3885-76$

Designed

Checked
Sheet i

File No.11-334-01

Date

Table of Contents

lev ol JSR 01/28/94

$A M B \quad 1 / 28 / 94$

Sheet No.

References

PURPOSE

MATERIAL BALANCE CHART $\ldots \ldots \ldots \ldots \ldots \ldots \ldots \ldots \ldots$

OOD MOBILIZATION

200 SITE PREPARATION

TAILINGS PILE

500 COVER

600 EROSION PROTECTION स12

700 DECONTAMINATION 
1. MKES, UMTRA-SRK, Calculation No. 11-330-02-80. Temporary Facilities - Material Quantities, Aprill1903 Jan 1994 Sep 1995

2. MKES, UMTRA-SRK, Calculation No. 11-333-01=08, Embankment Quantities Excavation,

3. MKES, UMTRA-SRK, Calculation No. 11-212-01-02, NC and UC Site Contaminated Materials - Excavation Quantities, April 1993

$$
\text { Cot } 032
$$

4.7. MKES, UMTRA-SRK, Calculation No. 11-330-01-00, Site Restoration - Earthwork Quantities and Seeding, March 1993 Jand $1994 \operatorname{Sep} 1995 / 2$

5. MKES, UMTRA-SRE, Calculation No.11-310-02-00, Embankunent Design-Excavation Stability, Jan 1994. 
MORRISON KNUDSEN CORPORATION

ENVIRONMENTAL SERVICES GROUP

Project UMTRA-SRK

Feature

Item
Contract No.

Designed

QUANTITIES AND MATERIAL BALANCE Checked
Sheet

File N0.11-334-01

Date

Date

$4 / 30 / 93$

PURPOSE

The purpose of this calculation is to summarize the bid schedule quantities which will be the basis of the engineer's estimate. The quantities have been obtained from previously completed calculations. Quantities for "Lump Sum" items are not estimated in this calculations.

Rev or Purpose

By JSR $1 / 28 \% 94$

AMD $1 / 28 / 94$

Sits 4-12,14 and 15 have been replaced with new sheets sits $i, 2,1,2$ and. 13 have been revised.

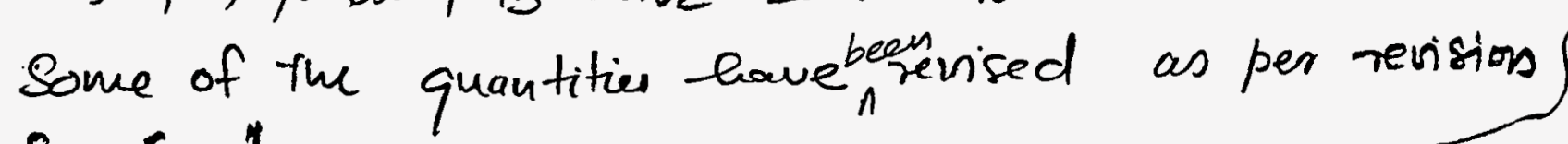
$B$ of digs...

Rev 02 Purpose

(a )Some quantities have been revised as per final design. sits 2,4-10,13-15 have been revised sit rot added (b) Radon Barrier thickness has becks changed.

Sheet

Rev 02 BY SR $9 / 14 / 95$

AMP $9 / 14 / 95$

F:|JSR|SRK|BID.TXT 


\section{CUT/EXCAVATION}

UMTRRA PF CONTI MATERIAL

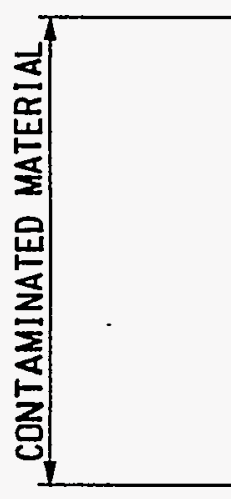

\begin{tabular}{|c|}
\hline NC TAILINGS \\
\hline NC SUBPILE \\
\hline NC OFFPILE \\
\hline
\end{tabular}

$31.700 \mathrm{CY}$

\begin{tabular}{|c|}
\hline UC TAILINGS \\
\hline UC SUBPILE \\
\hline 2 ' COVER \\
\hline
\end{tabular}

$34.300 \mathrm{CY}$

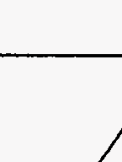

\begin{tabular}{|c}
\hline NC MATERIAL \\
\hline UC TAILINGS \\
\hline UC OFFPILE \\
\hline UC GAS LINE \\
\hline DEMOLITION OEBRIS \\
\hline VICINITY PROPERTY
\end{tabular}

$45,800 \mathrm{CY}$

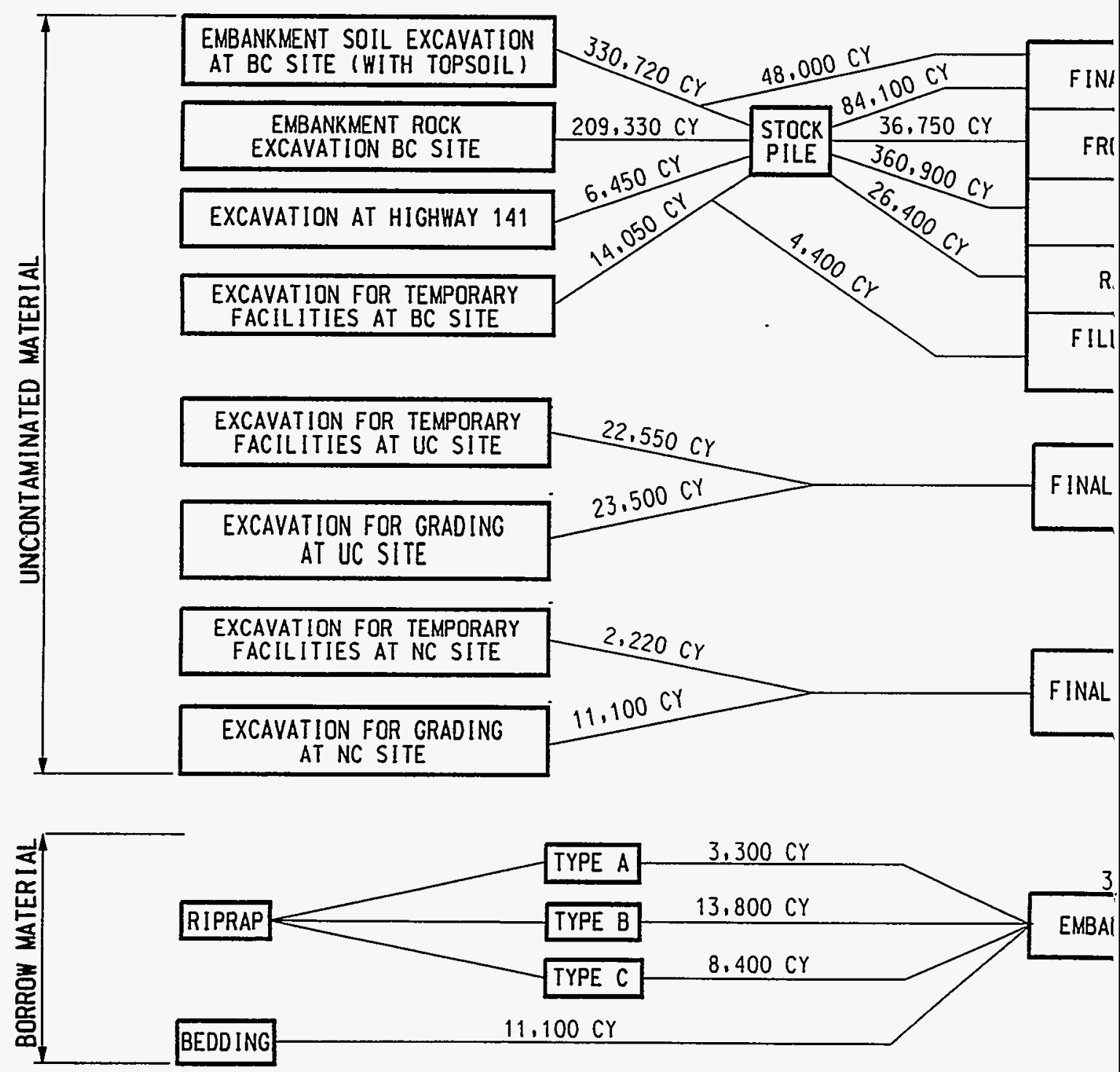


DICT - SLICK ROCK

ICT NO. 3885-76

BALANCE SUMMARY

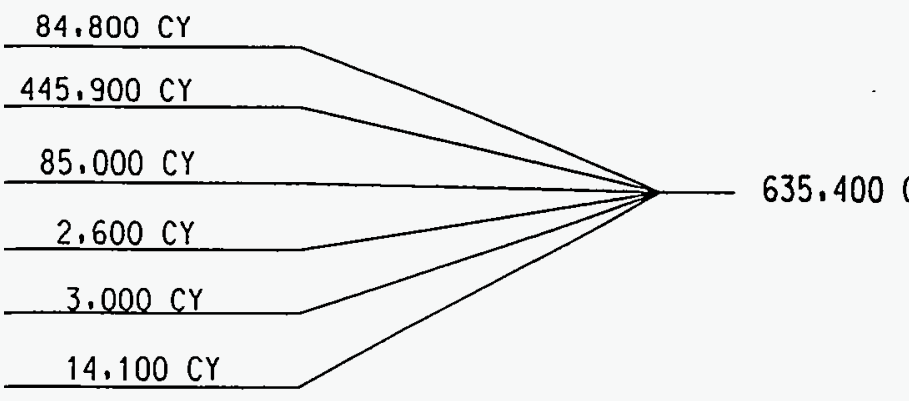

sheet 2

This entire sheet is Part of Revor.

FILL

By: ISR
AMP

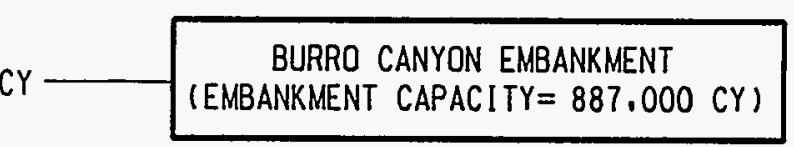

SITE GRADING

PROTECTION

rOCK PILE

IN BARRIER

OR TEMPORARY

ICILITIES

lITE GRADING

I TE GRADING

$600 \mathrm{CY}$

RENT COVER 
OOO - MOBILIZATION

\section{SUMMARY}

\begin{tabular}{|c|l|c|c|c|}
\hline \hline Item No & Description & Sheet No. & Unit & Approx. Quantity \\
\hline 001 & Mobilization & 3 & L.S. & N/A \\
\hline 002 & Payment for Bond Premium & 3 & L.S. & N/A \\
\hline
\end{tabular}

\section{1-Mobilization}

Bid item mobilization consists of subcontractor's mobilization of forces, materials, and equipment onto the job site. Also it includes the furnishings, installation, construction, maintenance, operation and subsequent removal and disposal of construction facilities not paid for separately under other sections of Subcontract documents.

Bid item 001 is a lump sum quantity and therefore will not be estimated in this calculation.

002 - Payment for Bond Premium

Bid item 002 is a lump sum quantity and therefore will not be estimated in this calculation. 
Sheet 4

File No.11-334-01

Date

Date

200 - SITE PREPARATION

SUMMARY

The entime sheet is Part of Rov ol ReV 02 By: JSR 9/14/95

\begin{tabular}{|c|c|c|c|c|}
\hline $\begin{array}{l}\text { Item } \\
\text { No }\end{array}$ & Description & $\begin{array}{l}\text { Sheet } \\
\text { No. }\end{array}$ & Unit & $\begin{array}{l}\text { Approx. } \\
\text { Quantity }\end{array}$ \\
\hline 201 & Temporary Offices & 5 & L.S. & $N / A$ \\
\hline 202 & Temporary Roads \& Parking Areas & 5 & L.S. & N/A \\
\hline 203 & Temporary Sanitary Facilities & 5 & L.S. & $N / A$ \\
\hline 204 & Temporary Electric Power & 5 & L.S. & N/A \\
\hline 205 & Temporary Water & 6 & L.S. & N/A \\
\hline 206 & Maintenance and Janitorial Services & 6 & MONTH & 1024 \\
\hline 207 & $\begin{array}{l}\text { Construction of desen facilities at } U C, N C \\
\text { Snow Removalsentees and -BC sites. }\end{array}$ & 6 & SEÁSON & MAL2 \\
\hline 208 & Furnish and install silt Fence & 6 & L.F. & 4510 \\
\hline 209 & $\begin{array}{l}\text { Demolition and Disposal of Existing } \\
\text { Structures and Facilities }\end{array}$ & 6 & L.S. & N/A \\
\hline 210 & Sealing of Monitor Wells & 6 & L.F. & 2700 \\
\hline 211 & $\begin{array}{l}\text { Excavation of Uncontaminated Material } \\
\text { for Access Control, Temporary } \\
\text { Drainage Ditches, Dikes \& wastewater } \\
\text { Retention Basin At UC, NC \& BC Sites } \\
\end{array}$ & 6 & C.Y. & 38,820 \\
\hline 212 & $\begin{array}{l}\text { Excavation of Uncontaminated Material } \\
\text { from the tailings Embankment } \\
\text { Foundation and Placement in } \\
\text { Spoil/Stock Pile and finish grading at } \\
\text { BC site. }\end{array}$ & 7 & C.Y. & 330,720 \\
\hline 213 & $\begin{array}{l}\text { Rock excavation from tailings } \\
\text { embankment formation and placement } \\
\text { in stockpile or for finish grading at the } \\
\text { BC site }\end{array}$ & 7 & C.Y. & 209,330 \\
\hline 244 & Pavement-Refleotion-Tests & 7 & t.S. & nta \\
\hline $\begin{array}{l}215 \\
214\end{array}$ & $\begin{array}{l}\text { Construction of County Road S8 } \mathrm{B}_{\Lambda} \text { at the } \\
\text { UC Site }\end{array}$ & ent 7 & L.F. & 920 \\
\hline
\end{tabular}




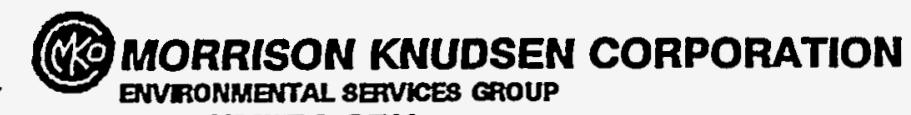

Project

ENVIRONMENTAL 8ERVICES GROUP

Feature BID SCHEDULE

Item

QUANTITIES AND MATERIAL BALANCE

Contract No. 3885-76

Designed

Checked
Sheet 5

File No.11-334-01

Date

Date $1128 / 94$

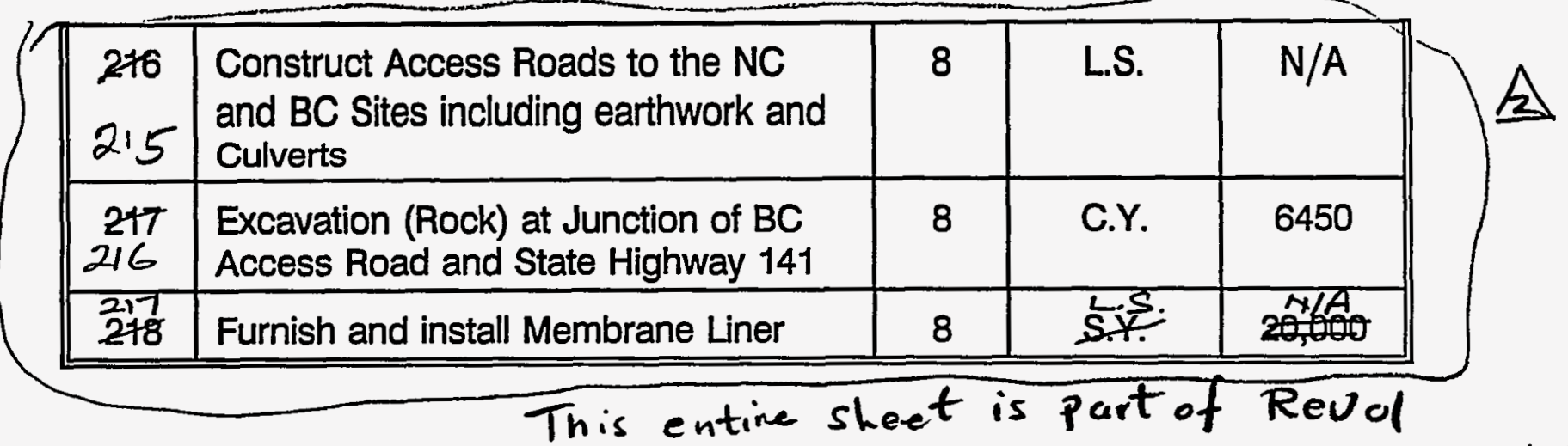

201 - Temporary Offices

ReN 02 By: JSR

The subcontractor shall furnish and install temporary trailers for the office staff as approved by the contractor. The locations and sizes are given in subcontract drawings and in information for bidders.

Each trailer shall be provided with running water, power and shall be properly lighted and temperature conditioned for summer and winter use.

Bid item 201 is a lump sum quantity and thus will not be estimated in this calculation.

\section{2- Temporary Roads and Parking Areas}

Subcontractor shall provide temporary roads and parking areas at each of the three sites. This item is a lump sum quantity and thus will not be estimated in this calculation.

\section{3 - Temporary Sanitary Facilities}

Subcontractor shall provide sanitary facilities including toilets and wash basins. This item is a lump sum quantity and thus will not be estimated in this calculation.

\section{4 - Temporary Electric Power}

Subcontractor shall provide and maintain all electric power and wiring requirements to facilitate the work. This item is a lump sum quantity and thus will not be estimated in this calculation.

\section{5 - Temporary Water}

All temporary water for potable and construction use shall be provided. This item is a lump sum quantity and thus will not be estimated in this calculation. 
206 - Maintenance and Janitorial Services

QUANTITIES AND MATERIAL BALANCE

Contract No. $\quad 3885-76$

Designed

Checked

JSR

This entime sheet is

Part of Revol
Sheet 6

File No.11-334-01

Date

Date $01 / 28 / 9$ $1 / 28 / 94$

Revo2 By:Ise

$A M B$

Subeontractor-shall provide daily janitorial and maintenance services.

A 19 month period of services is estimated; 12 months foreons

for winter-shtitown.

207 - Snow Removat Sonicos construction fumish, install, oberate, and Subcontractor shall provido-snow-removal-senvices for 80 hourf

facilitied. This item is a lump gum and thus a

208 - Furnish and install silt Fence

and thus

wi
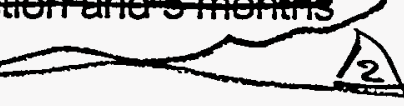
$9114 / 95$

Subcontractor shall provide silt fences at NC and BC sites to control sediments. The calculated length of silt fences from Ref 1 is 4510 linear feet.

\section{9- Demolition and Disposal of Existing Structures and Facilities}

Bid item 209 consists of existing structures and facilities to be demolished including decontamination pads, membrane liners, temporary fences and temporary foundations. This item is a lump sum quantity and thus will not be estimated in this calculation.

\section{0 - Sealing of Monitor Wells}

Measurement for sealing of monitor wells at $\mathrm{UC}, \mathrm{NC}$ and BC sites will be by the linear feet of wells sealed as measured from the bottom of well to the top of the completed well. The calculated linear footage is 2700 LF from Ref 1.

211 - Excavation of Uncontaminated Material for Access Control, Temporary Drainage Ditches, Dikes and wastewater Retention Basin At UC, NC and BC Sites

This item will be measured by the cubic yard of material excavated. The earthwork volume from Ref 1 is 45,270 for UC, NC and BC sites. This includes the rock excavation at HWY 141 and BC access road which is $6450 \mathrm{CY}$. Since bid item 215 covers that quantity, the total excavation for this bid item is $38,820 \mathrm{CY}$. 
MORRISON KNUDSEN CORPORATION ENVIRONMENTAL SERVICES GROUP

Project UMTRA-SRK

Feature

BID SCHEDULE

Item QUANTITIES AND MATERIAL BALANCE
This entive sheet is Part of Rev ol

Contract No. 3885-76

Designed

Checked

JSR

$A M B$
Sheet 7

File No.11-334-01

Date

Date
$01 / 28 / 94$

$01 / 28 / 94$

212 - Excavation of Uncontaminated Material from the tailings Embankment Foundation and Placement in Spoil/Stock pile or for finish grading at BC site

This item will be measured in cubic yard of material excavated for the embankment and for the toe apron at the BC disposal site and includes excavating, hauling, and placing the excavated materials in temporary stock piles or in spoil areas if excess or unsuitable for use as fill as required, including all clearing, stripping, shaping and compacting such stock piles.

The total embankment excavation including rock is $536,750 \mathrm{CY}$ (from Ref. 2). According to Ref. $5,61 \%$ of total excavation is soil. Therefore $327,420 \mathrm{CY}$ of soil will be excavated from the embankment area. Also 3,300 CY of soil will be excavated from the apron area (Ref. 2). Therefore total estimated embankment and apron excavation at the $\mathrm{BC}$ site is $330,720 \mathrm{CY}$.

213-Rock excavation from Tailings embankment formation and placement in stockpile or for finish grading at the BC site

This item will be measured in cubic yard of rock excavated for the embankment at the BC disposal site and includes excavating, hauling, and placing the excavated materials in temporary stock piles or in spoil areas if excess or unsuitable for use as fill as required, including all clearing, stripping, shaping and compacting such stock piles.

The total embankment excavation including rock is $536,750 \mathrm{CY}$ (from Ref. 2). According to item $215,327,420 \mathrm{CY}$ will be soil. Therefore $209,330 \mathrm{CY}$ of Rock will be excavated from the embankment area.

\section{4-Pavement-Beflection Tests-}

Biditem 214 is a lump-sum quantity and thus-will not be estimated in this caloulation.

\section{$215^{4}-$ Reconstruction of County Road S8 at the UC Site}

This item consists of reconstruction of the county Road to its original alignment and will be measured by the linear foot. The calculated length of the road is $920 \mathrm{LF}$.

Rev o2 By: JSR $9 / 14 / 95$

$$
\text { AMB } 9 / 14 / 95
$$




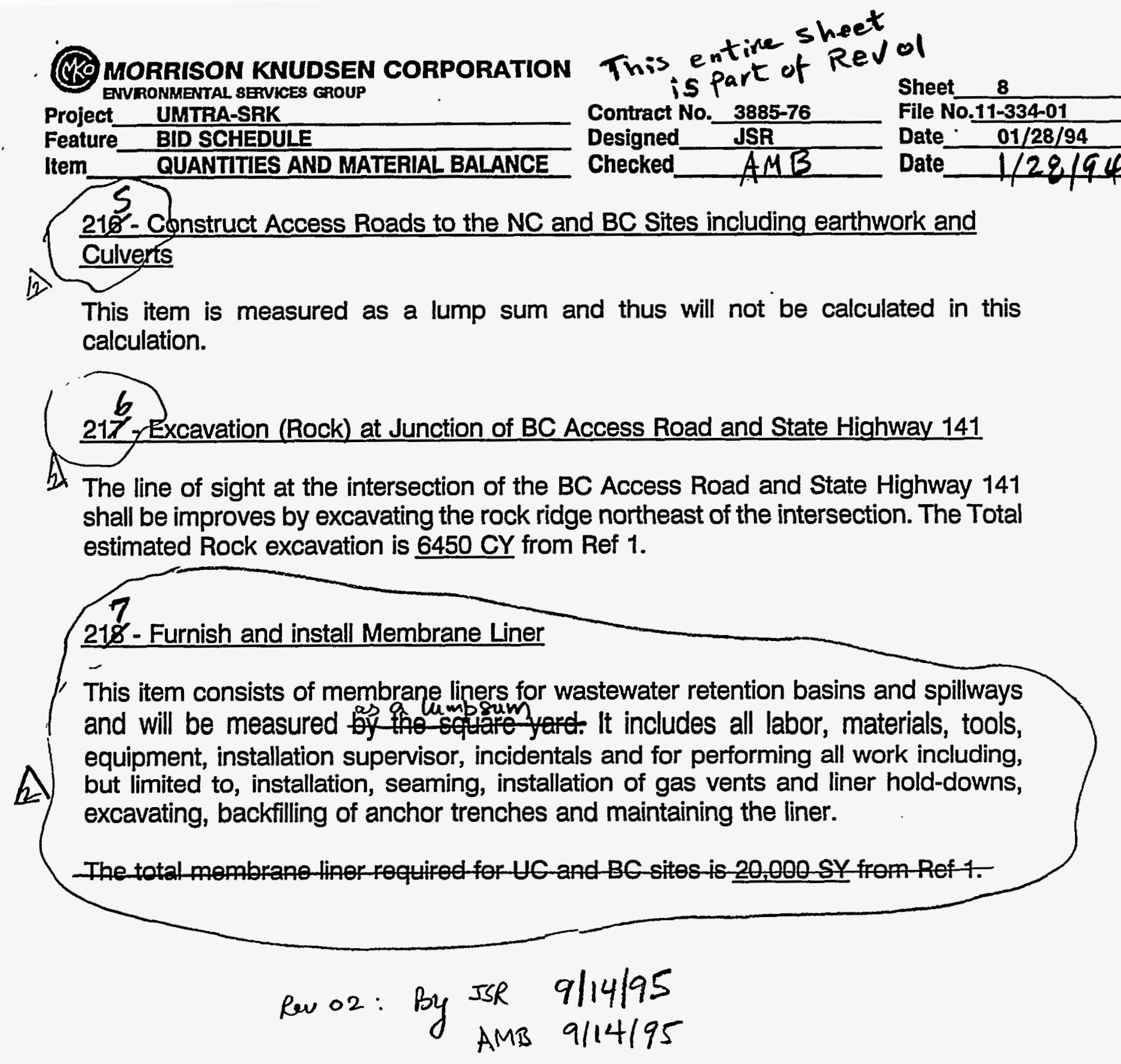


MORRISON KNUDSEN CORPORATION

GNVRONMENTAL SERVICES GROUP

$\begin{array}{ll}\text { Project } & \text { UMTRA-SRK } \\ \text { Feature } & \text { BID SCHEDULE } \\ & \text { QU em }\end{array}$

Contract No. 3885-76

Designed JSR

Checked

Sheet 9

File No.11-334-01

Date $\frac{01 / 28 / 94}{10}$

Date 1/28/44

400 -TAILING PILE This entire sheet is ReNO By ISR $9 / 14 / 95$

$A M B$ 9/14/95

SUMMARY Part of ReVal

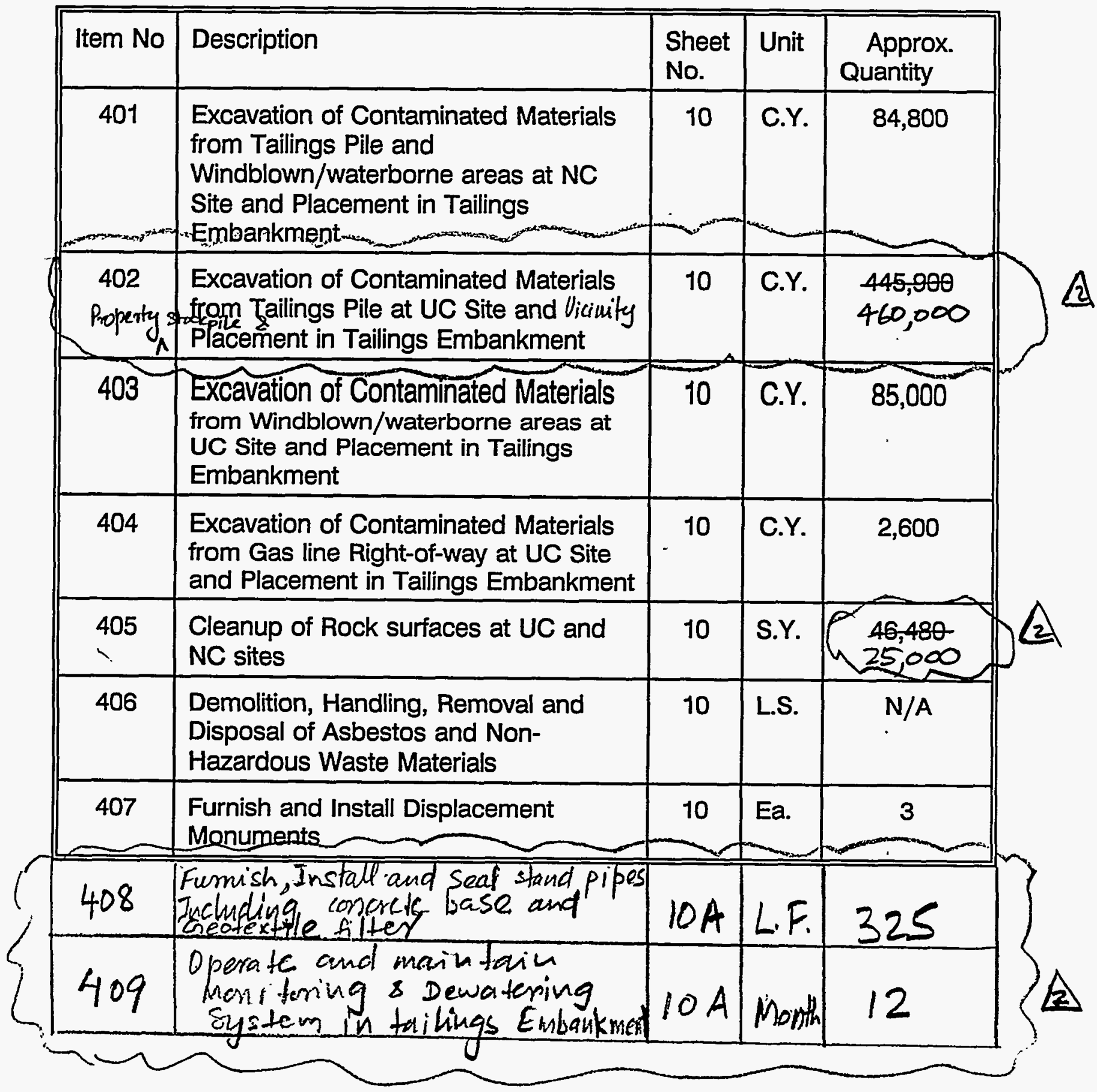

F:\JSR\SRK\BID.TXT 
MORRISON KNUDSEN CORPORATION ENVRONMENTAL SERVICES GROUP

Project UMTRA-SRK

Feature BID SCHEDULE

Item
This entive sheet is Part of Revol

Contract No. 3885-76

Designed

Checked
JSR

$A M B$
Sheet $\quad 10$ File No.11-334-01

Date Date

Revo

401 - Excavation of Contaminated Materials from Tailings Pile and Windblown/waterborne areas at NC Site and Placement in Tailing Embankment

The measurement for this item will be by the cubic yard of material excavated.

The tailings, subpile and Windblown/waterborne materials at the NC Site is $84,800 \mathrm{CY}$ from Ref 3.

\section{2 - Excavation of Contaminated Materials from Tailings Pile at UC Site and} Placement in Tailings Embankment

The measurement for this item will be by the cubic yard of material excavated. This item includes tailings pile A $(274,600 \mathrm{CY})$ \& B $(21,400 \mathrm{CY})$, subpile A $(88,200 \mathrm{CY})$ \& $B(15,900 \mathrm{CY})$ and $2^{\prime}$ additional material on both piles $(45,800 \mathrm{CY})$ at $U \mathrm{C}$ site from Ref 3. Therefore totatexeavation is 5,000 Af. Assums 14,100 CY from vicinity property stockpile. Therefore total contaminated material is $460000 \mathrm{cY}$. 403 -Excavation of Contaminated Materials from Windblown/waterborne areas at UC Site and Placement in Tailings Embankment

The measurement for this item will be by the cubic yard of material excavated. The total off pile excavation at UC site is $87,600 \mathrm{CY}$ from Ref 3 . This quantity includes the material from gas line R.O.W. $(2,600 \mathrm{CY})$ which is item 404 .

Therefore windblown/waterborne material at UC Site is $85,000 \mathrm{CY}$.

404 - Excavation of Contaminated Materials from Gas line Right-of-way at UC Site and Placement in Tailings Embankment

The measurement for this item will be by the cubic yard of material excavated.

The windblown/waterborne materials from Gas line Right-of-way at UC Site is $\underline{2,600}$ CY from Ref 3.

405 - Cleanup of Rock surfaces at UC and NC sites

The measurement of this item will be by the sq. The rock surface to be cleaned is 46,400-S.Y 2S,000 S.Y.

406 - Demolition, Handling, Removal and Disposal of Asbestos and Non-Hazardous Waste Materials

The measurement for this item will be by the lump sum and thus will not be estimated in this calculation.

\section{7 - Furnish and Install Displacement monuments}

The measurement of this item will by the number of monuments. There are 3 displacement monuments at the $\mathrm{BC}$ site. 
@MORRISON KNUDSEN CORPORATION

Project UMTRA-SRK

Contract No. $3885-79$

Feature Bid Schedule Designed J SR Date $9 / 14 / 95$ Material Balance Checked $A M B$ Date $9+14195$ This entire sheet is part of Rev on.

408 - Furnish, Install and Seal Stand Pipes Including Concrete Base and Geotextile filter for Monitoring and Dewatering system The measurement for this item will be by the linear feet. length of the stand pipes arc $325 \mathrm{ft}$.

409 - Operate and Maintain Monitoring \& Dewatering system in tailings Embankment, Provide Pumps and piping, and Pump Execs water to waste water Retention Basin as required: The measurement for this item will be by the months. The estimated. number of months are 12 .

ENG 375/91 
Contract No. $3885-76$ Designed Checked JSR $A M B$ This entive sheet is part 500 - COVER

SUMMARY of Rev ol
Sheet 11

File No.11-334-0

Date 01/28/94

Date $1 / 28+94$

Rev02: By J4R 9/14/95 $A M B$ 9/14/95

\begin{tabular}{|c|l|c|c|c|}
\hline Item No & Description & Sheet No. & Unit & Approx. Quantity \\
\hline 501 & $\begin{array}{l}\text { Furnish and Place Radon } \\
\text { Barrier Materials }\end{array}$ & 11 & C.Y. & (35,200 \\
\hline 502 & $\begin{array}{l}\text { Furnish and Place Frost } \\
\text { Protection Materials }\end{array}$ & 11 & C.Y. & 36,750 \\
\hline
\end{tabular}

\section{1 - Furnish and Place Radon Barrier Materials}

The measurement for this item will be by the cubic yard of material placed in fills.

The total quantity of Radon Barrier Materials is $35,200 \mathrm{CY}$ from Rof.

\section{$(26,400 c y) A$}

502 - Furnish and place Frost Protection Materials

The measurement for this item will be by the cubic yard of material placed in fills.

The total quantity of Frost Protection Materials is $36,750 \mathrm{CY}$ from Ref 2. 
Contract No. $3885-76$

Designed Checked
Sheet 12

File No.11-334-01

Date Date $01 / 28 / 9$

600-EROSION PROTECTION This entive sheet is

SUMMARY part of Rerol

\begin{tabular}{|c|l|c|c|c||}
\hline Item No & Description & Sheet No. & Unit & Approx. Quantity \\
\hline 601 & $\begin{array}{l}\text { Furnish and Place Riprap } \\
\text { Material, Type A }\end{array}$ & 12 & C.Y. & 3,300 \\
\hline 602 & $\begin{array}{l}\text { Furnish and Place Riprap } \\
\text { Material, Type B }\end{array}$ & 12 & C.Y. & 13,800 \\
\hline 603 & $\begin{array}{l}\text { Furnish and Place Riprap } \\
\text { Material, Type C }\end{array}$ & 12 & C.Y. & 8,400 \\
\hline 604 & $\begin{array}{l}\text { Furnish and Place Bedding } \\
\text { Material }\end{array}$ & 12 & C.Y. & 11,100 \\
\hline
\end{tabular}

601 - Furnish and Place Riprap Material, Type A

The measurement for this item will be by the cubic yard of material placed. The quantity of Riprap Material, Type A required on the embankment is 2,800 CY (from Ref 2) and $500 \mathrm{CY}$ on the finish grading (from Ref 4). Therefore total quantity is $\underline{3,300 \mathrm{CY}}$.

602 - Furnish and Place Riprap Material, Type B

The measurement for this item will be by the cubic yard of material placed. The quantity of Riprap Material, Type B required on the embankment is $12,900 \mathrm{CY}$ (from Ref 2) and $875 \mathrm{CY}$ on the finish grading (from Ref 4). Therefore total quantity is $13,800 \mathrm{CY}$.

603 - Furnish and Place Riprap Material, Type C

The measurement for this item will be by the cubic yard of material placed. The total quantity of Riprap Material, Type C is 8,400 CY from Ref 2.

604 - Furnish and Place Bedding Material

The measurement for this item will be by the cubic yard of material placed. The quantity of bedding Material required on the embankment is 10,400 CY (from Ref 2) and $700 \mathrm{CY}$ on the finish grading (from Ref 4). Therefore total quantity is $11,100 \mathrm{CY}$. 

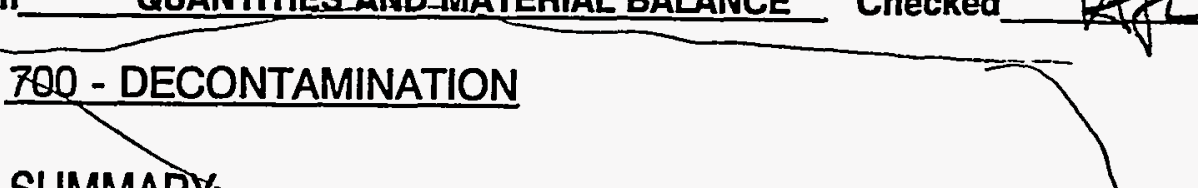

Date

$04 / 23 / 93$

Date $4 / 30 / 93$

SUMMARY

\begin{tabular}{|c|l|r|c|c|}
\hline Item No & Description & Sheet-No. & Unit & Approx. Quantity \\
\hline 701 & UC, NC and BC Sites & $\begin{array}{r}12 \\
13\end{array}$ & L.S. & N/A \\
& Decontamination Facilities & 13 & \\
\hline
\end{tabular}

701 - UC, NC and-BC Sites Decontamination Facilities

The subcontractor shall furnish, install, operate, and maintain decontamination facilities. This item is a lump sum and thus will not be estimated in this calculation.

Rev 02 By: ISR 8/10/94

$$
\text { AMB } 9 / 14 / 95
$$


Project

UMTRA-SRK

Feature

BID SCHEDULE

Item
Contract No. $3885-76$

Designed

Checked
JSR

$A M B$

800 - SITE RESTORATION

SUMMARY

This entire sheet is

Sheet 14

File No.11-334-01

Date

Date $01 / 28 / 94$

42879

Rev 02 By:JSR 8/10/94 AMB $9 / 14 / 95$

\begin{tabular}{|c|l|c|c|c||}
\hline Item No & Description & Sheet No. & Unit & Approx. Quantity \\
\hline 801 & $\begin{array}{l}\text { Common Fill from Stock pile } \\
\text { for Finish Grading of BC Site }\end{array}$ & 14 & C.Y. & $\begin{array}{r}132,100 \\
84,100\end{array}$ \\
\hline 802 & $\begin{array}{l}\text { Common Fill for Finish } \\
\text { Grading at UC and NC sites }\end{array}$ & 14 & C.Y. & 61,800 \\
\hline 803 & Seeding & 14 & acre & 211 \\
\hline
\end{tabular}

801 - Common Fill from Stock Pile for Finish Grading of BC Site

The measurement for this item will be by the cubic yard of material placed for finish grading from stock pile at BC site. The quantily roquired for ill is - 177,000-GY and

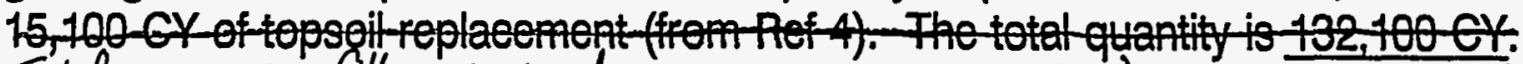
Total common fill required is $117,000 \mathrm{Cy}$. (Ref 4). 48,000 Cy will be directly placed from the excavation. Therefore common fill from stockpile is $64,000<y$.

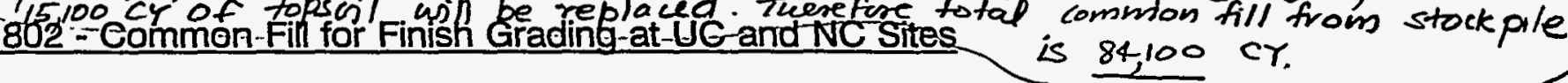

The measurement for this item will be by the cubic yard of material placed for finish grading at $\mathrm{UC}$ and NC sites. The quantities of fill required are $23,500 \mathrm{CY}$ for grading and $20,700 \mathrm{CY}$ for backfilling at UC site and $11,100 \mathrm{CY}$ for grading and 6,500 cy for backfilling at NC Site(from Ref 4). Therefore total quantity of fill is $61,800 \mathrm{CY}$.

\section{3 - Seeding}

This item consists of seeding at UC, NC, BC sites and Borrow sites which is measured in acres. The calculated area of seeding is 58 acres for UC site, 27 acres for NC site, 15 acres for BC site, 13 acres for BC stock pile and 98 acres for borrow sites from Ref 4 . Therefore total seeding area is 211 acres. 
MORRISON KNUDSEN CORPORATION

ENVRONMENTAL SERVICES GROUP

Project UMTRA-SRK

Feature

Item
Contract No. 3885-76

Designed JSR

Checked
Sheet $\quad 15$

File No.11-334-01

Date $\frac{\frac{01 / 28 / 94}{1+28 / 9 / 4}}{\text { Date }}$

is is: By JSR $9 / 1 / 4125$

900 - FENCING

This entire sheet is AMB 9 /44/195

SUMMARY part of Red of

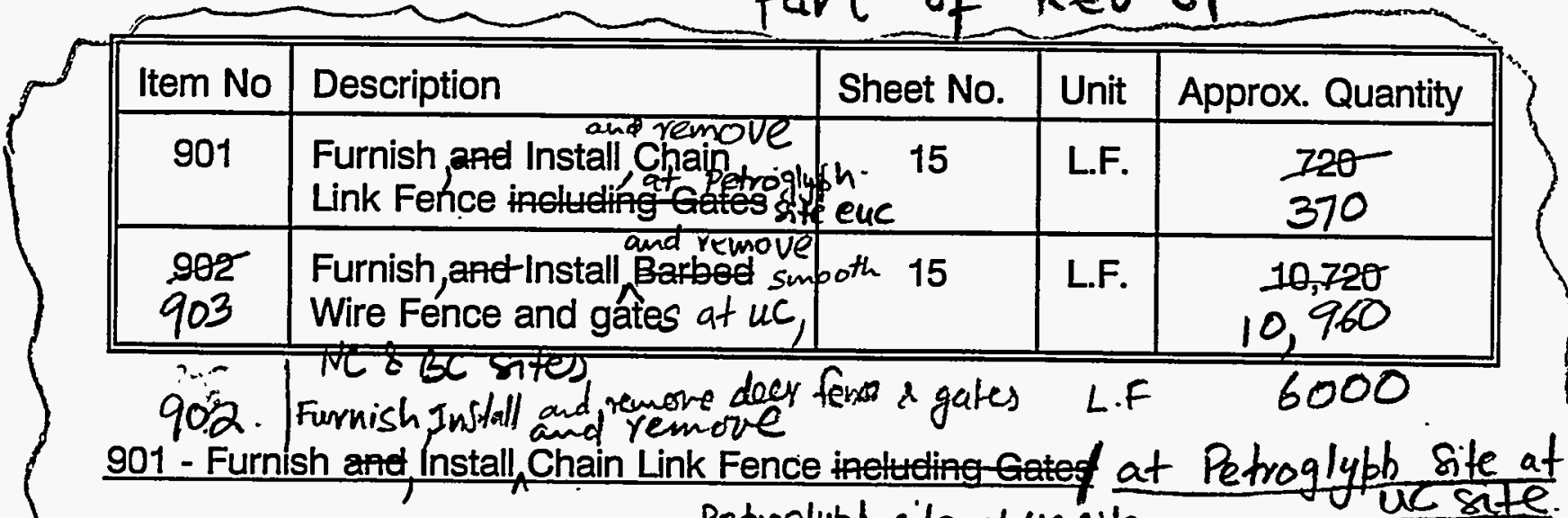
Petroglyph site at un site

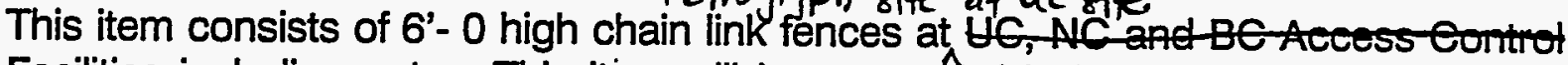

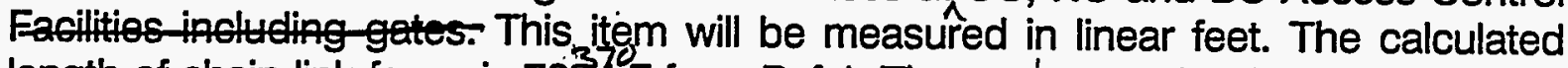
length of chain link fence is $723 \mathrm{ZF}$ from Ref 1 . Thereare-40

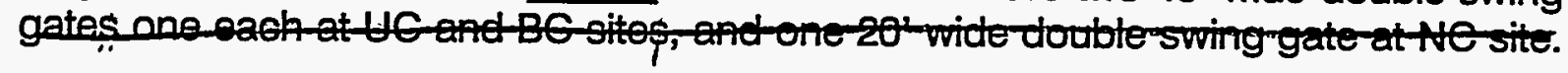

and remove smooth

903 - Furnish Install, Wire Fence and Gates

This item consists of 3'- B high barbed wire fences at UC, NC and BC sites and gates

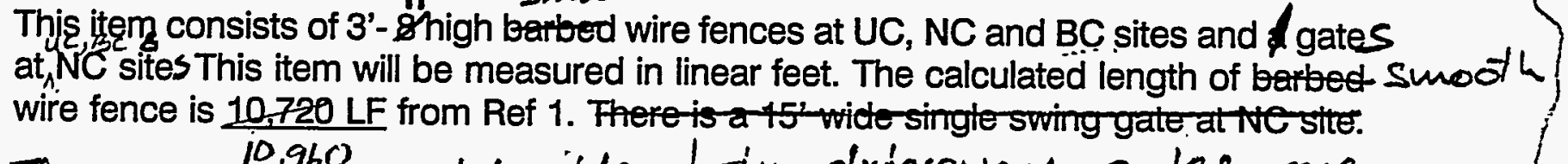
There are two $40^{\prime}$ wide twin driveway gates, one each at UC \& BC sites and one $20^{\circ}$ wide twin driveway gate at NC site.

902 Furnish, install and remove Deer Fence and Gates at $U C, N C$ and $B C$ sites.

This item consists of deer fences around retention basins in UC, NC and BC sites. The calculated length of deer fence is $6000 \mathrm{ft}$ from Ref 1 .

2

F:\JSR\SRK\BID.TXT 